\title{
COMPUTER SIMULATION OF SEDIMENTATION IN
} MEANDERING STREAMS

\author{
John S. Bridge
}

A Thesis Submitted for the Degree of PhD at the University of St Andrews

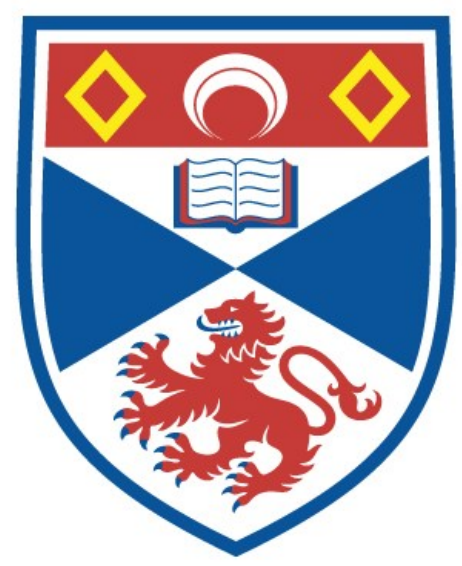

1973

Full metadata for this item is available in St Andrews Research Repository at:

http://research-repository.st-andrews.ac.uk/

Please use this identifier to cite or link to this item: http://hdl.handle.net/10023/15277

This item is protected by original copyright 
COMPUTER SIMULATION OF SEDIMENTATION IN MEANDERING STREAMS

by

JOHN S. BRIDGE

Ph.D. thesis

5t. Andrews, June 1973.

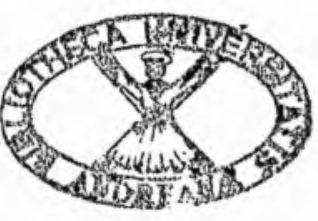


All rights reserved

INFORMATION TO ALL USERS

The quality of this reproduction is dependent upon the quality of the copy submitted.

In the unlikely event that the author did not send a complete manuscript and there are missing pages, these will be noted. Also, if material had to be removed, a note will indicate the deletion.

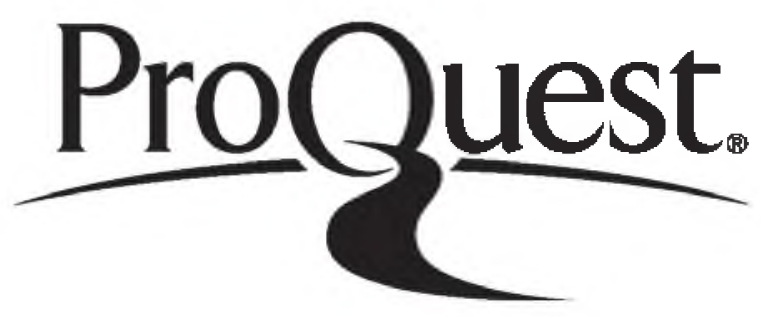

ProQuest 10171085

Published by ProQuest LLC (2017). Copyright of the Dissertation is held by the Author.

All rights reserved.

This work is protected against unauthorized copying under Title 17, United States Code Microform Edition (C) ProQuest LLC.

ProQuest LLC.

789 East Eisenhower Parkway

P.O. Box 1346

Ann Arbor, MI $48106-1346$ 
Th 8055 
I hereby certify that JOHN S. BRIDGE has been engaged in reseaxch for 9 terms at the University of $5 t$. Andrews, that he has fulfilled the conditions of Ordinance No. 12 and Resolution of the University Court, 1967, No. 1, and that he is qualified to submit the accompanying thesis in application fox the degree of Doctor of Philosophy.

I certify that the following thesis is of my own composition, that it is based on the results of research carried out by me, and that it has not previously been presented in application for a highex degrea. 
A $B S T R A C T$

A dynamic mathematical model has been constructed for the computer simulation of sedimentation in free meandering streams. The system is defined in terms of form and process, and component mathematical models (with mainly deterministic, but also probabilistic, characteristics) are formulated for the prediction of the following aspects of the system for a given physical situation and a single time increment: (1) The characteristics of the plan form of free meanders; (2) The movement of meanders in plan, and definition of cross sections across the meander in which erosion and deposition are considered in detail; (3) The hydraulio properties of the channel and the erosional and depositional activity within the channel as defined in specific cross sections; (4) Whether neck or chute cut off will occur;

(5) A relative measure of the discharge during seasonal high water periods, which is used in (3) and (4); (5) Aggradation. The 1imitations, qualifications and validity of the component mathematical models are discussed during their development, as is the input required. The overall model has been translated into a FORTRAN IV computer program and a set of experiments with selected input parameters has been performed. The results and their implications are rully documented and compared qualitatively with recent and ancient fluviatile sedimentation.

The shape cf simulated pointbar sediments, as controlled by channel migration over floodplains of variable sediment type, agrees broadly with the natural situation. Sheet deposjts cannot be simulated because large-scale meandex-belt movements are not accounted for; this also inhibits generation of thick sequences of alluvial sediments. When chamel migration is combined with a corstant ageradation rate the model predicts a genera.1 slope (relative to the land surface) of facies boundaries ard scoured. 
basal surfaces upward in the direction of channel movement. If aggradation sufficiently increases the thickness of fine grained overbank material, there is a channel stabilisation effect.

Epsiton cross-stratification, which represents the shape o: a pointbar surface before falling-stage deposition (lateral and vertical), may be picked out in the simulated sediments. The epsilon unit thickness is that measured rrom bankfull stage down to the lowest channel position existing prior to deposition.

The model records the characteristic fining upwards of grain sizes in the pointbar, and the systematic distribution of sedimentary structures. Channel migration combined with seasonal scouring and filling across the channel produces a characteristic relief in the basal scoured surfaces and the grain size and sedimentary structure boundaries. A related lensing and interfingexing of grain size and sedimentary structure facies may also present. The model also records large-scale lateral changes in grain size and sedjmentary structure associated with changes in the shape of developing meanders.

It is shown that a complete sequence of pointbar sediments capped by overbank sediments would rarely be preserved in the moving-phase situation. Such preservation only becomes likely when an aggrading section lies out of range of an eroding channel for a considerably longer time span than it takes a meander to move one half-wavelength downvalley. Deep channel-scours have a higher preservation potential than contemporary shallower ones. Where appropriate field data exist the model can be used il the more accurate recognition of ancient fluviatile sediments. Inferences may be made about the erosion-deposition processes operating in the ancient channel system, and the geometry and loydraulics of the system can be alluded to. A representative application of the model to the quantitative interpretation of an ancient pointbar deposit is illustrated. There is reasonable 
agrement between the natural and the simulated deposits, and a broad quantitative picture of the palaeoenvironment of sedimentation is obtained. 


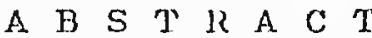

A dynamic mathematical model has been constructed for the computer simulation of sedimentation in free meandering streams. The system is defined in terms of form and process, and component mathematical models (with mainly deterministic, but also probabilistic, characteristics) are formulated for the prediction of the following aspects of the system for a given physical situation and a single time increment: (1) The characteristics of the plan form of free meanders; (2) The movement of meanders in plan, and definition of cross sections across the meander in which erosion and deposition are considered in detail; (3) The hydraulic properties of the channel and the erosional and depositional activity within the channel as defined in specific cross sections; (4) Whether neck or chute cut off will occur; (5) A relative measure of the discharge during seasonal high water periods, which is used in (3) and (4); (5) Aggradation. The limitations, qualifications and validity of the component mathematical models are discussed during their development, as is the input required.

The overal1 model has been translated into a FORTRAN IV computer program and a set of experiments with selected input parameters has been performed. The results and their implications are fully documented and compared qualitatively with recent and ancient fluviatile sedimentation.

The shape of simulated pointbar sediments, as controlled by channel migration over floodplains of variable sediment type, agrees broadly with the natural situation. Sheet deposits cannot be simulated because large-scale meander-belt movements are not accounted for; this also inhibits generation of thick sequences of alluvial sediments. When channel migration is combined with a constant aggradation rate the model predicts a general slope (relative to the land surface) of facies boundaries and scoured 
basal. surfaces upward in the direction of channel movement. If aggradation sufficiently increases the thickness of rine grained overbank material, there is a channel stabilisation effect.

Epsilon cross-stratification, which represents the shape of a pointbar surface before falling-stage deposition (lateral and vertical), may be picked out in the simulated sediments. The epsilon unit thickness is that measured rrom bankfull stage down to the lowest channel position existing prior to deposition.

The model records the characteristic fining upwards of grain sizes in the pointbar, and the systematic distribution of sedimentary structures. Channel migration combined with seasonal scouring and filling across the channel produces a characteristic relief in the basal scoured surfaces and the grain size and sedimentary structure boundaries. A related lensing and interfingering of grain size and sedimentary structure facies may also be present. The model also records laxge-scale lateral changes in grain size and sedimentary structure associated with changes in the shape of developing meanders.

It is shown that a complete sequence of pointbar sediments capped by overbank sediments would rarely be preserved in the moving-phase situation. Such preservation only becomes 1ikely when an aggrading section lies out of range of an eroding channel for a considerably longex time span than it takes a meander to move one half-wavelength downvalley. Deep channel-scours have a higher preservation potential than contemporary shallower ones.

Where appropriate field data exist the model can be used in the more accurate recognition of ancient fluviatile sediments. Inferences may be made about the erosion-deposition processes operating in the ancient channel system, and the geometry and hydraulics of the system can be aliuded to. A representative application of the model to the quantitative interpretation of an ancient pointbar deposit is illustrated. There is reasonable 
agreement between the natural and the simulated deposits, and a broad quantitative picture of the palaeoenvironment of sedimentation is obtained. 


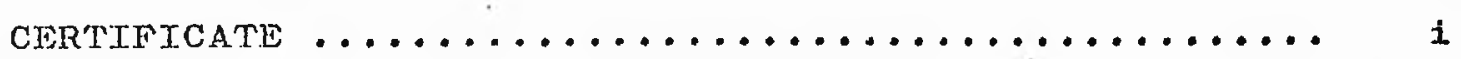

ABSTRACT ................................... ii

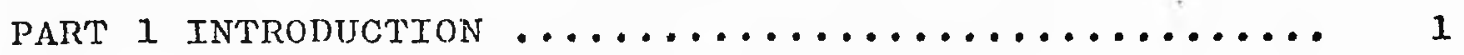

PART 2 DEVELOPMENT OF MUTENATICAL MODEL

1. INTRODUCTION: ENERGY DISTRIBUTION IN RIVER

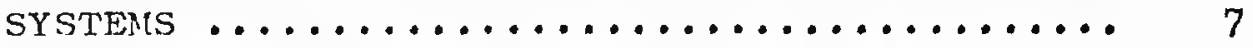

2. PLANIMETRIC GJOMETRY OE MEANDERS .......... 10

2.1. Theory of minimum variance .......... 10

2.2. Geometric characteristics of 'sine-generated' curve ........................ 13

2.3. Validity of 'sine-generated' curve .... 17

2.4. Initial input required by planimetric geometry mode1 ................... 17

2.4.1. Floodplain sediments ......... 17

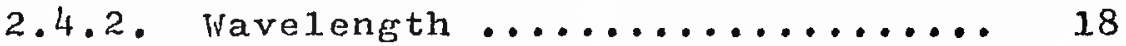

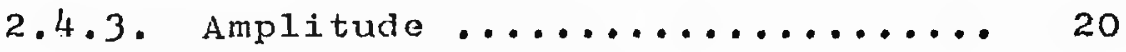

2.4 .4$, sinuosity .................. 22

3. MEANDERS IN A DYNAMIC FRAMEUORK ........... 25

4. CROSS SECTION DEFINITION ................ 27

5. MOJEL FOR DEPOSITION ON THE POINT BAR ..... 33

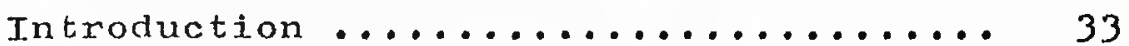

5.1. Qualitative features of a system involving lateral deposition ......... 33

5.2. Shape of cross profile ............. 35

5.3. Hydraulic properties of the system .... 36

5.4. Variation of grain size over the cross profilo ....................... 43

5.5. Variation of bed form and internal structure over the cross profile ...... 46

5.5.1. Allen's model ............. 47

5.5.2. Alternative mode1s .......... 51

5.5.3. Alternative model no. $1 \ldots . . . .52$

5.5 .4$, Alternative nodel no. $2 \ldots . . . .53$ 
5.5.5. Alternative mode1 no. $3 \ldots \ldots . .56$

5.5.6. Other alternative models ..... 66

5.6. Discussion of input parameters required in point bar mode1 ............... 68

5.6.1. Channel width and depth ...... 68

5.6.2. Mear radius of curvature, 1ongitudinal water surrace slope, and valley slope .......... 70

5.6.3. Resistance coefficients ...... 71

5.6.4. Fluid viscosity, fluid and sediment density ........... 74

5.6.5. Kennedy j factor .......... 75

5.7. Ixperiments to show variation of grain size and sedimentaxy structure with different input parameters and alternative bed-form models proposed ...... 76

5.8. Validity and limitations of point bar sedimentation mode1 ............... 79

6. MODLL FOR BANK EROSTON ............... 83

6.1. Factors affecting nature and rate of

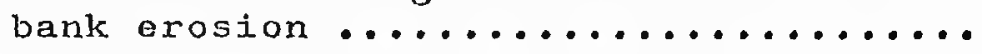

6.2. Mathematical model .............. 86

6.3. Validity of models proposed ......... 88

6.4 . Input ........................ 89

7. BANK RECESSION AND BAR GROITH ............ 91

8. EROSION AND DEPOSITION DURING HIGH WATER

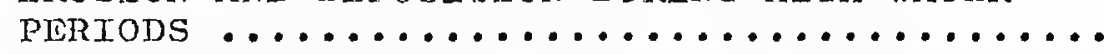

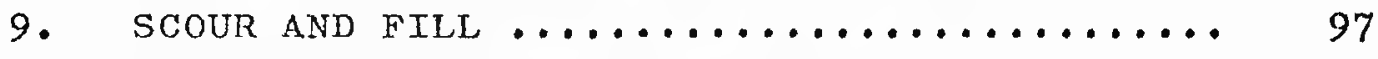

9.1. Preliminary discussion ............. 97

9.2. Mathematical model for scour depth ... 100

9.3. Deposition on falling stages ........ 102

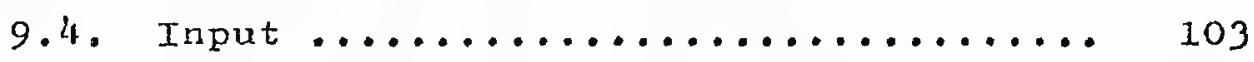

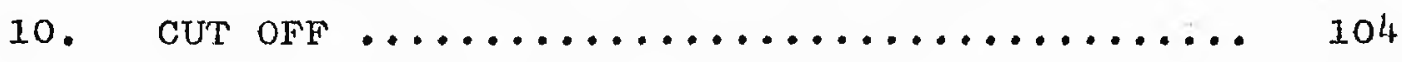

10.1. Model for chute cut-off ............ 104

10.2. Model for neck cut-off ............ 105

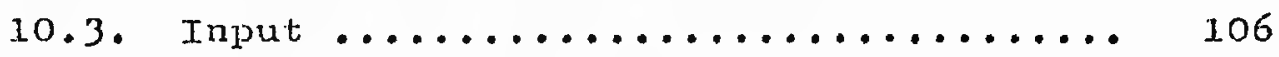


11. FLOND PERIOD VOLUME ................ 108

Introduction .................... 108

11.1. Sequential generation of stream-

flow data................... 108

11.2. Mathematical mode1 of hydrologic time series .................... 109

11.3. Input and experinentation with the

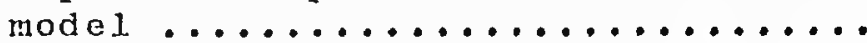

12. CONStRUCTION OF FLOODPLAINS ............ 115

12.1. Overbank deposition ........... 115

12.2. Aggradation ................. 118

PART 3 TIE COMPUTER PROGRAM

13. GTELRAL RLMARKS ................... 125

14. DESCRIPTION OF MAIN PROGRAMS AND SUB-

ROUTTRES ....................

14.1. Main program (no disc) ......... 128

14.2. Main program (using disc storage) . 136

14.3. Subroutine BAR (with entry point

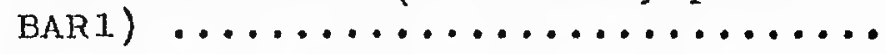

14.4. Subroutine MEANDR (with entry point MIEAND1) .................... 139

14.5. Subroutine SIMINT (and FUNC2) ..... 141

14.6. Subroutine RANSAM ............. 14.

14.7. Subroutine NEWRAP ............. 142

14.8. Subroutines RNDMIN and RNDM ...... 143

14.9. Subroutines PLOT and CHAR ........ 143

15. INPUT REQUTRTMENTS AND PROGRAM NODIFICATION

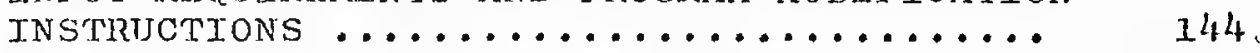

16. SAMPL RUN ........................ 154

PART 4. EXPERIMENTATION AND RESULTS

INTRODUCTTON ........................... 159

17. EXPERINENT 1 - MEANDFRS IN DYNAMIC EQUTLIBIRIUM .......................

18. EXPERIMENT 2 - MEANDERS IN DYNAMIC EQUILIBRIUM .........................

19. IEXPERIMENT 4 - DEVIELOPING MEANDERS ...... 171 
20. EXPERIMENT 5 - DEVELOPING MEANDIERS ........ 175 PART 5 DISCUSSION AND CONCLUSION

21. GENERAL REMARKS ................... 177

22. COMPARISON WITH MODERN FREE MEANDIERING

STREAM DEPOSITS .................... 179

22.1. Shape of pointbar deposits ........ 179

22.2. Epsilon cross-stratification ....... 181

22.3. Distribution of grain size and sedimentary structure ................. 182

22.4. Times taken to cut off .......... 186

23. COMPARISON WITH ANCIENT FLUVIATILE COARSE

MEMBERS .......................... 187

24. PRISERVATION OF POINT BAR SEDIMENTS ....... 191

25. APPLICATION OF MODEL TO QUANTITATIVE INTERPRETATION OF ANCIINT FLUVIATILE COARSE

MEMBERS ............................ 192

26. CONCLUDING REMARKS .................. 196

ACKNOTILEGEMNATS . . . . . . . . . . . . . . . . . . . . . 198

LIST OF SYMBOLS USED IN MATHEMATICAL MODHL ........ 199

REFERENCES CITED ......................... 206

APPENDTX 1 - MATHENATICAL METHODS ............ A1

A1.1. Newton-Raphson iterative formula ........ A1

A1.2. simpson's rule .................. A1.

A1.3. Generation of random samples from specified theoretical distributions ........... Az

APPENDIX 2 - STATISTICAL CURVE AND SURFACE FITSING.. A5

A2.1. polynomial regression .............. A5

A2.2. Polynomial surface fitting ........... $\Lambda 5$

APPENDIX 3 - DATA DECK SET UP FOR EXPJEIMENTS ..... A6 


\section{PART ONE,}

I N $\quad T \quad R \quad O \quad D \quad U \quad C \quad T \quad I \quad O N$ 


\section{INTRODUCTION}

It is well known that meandering streams flowing between exodible banks sweep across their floodplains as their loops migrate downvaliey and across the mean downvalley direction. Such migration Involves erosion of the outer, steeply sloping bank of the inflected channel and concomitant accumulation of layers of sediment on the Inner gently sloping bank. Such deposition lateral to the local current direction is sensibly termed lateral deposition. Lateral deposition is also important in tidal flats and estuaries, and is found to occur in comparatively straight channels with sinuous talwegs as well as those sinuous enough to be arbitrarily termed meandering. In all these cases the channel, or talweg, swings from one side to the other of the mean direction of fluid motion primarily because, at the high Reynolds numbers involved, the flow is unstable to centrifugal accelerations and is unable to assume a rectilinear path (Allen, 1970a).

It 1 s known that lateral sedimentation leads, in both fluvial and tidal situations, to a sequence of deposits marked by systematic vertical changes of grain size and sedimentary structure. This knowledge has been obtained from studies of ancient strata as woll as modern sedimonts. As regards fluviat1le doposits, vertical patterning of grain size and sedimentary structure has an important place in the familiar concept of the fining upwards sodimentary cycle, where the lower, coarse member of the cycle is known or thought to have accumulated through processes of lateral deposition. The finer members of such cycles are thought to have accumulated dominantly by processes of overbank deposition. 
As well as channel movements by erosion and lateral deposition in sinuous conduits, large-scale movements of a substantially discontinuous nature may occur in the form of cut-orf (the abandonment of all or part of a meander loop) or avulsion (the abandonment and relocation of a section of the meander belt). When all these channel movements are combined with a graduaj continuing net deposition within the floodplain, a complicated spatial distribution of lateral deposits, with a certain amount of overbank and channel fill deposits, will result within the preserved thickness of alluvium.

Although fining-upwards cyclothems have been widely recognised, our knowledge of them is still rather broad and unsupported by the detailed and comparative studies necessary for their full and correct interpretation. Several models of fluviatile sedimentation have been published and have formed a useful starting point in the recognition and interpretation of fining-upwards cycles (A11en, 1963c, 1964, 1965a, 1970a,b, 1971; Beerbower, 1964; Moody-Stuart, 1966; Potter, 1967; Potter and Blakely, 1967; Visher, 1965a,b). Most of these models are graphic and qualitative, and more than one of them embodies concepts that are physically suspect or oversimplified. Furthermore, they tend to be heavily biased towards study of single lateral deposits instead of including channel and overbank deposits within a three dimensional body of alluvium. Allen's (1970a,b, 1971) model of lateral deposition is the first quantitative approach to the interpretation of fining-upwards coarse members and, although static in nature, employs principles that may be extended for use in simple dynamic mathematical mode1s.

The purpose of this study is to develop a dynamic mathematical model for computer simulation of the nature of erosion 
and deposition in free meandering streams. It is anticipated that such a study will enable more accurate recognition and quantitativo interpretation of fining-upwards cycles than has hitherto been possible, as well as giving further insight into the processes involved in the natural system. The only previous attempt to simulate fluviatile sediments was by potter and Blakely (1967). This study made extensive use of Markov processes to generate stratigraphic successions, and the starting point was essentially the transition matrlx. Unfortunately it is of little use in the physical interpretation of ancient sediments, as it doesn't examine the processes at work.

The free meandering system under consideration is an open (in that it is being continually affected by external factors) system that is tending towards a steady state, or dynamic equilibrium (Leopold et al, 1964). The system must be arbitrarily defined by specifying its boundaries, its components and the structure of the inter-relationships among the components. A hierarchy of systems can be seen to exist here, with lesser systems nested within the overall framework of the free meandering river system, which is itself nested in the overall river system, and so on. Because the natural system consists of an assemblage of parts that are inter-related in a complex manner, it must be simplified conceptually before $t$ t can be represented by a model. Dynamic simulation is the operation of the model system in such a way that the behaviour of the real system is reproduced to some degree as the model moves through time.

The most powexful and flexible way of representing a system is with mathematical models, however one danger in their use is that a formal appearance may lend an unwarranted credibility Basically devolopment of the simulation model has nocessitated the 
construction of component mathematical models, with mainly deterministic, but also probabilistic, characteristics, for the sequential prediction of various aspects of the system for a given physical situation and a single time increment. These are:-

1) The characteristics of the plan form of the meander within which erosion and lateral sedimentation are taking place.

2) The definition of sections across the meander in which erosion and deposition will be considered in detail, given modes and rates of meandex movement in plan.

3) The hydraulic properties of the channel and the erosional and depositional activity within the channel in the bend, as defined in specific cross sections.

4) Whethex neck or chute cut-off will occur.

5) A relative measure of the discharge during the seasonal high water periods, which is used in 3) and 4).

6) Long term depositional trends due to time persistent changes in the independent system variables, i.e. aggradation.

The system has been defined in detail in terms of form and process as the individual component mathematical models were developed, and their limitations, qualifications and validity are discussed, as is the input required. It will be seen that the simulation model emphasises lateral sedimentation. This is partly because of the lack of data sufficient to make up models for the complicated overbank erosional and depositional processes, and partly because of the greater importance of channel sedimentation compared with other modes of sedimentation and exosion within the system. Unfortunately most of the deterministic relations are empirical, and these are generally less versatile than theoretical ones. As the mechanisms of the processes must be well defined before they can be reduced to sets of algebraic equations and logic 
statements, the development of mathematical models leads to deep insight into the system, and it is interesting to look at the modes of sedimentation expected on the basis of the analysis.

$\Lambda$ computer program of the mathematical model has been composed so that the model's behaviour can be reproduced with speed and ease with the progression of time. The programming language FORTRAN IV has proved sufficiently versatile for representation of the mathematical model and output from the program can easily be displayed in the form of graphs, tables and cross sections, using the line-printer and digital graph-plotter peripherals.

A fully comprehensive set of experiments with the program is not possible by virtue of the number of input variables involved, however examination of the expected behaviour of the system helped in designing a representative set of experiments. In supplying input variables, many are dependent system variables which must be mutualiy compatible in accordance with the natural system. The ideal situation would, of course, be to supply information only on the independent variables and be able to simulate the expected sedimentation and erosion patterns; such i.s not possible at present.

The sat of experiments with selected input parameters has been fully documented. By matching the abundance of output with real-world observations the model can be evaluated and its ability to provide a useful analogue to the real system can be judged, in the light of approximating assumptions made in the mathenatical model and the computer program. Difficulties exist in obtaining the comparative data from the real system; even when available, data may be sparse or unsuitable in form. Comparative studies are therefore, by necessity, only qualitative at present and concentrate on the broader implications of the model. The 
preservation potential of point bar and overbank sediments is discussed in the light of the model and a representative application of the model to the quantitative interpretation of an ancient point-bax deposit is performed.

In conclusion, the overall validity of the computer simulation model and its usefulness in the understanding and prediction of sedimentation aspects in meandering streams is discussed and suggestions are put forward for future development. 
PART TWO

DEVELOPMENT OF MATHEMATICAL MODEL, 


\section{INTRODUCTION: ENERGY DISTRIBUTION IN RIVER SYSTEMS}

The river network as a whole is an open system tending towards a steady state (dynamic equilibrium) and within which several. hydraulically related factors are mutually interacting and adjusting - specifically velocity, depth, width, hydraulic resistance and slope. These dependent variables adjust to the constraints applied by the independent variables of the system, that is, the quantity and character of runoff and sediment, valley slope and geological nature of the drainage basin.

The observed relationship between the dependent and the independent variables has been described using emplrical equations of mean tendency, which are assumed to represent the channel form in dynamic equilibriun (e.g. Leopold and Maddock, 1953). A more desirable theoretical solution may be obtained by considering also the energy distribution in the system. From its headwaters to its mouth a natural river channel essentially represents a system in which potential energy provided by quantities of water at given elevations is converted to kinetic energy of the flowing water and dissipated in friction created at the boundaries (Leopold et al, 1964). In analysing the behaviour of the channel system, primary interest lies not in the total energy in the system, but rather in the way in which energy is distributed throughout the system. This emphasis upon the distribution of energy within the system is in general analogous to a consideration of the entropy of thermodynamic systems (Leopold and Langbein, 1962; Scheidegger, 1970). From one point of view entropy may be said to be a measure of the energy in a system available for external work. The greater the entropy the less energy is available for external work. The natural process represented by the flow of water from the head- 
waters to the mouth of a river channel is an irreversible process in which energy is transformed with an increase in entropy.

Analogy with the thermodymanics of systems in a steady gtate led Leopold and Langbein (1962) to consider the way In which energy might be distributed and dissipated in the river system. They postulated the tendency toward minimum total rate of work in the system which is the same as uniform distribution, and minimum variance, of power expenditure per unit length. As discharge increases downstream this would tend to make the longitudinal prof $₫$ e concave upwards. They further postulated a tendency toward uniform distribution of power expenditure per unit bed area throughout the system, which tends to straighten the profile. The observed channel form is a 'quasimequilibrium' state which, while fulfilling the usual hydraulic laws, represents the most probable state between these two opposine tendencies. These tendencies are pronoted by exosion, deposition, variation In bed form, and related internal adjustments to energy utilisation (Langbein and Leopold, 1964). Langbein (1964) further showed that the adjustment in the hydraulic variables necessary to fulfil the energy and hydraulic requirements entails minimurn variance among the components of stream power such that no single varjable absorbs a disproportionate share of the required variation.

Theoretical solutions to the hydraulic geometry of fluvial systems, based on the above postulates, therefore represent the most probable behaviour of natural rivers satisfying the basic hydraulic equations. They are a measure of central tendency, therefore mutual adjustments of all the variables in every river system will not be expected to be the same, because of 1ocal physical constraints. The theorotical solutions agree well with the empirical mean measures of hydraulic geometry. The relevant 
application of these concepts to river meanders can be seen in the next and subsequent sections. 


\section{PLANIMETRIC GEOMETRY OF MEANDERS}

\subsection{Theory of Minimum Variance}

Many authors have attempted to explain the processes

involved in meander formation. None of these approaches, summarised by Leopold and Wolman (1960), Allen (1968, 1971), and Yang (1971b), can be used to calculate the characteristics of meander geometry adequately. Although various phenomena, particularly helicoidal flow, are known to be important in shaping meanders, there aro many diverse effects involved. Although each of the individual effects is deterministic in itself their interactions are too numerous to treat in a deterministic way. These may, however be treated stochastically.

The striking similarity anong meandering channels of various sizes in different settings is a result of cortain geometric proportions apparently common to all. Based on a large number of rlume and river data, Leopold and wolman (1960) found a conststent correlation between meander length, 1 , channel width, $w$, and mean radius of curvature, $r_{m}$, that is

$$
1=10.9 \mathrm{w}^{1.01}=4.7 \mathrm{r}_{\mathrm{m}}^{0.98}
$$

Al1 terms used to describe meander goometry are defined in fig. 2.1. Assuming the exponents in the above equation to be unity they arrived at the relation

$$
x_{\mathrm{un}} / \mathrm{w}=2.3
$$

These approximate mean relationships were considered to a Ereat extent to be indopendent of bed and bank materials, and It was concluded that a general mechanical principle was responsible for the observed meander geometry. Bagnold (1960) found the value of $x_{\mathrm{m}} / w$ to be that at which flow resistance is a minimum within the channel, subgesting that some principle related to 


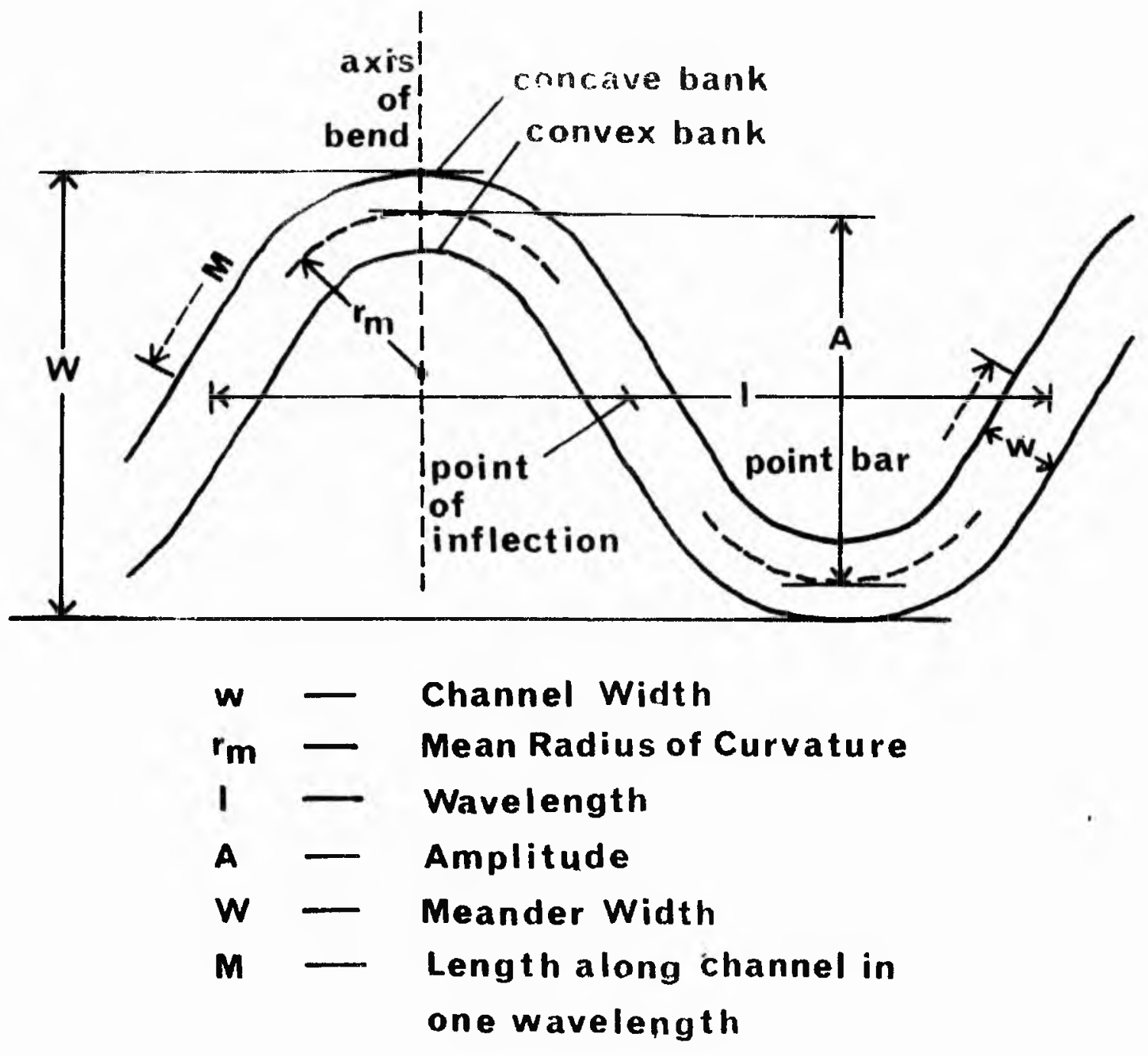

Fig. 2.1 Meander Geometry Definition. 
energy conservation operates in the meander mechanism.

In 1966 the previously developed theories of minimum variance were introduced to river meanders where 'meanders are the result of erosion-deposition processes tending toward the most stable form in which the variability of certain essential properties is minimised' (Leopold and Langbein, 2966; Langbein and Leopold, 1966). This minimisation involves the adjustment of the planimetric geometry and the hydraulic factors of depth, velocity and local slope.

In the context of the entixe river system a meandering segment, often but not always concentrated in the downstream rather than the upstream portions of the system, tends to provide a greater concavity by lengthening the downstream portion of the profile. Total work in the system is minimised therefore bocause, by increasing the concavity of the profile, the product of discharge and slope becomes more uniform along a stream that increases in flow downstream.

In the local context of a given sogment of channel the average slope of the channel is fixed by the relation of that segment to the whole profile. Any change in the channel must maintain that average slope. Between any two points on the valley floor, however, a variety of paths are possible, any of which would maintain the same slope and thus the same 1ength. This path is defined by a random walk model as follows.

A river has a finite probability, p, to deviate by an angle, $\Delta \phi$, from its previous direction in progressing an elemental distance, $\Delta s$, along its path. The probability distribution as a function of deviation angle is assumed normal. This has since been confirmed by Thakur and Schoidegger (1968). The actual meandex path corresponds to the most probable river path proceeding between two points $A$ and $B$, if the direction of 


$$
\text { सE }
$$


flow at point $A$ and the length of the path between $A$ and $B$ is fixed, and the probability of a charge in direction is given by the probability distribution above. This formulation of the problem is identical to that of a class of random walk problems for which solutions have been derived (Von Sche1ling, 1951, 1964). The exact solution is an elliptical integral but a sufficiently accurate approximation states that the most probable geometry for a river is one in which the angular direction of the channel at any point with respect to the mean downvalley direction is a sine function of the distance measured along the channel. The resulting curve minimises the sum of squares of the changes in direction in each unit length.

The equation of the 'sine-generated' curve is

$$
\varnothing=\omega \sin \left(\frac{s}{M} 2 \pi\right) \text {, }
$$

where $\varnothing$ is the deviation angle from the mean downvalley direction, $\omega$ is the maximum value of $\phi, s$ is the distance along the path, and $M$ is the total path distance. The equation yields a meander shape typically present in regularly meandering rivers and flumes and has the characteristic that the ratio of meander length to mean radius of curvature is about 4.7 . A definition diagram, fig. 2.2, shows the terms used in equation (2.3) and in the discussion of its development.

Furthermore, field observations have shown that depth, velocity and slope (the components of stream powex) are adjusted so as to decrease the variance of shear and the friction factor compared to that of an otherwise comparable straight reach of the same river. This is manifested in the more uniform water surface slope at high stage in a meander, which signifies a more unfform expenditure of energy for each unit distance along the chanel, aftex a slight correction for differences in velocity head (see $f i g \cdot 5 \cdot 4)$ 
since theory and observation indicate that meanders achieve the minimum variance postulated, it follows that for channels in which alternating pools and riffles occur, neandering is the most probable form of channel geometry and thus is more stable geometry than a nonmeandering reach. This has been independently demonstrated by Yang (1971a,b,c). Also using the thermodynamic analogy, he shows that the development of meanders, along with pools and riffles, fulfills the requirements of a natural stream evolving towards an equilibrium condition ; that is, minimum rate of potential energy expenditure per unit mass of water along its course. It should be noted that minimum vaxiance adjustment describes the net river behaviour, not the processes.

\subsection{Geometric characteristics of 'sine-generated' curve}

Various geometric characteristics of the 'sine-generated' curve have been defined by Langbein and Leopold (1966). Inspection of equation (2.3) indicates that at a relative distance $s / M$ equal to $\frac{1}{2}$ and $1, \varnothing$ has a value of zero, or the chamnel is locally directed in the mean downvalley direction. At distance $s / M$ equal to $\frac{1}{4}$ and $\frac{3}{4}$ the walue of $\varnothing$ has $i t s$ largest value $\omega$. This js indicated in fig. 2.3a,b which shows the curve of equation (2.3) for $\omega=110^{\circ}$ and also a plot of $\varnothing$ as a function of rolative distance along the channel path. Furthermore, the distance between $b$ and $f$ is twice the distance between $c$ and $e$ measured alone the mean downvalley direction as well as along the channel path. The tangent to the sine function at any point is $\Delta \phi / \Delta s$ which is the reciprocal of the local mean radius of curvature of the meandex. The sine curve is nearly stralght as it crosses the zero axis in fig. 2.3b, therefore the radius of curvature is nearly constant in a meander bend over two portions 


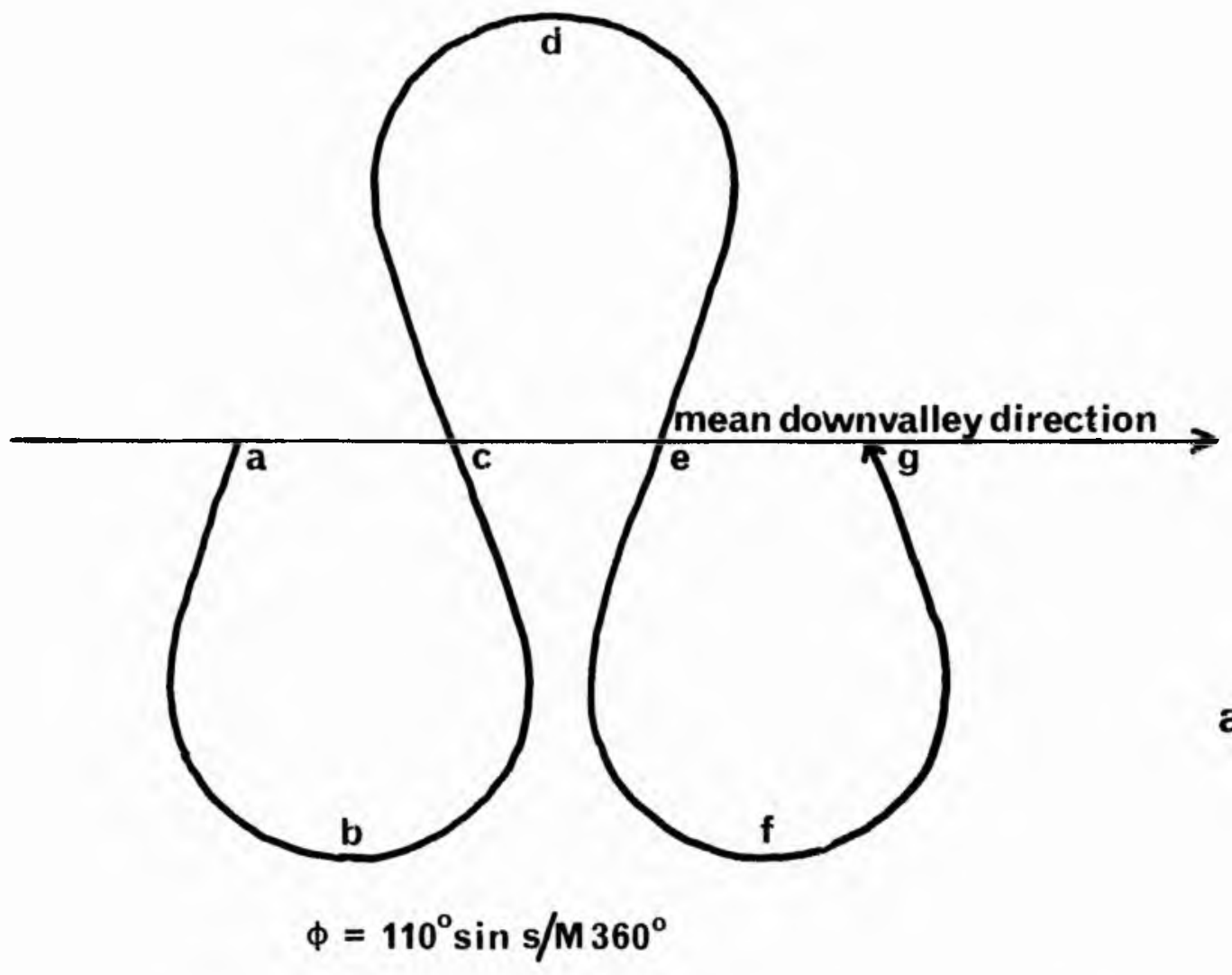

a

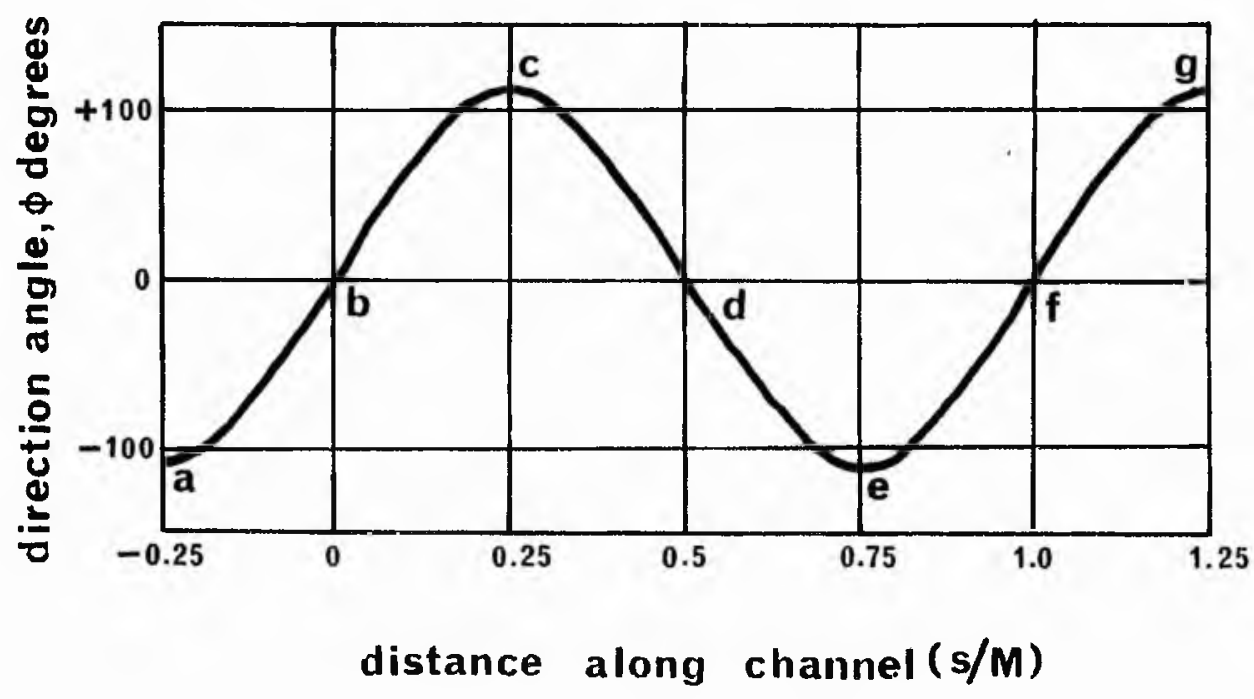

b

Fig.2.3 Sine-generated curve (a) and a plot of its direction angle as a function of distance along the channel path $(b)$. (after Langbein and Leopold,1966). 
covering fully a third of the length of each loop.

The angle $\omega$ is a unique function of sinuosity, sn, and an approximate algebraic expression is

$$
\omega=2.2 \sqrt{\frac{s n-1}{s n}} \text { or } \sin =4.84 /\left(4.84-\omega^{2}\right) \cdot(2.4)
$$

Average bend radius is related to wavelength and sinuosity. Defined as before, as the average over the $1 / 6$ of channel length for which $\varnothing$ is nearly linearly related to channel distance, bend radius is

$$
x_{\mathrm{m}}=\frac{1 / 6 \mathrm{M}}{\Delta \varnothing} \text {. }
$$

since $\varnothing$ ranges from $+0.5 \omega$ to $-0.5 \omega$ over this near 1 inear range, $\Delta \phi=\omega . \quad \Lambda s M=s n \cdot 1$, after substituting for $\omega$, we get

$$
r_{m}=\frac{1}{13} \frac{\left(\operatorname{sn}^{3 / 2}\right)}{(\sqrt{s n-1})} .
$$

Differentiating $r_{n}$ with respect to sn gives

$$
\frac{\mathrm{dr} m}{\mathrm{dsn}}=\frac{1}{13}\left[\frac{3}{2} \operatorname{sn} \sqrt{ }{ }^{2}(\sin \cdots 1)^{\frac{1}{2}}-\frac{1}{2} \operatorname{sn}^{3 / 2}(\operatorname{sn}-1)^{-3 / 2}\right] .
$$

At a turning point of the function $\mathrm{dr}_{\mathrm{m}} / \mathrm{d} s \mathrm{n}=0$, therefore

which reduces to

$$
\frac{3}{2}\left[\frac{s n}{\operatorname{sn}-1}\right]^{\frac{1}{2}}=\frac{1}{2}\left[\frac{s n}{\sin -1}\right]^{3 / 2},
$$

$$
(2 s n-3)(4 s n+3)=0
$$

The turning points are therefore at $s n=1.5$ and $-3 / 4$. The latter has no physical meaning, however, by inspection also of fig. 2.4 it can be seen that $x_{m}$ has a minimum value at the turning point snz1.5. The significance of this fact will become apparent latex. other relations can also be derived that are important in the development of the model. By inspection of fig. 2.2, the following may be written, 


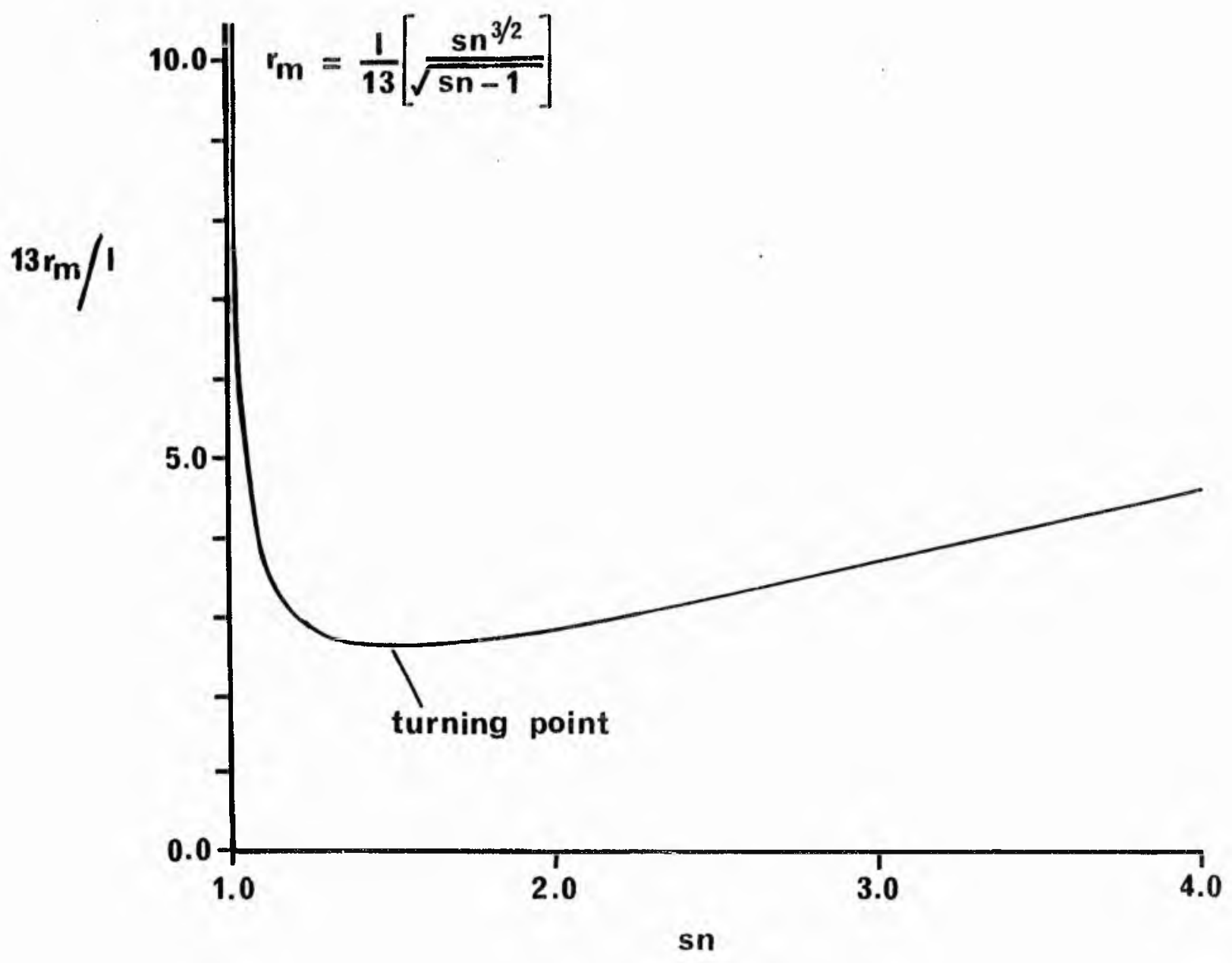

Fig. 2.4 Plot of sinuosity (sn) against dimensionless radius of curvature parameter. 


$$
x=\sum_{n \rightarrow \infty} \sum_{r=1}^{n} \Delta s \cdot \cos \phi_{n}
$$

and

$$
\lim _{\mathrm{n} \rightarrow \infty} \sum_{r=1}^{\mathrm{n}} \Delta \mathrm{s} \cdot \sin \phi_{\mathrm{r}} .
$$

This means that if a particular length of curve is divided into $n$ equal parts, then as $n$ tends to infinity, the projected length of the curve along the axis of abscissas, $\mathrm{x}$, or the ordinate axis, $\mathrm{z}$, is given by the sums of the product of arc length, $\Delta s$, and a cosine or sine function of the direction angle for each interval. In terms of integral calculus

$x=\int_{0}^{\varnothing} \cos \phi \mathrm{d} s$ and $z=\int_{0}^{\phi} \sin \phi \mathrm{d} s$

To integrate these functions, ds must be expressed in terms of $\varnothing$. Rearranging equation (2.3), we get

$$
s=\frac{M}{2 \pi} \sin \quad-1 \quad(\varrho) \text {. }
$$

Differentiating with respect to $\varnothing$,

$$
d s=\frac{M}{2 \pi} \frac{d \emptyset}{\sqrt{\omega^{2}-\emptyset^{2}}}
$$

Finally, substituting for $M$, the integrals may be written as,

$$
x=\frac{\operatorname{sn} .1}{2 \pi} \int_{0}^{\phi_{0}} \frac{\cos \phi a \phi}{\sqrt{\omega^{2}-\phi^{2}}}
$$

and

$$
z=\frac{\operatorname{sn} \cdot 1}{2 \pi} \int_{0}^{\phi} \frac{\sin \phi \mathrm{d} \phi}{\sqrt{\omega^{2}-\phi^{2}}}
$$

It is now easy to find expressions for lneander amplitude, $\Lambda$, and the width of the meander neck, GAP, measured to channe1 centre 1ines. The last parameter is of course physically meaning less if $\omega$ is less than $\pi / 2$. The expressions axe 


$$
A=\frac{\sin .1}{\pi} \int_{0}^{\omega} \frac{\sin \phi d \phi}{\sqrt{\omega^{2}-\phi^{2}}}
$$

and

$$
\operatorname{GAP}=1\left\{1-\frac{\sin }{\pi}\right\} \int_{0}^{\pi / 2} \frac{\cos \phi \mathrm{d} \phi}{\sqrt{\omega^{2}-\phi^{2}}} .
$$

No analytical solutions are possible for these integrals and so approximate solutions are obtained numerically by simpson's rule (see appendix 1 and program specifications).

An expression for $\sin ($ hence $\omega)$ in terms of amplitude and wavelength was obtained from equation (2.10) by evaluating the integral numerical1y over a range of $\operatorname{sn}(1.1$ to 4.5$)$ and performing a polynomial regression analysis with sn as the dependent variable and the ratio $A / 1$. as the independent variable. The resulting best fit equation is

$\operatorname{sn}=0.96+0.34\left(\frac{\mathrm{A}}{1}\right)+1.67\left(\frac{\mathrm{A}}{\mathrm{I}}\right)^{2} \mathrm{k}=0.43\left(\frac{\mathrm{A}}{1}\right)^{3}$.

Full details of the analysis can be seen in appendix 2 . As will be seen from fig. 2.5 and the analysis of variance table in appendix 2 , the relation is almost linear, except for a small part of the curve at small values of $\mathrm{sn}$.

It can be seen from the above expresisions that by specifying any two of sinuosity, amplitude and wavelength, all of the other geometric parameters discussed can be derived. This is an important point in the analysis because, given only two geometric characteristics, much of the planimetric geometry can be defined a a definition which also implicitly specifies mutual internal adjustment of the dependent hydraulic variables to the independent system variables, according to the minimum variance theory previously outlined. A discussion of the dependence of wavelength, amplitude and sinuosity on the independent variables will follow later for the purposes of input to the model. 


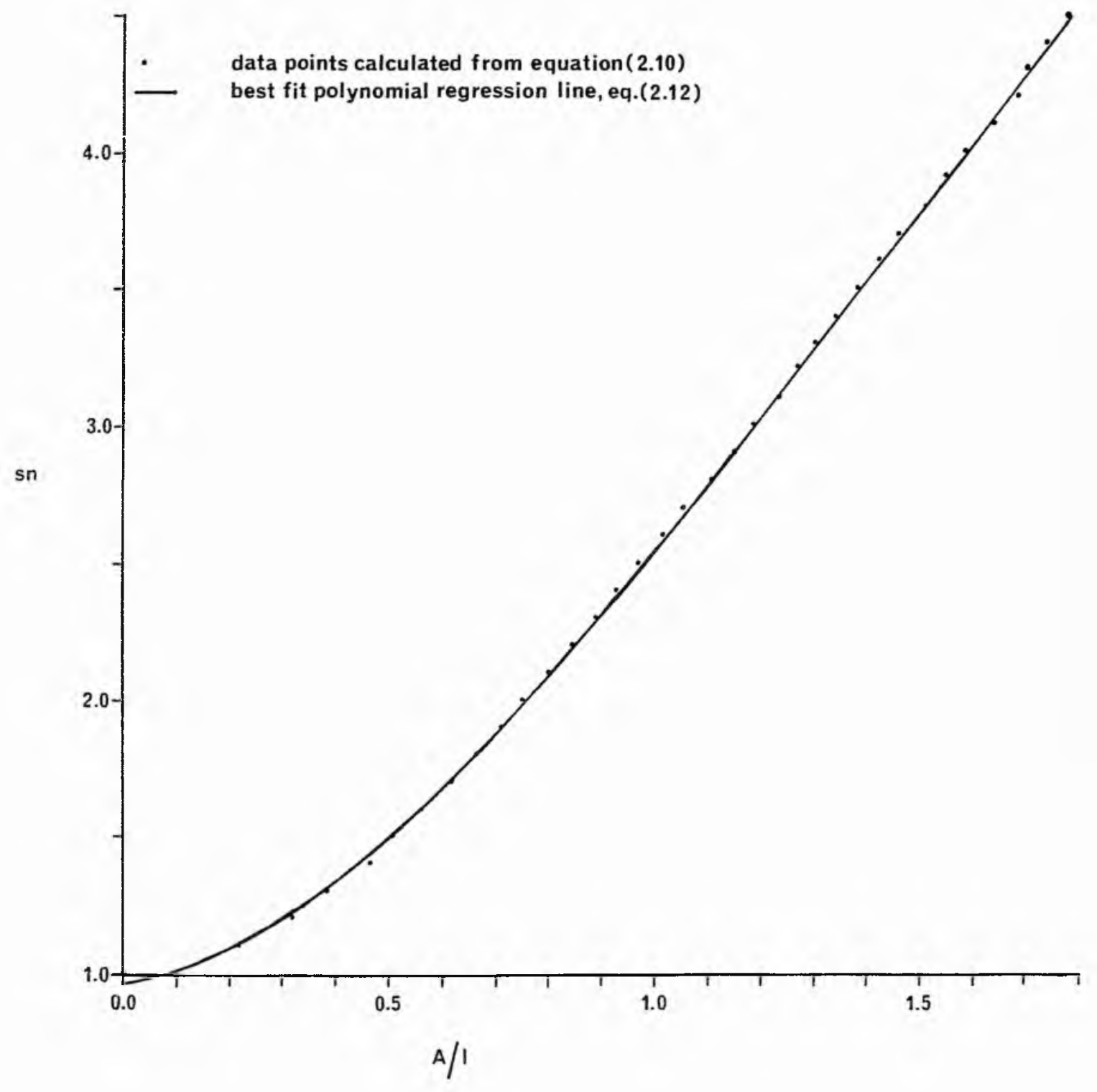

Fig.2.5 Plot of polynomial regression between sinuosity(sn) and ratio of amplitude over wavelength ( $A / I)$. 


\subsection{Validity of the sine-generated curve}

Schoidogger $(1967,1970)$ points out that the derivation of the sine genexated curve is not in conformity with the commonly accepted principles of statistical mechanics. Usually the expected value of an observable is taken as its average over all the configurations of the ensemble in question, whereas in Langbein and Leopold's theory the characteristic pattern is taken as that pattern of the observable which occurs in the most probable configuration of the ensemble.

The curves are in general too regular to describe a whole system of river meanders, and the reason is sought in the fact that the most frequent are not the expected randon ralks. Moro recent studies have used models to generate constrained random walks whose expected paths cannot be distinguished statistically from the paths of natural meandering streams (Surkan and Van Kan, 1969; Thakur and Schoidogger, 1970; Ghosh and Scheidegger, 1971).

A1though these studies may be more statistically rigourous and more authentic than langbein and Leopold's model when applied to meandering reaches in general, the sine-generated curve has been shown; using ompirical and theoretical considerations, to underlie the stable form of meandexs and describes regular forms very we11. $\Lambda$ s the present study may only treat regular shapes with definable goometric charactexistics the sine-generated curve has been adopted, and will be used tn the model to represent the channel centre line.

2.4. Initial input reguired by planimetric Geometry model 2.4.1. Itoodplatin sediments

That actual meanders axe often irregular is well known, and deviations from the tdealised case are caused by heterogen-ities in bank materials, structural controls, and other random 
actions causing varying flow.

In order that the modol meanders conform to the ininimum variance equations, it will be assumed that the bank materials are reasonably homogeneous laterally, and there are no random actions of any nature causting shifts from stable to unstable forms. The meanders will therefore be 'free' meanders. Lateral homogeneity in bank materials is built into the model, the vertical variation of sediments in the floodplain, however, will be specified as input. 2.4 .2 . Havelength

Meander wavelength, like othex form characteristics of stable alluvial channels, is a function of the independent variables. It has been recognised empirically for years by many authors that wavelength, 1 , increases with strean size according to

$$
1=\mathrm{c}_{1} \mathrm{Q}^{\mathrm{N}}
$$

in which stream size is measured by a discharge $Q, c_{1}$ is a coefficient and $N$ is an exponent close to 0.5 . Because channel width, w, and mean depth, d, depend on discharge relations can also be formed between $w$ and 1 and $d$ and 1 . It was shown in equation (2.1) that 1 and $r_{m}$ are closely related.

As will be expanded in section 8 , the precise interpretation of the discharge, or the range of discharges, that defines the channel form in natural streams is a major source of disagreement (Carlston, 1965; Ackers and Charlton, 1970c). This is not a problem under controlled laboratory studies with constant discharge. Although exponent $N$ does not appreciably vary, $c_{1}$ varies considerably according to different authors depending on the data used, suggesting that one equation of the form given above cannot doscribe the wavelength of all froe meandering streans. Indeed, discharge is but one of the independent 
variablos and the control of wavelength is undoubtedly more complex than equation (2.13) would suggest.

The dependence of wavelength on some or all of the other Independent variables has been empirically examined by many authors, often involving the arranging of the independent variables into dimensionless groups (e.g. Ackers and Charlton, 1970a,b; Carlston, 1965; Chang et a1, 1971; Charlton and Benson, 1966; Freidkin, 1945; Kinosita, 1961; Schumn, 1967, 1969; Shahjahan, 1970). Theoretical studies of meandering have also yielded relationships for wavelength (e.g. Anderson, 1967; Ca1lander, 1969; Engelund and Hansen, 1967; Fujlyoshi, 1950; Hansen, 1967). There appears to be some confusion and apparent1y conflicting views concerning the wavelength of meandering laboratory and natural streams of different sizes and types. It is clear that wavelength cannot be taken as uniquely related to discharge. Although the exponent in equation (2.13) appears to represent the effect of discharge fairly well when about 0.5 , the coefficient $c_{1}$ obviously represents the net effect of the other independent variables. Although various investigators have attempted to account for the effects of some of these variables in their equations, valley slope has not béen accounted for by any of them. None of the equations uniquely describes the effect of the independent variables, and even the theoretical studies require empirical information. It follows that in a natural stream, for a given discharge pattern, a number of different wavelengths may occur depending on the variation in the other dependent variables, either along the same stream or between different streams. The existence of a number of wavelengths in a given stream has been confirmed by speight (1965a,b, 1967), Toebos and Chang (1967) and chang and Toebes (1970). 
In view of the aforesaid it appears that the theoretical or empirical relationships developed to date can only be used for an approximate estimate of the effect of the independent variables on wavelength. The problem of multiple wavelengths will not be encountered because of the choice of model conditions (see section 2.4.1). If 1 is being defined for input using one of the equations cited in the literature, estimates of empirical constants would necessarily be subjective. Furthermore, a time integral of the discharge hydrograph is more preferable than a single measure of discharge (soe section 8). It will be seen later that channel width must be specified as input to the model. Leopold and Wolman's (1960) relation, that wavelength is approxImately seven to ten times the channel width is a useful approximation 1inking these two parameters.

\section{$2.4 .3 \quad$ Amplitude}

Freldkin (1945) showed in flume studies that in uniform material, at constant discharge, amplitude did not continue to increase nor did meander loops cut off as the meanders migrated downstream. After the initial development of the bends, the wavelength reached a limiting value, amplitude increased due to erosion at the concave banks but was checked by the formation of chutes when flow resistance was less over the bar than in the channel. After formation of a chute the bend formed a little further downstream, and again started to increase in amplitude to a limiting value, wavelength remaining constant, until another chute formed.

It is to be expected that this limiting amplitude, like wavelength, will be a function of the independent system variables. The variation of the limiting amplitudes (and the similar parameter, meander width) with the independent variables are best obtained from laboratory meanders that have been allowed to 
develop freely to a stable form (e.g. Shahjahan, 1970). However, the measured amplitude may not represent a stable limiting value in laboratory meanders that were not allowed to develop freely, or in natural rivers where meandering was developing, say, after cut off, and thexefore not in dynamic equilibrium. A further problem in natural rivers is the variation of the independent variables along the length of the stream (i.e. tributaries, local variations in stream banks, etc.) which makes an objective measure of meander amplitude for a reach difficult to obtain. These points explain the poor correlations of amplitude separately with discharge, wavelength and channel width (Leopold and Wolman, 1960; Carlston, 1965; Ackers and Charlton, 1970a), and the poor multiple correlations, including various other hydraulic variables (Chitale, 1970). One interesting study (Nagabhushanaiah, 1967) expresses meander width, $W$, of laboratory meanders in terms of discharge, critical discharge at which bed load movement begins, $Q_{c}$ ' mean diameter of bed material, $D$, bed slope, $s_{b}$, and time, $t, i . e$.

$$
\frac{\mathrm{V}}{\mathrm{D}}=0.76 \frac{\left(\mathrm{QS}_{\mathrm{b}}^{2}-\mathrm{Q}_{\mathrm{c}} \mathrm{s}_{\mathrm{b}}^{2}\right) \mathrm{t}^{0.5}}{\mathrm{D}^{3}}
$$

It is interesting to note that the time term describes the progressive development of the meander amplitudes from zero up to a limiting value (see fig. 2,6). The shapes of the curves broadly agree with the data on natural streams obtained by Handy (1972). Nagabhushanaiah further noted that the quantity and size of transported sediment increases with discharge and slope, and decreases with time; that is, in a developing meander, as amplitude increases, water surface slope decreases and rate of sediment transport decreases. 


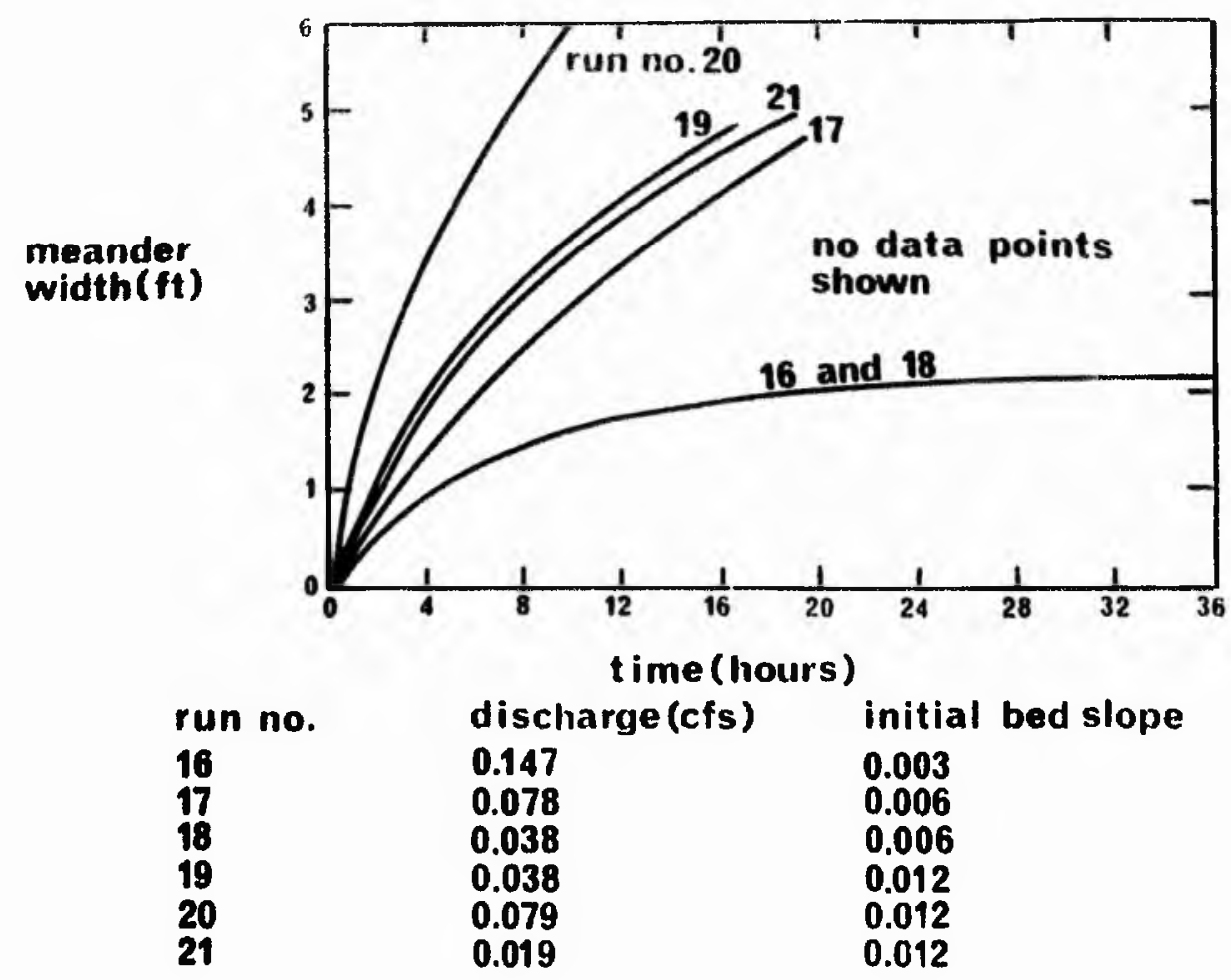

Fig.2.6 Variation of meander width with time.

(after Nagabhushanaiah, 1967).

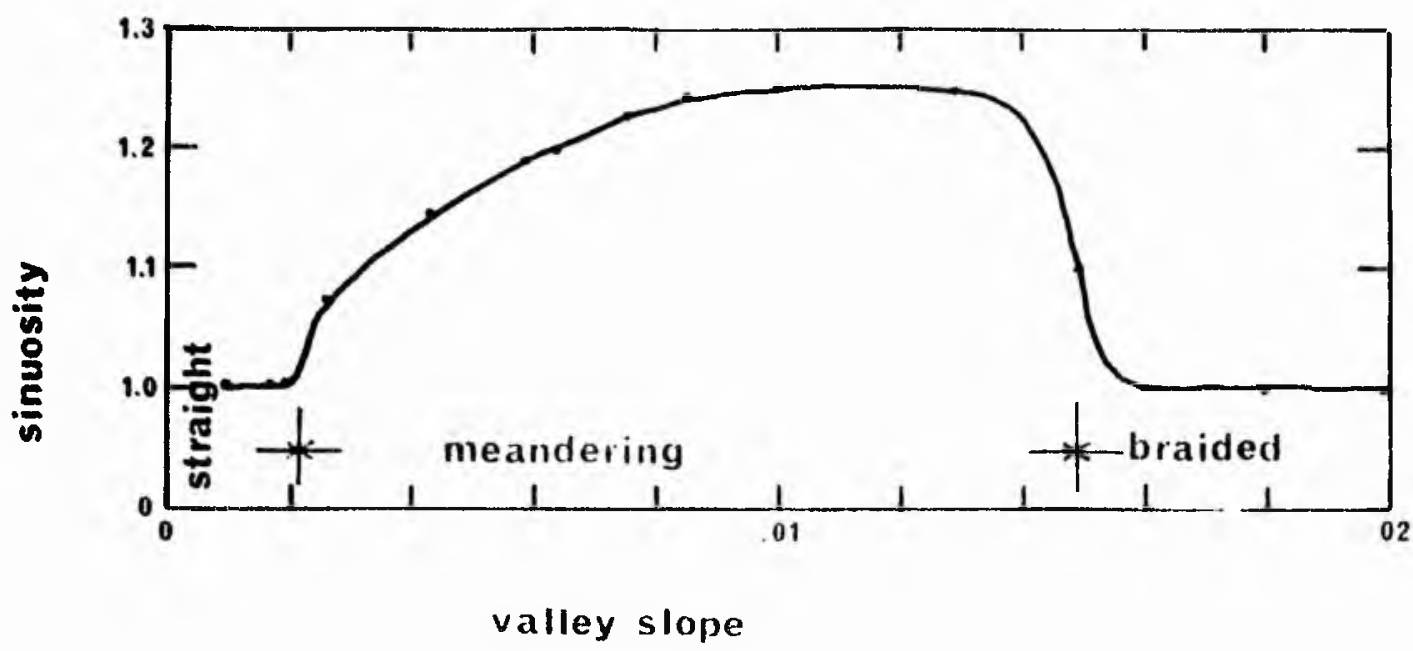

Fig.2.7 Relation between valley slope and sinuosity for experimental studies' 'fter Schumm et al, 1972). 
In general, limiting amplitude of meanders increases with increasing discharge, valley slope and sediment discharge, and decreases with increase in size of bed matertal. Some of the empirical derived relatjonships in the literature should be, examined with caution as the values of amplitude used in their derivation may not be limiting values. obviously the amplitude of a meander doveloping to a limting value may have any value depending on the progression of time from any initial situation. Amplitude is not actually required as physical input to the model, however its relationship with other dependent variables, notably 1 and $s n$ in equation (2.12), is useful if a particular amplitude is required in the model.

\section{4 .4 sinuosity}

In this study sinuosity is defined as the ratio of length along the channel centre line to length along the valley axis. This definition is sometimes altered slightly by various authors to suit particular situations.

Schumm (1963, 1969) has shown, using data from natural streams, that sinuosity is related to width-depth (max.) ratio, $F$, and the woighted mean percentage of silt and clay in the. perimeter of the channel, M, by the following regression equations

$$
\begin{aligned}
& \operatorname{sn}=3.5 \mathrm{~F}^{-0.27} \\
& \operatorname{sn}=0.94 \mathrm{M}^{0.25}
\end{aligned}
$$

where the lower limit of the sand sizes is defined as $0.074 \mathrm{~mm}$. M I.s considered as an index of the ratio of bed material load to sediment load, that is the type of sediment load moved through the channels. For channels in the Great Plains of the United States and the Riverine Plain of New South Fales, Australia, $M=55 / Q_{t}$ where $Q_{t}$ is the total sediment load that is sand or bed load at mean annual dischaxge. Although the dimensions of 
meanders (wavelongth, limiting amplitude, channel width, etc.) aro rolatod primarily to discharge, thene is no significant relationship between sinuosity and discharge. However, a change in discharge may cause a modification through its effect on type of sediment load transported in the channel.

Valley slope has been found to control sinuosity (Freidkin, 1945; Ackers and Charlton, 1970a,d; Schumn, 1971; Schumn and Khan, 1972; Schumm et al., 1972), however some qualification is noeded hero. Fig. 2.7 relates to tho experimental work of Schumm and Khan (1972). It should be noted that in this study the sodiment load was increased to maintain a stable channel (nonscouring and nonaggrading) as valley slope was increased. If valley slope is too small or large for a glven introduced sediment load general agradation or degradation, respectively, will tend to occur, hence changing valley and channel slope (Ackers and Charlton, 1970a; Schumm and Khan, 1972). Sediment load and valley $310 \mathrm{pe}$ therefore cannot be viewed as mutually independent variables in this respect. Valley siopo may be largely independent of sediment load when there are tectonic influences, that $1 \mathrm{~s}$, uplift, depression or tilting of tho valley. Fig. 2.7 shows sinuosity increasing with increasing valley slope (tucrease in sediment load is not shown). If, however, the valley glope is too steep for a given sediment discharge the river may efther degrade in ordex to reduce the ralley slope or reduce the channel gradient by increasing sinuosity (Schumm, 1971). The latter situation and ff.f. 2.7 thereforo represont two apparently irreconcilable situations, The channel slope and sediment load in the latter situation nust however be above that critical for the existence of meanders. There axe obviously limits to the amount of degradation possible, and the relative amounts of adjustment will depend on the distribution of energy expenditure. It should be noted that sinuosity in these relations, 
derived from natural streams, is that limiting sinuosity assoclated with a stable wavelength and limiting amplitude, 1.e. in dynamtc equilibrium. However sinuosity will vary somewhat with time about these measures of mean tendency depending on the occurrence of cut-offs and subsequent growth to a stable form. In the model limiting sinuosity and initial sinuosity are required as jinput. These will be synonymous if the meander is in a stable form. If the meander is developing to a stable form initial sinuosity may take ary desired value. 


\section{MEANIERS IN A DYNAMIC FRAMEWORK}

Although the meandering; behaviour may be stable through time, meanders shift continuously in the mean downvalley and normal to the mean downvalley directions by the orderly erosion of the concave banks and deposition on point bars. The spatial and temporal distribution of erosion and deposition around a meander is determined by the interaction between the flow pattern and the sediment forming the perimeter of the channel. Inherent difficulty lies in expressing the magnitude and the direction of the forces involved at every point along the channel. These forces are discussed later in section 6 .

Although theoretical studies make it possible to predict the flow axound a channel bend given channel shape and discharge (e.g. Rozovskii, 1961; Yen, 1971), the flow may mould a loose sediment bed, which in turn will alter the flow pattern. The interactive relation between the shape of a loose sediment bed and the flow cannot be described adequately for every point in the bend. Difficulty also is expexienced in describing the other forces acting on the bed and banks within the context of the whole meander. Furthermore, from a practical viewpoint, there would be severe limitations imposed by the availability of computer memory if the erosional and depositional activity of a meander was to be completely described and recorded in three spatial dimensions.

By using the sinemgenerated curve in a dynamic framework, however, the movement of meanders in plan can be referred to specific moving reference axes (see fig. 3.1). The net river behaviour can be described simply by looking at the movement of the refexence axes and the changes in the shape of the sine gencrated curvo relative to the reference axes. 


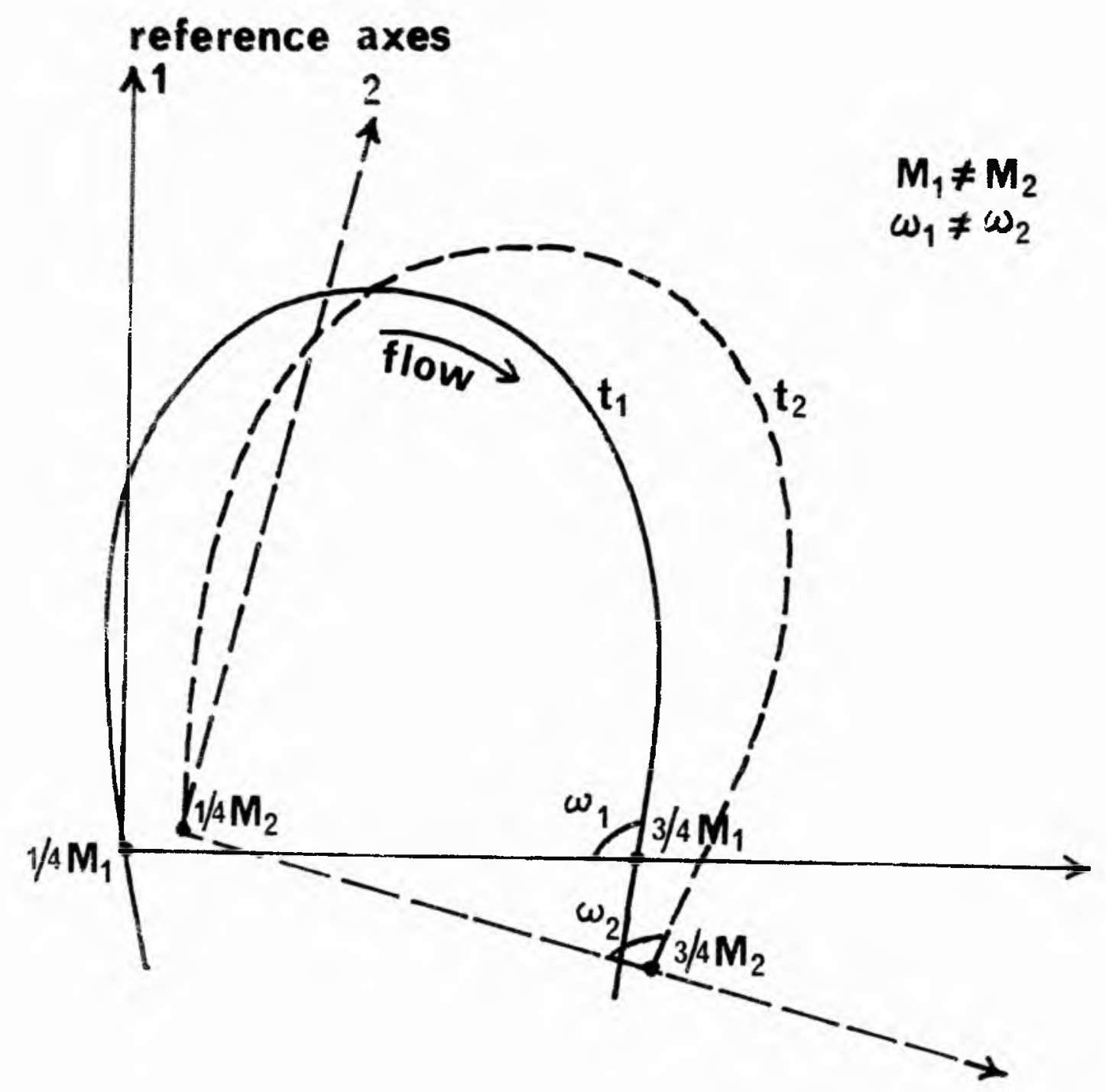

Fig.3.1 General mechanism of meander loop movement. 

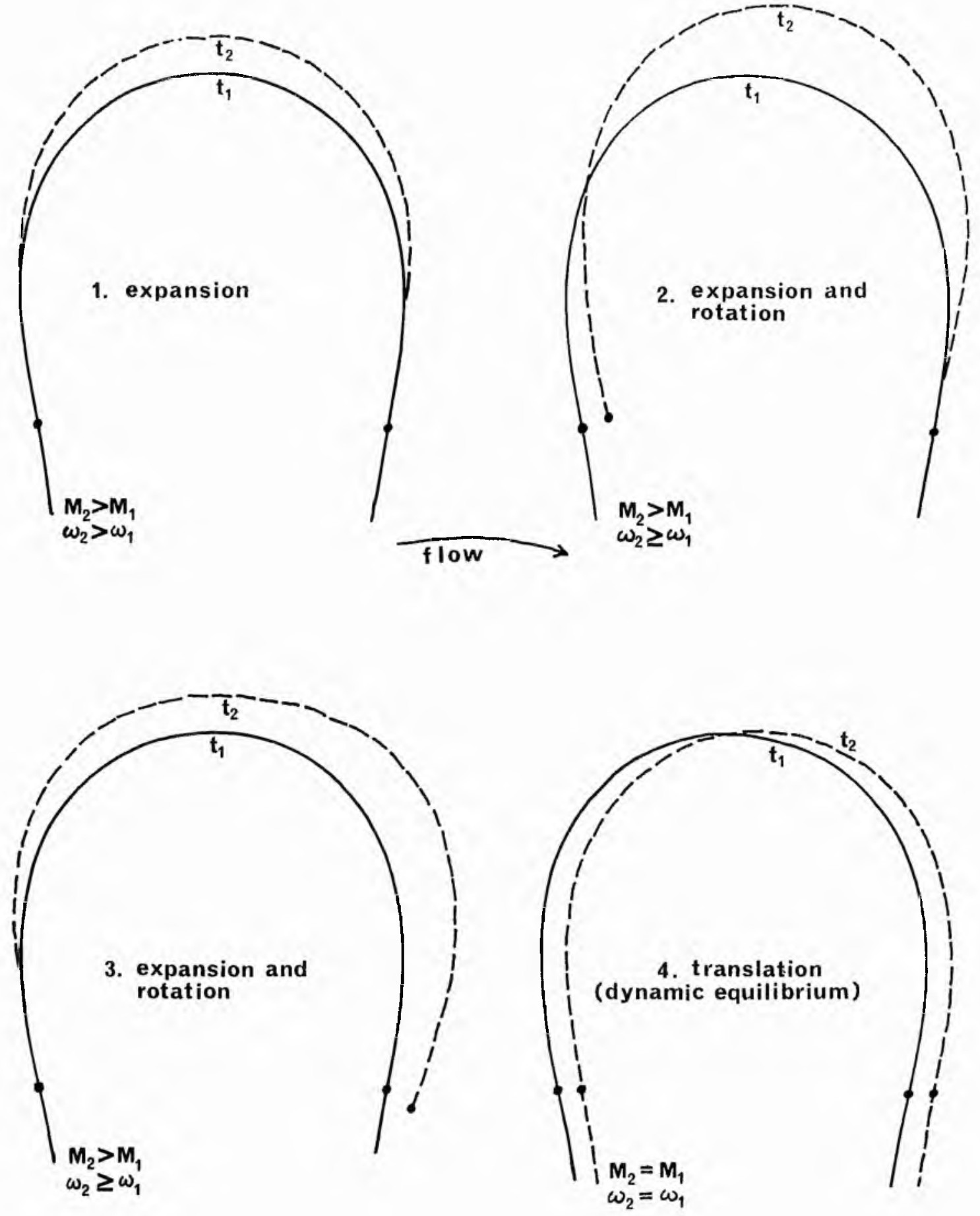

Fig.3.2 Observed modes of movement in meander loops (modified from Daniel, 1971). 
Daniel (1971.) used this method in studying the movement of meandering streams in Indiana, and observed various modes of change, as shown in fig. 3.1 \& 3.2. In the first case of fig. 3.2, the meander is developing to a stable limiting amplitude, the lack of downstream migration indicating a restriction to bank movement in the downvalley direction, perhaps due to clay plugs. In examples 2 and 3 the situation is essentially similar except one arm of the meander in each case is not having its downvalley migration hindered. Where erosion rates differ greatly within a loop in this way, rotation of the reference axes occurs and the meander wavelength changes. Example 4 represents the stable situation whereby path length is not increasing, wavelength, amplitude and sinuosity are constant and the meander is migrating downvalley. Danial states that the three forms of movement, increasing path length, rotation, or translation, should have application to a 11 forms of meanders, the dominance of any single mechanism depending on the local physical constraints. In natural streams the usual condition would be some combination of al1 three, as in fig. 3.1.

In the model, rotation will not occur because of the 'built in' lateral homogenelty of the bank materials. The only modes of movement will be (a) translation (downvalley migration of a meander in a stable form), fig. 3.2, example 4, and (b) translation and expansion together. The lattex will be the situation in the case of a meander developing to a limiting stable form. Duxing the development of free meandering from a strajght natural or laboratory channel, the meander length remains essentially constant although the meander amplitude increases (Charlton and Benson, 1966; Ackers and Charlton, 1970a; Kinosita, 1961; Anderson, 1967). See fig. 3.3. Tn the model, therefore, meanders can be allowed to develop to a limiting sinuosity/ amplitude according to mode (b), while wavelength remains constant. 


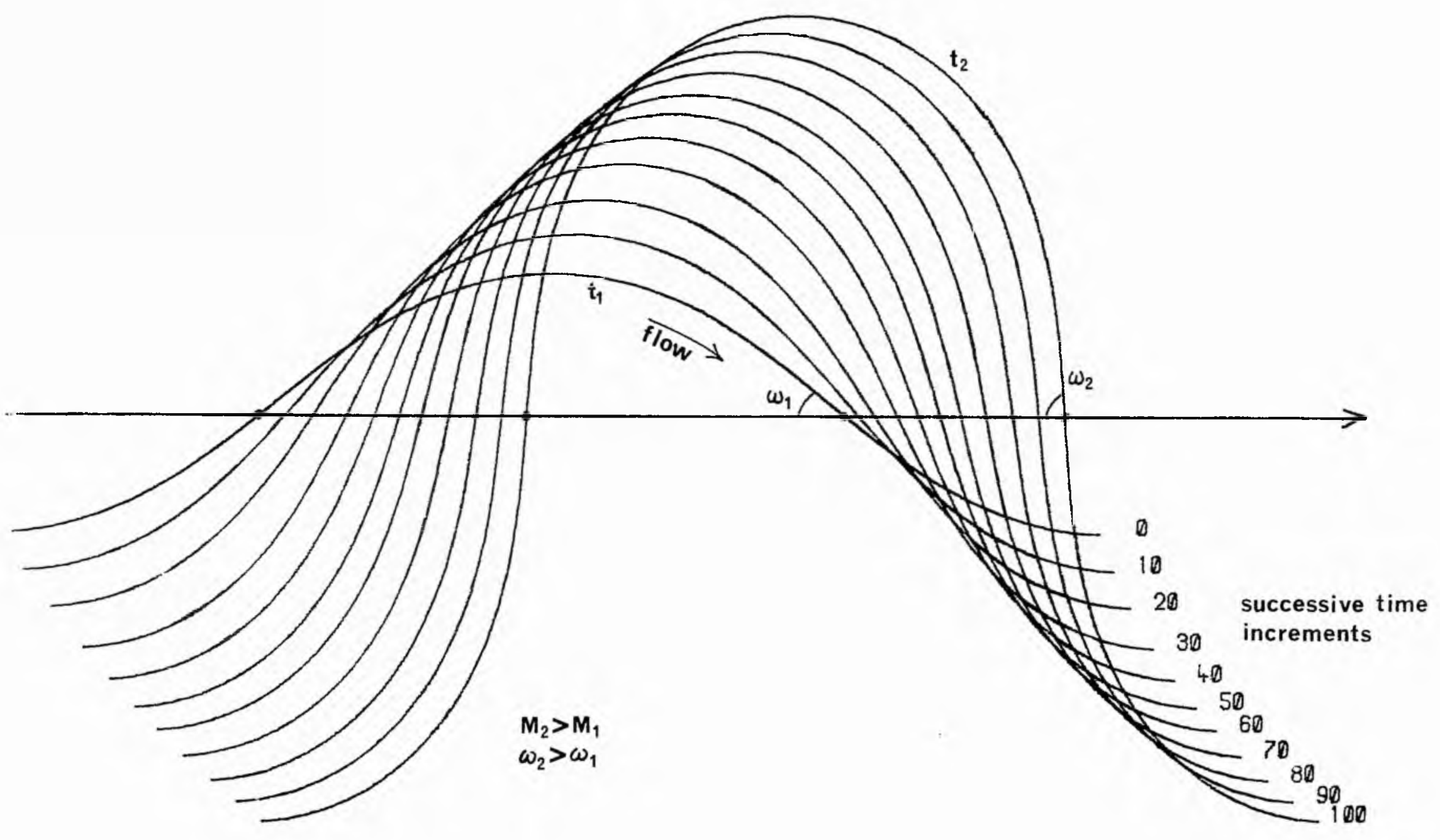

Fig.3.3 Meander loop migration by translation and expansion (developing meander). 
Assuming these modes of movement, it can be seen that by specifying only the downvalley rate of bank migration and the rate of expansion (path length increase) of the meanders, the movement of the whole meandex in plan form can be accounted for. Furthermoxe, as will be shown later, path leneth increase can be expressed readily in terms of rate of bank erosion in a direction through the axis of the bend. 


\section{CROSS SECTION DEFINTTION}

It has already been pointed out that it is not possible to analyse the detailed spatial and temporal distribution of erosion and deposition around the whole of a meander bend. This is quite possible, however, if dealing with specific cross sections across the channel. Because of the modes of movement of the meander in plan that are being considered, it it convenient to define cross sections across the channel in the downvalley direction and approximately normal to this direction.

In the model it is possible to look at the processes of erosion and sedimentation in three types of cross section. These are:-

(1) Through the axis of the bend at the inner (convex) bank in a direction approximately normal to the mean downvalley dixection. The actual direction will depend on the successive positions of the bend axis at the inner bank as the meander migrates. This is the LATERAL section.

(2) In a direction parallel to the mean downvalley direction through one arm of the meander loop. This is the ONE-CHANNEL DOWNVALLEY section. In the case of a developing meander this section is located through the point of inflection of the meander $1 \mathrm{imb}$.

(3) In a direction parallel to the mean downvalley direction through both arms of the meander, and located through the points of inflection of both meander limbs. This is the TWO-CHANNEL DOINVALLEY section.

The best type of section to use will often depend largely on the type of migration that is occurring. In case (a) of section 3, downvalley migxation of meanders in dynamic equilibrium, there is no point in using a lateral section as no deposition or erosion is occurring normal to the mean downvalley direction. 


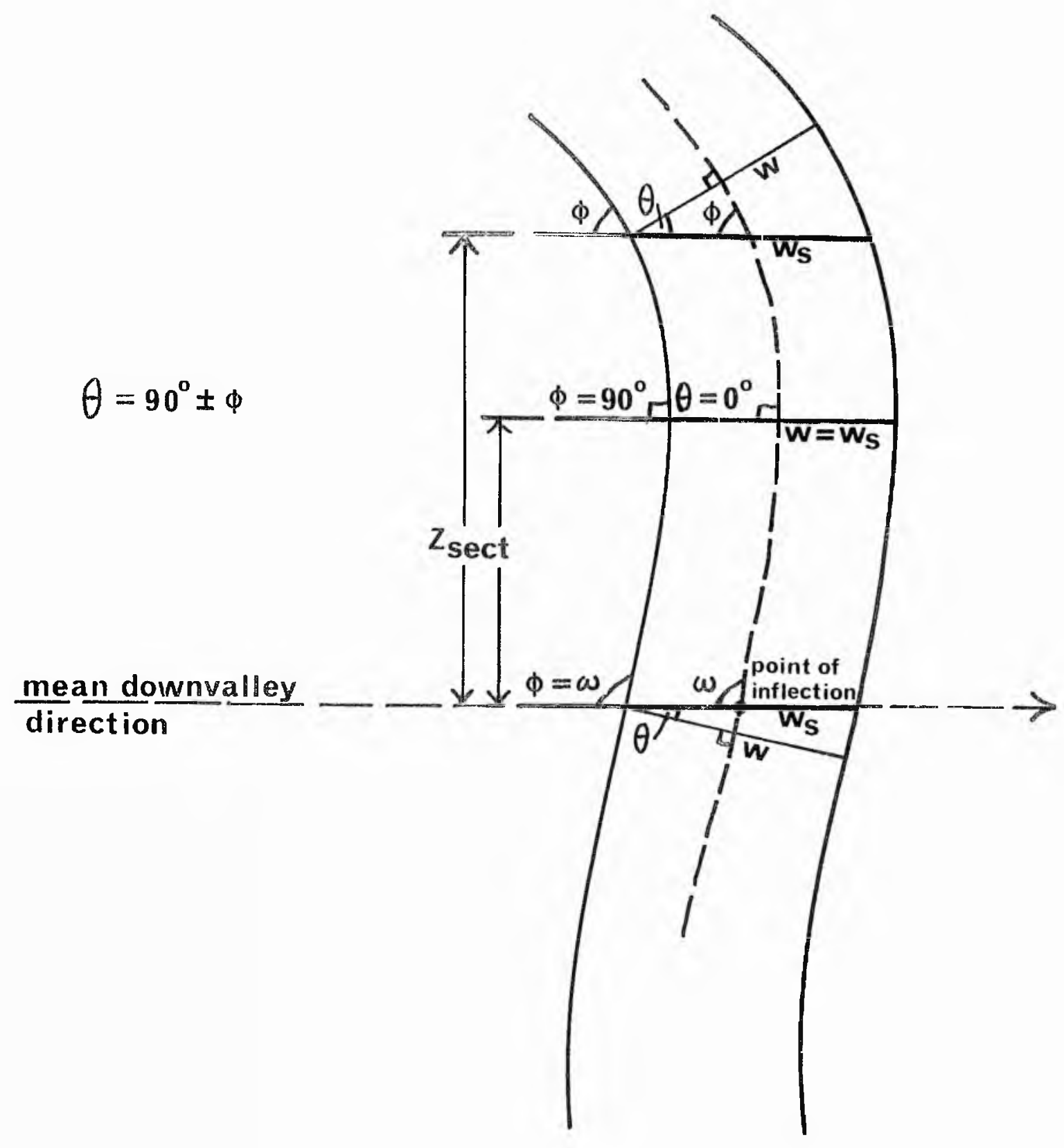

Fig.4.1 Cross section definition-downvalley sections. 
Cross section types 2 and 3 are the obvious chotce. Type 2 could be located anywhere on a meander limb (except near the axis), however type 3 is always located through the points of inflection of the loop. This is largely because along this section the straight line distance between the channel centre lines can easily be calculated as half the wavelength.

It can be seen from fig. 4.1 that the angle the channel centre line makes with the mean downvalley direction, and hence the projection of the actual channel width in this direction, will depend on the distance of the cross section from the parallel line joining the points of inflection of the loop and, of course, the shape of the loop. In the model the width of the channel as represented in the chosen cross section must be adjusted with respect to the actual channel width. In this case of translation only, the adjusted channel width remains constant as migration proceeds, and the relation between actual width and width projected in the cross section, $w$, is given by

$$
w_{s}=w / \sin \phi
$$

The value of the angle $\varnothing$ at a normal distance, Z sect, from the line joining the points of inflection of the 1oop, is obtained using an equation of the form of equation (2.9b), i.e.

$$
A / 2.0-Z_{\operatorname{sect}}=\frac{\sin .1}{2 \pi} \int_{0}^{\emptyset} \frac{\sin \phi \mathrm{d} \phi}{\sqrt{\omega^{2}-\phi^{2}}}
$$

The integral in equation (4.2) was evaluated numertcally by Simpson's rule for various values of $\omega(=f(s n))$ and $\varnothing$. Polynomial surfaces of degroe 1,2 and 3 were then ritted by least squares to the values of $\mathrm{sn}, \phi$, and the integral. as the dependent variable. It was found that the cubic $f i t$ is statistically best, which yields the following equation. 


$$
\begin{aligned}
& \frac{\pi\left(A-2 z_{\operatorname{sect}}\right)}{\operatorname{sn} .1}=0.2804 \phi^{3}-0.1713 \sin \phi^{2}+0.1139 \phi \mathrm{sn}^{2} \\
& \ldots 0.0292 \mathrm{sn}^{3}+0.2244 \phi^{2}-0.552 \phi \operatorname{sn}(4.3) \\
& +0.2123 \sin ^{2}+0.8895 \varnothing-0.4651 \mathrm{sn} \\
& +0.2668 \text {. }
\end{aligned}
$$

In the model, $\varnothing$ can be found, given A, sn, 1 , and $z_{\text {sect, by }}$ solving equation (4.3) using the Nowton-Raphson method (soe appendix 1). A good initial estimate of $\phi$, which is required by this method, is obtained using the equation of the fitted polynomial surface of degree 1 (plane surface). Full details of the trend surface fitting are given in appendix 2 .

In case (b) of section 3, the developing meander, any type of cross section can be used, however the channel width projected in any of these cross sections does not remain constant as migration proceeds. Whichever of the three types of cross section is used, continual adjustment of channel width is necessary as outlined below.

In a developing meander the rate of downvalley migration must be referred to a specific axis, as each point in the meander is migrating downvalley at a different rate (see fig. 3.3). This reference axis is conveniently taken as the line joining the points of inflection of the loop. Cross section types 2 and 3 are therefore taken as lying along the line of this reference axis. $Z_{\text {sect }}$ is therefore equal to zero, and the angle at which the channel centre line crosses the reference axis is of course $\omega$. The width adjustment is given by

$$
w_{s}=w / \sin \left\{2 \cdot 2 \sqrt{\frac{s n-1}{s n}}\right\} \text {. }
$$

The lateral section is always defined through the inner bank at the axis of the bend. The direction therefore depends on the relative bank migration $t n_{\mathrm{e}}$ the downvalley direction and normal to the downvalley direction (see rif. 4.2). The net amount of 


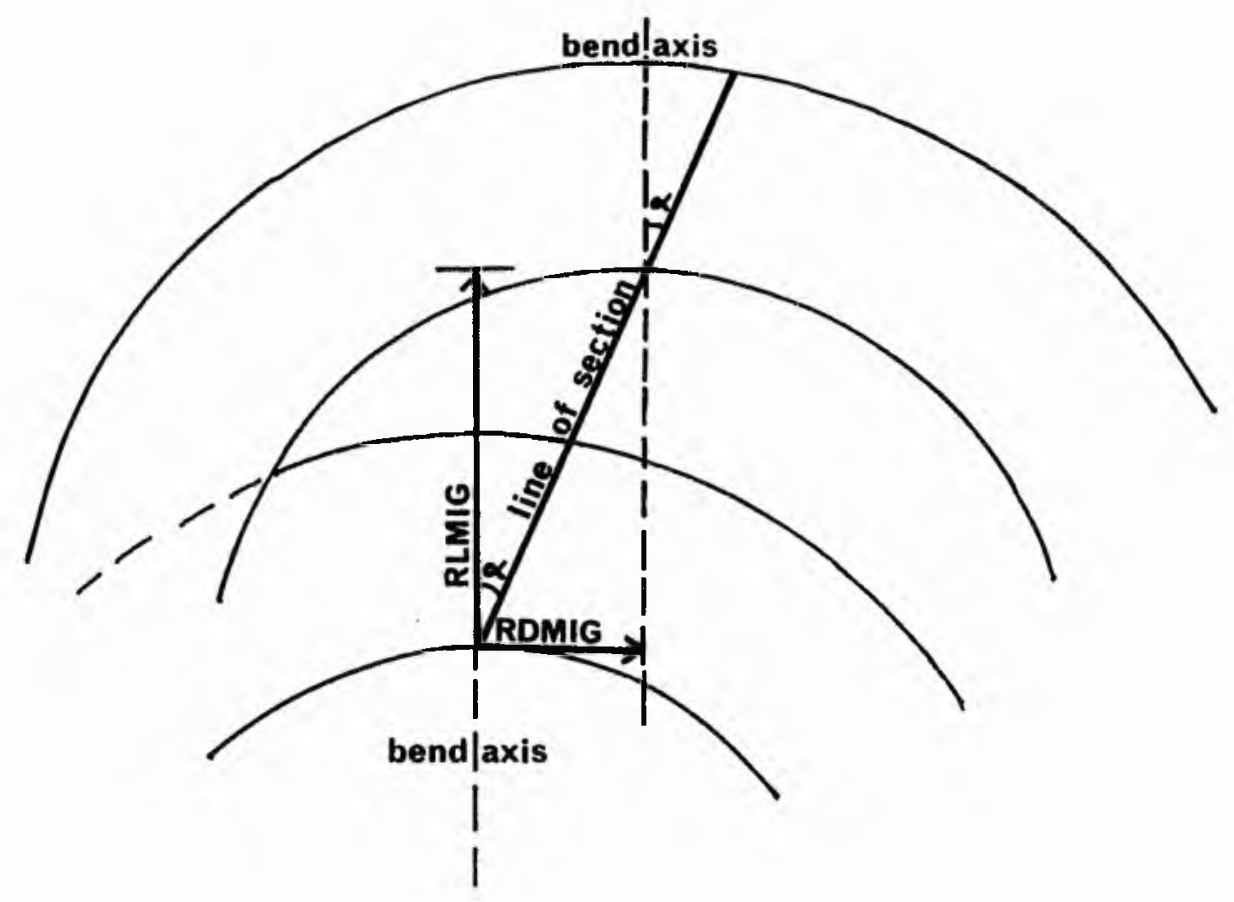

Fig.4.2 Definition of direction of line of section in a lateral section.

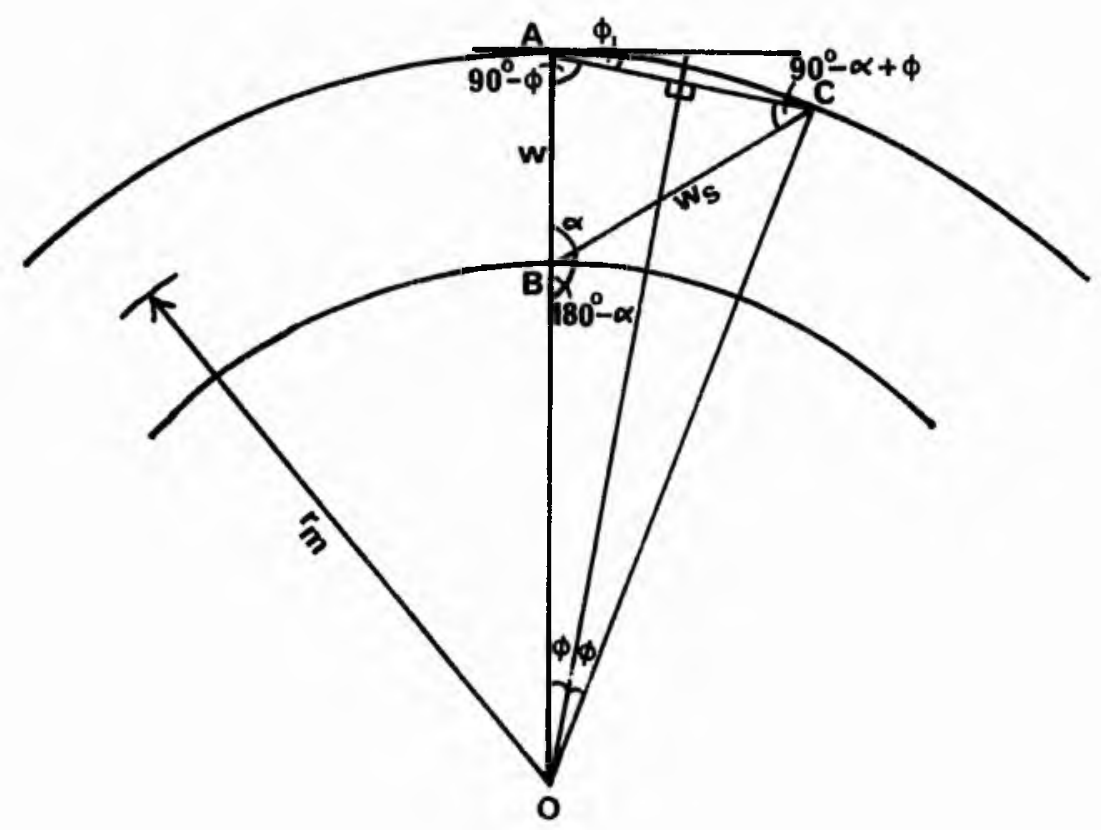

Fig.4.3 Definition of projected channel width $\left(w_{s}\right)$ in a lateral section. 
bank migration in this section, RMIG, is given by

$$
\mathrm{RMIG}=\sqrt{\mathrm{RLMTG}^{2}+\mathrm{RDMIG}^{2}},
$$

where RLMIG and RDMIG are the amounts of bank migration normal to and in the downalley direction in any given time increment. The angle, $\alpha$, that the line of section makes with the normal to the downvalley direction is given by

$$
\alpha=\tan ^{-1} \quad\left(\frac{\text { RDMIG }}{\text { RLMIG }}\right) \text {. }
$$

It can be seen that this angle will vary depending on the relative rates of bank migration. As in section types 2 and 3 , the channel width represented in the section must be continuously adjusted as the meandex develops. The adjustment formula is developed below.

Dy inspection of $f i g .4 .3$, it can be seen that the sine rule applied to triangle OBC gives

$$
\frac{o C}{\sin (180 \cdots \alpha)}=\frac{w_{s}}{\sin 2 \beta}
$$

and for triangle $A B C$ gives

$$
\frac{w}{\sin (90-\alpha+\phi)}=\frac{w_{s}}{\sin (90-\varnothing)}
$$

Eliminating $w_{s}$ in the above equations, we obtain

$$
\begin{aligned}
& \frac{\alpha \sin 2 \phi}{\sin \alpha}=\frac{w \cos \phi}{\cos \alpha \cos \phi+\sin \alpha \sin \phi} \\
& \therefore \quad \tan \alpha \quad=\frac{0 \operatorname{cstn} 2 \phi}{w-20 \sin \sin ^{2} \phi} \\
& \text { As } 1+\cot ^{2} \phi=\operatorname{cosec}^{2} \phi \text { and } \sin 2 \phi=\frac{2 \tan \phi}{1+\tan ^{2} \phi} \text { wo can write } \\
& \tan \alpha=\operatorname{OC}\left(\frac{2 \tan \phi}{\left.1+\tan ^{2} \phi\right)} / w-\frac{\left(20 \mathrm{tan} \tan ^{2} \phi\right)}{1+\tan ^{2} \phi}\right)
\end{aligned}
$$




$$
\begin{aligned}
& \therefore \tan ^{2} \phi(w-2 O C) \tan \alpha-\tan \phi 2 O C+w \tan \alpha=0 \\
& \therefore \tan \phi=\frac{O C \pm \sqrt{O C^{2}-(w-2 O C) w \tan ^{2} \alpha}}{(w-2 O C) \tan \alpha}
\end{aligned}
$$

The physically meaningful value of tan $\varnothing$ involves the negative of the square root term. Therefore $\varnothing$ is given by

$$
\phi=\tan ^{-1}\left[\frac{O C-\sqrt{O C^{2}-(w-2 O C) w \tan ^{2} \alpha}}{(w-2 O C) \tan \alpha}\right]
$$

A.s OC $=r_{m}+w / 2.0$

$$
\phi=\tan ^{-1}\left[\frac{x_{m}+w / 2.0 \cdots \sqrt{\left(x_{m}+w / 2.0\right)^{2} w 2 x_{m} \tan ^{2} \alpha}}{2 r_{m} \tan \alpha}\right]
$$

The projected width, $w_{3}$, is then given by

$$
w_{3}=\frac{w \cos \emptyset}{\cos (\alpha-\varnothing)}
$$

If the rate of downvalley migration is large relative to the migration normal to this direction, the angle, $\propto$, Is large and the width of the channel represented in the cross section will be considerably greater than the actual channel width. This will also dopend on the radius of curvature of the bend. Furthernore, in this situation the point bar deposits produced in the cross section will. very quickly be wiped out by the meander limb immediately upstream. Normally, in a developing meander, across valley migration of the channel will be several times grreater than downalley migration and angle $\propto$ is small. But as the meander develops to its limiting amplitude, the across valley migration gradually slows down, while downstream migration remains about constant (see section 6). Therefore, untess cut off occurs before the rate of downvalley migration becones large relative to the across valley migration, much of the deposit produced in type 1 section will be wiped out by the upstream 
meander limb. The model does not take account for the erosive effect of this upstream meander limb in section types 1 or 2 , therefore care should be exercised when examining these sections. 


\section{MODEL FOR DEPOSITION ON THE POINT BAR}

\section{Introduction}

The model adopted is based largely on Allen's (1970a,b) quantitative semi-empirical theory of lateral deposition which relates grain size and bed form across a curved channel to tho oxisting flow conditions. If the changes across such a channel can be arrived at, the vertical variation within the sediment bar produced by lateral deposj.tion at once becomes known. The tern 'lateral deposition' hexe implies deposition lateral to the local mean downcurrent direction.

In the model it is assumed that the channel geometry is known and that the strean flow can be described by the conventional hydraulic equations, supplemented by a single additional relationship for the helicoidal secondary flow in the channel bend. It will be assumed that each type of bed form in the channe1, leading to a distinctive type of sedimentary structure, is characterj.sed by a unique value of the friction coefficient.

\subsection{Qualitative ceatures of a system involving lateral} deposition

Figure 5.1 shows the main features of the physical situation in which lateral deposttion occurs. The curved chame1, containing a water stream powerful enough to ontrain and transport sediment, is bounded by a steep outer bank and a gently inclined inner bank with a sigmoidal cross profile. The dimensions of the channel are discussed in section 5.6.1.

A water particle travelifn along the channel follows a holicoidal spiral path taking tt from lnner to outer bank when close to the water surface, and from outer bank to Inner bank when near the channel bed. The pitch of the spiral path taken by the particle is large even compaxed with the chancl width, 


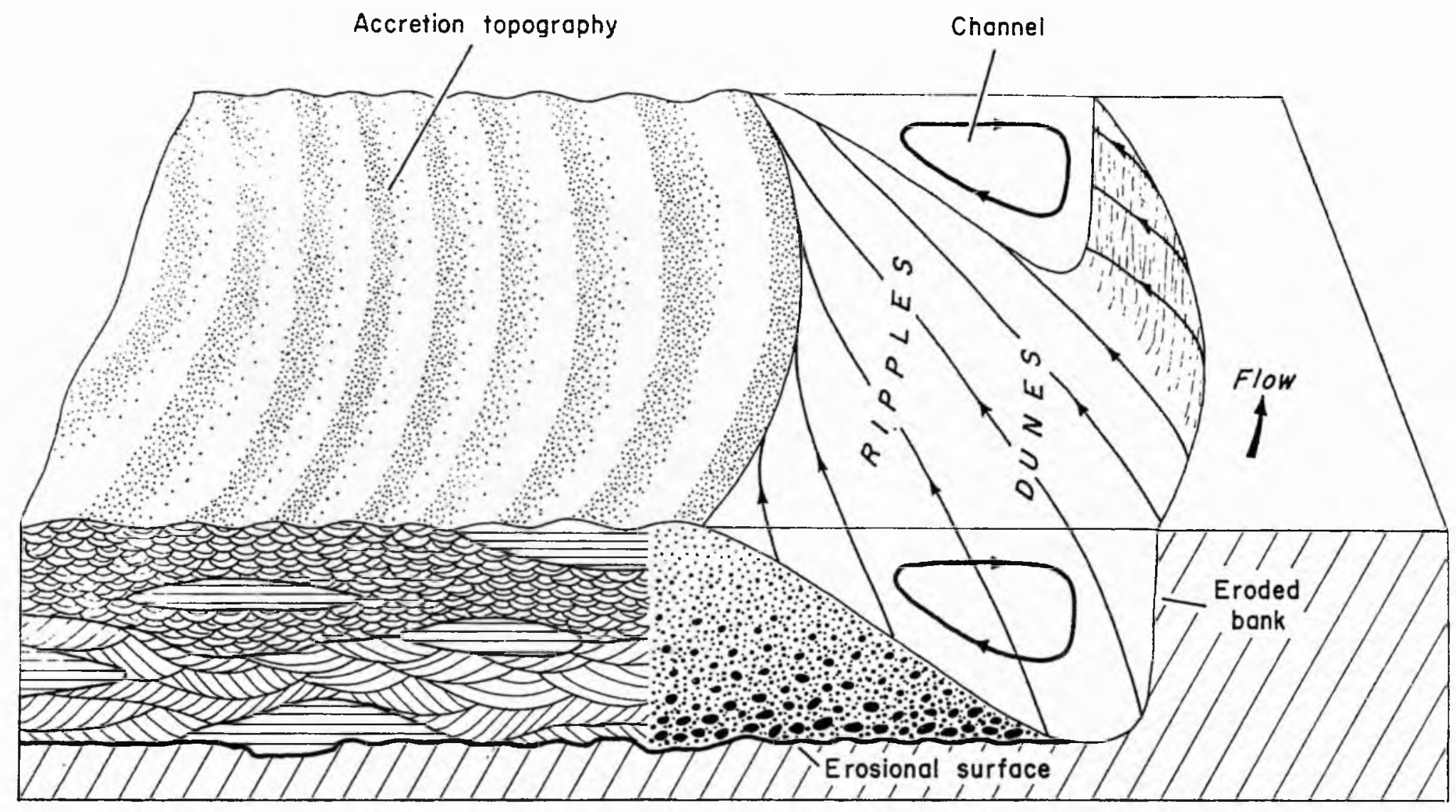

\section{8... Decreasing grain size Chass-bedding Flat-bedding}

\section{$\longrightarrow$ Skin-friction line}

Fig. 5.1. Qualitative features of an ideal channel bend migrating by processes of lateral deposition. Vertical scale exaggerated. (from Allen, 1970a). 
so that the transverse component of the particle velocity is small compared with the downstream component. The watex particles moving exceedingly close to the channel. bed can be represented by special limiting stream lines (skin friction lines) to which the bed shear vector is everywhere tangential. From the pattern of skin friction lines it can be seen why sediment accumulates on the inner bank father than the outer, lateral deposition building up the inner bank of the chanmel to balance the erosional 1osses on the outer bank. Because of its migration in this way, the talweg of the channel sweeps out laterally an erosional surface on which is lajd sediment deposited on the innex bank.

It is evident from the pattern of skin friction lines that the fluid flow must exert a component of bed shear stress dirocted tangentially up the slope of the cross profile. For equilibrium, this upslope force must be balanced by a force of equal magnitude acting tangentially down the slope of the cross profile. Allen $(1970 a, b)$ states that the balancing force is purely the body force associated with the sedinent travelling over, and in substantially continuous contact with, the channel bed. However the work of Bagnold (1956, 1966) has established the existence also of a dixect frictional opposition to tho impulsion of the bed load, in the direction of motion, which is proportional to the excess weight of the sedinent. The dynamic Priction coefricient is defined as tane and is of the same oxder as the static solid friction coefficient, not only when the grains. are closely packed but also when they are consiclexably dispersed. In this dynamic condition when the mass of grains ts under contimuing shear, with untual jostling notions in all directions, the angle $\theta$ is associated with the average angle of encounter between Indlvidual grains, and tan 0 is the ratio of the tangential to the normal components of gxain momentum resulting from tho 
encounters. By equating these foxces, the way grain sizes of bed material varies over the cross profile may be predicted for oquilibrium conditions.

When considering what bed form arises it is assumed that longitudinal slope of the water surface is constant over the cross profile, whence, from the conventional hydraulfc oquations, the bed shear stress and stream power must both in greneral decrease tnwards from the talweg. Selection of the bed structure will be seen to follow, given empirical data on the hydraulic limits and friction coefficients of different bed forms. 5.2. Shape of cross profile

To doscribe the seometry of the cross profile it is assumed that the local flow depth varies as

$$
\frac{y}{h}=\frac{1}{2}\left[\cos \pi\left(\frac{z}{w_{1}}\right)^{n_{1}}-1\right],\left(z \leqslant w_{1}\right),
$$

where $y$ is the flow depth, (measured positively downward from water surface), at any transverse distance z acooss the channel, $h$ is the maximun flow depth measured above talweg, $z$ is the perpendicular transverse distance across the water surface measured from edge of water at inner bank, and $w_{1}$ is the width of flow between inner bank and talweg (soe fig. 5.2). The exponent $\mathrm{n}_{1}$ prescribes the degree of concavity or convexity of the cross profile (see fif. 5.3). The cross profiles of natural channel bends are closely approximated by choosiner $n_{1}$ stmilar to or a little larger than unity (A11en, 1970a).

The shape of the cross profile in bends has also been descrtbed using empirical expressions (e.g. Riploy, 1927), and theorotical expressions which attempt to describe the interactivo effect between the loose sediment bed and the fluid flow (e.g. Yon, 1970; Ibade-Zade and Kiyasbei11, 1967; Pokhsraryan, 1957, 1958). An option will exist in the model to enable the use of an altornative expresston to equation (5.1). However, a 


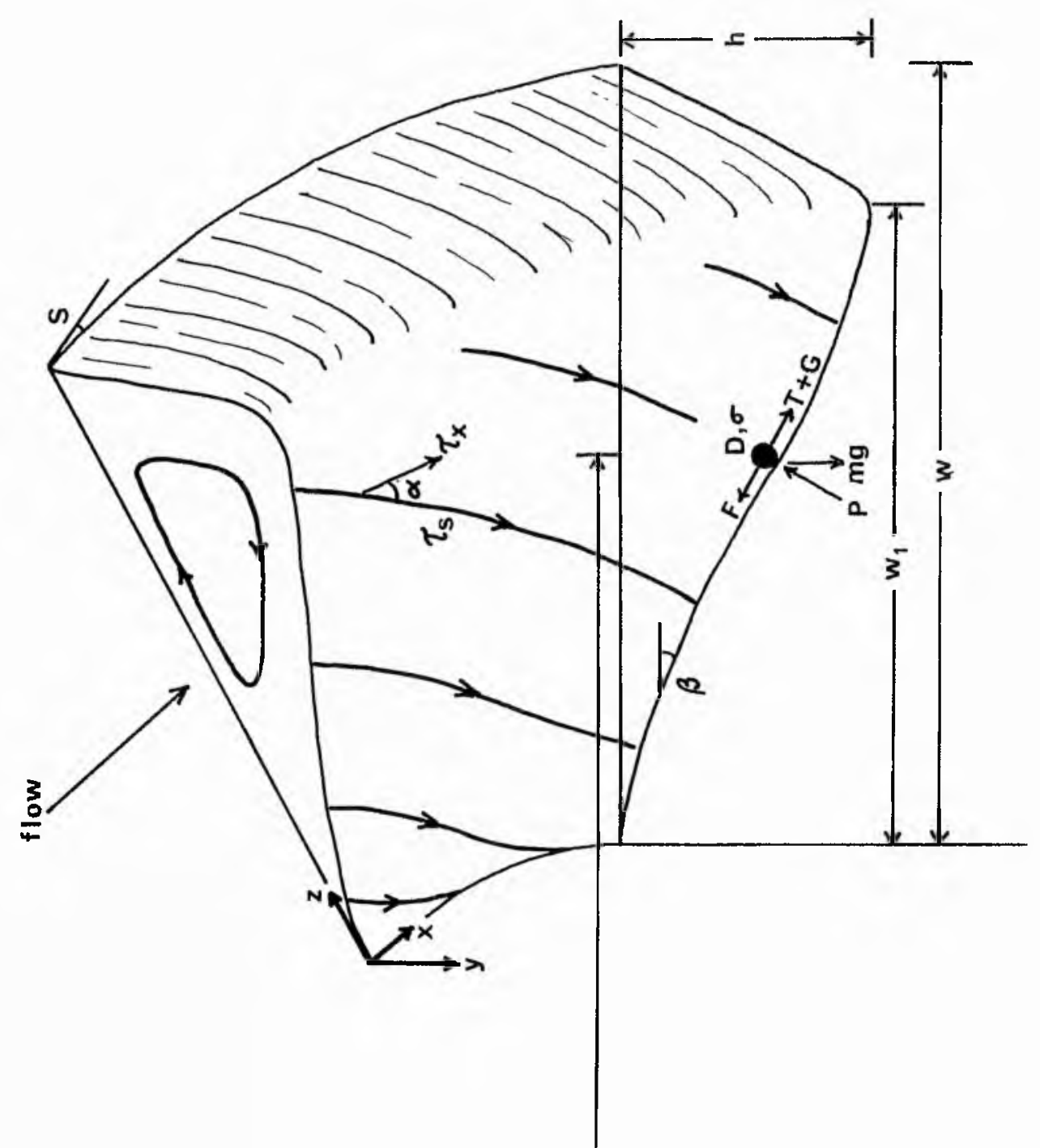

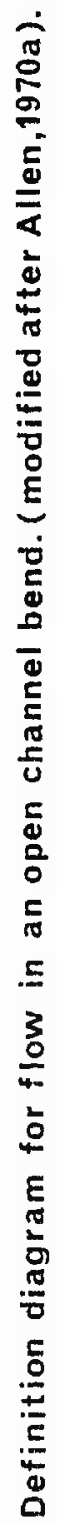

กั 


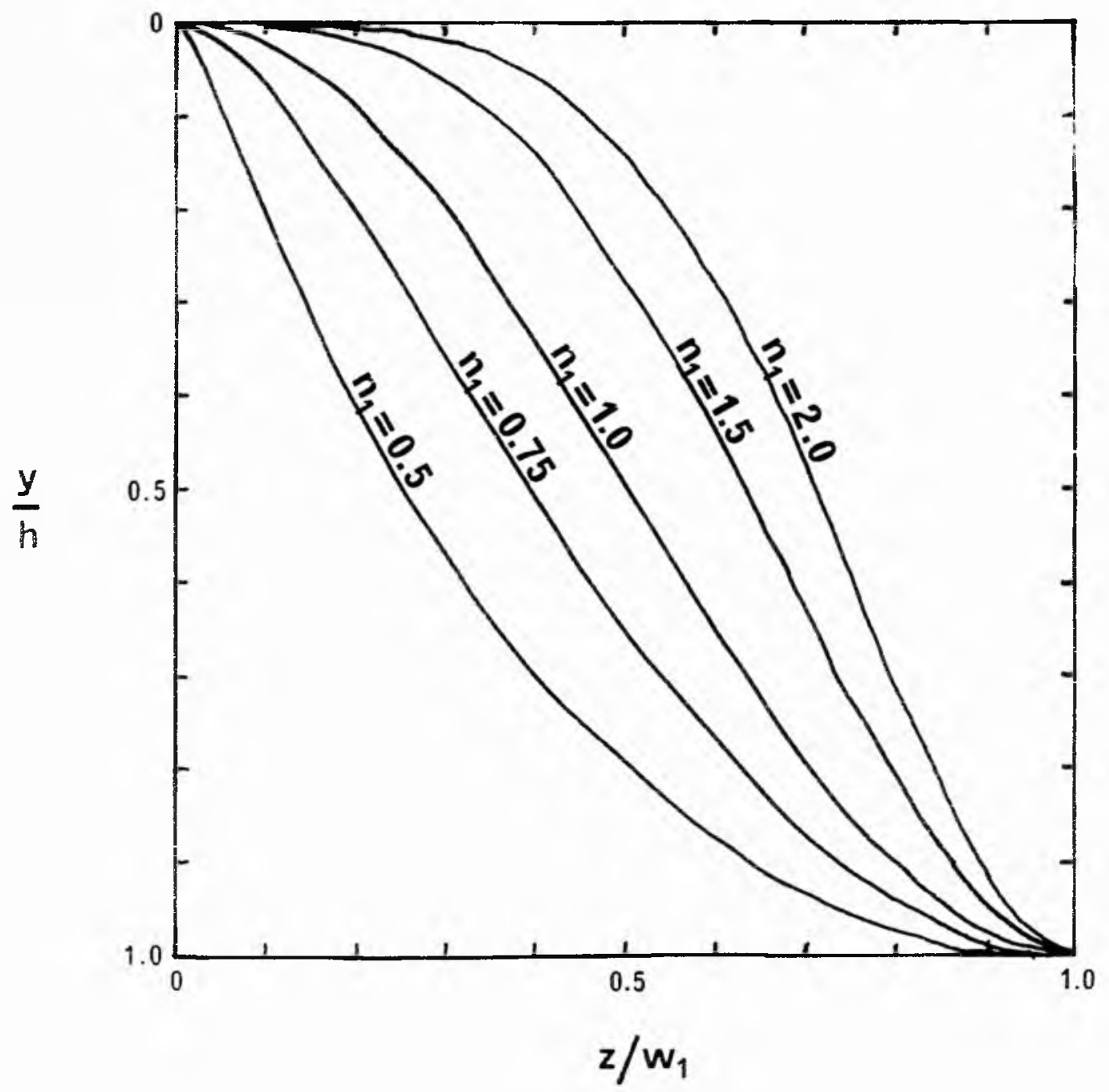

Fig. 5.3 Dimensionless channel cross profiles, according to equation(5.1). (after Allen,1970a). 
prerequisite of an alternative expression is that the value of $d y / d z$ and any other defining parameters can be readily supplied. Some of the theoretical equations are unfortunately very cumbersome and others require parameters whose values cannot be readily obtained.

The above expression describes only the inner channel bank on which deposition takes place. An accurate description of the outer bank is not needed, but it is essential to relate channel width, w, to width measured outwards to the talwog. Thus

$$
w_{1}=k_{1} w
$$

where normal1y $0.70 \leqslant \mathrm{k}_{1} \leqslant 0.95$. In the model the shape of the outer bank is defined, for simplicity, with an equation of the form of equation (5.1) with the exponent assuming a value of unity.

\subsection{Hydraulic properties of the system}

The flow in the cross section of the channel bend can be described usine the conventional hydraulic equations. At any transverse distance, $z$, the bed shear stress parallel to the $x-$ direction (see fig.5.2) is

$$
\tau_{\mathrm{x}}=\rho \mathrm{gs} \mathrm{ycos} \beta \text {. }
$$

This reduces to

$$
\tau_{\mathbf{x}}=\rho \mathrm{gsy}
$$

since $\beta$ is a small angle and $\cos \beta$ is near unity. Here the slope, S, in the general case represents the slope of the energy grade line. In the special case of uniform flow, the slope of the energy grade line is equal to the bed slope and the longitudinal water surface slope. In this study, $S$ is actually taken as the Longitudinal water surface slope, the rationale for which is discussed later. 
The bed shear stress at any station $z$ can also be expressed in a form which includes the fluid flow velocity averaged over the vertical at that station, thus

$$
\tau_{x}=\frac{1}{8} f p v^{2}
$$

in which $V$ is the mean fluid flow velocity parallel to the $x$ direction and $f$ is the Darcy-Wejsbach friction coefficient, both at the given station $z$. The value of $f$ depends on the character of the bed and the flow and describes the flow resistance of the channe1. The flow resistance in open channels is a complex problem. The shape of the channel in alluvium changes with flow conditions, bed features of various scales may form, and various degrees of channel sinuosity may develop. These changes affect the drag caused by the surface roughness and introduce form drag caused by bed features, as well as energy lossas due to secondary currents. Furthermore, the fluid properties and turbulent characteristics of the flow are changed by moving sediment along the bed and in suspension (Raudkivi, 1967). A further discussion of flow resistance is warranted at this point. Experimental investigations confirm the conclusions of dimensional reasoning that $f$ is a function of Reynolds number and boundary roughness, as measured by the ratio of the size of roughness elements to the flow depth, or relative roughness. For fully turbulent open channel. flow, f no longer depends on the flow Reynolds number, (Allen 1970c). However the application of the Darcy term 'friction coefficient beyond the context within which it was developed (i.e. uniformly distributed wall friction in pipes) has tended to encourage the tacit assumption that flow resistance in open channels is due principally to friction associated with distributed boundary roughness. This simplified and traditional view of open channel resistance 
disregards the fact that the 'square law' resistance, described by $f$ as in equation $(5.5)$, may be appreciably increased by the distortion of the low at discrete bends and other large scale channel irregularities. A1so, such internal distortion is accompanied, inevitably, by some deformation of the free water suxface, invalidating the required condition that the whole boundary remains fixed.

With steady, nonuniform flow, tangential accelerations occur when velocity is changed in magnitude, and normal accelerations when the velocity is changed in direction. These changes in velocity result in changes in momentum flux, which is accomplished only by pressures against the fluid in addition to pressures which would be associated with uniform flow (i.o. not a Inydrostatic pressure distribution). When such changes in velocity occur, zones of separation and secondary flow (i.e. helicoidal flow) frequently result, and this consequently Increases the shear and turbulence at the expense of the piezometric head. Honce head losses result. Since the foregoing changes in velocity and the resulting head losses are caused by nonuniform distribution of pressures on the boundary, the losses are termed form losses because of the pressure resistance and the associated changes, usually increases, in shear (Albertson and Simons, 1964).

The components of resistance to flow in a non-prismatic free boundary chamel can therefore be stated as:(a) Surface resistance (due to grain roughness). Where surface resistance occurs, the flow does not separate from the macroboundary but does separate from the grains, or microroughness. This type of resistance occurs on a plane bed, on the back of dunes, and in antidune flow (Simons and Richardson, 1966).

(b) Pressure resistance (due to form roughness). On the 
smallex scale, flow separates from the macroboundary in the case of ripples, dunes, and, to a limited extent with antidunes. The result is a pressure reduction in the separation zones (form drag) and the generation of large scale eddies (Simons and Richardson, 1966). A further source of energy dissipation is associated with the nonumiform flow over backs of dunes, and when antidunes gxow and subside. on a larger scale, nonuniform flow in meanders gives rise to pressure resistance due to changes in width and depth and changes in alignment, which set up helicoidal flow and sometimes eddies. As already stated pressure resistance normally involves increases in shear.

(c) Spill resistance (Leopold et al., 1960). Occurs 1ocal1y at particular placos in open channels under some conditions: Energy is dissipated by local waves and turbulence when a sudden reduction in velocity is forcibly imposed on the flow. Spill resistance is associated with local high velocities as when water backs up behind an obstruction and spil1s into lower velocity flow. This type of resistance occuxs with breaking waves in chute and pool flow, and sometimes in antidune flow. Blocks of bank material slumped into a channel cause such splils as do some bends of sharp curvature.

If the types of resistances (a) and (b) are described in terms of a mean distributed boundary stress, $p$ RR (where $R$ is the hydraulic radius), they will vary as the square of the flow velocity (Loopold et al., 1960). However, when energy dissipat.ion due to spill Is introduced the oquivalent distributed boundaty stress can no longer be expected to vary as the squaro of the mean velocity because spill resistance in an open channel. 
cannot exist at low velocities but must start increasing from zero at some finite mean velocity at which parts of the flow become locally supercritical. Such resistances cause foci of intense enexgy dissipation. Leopold et al. (1960) state that Froude numbers in natural streams show a distinct cut off below the crtical value at which spill resistance occurs. They suggest that there exists 30 me threshold beyond which processes operating in a natural channel atter the hydraulic relations at channel cross sections in such a way that the velocity depth ratio is reduced and thus the Froude number is limited.

Discussing the resistance to flow in terms of the DarcyWeisbach friction coefficient, Ackers and Charlton (1970d) separated the overall friction coefficient, $f$, into that part representing form losses introduced due to the addition of benda, $f_{b}$, and another part representing the resistance due to bed friction of a comparable straight channel, $f_{s}$, thus

$$
f=r f_{s}+t_{b},
$$

where $r$ is a factor by which the straight channel friction factor would have to be multiplied to account for the change in relative roughness (arising from bed features) due to change in hydraulic radius with meandering. This subdivision is convenient for the present study, and the values of $f_{b}, f_{s}$ and $r$ to be used in the model are discussed in section 5.6.3.

Bearing in mind these points concerning flow resistance, we can proceed by combining equations (5.4) and (5.5) to give for each station $z$

$$
v=\sqrt{(8 E S y / f)} .
$$

The rroude numbor, Fr, an important parameter describing open channel. flow, is dofined as

$$
F x=V / \sqrt{(f y)},
$$


whence from equation (5.7) the froude number at each station becomes

$$
F r=\sqrt{(8 S / x)}
$$

The stream power, a significant quantity doternining the sediment transport rate and the exjstence of certain bed forms, is defined as

$$
\omega=v \tau_{x}
$$

where $w$ is the stream power. From equations (5.4) and (5.7), we can write

$$
\omega=\rho \sqrt{(8 / x)} \cdot(\mathrm{gsy})^{3 / 2}
$$

In the computations of velocity, Froude number, boundary shear stress and stream power, the longitudinal water surface will be used as an approximation for the required enexgy slope. In steady, uniform flow this is only approximately true. In the nonuniform flow associated with pools and riffles and horizontal bends, the water surface slope would be expected to vary alonf the length of the river, and not be paraliel to the energy slope. Leopold et al. (1964) have shown, however, that in a meandering reach the energy slope and water surface slope at high stage are moxe uniform than in a compaxable straight reach. The effect of the bend is to increase energy losses due to secondary circulation, thus locally increasing the slope, which would otherwise be lower than over the crossover. This tendency towards uniform distribution of energy expenditure is discussed eaxijex. Fig. 5.4 from Leopold et al. (1964) shows the uniform water surface slope and energy slope, also that they are practically parallel, the velocity heads being virtually constant along the reach in question. 


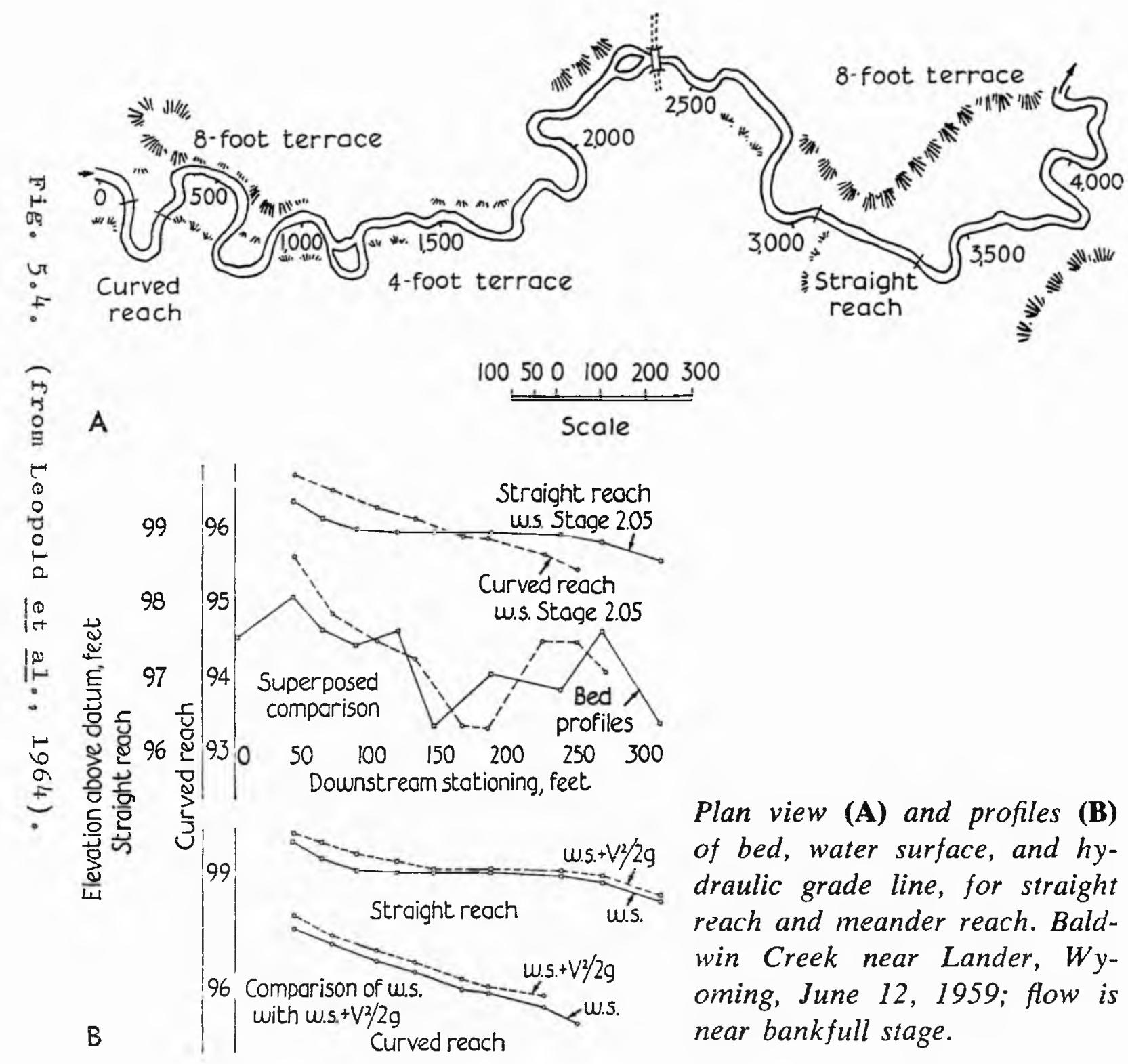


Leopold et a1. (1960) comment that an appreciable part of the whole flow resistance pgles of an irregular channel is due to internal energy losses in eddies and vortices at local deflections, and therefore the friction slope is not equal to the enexgy slope. The stresses are probably borne by the projecting portions of the flow boundary. A considerable portion of this stress will consist of components normal to the 1 ocal flow boundary and therefore of a nonerosive nature. As a result, the river bed on the whole must be relieved of a portion of the overall bed shear as given by perss. This does not detract from the fact that shear is increased with nonuniform flow, as previously discussed, and that the maximum bed shear is higher in bends than in comparable straight reaches (Ippen and Drinker, 1962; Ackers and Charlton, 1970d). It should be noted, however, that increase In shear due to transverse circulation alone was not found to be very great by Shukry (1950) in his experiments. Rozovgkii (1961) derives an expression for these losses.

Assuming that the longitudinal slope of the water surface $(\approx$ energy slope) is constant across the channel cross section we lind that in a given cross section (a) local bed shear stress parallel to the channel centre line depends only on the local flow depth (b) the local mean flow velocity and stream power dopend only on the local flow depth and the Darcy-Weisbach coefficient and (c) the local Froude number depends only on the local friction coefficient.

It remains necessary to account for the helicoidal motion of rluid particles carried through the channel bend and past a cross seotion of interest. We aro chiefly concorned with the flow exceedingly close to the bed on the inner bank of the channel, that is, with the skinwfriction lines, or limiting streamines, of the motion. Rozorskii (1961) found theoretically and empirically that 


$$
\tan \alpha=11 \mathrm{y} / \mathrm{r}_{1}
$$

where $\alpha$ is the angle on the bed between the channel centre line and the skin friction line of the helicoidal flow, at any station z. Here, $r_{1}$ is the local radius of curvature. This expression will be found sufficient to take account of the helicoidal flow.

\subsection{Variation of prain size over the cross profile}

Because of the helicoidal flow in the channel, the fluid exerts a shear stress component directed upsiopo in the plane of the cross profile. However, a sediment particle moving over the bed in substantially continuous contact with it must be affected by the downslope component of the body force and the dynamic frictional stress due to shearing over other grains. Becauso the speed of lateral movement of the channel cross profile due to bank erosion and deposition is small compared with the speed of advance in the bed load layer of a slowly moving sediment particle, it can be supposed that equilibrium is achieved when the downslope force components are equal to the upslope component of the fluid force. The particle will then follow a path parallel to the channel centre line and, by oquating the three force components, we can find the variation of particle size over a cross protile whose geometry is specified.

With the conventions of $\mathrm{fig} .5 .2$, the body force component, G, acting on a particle of diameter D at a station on the cross profile is

$$
G=\frac{4}{3} \pi\left(\frac{D}{2}\right)^{3}(\sigma-\rho) g \sin \beta
$$

where $\beta$ is the angle of slope of the cross profile, and $\sigma$ and $p$ are the sediment and fluid density respectively.

The frtctional force opposing motion, $T$, in the plane or the cross profile is given by

$$
\begin{aligned}
T & =P \tan \theta \\
& =\frac{4}{3} \pi\left(\frac{D}{2}\right)^{3}(\sigma-\rho) \operatorname{toc} \beta \tan \theta
\end{aligned}
$$


where $P$ is the normal. stress, and $\tan \theta$ is the dynamic friction coefficient of solid friction as previously described.

The upslope componenent of the fluid force is

$$
F=\pi\left(\frac{D}{2}\right)^{2} \cdot \tau_{s} \sin \alpha=\pi\left(\frac{D}{2}\right)^{2} \cdot \tau_{x} \tan \alpha
$$

where $\tau_{\text {s }}$ is the bed shear stress measured tangentially to the skin friction line at the station considered. For equilibrium, $F=T+G$ and

$$
\sin \beta+\cos \beta \tan \theta=\frac{3 \tau \tan \alpha}{2\left(\frac{x}{\sigma}-\rho\right) g D}
$$

As $\beta$ is very small, we may write $\sin \beta \approx \tan \beta$ and $\cos \beta \approx 1$, whence

$$
\tan \beta=\frac{\mathrm{dy}}{\mathrm{d} z}=\frac{3 \tau \tan \alpha}{2(\sigma-\rho) g \mathrm{D}}-\tan \theta
$$

A second expression for dy/dz is obtained by differentiating with respect to $z$ the equation for the variation of local flor depth in the cross profile, $y$. If this is taken as equation (5.1), neglecting the negative sign, we obtain

$$
\frac{d y}{d z}=\frac{n_{1} z^{\left(n_{1}-1\right)} \pi h}{m_{w} w_{1}} \sin \pi\left\{\frac{z}{w_{1}}\right\}^{n_{1}}, \quad\left(z \leqslant w_{1}\right) \cdot(5.18)
$$

Eliminating dy/dz between equations (5.17) and (5.18), and after substitutions from equations (5.4) and (5.12), an axpression for D can be obtained as

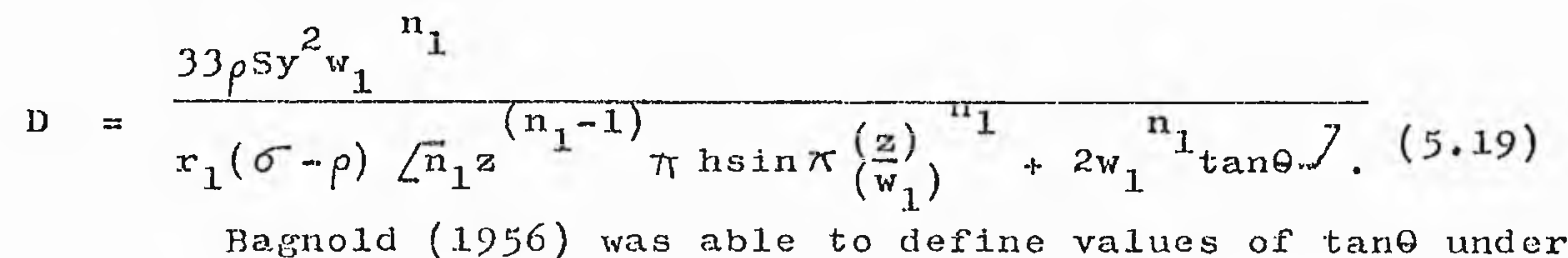
conditions in which the moving bed load solids are sufficiently numexous to interpose an effective flow bounctary between the free fluid flow above and the stationary bed below. This critical stage 1 approximately when bed features disappear, or at 1 east 
cease to create appreciable forn drag. Above this critical stage experiments show $\tan \theta$ to vary from 0.375 to 0.75 according to the conditions of shear owing to the variation of fluid-viscosity effects with variation of grain size and mass. Below this critical stage, Bagnold gives grounds for assuming values of $\tan \theta$ over a similar range of values, depending on grain size only.

Grain size profiles were calculated using constant values of tane of $0.0,0.3$ and 0.6 for six separate cross sections in order to assess the importance of the dynamic frictional stress. The values of the other parameters used in equation (5.19) were conveniently taken from Allen (1970a). In reality the value of $\tan \theta$ will vary over each profile with the conditions of shear, as defined by Bagnold $(1956,1966)$, however, by inspection of fig. 5.5 It can be seen that the effect of $\tan \theta$ on the grain size distributions is so small that its variation over the cross profile can be ignored.

For simplicity, therefore, it is considered justifiable to omit the effect of $\tan \theta$ and assume a value of zero. Equation (5.19) then becomes

$D=\frac{33 \rho \mathrm{Sw}_{1}{ }^{n_{1}{ }^{2}}}{\pi n_{1}(\sigma-\rho) r_{1} \quad h_{z}\left(n_{1}-1\right) \sin \pi\left(\frac{z}{w_{1}}\right)^{n_{1}}}$

Equation (5.20) has implications about the general calfure of the load that can be carried through a specified channel bend, as well. as about the variation of particle size over a given cross profile in the bend. The general calibre of the load increases with ascending water surface slope, maximum channel depth, and channel width between the inner bank and the talweg. The general. 

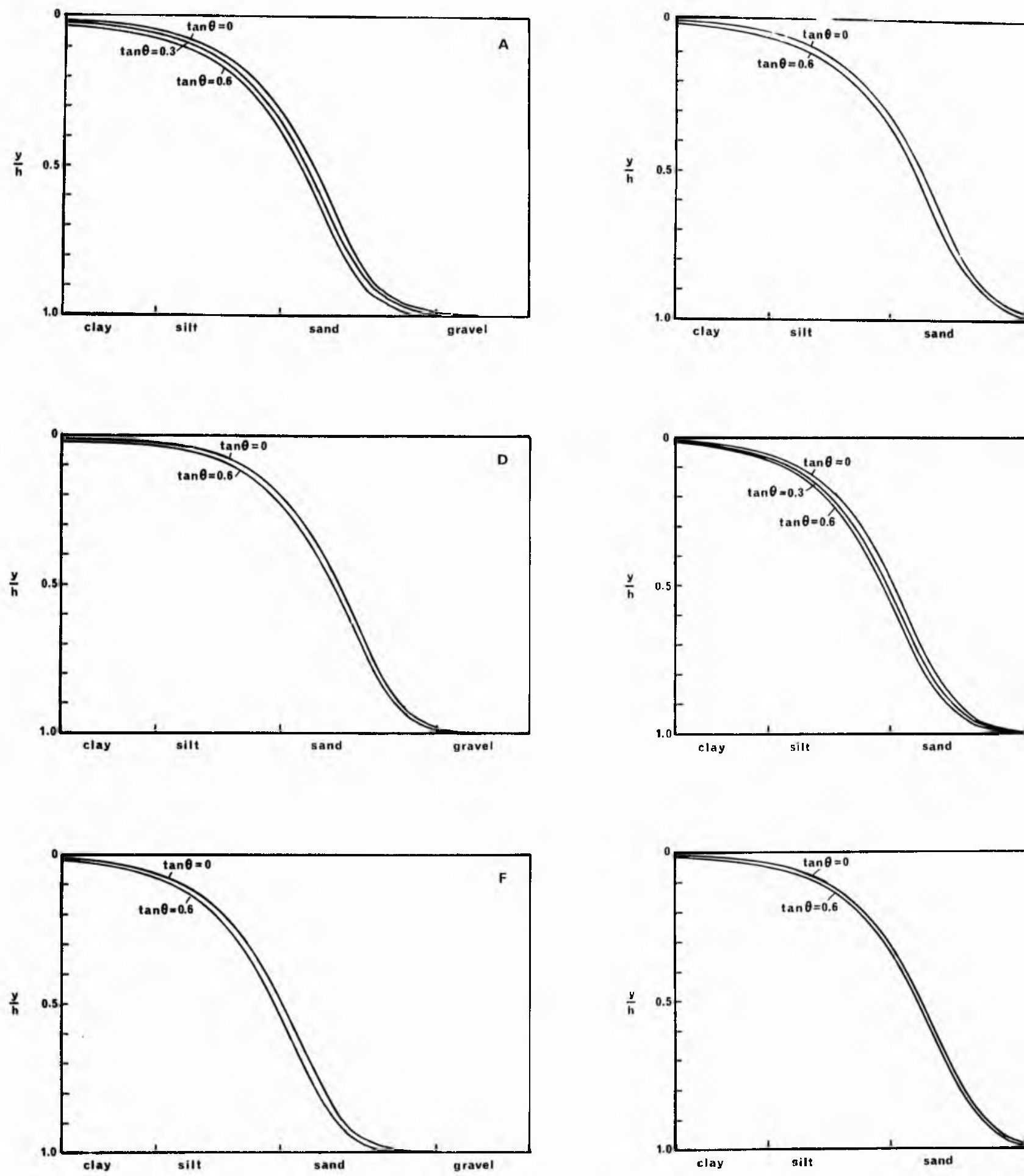

\begin{tabular}{|l|l|l|l|r|l|}
\hline & $h$ (metres) & $w_{1}$ (metres) & $r_{m}$ (metres) & $s$ & \\
\hline A & 1.05 & 405.0 & 4050.0 & 0.000285 & $n_{1}=2$ \\
C & 3.2 & 96.0 & 336.0 & 0.000125 & $k_{1}=0.8$ \\
D & 1.4 & 110.0 & 228.8 & 0.0000973 & $\sigma=2.65 \mathrm{gm} / \mathrm{cm}^{i}$ \\
E & 1.92 & 48.0 & 201.6 & 0.000124 & $\rho=1.0 \mathrm{gm} / \mathrm{cm}^{3}$ \\
F & 3.2 & 80.0 & 300.0 & 0.0000666 & \\
G & 5.55 & 138.0 & 520.0 & 0.0000768 & \\
\hline
\end{tabular}

FIG. 5.5 GRAIN SIZE PROFILES, ACCORDING TO EQUATION(5.19), C FOR DIFFERENT VALUES OF DYNAMIC FRICTION COEFFICIE OTHER INPUT PARAMETERS TAKEN FROM ALLEN, 1970a. 


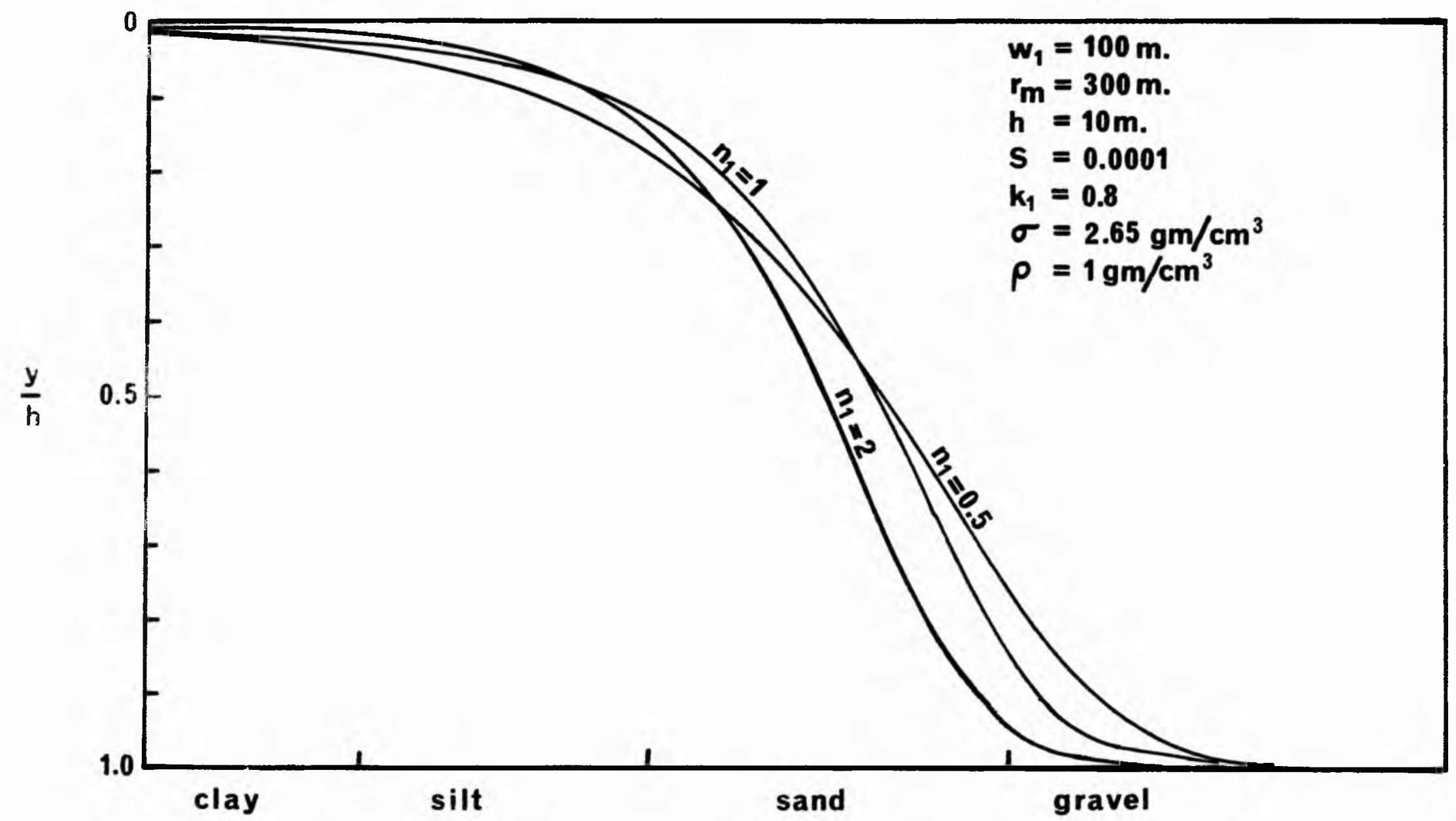

Fig.5.6 Grain size profiles, according to equation(5.20), calculated for different values of $n_{1}$. 
calibxe decreases with increasing radius of curvature of the bend and convexity of the cross profile. As regards the cross profile, particle size increases from $D=0$ on the inner bank of the channel, to $\mathrm{D}=\infty$ at the talweg. In practice $\mathrm{D}$ is not equal to infinity at the talweg, though it is commonly large, gravel being present. The steepness of the grainsizo profile is very sensitive to changes in $n_{1}$ and fig. 5.6 illustrates some of the alternative grain size profiles obtained with different values of $\mathbf{n}_{1}$

5.5 Variation of bed form and internal structure over the cross profile

In the absence of large bars, the bed forms that occur in flume experiments and natural rivers (depending on flow, fluid, geometry and sediment characteristics) are ripples, ripples on dunes, dunes, plane beds, antidunes, and chutes and pools. Theso bed forms are classipied into a lower flow regimo, an intermediate trangition zone, and an upper flow rogime (simons et al., 1961, 1965; Simons and Richardson, 1966, 1971). Classification is based on similarity of bed form, mode of sediment transport, and magnitude of resistance to flow (see fig. 5.7).

The primary sedimentary structures associated with ripples, dunes, flat beds and antidunes are respectively, cross lamination, cross bedding, flat bedding, and cxoss beds inclined at low angles upstream (A11en, 1968; Harms and Fahnestock, 1965). Although trough cross bedding is generally thought to be associated with dune migration, controversy exists over the exact nature, of the sedimentation process and the type of clunes responsible (Allen, 1968). Tabulax cross beds are thought to be associated with straight crested dunes, although rlat topped transverse bars are probably responsible for some of the larger scale varietles $($ Alen, 1968). 


\begin{tabular}{|c|c|c|}
\hline Flow regime & Bed form & $\begin{array}{c}\text { Bed Material } \\
\text { concentrations } \\
(\mathrm{ppm})\end{array}$ \\
\hline \multirow{3}{*}{ Lower regime } & Ripples & $10-200$ \\
\hline & Ripples on dunes & $100-1,200$ \\
\hline & Dunes & $200-2,000$ \\
\hline Transition & $\begin{array}{l}\text { Washed out } \\
\text { dunes }\end{array}$ & $1,000-3,000$ \\
\hline \multirow{3}{*}{ Upper regime } & Plane beds & $2,000-6,000$ \\
\hline & Antidunes & $2,000 \rightarrow$ \\
\hline & $\begin{array}{l}\text { Chutes and } \\
\text { pools }\end{array}$ & $2,000 \rightarrow$ \\
\hline
\end{tabular}

Fig. 5.7. Classification of flo 


\begin{tabular}{|c|c|c}
\hline $\begin{array}{c}\text { Mode of sedi- } \\
\text { ment transport }\end{array}$ & $\begin{array}{c}\text { Type of } \\
\text { roughness }\end{array}$ & $\begin{array}{c}\text { Phase relation be- } \\
\text { tween bed and } \\
\text { water surface }\end{array}$ \\
\hline Discrete steps & $\begin{array}{c}\text { Form roughness } \\
\text { predominates }\end{array}$ & Out of phase \\
\hline Continuous & $\begin{array}{c}\text { Variable } \\
\text { predominates }\end{array}$ & In phase \\
\hline &
\end{tabular}

regime, (from Simons et al., 1965). 


\section{5 .1 Allen's mode1}

To determine the variation of bed form, hence sedinentary structure over the cross profile, Allen (I970a,b) draws heavily on empirical information. The results of Guy et al. (1966) and Wiliams (1967) lead to fig. 5.8 showing that the occurrence of ripples, dunes and lower phase plane beds in quartz density sands is determined by stream power and calibre of load. It will be noted that a plane bed, and not ripples, is generated at conditions just a little more severe than the threshold of movement in the case of quartz density sands for which $\mathrm{D} \geqslant 0.065 \mathrm{~cm}$. If, in the case of a given rlow, the flow conditions are made severe enough, a plane bed referable to an upper phase of such beds wili appear. Upper phase plane beds depend fox their appearance simply on a relationship between the bed shear stress and the body force exerted by the particles of the load, and not uniquely on stream power, as seen below.

Fig. 5.9 shows the variation of the Daxcy-Weisbach friction coefficient with stream power and bed form, using the diata of Guy et al. (1966). It can be soen that there is a woll defined value of the stream power at which ripples or lower phase plane beds change to dures for a given ealibre of load, but that plane beds overlap with dunes as regards stream power. Under very severe flow conditions in an open channel, antidunes appear when the Froude number is in the neighbourhood of unity. These bed forms also are not uniquely determined by stream powex.

Roferring to $f i g .5 .9$ it can be supposed that rlpples and dunes are associated with a constant value of the friction coefficjent. This will be designated as $f_{1}(=0.08$ in $f i g .5 .9)$. It can also be supposed that plane beds of either phase and anticiunes also take a constant value $f_{2}(=0.02$ in $f i g .5 .9)$. In practice, the friction coefficient for a given bed form is not 


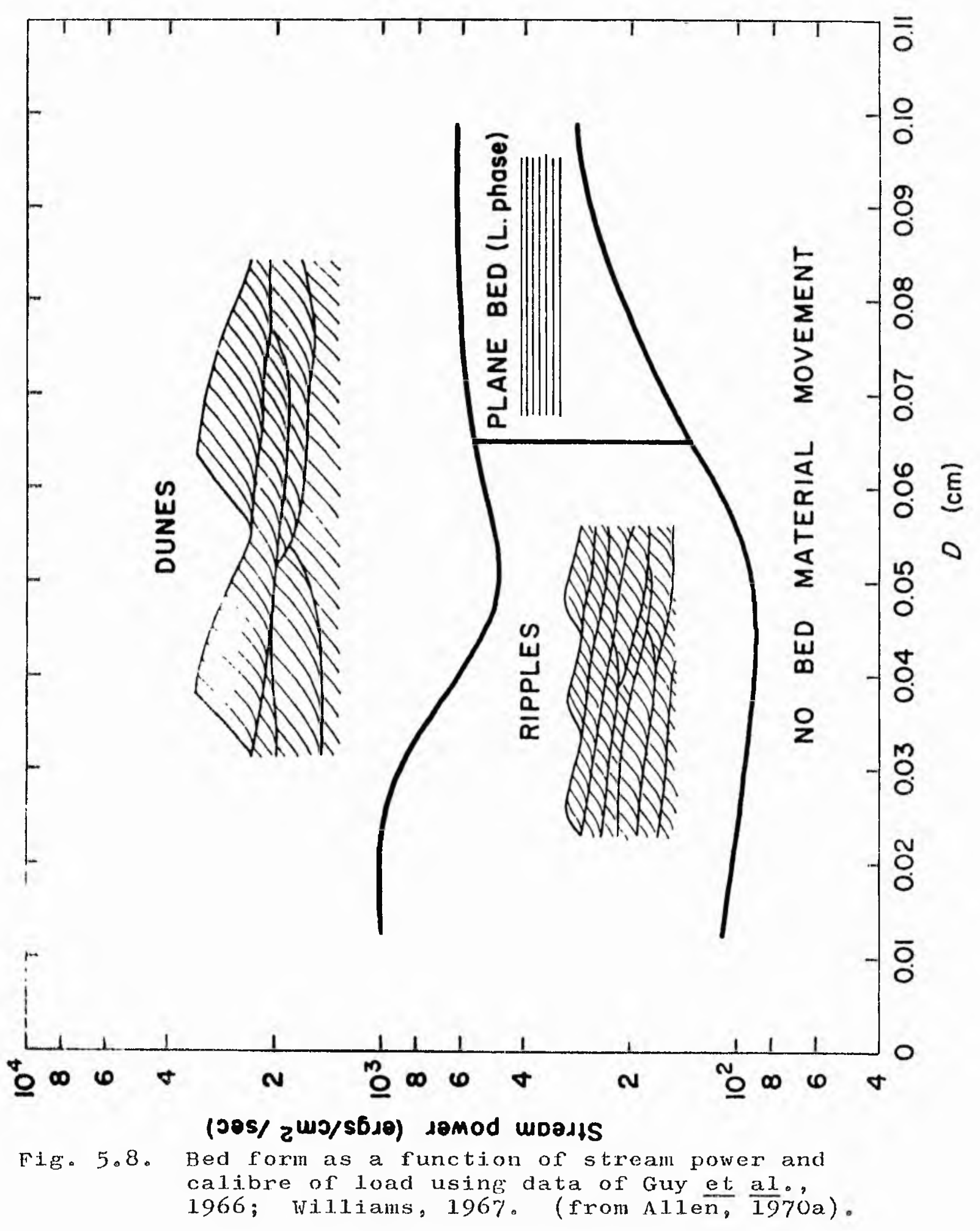



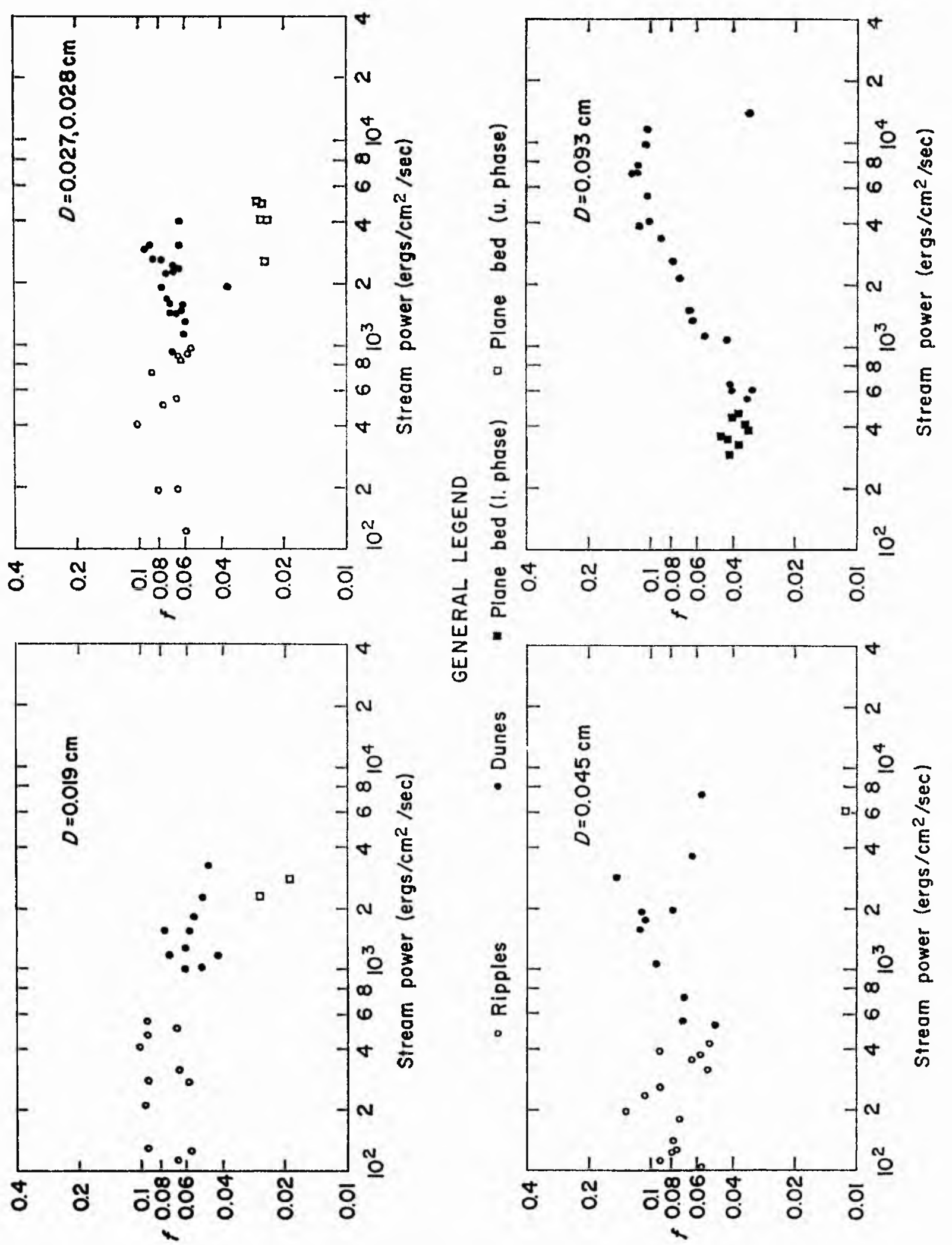

Fig. 5.9. Darcy-Weisbach friction coefficient as a function of bed forms, stream power and calibre of load using data of Guy et all. 1.966. (from A11en, 1970a)。 
unique, being subject to a $20 \%$ variation or thereabouts in the diagram shown. Allen (1970a) considers the assumption of a constant value of the coefficlent acceptable at the level of accuracy desired in the study. One further point is that diagram 5.9 refers to experiments in straight flumes, therefore the values of $f_{1}$ and $f_{2}$ quoted by Allen must be adjusted to account for the additional lossos associated with bends, according to equation (5.6). This is discussed fully in section 5.6.3. Kennedy (1963) showed that the minimum Froude number fox the appearance of antidunes 1 is $\mathrm{Fr}=0.84 \%$ and that the Froude number at which antidunes are the bed forms is insensitive to changing flow conditions. Therefore the bed form in the channel is antidunes if

$$
F r=\sqrt{\left(8 S / r_{2}\right)} \geqslant 0.844
$$

but is either a plane bed, dunes or ripples if

$$
\sqrt{\left(8 S / r_{2}\right)}<0.844
$$

If the bed form is ripples or dunes by the inequality (equation (5.22)), then the friction coefficient $f_{1}$ is used in the calculation of actual mean flow velocity, froude number and strean power.

In order to say whether a planc bed, dunes or ripples appear as the bed form at a given station, we first write for that station the dimensionless shear stress

$$
\theta=\tau_{x} / g D(\sigma-\rho)
$$

where $\theta$ is the dimensionless shear stress and $\tau_{\mathbf{X}}$ and $D$ are the bod shear stress parallel to the channel centre line and the particle diameter, respectively, at the given station.

Bagnold (1966) and Hill (1966) showad theoretically, with an experimental justification, that granular solids driven over the bed of a fluid stream will exist as an upper phase plane bed 
provided that

$$
\text { (1) } \geqslant \theta \operatorname{crit}
$$

wherein $\theta_{\text {crit }}$ is the critical value of the dimensionless shear stress, dependent on particle size. When this inequality is not satisfied then either ripples, lower phase plane beds, or dunes will appear, depending on the local stream power. Substituting for D from equation (5.20), equation (5.23) can be written as

$$
\theta=\frac{\pi n_{1} x_{1} h^{\left(n_{1}-1\right)} \sin \pi\left(\frac{z^{2}}{w_{1}}\right)^{n_{1}}}{33 w_{1}{ }^{n_{1}} y}
$$

which, like equation (5.20) itself, depends strongly on exponent $\mathbf{n}_{1}$

Inspection of equation $(5.25)$ will show that the value of 9 for a channel bend increases in general magnitude with ascending radius of curvature, but decreases with increasing flow width. Thus large ratios of radius of curvature to channel width favour upper phase plane beds as the bed form, whereas small ratios favour ripples and dunes. There is, however, a critical range of values of the ratio which could permit upper phase plane beds at restricted levels in the channel cross profile, depending on values of the exponent $n_{1}$ (see fig. 5.10).

It remains explicitily to assign numerical values to o crft. According to the results of Bagnold $(1954,1966)$ these are, approximately

$$
\begin{aligned}
& \theta_{\text {crit }}=0.52(\mathrm{D}<0.025 \mathrm{~cm} .) \\
& \theta_{\text {crjt }}=(0.56-1.430)(0.025 \leqslant D \leqslant 0.20 \mathrm{~cm} .) \\
& \theta_{\text {crit }}=0.27(\mathrm{D}>0.20 \mathrm{~cm} .)
\end{aligned}
$$

for quartz density sands in watex, depending primaxily on how the gratns behave when sheared in dense array over the stream bed, which was discussed proviously in soction 5.4 . 


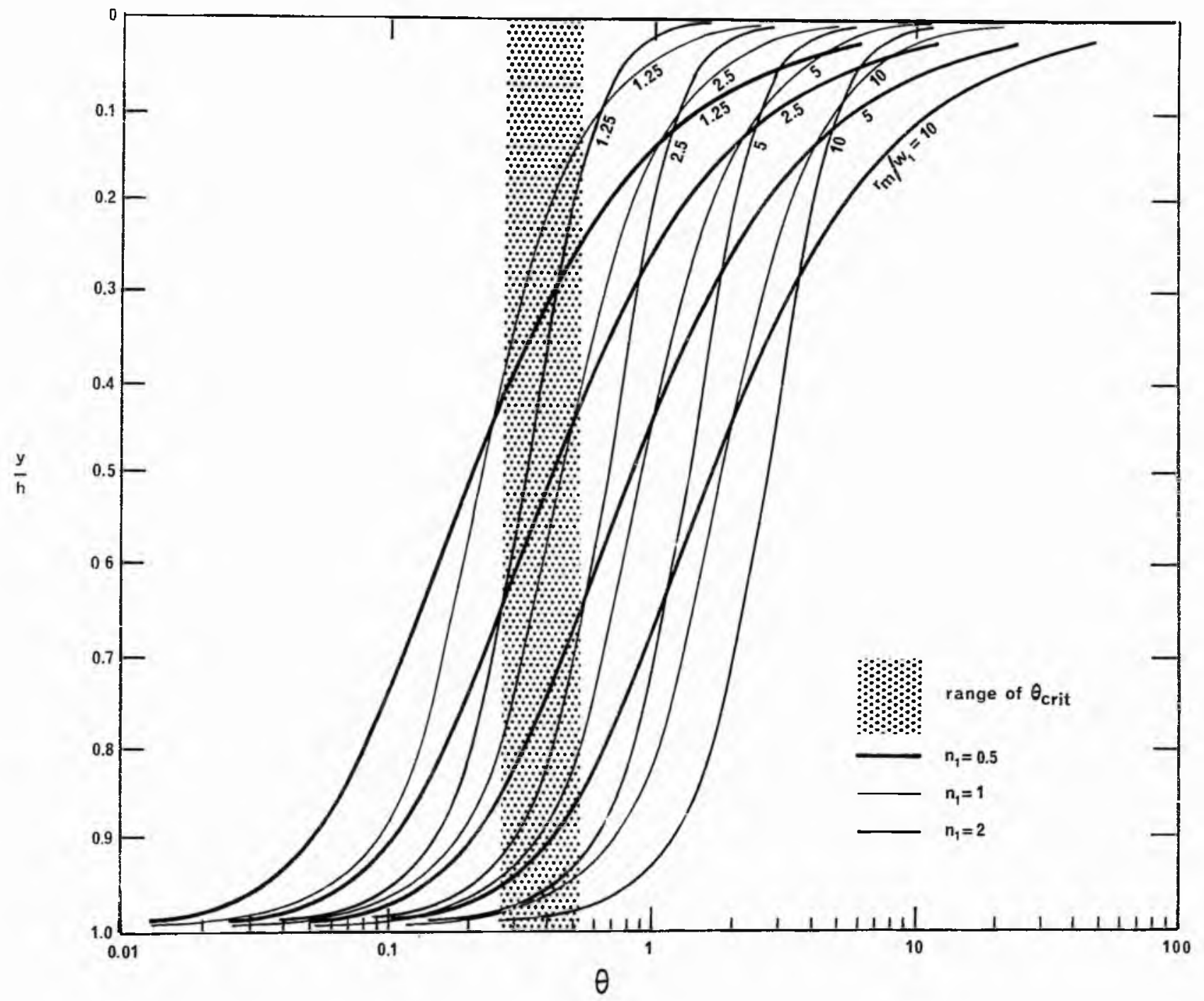

FIG. 5.10 PROFILES OF DIMENSIONLESS BED SHEAR STRESS AS A FUNCTION OF RATIO OF RADIUS OF CURVATURE TO CHANNEL WIDTH, ACCORDING TO EQUATION (5.25). 
Either ripples, lower phase plane beds, or dunes are the bed form if the inequality, equation (5.24), is not satisfied. The choice may be made on the basis of the local stream power, for, as can be seen in figure 5.9, there is a definite value of the power for a given calibre of load at which ripples or lower phase plane beds give place to dunes. The bed foxm is dunes if

$$
\omega \geqslant \omega_{\text {crit }}
$$

where $\omega_{\text {crit }}$ is the critical power for the transition from ripples or lower phase plane beds to dunes, but ripples or lower phase plane beds if

$$
\omega<\omega_{\text {crit }}
$$

values of $\omega_{\text {crit }}$ can be obtained from the experimental data summarised in fig. 5.9, thus

$$
\begin{aligned}
& \omega_{\text {crit }}=750(D \leqslant 0.023 \mathrm{~cm} \cdot) \\
& \omega_{\text {crit }}=950(0.023<D \leqslant 0.036 \mathrm{~cm} \cdot) \\
& \omega_{\text {crit }}=475(0.036<D \leqslant 0.069 \mathrm{~cm} \cdot) . \\
& \omega_{\text {crit }}=520(D>0.069 \mathrm{~cm} \cdot)
\end{aligned}
$$

where $\omega_{\text {crit }}$ is in the units ergs $\mathrm{cm}^{-2} \mathrm{sec}^{-1}$. If the inequality, equation $(5.27)$, is not satisfied, then from fig. 5.8 ripples are the bed form if

$$
\mathrm{D} \leqslant \mathrm{D}_{\text {crit }}
$$

but lower phase plane beds appear when

$$
\mathrm{D}>\mathrm{D}_{\mathrm{crit}}
$$

where $D$ is the particle diameter of the local bed material and $D_{\text {crit }}=0.065 \mathrm{~cm}$.

Thus the bed form is selected by the application of a series of inequalities to stations on the channel cross profilo. 
Whether antidunes appear is determined by the Froude number, controlled primarily by the longitudinal slope of the water surface. It may be noted that since in the present model the slope is assumed constant in each cross section, antidunes either fill the whole channel width or do not appear at all. In the field, however, antidunes can occur in the same reach of the river as other bed forms (Kennedy, 1963). If the Froude number of flow is less than that required for antidunes, either plane beds, dunes or ripples may occur. Distinction between an upper phase plane bed and dunes, ripples or lower phase beds is made on the basis of an inequality involving the bed shear stress and the callbxe of load combined in the form of a dimensionless stress. When the dimensionless stress falls below a critical value for an upper phase plane bed, either dunes, ripples or lower phase plane beds may be the bed form. The choice between the latter three is made using the knowledge that ripples or lower phase plane beds give place to dunes at a critical value of the stream power, and that ripples occur only when the calibre of the load material is less than a certain value.

\section{5 .2 Alternative models}

Allen's (1970a,b) model for the prediction of bed form across the channel cross profile draws on the results of both theoretical and experimental work. Over the years many authors have attempted to predict the hydraulic limits for the existence of the various bed forms, and consequently a large body of experimental and field data exist. The predictive methods used have been by graphical or multivariate statistical analysis of empirical data, based on some theoxetical reasoning, ox by purely theoretical approaches. These methods are summarised, for example, in Allen (1968), Graf (1971), Raudkivi (1967), and Simons and Richardson (1971).

It is not intended here to go into the analysis of 
alluvial bed form wechanics (see, for instance, Allen (1968), Mercor (1971), Raudkivi (1967)), or perform a cxitical assessment of the many different approaches to the problem of bed form prediction. It is intended to describe some alternative models to that of $\Lambda 1$ ien, which are thought to be equally acceptable in view of the prevailing state of knowledge. These alternative models inevitably contain certain elements in common with each other and with Allen's model. The differences that exist lie essentially in the prediction of the change from lower regime forms to upper regime forms.

\section{5 .3 Altexnative model no. 1}

In discussing the graphical method of prediction, simons and Richardson (1971) point out its inabllity to consider all the variables involved in the problem, as opposed to the multivariate statistical technique. They point out the failures of some of the graphical methods proposed, and conclude that the rolation between bed form, stream powex, and median fall dianeter of bed matexial fits the field and flume data fairly well. In fact the Jower flow regime part of this relation is used in Allen's model previously discussed, with the substitution of median grain size for fall diameter.

Simons and Richardson (1971) also favour a Fr,R/D plot proposed by Athaullah and Simons (1970), however this plot only distinguishes between regimes cather than specific bed form types (see $\mathrm{fIE} \cdot$ 5.11). In this model, therefore, aditional criteria are required to distinguish the different bed forms within the regimes. The delineation of the transition regime constitutes an improvement on $\Lambda 11$ en's model.

From fig. 5.11, the equations of the lines dividing the upper regime, transition and lower reglme can be obtained. The line dividing the upper flow regime from the transition is given 


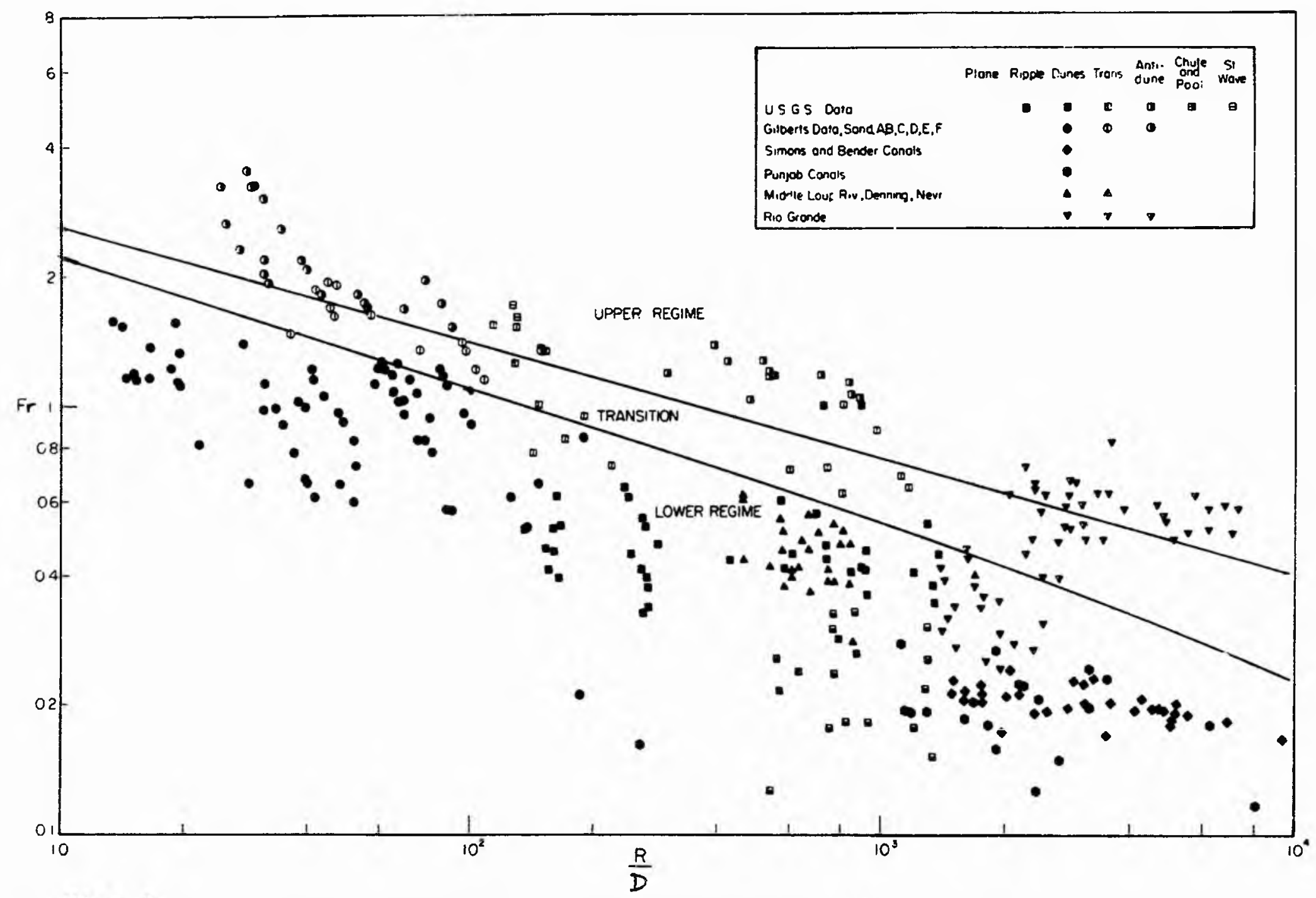

Fig. 5.11. Froude number, Fr, versus R/D criterion. (from simons and Richardson, 1971). 


$$
\log _{1.0} F x_{u}=0.75-0.27 \log _{10}(R / D)
$$

where $\mathrm{Fr}_{\mathrm{u}}$ is the Froude number at the change from transition to upper flow regime, $R$ is the hydraulic radius and $D$ is the particle diameter. Upper flow reglme forms, upper phase plane beds or antidunes, will therefore form at a station if

$$
\log _{10} \mathrm{Fr}=\log _{10}\left(\sqrt{8 \mathrm{~S} / \mathrm{f}_{2}}\right)>\log _{10^{\mathrm{Fr}}} \text {. }
$$

In order to separate the antidunes and plane bed fields, the critical Froude number of 0.844 is used, as in Allen's model. The line dividing the transition from the lower flow regime is given approximately by

$$
\log _{10} \mathrm{Fr}_{\mathrm{t}}=0.67-0.33 \log _{10}(\mathrm{R} / \mathrm{D})
$$

where $F r_{t}$ is the Froude number at the change from lower flow regfime to transition. Lower flow regime bed forms, dunes, ripples, or lower phase plane beds, will therefore form at a station if

$$
\log _{10} F x=\log _{10}\left(\sqrt{8 \mathrm{~S} / \mathrm{f}_{1}}\right) \leqslant \log _{10} F r_{t} .
$$

The lower flow regime bed foxm fjelds will be separated by the stream power, nedian diametex of bed material criterion, fig. 5.8 , a.s in Allen's model.

It should be noted hexe that extrapolation of boundary lines separating bed forms or regimes, outside the data fields to which they relate, is not strictly valid. This should be borne in mind when using equations (5.32) and (5.34) above, and in any other cases where a limited range of data points is used.

\section{5 .4 Alternative model no. 2}

This model is the same as Allen's except that the existence of an upper phaso plane bed instead of ripples or dunes is determined using the criteria proposed by Hill et al. (1969). 
From dimensional analysis and theoretical considerations, they produce a general functional relationship applicable for the instabiltty of an upper phase plane bed,

$$
\frac{v_{* \operatorname{crit}} \mathrm{D}}{\nu}=f\left(\frac{G D^{3}}{\nu^{2}}\right)
$$

where $V_{*}$ crit is the critical shear velocity for the instability and $\mathcal{\nu}$ is the kinematic viscosity $\left(\mathrm{cm}^{2} / \mathrm{sec}\right)$. Shear velocity, $v_{*}$, is defined here as $\sqrt{\tau_{x} / \rho}$, and has the dimensions of velocity. They further state that the two instabilities of upper phase plane bed to dunes and upper phase plane bed to ripples would represent two distinct functional relationships. Fig. 5.12 represents the stability diagram drawn from their own experimental data combined with that of other investigators, and demonstrates the existence of the two apparentyy distinct trends. The greater scatter of points shown by the data of 'other investigators' is due to the fact that the lowest obsexved values of shear on a plane bed were used. It should be realised that no transition regime is explicitly recognised in this model.

For high values of $\mathrm{gD}^{3} / \nu^{2}$ the plane bed is replaced by dunes while for low values the plane bed changes over to ripples. The authors explain this situation in terms of a dominant force at the particle level. The parameter $\mathrm{gD}^{3} / \nu^{2}$ can be 1ooked upon as a ratio of the gravitational force to the viscous force on the particle. Then it simply follows that when the gravitational force dominates compared to the viscous force dunes result on the plane bed. On the other hand, if the viscous forces are more dominant than the gravitational forces, ripples seem to develop on the plane bed.

Hill et al. (1969) then tried to fit equations of the form 


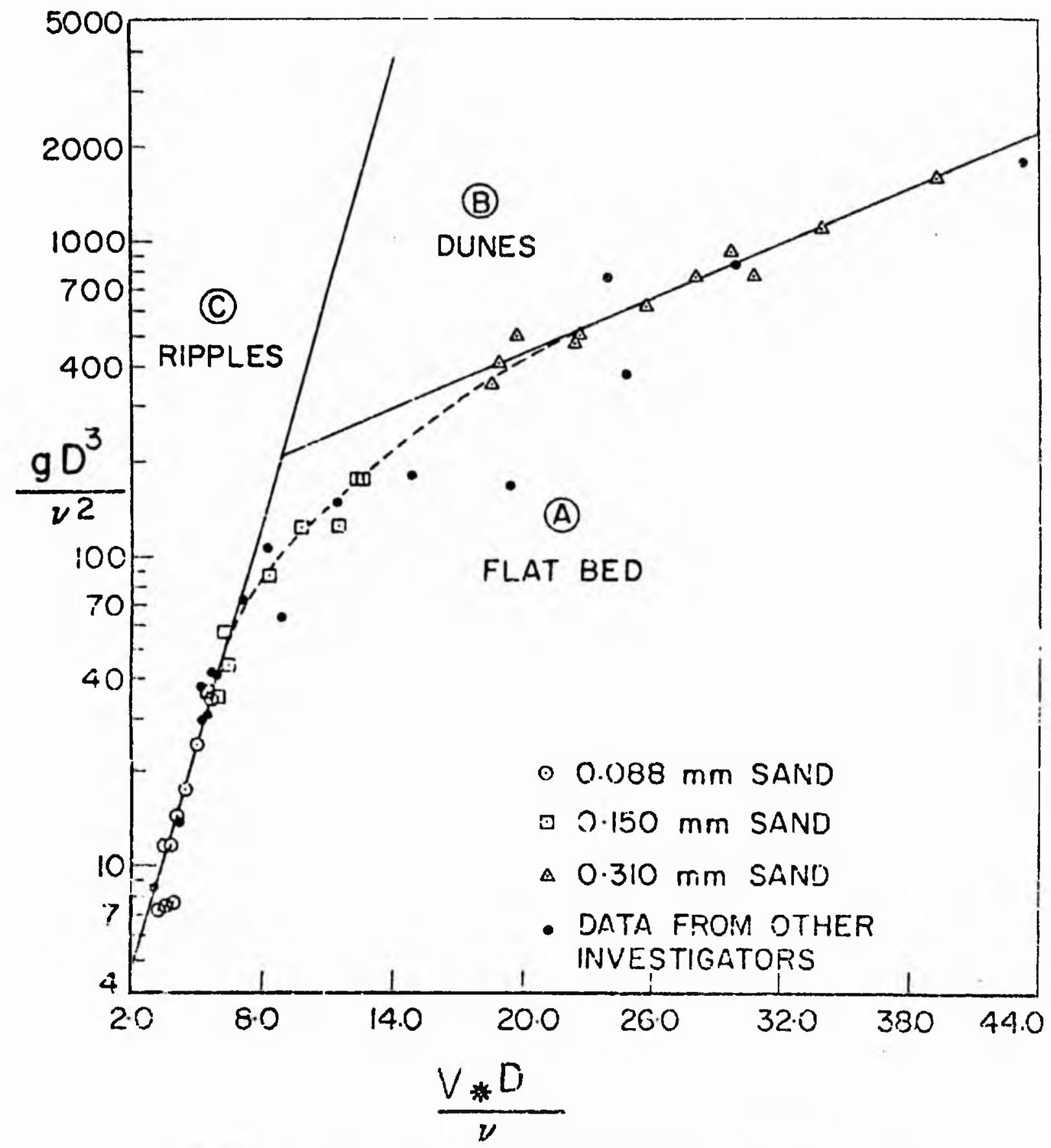

Fige 5.12. Stability diagram from Hill et al., 1969: solid lines indicate limiting stability condition; broken lines indicate zone of transition. 


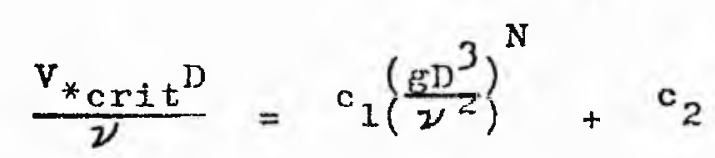

to the straight lines of fig. 5.12, where $c_{1}$ and $c_{2}$ are constants and $N$ is an exponent. In general it is not possible to fit an equation of the form given above to straicht lines drawn on semilog scales. In fact subsequent examination has revealed that the best fit equations derived by the authors were fitted to all tize data for each type of instability and not to the straight lines marked on fir. 5.12 (II111, 1972, pers. comm.).

The intersection point of the equations derived by HiII et al. $(1969)$ is at $v_{* \text { crit }} \mathrm{D} / 2 \approx 6.6, \mathrm{gD}^{3} / \nu^{2} \approx 60.63$. As can be seen from fig. 5.12, this point is neither at the intersection of the two straicht lines marked, nor at the point where the experimental data show the transition from one type of instability to the other, $1 . e . V_{* \operatorname{crit}} \mathrm{D} / \nu \approx 10.63, \operatorname{rg}^{3} / \mathcal{L}^{2} \approx 121.51$. The equations cannot be used therefore to determine when either type of instability will occur, because or this considerable inecouracy In the 'transition' area. In order to owercome this difficulty, a polynomial regression analysts was performed for all the data points available, as the data appears to vaxy as a smooth function. The resulting best fit oquation is

$$
\begin{aligned}
& \frac{V_{* c r i t^{D}}}{\nu}=3.13+0.073\left(\frac{\mathrm{rD}^{3}}{\nu^{2}}\right)-0.92 \times 10^{* 4}\left(\frac{\left.g D^{3}\right)^{2}}{\nu 2}\right)^{2} \\
& +0.62 \times 10^{-7}\left(\frac{g D^{3}}{\nu}\right)^{3}-0.15 \times 10^{-10}\left(\frac{g D^{3}}{\nu^{2}}\right)^{4}
\end{aligned}
$$

As can be seen from fig: 5.13 , equation (5.38) describes the position of the transition between the two types of tnstabilities feixly vell, and coxresponds to a value of $\mathrm{wD}^{3 / 2} \mathrm{z}^{2}$ of about lao. Ful1 details of the polynomial regxession axe given in appendix 2. Thus, in the abserace of antidunes, uppex phase beds wis. form at: a station ix 


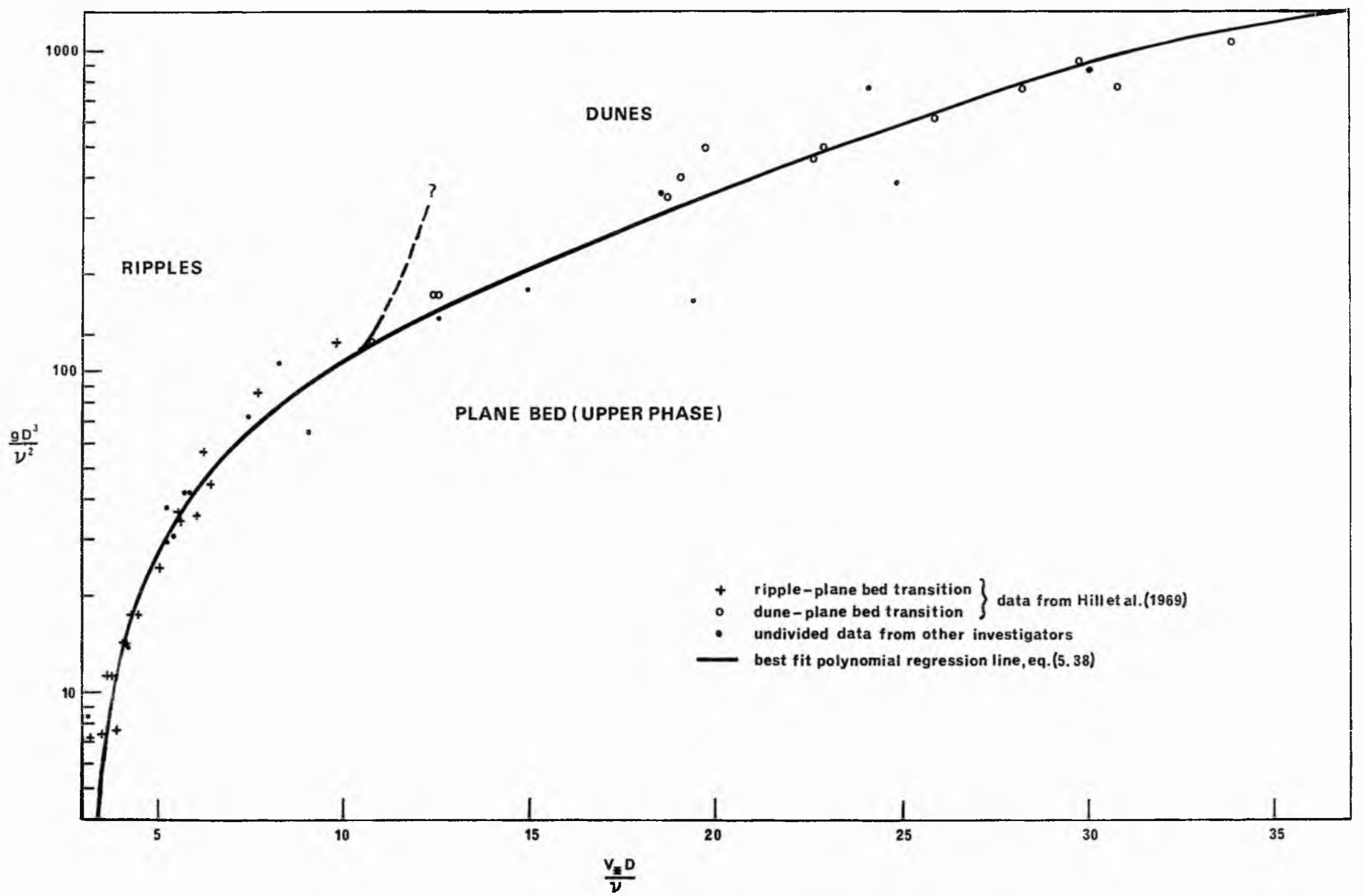

FIG. 5.13 STABILITY DIAGRAM FOR RIPPLES, DUNES AND UPPER PHASE PLANE BEDS, ACCORDING TO CRITERIA OF HILL ET AL, 1969. STABILITY BOUNDARY ACCORDING TO EQUATION (5.38). 


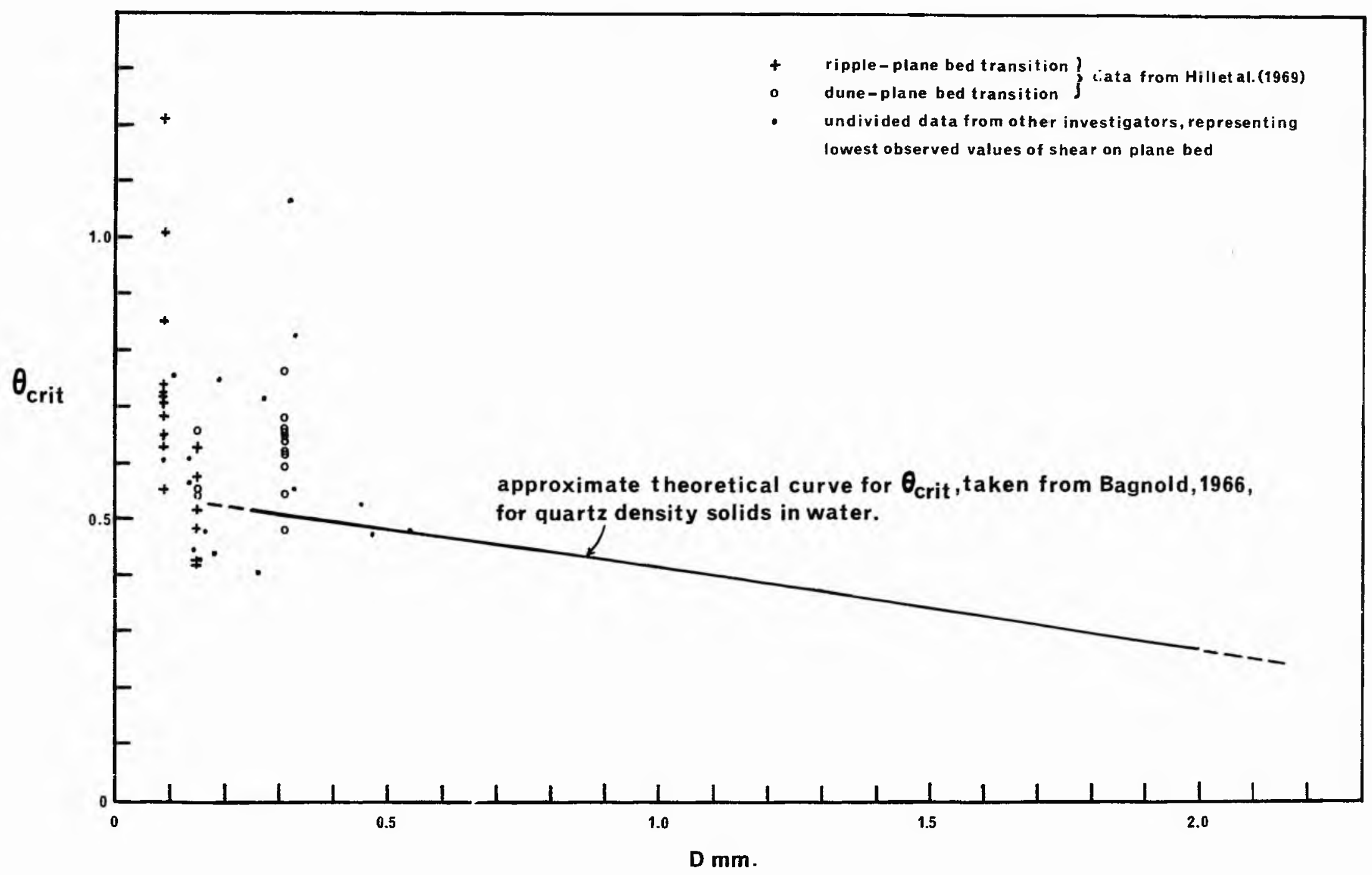

Fig. 5.14 Comparative plot of theoretical and empirical values of $\theta_{\text {crit }}$ against grain size. 


$$
\frac{v_{*} \mathrm{D}}{\nu}>\frac{\mathrm{v}_{* \operatorname{cr} i t^{\mathrm{D}}}}{\nu}
$$

otherwise dunes or ripples will form, depending on whethex $\mathrm{s}^{3} / \nu^{2}$ is greater or less than 120, respectively. By way of comparison of this method of prediction for upper phase plane beds and that used in Allen's model, we can rewrite $v_{*} \operatorname{crit}{ }^{D / \nu}$ as follows

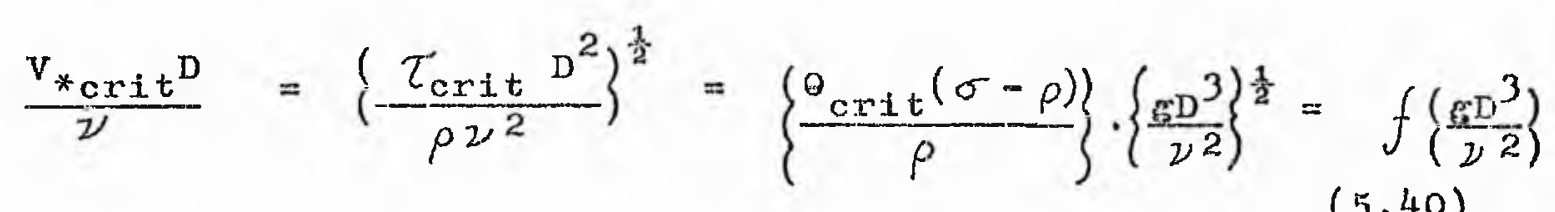

This is similar to the Bagrold cxiterion used in Allen's model except that,in addition, the viscosity of the fluid is taken into account. Fig. 5.14 shows orit plotted against D, using the data from Hill et a1's (1969) compilation and the values taken from Bagnold $(1954,1966)$ which Allen cites. It can be seen

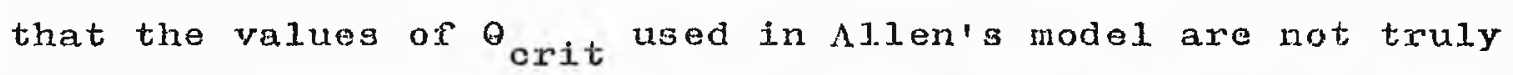
representative of the observed values for the ranie of $D$ covered.

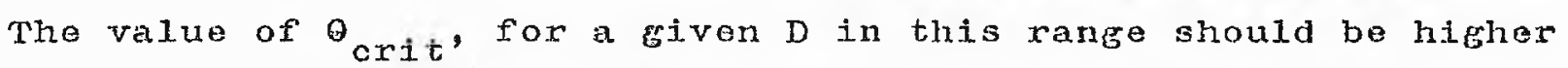
than is shown, and clearly the excessive scatter of the data is an indication of the omission of tmportant controlling factors. Indeed, Bamnold (1966) states... though the value of $\theta$ is an approximate guide in default of a better one, it is not, as polnted out earlier, a preciso criterion for either the disappearance of dune features on the bed of the change of trend in the transport rate versus power curves...' and '...dune reatures often pexsist at the higher flow stages...'.

\section{5 .5 Alternative mode1. no. 3}

One of the theoretical models that has received a lot of attention is that of Konnedy $(1.963$, 1969). Ho made an elaborate stability analysis of the bed forms on lines similar to those adopted by Anderson (1953), but recognised phase differences hetween bed and surface waves. He also rocognised that a change 
In the local transport rate might lag by a certain amount the causative change in the local fluid velocity.

Development of the model proceeds in the usual manner of fluid-stability analysis by tracing the development of sinesoidal shaped bed forms from a nearly flat bed on which thexe is an initial small sinesoidal disturbance. The flow over the develop* ing bed forms is assumed to be two mdimensional, irrotational and incompressible, and the free surface is assumed to adjust itself continuously according to the requirements of the Bernoulli equation. The Bernoulii equation requires that the surface disturbance also be sinesoidal with the same wavelength as the bed form and have an amplitude given by

$$
\frac{a(t)}{A(t)}=\left[1 . \cdot\left(1 / F r^{2} k d\right) \tanh k d\right] \cosh k d .
$$

where $a(t)$ and $\Lambda(t)$ are the amplitudes of the bed and surface disturbances respectively, d is the mean depth of flow, Fr is the Froude number based on the velocity for mean depth, and $k$ is the wavo number.

Equation (5.41) can be used to show the conditions under which, theoretically, dunes and antidunes can form. For dunes, the bed wave and surface wave axe $180^{\circ}$ out of phase and $a(t) / A(t)$ is less than zero; alternatively, fox antidunes the two wave forms are in phase and $a(t) / A(t)$ is greater than zero. setting $a(t) / \Lambda(t)$ to zero abóve gives

$$
\mathrm{Fr} \mathrm{r}^{2}=\tanh \mathrm{kd} / \mathrm{kd}
$$

which is shown platted in fig. 5.15 along with data accumulated by Kennedy (1963) from a number of sources. It does appear to separate the two types of bed forms successfully.

The sedinent transport relation was then formulated, which rolates transport rate to some power of the difference between flow 


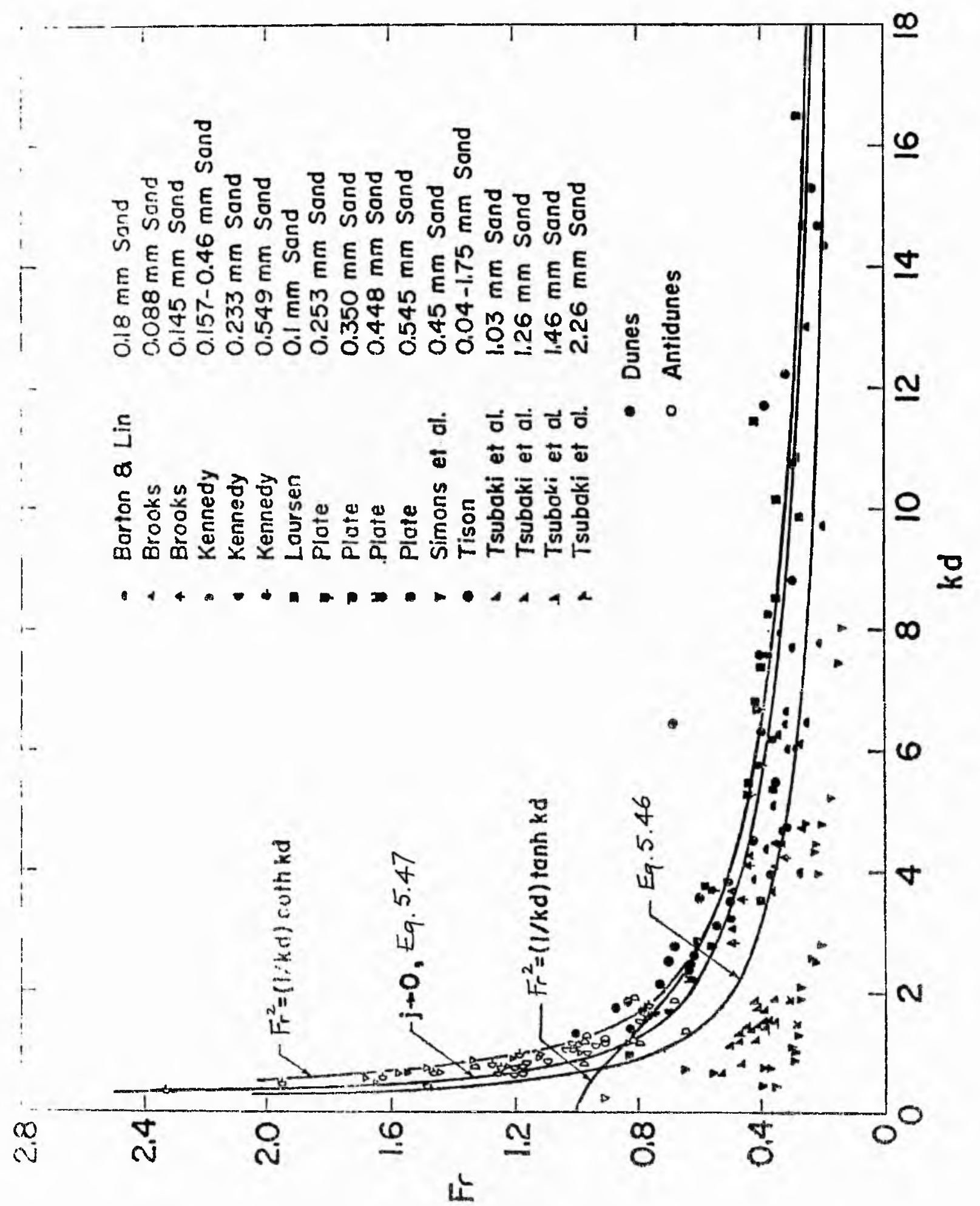

Fig. 5.15. Comparison of predicted and observed ranges of kd. (after Kennedy, 1969). 
velocity and the critical velocity for the initiation of motion (Kennedy, 1969). The problem here is, if a simple bed load equation is used relating sediment transport to velocity alone, the transport of material will bo symmetric about the crests and troughs so that the bed form will migrate but not grow. The asymmetry required for bed form growth is provided In Kennedy's model by introducing a lag distance $\delta$ whereby changes in bed load transport $\mathrm{lag}$ changes in velocity, as must be the case when particles at rest are picked up and accommodated in the main flow and moving particles are dropped and deposited in decelexating flow that can no longer move them (trangport relaxation distance). Anothex factor contributing to $\delta$ is the phase shifts between the bed displacement and the longitudinal distributions of the local flow properties (Kennedy, 1969). Using the sediment transport relations, the expressions for the velocity potential and the shape of the sinesoidal wave, and equation (5.42), given above, Kennedy (1969) dexived the following relation for the bed form velocity

$$
\mathrm{v}_{\mathrm{b}}=\overline{\mathrm{T}}_{\mathrm{n} k} \cdot \frac{\mathrm{V}}{\mathrm{V}-\mathrm{V}_{\mathrm{c}}} \cdot \frac{1-\mathrm{Fr} \mathrm{r}^{2} \mathrm{kd} \operatorname{tonh} \mathrm{kd}}{\tanh k \mathrm{k} \omega \mathrm{Fr} \mathrm{r}^{2} \mathrm{kd}} \cos \mathrm{k} \delta
$$

and for the bed amplitude

$$
\begin{aligned}
a(t)=A(0)\left(1 / F r^{2} k d\right) \cosh k d\left(F x^{2} k d-\tanh k d\right) . & (5.44) \\
& \exp \left\{t n \bar{T} k^{2} \cdot \frac{V}{V-V_{c}} \cdot \frac{1-F x^{2} k d \tanh k d}{F x^{2} k d-\tanh k d} \sin k \delta\right\}
\end{aligned}
$$

where $V_{b}$ is the bed form velocity, $v_{c}$ the cxitical velocity for initiation of motion, $v$ the mean flow velocity, $\vec{T}$ is the net forward sediment transport rate for the whole stream, $n$ is an exponent from Kennedy's transport law, and $t$ is time.

Iquation ( 5.44$)$ shows that the amplitude of small bed waves on an otherwise flat bed caused by any arbitrary disturbance 
will increase exponentially with time provided that $k$ and $\delta$ axe such that the exponential term is positive. In reality, factors not accounted for in this linearised model must intervene and fix the equilibrium height of the bed forms. Table 5.1 summarises the various classes of bed forms predicted by equations $(5.42),(5.43)$ and $(5.44)$, and the conditions of occurrence of each. The configurations are classed as antidunes or as ripples or dunes according as the bed and surface waves are in phase or out of phase, as already discussed. The sign of the exponent in equation (5.44) constitutes the stability criterion: positive, zero and negative values of the exponent correspond to unstable, neutrally stable, and stable configurat-ions respectively.

The possibility of an instability occurring at Froude numbers for which $F x^{2} k d$.tanh kd $>1$ was pointed out by Reynolds (1965). At these higher Froude numbers the horizontal component of the velocity perturbation changes sign between the bed and the free surface, whereas at lower values of Fr it retains the same sign over the full depth at each station. This high froude number region is of little practical importance, as for a given Froude number, values of $\mathrm{kd}$ less than that corresponding to $F x^{2} k d, \tanh k d=1$ have a greater initial growth and hence are dominant (Kennedy, 1969). The curve of $\mathrm{Fr}^{2} \mathrm{kd} \cdot \tanh \mathrm{kd}=1$ is therefore the upper limit of two dimensional waves (Reynolds, $1965)$

So far, no restrictions have been imposed on the wave1ength. However, it is observed in both laboratory flumes and natural streams that flow-generated bed configurations have characteristic wavelengths and amplitudes that depend on the properties of the flow, fluid, and bed material. It is now assumed, as is customary in classical fluid stability analysis, 


\begin{tabular}{|c|c|c|c|}
\hline Case & Froude Number & $\begin{array}{c}\text { Bed and } \\
\text { Surface Profiles }\end{array}$ & $k \delta$ \\
\hline 1 & $F_{r}^{2} k d>\tanh k d$ & In phase & \\
\hline $\begin{array}{l}\text { la } \\
1 \mathrm{~b} \\
\text { ic } \\
\text { id } \\
\text { le } \\
\text { if }\end{array}$ & $\begin{array}{l}F_{r}^{2} k d \tanh k d<1 \\
F^{2} k d \tanh k d<1 \\
F_{r}^{2} k d \tanh k d<1 \\
F_{r}^{2} k d \tanh k d<1 \\
F_{r}^{2} k d \tanh k d<1 \\
F_{r}^{2} k d \tanh k d<1\end{array}$ & $\begin{array}{l}\text { In phase } \\
\text { In phase } \\
\text { In phase } \\
\text { In phase } \\
\text { In phase } \\
\text { In phase }\end{array}$ & $\begin{array}{c}0,2 \pi \\
0<k \delta<\pi / 2 \\
\pi / 2 \\
\pi / 2<k \delta<\pi \\
\pi \quad \pi \\
\pi<k \delta<2 \pi\end{array}$ \\
\hline $1 g$ & $F_{r}^{2} k d \tanh k d=1$ & In phase & - \\
\hline $\begin{array}{l}1 \mathrm{~h} \\
1 \mathrm{i} \\
1 \mathrm{j} \\
1 \mathrm{k} \\
1 \mathrm{k} \\
\mathrm{im}\end{array}$ & $\begin{array}{l}F_{r^{2}} k d \tanh k d>1 \\
F_{r}^{2} k d \tanh k d>1 \\
F_{r}{ }^{2} k d \tanh k d>1 \\
F_{r}^{2} k d \tanh k d>1 \\
F_{r}^{2} k d \tanh k d>1 \\
F_{r}^{2} k d \tanh k d>1\end{array}$ & $\begin{array}{l}\text { In phase } \\
\text { In phase } \\
\text { In phase } \\
\text { In phase } \\
\text { In phase } \\
\text { In phase }\end{array}$ & $\begin{array}{c}0,2 \pi \\
0<k \delta<\pi \\
\pi \\
\pi<k \delta<3 \pi / 2 \\
3 \pi / 2 \\
3 \pi / 2<k \delta<2 \pi\end{array}$ \\
\hline 2 & $F^{2} k d=\tanh k d$ & $\begin{array}{c}\text { Indeterminate from } \\
\text { potential } \\
\text { formulation }\end{array}$ & \\
\hline 3 & $F r^{2} k d<\tanh k d$ & Out of phase & \\
\hline $\begin{array}{l}3 \mathrm{a} \\
3 \mathrm{~b} \\
3 \mathrm{c}\end{array}$ & $\begin{array}{l}F_{r^{2}} k d<\tanh k d \\
F^{2} k d<\tanh k d \\
F_{r}^{2} k d<\tanh k d\end{array}$ & $\begin{array}{l}\text { Out of phase } \\
\text { Out of phase } \\
\text { Out of phase }\end{array}$ & $\begin{array}{c}0,2 \pi \\
0<k \delta<\pi \\
\pi\end{array}$ \\
\hline $\begin{array}{l}.3 \mathrm{~d} \\
3 \mathrm{e} \\
3 \mathrm{f}\end{array}$ & $\begin{array}{l}F_{r^{2}} k d<\tanh k d \\
F_{r}^{2} k d<\tanh k d \\
F_{r}^{2} k d<\tanh k d\end{array}$ & $\begin{array}{l}\text { Out of phase } \\
\text { Out of phase } \\
\text { Out of phase }\end{array}$ & $\begin{array}{c}\pi<k \delta<3 \pi / 2 \\
3 \pi / 2 \\
3 \pi / 2<k \delta<2 \pi\end{array}$ \\
\hline
\end{tabular}

Table 5.1. Summary of conditions for occurre Kennerly, 1969). 


\begin{tabular}{|c|c|c|}
\hline Bed Stability & $\begin{array}{l}\text { Movement of } \\
\text { Bed Forms }\end{array}$ & $\begin{array}{c}\text { Bed } \\
\text { Configuration }\end{array}$ \\
\hline $\begin{array}{l}\text { Neutral } \\
\text { Unstable } \\
\text { Unstable } \\
\text { Unstable } \\
\text { Neutral } \\
\text { Stable }\end{array}$ & $\begin{array}{l}\text { Upstream } \\
\text { Upstream } \\
\text { None } \\
\text { Downstream } \\
\text { Downstream } \\
\quad\end{array}$ & $\begin{array}{l}\text { Antidunes } \\
\text { Antidunes } \\
\text { Antidunes } \\
\text { Antidunes } \\
\text { Antidunes } \\
\text { Flat bed }\end{array}$ \\
\hline Neutral & None & Antidunes \\
\hline $\begin{array}{l}\text { Neutral } \\
\text { Stable } \\
\text { Neutral } \\
\text { Unstable } \\
\text { Unstable } \\
\text { Unstable }\end{array}$ & $\begin{array}{l}\text { Downstream } \\
\text { Upstream } \\
\text { Upstream } \\
\text { None } \\
\text { Downstream }\end{array}$ & $\begin{array}{l}\text { Antidunes } \\
\text { Flat bed } \\
\text { Antidunes } \\
\text { Antidunes } \\
\text { Antidunes } \\
\text { Antidunes }\end{array}$ \\
\hline & $\begin{array}{c}\text { Indeterminate from } \\
\text { potential } \\
\text { formulation }\end{array}$ & $\begin{array}{c}\text { Indeterminate from } \\
\text { potential } \\
\text { formulation }\end{array}$ \\
\hline $\begin{array}{l}\text { Neutral } \\
\text { Stable } \\
\text { Neutral } \\
\\
\text { Unstable } \\
\text { Unstable } \\
\text { Unstable }\end{array}$ & $\begin{array}{l}\text { Downstream } \\
\text { Upstream } \\
\text { Upstream } \\
\text { None } \\
\text { Downstream }\end{array}$ & $\begin{array}{l}\text { Ripples or dunes } \\
\text { Flat bed } \\
\text { Ripples or dunes } \\
\text { (Transition) } \\
\text { " } \\
\text { Ripples or dunes }\end{array}$ \\
\hline
\end{tabular}

nce of various bed configurations (after 
that the characteristic or dominant wavelength is that for which the growth rate of the small amplitude disturbances is a maximum. The initial rate of amplixication is obtained by differentiating equation ( 5.44 ) with respect to $t$, then putting t to zero, i.e.

$$
a_{t}(0)=A(0) n \bar{T} k^{2} \frac{V}{V-V} c\left[\left(1 / F r^{2} k d\right) \cosh k d-\sinh k d \sin k \delta\right]
$$

Before the value of kd for which $a_{t}(0)$ is a maximum can be determined it is necessary to refine the specification of the lag distance. As previously mentioned, at least two factors contribute to $\delta$ : the phase shifts between the bed displacement and the longitudinal distributions of the local flow properties, and the transport relaxation distance. The relative importance of each for a given flow cannot presently be assessed, however it is possible to examine the dominant wavelengths corresponding to the two limiting cases in which one or the other of these contributors to $\delta$ can be disregarded (Kennedy, 1969).

Where the lag distance results only from phase shifts between the local flow properties and bed displacements, $\delta$ can, as a first approximation, be treated as a constant multiple of the wavelength. Introducing $\delta=\mathrm{c}_{3} 2 \pi / \mathrm{k}$ into equation ( 5.45$)$ and equating to zero the derivative of $a_{t}(0)$ with respect to $k$ gives

$$
F x^{2}=\frac{\cosh ^{2} k d}{k d(\sinh k d+k d)}
$$

In the other limiting case where the transport relaxation distance plays the predominant role, $\delta$ would be constant and independent of wavelength. It is then convenient to normalise by the flow depth and introduce $j=\delta / d$ into equation $(5.45)$. Differentiating the resulting cxpression for a ${ }_{t}(0)$ with respect to $k$ and equating to zero yields the following implicit equation for the dominant values of kd. 


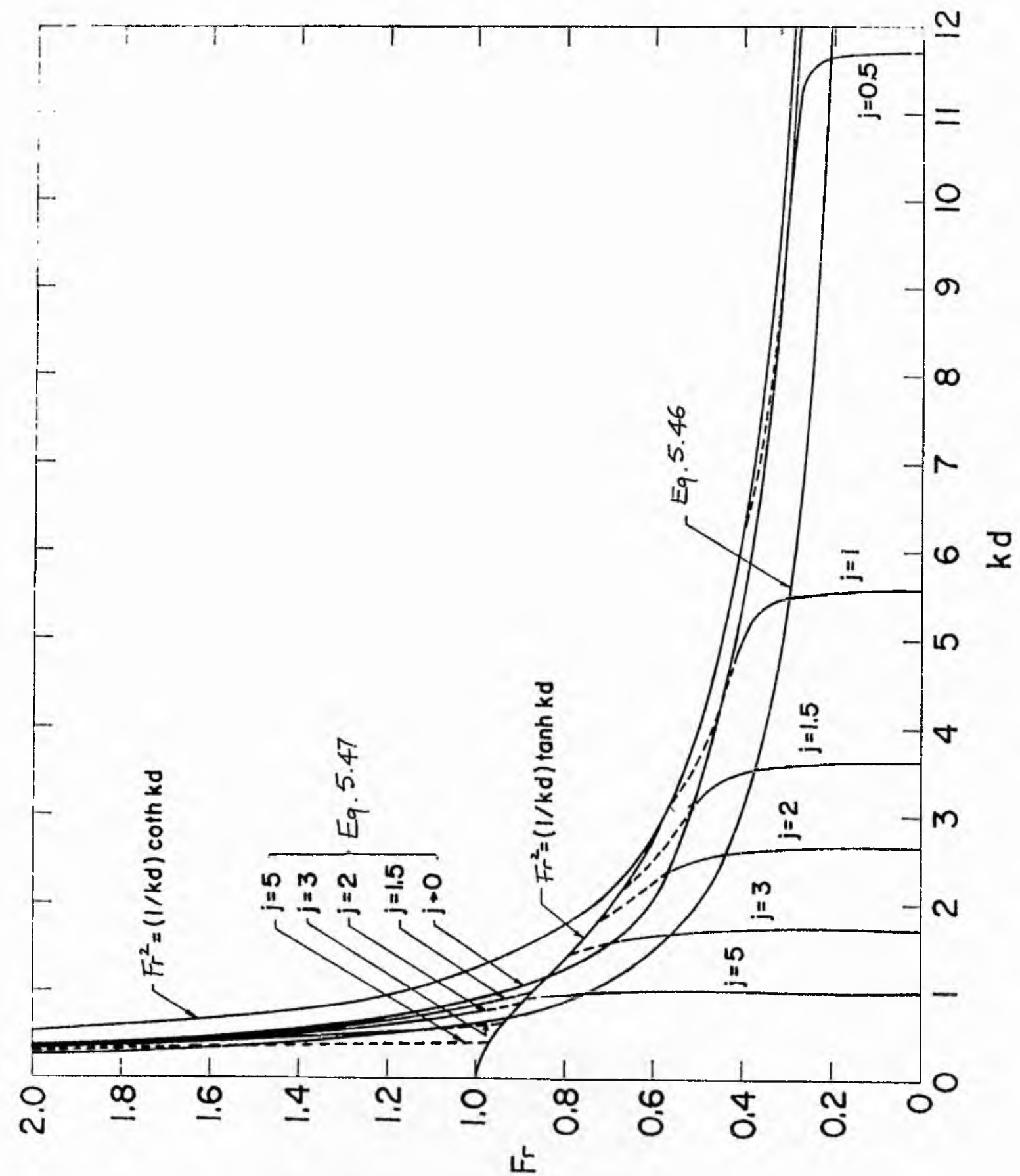

Fig. 5.16. Dominant kd given by equations (5.46) and $(5.47)$, and regions of occurrence of various bed forms. The various configurations are identified by the character of the Iines representing equation $(5.47)$, except $j \rightarrow 0$, as follows. Fr $\mathrm{r}^{2} \mathrm{kd}<$ tanhkd: solid lines correspond to ripples and dunes, dashed lines to transition. Fr2kd>tanhkd: solid lines correspond to antidunes moving upstream, dashed lines to antidunes moving downstream. (after Kennedy, 1969). 


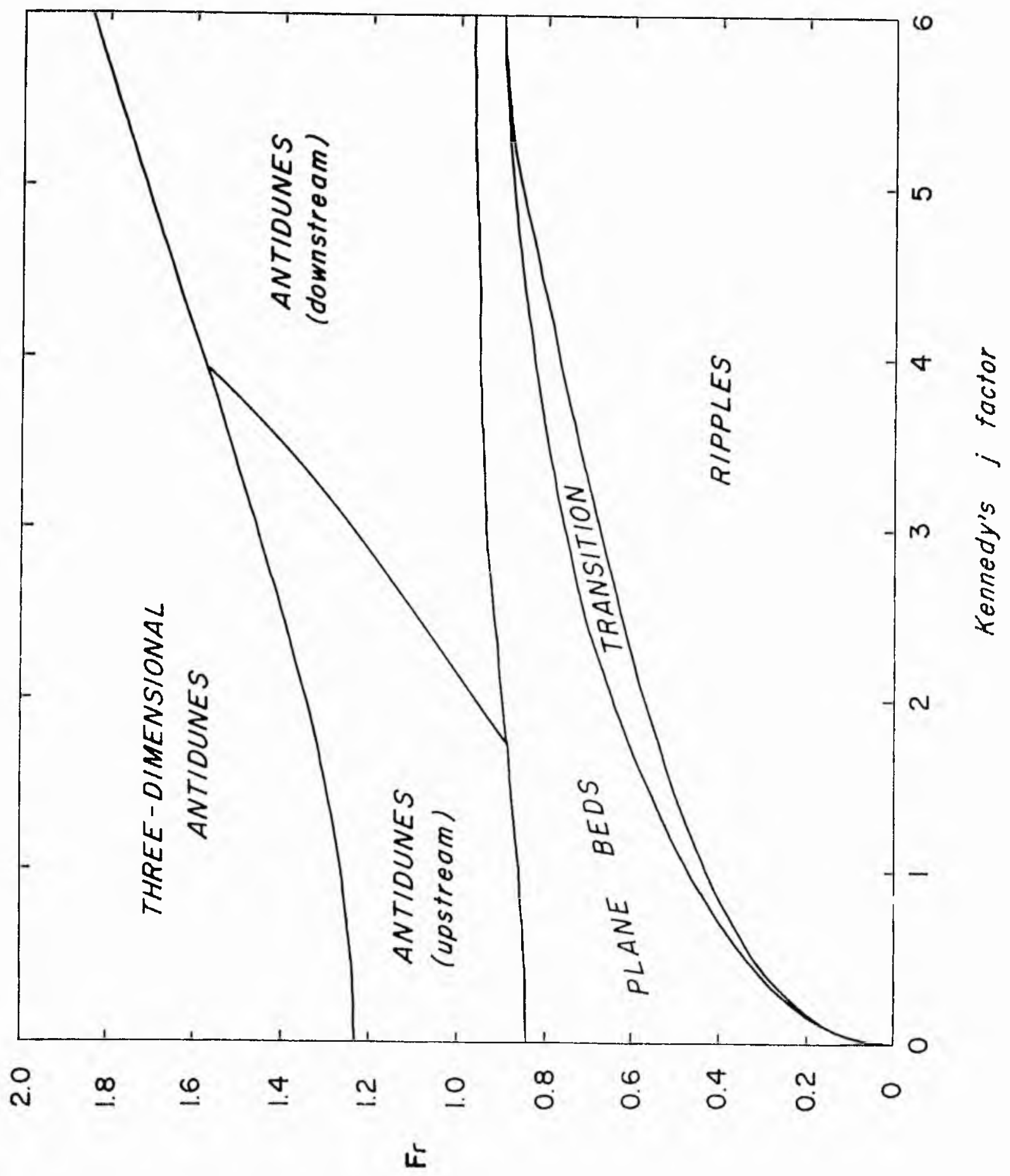

Fig. 5.17. Occurrence of bed forms according to analysis of $J . r$. Kennedy. Antidunes are distinguished according to whether they move upstream or downstream. (after Kennedy, 1963)。 


$$
F r^{2}=\frac{1+k d t a n h k d+j k d \cot j k d}{(k d)^{2}+(2+j k d \cot j k d) k d \tanh k d}
$$

Fig. 5.16 shows the relation between Fr and kd given by equations $(5.46)$ and $(5.47)$. Only the first two maxima of equation (5.44) are shown for each value of $j$; one each for

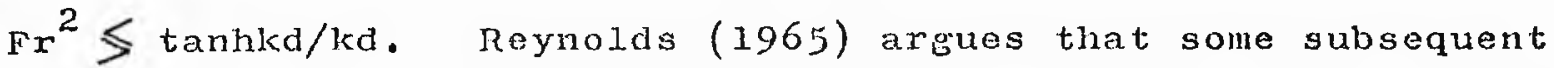
maxima actually have a higher growth rate, and hence should be retained. However, Kennedy (1969) states that it soems physically unlikely that the transport distribution over an individual bed form would be more strongly affected by more remote bed waves than by adjacent ones, and hence the higher harmonics are disregarded. As was noted above, all maxtma of interest fall below the Froude number given by $\mathrm{Fr}^{2} \mathrm{kdtanhkd=1}$. The expected bed configurations summarised in table 5.1 axe indicated by the character of the lines representing equation ( 5.47$)$.

It is now therefore possible to define the conditions of occurrence of the various bed forms in table 5.1 , for different values of Fr and $j$, by eliminating kd between equation (5.42) and $(5.47)$, (thus assuning that $\delta$ is a constant rather than a function of $\mathrm{k}$ ). An expression for the value of $\mathrm{kd}$ at the critical intersection of equation (5.42) and (5.47) is found to be the solution to

$$
\sinh ^{2} k d-j k d \cot j k d=1
$$

For any given $j$ there are two solutions to equation (5.48) giving the two values of Fr which bound the plane bed field, as shown in fig. 5.17. In the mode1, antidunes will occur at a station if

$$
F r^{2}=8 s / f_{2}>\mathrm{Fr}_{\mathrm{a}}^{2}=\operatorname{tanhkd} / \mathrm{kd} \text {, and } 0<j k d<\pi
$$


where Fra is the minimum Froude number for the formation of antidunes. The value of $k d$ needed in this case by equation (5.49) cannot be easily obtained, as this involves finding the solutions to equation (5.48) numerically. The Newton-Raphson method breaks down here because the roots are very close at some values of $j$ and an initial estinate of a root has to be very close to the actual root. To overcome this difficulty within the model, the roots of equation (5.48) were found by trial and exror for various j values and a polynomial regression was performed with Fra as the dependent, and $j$ as the independent, variable. The resulting best fit equation, which gives the value of Fra for use in the inequality $(5.49)$, is

$F r_{a}=0.84+0.27 j+0.0047 j^{2}-0.00089 j^{3}$

Full details of the analysis can be seen in appendix 2.

The plane bed configuration will therefore occur if the value of Fr lies below the curve of equation (5.50), for a given $j$, or lies above the curve which was obtained by performing a simtlar regression analysis using the lower Fr values $\left(F r_{u}\right)$, corresponding to the alternative roots of equation (5.48). The resulting polynomial regression equation of this lower boundary for plane beds is $F r_{u}=0.0049+072 j-0.25 j^{2}+0.04 j^{3}-0.0024 j^{4}$. If Fr, as defined in inequality (5.49), is less than Fr given in the above equation, a transition regime will exist at a station, provided $\mathrm{Fr}$ is greater than $\mathrm{Fr}_{1} \cdot \mathrm{Fr}_{1}$ is the maximum Froude number for the formation of dumes or ripples. Fr 1 is defined by substituting $j \mathrm{kd}=3 \pi / 2$ in equation $(5.47)$, thus

$$
\operatorname{Fr}_{1}^{2}=\frac{1+\frac{3 \pi}{2 j} \tanh \frac{3 \pi}{2 j}}{(3 \pi / 2 j)^{2}+(3 \pi / j) \tanh \frac{3 \pi}{2 j}}
$$


As can be seen from fig. 5.17, there is no transtion field above a value of $j$ of 5.35 .

Therefore, dunes or ripples will be the bed form if

$$
\mathrm{Fr} \mathrm{r}^{2}=8 \mathrm{~S} / \mathrm{f}_{1}<\mathrm{Fr} \mathrm{r}_{1}^{2}
$$

which fulfills the requirements, shown in table 5.1 , that $\mathrm{Fr}^{2}<$ tanhkd $/ \mathrm{kd}$ and $3 \pi / 2<j \mathrm{kd}<2 \pi$. To separate the fields of dunes, ripples and lower phase plane beds, the stream power and median diameter of bed material criteria are adopted, as used in Allen's mode1.

Fig. 5.15 presents a comparison of the experimental data summarised by Kennedy (1963) and the four reference curves shown in fig. 5.16. The agreement is seon to be very satisfactory. practically all points fall below $\mathrm{Fr}^{2}$ kdtanhkd=1, which is taken as a justification for not including in figs. 5.16 and 5.17 case In to $1 \mathrm{~m}$ of table 5.1 (Kennedy, 1969). A comparison of figs. 5.15 and 5.16 indicates that some dunes have values of $j$ of five and greater. Such large values of $\delta$ seem very large for the bed load, but are readily concelvable for the suspended load, in which the transported material must settle significant distances to the bed as the flow decolerates, and be diffused upward as the local transport capacity increases. Accordingly, kennedy (1969) axgues here that dunes are formed by a perturbation of the longitudinal distribution of the suspended load transport, which has a relatively "lare value of $\delta$, while ripples are formed by a perturbation of the bed load transport for which 8 is much smaller. This would explain the simultaneous occurrence of rtpples and dunes in some flows as resulting from two different modes of instability, the ripple instability associated with the bed 1oad, and the duno instability with the suspended load. 
In the model, the value of $j$, which depends on the depth and velocity of flow and the sediment and fluid properties, must be specified. However, nothing is really known about this parameter, upon which the appearance of the different bed forms depends. In this respect, a constant value of $j$ is not really justifiable, but represents a best approximation for our present purposes. This problem is returned to in section 5.6.5. Ono fuxther point is that, in Kennedy's formulation, the occurrence and effects of the separation zone when dunes and ripples occur is not accounted for.

Hayashi (1970) built upon Kennedy's model retaining his basto concepts and his results but mainly improving on the sediment transport flow relationship. Besides the lag distance $\delta$, he introduced the local bed slope as a parameterinfluencing the sediment transport. The $\delta$ here is the distance by which the local sediment transport rate lags behind the local tractive force at the noan level of the bed. This will be a small distance and differs essentially from the quantity $\delta$, in Kennedy's work. His analysis yields the following expression for the rate of growth of the waves.

$a(t)=a(0) \exp \left[\frac{m g^{2} C_{4}}{c} F r^{4} k^{2} d^{2}\left\{c-2 F r^{2} k d \frac{\left(1-F r^{2} k d \tanh k d\right)}{t a n h k d-F r^{2} k d}\right\} t\right]$

where $\mathrm{m}$ is a dimensional coefficient in Hayashi's sediment transport relationship, $c_{4}$ is a constant and $c$ is a dimensionless parameter defined by

$$
\frac{c_{4}}{\delta}=c \frac{v^{2}}{26}
$$

Equation (5.54) shows that the amplitude of bed waves will increaso with time when the sum in the parentheses is positive; in this case a flat bed is unstable. putting $\Gamma=\mathrm{C}-2 \mathrm{~F} \mathrm{r}^{2} \mathrm{kd}$. 
$\left.\left(I-F x^{2} k d \tanh k d\right) /\left(\tanh k d \cdots F r^{2} k d\right)\right], \Gamma>0$ gives the regions of occurrence of sand waves and $\Gamma<0$ gives the regions of flat bed. The limits of the regions of occurrence of sand waves are given by $\Gamma=0$ and tanhkd-Fr ${ }^{2} k d=0$, and the 1 imiting values are

$F x^{2}=\left(\begin{array}{r}F r^{2} \\ 2 \\ \left(F x_{1}^{2}\right.\end{array}\right)=\frac{1}{\text { 4kdtanhkd }}\left[C+2 \pm \sqrt{(C+2)^{2}-8 c \tanh ^{2} k d}\right]_{5.56)}$

where $F r_{1}$ is the maxlmuin $F r$ for the formation of dunes, and $F r_{2}$ is the maximum ir for the formation of antidunos, and

$$
F r^{2}=F x_{a}^{2}=\operatorname{tanhkd/kd}
$$

which divides dunes from antidunes as in Kennedy's work.

The region of occurrence of dunes is delineated in the (Fr,kd) plane by $0<F x<F x_{1}$, that of antidumes by $F r_{a}<F r<F r_{2}$, and the regions of flat beds are delineated by $F x_{1}<F r<F r_{a}$ and $\mathrm{Fr}_{2}<\mathrm{Fr}$. It can be seen from equation (5.54) that in the case of $\mathrm{C}=0$, instability occurs only in the region the linits of which are given by equation $(5.57)$ and

$$
F r^{2}=F r_{m}^{2}=\operatorname{cothkd} / k d
$$

It is to be noted that if $\mathrm{C}$ were zoro, no dunes, only antidunes, would occur on erodible beds. As already mentioned, Reynolds (1965) argues that equation (5.58) marks the end of the region of instablifty of bed waves of small amplitude (antidunes) and the beginning of anothex region of instability. However, Hayashi's analysis indicates, in general, that equation (5.56) dolineates the upper limits for antidunes, and beyond this flat beds will occur. Inspection of the doninant wave number in fig. 5.19 will Indicate that Reynolds' (1965) criterion appears to be correct. The magnitude of $\mathrm{C}$ has not been determined experimentally, nevertheless a comparison of experimental clata sumarised by Kennedy (1963) and the regions of occurrence of sand waves for 
the case $C=2.0$ provides the best agreement between theory and experimental data (Hayashi, 1970).

The initial rate of growth of sand waves is given, from equation $(5.54)$, as

$a_{t}(0)=a(0)\left(\mathrm{mg}^{2} c_{4} / C\right) F r^{4} k^{2} d^{2}\left\{\mathrm{C}-\left[2 F x^{2} k d \quad \frac{\left(1-F r^{2} k d t a n h k d\right.}{\left(\text { tanhkd }-F^{2} x^{2} k d\right.}\right\}\right\}_{5}$

The initial rate of growth, normalised by a $(0) \mathrm{mg}^{2} \mathrm{c}_{4} / \mathrm{C}$ for the case $C=2.0$ is plotted $1 n$ Fig. 5.18 for different values of kd and Fr.

By way of explanation of Fig. 5.18, singularities have occurred in equation (5.58) for values of Fr from 0.5 to 0.9 at the cesonant point, $F r^{2} k d=t a n h k d$. Through the artifice of specifying the initial amplitude of the surface wave rather than that of the bed wave, the resonancy is replaced by a null point (see Kennedy, 1969). Tt can therefore be assumed, for practical purposes, that the maximum jnitial rates of growth in the dune field gradually decrease from Fr $=0.1$ to zexo at about $F x^{2}=0.7 . \quad$. And above about $F r=1.0$ the maximum initial rates of growth occupy the antidune field. As already stated, the dominant wavelength is that for which the injtial rate of growth is a maximum. The maximum initial rates of growth are plotted on f'ig. 5.19, which indicates that dunes or ripples rill exist as bod forms from $r x=0$ to about $F x=0.7$. Flat beds will exist rrom Fr $\approx 0.7$ to $F r \approx 1.0$. Above Fr $\approx 1.0$ antidunes are the stable bed form. It is worth noting that the line of maximum initial rates of growth is broadly similar to equation (5.46) of Kennedy, as previously cited. Furthermore, Hayashi's model for $\mathrm{C}=2 . \mathrm{O}$ is consistent with $\mathrm{flg} .(5.17)$ and ropresents a valize of $j$ of about 2.5 .

\section{5 .6 othex atternative models}

As pointed out by Simons and Richardson (1971) the 


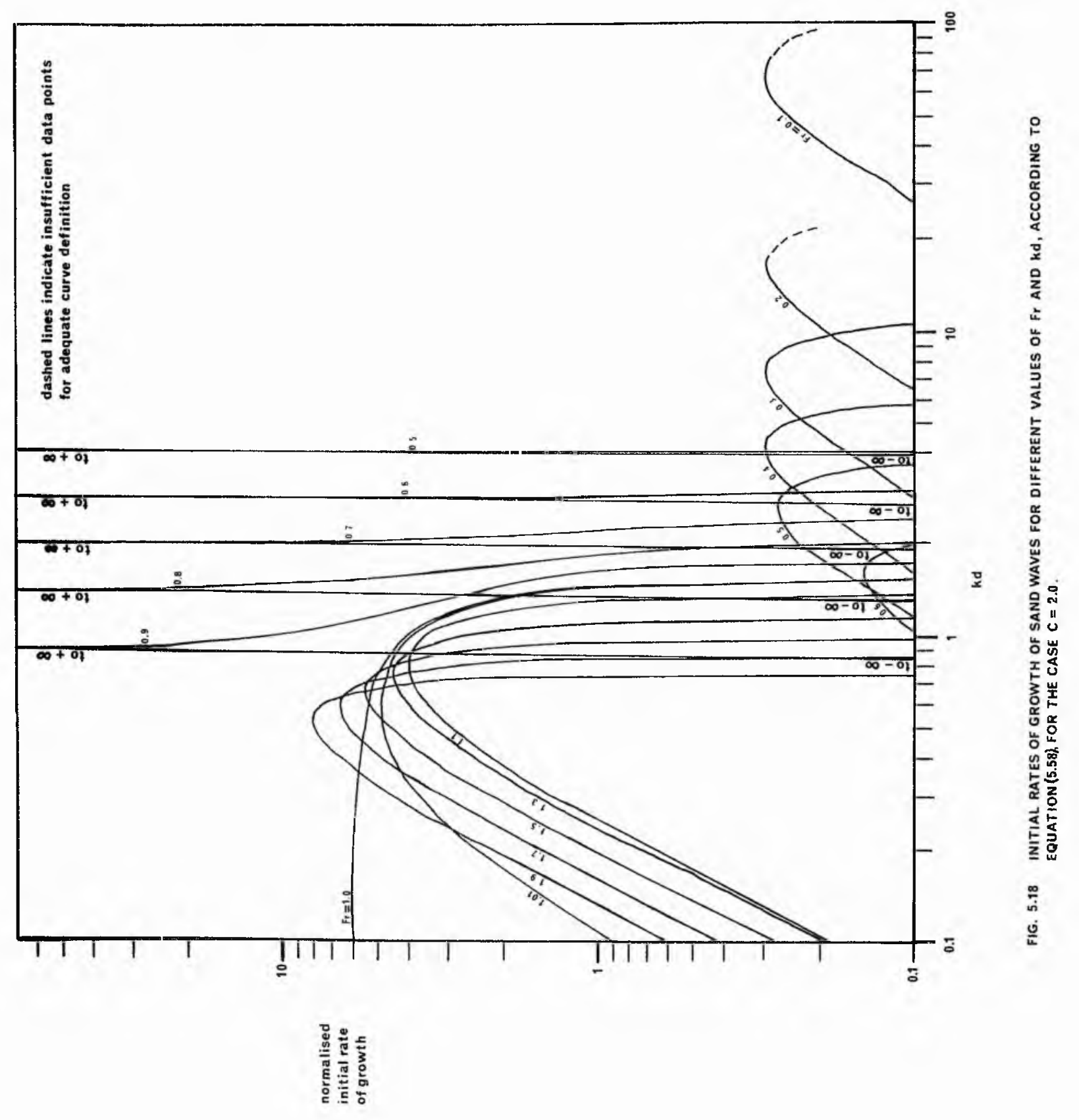




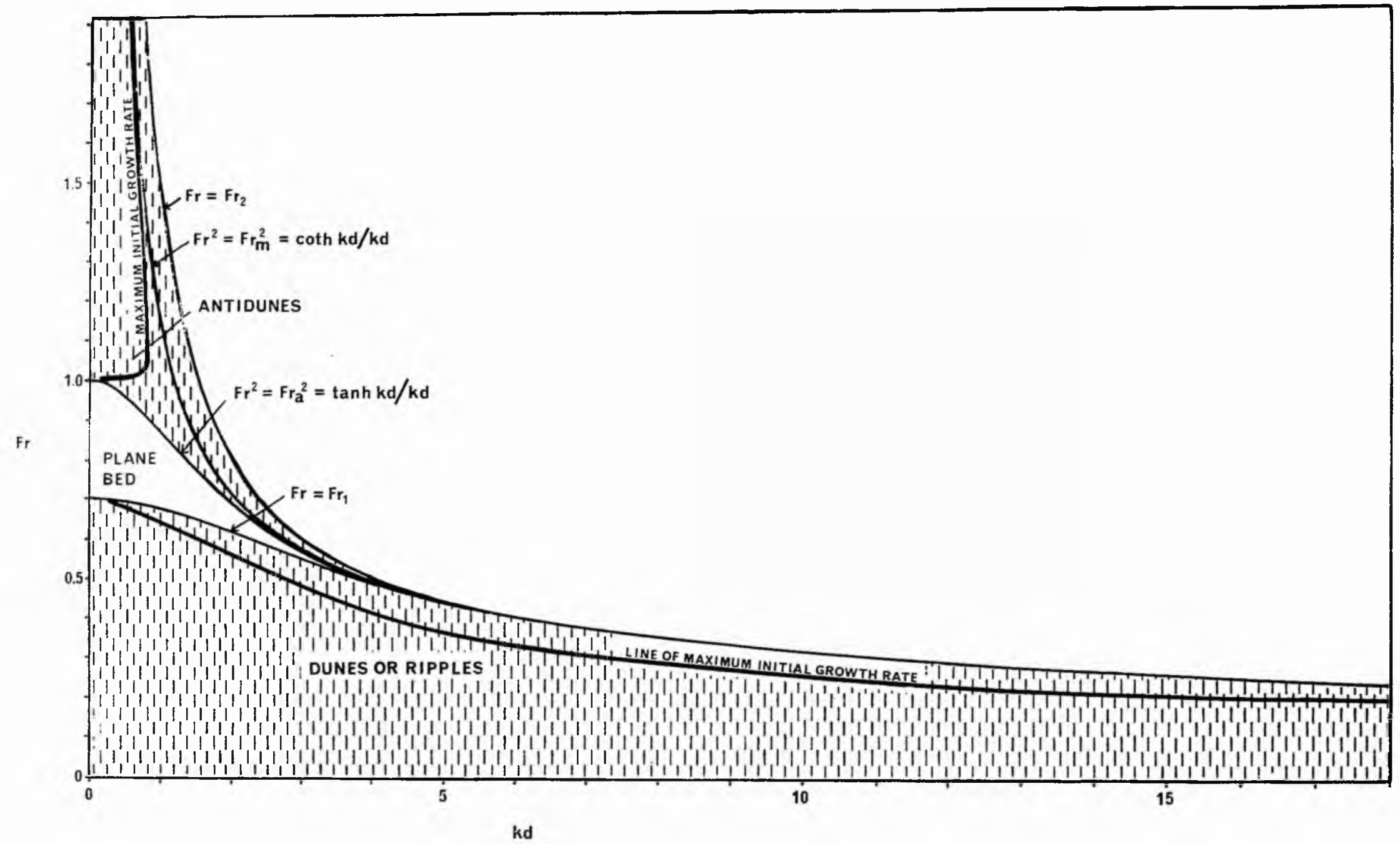

Fig. 5.19 Regions of occurrence of sand waves for the case $C=2$, according to Hayashi (1970), with lines of maximum initial growth rate indicated. 
multivariate statistical apjroach, specifically discriminant analysis, shows considerable promise in the field of bed form prediction. Its usefulness lies in its ability to consider the many variables which affect the existence of a particular bed form. Thus far, discriminant analysis has been used only to classify bed forms into lower, transition, or upper flow regimes based on four dimensionless hydraulic parameters that are assumed to control bed forms (Athaullah and Simons, 1970). Obviously considerable scope for development exists. For instance, Southard (1971) argues that dimensionless measures of depth, mean velocity, and sediment size (or these three parameters themselves) can be used as coordinates in three dimensional diagrams to characterise the various bed configurations. These have the property of one to one correspondence between possible bed configurations and points in the diagrams, thus eliminating overlapping fields in diagrams involving bed shear stress. Depthvelocity diagrams plotted primarliy from the data of Guy et al. (1966) and Williams (1967, 1970) for five sediment sizes ranging from fine to very coarse sand show contiguous but nonoverlapping fields for the various bed forms. Discriminant analysis could be used to advantage here by classifying the bed forms according to the three parameters mentioned. Alternatively, examination of the boundaries between bed form fields in three dirnensions could be analysed using multiple nonlinear regression. With reirard to theoretical ipproaches to bed form prediction, similar shear flow models to that of Kennedy (1963, 1969) have been developed (Reynolds, 1965; Gradowczyls, 1968). but the small additional information that they yield is obtained at the price of considerable analytical complexity (Kennedy, 1969). Engelund and Hansen (1966) developed a comprehensive stability theory based on the flow of a real fluid over a sinusoidal 
movable bed and. were able to present stability diagrams for two and three dimensional bed waves. Improved account of the physical mechanisms involved in formation of bed waves in real fluid flow over movable beds has recently been made by Engelund (1970) and Engelund and Fredsoe (1971) with encouraging results. It is worth noting here that these two studies confirm Reynolds' (1965) upper stability limit for two dimensional bed waves (see equation (5.58)), but that the transition from antidunes to dunes from potential theory (see equation (5.42)) is a stability boundary marking the lower limit for antidunes.

5.6 Discussion of input parameters requixed in point bar model

\section{6 .1 Channe1 width and depth}

The greater quantity of water that moves through a channel, the larger the cross section of that channel will be. Preceded by numerous studies of canal morphology and stability, Leopold and Maddock (1953) demonstrated that for most rivers the width and depth increase with mean annual dischargo as

$$
\begin{aligned}
& w=c_{5} Q_{m}^{0.5} \\
& d=c_{6} Q_{m}^{0.4}
\end{aligned}
$$

where $c_{5}$ and $c_{6}$ are coefficients and $Q_{m}$ is mean annual discharge. The coepictents vary for each river, however, in some cases, with a downstream increase in discharge width or depth decreases. It is probable therefore that another independent variable is influencing channel dimensions, and this must be sediment load (Schumm, 1971). Analysis of data from the stable sand bed streams of the Great plains of the United states and the Riverine Plains of $\mathrm{New}$ South Wales, Australia (Schumm, 1969, 1971) has produced the following relations 


$$
\begin{aligned}
& w=2.3 Q_{m}^{0.38} / M^{0.39} \\
& d=0.6 Q_{m}^{0.29} M^{0.34}
\end{aligned}
$$

where $M$ is schumm's weighted mean percent silt and clay in the perimeter of the channel. The relation for channel width indicates that $88 \%$ of the variability of width can be accounted for by mean annual discharge and type of sediment load (M being an Index of the latter), both being about equally important. The relationship for channel depth is not quite as good with about $81 \%$ of the variability of channel depth accounted for by discharge alone. Similar equations with equivalent correlation coefficients were obtained using mean annual flood discharge instead of mean annual discharge (Schumm, 1969).

When discharge is used with $M$ to develop a multiple regression equation for width-depth ratio, that is

$$
F=56 \mathrm{Q}_{\mathrm{m}}^{0.10} / \mathrm{M}^{0.74}
$$

only a slight improvement over Schumm's earlier relation

$$
F=255 \mathrm{M}^{\cdots 1.1 .08}
$$

is obtained.

Hence schumm (1971) concludes that variations in channel dimensions with constant discharge are attributable to changes in sediment load. Local variations may be strongly affected by Local. variations in bank resistance, but the width-depth ratio of alluvial chandels appears to be primarily determined by the nature of the sediment transported through the channe1. This conclusion is supported by the observation of Leopold and Maddock (1.953) that '...decreasing width at a constant velocity.... results in increased capacity for suspended load at constant discharge', and '.oat constant velocity and discharge, an increase 
in width is associated with a decrease of suspended load and an increase in bed load transport' Therefore a high width-odepth ratio is associated with lạage bed materiaj load.

It is to be expocted that width and depth are significantly related to the other clependent morphologlcal variables, which are also controlled by the independent system variables. Many authors have formed such relations. Some of the most useful ones in the present context are those linking wavelength and simuosity with width and depth, i.e.

$$
\begin{aligned}
& 1=10.9 w^{2.01} \text { from Leopold and wolman (1960) } \\
& 1=18 \mathrm{~F}^{0.53} \mathrm{w}^{0.69} \text { from Schumm (1972) } \\
& \sin =3.5 \mathrm{~F}^{-0.27} \text { from Schumm (1963) }
\end{aligned}
$$

These equations can be used to define approximate relations between the dependent input variables, without making reference explicitly to the independent variables, specifically discharge and sediment load. An important point in this respect is that width and maximum depth (at specific cross sections) have to be defined in the model. It is well known that width and depth vary along the length of a meandering reach, as well as across the charnel, with the alternating occurrence of pools and riffles. Averaged values of width and depth, either in the downstream direction or across the stream, will therefore not be truly representative of the channel dimensions at any specific cross section. Here, interest is centred around the pool areas, where width is normally less than over the riffles and maxtmum depth is normally greater.

Finally, anothex study of interest graphically relates depthwidth ratio to vaxious dependent and independent hyclxalic variables. This work is summarised by simons (1971).

5.6.2 Mean xadius of curvaturo, longitudinal water suxface 3lope and valley slope

Mean radius of curvature is defined in the planinetric 
geometry mode1, section 2,2, through its relationship with sinuosity and wavelength. Longitudinal water surface slope is obtained indirectly from the planimetric geometry model and is defined as valley slope divided by sinuosity. Valley slope is one of the substantially independent variables that is required as input to the model. The only specification restrictIon for the valley slope value is that, for a given discharge, the channel pattern is meandexing. Straight, meandering and braided channels can be distinguished empirically in terms of slope and discharge (Leopold et al., 1964; Ackers and Charlton, 1970a;d; Schumm and Khan, 1972).

\subsubsection{Resistance coefficients}

As already stated, the Darcy-Visbach friction coefficient can be conveniently separated into that part representing form losses introduced due to the addition of bends, $f_{b}$, and another part representing the resistance due to bed friction of a comparable straight channel, $f_{s}$. This subdivision involves the introduction of a factor, $r$, by which the straight channel friction factor must be multiplied to account for the change in relative roughness (arising from bed features) due to change in hydraulic radius with meandering.

Because of the large range of bed forms that may occur in an alluvial channel, the large variation of resistance to flow among the different bed forms, and the large number of interrelated independent variables affecting the bed form, it has not been possible to write a generalised function to predict resistance to flow or the velocity of flow (Simons and Richardson, 1966). As Simons and Richardson (1966) point out, 'A generalised function may not exist, because (1) more than one resistance to flow may occur for a given slope, depth, and bed material, (2) hysteresis exists in the change in bed confjeuration and resistance to flow depends on the preceding filow conditlons, and (3) the bed configux. 
ation will oscillate between a dune bed and a plane bed for a given bed material at certain siopes and discharges. This problem is funther complicated by three-dimensional flow, varying depth, varying bank roughness, and nonuntformity of flow in alluvial channels'. In this discussion of $f_{s}$ only, however, we can assume that the complications due to the three dimensional helicoidal and nonuniform flow associated with meanders are accounted for in $f_{b}$, the discussion of which will follow.

In predicting the bed configuration present under differont hydraulic conditions, it is necessary to know the value of $f_{s}$ for the different bed forms that may exist. There are, in fact, various methods for estimating the resistance to flow under various conditions, and these are described in, for example, Graf (1971), Raudkivi (1967), Simons and Richardson (1966, 1971). Unfortunately, these methods normally require some information on the bed forms present, which precludes their general use in the model. With the appreciation that $f_{s}$ varies for different bed forms and with the same bed form, due to the reasons outlined in Simons et al. (1965) and simons and Richardson (1966, 1971), a single value of $f_{s}$ may be assumed for each bed form as a reasonable first approximation. As mentioned in section 5.5.1, plane beds of either phase and antidunes take on one constant value, as do ripples and dunes. When these two constant values are multiplied by the factor $x$ and combined with the component of the total coefficient which is due to the addition of bends, they become $f_{2}$ and $r_{1}$ respectively. Table 5.2 shows some of the observed ranges of $f_{s}$ for different bed forms in flumes and natural rivers (in the absence of meanders). Loopold et al. (1960) performed experiments in order to find the relative magnitudes of the resistance elements in straight and sinuous channols with fixed banks. The additional 


\begin{tabular}{|c|c|c|}
\hline Lower phase plane bed & $0.02-0.035^{1}$ & $0.019-0.14^{5}$ \\
\hline Ripples & $\begin{array}{l}0.052-0.13^{1} \\
0.0693^{2}\end{array}$ & \\
\hline Dunes & $\begin{array}{l}0.042-0.16^{1} \\
0.056-0.099^{3} \\
0.048-0.08^{4}\end{array}$ & \\
\hline Upper phase plane bed & $\begin{array}{l}0.02-0.03^{1} \\
0.014-0.022^{3} \\
0.018-0.025^{4}\end{array}$ & $0.011-0.034^{5}$ \\
\hline Antidunes & $\begin{array}{l}0.02-0.35^{1} \\
0.03-0.07^{1}\end{array}$ & $\begin{array}{l}\text { nd- } \\
\text { es } \\
\text { es }\end{array}$ \\
\hline
\end{tabular}

1. Simons and Richardson (1966)

2. Ackers and Charlton (1970d)

3. Nordin (1964)

4. Culbertson et a1 (1972)

5. Culbertson and Dawdy (1964)

Table 5.2. Some observed ranges of the friction coefficient in straight flumes and natural rivers. 
losses due to bends were obtained using a constant cross section and boundary roughness in the straight and curved channels. In these experiments, therefore, there was no need to introduce the factor $r$, as there were no changes in relative roughness due to changes in hydraulic radius. In the straight channel the resistance to flow was wholly surface resistance, measurable by peRs. For a sinuous channel pgRs no longer gives the surface resistance only but includes energy losses due to the addition of bends.

The experimental results confirmed their anticipated relation between resistances in the curved and straight channels and the square of the flow velocity (see fig. 5.20). In fig. 5.20 the overall resistance coefficient $(=f / 8)$ is given by the slope of the straight lines, except where spill resistance begins and resistance no longer varies as the square of the flow velocity.

The data indicate that the channel curvature alone can account for energy loss of the same order as that due to the surface resistance in straight channels in the absence of bed forms, and in tight curves may be double that quantity. Furthermoxe, Ackers and Charlton (1970d) find in their experiments on small meandering streams with rippled beds that some 60\% of the head 1osses aro due to bends and variations in cross section. Similar results were obtained by Allen (1939) and Allen and Shahwan (1954) from their model experiments and field studies, Grave uncertainties exist in the relation between the extra 'squaxe law' resistance introduced by channel bends and the Geometric characteristics of the bends (Bagnold, 1960; Jeopold et al, 1960; Shukxy, 1950; Yen, 1.965, 1971). Figure 5.21 shows the values of $f_{b}$, from the data of Ackers and charlton (1970d), plus additional data on $r_{m}$ from their records, and Leopold et al. 


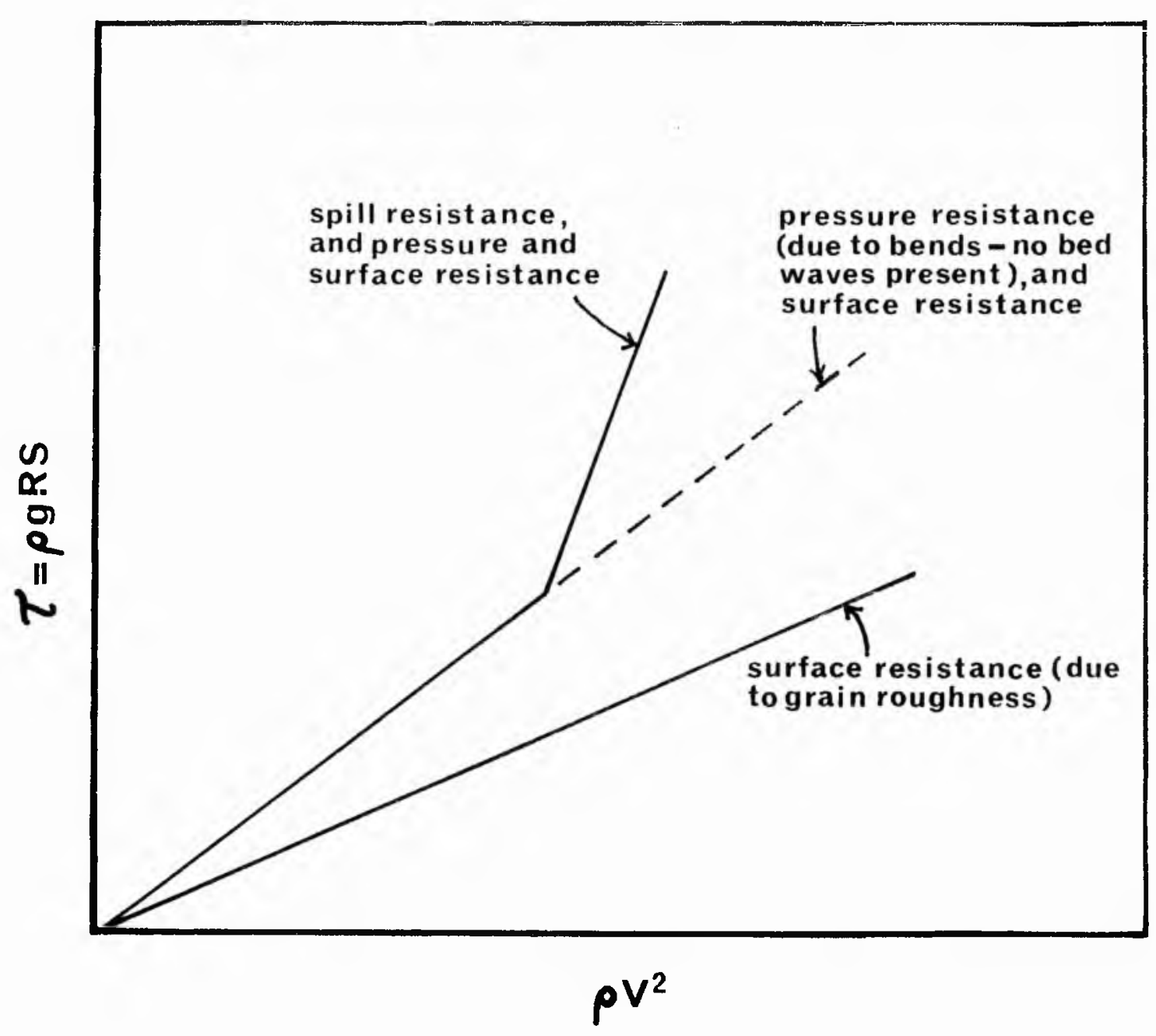

Fig. 5.20 Postulated relation of bed shear stress to square of flow velocity in sinuous fixed bed channel. (after Leopold et al.). 

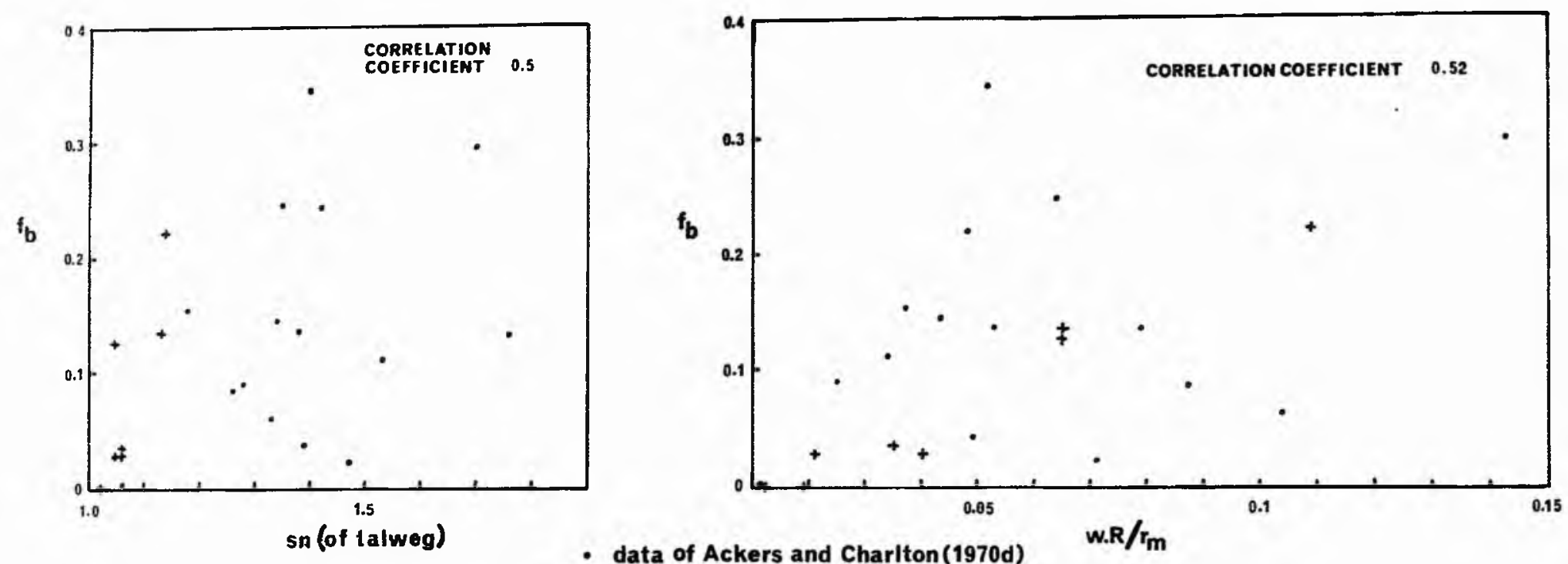

data of Ackers and Charlton (1970d)

- data of Leopold et al.(1960)
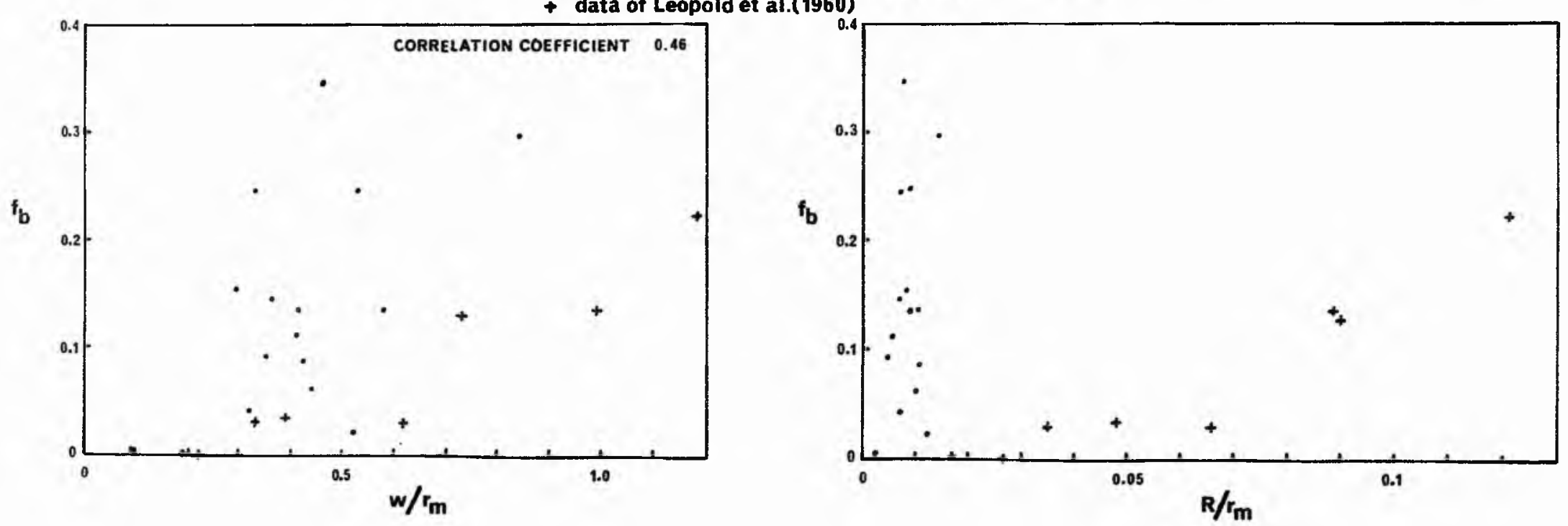

Fig. 5.21 Plots of $f_{b}$ against various geometric proportions. 
(1960), plotted against various geometrlc proportions. The values of $f_{b}$ computed from the Ackers and Charlton data were derived by comparing the losses in the meanders at crossings with only one averago straight reach with a corrected friction coefficient, $\mathrm{rf}_{\mathrm{s}}$, of 0.036 . Due to the excessive scatter of the data available and the restricted range of experimental conditions, no general relation for the value of $r_{b}$ is available. If the value of $f_{b}$ is drawn from $f i g .5 .21$ it must only be approximate, and there nay be inherent danger in extrapolation based on these restricted data. Ackers and charlton (1970d) used the following average values in their analysis based on small meandering streams; $f_{b}=0.135 ; \quad r=1.249 ; \quad f_{B}=0.0693$

\section{6 .4 Fluid viscosity, fluid and sediment density}

A value of flutd viscosity is required for alternative model no.2 (section 5.5.4), and $f i g .5 .22$ shows the effect of fine sediment (bentonite) and temperature on the apparent kinomatic viscosity, $\nu$ (Simons and Richardson, 1971). This is an apparent viscosity because aqueous dispersions of fine sediment are non-Newtonian. The magnitude of the effect of the fine sediment on viscosity is large and depends on the chemical makemup of the fine sediment. The changes in fall velocity of the median diameter as a result of the changes in the viscosity and the fluid density can be noted in fig. 5.23 (see simons and Richardson, 1971). Therefore, by specifying a particular value of $\nu\left(\mathrm{cm}^{2} / \mathrm{sec}\right.$ units $)$ in the model, implicit mention is made of the amount and nature of the suspended sediment concentration and the temperature of the fluid, which constitute substantially independent variables.

In addition to changing the viscosity, fine sediment 


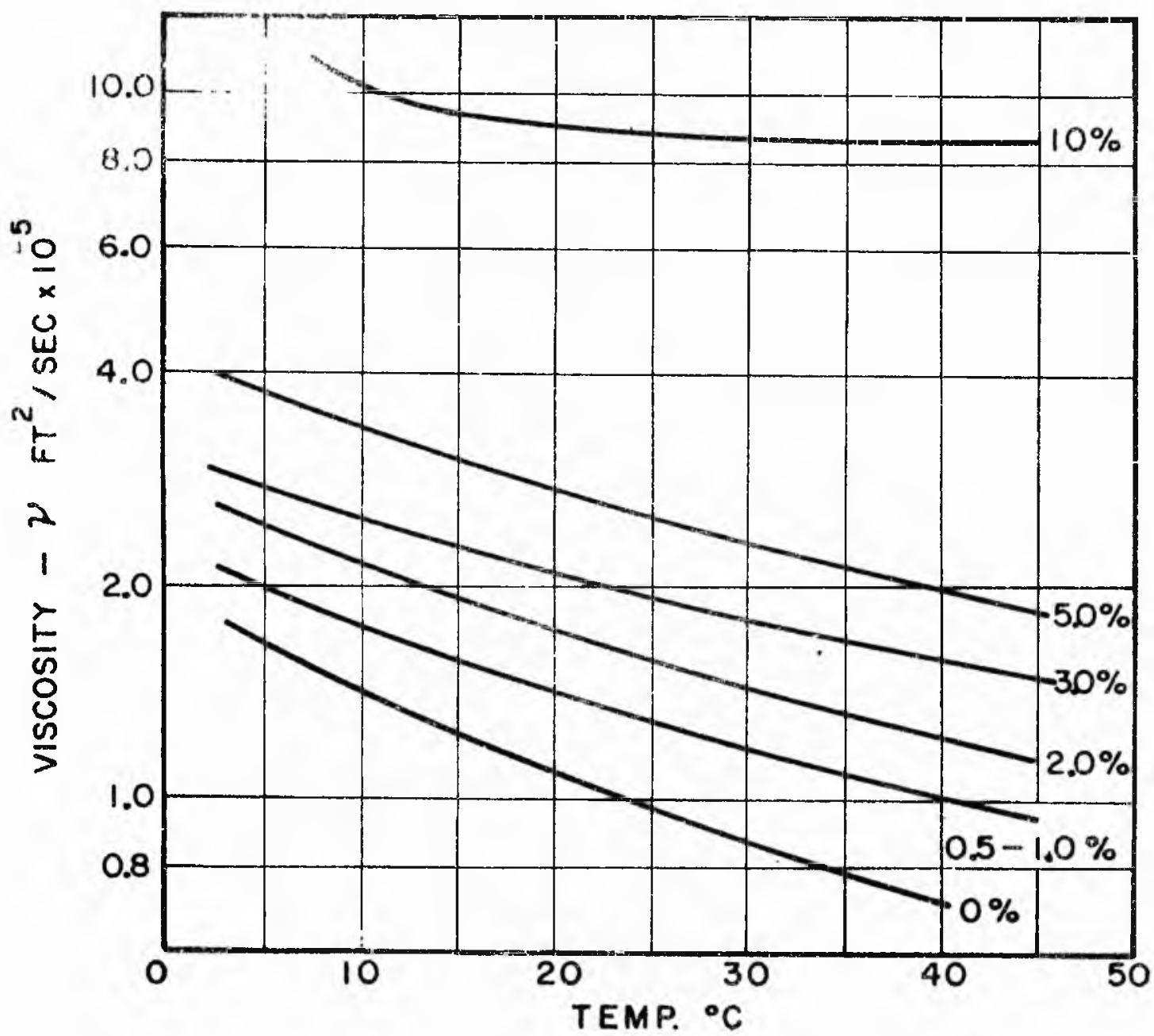

Fig. 5.22. Apparent kinematic viscosity of waterbentonite dispersions. (from simons et aI. 1965). 


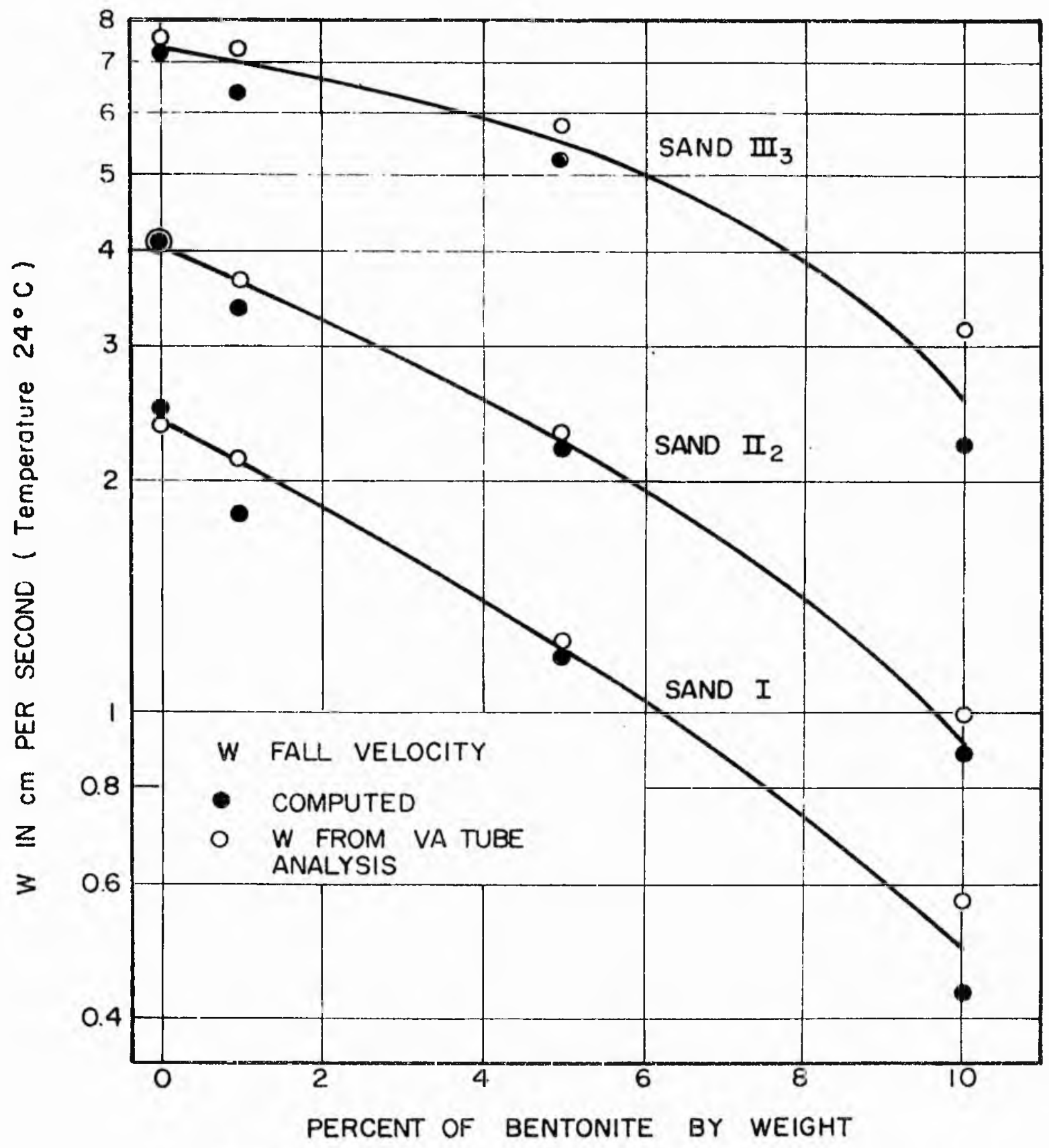

Fig. 5.23. Variation of rall velocity with percentage of bentonite in water. (from simons et al., 1965). 
suspended in water increases the mass density $\rho$ of the mixture. The mass density of a watex sediment mixture can be computed from

$$
\rho=\frac{\rho_{w} \sigma_{s}}{\sigma_{s}{ }^{m-C} c_{s}\left(\sigma_{s}-\rho_{w}\right)}
$$

where $\rho_{w}$ is the density of pure water, $\sigma_{s}$ is the density of the suspended sediment, $C_{s}$ is the suspended sediment concentration in $\%$ by weight (Simons and Richardson, 1971). As $\rho_{w}=1 \mathrm{gm} . / \mathrm{cm}^{3}$.

$$
\rho=\frac{\sigma_{s}}{\sigma_{s}-c_{s}\left(\sigma_{s-1}\right)}
$$

The density of the sedimentary particles in the bed load is an independent system vaxiable and is normally taken as the value for quartz grains, i.e. $2.65 \mathrm{gm} / \mathrm{cm}^{3}$.

\section{6 .5 Kennedy $\mathbf{j}$ factor}

It was pointed out in section 5.5 .5 that the value of $\mathbf{j}$ depends on the depth and velocity of flow and the sediment and rluid properties, also that nothing is really known about the parametes. In alternative model no.3, it is required to specify a single value of $j$ for the whole cross profile. This constitutes only a first approximation.

As there is no theoretical definition available for the value of $j$, it will be necessary to turn to the body of experimental datia that exists. As an example, from the data of Guy et al. (1966), $j$ appears to vary between about 2 and 3 for their finer sand grades. 


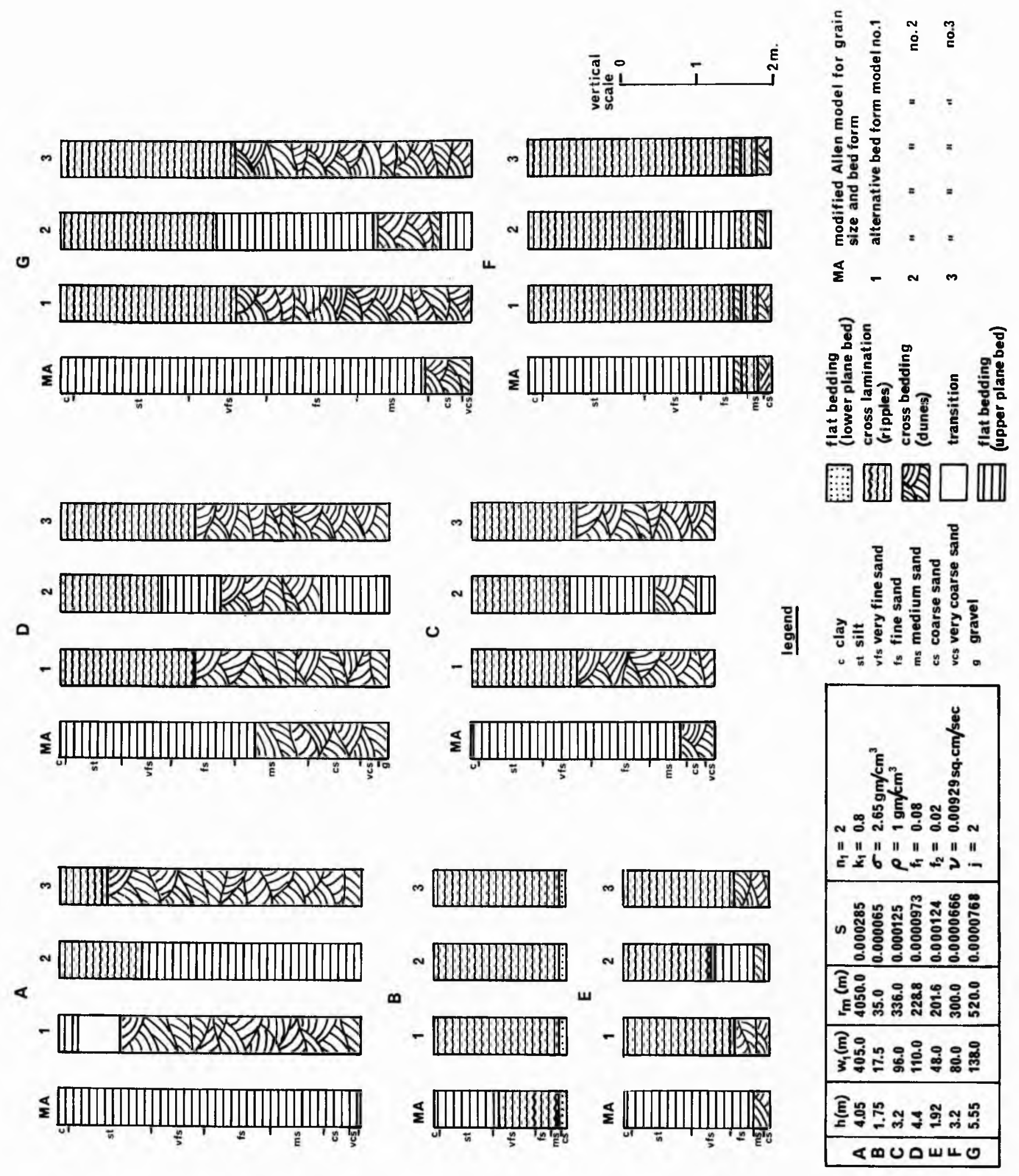




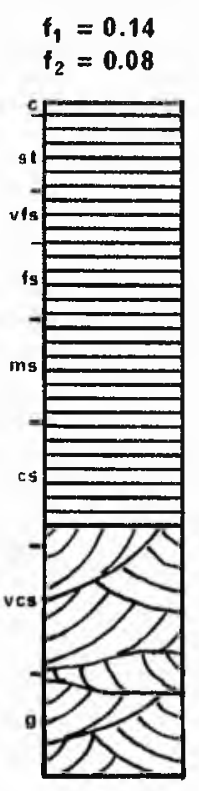

$$
\begin{aligned}
& f_{1}=0.08 \\
& f_{2}=0.02
\end{aligned}
$$
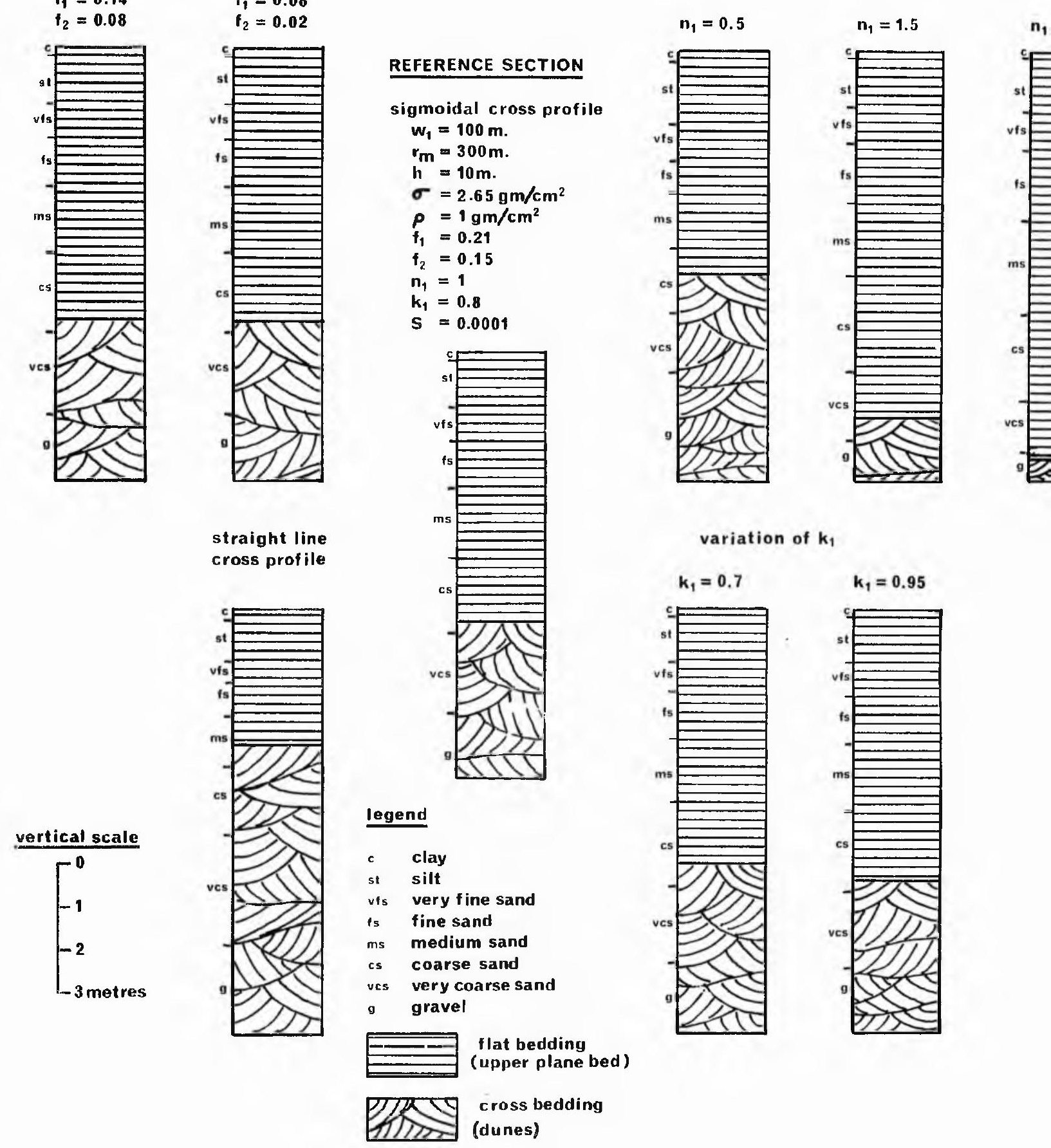

FIG. 5.24

VERTICAL VARIATION OF GRAIN SIZE AND SEDIMENTARY STRUCTURE WITHIN POINT BAR, AC TO MODIFIED ALLEN $(\mathbf{1 9 7 0 a , b )}$ MODEL, FOR SELECTED VARIABLE INPUT PARAMETERS. 
5.7 Experiments to show variation of grain size and

sedimentary structure with different input parameters

and altexrative bed-form mode1s proposed

Fig. 5.24 shows the vertical variation of grain size and sedimentary structure within a point bar, calculated using Allen's bed-form model. The figure is designed to show the effect of variation of selected parameters on the sedimentary deposit over a point bar of constant dimensions. The sections shown should therefore be compared with the reference section in the centre of the figure. They all have the same input parameters listed except for the specifle paraneters which are being varied in each case. The effect of variation of $k_{1}$, with all other input variables constant, is to vary the full width of the channel, which affects the local radius of curvature at a particular station. Increase in $k_{1}$ effectivoly increases the local radius of curvature. The effect on the grain size and sedimentary structure profiles is slight, but with an expected decrease in general calibre of the Load as $k_{1}$ increases, and a sljeght downward extension of upper phase beds due to the decreased grain size.

Variation of the friction coefficients will obviously have no offect on the grain size, by virtue of the model used, hovever, in these particular cases, there is no effect on the sedimentary structure profiles eithex. This is by virtue of the criteria used to predict upper phase plane beds, and that the hydraulic conditions pertaining to the lower part of the profiles were not near the limiting conditions for the existence of ripples of lower plane beds. In the case of the hydraulic situation being near to these boundary conditions, dunes will be expected to occur at the expense of ripples or lower plane beds with decrease in the 
friction coefficients. The appropriate choice of friction coefficients for a particular meander is obviously an important consideration.

As already noted, the grain size profiles, and hence the sedimentary-structure profiles, are very sensitive to changes in the shape of the cross profile (e.6. fig. 5.6). Fig. 5.24 shows, as expected, a decrease in general calibre as the convexity, $n_{1}$, increases, also a steepening of the grain size profile. This variation in grain size has the effect of extending the upper phase plane bed field downwards, effectively doubling the thickness from $n_{1}=0.5$ to $n_{1}=2$. Substitution of a straight point-bar cross profile for the sigmoidal curve (equation (5.1)) has the effect of increasing the amount of coarse sediment grades relative to the finex grades, when compared with the reference section. This has the effect of upward extension of cross beds in the section at the expense of upper plane beds. In fact, with a straight profile, D varies approximately as the square of the local depth when $r_{m}$ is large, but has an approximately linear relation when $r_{\mathrm{m}}$ is small.

Figuce 5.25 is designed to show the effect of variation of the channel and meander dimensions on the grain size and sedimentarymstructure profiles, and to compare the alternative bed-form models proposed. The parameters that were varied in fig. 5.24 are kept constant in this figure. The actual data used were taken from A.lien (1970a).

From equation $(5,20)$ it is expected that, apart from the effects of $n_{1}$, the general calibre of the load increases with longitudinal water-surface slope, maximum channel depth, and channe1. width between the inner bank and the talweg, but decreases With increasing radius of curvature. These constituto a sufficiently large number of variables such that the general calibre can be very stmilar for many different combinations. 
However, fig. 5.25 does show, for instance, in the case of $\mathrm{D}$ that a generally high calibre of load exists due to a high ratio of $w_{1}$. h.s over $r_{1}$. Case has a relatively low ratio and subsequently has a low general calibre.

The value of given in equation (5.25) in Allen's bedform model depends, apart from $n_{1}$, on the ratio of $r_{1}$ over $w_{1}$. The laxge ratio of $x_{1} / w_{1}$ in the case of A therefore gives a dominance of upper-phase plane beds, whereas low ratios in cases $B$ and $D$ give considerably less, as was expected.

The sedimentary structure profiles obtained using the different bedoform models are bxoadly in agreement, except Allen's model predicts more upper plane beds than the others. All of the alternative models concur faixly well in their prediction of the change from ripples to dunes or upper plane beds, however there is Eenexally disagreement in the prediction of dunes or upper plane beds (except in the cases of models 1 and 3). Part of this disagreement may be due to the fact that the transition regime has been ignoxed in all the models except no.1. In the last case the sodimentary structures will be expected to be those resulting from washed-out dunes. Where only lower-regime forms are predicted, protiles will be the same, as the models differ fundamentally only in their prediction of the transition from lower-to upper-regime rorms. It is worth noting that neither the grain size nor sedimentaxy structure can be assumed to be wholly correct at the top of the proflles where the grain-size model predicts fine silt an clay. The grain-size and bed-form models are based on a consideration of cohesionless particles and cannot take account of the cohesive forces involved with fine sediment. Nevertheless, although not theoretically correct, the profiles may be qualitatively accoptable in this range.

The alternative model no.3 is unfortunately j.inited by the lack of data on the variation of $j$, and in altexnative no.2 the 


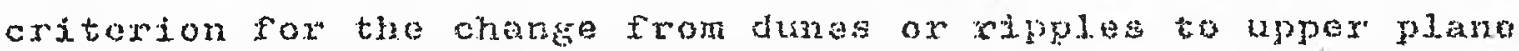
beds is deflnod only for a small range of grain size ranging up to medium sand. Alternative no.1 apreans to be generally applicable and has the advantage of being abje to prediot transibional bed forms. Allen'b model is genexally appltcable and, although quoted values of 0 crit are only approximate, they covex a wide range of grain sizes. In default of a bettex one this model j.s most favourable because it combines a sound theoretical basis with a strone ampirical justification. Allen's model has therefore been adopted in the simulation model, although any a]ternative may be easily incorporated if preferred.

\subsection{Validity and Iimitations of the point-bar sedimentation mode1}

The model is a considerable simplification of a physically complex natural situation and it contains a sufficiently large number of variables to produce closely corresponding deposits in a number of ways (Allen, 1970a). The only really independent variables are amount and character of fluid and sediment discharge and the valiey slope. It has not been possible to produce a model which has only the independent variables as the starting point. Some of the input variables are system dependent, some are independent. For instance, the channel shape and dimensions are made 'independent' variables by specifying then as input, where they are in fact dependent. The bed sediment-size is treated as dependent where ir fact it should be independent. Furthermore, availability of all size grades is assumed.

The dependent morphological variables to be specified as input (i.e. wavelength, width, depth, etc.) share an inter-dependency, as shown previously, because of their common link with the Independent variables. These system dependent input variables must ve mutually compatible, and, without specific reference to the independent variables, the relationships given by equations (5.63), 
$(5.64)$ and $(5.65)$ axe the most useful way of monitoring the compatibility of the input variables.

Naturally the choice of any of the dependent variables has Impltcations relating to the independent ones. Moreover, tho amount and character of fluid and sediment discharge may be predefined explicitly in an approximate way by using the various relationships betwoen the dependent and independent variables that exist in the 1iterature and that have been mentioned hitherto.

The use of uniformmlow equations constitutes somewhat of a simplification, however uniform-flow formulao may be used for nonuniform flow if the flow is 'gradually varied' (Chow, 1959). As already stated, this involves using the energy slope in the computations, and assuming that the hydrostatic distribution of pressure prevails over the channel section. Yen (1965) has shown that the pressure distribution along any vertical is virtually hydrostatic as long as the ratio of depth to radius of curvature is sma1l. The use of these formulae becomes increasingly suspect as the curvature of flov becomes more pronounced, producing non.. hydrostaticmpressure distributions, separation zones, and a state of high turbulence (1.e. 'rapidly varied flow'). Yen (1965) and Rozovskii. (1961) state that stream separation and eddy-zone formation is only encouraged at very sharp bencls as depth increases relative to width, and particularly when the banks have gentler slopes; that is, the greater the influence of rall friction. An analysis of this problem does not therefore seem warranted. Because of the secondaxy currents associated with flow in bends the subdivisjon of the channel crossmsection into discrete subsections will 1ikely result in the continudty principle being violated. Fowever Yen (1965) has shown that the lateral dischargo j.s very small compared with the downstream (1ongitudinal) discharge. It should be noted that the mean fluid velocity and bed stress calculated in the model, and used in the delineation of bed-form 
fields, are. those measured in the longitudinal direction. Assumptions have been made about the uniqueness of the longitudinal water-surface slope and no account has been taken of the minor, yet characteristic superelevation of the transverse water surface. The substitution of the longitudinal water-surface slope for the energy slope was rationalised earlier.

Assumptions have also been made regarding the friction factors, hysteresis effects have not been analysed, and, in general, the effects of temperature and fine sediment on the development of bed-forms have been ignored. Subsequent refinements to the mode1 may describe variations in the shape and dimensions of bed forms with hydraulic conditions. For instance, it is to be expected that, if present, dune height will vary with cepth across the point-bar profile.

only events at the bankfull stage of the stream have been considexed, whereas in reality stage varies with time and deposition may occur over a range of stages. This is discussed more fully below (section 8). Associated with this is the fact that the model does not account for the deposition of 'clay drapes' during the late stages of the flood period, when suspended fines settle from the flow. This is seldom seen, however, because bax deposition is greatest when stage is high and scouring by successive floods may remove the mud drapes and much of the previous deposits. By virtue of the scales involved, and the fact that most deposition is assumed to occur around bankfull. stage, much of this finer detadl will be lost in the model. Although the shape of the cross profile approximates the shape of sandy point baxs described by many authors, others have noted distinct levels, particularly associated with chutes and chute bars. (McGowan and Garner, 1970; Bluck, 1971). The shape of the cross profile will be expected to vary along the length of the channel, not only due to chutes and chute bars if present, but 
with the natural occurrence of pools and riffles.

$$
\text { Despite the simplifications involved, Allen (1970a) shows }
$$

the model. to agree with the overall characteristics of known or inferred lateral deposits, and he goes on to make some generalisations based. on the model. Ire points out that the abundance of erosional contacts between sedimentary units testifies to the incompleteness of the depositional record. The applicability of Allen's model as an entity is however limited to 'complete' point bax sections. It is anticipated that by embodying this component model in a real dynamic situation, and taking account of other important processes, that a fuller interpretation and understanding of lateral deposits will appear. 


\subsection{Factors effecting the nature and rate of bank erosion} Recent work by Turnbull et al. (1966) on the Mississippi has shed much light on the detailed processes involved in bank erosion. During rising flood stages the river erodes a deep pool in its talweg. An oversteepening occurs at the toe of the concave bank slope, resulting in a subaqueous failure. Sometimes nothing further occurs but at other times the subaquoous bank failure triggers a further upper-bank fallure, The upperbank failures may be either by shear or by partial to complete liquefaction of the soil, resulting in a flow failure. The type of failure is determined by the type of sediment composing the river bank, but the initiating process was believed to be the same. The study indicates that when long spans of time are considered the subaqueous and upper-bank failures appear to be continuous. Within the period of a year bank failure is a discontinuous process which is seasonally controlled.

The accompanying diagrams, fig. 6.1, show the exact mature of bank failure in different types of exposed deposits, as the hydxaulic and gravity forces acting are offered varying degrees of resistance to exosion. The nature of the applied and resisting forces are discussed below.

With regard to hydraulic forces, many authors describe the formation of a scour pool associated with tho high velocities and bed shear stresses during high-water periods. Rozovskii (1961.) lias shown that the downward vertical component of velocity at the concave bank associated with the helicoidal flow is not very great, and so its erosive effect is not considered a dominant hydraulic factor. The impact of fluid on the concave bank is also considered untmportant (Kondratev, 1962). 


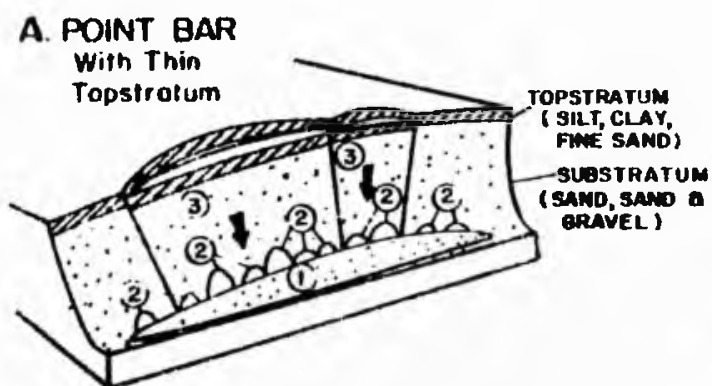

(1) Scour Pool

(2) Numerous Smoll Subaqueous Failures by Flow or Shear

C) Sloughing olid Thin Upper Bank Fallures by Shear

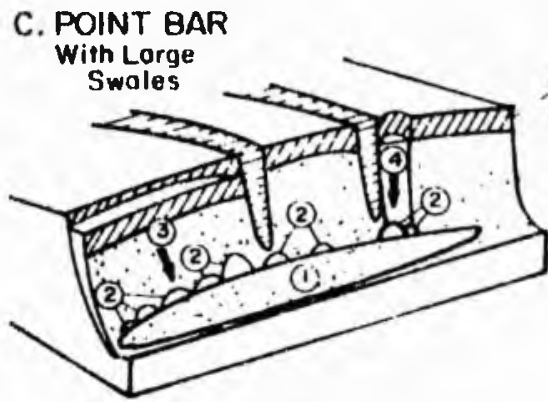

(1) Scour Pool

(2) Small Subaqueaus Farlures by Flow or Sheor

3) Thin Upper Bank Follure Termınated by Swale

(A) Upper Bank Fallure by Flow or Shear Localızed Adjacent to a Swole

E. CHANNEL FILL

\section{Clay Pluo}

(Clay Deposition in

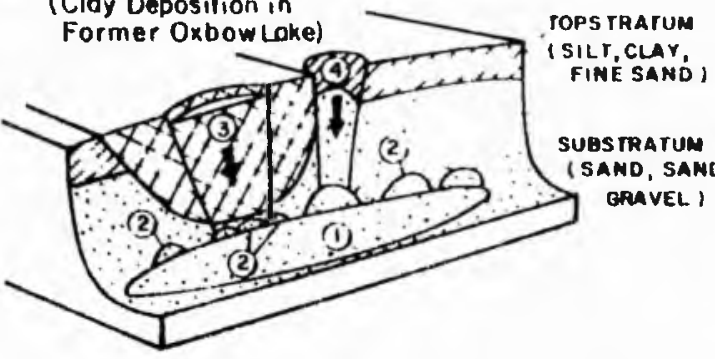

(1) Scour Pool

(2) Suboqueous Bonk Follure by Flow or Shear

(3) Upper Bonk Failure by Shear in ClayPlug

(-) Upper Bonk Failure byFlow or Sheor

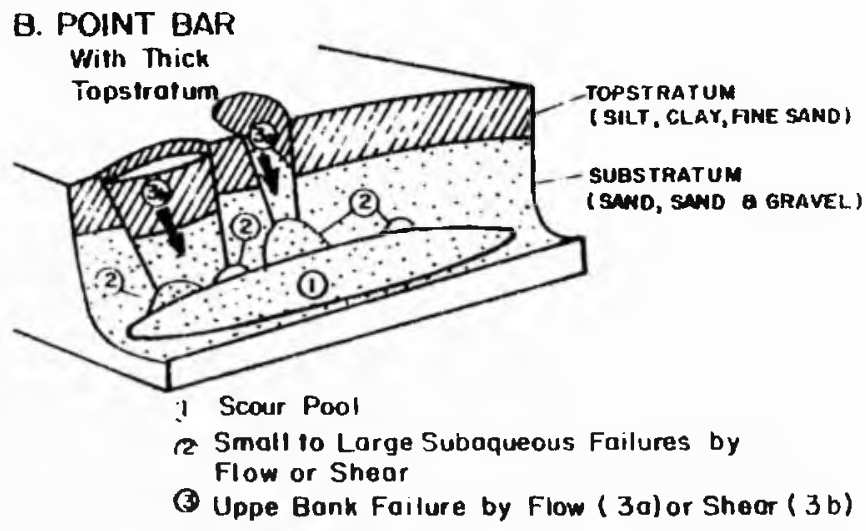

D. CHANNEL FILL *

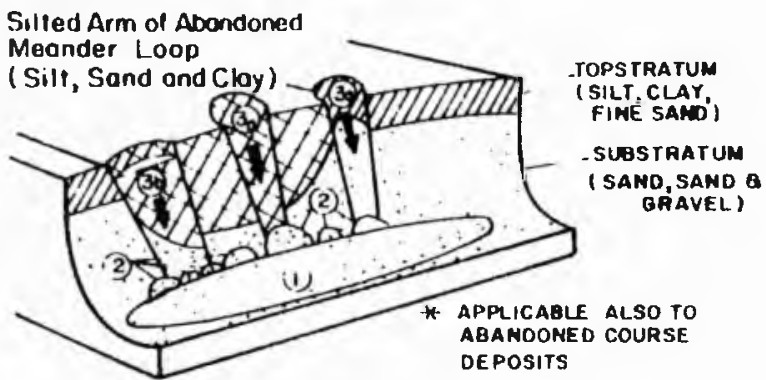

(1) Scour Pool

(2) Suboqueous Fallures by Flow or Shear

(3) Upper Bank Fallures by Flow or Shear

(3a) (3b)

F. BACKSWAMP

Clay and Silty Clay

of Vorying Thickness

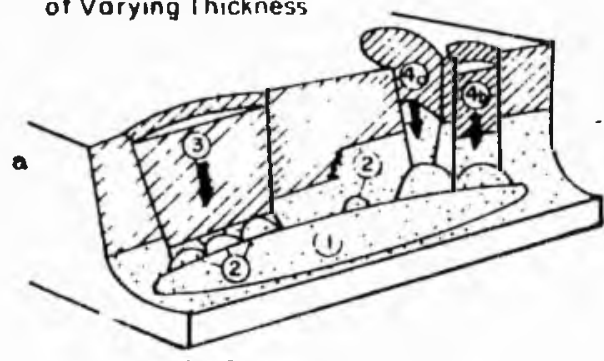

TOPS TATUM

(Clat a silt)

- SI JOS TRATUM

SANG, SAND

GRAVEL)

$\begin{aligned} \text { Fig. 6.1. General influences } & \\ & \text { bank sediments on th } \\ & \text { failure, according to } \\ & \text { (from simons, 1971). }\end{aligned}$

General influences of the geology or river bank sediments on the mechanics of bank failure, according to Turnbull et al.. 1966.

$\begin{aligned} \text { Fig. 6.1. General influences o } & \text { bank sediments on th } \\ & \text { failure, according t } \\ & \text { (from simons, 1971). }\end{aligned}$

(1) Scour Pool

(2) Subaqueous Barktulure by Fll..... Finear

(3) Upper Bonk Failuse hy She or

(4) Upper Bank Fallue ty Flow or Shear

(4a) (4b) 
A substantial diflerence exists between cohesionless sands and gravels and cohesive silt and clay in their interaction with these flow-induced hydroctynamic forces. For the cohesionless sediment the resistance to erosion will depend on the submerged weight of the particles and their angle of repose. In the cohesive beds, surface forces and electrochemical forces control the resistance to erosion. These cohesive forces are only partially understood, however it is known that they are not constant forces but are functions of the fluid quality and have time-dependent strength properties (Parthenjades and Paswell, 1970). A large number of physico-chemical factors of cohesive soils control erosion resistance, and various gross soil properties have been used as indices of erodibility, i.e. shear strength, plasticity index, mean particle size, percent clay, Atterberg limits, dispersion ratio etc. Some of the indices are unsatisfactory for various reasons (e.g. not unique measures, do not accurately convey the state of the soil at the surface, etc.), and although single properties are probably undesirable in such a complex system, better indices may be surface moisture content, density, potential swell, or particle orientation. Unfortunately there is no quantitative standard of erodibility by running water yet developed (Leopold et al., 1964; Task commitbee on exosion of collesive material, 1968; Partheniades and Paaswe11, 1970; Partheniades, 1971.).

In the talweg, the material is noxmally cohesionless sand or gravel, however the concave bank may have varying a'nounts of cohesive naterial exposed to the rlow. Where the banks are noncohesive, sloughing (the continual and general movement of particles) occurs, as well as slumping. In cohesive sediment, although lumps of cohosive sediment are removed by direct action of fluid forces and impact of suspended sediment, the dominant 
mode of erosion here is slumping (Fisk, 1947).

The bank failures (slumping) by shear or flow are caused by gravitational instability, whlch has been shown to be a result of oversteepening of the bank due to the formation of a scour pool or due to undexcutting of more easily erodible sediment deop on the exoced bank. Failures by flow or shear occur in the dominantly noncohesive point-bar deposits, and by shear in the thick backswamp clays and clay plugs (see fig. 6.1). Failure is greatly influenced by wetting of bank materials. Arroyos cut in fine-grained alluvium experience most bank cutting after, not duxing, flow, with wetting causing later slumping (Leopold and Miller, 1956). Wolman (1959) showed that a combination of thoraugh bank wetting and freeze and thaw promoted the greatest bank erosion in winter, despite large discharges in summer. Failure is also enhanced by the return seepage of water which infiltrates the banks during high flow. Upon lowering the stage the balancing pressure of the water in the channel is released and failure may occur (Jahns, 1947; Fisk, 1947; Jngris, 1949). The slumped blocks are broken up by the river and then subsequently become swept away and incorporated in the floodplain and channel deposits.

As far as slumping is concerned, therefore, the shear strength and permeability are important factors controlling erosion resistance, as are the spatial distribution of sedinent types in the bank. Vegetation in the stream bank will inhibit sloughing and slumping, and Jahns (1947) cites the particular example of a vegetated slumped block on which trees re-established thomsolves at a lower level and severely inhibited crosion. 


\subsection{Mathematical mode1}

Due to the complexity of the processes of bank exosion, an analytical treatment is not yet possible, however it is posstble to look at bank erosion in the required directions using semi-empirical deterministic models. Rate of bank erosion in the direction nomal to the mean downvalley direction is discussed first, followed by the development of an expression for downvalley migration .

Handy (1972) has shown that a fixstmorder rate equation of the form

$$
S / S_{0}=e^{-c_{1} t}
$$

was found to describe the distance $s$ of the channel at the bend axis from an assumed equilibrium position (i.e. from the position at limiting amplitude) as the meander was developing after cut off. $S_{0}$ is the initial distance from equilibrium, and $t$ is the tinc

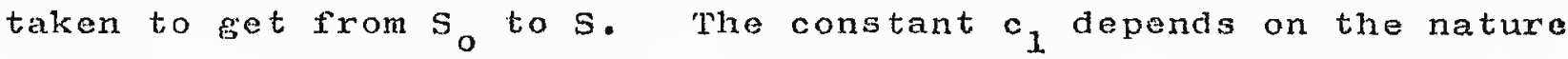
of the bank materials and the size of the river. From his analysis, the average rate of exosion at the bend axis, in a direction normal to the mean downvalley direction, can be expressed as

$$
\text { RLMIG }=\mathrm{c}_{1} \mathrm{~S}
$$

The equation describes the net rivex behavioux and indicates that in Eeneral the rate of erosion, RLMIG, will decreaso as the meander amplitude increases. This is substantiated further by the flume study of Nagabhushanaioh (1967) discussed proviously (see fig. 2.6). Although the equation (6.2) describes the gradual reduction in eroding ability in the direction normal to the mean downvaliey dixection, this will also vary on a different level as dischaxge varies with time, and as the erosion resistance varies with changes in bank materia1.3. 
As already mentioned, Daniel (1.971) fitted the sine generated curve to successive meander shapes as the meanders migrated. He also correlated the increase in path length with flow volume, Q vol (time integral over a year of all daily flows above mean annual $f(10 w)$, and percent of silt and clay in the banks. The flow volume showed a linear, and the grain size Index a nonlinear, relation with increase in path length. It should be noted that the number of points used to define these relations was very sma11. Inspection of equation (2.12) and $f i g$. 2.5 will show that path length (equals sn.1) increase has an approximate linear relation with amplitude increase, wavelength rematning constant. The exosion rate, RLMIG, will therefore also have a line relation with flow volume and a nonlinear relation with percent silt and clay. An expression for the erosion rate may therefore be written

$$
\text { RLMIG }=S k_{2} Q_{v_{01}} / G S I^{n} \cdot 2 .
$$

where GSI is a grain size index (\% silt and clay in the outer bank of the stream), $n_{2}$ is an exponent, and $k_{2}$ is a constant. Here the term $k_{2} \mathrm{Q}_{\text {vol }} / \operatorname{GSI}_{2}$ replaces $c_{1}$ of equation $(6.2)$. Q vol will not be expected to vaxy very much because the annual flow volumes for days above average dischargo are relatively constant (Daniel, 1971). $\Lambda$ discussion of the variability of Q vol is given in section 11.3. It may be possible to replace Q vol by some moasure of stage, as $l_{0}$ as the relation with erosion rate is ascertained. This may, however, involve complications involved with discontinuities in the stage-dischaxge rolation (shifts in rating).

Flume studies (e.g. Freidkin, 1945; Charlton and Benson, 1966; Ackers and Char1ton, 1970a) and the field work of Handy (1972) have shown that the downvalley rate of meandox migration $i$ s 
Independent of the amplitude growth and development. Given lateral homogeneity in bank materials and a constant discharge pattern, thexefore, the rate of downvaliey migration (RomIG) will be constant. A general expression for the rate of downalley migration, of the same form of equation (6.3) is therefore

$$
\text { RDMIG }=\mathrm{k}_{3} \mathrm{a}_{\text {vol. }} / \mathrm{GSI}^{\mathrm{n}} 2
$$

By specifying the constants $k_{2}$ and $k_{3}$ in equations (6.3) and (6.4), the overall physical limitations to erosion are indicated, and the overall effects of tho bank materials and variation in discharge pattern on bank erosion are accounted for. The exponent $\mathbf{n}_{2}$ describes the nature of the variation in the 'bank materials term' with grain size. RLMIG and RDMIG refer to average erosion over the period of time covered in $Q_{\text {vol. }}$

\subsection{Validity of models proposed}

It has not been possible to construct generalised models which take account of the numerous controliing factors of bank erosion that have been described, particularly the specific distrjbutions of bank materials that give rise to characteristic modes of bank failure. Instead a simplified view of the 'subsystem' has been taken, and a moxe practical empirical approach has been substituted for a more desirable, yet impracticable, analytical approach. Thus, the flood period volume has been used to account for the hydraul.jc forces acting. This may be assumed to account not only for direct fluid stress in the talweg and over the concave bank, but also the effect of the flood watiers on the condition of the bank sediment and the return soopage of water on falling stages. A grain size index has been used as a measure of the resistance to exosion by direct fluid forces and failuxe due to eravitational instability. In reality, a number of different parameters of the sediment forming the banks will be expected to affect resistance to erosion, as previously discussed, and the 
spatial distribution of sediment types has been shown to be important. Furthermore, the effects of vegetation have not been accounted for explicitly.

The models do, however, maintain the general relationship that exists in natural streams, which is that bank erosion and recession is most rapid in the case of banks of loose sand and gravel and streams of lare power, and is least rapid in the case of silt of clay and low powered streams (e.g. Jahns, 1947; Kolb, 1963; Allen, 1970c). Furthermore, equation (6.3) adequately accounts for the reduction in bank erosion normal to the mean downalley direction as amplitude reaches a limiting value. The constants of proportionality, $k_{2}$ and $k_{3}$, and exponent $n_{2}$, give sufficient flexibiljty in the equations such that there is adequate representation of the controls of bank erosion in specific cases. As an example in this respect, a factor like vegetation may be accounted for implicitly by choosing the appropriate empirical constants.

\subsection{Input}

Actual rates of erosion in natural streams vary from a few decimetres to many tens of metres a year. Some of the observed rates of erosion are complied by folman and Leopold (1.957).

Fig. 6.2 shows the nature of the variation of bank inigration rate, using equation (6.4), for various values of GSI, Q vol and constants $\mathrm{k}_{3}$ and $\mathrm{n}_{2}$. Sinilar curves would be obtained for equation (6.3) with $\mathrm{sk}_{2}{ }^{2}$ vol replacing k $3^{\mathrm{Q}}$ vol

No quantitative measure of $\mathrm{n}_{2}$ is available, however, by inspection of fig. 6.2, a value close to 0.5 seens appropriate. obviously more specific empirical data are reguired to define $\mathrm{n}_{2}$ adecuately. More empirical information is also required to define the relative rates or lataral and downalley migration in the case of a developing meandex, ard thus to define the redative 


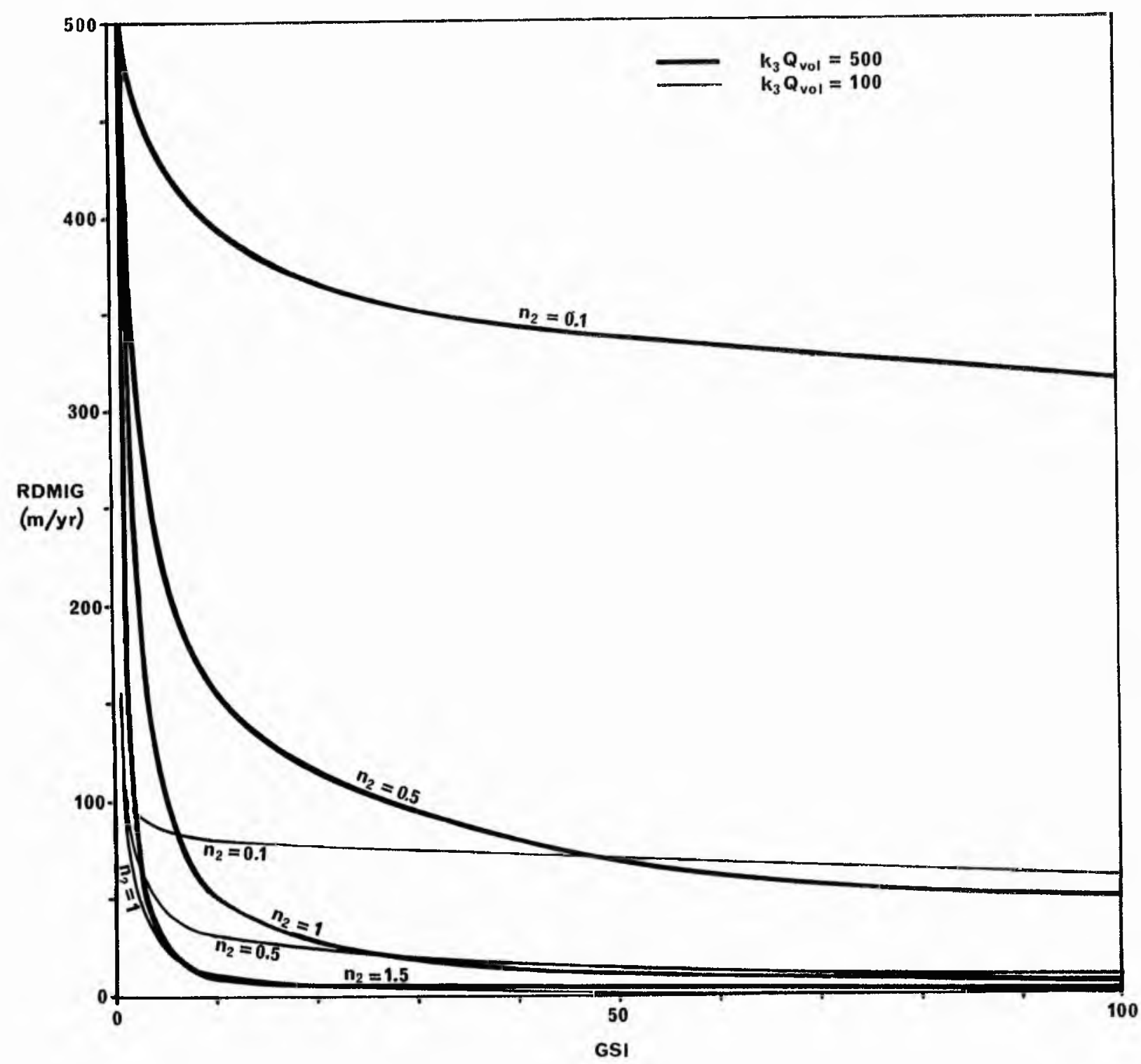

FIG. 6.2 PLOT OF BANK EROSION RATE AGAINST GRAIN SIZE INDEX, ACCORDING TO EQUATION (6.4), FOR DIFFERENT VALUES OF $k_{3} Q_{\text {vol }}$ and $n_{2}$. 


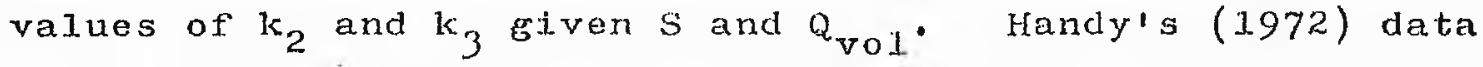
suggest that the maximum lateral migration is more than three times the downvalley migration. The absolute values of $k_{2}$ and $k_{3}$ will depend on the values of Q vol . In this respect, it should be noted that the absolute values and units of Q vol for a particular channcl are not explicitly specified (see section 11), therefore $k_{2}$ and $k_{3}$ assume the role of sealing constants. 
Bank erosion in the bend of a meander is usually counteracted by an approximately equal amount of deposition on the opposite bank, thus explaining why the width and cross sectional area remain about the same as the channel moves laterally across the floodplain (Wolman and Leopold, 1957; Leopold and Wolman, 1960; Leopold et a1.., 1964). This ts the basis for determining the amount of lateral sediment deposition in the model. Ifowever, certain important points must not be overlooked when using this simplified approach.

In the particular case of a meander developing to a stable form, under conditions where the independent variables are constant, the various deperident variables will be expected to change. The direction of the changes at a cross section may be discussed by examining the Darcy equation,

$$
Q=a \sqrt{8 g R S / f}
$$

where a is the cross sectional area of flow and $Q$ is discharge. For the case of constancy in the independent variables of the channel, at bankfull stage, of a stable meander, all of the parameters on the right hand side of the equation (7. 1) are constant (assuming that the 'friction' coefficient is the same for all occurronces of bankfull stage). However, as a meander increases in amplitude at constant waveleneth, sinuosity increases and longitudinal water surface slope will decrease. Reference hexe is bejng made to development of meanders in which the initial watox-surface slope is too steep for a given constant aqueous and sediment discharge (e.g. after the cut off or avulsion situations), and not the sttuation where water-surface slope is fncreasing during the development of meanders due to general ageradation which results from too high a sediment load for a given slope (see Ackers and Charlton, 1970). Tnvolved with 
increase in sinuosity is a characteristic variation in the mean radius of curvature of the bend (see fig. 2.4). Radius of curvature decreases with sinuosity up to a value of 1.5 ; above this sinuosity, the radius of curvature gradually increases. To counteract the decrease in $S$ on the right hand side of equation (7.1), $f$, and $R$ may vaxy in any combination, however f has been shown to be intimately related to $\mathrm{a}, \mathrm{R}$ and $\mathrm{x}_{\mathrm{m}}$.

In general, $f_{b}$ and $f_{s}$ rill increase with sinuosity. With a and $R$ constant, $f_{s}$ will increaso with decxeasing bed shear stress and stream power and increase in the degree of form roughness with the lower flow regime. $f_{b}$ has been shown to increase with sinuosity, however it also depends on the ratio $r_{n} / w$. With width constant, $f_{b}$ will be expected to increase up to a sinuosity of 1.5 , and then gradually decrease. This anomaly may be spurious but at the moment no further light can be thrown on the problem.

If it is assumed that $f$ is increasing with development of the meandex, a and/or $R$ must increase. The effect of increasing $R$, keeping width constant, would be to increase the spiral motion (Yen, 1965; Rozovskii, 1961), thus increasing $f_{b}$. However $f_{s}$ may be decreased due to relative roughness effects ox increase jin bed shear stress and flow power.

Increase in $a, k e o p i n g R$ and the shape of the cross section constant, jnvolves increasing width. The effect or increase in width on spixal flow appears to be somewhat confused in the literature. Yen (1965) infers that an increase in width at constant depth reduces the strength of the spiral flow; Shukry (1.950) says the opposite but is dealing with channols of smal1 width/depth ratio. Rozovskii (1961) says the losses due to spiral flow are independent of width.

As can be seen the relationship between $f, a, k$ and $r_{m}$ is 
very complex and is not properly understood. Furthermore, despite the difficulty in determining the dixection of change $1 n$ $f, a$ and $R$, the relative amounts of change are indeterminate at present. Suffice it to say that there will be adjustinents, however slight they may be, in the dimensions and resistance of the channel as the meander increases in sinuosity. Unfortunately these cannot be accounted for at present. 
It has been noted by many authors that most of the erosional and depositional activity of rivers is limited to pexiods of high water (Leopold et a.1., 1964), and the dimensions of vaxious morphological characteristics of meandering rivers have been related to various measures of discharge that are considered to be 'dominant' discharege (e.g. equation (2.13)). Wo1man (1959) states that $85 \%$ of observed erosion occurred during the winter floods, supporting Carlston's (1965) conclusions that the dominant deposition and exosion controlidng meander wavelength occurred during flows that are equalled or exceeded $10.40 \%$ of the year. Schumm $(1968,1969)$ found that when average, bankfull, and mean annual flood discharge are each correlated with the percentage of silt and clay in the channel perimetex and the channel

properties, the results are, to nearly equal degrees, significant explanations of meander wavelength and channel cross-section properties. Stall and fok (1968) found that hydraulic geometry was best explained using the discharge that is exceeded 10\% of the year. Recent work by Ackers and Charlton (1970c) supports some of the earlier workers in their contention that bankfujl discharge determines meander pattern.

These facts show that no single measure of discharge can be assumed to control meander dimensions but that a range of discharges are involved. Indeed, Freldkin (1945) shows the position of the main velocity thread in a meander loop at varying discharge, and indicates that low flow, half-bankfull flow and bankfull flow, respectively, attack the upstream, mid, and downstream parts of the concave bank. At discharge greater than bankfull the meandering pattern is not lost, and vigorous exosional and depositional activity within the channel continues, however with a much moxe complicated flow field prevailing 
(Toebes and Sooky, 1967).

Daniel (1971) submits evidence to support the assumption that channel formation will begin at just about the average discharge and continue for all higher discharges (not, however, making any distinction with respect to what part of this range will have the greatest effoct). In the model this quantity, flood pertod volume, is calculated for every year and used in various computations involving erosional and depositional activity. For the reasons outlined above, this 'time integral' of the discharge hydrograph is much more preferable than an instantaneous measure of dischargo (c.f. Allen, 1971). Despite the range of discharges influencing channel formation much of the erosional and depositional activity is expected to occur at discharges closely associated with bankfull stage. This lends support to the study of events within the channel at bankfull stage only (see section 5.8). Bankfull stage recurs on average once or twice every year or so according to climatic regime (Leopold et al., 1964; Woodyer, 1968). It can be assumed, therefore that bankfuld stage will be attained, or neaxly attained, at least once a year, associated with the seasonal high water periods.

During the rising stages of a high water pexiod, increase in velocity and bed shear stress bring about an increase in bed sediment transport and bank erosion. The outer concave bank, together with previously slumped material, is scoured outwards and noxmaljy net erosion will occur in the pool and over the point bar. Chutes may develop over the top of the bax and permanent chute or neck cut of may occux when cextain limiting conditions cxist. Avulsion is also a flood stage phenomenon. 
On falling stages sediment is normally deposited on the bax and in the pool and, ultimate1y, approximately the same channel. width and cross sectional area that existed before the flood period will be attained. The position of the bar will be different from that before the flood period, due to the recession of the outer bank. Bank caving following scouring in the pool is often a falling stage phenomenon (Matthes, 1941; Jahns, 1947; Inglis, 1947; Russell, 1967), and those caved blocks left after the end of the flood perjod will be swept away in subsequent flood perlods. A detailed discussion of iscour and fill' and cut-off follows. 


\subsection{Preliminary discussion}

Colby (196/) discusses two principles which are helpful In understanding scour and fill in sand bed streams. One is the principle of continuity of volume of bed material along a stream reach. This principle simply states that changes in the average elevation of a bed in a reach result from the diffexence in the rates at which sand enters and leaves the area. The second principle is that a relation exists between the discharge of sands and the characteristics of flow and available sodiment (e.g. Bagnold, 1966). Thereforo in the case of steady uniform flow the average sediment transport rate at a point remains constant with time and remains unchanged with distanco along a streamline. In such a case there will be no progressive erosion or deposition of sediment and the stream bed elevation will remain constant. However, when the flow is unsteady and nonuniform, the abjlity to transport sediment varies and erosion and deposition can occur. For gradually changing flow, Allen (1970c) expressed the rate of erosion or deposition as

$$
\underset{\text { rate of erosion }}{\text { depition }}=\frac{\partial t}{\partial x}+\frac{l \partial t}{v \partial t}
$$

where $i$ is the sediment transport rate (imnexsed weight passed per unit width per unit time), $V$ the mean fluid flow velocity, $t$ is time, and $x$ is the distance measured in the local downstream dixection. The first term represents the contribution from the nonuniformity of flow and the second term the contribution from the unsteadinesi. Whethex exosion or deposition occurs depends on whether the right hand side is positive or negative respectively, which depends on the relative magnitudes and signs of the two terms. In fact, on furthex study of equation (9.1), it can be seen that the unsteady term indicates that exosion or deposition 
may occur at a point only because of changing transport rato at that point. This is not in agreement with Colby's principle of continuity of sediment movement and so in studies of scour and fill the meaningful use of this term is precluded.

The proviso of gradually varying flow is partly due to the lag between sediment transport rate and changes in flow. The quantities determining local sediment transport rate do not change instantaneously with chaneses in flow but instead, at any point, these quantities are strongly influenced by the flow conditions prevailing upstream from this location. Furthermore, a finite time and corresponding distance are requixed for the excess entrained sediment to settle to the bed as the flow power decreases, and for additional sediment to be entrained as rlow power increases (Kennedy, 1963).

study of the hydraulic geometry shows that, during the passing of a flood wavo at a cross section, changes in water djscharge and energy gradient will result in changes in flow resistance, width, depth, watex suxface slope, and velocity (Leopold et al., 1964). In particular width, depth, slope and velocity generally increases. Flow resistance is affected by the changing concentration of fine sediment or, in a more discontinuous way, due to changing bed forms or overbank flow. Turbulence, fluid density and apparent viscosity, hence effective fall velocity of particles, are affected by changes in temperature and fine sediment concentration. This in turn affects bed configuration. The changes in hydraulic variables are inter.related in a complex way, however, in general, it can be said that sediment transport rate at a section increases with the flood wave.

The principles involved in scour and fill can be applied to meandexing streams in two contexts; in texms of a reach and. 
in terms of a single bend. Tn the first case, for simplicity, we will assume that there is no overbank flow and that the general characteristics of flow and available sediment are the same at each end of the reach. Duxing a flood wave the hydrograph will lag a little from one end of the reach to the other, henco average sediment discharge will lag in a similar way. This will result in net deposition in the reach during the rising stages, i.e. $\partial i / \partial x$ is negative, and net erosion during the waning period, i.e. $\partial j / \partial x$ is positive. If however, for some reason, the inflow to the reach is stopped, scour over the distance required to entrain an equilibxium sediment load would occur at the beginning of the reach: Also, if the characteristics of flow and available sediment are not the same fow both ends of the reach, perhaps due to difrerences in slope or flow resistance, then $\partial i / \partial x$ would not be zero and scour or deposition may occur. Colby has shown that the thickness of sediment eroded or deposited in these cases in very small when averaged out over the stream bed. These points indicate that the average bed elevation in a reach, under the conditions specified, is usually fairly stable during the passage of a flood period, and that there is not general scouring and filling over the whole reach during rising and falling stages.

In the context of a single bend, the characteristics of flow and available sediment during the low water stages can be expected to be different between the pool and the riffle, and thus sediment transpoxt rates will be different. However sediment transport rates will be very small at low discharges, so they warrant little attention. Lane and Borland (1.954) have shown that as the water surface rises the cross sectional area of flow increases faster at the riffles than in the pools, and this is formally accompanied by changes in the hycraulic parameters in a different way in the pool from at the riffle. In general 
on rising stages $\partial i / \partial x$ becomes a positive number at the pool and scouring occurs in the pool. At the riffie $\partial i / \partial x$ becomes a negative quantity and deposition occurs on the riffle. on falling stages the opposite will happen (Freidkin, 1945; Sundborg, 1956; Kondratev, 1962). Such bed changes will be significantly more than in the cases previously mentioned (colby, 1964). See fig. 9.1 .

\subsection{Mathematical mode1 for scoux depth}

Scour and fill in a neander can be treated empirically on a deterministic basis with cextain stochastic properties.

The average net scour (in terms of immersed weight per unit width) at a cross section in the pool, NS, can be written

$$
N S=\int_{0}^{T} \frac{\partial i}{\partial x}(t) d t
$$

where $d i / d x(t)$ is the time varying rate of exosion or deposition at a particulax section. $T$ is the time from the beginning of appreciable sediment movement on rising stages ( $\partial_{1} / \partial x(t)$ may not necessarily be positive at this time) through the positive range of $\partial i / \partial x(t)$ until it becomes zero. $\partial i / \partial x(t)$ will then become negative and deposition will occur until it becomes zero again (see tig. 9.2)

It can be seen that the net scour (area $A-B$ in fig. 9.2) will depend on $T$ and the shape charactoristics of the curve of $\partial i / \partial x(t)$ as $t$ varies between 0 and $T$. The shape of the curve of $\partial i / \partial x(t)$ depends, as already indicated, mainly on (a) the hydraulic nature of the cross sections within the pool and riffle at a given stage, which controls the relative spatial and temporal distribution of sediment transport rates, torether with (b) the time variation of the changes in stage. Fig. 9.1 shows a tendency for $\partial_{i} / \partial x(t)$ to change from positive to negalive 
Absolute bottom, Level above graph elevation datum

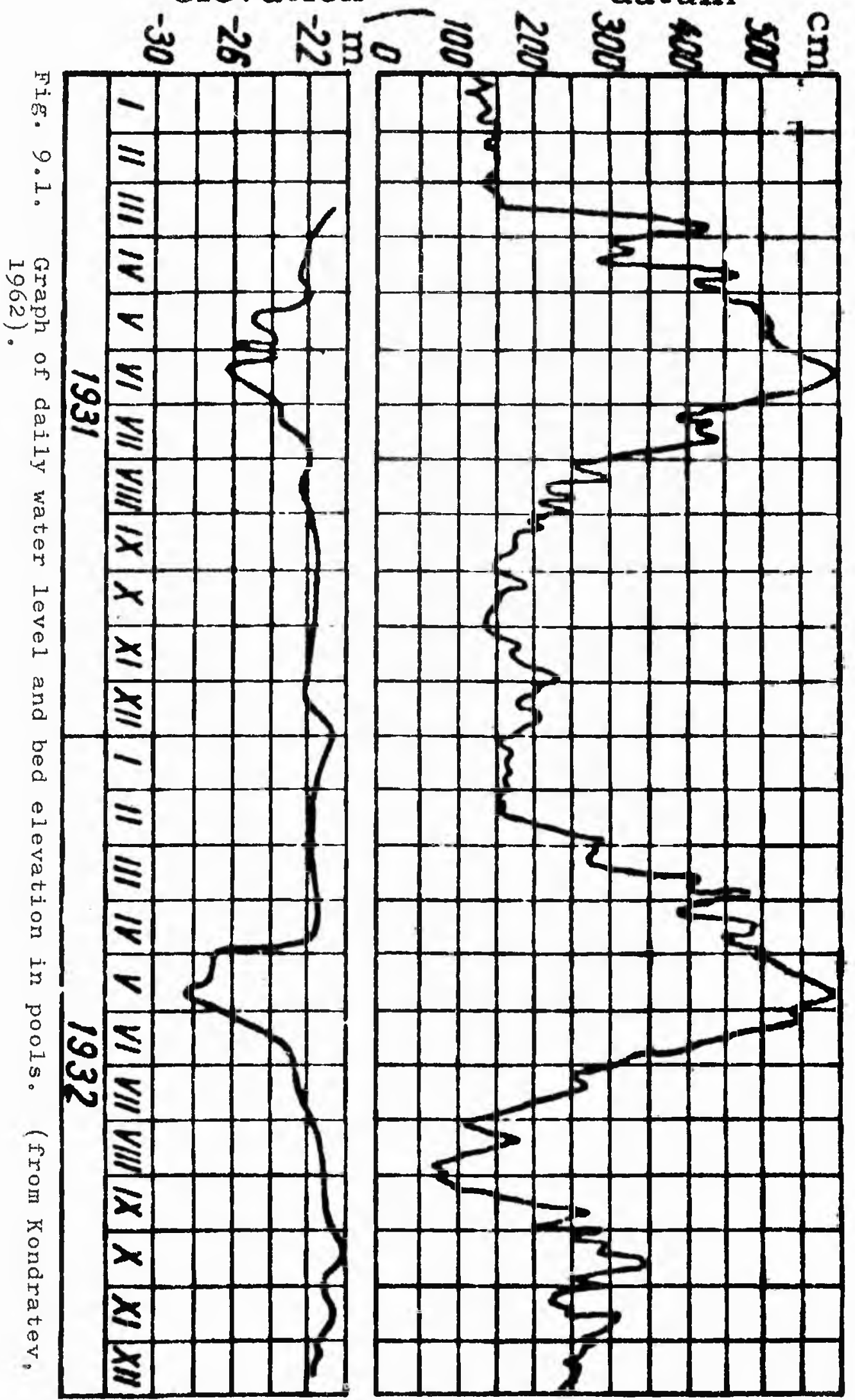




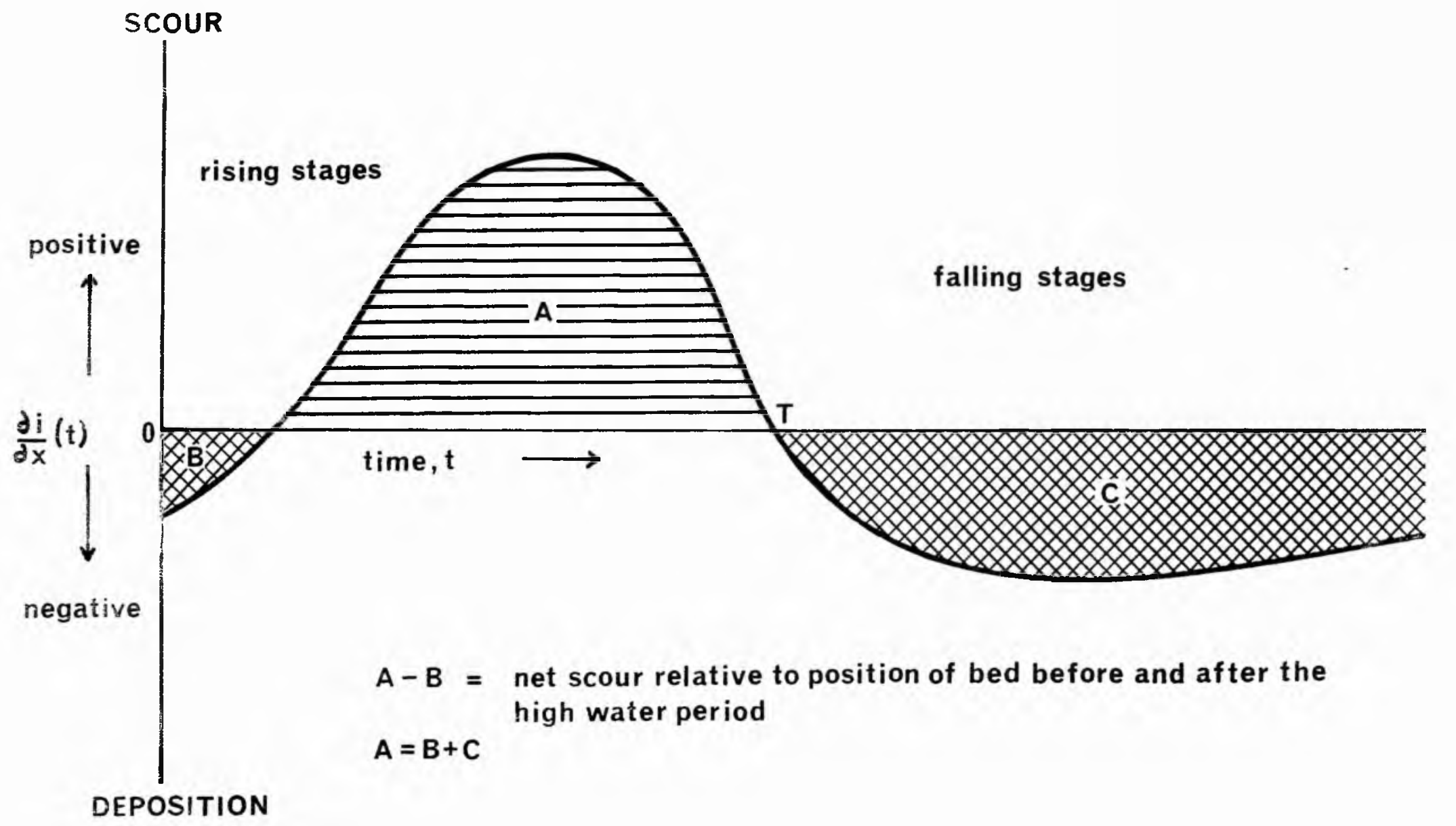

FIG. 9.2 IDEALISED VARIATION OF EROSION AND DEPOSITION AT A CROSS SECTION IN A CHANNEL BEND(POOL) WITH THE PASSING OF A HIGH WATER PERIOD. 
(i.e. $t=T$ ) at about the peak stage for each flood pexiod and that there is a tendency for a change from negative to positive (deposition to scouring) in the early rising stages. $T$ is also expected to depend on (a) above, as well as the flood period hydrograph. The curve shown in $f i g .9 .2$ is assumed to be smooth for simplicity, although by virtue of the controling factors it would probably have fluctuations imposed on the general trend. We may now say qualitatively that for any high water period, the average net scoux at a cross section is a sole function of the cross section characteristics around the meander and the shape of the flood pertod hydrograph. An analytical. representation of this functional relation cannot be determined at present; however a somewhat approximate relation will be determined for this study.

A measure of the flood period hydrograph can be obtained as the integral of the flood period discharges with rospect to time, that is, the flood period volume, $Q_{\text {voll }}$ as defined earlier. It will now be assumed that the hydraulic nature of the cross sections within the pool and riffle at any given stage is constant for every flood period. This assumption may not be valid, especially when meandex sinuosity and amplitude is increasing, however much further research is needed to test this assumption adequatiely. For the present the variation of $N S$ can be described using an equation of the form

$$
\operatorname{DSCR}=\mathrm{k}_{4}\left(\mathrm{Q}_{\text {vo1 }}\right)^{\mathrm{n}} 3
$$

where DSCR is a measure of NS and is the net depth of scour below the bed elevation before and after the flood period, measured at the talweg. The empirical values of the constants $k_{i_{4}}$ and $n_{3}$ for a particulax cross section are imposed by the characteristics of 
the channel cross sections around the meandex, for the range of

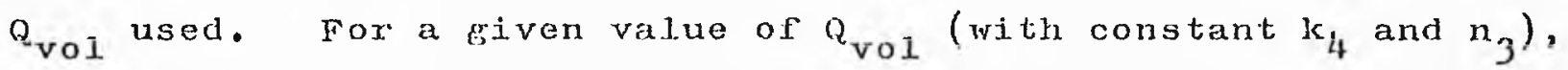
NS (and DSCR) may vary for some combination of the following reasons; (a) Apart from the approximating assumption of using $Q_{\text {vol }}$ as the only variable, approximations axe also involved in the use of $Q_{\text {vol }}$ as a parameter and effectively ignoring separate flood events within a flood period.

(b) Local effects of scouring, i.e, in the lee of dunes. (c) General scouring or deposition affecting the whole reach as previously discussed. It will therefore be assuned that all fluctuations about the mean curve (equation (9.3)) can be treated stochastically by introducing a texm, ex, which is a normally distributed random variable with mean $o$ and standard deviation, stdrn, some function of the absolute limits of scour depth. Therefore, we write

$$
\operatorname{DScR}=k_{4}\left(\mathrm{Q}_{\mathrm{vol}}\right)^{\mathrm{n}} 3+\operatorname{er}(0, \operatorname{stdvn})
$$

Unfortunately, at present the general validity of equation (9.4) cannot be assessed, however it is sufficientiy flexible to account for scouring in the specific case, as defined by the empirical input variables, $k_{4}, n_{3}$ and stdvn.

In the model the overall. shape of the scour hollow is obtained by assuming no scour at the junction of the bankfull water surface and the innex bank. The scoured bed profile is then defined using the equation for the point bax profile (e.E. equation (5.1)) but, at the talweg, the bed is a distance DSCR below the oxiginal depth. This is illustrated in fig. 14.2. The shape of this scoured bed is purely heuristic, howevex inspection of fig. 9.3 lends a faix degree of credibiltty to the method used.

\subsection{Deposition on ralling stages}

on falling stages, in the absence of any extexnal disturbing factors, deposition will normally fill the bed to its original depth, however probably at a slower rate because the hydrograph is 


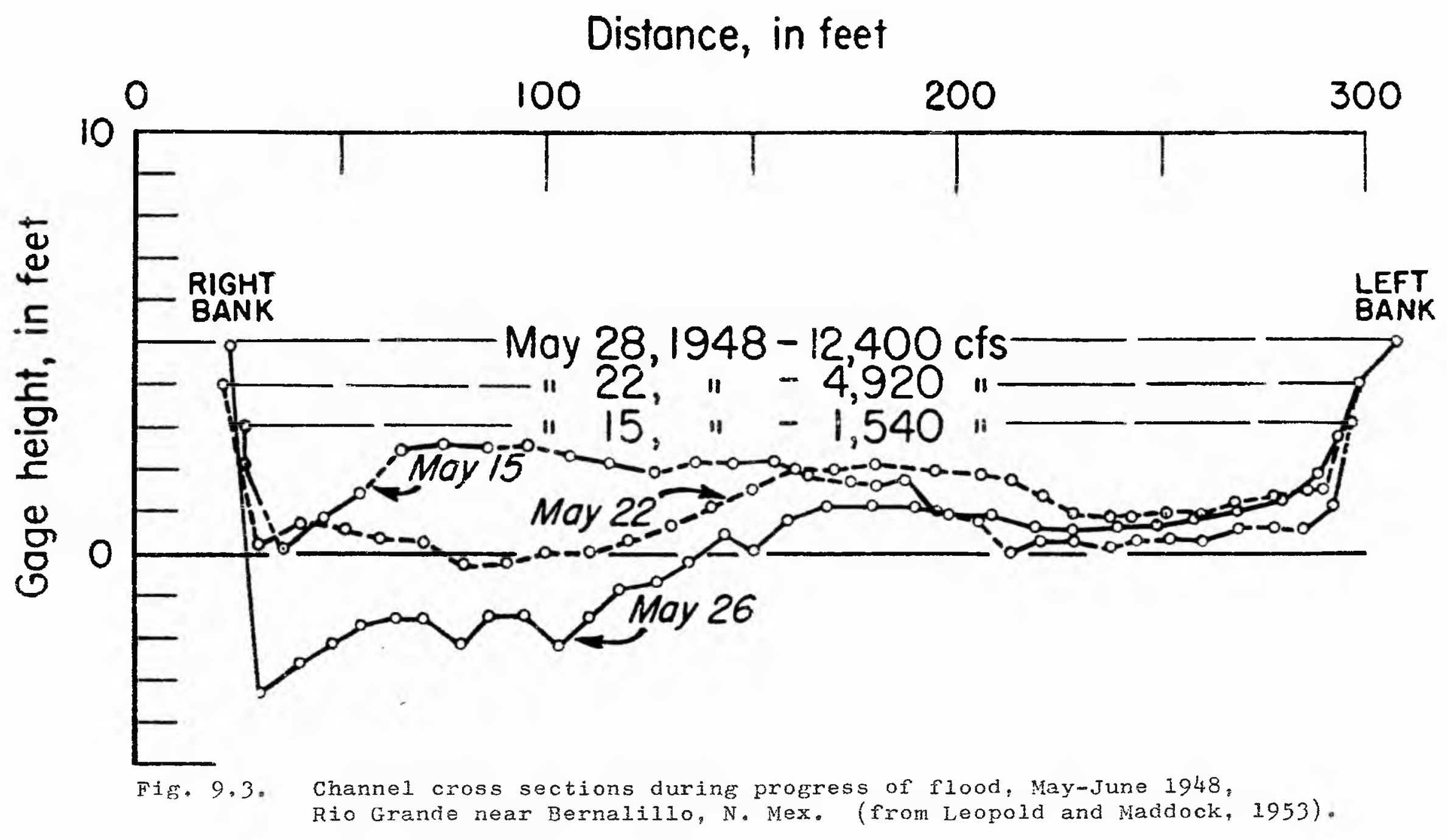


not as steep on falling stages (see fig. 9.1). In the model it will be assumed that, after scour, most of the filling to the original bed profile is accomplished on falling stages, or at least before the next flood period. The scoured bed is filled by incrementally reducing the depth at the talweg and redefining the bed profile at each increment using the equation of the point bar profile. The grain size and sedimentary structure in the fil1 are obtalued by repeated application of the point bar sediments model. (section 5) across the profile as the depth is incrementally reduced to the original (see section 14 for details). It is thexefore assumed that the 'equilibxium' bed forns, corresponding to the different flow conditions, have time to develop at each level of the bed as filling proceeds. Throughout this operation it is assumed that the water level and slope are constant at bankfull level. Thus, as in the point bar sediments model, it is assumed that all the depositional activity occurs at bankfull stage.

\section{$9.4 \quad$ Input}

The values chosen for $k_{4}, n_{3}$ and stdvn can only be heuristic at present, and they will be expected, because of their empixical nature, to be limited for use on any one specific cross section under discussion. As prevfously stated, the absolute values and units of $Q_{\text {vol }}$ are not explicitly specified as they are only being used in empirical equations which are scaled by proportionality constants, in this case $\mathrm{k}_{4^{*}}$ Having chosen the nocessary empirical values, the variation of scour depth will be described, whilst also making a statement describing the 11mitations Imposed on scouring by the whole hydraulic makeup of the meandex in question. The shape of the curve of DSCR plotted agrainst Q vol is described by exponent $n_{3}$. It is expected to be a positive number but little else can be said at present. Stdrn is expected 

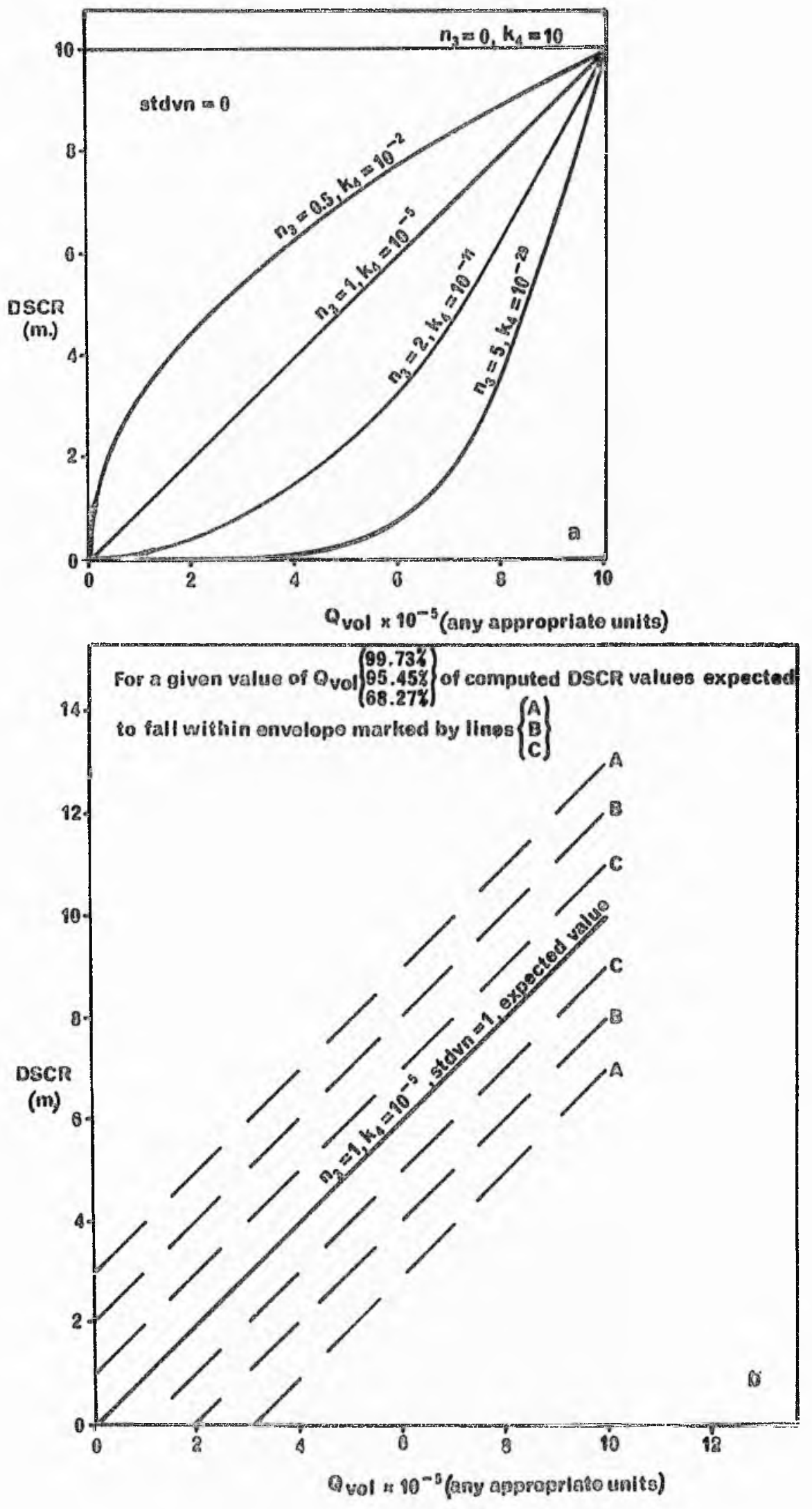

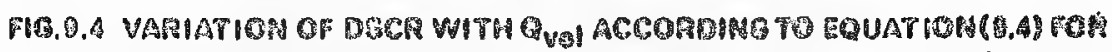

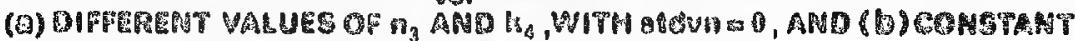

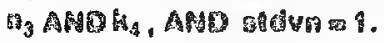


to be some fraction of the limiting scour depth. The value of $k_{4}$ for a given range of Q vol can be allurled to by using as a general guide the fact that the bed comonly recedes about the same order of magnitude as the water surface rises (Leopold et al., 1964; Lane and Borland, 1954). Fig. 9.4 shows the variation of DSCR with $Q_{\text {vol }}$ for various values of the parameters. It is to be noted here that if $\mathrm{n}_{3}$ is required to be vaxied, for a given range of $Q_{\text {vol }}$ and DSCR, $k_{4}$ must be adjusted also.

The phenomenon of scour and fill is characteristic of ephemeral sand bed streams and large rivers in semi-arid climates; it is less typical of rivers in humid areas or thoso In high mountains, presumably because perennial flow tends to winnow away the fine material and the bed becomes armoured with coarse materials (Leopold et al., 1964). In the sand bed streams of the present study scoux and $f i l l$ is to be expected, however an option will exist jn the model if scour and fill is not required.

\section{CUT-OFF}

Cut-off occurs whenever the meandering strean can shorten its course and thus locally increase its slope, the frequency of cut-off increasing with channel sinuosity (Al1en, 1965a).

\subsubsection{Model for chute cutwoff}

As already stated, the formation of chutes is associated with a liniting sinuosity and amplitude to the extent that flow reststance is less ovex the bax than around the bend (Freidkin, 1.945). An increase in amplitude and sinuosity towards limiting values, wavelength constant in the model, thus involves an increasing tendency for the formation of chutes. Durinf flood stages, the directing of a greater part of the flow across the bar enhances the tendency for chute formation. Due to the assumed homogeneity of the bank matertals, there will be no other causo of change in alignment of the flow upstream. 
permanent chute cut-off can thexefore be treated as a probability function of the magnitude of the flood volume and the sinuosity, a higher probabiltty of occurrence being associated with high floods and with a liniting value of sinuosity, thus

$$
p(c)=\left(Q_{\text {vol }} / Q_{\text {volmax }}\right)^{e c_{1}}\left({\mathrm{sn} / \mathrm{sn}_{1 i m}}\right)^{\mathrm{ec}}
$$

where $p(c)$ is the probability of chute cut off, $\mathrm{sn}_{1 i \mathrm{~m}}$ is the limiting sinuosity, and $Q_{\text {volmax }}$ is the value of $Q_{\text {vol }}$ at which cut off would be a certainty if $s n_{\text {is }}$ also equal to $\operatorname{sn}_{1 i m}$. By increasing the empirical exponents $\mathrm{ec}_{1}$ and $\mathbf{o c}_{2}$ ' the probability of cut-off becomes very small unless Q vol and sinuosity are close to their limiting values.

The occurrence of chute cut $\ldots$ of in the model is determined by generating a pseudorandom number in the range 0 to 1 . This number is then compared with the value of $p(c)$ for the particular high water period under consideration. If $p(c)$ is greater than the numbex, chute cut-off will occur. In fact the process of chute cut-off takes place slowly because the angle of diversion of the water down the shorter course is small and the increase In flow is gradual (Fisk, 1947). The occurrence of chute cut-off is therefore defined as the beginning of the process which is assumed to go to its end point. The model will be stopped after the initiation of cut-off because of the complicated flow patterns that result, the inability to predict where the new channel will form, and the inability to account for the deposition in the old channe 1.

\subsection{Model for neck cut-off}

Neck cut... off will tend to occur when the meander neck, the shortest distance from the arjacent banks of closing meander limbs (therefore only defined in meanders in which the local downstream direction makes an angle greatex than $90^{\circ}$ with the 
mean downvalloy diroction), approaches close to a limiting width, necessarily a small quantity, Neck cut-off could occur if sinuosity could increase, wavelength remaining constant, to such a value that the meander neck, GAP, becomes very small. This is the situation in the meandering tidal creeks of the Nifer Delta region (Nedeco, 1959). Decreasing wavelength of a meander due to a clay plug, and the associated distortion of the loop, will cause sinuosity to increase rapidly and the meander neck to become smal1. Neck cut... oft in this situation is described by risk (1944, 1947) from the Jower Mississippi. Because of the conditions imposed in this study, the effects of clay plugs cannot be accounted for and the latter mechanisin cannot be simulated. A probability function similar to that used for chute cutmoff may be used fox neck cut-off, i.e.

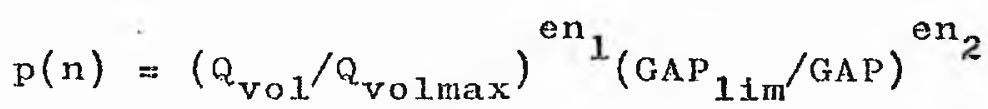

where GAP lin $_{\text {is }}$ is the limiting value of GAP, and $e n_{1}$ and $e n_{2}$ are empixical exponents. The occurrence of neck cut-off will be determined as outlined above, and the model will again be stopped after neck cut off has been initiated.

\subsection{Input}

The expressions piven in equation (10.1) and (10.2) above are necessarily of a heuristic nature because of the lack of precise knowledge on the subject. The values of $e_{1}, e_{2}$, on and on will. be intuitive and will be expected to be relatively large positive numbers. Their values can only be inferred by trial and error, and in this respect it is noteworthy that average times taken to cut-off from inception of a meander loop to cut-off are of the oxder of hundreds of years (see Handy, 1972; Lathrap, .2968). This will be expoctod to vary with tho general hydraulic setting. Specifically, the time taken before cut-off is intilated will be expected to depend on the general 
calibre of sedinent in the perimeter of the chamel, which will influence the rate of lateral erosion relative to the size of the stream, the 'oquilibrium' struosity, and the susceptibility to deep scouring. It will also depend on the variability of the flood period volume above that which exexts most control on the channel formation.

Ixamination of the maps produced by Fisk (1947) of the Mississippi Valley shows that natural chute cut-off occurs at sinuosities up to about 2.0 , and before the angle between the local direction of the channel and the mean downvalley direction exceeds about $90^{\circ}$. If the value of the limiting sinuosity is greater than about 2.0 (approximately) chute cut-off will not normally be expected and mutual adjustmenis of the exponents nay be required here.

The limiting value of sinuosity has been discussed earlier, and expected to depend on the grade of sediment in the meander and, related to thts, the width/depth ratio of the channel. Fisk's maps furthex show that neck cut-off involves meanders wi.th considerably greater sinuosity than 2.0, and the limiting value of the meander neck is expected to be very small relative to the dimensions of the meander.

The relationship between any $Q_{\text {vol }}$ and the value of $Q_{\text {volmax }}$ (presumably based on long period records) depends on the character of the river regime under consideration. Presumably adjustments f.n $e c_{1}$ and $e_{1}$ will be required as the variability of $Q_{\text {vol }}$ varies. 
11 FLOOD PEIIIOD VOLUIE

\section{Introduction}

It is now necessary to compute the flood period volume, Qvol, which is defined as the sum over a year of all daily flows above the mean daily flow for a particular hydrograph. This is done by sequential generation of daily streamflows using the apparatus of operational hydrology. The absolute values of daily flows, hence flood period volumes, are not important as they are used only in empirical equations which are scaled with proportionality constants. The important point therefore is the shapes of the hydrographs and not the absolute scales. It would in fact be difficult to find an absolutely rigorous discharge hydrograph (dimensionwise) to fit a particular channel section, as the discharge pattern, specifically bankfull discharge, is not explicitly defined. By virtue of model construction, discharge, an independent system variable, is made dependent by specifying the channel and meander dimensions.

\subsection{Sequential generation of streamflow data}

one approach to streamflow simulation involves analysis of the hydrological system in order to find the causal relation between streamflow and its controlling factors. Numerous deterministic methods have been proposed and developed to empirically relate one or more climatic and physiographic factors to the streamflow hydrograph or some other streamflow characteristic, with considerable variation in the number of factors used (see Chorley and Kennedy, 1971; Crawford and Linsley, 1966). Although such methods of streamflow generation may be useful in reconstructing the climatic and physiological characteristics of the basin to which a particulax streamflow record is related, their use is precluded by the anount and nature of the input data required. 
Other approachos seek only to amalyse the observed streanm flow record. The flood record is orten analysed and fitted with a certain probability distribution to determine the recurrence intervals of the flood or the flood frequencies. This type of analysis cannot be used for sequential generation of streamflow data. Because the hydrologic process is stochastic (Chow, 1964, 1967) the streanflow hyclrograph may be thought of as a continuous time series, and daily, monthly or annual discharges (or stages) represent discrete time series. A time series may be approximated by a mathematical generating model, the choice of which is based on how well the mathematical structure of the model fits the physical characteristics of the time series. Hydrological processes and time series are generally treated as stationary, sometimes after simple transformations on the original time series, in ordor to simplify the mathematics. Various mathematical models, or combinations of models have been used in hydrology, and, in order to decide which provides the best fit, the sample correlogram and power spectrum have been used (e.g. Chow and Kareliotis, 1970; Dawdy and Matalas, 1964; Quimpo, 1967, 1968a,b).

11.2 Mathematical model of hydrolopic time series In this study it is intencled to generate a pattern of daily flows at a given stream section (absolute values being irrelevant) using the mathematical representation of the time series pextainjng to that section. Such a series will be the combined eftect of a deterministic conponent and a. stochastic component. In general, the deterninistic component may be composed of a trend and an oscillatory component. Trends may be removed from the time series by such methods as moving averages or polynomial regression, however, in this study the trend will, for simplicity, be assumed absent. 
The mathenatical model. used is a combination of the sum of harmonics and autoregressive models (e.g. Roesner and Yevjevich, 1966; Ruimpo, 1967, 1968a; Rodriguez-Iturbe, 1968; Adamowski, 1971). If $x_{t}$ is a nonstationary time series of daily flows, that is assuming the observed value of each of the $365^{\text {days in }}$ the yoar is to be drawn from a different population, stationarity can be approximated and the components of $\mathrm{x}_{\mathrm{t}}$ can be separated by the following transformation,

$$
z_{t}=\left(x_{t}-m_{\tau}\right) / s_{\tau}
$$

where ${ }^{\mathrm{m}} \tau$ is the daily mean value of the day $\tau,{ }^{3} \tau$ is the standard deviation of day $\tau$, and $\tau$ xuns from 1 up to 365 . The 'standardised series', $Z_{t}$, is second order stationary, being distributed with zexo mean and standard deviation unity for all daily values.

Using Fouriex analysis, a mathematical representation of the ${ }^{m} \tau$ and the $s_{\tau}$ may be expressed as continuous functions, $m_{t}$ and $s_{t}$, by the expressions

$$
\begin{aligned}
& m_{t}=\vec{m}_{\tau}+\sum_{k}\left(A_{k} \cos \frac{2 \pi k}{L} t+B_{k} \sin \frac{2 \pi k}{L} t\right) \\
& s_{t}=\vec{s}_{\tau}+\sum_{k}\left({ }_{s} A_{k} \cos \frac{2 \pi k}{L} t+s_{k} B_{k} \sin \frac{2 \pi k}{L} t\right)
\end{aligned}
$$

where $\bar{m}_{\tau}$ and $\bar{s}_{\tau}$ are the means of the $m_{\tau}$ and $s_{\tau}$ respectively, and $A_{k}, B_{k} s^{A} A_{k}$ and ${ }_{s} B_{k}$ are Fourder coefficients. Ixperience shows that a plot of the expected daily values of the time series $x_{t}$ over a number of years results in a pexiodic movenent with a fundamental period of one year. L therefore becones 365. It should be noted that in order to fit the trigonometric functions of the fourier series to the shape of the observed periodic movements, the number of hamonics requirod varies depending on the shape of the periodic movements in question. 
For instance, physical considerations of hyclrologic pexiodicities normally indicate a yearly cycle, often with a 6rmonth cycle as we11. If the yearly pexiodic movement is far from a sine function, some or all of the other subharmonics may be needed, depending on their contribution to the observed variance. Thus, because of the method of analysis used, more harmonics than those corresponding to the basic astrononomical cycles may be required. In this study, no more than five subharmonics of the yearly cycle will be used, that is, $k=1,2, .6$.

If equation $(1, .1)$ is rewritten using the harmonic representation of $m_{\tau}$ and $s_{\tau}$ the resulting series

$$
\mathrm{Y}_{\mathrm{t}}=\left(\mathrm{x}_{\mathrm{t}}-\mathrm{m}_{\mathrm{t}}\right) / \mathrm{s}_{\mathrm{t}}
$$

in the general case is no longer aistributed with zero mean and standard deviation unity. A further transformation is necessary, i.e.

$$
z_{t}=\frac{Y_{t}-\vec{Y}}{s_{y}}=\frac{x_{t}-\vec{Y} s_{t} t_{t}^{-m}}{s_{y} s_{t}}
$$

where $\vec{Y}$ and $s_{y}$ are the mean and standard deviation of $Y_{t}$, respect. ive1y. This is the 'standardised fitted series' or just 'fitted series'. It can be seen that $Z_{t}$ may be described by equation (11.1 or (11.5), however the number of paxameters required will always be much less using equation ( 11.5$)$, thus making this expression more desirable.

Now that the perjodic movement has been isolated, the residual series $Z_{t}$ can be fitted with a mathematical model. The shape of the correlogram of $\mathrm{Z}_{t}$ will indicate the type of model to be used. If, on a given level of significance, it can be said that $\mathbb{E}\left(x_{L}\right)=\rho_{L}=0$, where $x_{L}$ and $\rho_{L}$ are the Lth order serlal correlation coefficients of sample $z_{t}$, and the population from which $z_{t}$ is dram, respectively, then the time series $z_{t}$ may be considered as a sequence of stochastic variables which are independent 
to test for independence.

It has been shown that in general the series $\mathrm{z}_{t}$ for daily values cannot be represented by the independent model, but moy be represonted by a linear autoregressive (Markov) model of the second order (Quimpo, 1967, 1968a). The adequacy of the mode1 is based on the fact that the computed variance of the model. based on the estimated parameters agreed well with the computed residual varjance. The model is given as

$$
z_{t}=a_{1} z_{t-1}+a^{z} z_{t-2}+\epsilon_{t}
$$

Estimates of $a_{1}$ and $a_{2}$ aré given as

$$
a_{1}=\frac{x_{1}\left(1-x_{2}\right)}{1-r_{1}} \quad \text { and } \quad a_{2}=\frac{r_{2}-r_{1}{ }^{2}}{1-r_{1}{ }^{2}}
$$

The residual series $\epsilon_{t}$ is independent of $z_{t-1}, z_{t-2}$, and other E's. For the model adopted

$$
\epsilon_{t}=\frac{1+a_{2}}{1-a_{2}}\left\{\left(1-a_{2}\right)^{2}-a_{1}^{2}\right\} \eta_{t}
$$

where $\eta_{t}$ is a standardised independent stochastic variable (the primary variable).

It is necessary to know the distribution of $\epsilon_{t}$ as this may be crucial if it contributes much of the variance of the whole series. However the model only accounts for second order stationarity in $Z_{t}$, thererore the frequency distribution of $\epsilon_{t}$ cannot be simply determined bocause the expected values of the centr al moments greater than two may not be constant. If they were constant, for instance if the residual series $\epsilon_{t}$ follows a Gaussian distribution, then $\mathrm{z}_{t}$ would be strictly stationary. In practice the residual values are positively skewed because of the rostraint imposed by a minimun flow of zero. Evaluation of the higher moments is unrealistic and so stationarity of order higher than two is best approxinated by assuming that tho residual series, 
$\epsilon_{t}$ or $\eta_{t}$, is distributod with a positjve skew, $i$.e. lognomal or gamma (Poaxson type IIT) (Hamlin, 1971). Upon the determination of the probability distribution, a serjes $x_{t}$ may be senerated by genexating random vaxiables $\eta_{t}$ with the appropriate distribution using Monte Carlo techniques (see appendix 1).

It is obviously Important to know the relative influence of each component of the original time series in order to assign limits of accuracy when one or more of the components are neglected or approximated in a simplified synthesis of hydrologic data. In this study simplified models have been used at the cost of generality. The number of harmonics used to describe the periodicity has been limited to six, and the order of the autoregressive model has not been tested boyond oxder three. Furthermore, the trend component, which may include a 'persistence' effect, has been ignored.

\subsection{Input and experimentation with the model}

Once the series of daily flows for a year has been generated it is a simple matter to find the flood period volume. The parameters used in the hydrology model adopted will depend on the shape of the hydrograph that is selected for use in the model, or the 'river regime' that is desired.

Fig. 11.1 represents the daily means and standard deviations about the means for selected rivers in the U.S.A., taken from Quimpo (1.96\%). They are classified into different river regimes according to the classification given by Beckinsale (1969). Table 1. I shows the values of the parameters used in the stochastic nodels of the daily river flows, as calculated by Quimpo (1967).

500 years of reconds were genexated using the parameters given in table 1.1.1 for each station, using three different distributions for the primary variable $\eta_{\mathrm{t}}$. The values of Q vol 


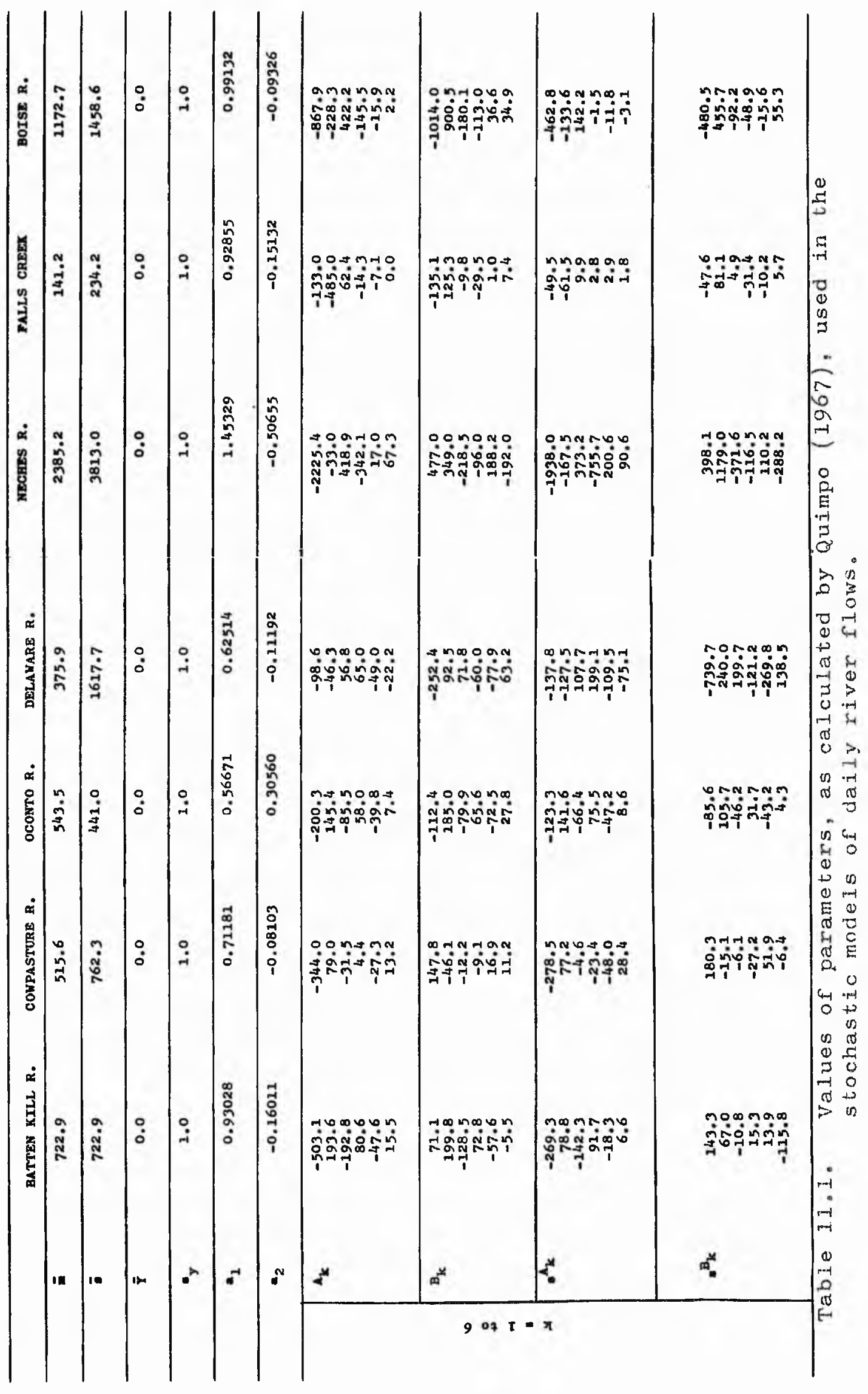



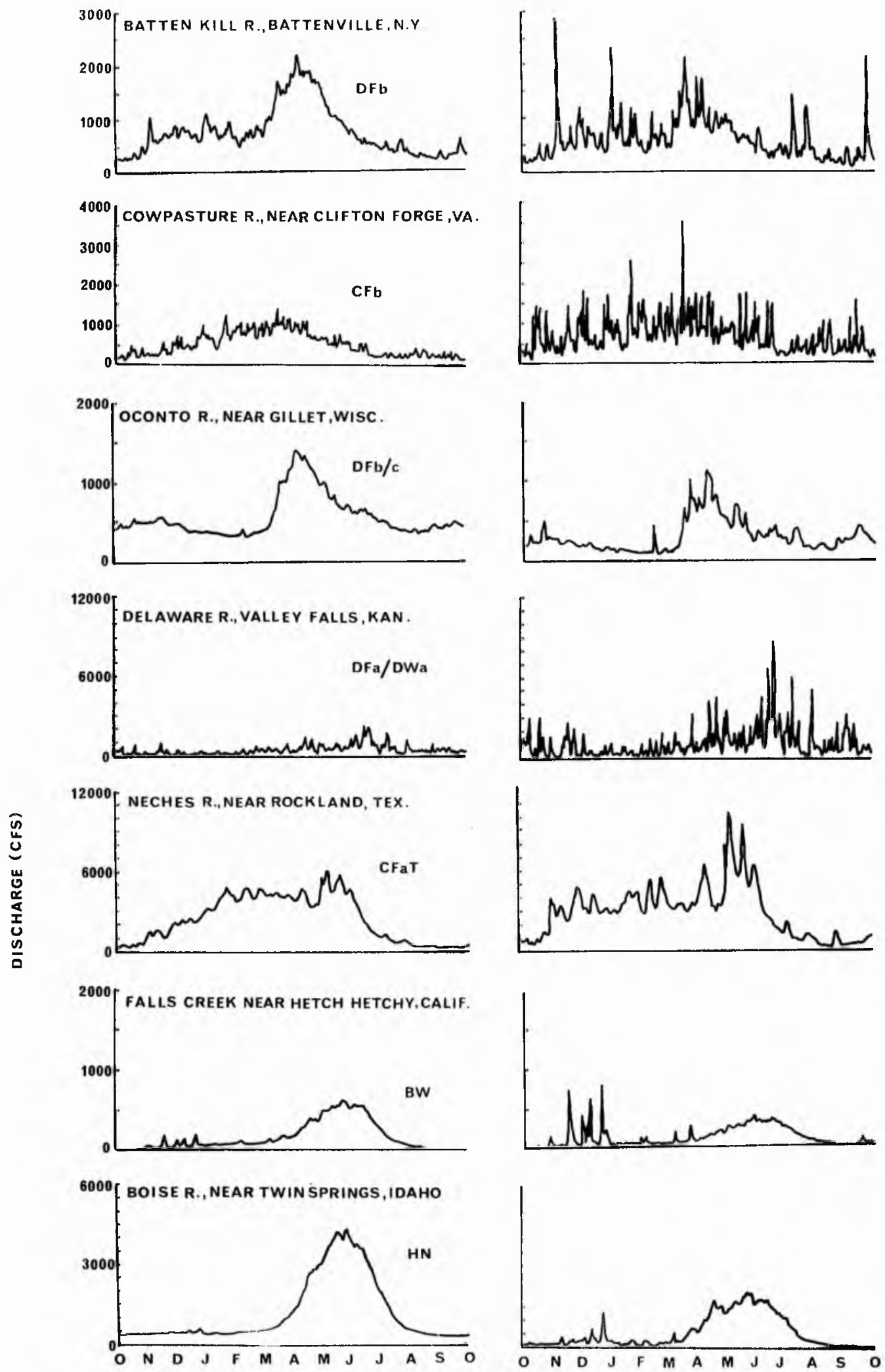

Mean daily flows of annual hydrograph

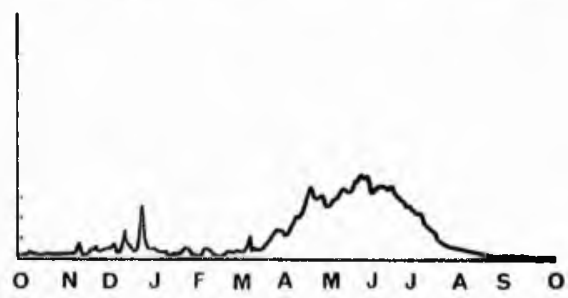

Standard deviations about individual values of daily means of annual hydrograph

Fig.11.1 Daily means and standard deviations about means for selected stream flows in the U.S.A.. (taken from Quimpo, 1967). 


\begin{tabular}{|c|c|c|c|c|}
\hline & \multirow{2}{*}{$\begin{array}{l}\text { coefficients } \\
\text { of variation }\end{array}$} & \multicolumn{3}{|c|}{ variances explained by } \\
\hline & & periodic & autoregressive & primary series \\
\hline BATTEN KILL RIVER & $\begin{array}{l}0.143(\mathrm{~N}) \\
0.151(\mathrm{G}) \\
0.186(\mathrm{~L})\end{array}$ & 0.2904 & 0.4627 & 0.2469 \\
\hline COWPASTURE RIVER & $\begin{array}{l}0.119(\mathrm{~N}) \\
0.132(\mathrm{G}) \\
0.182(\mathrm{~L})\end{array}$ & 0.1051 & 0.3912 & 0.5037 \\
\hline OCONTO RIVER & $\begin{array}{l}0.234(\mathrm{~N}) \\
0.245(\mathrm{G}) \\
0.297(\mathrm{~L})\end{array}$ & 0.3525 & 0.4514 & 0.1961 \\
\hline DELAWARE RIVER & $\begin{array}{l}0.116(\mathrm{~N}) \\
0.129(\mathrm{G}) \\
0.195(\mathrm{~L})\end{array}$ & 0.0216 & 0.3170 & $0.6614^{\prime}$ \\
\hline NECHES RIVER* & $\begin{array}{l}0.271(\mathrm{~N}) \\
0.282(\mathrm{G}) \\
0.332(\mathrm{~L})\end{array}$ & 0.1969 & $0.76,13$ & 0.0418 \\
\hline FALIS CREEK & $\begin{array}{l}0.116(\mathrm{~N}) \\
0.119(\mathrm{G}) \\
0.121(\mathrm{~L})\end{array}$ & 0.5387 & 0.3035 & 0.1578 \\
\hline BOISE RIVER & $\begin{array}{l}0.235(\mathrm{~N}) \\
0.244(\mathrm{G}) \\
0.285(\mathrm{~L})\end{array}$ & 0.6725 & 0.2699 & 0.0576 \\
\hline \\
\hline \multicolumn{5}{|c|}{$\begin{array}{l}\text { G - Gamma distributed primary variable, skewnessel } \\
\text { L - Lognormal distributed primary variable } \\
\text { * Not shown in fig. } 11.2\end{array}$} \\
\hline \multicolumn{5}{|c|}{$\begin{array}{l}\text { Table 11.2, Coefficients of variation of Qvol for simulated stream flows. Variances } \\
\text { explained by different components of the stochastic generating model } \\
\text { (taken from Quimpo, 1967). }\end{array}$} \\
\hline
\end{tabular}



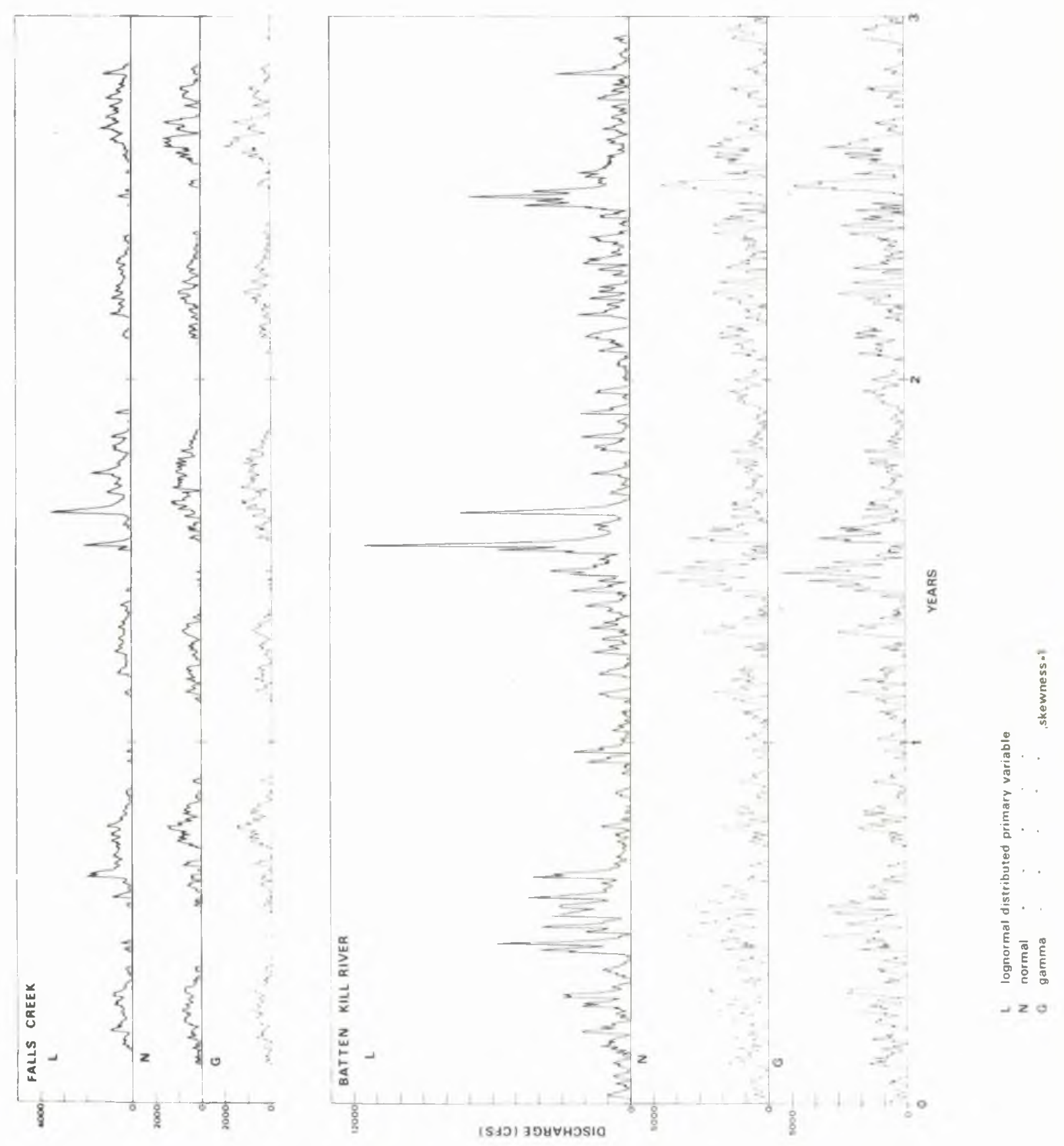

Fio. 11.2. Simulated daily river flows over a three year period, using data given in table 11.1. 

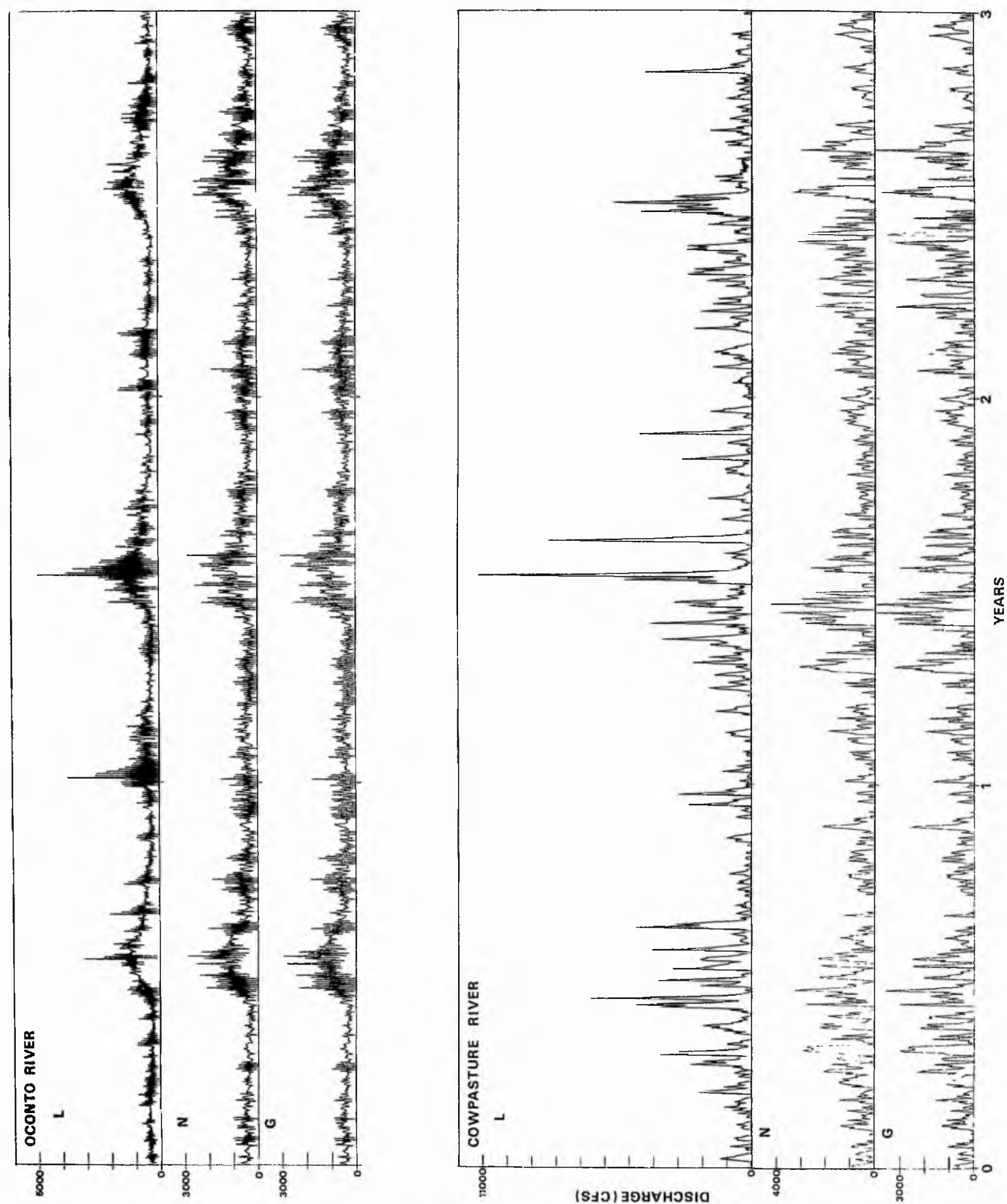

Fig. 11.2. - continued。 


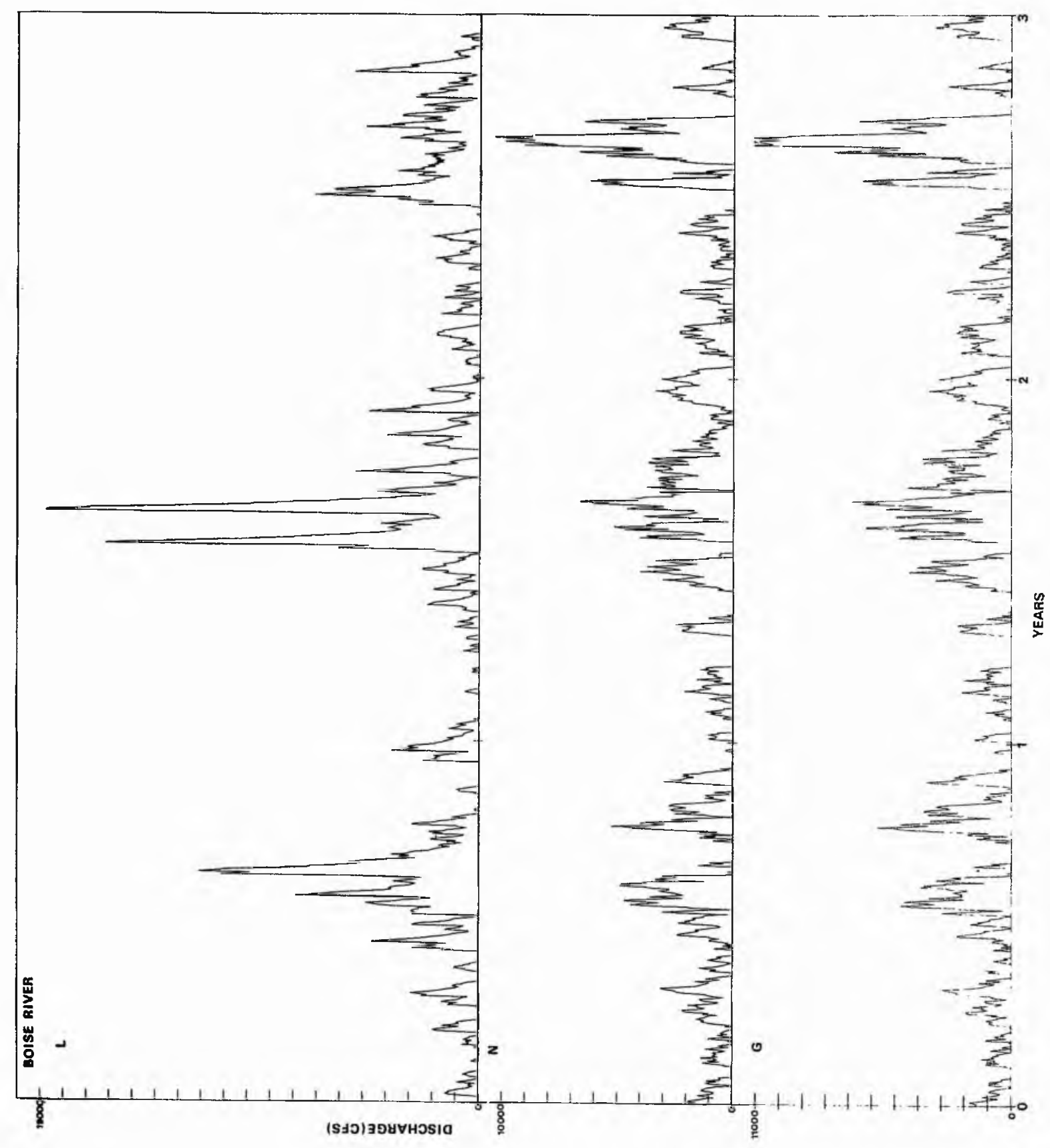

Fig. 11.2. - continued。 


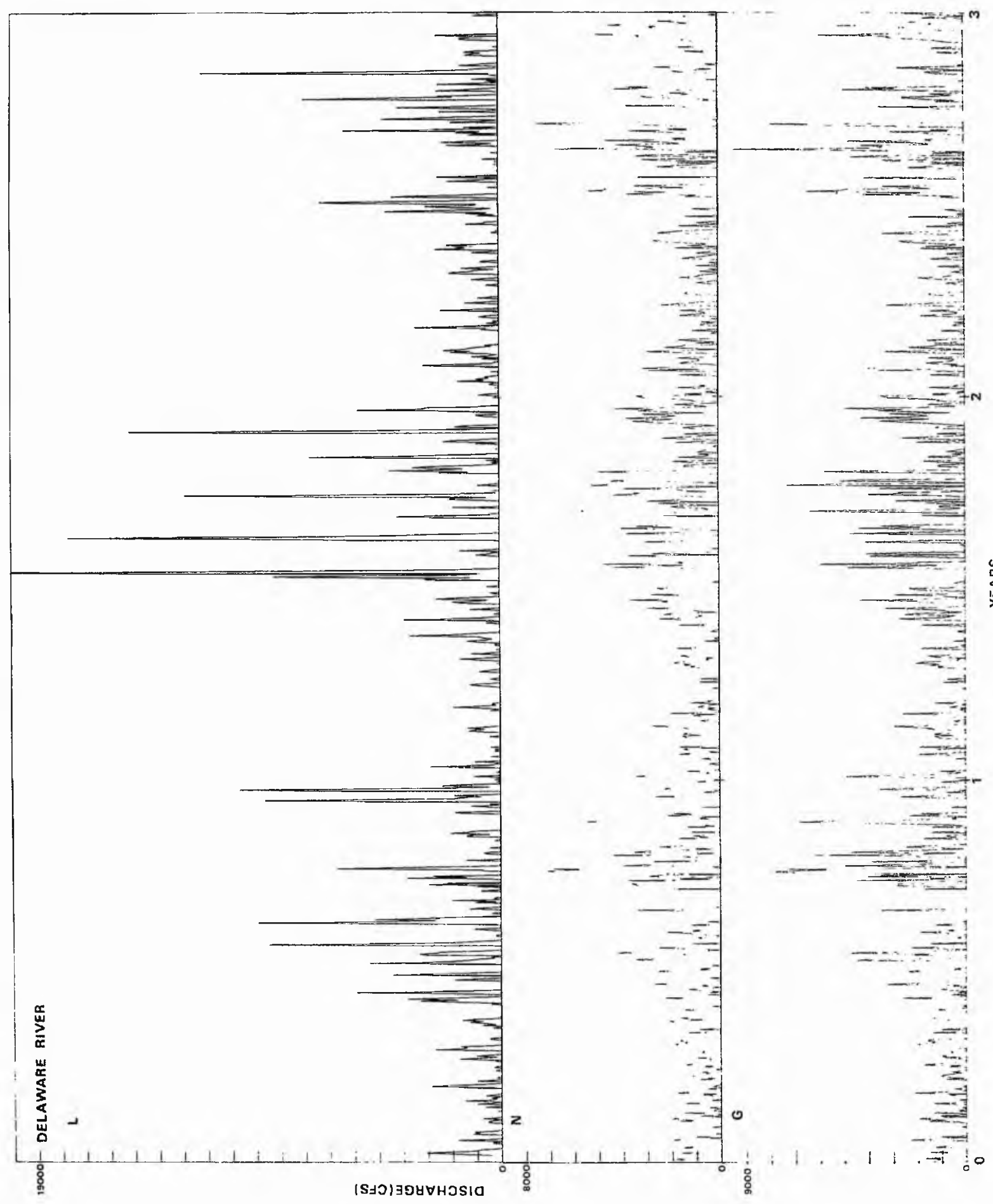

Fig. 11.2. - continued. 
were calculated in each case, and the moans, standard deviattons and coefficients of variation over the poriod of 500 years were found. rigs. 1.1.2 show sample plots of the simulated flows over a three year period, Table 11.2 shows the coefficient of variation of $Q_{\text {vol }}$ for each station and for each differently distributed primery variable. Also shown on the table are the variances explained, as fractions of unity, by the periodic and autoregressive components of the stochastic models adopted. As can be seen the coefficients of variation are closely dependent on the primary variable distribution. No obvious relation of the coefficients of variation with the other parameters in table 11.2 can be soen, however a detailed statistical analysis with additional data may yield one.

It is obvious that the variability of Qvol, hence the variability of the processes it influences, is not only a function of the flow regime in question but also on the method of flow synthesis used. 


\subsection{Overbank deposition}

The annual high-water periods will equal or exceod the elevation of the floodplain just about every year. That the frequency of overbank flow is nearly the same in regions of very diverse runoff, from tropics to semi-arid regions, implies that the size of the rivex channel is appropriate to the quantity of flow provided by the drainage basin. It is also apparent, however that if overbank flooding by sediment-laden waters does occur, some deposition will in all likelihood bo associated with 1t. If there were continuous deposition the channel would gradually appear to become depressed within its own allurium. The regular frequency of flooding indicates that this is not the case, and hence some mechanisms must counteract this tendency (Wolman and Leopold, 1957; Leopold et al., 1964). It will be necessary to consider the nature of the deposits making up the flood plain and some possible explanations of the inferxed lack of importance of overbank deposition in flood plain formation. In their manner of construction and in the nature of the deposits which make them up, flood plain deposits form two fundamentally distinct groups (see wolman and Loopold, 1.957). point bars, channel bars and alluvial islands result from the ' lateral' accretion of stream bed load on the sideways migration of channels. As a meandexing stream shifts laterally, deposition on point bars is concomitant with erosion of the opposilie concave bank. Wolman and Leopold (1957) furthex state that the surface of the material deposited approaches the level of the older part of the flood plain.

The 'vertical' accretion of suspended load after overbank flow leads to the construction of levees, crevase splays, and floodbasins on top of lateral acctetion deposits. Averaged 
thicknesses of sediment that have been deposited on flood plains by great floods is of the order of mm. and cm.. Observations show, however, that widespread deposition of sediment by overbank flows is not the case. In fact there is large variation in the thickness of deposits locally (with some deposits ranging up to metres in thickness); fuxthexmoxe, velocities axe often large enough to produce scour (see Wolman and Leopold, 1957; Wolman and Eiler, 1958; Mckee et al. , 1967).

Observations of Wolman and Leopold (1957) on U.S. rivero indicate that as much as $80-90 \%$ of a normal rloodplain may be composed of deposits of lateral accretion, and the remaining 10$20 \%$ consists of overbank deposits. Whether significant vertical accretion occurs or not depends on intermal factors, inheront in the stream regime, and on others external to the stream.

There are three 'internal' factors which help to explain the relative umimportance of overbank deposition in flood plains, and why the elevation of the surface of a flood plain remains stable relative to the level of the channel bed, despite frequent flooding (Wolman and Leopold, 1957). First, the highest discharges are often charactexised by lower concentrations of suspended sediment than discharges of intermediate sizes. Second, periodic removal of the flood plain by lateral erosion holps to control its height. Third, velocities of the overbank flow may be high enough to move sediment of appreciable size.

Suspended sediment load is a substantially independent quantity within the floodplain system, and although correlated with discharge, is not a function of discharge itself. This explains the observations of Wolman and Leopold (1957) that many streams ghow a maximum concentration of suspended load at stages well below the bankfull and not, as might be expected, at the flood stage. Fisk (1947) xefers the poor development of lovees 
along certain Mississippi tributaries to small suspended loads. The extent to which periodic removal and replacement is effective in limiting the height of the floodplain surface depends on the relative rates of channel migration and overbank accretion. Relatively steep braided streams with coarse loads axe notorious for the rapidity with which theix channels move across a floodplain, so keeping floodplain relief low and minimising the effect of overbank deposition. Many such streams are aggrading xapjdly, although solely by net deposition followIng lateral accretion (e.g. Goleman, 1969). Meandering streams are also free to range and level their floodplains. It is noteworthy that with very sinuous streams, cut off and subsequent development of channel fills of fine sediment may lead to meander bolt fixation, and an 'alluvial ridgel may form. Arulsion may occur, producing a surface of complex and appreciable relief. This will have the effect of hampering overbank flows in their movement downvalley, as well as inhibiting rapid channel migration. When floodplain relief is kept low by the 'ploughing' action of shifting channels, overbank flows are able to move down the plains when floods occux. Wolman and Leopold (1957) repoxt mean velocities of overbank flow in such situations to range between 0.15 and $2.7 \mathrm{ft} / \mathrm{sec}$, and to average $1.6 \mathrm{ft} . / \mathrm{sec}$. Mckee et a1. (1967) compute mean velocities about five times greater than these. The downvalley slope may be considerably greater than that of the channel, and this higher gradient, topether possibly with less roughness in the flood plain section, tends to keep the velocity high and reduces the probability of deposition of fine material on the flood plain. Indeed, widespread scouring has been observed.

The external factors inclucle changes in stream base level 
and changes of land level due to subsidence (tectonic, compactional), or uplift (tectonic, isostatic). These are discussed more fully in the following section on aggradation.

Bearing in mind the aforesaid, it will be assumed in the model that the elevation of the surface of the floodplain remains stable relative to the goneral level of the channel bed. Also, sediment deposited on the point bar will extend up to the level of the floodplain, taken here obviously as the bankfull level of the channel. The surface of the flood plajn will be assumed plane and horizontal in the direction normal to the mean downvalley direction. The relief of the floodplain surface will therefore be 1ost. The grain size of the pre-existing floodplain sediments must be specified in the model, assuming that, at any level, they are laterally homogeneous. In this respect, it should be realised that a specified proportion of the total floodplain thickness may have the character of overbank deposits. Processes of overbank deposition will not be treated in the model because of (1) the negligible rates of erosion and deposition, (2) the complicated flow patterns within the channel and over the floodplain, which will party determine the spatial and temporal distribution of exosion and deposition, (see sellin, 1964; Toebes and Sooky, 1967), and (3) indeterminate concentrations of suspended sediment. The process of avulsion also cannot be treated at this stage.

\subsubsection{Aggradation}

There are various 'external' ractors which influence the relative proportions of overbank and point-bar deposition within the floodplain. Variation of these external factors bring about persistent long tem erosional and depositional tronds over the floodplain. These are termod degradation and aggradation respect- 
ive1y. Degradation wilj not be considered here, as interest lies at present only in net deposition. By definition, profressive long term deposition both within the channel and on the floodplain is aggradation. Under these conditions overbank deposition may be expected to comprise a significant part of the floodplain deposits, but this will depend on the rate of channel migration relative to the rate of ageradation.

As already stated, the dimensions, shape, slope and pattern of stable alluvial streans are delicately adjusted to transport the anount of water and sediment supplied by the headwaters. Aggradation occurs when the production of sediment exceeds the amount that can be carried away by the processes of transportation (Leopold et al., 1964). Up to this point we have considered meandering streams in the stable nonaggrading, nonscouring situation with the independent variables remaining constant. Various external factors can affoct the independent system variables as defined previously, leading to aggradation in certain cases; that is, climatic changes and river diversions can modify the balance between sediment and water discharge. Also structural movenents, sediment compaction, or eustatic sea level changes will cause valley slopo to vary independently. In the recent past the combination of subsidence and rising base level has led to deep alluviation by overloaded streams in the lower valley of the Mississippi (Fisk, 1944, 1947). Here the alluviation took place because rise in base level decreased the overall sjope of the valley, which was reflected in the progressive upstream loss in carrying power. The gradational nature of the sodiments throughout the valley and the occurrence of coarse clastics at depth at the present coastline incicate that aggradation kept pace with riso in sea level throuphout most of the aggrading period. The constant generat aggradation of the 
presently overloaded Brahmaputra causes the channel to become wider and shallower and to cause the main current to seek bettex gradients, new alignments and paths of least resistance (coleman, 1969). Local faulting is partly responstble for these changes in direction. Slope oversteepening due to structural movements, with subsequent flatteninf due to aggradation, has also been recorded elsewhere (Leopold et al, 1964).

When changes in the independent factors cause argradation the various dependent hydraulfo variables may adjust in a vide variety of ways in ordor to maintain continuity of sedinent and vater transport. Schumm (1969, 1971) has formod generalised expressions xelating water discharge and ratio of bed load to total load to various hydraulic variables, in order to illustrate expected directions of change of the dependent variables to changes in water and sediment discharge. Many other authors have noted changes in different hydraulic variables in response to changes in discharge and slope. However the precise forn taken by the adjustments cannot be described quantitatively. They will probably be such that the rate of work expended in the system is minimised, the local conditions determining their exact nature.

Leopold et al. (1964) state that the tendency for the maintenance of quasi-equilibrium in stream channels js sufficiently pervasive that only slight deviations, if sustained for a long enough period of time, may account for aggradational features of considerable magnitude, but the deviation from equijibrium conditions necessary for the construction of such depositional features cannot be recognised or identified by any critexia now avallable. Stratisraphic studies of alluvial sequencos indicate that large scale aggradation in valley systems results from procosses which act relatively slowly. For example, during the 
aggradation of the Mississippi valley since late Wisconsin times the average valley 3 lope has only changed on the order of $10^{-9}$ $\left(10^{-3}\right.$ of a percent).

It does not seem unrealistic, by virtue of the very small average changes in the hydraulic vaxiables over the periods of time to be simulated in the model (up to the order of hundreds of years), to assume that the hydraulic parameters are constant during aggradation. Account cannot therefore be taken of large scale channel and flood plain changes due to sudden short-term variation in the independent variables, as happened, for example, in the Cimarron River of southwestern Kansas between 1914 and 1939 (Schumm and Lichty, 1963). Such changes in the independent variables are not persistent and the channel changes are not pexmanent.

Although the stability of the absolute elevation of the surfaces of most flood plains cannot be proven, evidence indicates that even during aggradation the difference in elevation between the river bed and surface of its floodplain does in many instances remain constant over long periods of time (Wolman and Leopold, 1957). Wolman and Leopold (1957) further state that In those cases where continual aggradation produced the valley fill, it is difficult to explain how the relative position of the channel to the floodplain remained fixed during aggradation if overbank deposition is considered the principle mechanism of laying down the valley fill. Rather, concomitant rise of both stream bed and floodplain surface appears to be best explained by attributing the bulk of the deposited material to the process of point bar formation'.

The uniform frequency of flooding of rlood plains does not rule out the possibility that both tho surface of the flood plain and the bed of the channel are being built simultaneously. 
Gages on the Nile river, which provide the longest periods of recoxd of any river in the world, indicate that both the bed and banks of the Nile are being raised at a rate of about one metre in 1000 years. The maximum thickness of recent valley alluvium in the Mississippi valley varies from about $200 \mathrm{ft}$. (av. $125 \mathrm{ft.}$ ) in the north to over $350 \mathrm{ft}$. (average $138 \mathrm{ft.}$ ) in the south, and this was deposited in 25,000 30,000 years (Fisk, 1944). other data from Leopold et aI. (1964) suggest comparable rates of aggradation.

In the mode1, it will be assumed that during aggradation the elevation of the surface of the floodplain remains stable relative to the level of the channel bed. As in the last section, the suxface of the floodplain will be assumed plane and horizontal in the direction normal to the mean downvalley direction. It is not intended to look at the processes influencing aggradation, but to assume that the whole floodplain is aggrading at a specified constant rate due to one or more of the previously discussed factors, without taking account of them explicitly. The rate of aggradation will be specified as input and it will bo assumed that progressive aggradation is continuing at this constant rate, without interruption, for the whole cross section represented in the model, irrespective of its direction. Due to the fact that the processes of overbank deposition in nonagrading and aggrading situations are too complex to treat here, the nature and surface relief of the overbank deposit cannot be determined in detail. Duxing aggradation much of the overbank deposit will be expected to be crevasse splay and levee deposit. However, by virtue of the observed rates of aggradation mentioned earlier, and the expected rates of chanrel migration, much of the total floodplain deposit is expected to be produced in the channe1. In such an instance, relief will be kept low and the formation of 'alluvial 


\section{ERRATUM}

There is no page number 122 
ridges' would be inhibited. Movement across the floodplain of the meander belt continuously ox discontinuously (avulsion) cannot be accounted For in the model. Avulsion and continuous meander belt migration may be expected to assume nore importance in this aggradine situation with local slope oversteepening, perhaps due to tilting; of the valley associated with tectonism, (e.g. Russel1, 1954; Coleman, 1969). In the mode1, overbank deposits produced during aggradation will be sepaxately desifnated, although their detailed structure and texture will be indeterminate For the purpose of defining their erosion resistance in exposed cut banks they will be assumed to be predominantly silt and clay, although sand nay be present also (e.g. Allen, 1965a). 
PART THREE

TIE COMPUTIER PROGRAM 
13. GENERAL REMIRKS.

Once the structure of the mathematical model is established the next step is to develop a computex program that represents the vartous components and processes to be simulated. The programmg language FORTRAN IV was found sufficiently versatile. Numerous texts deal with the definition and efficient use of the language (e.r. IBM, 1968; Cress et a1, 1970; Kreitzberg and Shneiderman, $1.972)$.

Flow of time is implicj.tin any dynamic system where all the processes are time dependent. In dealing with digital models, time can be moved forward in a series of discrete steps, the state of the system being altered by an increment at each step. Continuous time would be nore closely approximated as the time increment is decreased. The choice of time increment of a year is purely for convenience, in that there is normally one major flood period a year during which most of the erosional and depositional activity takes place, ignoring the separate flood events that inevitably constitute a high water period. There may, however, be two flood periods (i.e. double equatorial maxima) where exosional. and depositional activity are vigorous. This does not affect the model, as quantities involving the flood volume are defined bearing in mind that the time span involved is a year. For instance, in the case of scour and fill, if there were two equally important discrete flood periods in a year, then the net depth of scour for a given meander will be expected to be less for each one than for a single flood period with the same annual flood volume. If \pm t is required to look at these flood periods within one year separately, it would be an easy matter to do 80 , as a mathematical model of the hydrograph has been made. By vintue of the model construotion the time increment cannot be smaller than the length of time between the major seasonal periods of vigorous erosional and dopositional activity which the model 
toonde.

Accompanying the examination of erosion and deposition jin vertical cross sections arises the need to represent two dimensional space within the computer program. Not only is it required to locate a particular type of material (i.e. sand, water, dunes, etc.) in a cross section, but also discrete quantities of these materials must be transported to and from different locations within the section as erosion and depostion in the meander proceed. Because of the need to incorporate this accounting system space is represented by a fixed grid of rectangular (or square) cel1s. Two-dimensional FoRTRAN arrays readily allow this, the accounting information is easy to handle, and can be displayed easily. The scale of the cells is a critical factor, which depends on the dimensions of the cross sections and the availability of computer time and storage. Clearly the greater the resolution required for a given cxoss section, the greater the number of cells are required, thus increasing computer time and storage requirements. In order to give the greatest number of cells possible the information in the two-dimensional FORTR $\leq N$ arrays 15 accommodated in 'half length integer' form. Furthermore, two programs were written, one using only the addressable storage (core store) of the computer, and the other using additional disc storage.

Both programs have been run successfully many times on the IBM $360 / 44$ computer in st. Andrews University computing Laboratory. A CIL off-line graph plotter was used to plot channel-centre lines of meander planforms, but otherwise all output was produced on an IBM 1403 line printer. A disc is required by one of the programs. The maximum core store requirements depend on the number of cells, and whether a disc is used. With cross sections of 200 cells by 60 cells the program 
with a disc uses $78 \mathrm{k}$ bytes, and $129 \mathrm{k}$ bytes without a disc. The approximate ruming time (CPU tino) depends on the number of time increments, the selection of various options within the program, and on the number of cells in the cross sections. When a disc ts used the running time is considerably increased by the large number of input/output opexations. Running times will accordingly be reported for the particular conditions of the individual experiments conducted. 
The following descriptions are given in confunction with the simplified flow cliagrams, and the program listings. The programs are listed in tables 1.4 .1 and 14.2 . Table 14.1 ists the program that uses only core storage. The same subroutines listed here are required by the program using additional disc storage and are therefore omitted from table 14.2. In the following sections words in capjtal letters are rorrRAN variables, arrays or subroutine names.

\section{I4.1 Main Propram (no disc)}

1. Reads input parameters. The job ts terminated if (a) NPRINT, NFPLOT, or NTPLOT equal zero, (b) the initial bankfull stage measured from the section base, wS, is greater than the section thickness, YTOT, (c) initial sinuosity, $S N$, is greater than the limiting sinuosity, SNLIM, (d) maximum unscoured flow depth measured above talweg, H, is greater than WS.

Some of the texms used within the program in the definition of the cross sections, and which are refexred to above and subsequently, are shown in $\mathrm{fig} .14 .1$.

2. Calls subroutine RNDMIN.

3. Finds cell depth (size in vertical direction), YCEL and cell width (size in the horizontal direction), zCEL. If amount of aggradation per year, DWS, is greater than YCEL the job is terminated.

4. Finds bankfull stage rolative to base of section, IWS, in cell depths/rows, and distance of inner bank of channel from left hand side of section, IBANK, in cell widths/columns. See fig. .24 .1$.

5. Finds initial amplitude, AMP, and the limiting amplitude, AMPLIM, by calling subroutine NEWRAp: Checks that a one-. channel downvalley section is not located near the bend axis. 


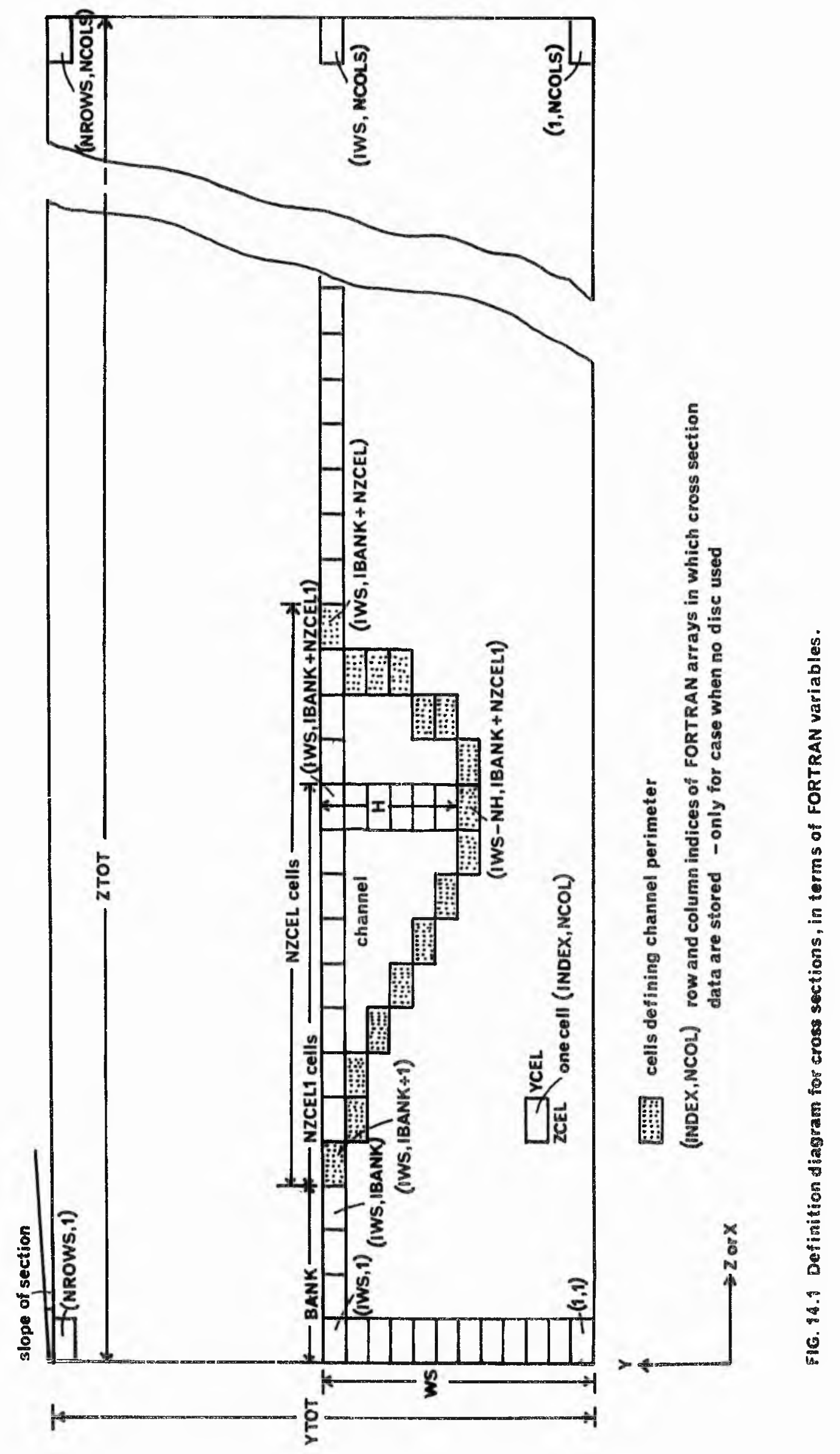


That is, if the normal distance of the line of section from the line joining the points of inflection of the loop, ZSECT, is gxeater than the arbitrary value of AMP/3.0, the job is stopped. Finds initial distance of channel at tho bend axis from the limiting amplitude position, ss. If SS $\leqslant 0.0$, the indicator, LIM, is set to 1 .

6. Alphameric characters are read into array $\operatorname{SEDIN}(I)$, and the arrays $\operatorname{SEDGS}(T, J)$ and $\operatorname{SEDSTR}(I, J)$, which hold the grain size and sedimentary structure cross sections respectively, are filled with alphanexic characters. SEDGS is filled from the bottom of the section up to row IWS with the characters read into sEDIN(I), one character of this array specifying the character for one complete row, $I$, of SEDGS. The remaining rows are filled with blanks. Similarly, SEDSTR is filled with the old sediment character, OLDSED, and the array holding the time line cross section, $\operatorname{TLPI} O T(I, J)$, is filled with blanks.

7. Initialisation of synthetic hydrology parameters. Initialises parameters involving skewness, SKEW, the autoregressive mode1 parametexs, ZTM1, ZTM2, and COEFF (calling subroutine RANSAM), and stores the daily mean flow values and daily standard deviations of flow in FMSUM(NDAY) and FSSUM(NDAY), respectively, after evaluating them with the harmonic representation of equations $(1.2 .2)$ and $(11.3)$.

8. Finds full width of flow between inner and outer banks, WW. Fincls values of WW and the width of flow between inner bank and talweg, W, measured in cells, NZCEL and NZCEL1. NZCELI is also expressed in real mode, FLT?. Finds limiting width of meander neck measurod fxom channel centre lines, GAPLIM. GAPLIM is initially read in as the limiting meandex neck measured between immediately adjacent banks. Finds value of 
H measured in cells, NH. Finds parameters used in subm routine MrANDr for use in calculating proude numbers and for scaling the plot of the meander plan. Finds parameters used in subroutine BAR. Note VAR1 and EXNMl are only required if the sigmoidal cross profile is used. The number of the time increment, NFLD, is set to 0 , the time keeping devices, IPRINT and ITPLOT, are initialised, and the ratio YCEL/ZCEL is calculated.

9. Write scales and titles on graph containing traces of channel centre line. Calls subroutines CHAR and PLOT, for this operation.

10. Prints out cross section, channel, synthetic hydrology, bank migration, scour and fill, and cut-off control parameters. Prints out whether cross sections are lateral or downvalley sections, and the value of ZSECT for downvalley sections. Prints legend.

11. Ca11s subroutine MEANIR to calculate and plot initial planimetric form of the meander, and calculato other parameters for use in subroutine BAR.

12. Tnitial operations axe perfoxrned concerning the projected channel widths in cross sections, including redefinition of NZCEL \& NZCEL1. If a two-channel downvalley section is being used (i.e. if IFCOD6 $=2$ ) the straight line distance between points of inflection of loop, NDAVA, is calculated. Then jumps to step 19 to initialise and print channel section using subroutine BAR.

Al1 the above operations (1-12) are performed only once. Now begins the major loop of the program which is entered once every time increment. Steps 13 to 18 are omitted during initialisation (i.e. NFLD=0). 
13. Reinitialises time keeping devices. If IPRTNT and/or ITPLOT equal o, printed output and/or a time line will be produced this time increment, NFLD.

14. Finds flood period volume, QVoL, by summing all daily flows, $\mathrm{XT}$, in the year (generated using equation (11.5)) which are above the value of the mean dally flow, DM. Calls subroutine RANSAM for this operation.

15. Tests to see if the meander has a neck or clute cut-off during this year. Calls subroutine RNDM for these tests. If cutoff occurs (ICUT put to 2 or 3 ) various parameters are printed, the meancler trace j.s plotted (MEANDR is called), and the job is terminated.

16. Finds amount of bank migration in downvalley direction, RDMIG, and nomal to this direction, RLMIG. Finds respective total amounts of mieration, TDMTG and TLMIG. RLMIG and TLMTG are not calculated if the limiting sinuosity has been reached (LIM $=1$ ) . Prints various parameters if IPRINT=0.

17. Aggrades the flood plain if required. As amount of aggradation, $\Lambda$ GG, fills the cells corresponding to a particular row, the row elements are allocated an alphameric character, FLOOD, for every column in the sedimentary structure and grain size cross sections. As the cells become filled, bankfull stage, IWS, is adjustod accordingly by adding 1. Thus although aggradation continues at a constant rate, the program represents it as a discontinuous process within the cross sections. However, a record is kept of $\mathrm{AgG}$ which is printed out if IPRINT $=0$.

18. Finds amount of bank migration in cross section represented, RMIG .

The following operations, as fax as step 32, are involved with recording on SEDGS (INDEX, NCOL) and SEDSTR (INDEX, NCOL) the resulting erosion and deposition after this year's 'floods', and 
putting a time line on TLPLOT (INDEX, NCOL) if T'TPLOT=0.

19. Adjusts channel width (in cel1s), N7CEL \& NZCEL1, represented in the cross section, and related parameters, depending on type of cxoss scction and changes in shape of the meandex. The exror due to smoothing in the amount of bank inigration for the preceding time increment, DIV, is added to RMIG. $\mathrm{DEV}$ is then recalculated for the next time increment. The amount of concomitiant point bar mirration (in cells), NRMIG, is then defined depending on changes in channel width in the cross section represented.

20. Finds total number of cell widths/colums required for the channe1 section, NZCELT, and test to see if the right hand edge of the cross sections have been exceeded. If so, the job is terminated.

Scour and fill operationswif. NFLD=0 or the scour and fill process is not required (IFCOD 5=0), steps 21 to 24 are skipped. 21. Maximum depth of scour bolow unscoured depth measured above old talweg (i.e. position of talweg at end of last time increment), DSCR, is calculated using equation (9.4). Subroutine RANSAM is called during this operation. A test is made to ensure DSCR $\geqslant 0.0$. If IPRINT $=0$, DSCR is printed out.

22. Maximum depth of water measured above the old talweg, In, is calculated. If this exceeds the bottom of the specified section the job is terminated.

23. Every column of the inside bank of the channel section (IBANK+1 to IBANK+NZCEL1) is now filled to the original depth by successive recalculation of the transverse profile, the deptin at the old talweg being progressively decreased by one cell depth/row until filling is complete. Subroutine BAR or BARL is called during these operations in order to fill the appropriate elements of SEDGS and SEDSTR with alphameric characters. 
24. The area bounded by the position of the old talweg, the maximum scour depth below the old talweg, and the position of the new talwer (i.e. the base of the outer channel bank at the end of this time increment) is now filled by allocating for each row in this area the grain size and bed form symbols calculated for the row elements of the old talweg column, IBANKNNZCELI, in step 23.

Fig. 14.2 illustrates the sequence of events in the scouring and filling operation described above. Steps 25 to $32^{\circ}$ constitute a major $100 \mathrm{p}$ and are concerned with erosion of the outer bank and deposition on the point bar. As a result of the erosion of the outer bank and changres in the projected channel width in the cross sections, the whole transverse profile is shifted accordingly, and the left hand side of the new point bar profile is started at column IBANK +NRMIG+1 of the cross section.

25. Parameters are initialised and if IPRIN $\mathrm{T}=0$ headings are written for the printed output from subroutine BAR.

For every column, NCOL, of the new point bar (up to IBANK NZCEL 3. NRRIG) steps 26 to 31 aro executed.

26. The depth of water, sediment size and bed form are calculated by calling subroutine BAR or BARl and the appropriate elements of SEDGS (INDEX, NCOL) and SEDSTR (INDEX, NCOL) that describe the profile of the new point bar are filled with alphameric characters.

27. If the grain size at a particular station is silt or clay this is recorded.

28. The alphameric character for the time line is allocated to the appropriate element in TLPLOT (INDEX, NCOL) if ITPLOT=O.

29. The area between the old and new point bax is 'ritled by filling each row, INDEX, with the alphamerio character as just allocated, in step 26, to the coxresponding row of the new point bar profile. See fig. 1.4.2. 

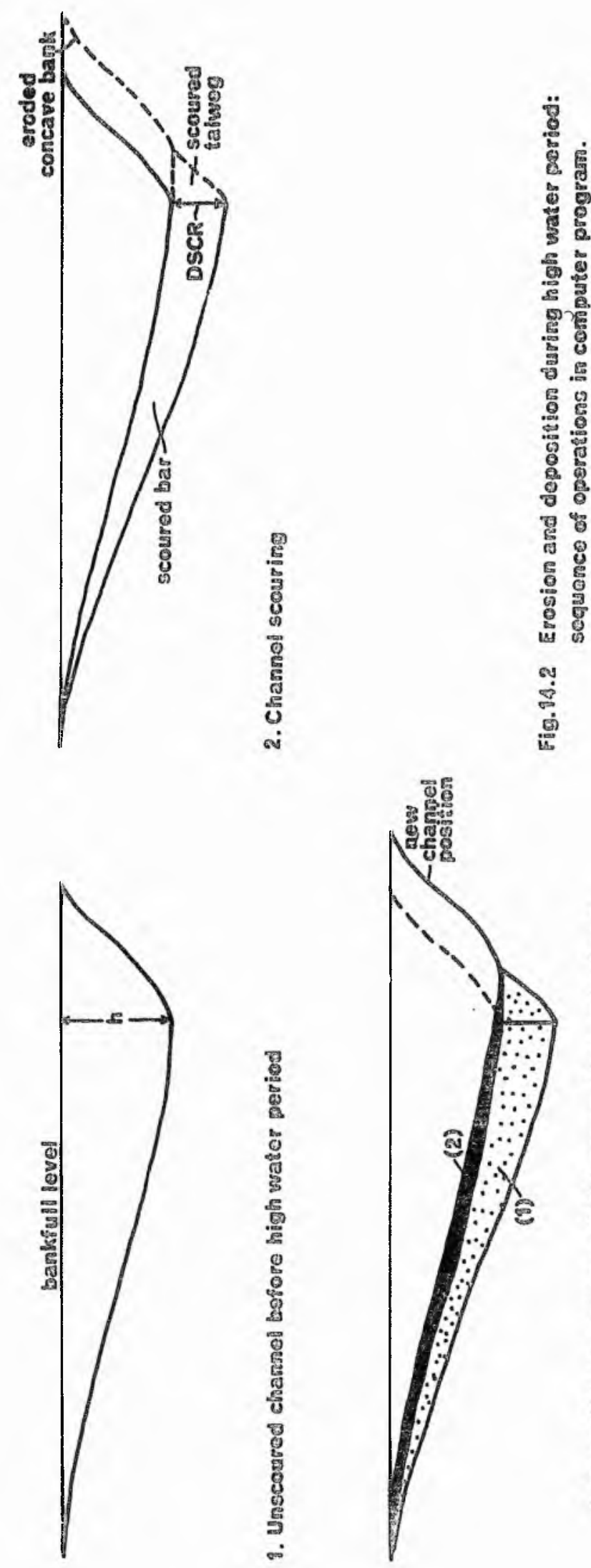

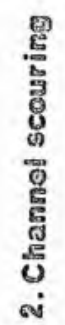

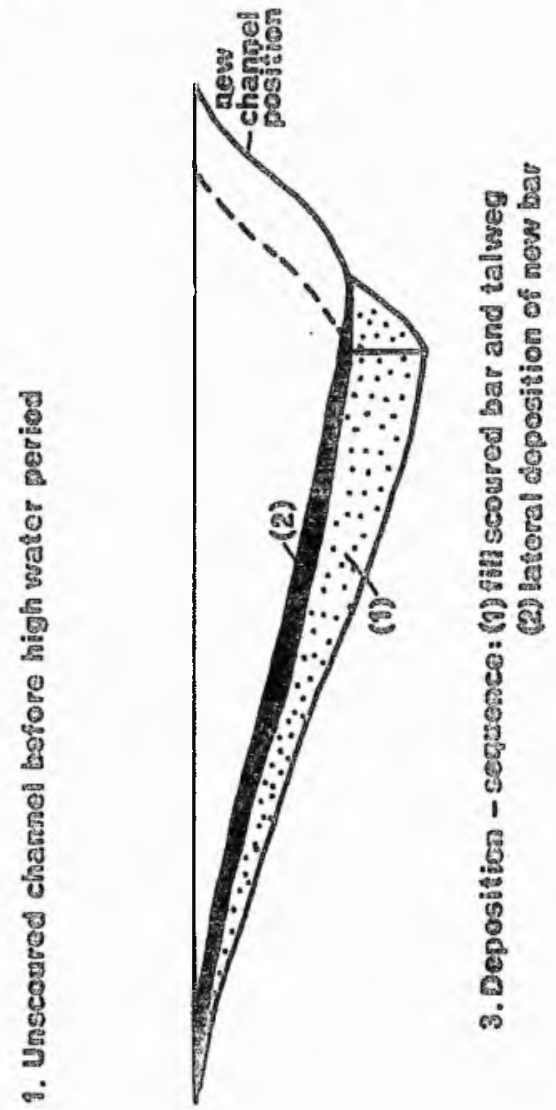


30. If the section scaling is such that not all of the rows in the point bar section had a character allocated j.n 26, the row(s) with 'missing' symbols is (are) filled with the same symbols as axe on the next 'full' row beneath.

Steps 29 and 30 are omitted if there is no filling to bo

done.

31. Each column is finally filled with water.

For every column of the outer bank (IBANK+NZCEL1+NRMIG+1 up to IBANK+NXCELT) the following step is executed.

32. The depth is calculated using a similar equation to that of the sigmoidal profile of the point bar. The indices of the cells, (INDEX, NCOL), corresponding to the position of the bank are calculated and, if ITPLOT=0, alphameric characters for the time line are allocated to the appropriate elements in TLPLOT (INDEX, NCOL). The amount of exposed silt, clay, overbank deposit, and gravel are recorded by scanning each row in the outer bank. Each column is finally filled with water.

33. The weighted percentage of silt and clay (including overbank deposits) in the perimeter of the projected channel, SCHUM, is calculated. This is not the same index as that used by Schumm (1960) which is calculated in a different way and uses $0.074 \mathrm{~cm}$. as tho lower limit of sand sizes.

34. Percentage of silt and clay in inner bank, BGSI, and the outer bank (including overbank deposits with the silt and clay), OBGSI, and the percentage of grave1, GRAVI, in the outer bank are calculated. If OBGST $=0$ it is set to 1.0 for the purposes of equations $(6.3)$ and $(6.4)$. If GRAVI is greater than the limiting value, GRAVLM, the job is terminated.

35. If a two-channel downvalley section is being used, the channel section data just computed and stored in SEDGS, SEDSFR and 
TLPLOT, are used to fill these arrays with the same information, NDAVA columns further on. NDAVA is the distance between the two channels measured in columns/cell widths along the valley axis.

36. If IPRINT $=0$ the cross sections showing grain size and sedimentary structure (bed form) distribution are printed out.

37. IBANK is incremented by NRMIG and a test is made to see if XNAX has been exceeded, before trying to plot the planimetric geometry with the graph plotter.

38. The new amplitude, AMP, and the distance of the channel at the bend axis from limiting amplitude, SS, are now calculated if the liniting sinuosity/amplitude has not already been reached, i.e. $L I M=0$. If $L I M=0$ subroutine MEANDR is called and a test is made to see if the two limbs of the meander have closed on each other. If the limiting sinuosity is just reached after the recalculation of $A M P$ and $S S$, LIM is set to 1 , AMP is set to AMPLIM, RLMIG and $S S$ are set to 0.0 , and MEANDR is called. Also a message is written saying that the limiting sinuosity/ amplitude has been reached, and if a lateral section is represented the program is stopped. If LIM is already equal to 1, AMP and SS are left unaltered and MEANDl is called, unless the downvalley migration is zero in which case neither MEANDR or MEAND1 are called.

39. NFLD is incremented by 1 and, if less than or equal to the number of required time increments, NTTM, control is transferred back to step 13 .

The job jis stopped either with an exror message or due to the execution of the requixed number of time increments.

A simplified flow diagram of the main progran is shown in fig. 14.3. This diagram relates to both main programs. 


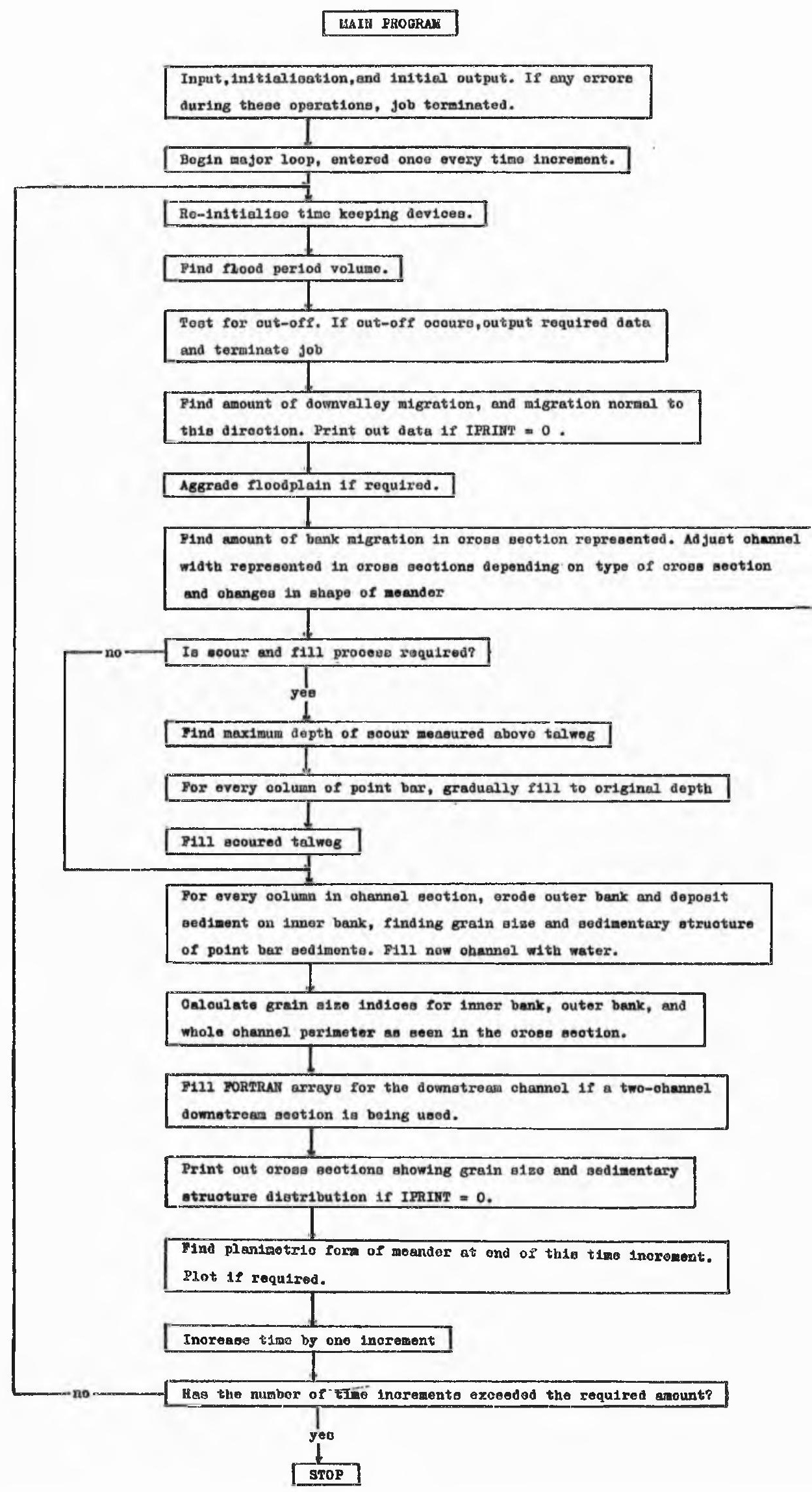


$c$

C CONTROL STATEMENTS

0001

0002

0003

0004

0005

0006

0007

0008

0009

0010

0011

0012

0013

0014

0015

0016

0017

0018

0019

0020

0021

0022

0023
REAL NEWRAP

DIMENSION FMT 4 (5), A (6), B(6), SA (6), SB $(6)$, FMSUM $(365)$, FSSUM $(365)$

INTEGER *2 SEDGS, SEDSTR, TLPLOT $(60,200)$, GRAVEL, SAND, S ILT, CLAY, UPPB,L 1PPB, ANTIDN, RI PPLE, DUNES, OLDSED, WAT ER, DOT, BL ANK, SEDIN $(60)$, FLDOD COMMON/COML / I PRINT, RINB, FROUD1, FROUD2, VAR 2S, RC

COMMON/COM2/I NDEX, NCOL, H, EXN, IWS, Y, NYCEL, D, VAR 1, EXNM I, YCEL, SIGRO, 2 1 , GRAVEL, SAND, SI LT, CLAY, UPPB, LPPB, ANT I DN, R I PPLE, DUNES, SEDSTR 160,200 2), SEDGS $(60,200)$

COMMON/COM3/NFLD, TI TLE (15), WVL, AMP, VS, GAP, NFPLOT, CHS, WW, R, RO, VAR 2 , IF2 2, F18,SS, TDMIG, SN

COMMON/COM4/SKEW2, SKEW6, SKEW62

DATA RMIG, TLMIG, AGG, DEV, NDAVA, LIM, MARK, ICUT $/ 4 * 0,0,2 * 0,2 * 1 /$

FORMAT STATEMENTS

1 FORMATI $15 A 4,110)$

2 FORMAT $(11,3 F 12,0,11)$

3 FORMAT (414)

4 FDRMAT $(314,6 F 8.0,5 A 4)$

5 FORMAT $\{80 A 1)$

6 FORMAT (6F 12.0$)$

7 FORMAT I 8F 8.0.14)

21 FORMATIIHI, $1 X, 15 A 4 / / 1 X$, 'CROSS SECTION PARAMETERS',49X, 'METRES', $5 X$, 1 'CELLS'//6X, 'WIDTH OF SECTION',48X,F10.3, I 10/6X. "THICKNESS OF SEC $210 N \cdot, 44 X, F 10.3,110 / 6 X, '$ INITIAL DISTANCE OF INNER CHANNEL BANK FROM 3 L.H.S. DF SECTION , 3X,F10.3,110/6X,' INITIAL BANKFULL STAGE MEASUR $4 E D$ FROM SECTION BASE, 15X,F10.3,I10/6X, 'CELL SILE IN VERTICAL(Y) D 5IRECTION", 30X,F10.3/6X, "CELL SIZE IN HORIZONTAL( $Z$ OR $X)$ DIRECTION" $6,23 x, F 10.3 / 1 / 1$

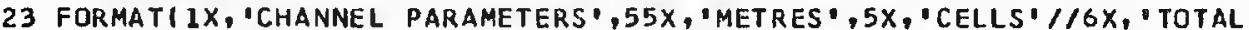
IWIDTH OF CHANNEL $(W), 39 x, F 10,3,110 / 6 \mathrm{X}$, ' $W$ IDTH OF FLOH BETHEEN INNER 2 BANK AND TALHEG(WI) $, 17 X, F 10,3,110 / 6 X, ' R A T I O$ OF $W 1$ TO $W 1,68 X, F 10$. $33 / 6 X$, MAXIMUM FLOW DEPTH MEASURED ABOVE TALWEG', 24X,F10.3/6X, 'DENS 41 TY OF SEDIMENTARY PARTICLES $, 52 X, F 10.3,{ }^{\circ}$ GM/CM $3 \% / 6 X, ' F L U I D$ DENSIT $5 Y 1,71 X, F 10.3, \cdot$ GM/CM3 $/ 6 X,{ }^{\circ}$ DARCY-WEISBACH FRICT ION COEFFICIENT FOR 6 DUNES AND RIPPLES $, 27 X, F 10.3 / 6 X, \cdot D A R C Y-W E I S B A C H$ FRICTION COEFFICI 7ENT FOR PLANE BEDS AND ANTIDUNES', $20 X, F 10.3 / 6 X,{ }^{\prime}$ EXPONENT $N 1,73 X, F$ $810.3 / / / 1$

24 FORMATI $1 X$, SYYNTHETIC HYDROLOGY PARAMETERS(UNITS NOT NECESSARY) $/ 16$ $1 X$, 'MEAN OF ALL DAILY MEAN YALUES', 25X,F10,3/6X, 'STANDARD DEVIATIDN 2 DF DAILY MEAN VALUES $, 15 X, F 10.3 / 6 X, 1$ MEAN OF YT SERIES $, 37 X, F 10.31$ $36 \mathrm{X}$, STANDARD DEVIATION OF YT SERIES, $23 \mathrm{X}, \mathrm{F} 10.3 / 6 \mathrm{X},{ }^{\circ}$ COEFFICIENTS IN 4 AUTOREGRESSI VE MODEL $\cdot, 15 X, \cdot A 1=1, F 10,3,7 X, \cdot A 2=\bullet, F 10,3 / 60 X, \cdot$ HARMONI 5CS FROM 1 TO $6 \% / 6 X$, 'FOURIER CDEFFICIENTS FOR DAILY MEANS $(A) 1,15 X, 6$ 6F 10.3/42X, $(B),, 15 X, 6 F 10.3 / 6 X, \cdot F O U R$ IER COEFFICIENTS FDR DAILY STD TDEVIATIONS (SA) . 5X, 6F $10.3 / 51 X, \cdot(S B), 5 X, 6 F 10.3 / 6 X$, MAXIMUM VALUE O 8F QVOL $.33 \times, F 10,3 / / 11$

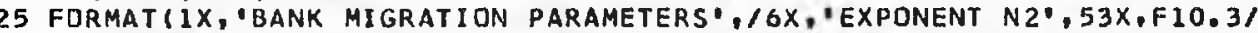
$16 \mathrm{X}$, 'VALUE OF CONSTANT IN LATERAL MIGRAT ION RELATION", $14 X^{\circ}, \mathrm{K} 2=\circ$, EI 10 2.3/6X, 'VALUE OF CONSTANT IN DONNVALLEY MIGRATION RELATION", $12 X, ' K 3$ $3=1, E 10.3 / 6 \mathrm{X}, 1$ LIMITING PERCENTAGE DF GRAVEL ALLOHABLE IN OUTER BANK $4^{\circ}, 11 \times, F 10.3 / 1 / 1$

26 FORMATI $1 X$, 'SCOUR AND FILL PARAMETERS $/ 6 X, \cdot$ CONSTANT $K 4^{\prime}, 43 X, E 10,3 / 6$ $1 X$, EXPONENT $N 3,39 X, F 10.3 / 6 X$, STANDARD DEVIATION OF ERROR TERM: 18 $2 X, F 10,3 / 1 / 1$

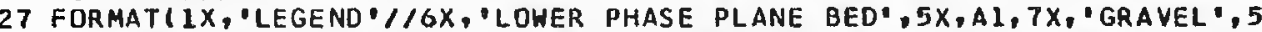

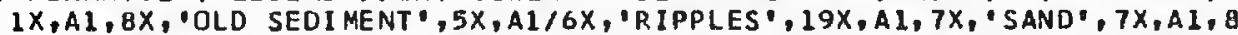
$2 X, '$ 'WA TER', $12 X, A 1 / 6 X, '$ DUNES', $21 X, A 1,7 X, ' S I L T ', 7 X, A I, 8 X$, 'TIME LINE', $38 X, 5 A 1 / 6 X$, UPPER PHASE PLANE BEO',5X,A1,7X, 'CLAY', TX, A1, 8X, 'AIR', 1 $44 X, ' B L A N K \cdot / 6 X$, 'ANT I DUNES', $17 X, A 1,7 X$, 'OVERBANK' $3 X, A 1 / 40 X$, 'DEPOSITS $5 \cdot 1 / 1$

28 FORMAT $1 X,{ }^{\circ}$ CUT-OFF CONTROL PARAMETERS $/ 6 X,{ }^{\prime} L I M I T I N G$ HIDTH OF MEAND IER NECK',24X,F10.3," METRES'/6X, "EXPONENTS IN NECK CUT-DFF RELATIO

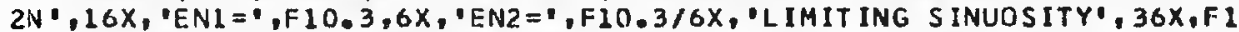
30.3/6X,'LIMITING AMPLITUDE',36X,F10,3,' METRES $/ 6 X$, 'EXPONENTS IN C 4HUTE CUT OFF RELATION', $15 X,{ }^{\prime} E C 1={ }^{\prime}, F 10.3,6 X, 0^{\circ}$ EC $2=1, F 10.3 / 1 / 1$

29 FORMATI LHI, $1 X, 15 A 4$, TIME INCREMENT', I5//1X, 'FLODD PERIOD VOL UHE FO IR THIS YEAR" $26 X, F 10.3 / 11 X$, OUTER BANK GRAINSILE INDEX AT BEGINNIN 26 OF YEAR', $12 X, F 10.3 / / 1 X, '$ INNER BANK GRAINSIZE INDEX AT BEGINNING 30F YEAR', $12 X, F 10.3 \% 1 \%, \%$ SILT-CLAY IN CHANNEL PERIMETER AT BEGINN $4 I N G$ OF YEAR',6X,F10,3//1X, DISTANCE FROM LIMITING AMPLITUDE AT BEG 51 NNING OF YEAR',6X,Fl0.3, METRES $/ / 1 X, \cdot L A T E R A L$ MIGRAT ION DURING T 6HIS YEAR', $25 X, F 10,3,0^{\circ}$ METRES $\% / 1 X$, 'TOTAL LATERAL MIGRATION AT END TOF THIS YEAR", 16X,F10.3," METRES $\% / 1 X, "$ DOWNVALLEY MIGRATION DURING 8 THIS YEAR",22X,F10.3," METRES $/ / 1 X, "$ TOTAL DOWNVALLEY MIGRATION AT

'Table 14.1. Listing of main program (no disc) and subroutines. 
0038

0039

0040

0041

0042

0043

0044

0045

0046

0047

0048

0049

0050

0051

0052

0053

0054

0055

0056

0057

0058

0059

0060

0061

0062

9 END OF THIS YEAR', $13 \times, F 10.3, \cdot$ METRES $\% / 1$

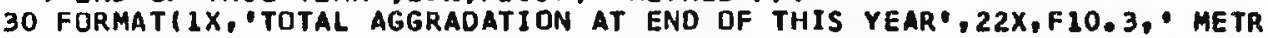
IES://

31 FORMAT $/ / / / 1 X$, A UOWNVALLEY SECTION IS REPRESENTEO IN THIS TEST'/1X 1, DISTANCE OF LINE OF SECTION FROM POINT DF INFLECTION OF LODP IS* 2,F10.3, METRES $\% / 1$

33 FDRMAT $/ / / 1 \times$, 'A LATERAL SECTION IS REPRESENTED IN THIS TEST $/ /)$

35 FORMAT I IHI, 1X, 15 A4, TIME INCREMENT', I5//1X, "CROSS SECTION SHDNING 1 DISTRIBUTION OF GRAIN SIZE ACROSS MEANDERING RIVER FLOOD PLAIN $/ /$ 2/1)

36 FORMAT 1 IHI $, 1 X, 15 A 4$, TIME INCREMENT', I5//IX, 'CROSS SECTION SHDHING 1 DISTRIBUTION OF SEDIMENTARY STRUCTURE ACROSS MEANDERING RIVER FLO 2OD PLAIN / / /

39 FORMATIIX, "THE SPECIFIEO SECTION WIDTH HAS BEEN EXCEEDEO-THE HIOTH 1 MUST BE INCREASED IF MIGRATION IS TO PROCEED')

41 FORMATI $1 X$, 'DEPTH OF SCOUR AT TALHEG FOR THIS YEAR', $21 X, F 10.3,{ }^{\prime}$ MET LRES $/ / 1$

42 FORMAT I IHO, 1X,15A4,' TIME INCREMENT', 15//1X, 'VARIATION OF GRAINSIZ IE AND BED FORM OVER CHANNEL CROSS PROFILE $~ / / 7 X, ' D E P T H ', 3 X$, 'GRAINSI 2ZE", $8 X$, 'BED FORM",6X,"LOCAL MEAN", 4X, 'LOCAL", 10X, 'LOCAL STREAM", 2X

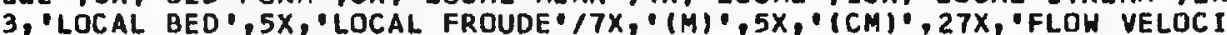

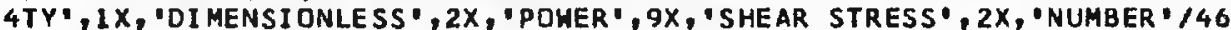

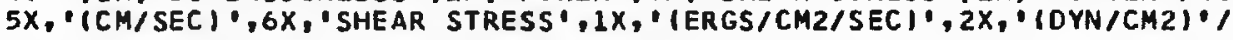
6)

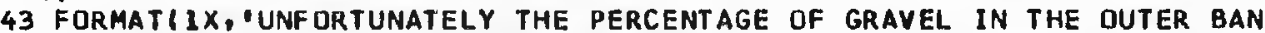
IK IS ,F10.31

45 FORMAT 'SCALE-1 INCH TO',F10.2," METRES , 100X)

47 FORMAT ("MEANDER GEOMETRY", 100X)

49 FORMATI////1X, 'TIME INCREMENT ' 15, LIMITING SINUOSITY/AMPLITUDE IHAS BEEN REACHED 1

972 FORMATI//1X," TIME INCREMENT "15,/1X,"LIMITING SINUOSITY/AMPLITU 1DE REACHED IN A LATERAL SECTION - TEST TERMINATED'I

977 FORMAT $/ / / 1 x_{1}$ IINITIAL BANKFULL STAGE MEASUREO FRDM BASE DF SECTION 1 EXCEEDS SPECIFIED SECTION THICKNESS - TEST TERMINATED'I

979 FORMAT $/ / / 1 X$, 'ERROR IN SECOND DATA CARD - LAST THREE VARIABLES MUS IT BE NONZERO')

981 FORMAT $11 X$, THE LOHER BOUNDARY OF THE CROSS SECTION HAS BEEN EXCEED IED - $/ 1 X, \cdot A D J U S T M E N T$ IS REQUIRED IN EITHER INIT IAL BANKFULL STAGE. 2DEPTH AT TALHEG, OR SCOUR AND FILL PARAMETERS'I

983 FDRMAT $1 X$, ,INITIAL SINUOSITY IS OUTSIDE THE SPECIFIED LIMITS - TES $1 T$ TERMINATED')

985 FORMATIIX,'THE SPECIFIED SECTION THICKNESS HAS BEEN EXCEEDED - RES ICALING IS REQUIRED IF AGGRADATION IS TO CONT INUE')

987 FORMATI $1 X$. THE SPECIFIED LENGTH DF THE $X$-AXIS ON THE GRAPH PLDTTER 1 HAS BEEN EXCEEDED - RESCALING IS REQUIRED')

989 FORMATI///IX, 'RATE OF AGGRADATION PER FLOOD INCREMENT IS GREATER T IHAN DNE VERTICAL CELL - RESCALING IS REQUIRED')

991 FORMATI///X," TIME INCREMENT ",15," - TEST TERMINATED DUE TO CHUT IE CUT OFF 'I

993 FORMATI//1X," TIME INCREMENT " I5," - TEST TERMINATED due to NECK 1 CUT OFF')

995 FORMATI///1X, DEFINITION DF LINE OF SECTION IS IN ERROR - TEST TER IMINATED')

C

C READ INPUT PARAMETERS

READ (5, 1)TITLE, IX

READ $(5,3)$ NT I M, NPRINT , NF PLOT, NTPLOT

IF (NPRINT.EQ.0. OR. NFPLOT.EQ.0. OR. NTPLOT.EQ.0IGO TO 978

REAO $(5,4)$ NC OLS, NROWS, I FCOD6, $2 T$ OT, YTOT, BANK, HS, DHS, ZSECT, FMT 4

IF (HS.GT.YTOT)GD TO 976

READ $(5,6)$ SN, HVL, VS, XMAX

READ $(5,6) C 1, C 2, E 1, G R A V L M$

READ $(5,6) E C 1, E C 2, E N 1, E N 2, G A P L I M, S N L I M$

IF ( SN.GT. SNLIM)GO TO 982

READ 15,7 I $, H, E X N, C 5, F 1, F 2, S I G M A, R D$, IFCOD 7

IF (H. GT. WSIGO TO 980

READ 15,5 ) GRAVEL, SAND, SI LT, CLAY, UPPB, LPPB, ANT IDN, RIPPLE, DUNES, DLDSE

1D, HATER,DOT, BLANK, FLODD

READ $(5,2)$ IFCOD 3, QVOLMX, SKEH

READ (5,6)DM,DS, YM, YS, AL, A2, A, B, SA, SB

READ $(5,2)$ IFCOD5, C6,E2, STDVN, IFCODI

$\mathrm{C}$

C CALL RNDMIN

CALL. RNDMIN(IX)

C FIND CELL DIMENSIONS 
C

C IFIDWS.GT, YCELIGD TO 988

0067

0068

0069

0070

C FIND BANKFULL STAGE RELATIVE TO BASE OF SECTION AND DISTANCE OF INNER C BANK OF CHANNEL FROM LEFT HAND SIDE OF SECTION (IN CELLS)

IBANK $=$ BANK / ZCE L

IWS =WS $/ Y C E L$

C

C FIND INITIAL AMPLITUDE

AMP $=W V L * N E$ WRAP $(S N, 0,0000001)$

IF ( ZSECT.GT. (AMP/3.0) )GO TO 994

C

C FIND LIMITING AMPLITUDE

0071
0072

0073
0074
0075
0076
0077
0078
0079
0080
0081
0082

AMPLIM $=W V L * N E W R A P(S N L I M, 0.0000001)$
C FIND INITIAL DISTANCE FROM LIMITING AMPLITUDE

SS $=($ AMP LI M-AMP $) / 2.0$

C IF (SS. LE. 0.0$)$ I I M=1

C INITIALISE CROSS SECTION ARRAYS

C

READ (5,5) (SEDIN(I), I =1, IWS

DO $146 \mathrm{~J}=1$, NCOLS

DO $146 \quad I=1$, NROWS

IF(I.GT.IHSIGO TO 145

SEDGS $(I, J)=$ SEDIN(I)

SEDSTR $(I, J)=$ DLDSED

GO TO 146

145 SEDGS $(I, J)=B$ LANK

$\operatorname{SEDSTR}(I, J)=B$ LANK

c

$146 \operatorname{TLPLOT}(I, J)=B L A N K$

C INITIALISE SYNTHETIC HYDROLOGY PARAMETERS

C

C SKEWNESS PARAMETERS

0083

0084

0085

0086

0087

0088

0089

0090

0091

0092

0093

0094

0095

0096

0097

0098

0099

0100

0101

0102

0103

IF (SKEW.EQ.0.0)GO YO 162

SKEW2 $=2.0 / S K E W$

GO TO 163

162 SKE $W 2=0.0$

163 SKE $66=$ SKE $W / 6.0$

SKE W62 = SKE W6 * SKE W6

C

C AUTOREGRESSIVE MODEL PARAMETERS

2 TM1 = RANSAM (IFCOD 3$)$

ZTMZ =RANSAM (IFCOD 3$)$

COEFF $=S Q R T((1,0+A 2) /(1,0-A 2) *(1,0-A 2) * * 2-A 1 * 2)$

C CALCULATE MEAN AND STANDARD DEVIATION OF FLOW FOR EACH DAY OF THE yeAR C AND STORE IN ARRAYS

DO 150 NDAY $=1,365$

F SSUM (NDAY) $=0.0$

$F M S U M(N D A Y)=0.0$

VAR $=0.017211 * F L O A T$ (NDAY)

DO $149 \mathrm{~K}=1,6$

$A R G=F L O A T(K) * V A R$

FMSUM(NDAY) $=F M S U M(N D A Y)+A(K) * C O S(A R G) * B(K) * S I N(A R G)$

149 F SSUM $(N O A Y)=F S S U M(N D A Y)+S A(K) * C O S(A R G)+S B(K) * S I N(A R G)$

150 CONTINUE

$c$

C FIND FULL WIDTH OF FLOW BETHEEN INNER AND DUTER BANKS

$W W=H / C 5$

C FIND Values of $W$ AND HH IN CELLS

C

NZCEL $=W W / Z C E L$

NZCELI $=H / Z C E L$

$c$

FLT2=FLOAT (NZCELI) 
C FIND LIMITING WIDTH OF MEANDER NECK MEASURED FROM CHANNEL CENTRE LINES

0105

0106

0107

0108

0109

0110

0111

0112

0113

0114

0115

0116

0117

0118

0119

0120

0121

0122

0123

0124

0125

0126

0127

0128

0129

0130

0131

0132

0133

0134

0135

0136

0137

0138

GAPLI $M=$ GAPLIM+WW

C

C. Find value of H IN CELls

NH=H/YCEL

C PARAMETERs useo to Calculate froude nOS. IN MEANDR

c

$F 18=8.0 / F 1$

$F 28=8,0 / F 2$

C PARAMETERS USED IN BAR

SIGRD $=S I G M A-R O$

EXNH1 $=E X N-1.0$

VAR $1=3.14 * E X N /(200.0 * W * * E X N)$ VAR $2=16.5 * R O /$ SIGRO

C PARAMETERS FOR SCALING PLOT OF MEANDER GEOMETRY IN MEANDR

$S C A L E=A M P L I M / 9.0$

SCALE $2=S C A L E / 2.0$

$X L=X M A X / A M P L I M * 9.0$

C TDMIG $=0.0$

C INITIALISE TIME KEEPING DEVICES AND NFLD NFLD $=0$
IPRINT $=$ MOD ( NFLD, NPRINT $)$
ITPLOT $=$ MOD (NFLD, NTPLOT)

C

C RATID OF YCEL/ZCEL

C

YCOZC $=Y C E L / Z C E L$

C WRITE SCALES AND TITLES ON GRAPH

c

CALL PLOT $\{1,0.0, X M A X, X L, X M A X, 0.0, A M P L I M, 9.0, A M P L I M)$

CALL PLOT 1991

CALL PLOT $(90$, SCALE2, -SCALE2)

WR ITE $(3,45)$ SC ALE

CALL CHAR $(0.2,0)$

CALL PLOTI99)

CALLPLOT (90, SCALE2, AMPLIM)

HR I TE $(3,47)$

CALL CHAR $(0.2,0)$

CALL PLOTI99)

C

C PRINT DUT CROSS SECTION PARAMETERS

WR ITE $(6,21)$ TITLE, ZTOT, NCOLS, YTOT, NROWS, BANK, IBANK, WS, IWS, YCEL, ZCEL

C PRINT OUT CHANNEL PARAMETERS

C

WR ITE $(6,23) \mathrm{WH}, N Z C E L, H, N Z C E L 1, C 5, H, S I G M A, R O, F 1, F 2$, EXN

C PRINT OUT SYNTHETIC HYDROLOGY PARAMETERS

C WRITE $(6,24) D M, D S, Y M, Y S, A 1, A 2, A, B, S A, S B, O V D L M X$

C C PRINT OUT BANK MIGRATION PARAMETERS

C

WRITE $\{6,25) E 1, C 1, C 2$, GRAVLM

C PRINT OUT SCOUR AND FILL PARAMETERS

C WRITE $(6,26) C 6, E 2, S T D V N$ C PRINT OUT CUT OFF CONTROL. PARAMETERS

WRITE $(6,28)$ GAPLIM,ENL, EN2, SNLIM, AMPLIM, ECI, EC2

C PRINT OUT TYPE OF SECTION

C

IF IIFCOD6.GT.0IGO TO 167

WR I TE $(6,33)$ 
C 167 WR I TE $(6,31)$ ZSECT

0141

c

168 WR I TE $(6,27)$ LPPB, GRAVEL, OI.OSED, RIPPLE, S AND, WATER, DUNES, SILT, DOT, DOT $1, D O T, D O T, D O T, U P P B, C L A Y, A N T I D N, F L O O D$

C FIND AND PLOT INITIAL PLANIMETRIC FORM OF MEANDER

CALL MEANDR

C

C INITIAL OPERATIONS CONCERNING CROSS SECTION DEFINITION - THEN BRANCM

C TO INITIALISE AND PRINT CHANNEL SECTION

0143

0144

0145

0146

0147

0148

i. 149

0150

0151

0152

0153

0154

0155

0156

0157

0158

0159

0160

0161

0162

0163

0164

0165

0166

0167

0168

0169

0170

0171

0172

0173

0174

0175

0176

0177

0178

0179

0180

0181

0182

0183

0184

0185

0186

C

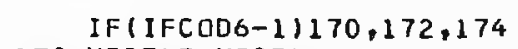



C IF (IPR I NT.EQ. 0) WRI TE $(6,30)$ AGG NAGG =AGG /YCEL IF(NAGG - LT. MARK)GO TO 210

C

C IF ROW OF CELLS IS FILLED, ADJUST BANKFULL STAGE AND FILL ROW WITH C ALPHAMER IC CHARACTERS

C

I HS $=$ I HS + I

IF (IHS.GT. NROWS)GO TO 984 MARK $=$ MARK+1

DO $200 \mathrm{~J}=1, \mathrm{NCOLS}$ SEOGS $(I W S, J)=F L O O D$

$C$ 200 SEDSTR (IHS, J) =FLOOD

C RECORD ON 2-D ARRAYS THE RESULTING EROSION ANO DEPOSITION AFTER THIS C YEAR

C

C FIND AMOUNT OF BANK MIGRATION IN CROSS SECTION REPRESENTED.ADJUST

C CHANNEL WIDTH(IN CELLS) REPRESENTED IN CROSS SECTION (AND RELATED

$C$ PARAMETERSI, DEPENDING ON TYPE OF CROSS SECTION AND CHANGES IN SHAPE C DF MEANDER

C

210 IF(IFCOD6.GT.0)GO TO 215

$R M I G=S Q R T(R L M I G * R L M I G+R D M I G * R D M I G)$

$A A=A T A N(R D M I G / R L M I G)$

TANA $=$ RDMIG/RLMIG

$P=A T A N((R+W W / 2.0-S Q R T((R+W W / 2.0) * * 2+2.0 * R * W W * T A N A * T A N A)) /(-2.0 * R * T$ IANAII

$Z C E L)=Z C E L * \operatorname{COS}(A A-P) / \operatorname{COS}(P)$

GO TO 216

215 RMIG=RDMIG

SINPHI $=\operatorname{SIN}(2.2 * \operatorname{SQRT}((S N-1 \cdot 0) / S N)$

ZCELI $=$ ZCEL*SI NPHI

216 NZCELO $=$ NZCEL

NZCLIO=NZCELI

$N Z C E L=W W / Z C E L I$

NZCELI $=W / Z C E L 1$

FLT2 =FLOAT (NZCELI)

$Y Z O Z C=Y C E L / Z C E L 1$

IF (NZCELI-NZCL1O) 218,218,217

217 NZDIF=NZCELI-NZCLIO

GO TO 225

C

218 NZDIF $=0$

C ADD LAST YEAR'S SMOOTHING ERROR TO THIS YEAR'S BANK MIGRATION

$C$ 225 RMIG $=R M I G+D E V$

C FIND BANK MIGRATIONIIN CELLSI IN CROSS SECTION, AND CALCULATE ERROR C DUE TO SMOOTHING, DEV 
C 227 FNRMIG =FLOAT (NRMIG)

C FIND TOTAL NUMBERS OF CELLS REQUIRED FOR CHANNEL SECTION AND CHECK

C THAT DOES NOT EXCEED SPECIFIED LIMITS

NZCELT $=$ NZCEL+NRMIG

IF( (IBANK+NZCELT+NDAVA),GT.NCOLS)GO, TO 585

$c$

C IF NO SCOUR AND FILL GO TO 400

IF (NFLD.EQ.0)GO TO 400

IFIIFCODS. NE. IIGO TO 400

C FIND MAXIMUM DEPTH OF SCOUR MEASURED ABOVE TALHEG

0235

0236

0237

0238

0239

0240

0241

0242

0243

0244

0245

0246

0247

0248

0249

0250

0251

0252

0253

0254

0255

0256

0257

0258

0259

0260

0261

0262

0263

0264

0265

0266

0267

0268

0269

0270

0271

0272

0273

0274

DSCR $=$ C6*QVOL**E2+RANSAM(IFCOOI)*STOVN

IF IDSCR. LT. O. O) DSCR $=0.0$

IF ( I PRI NT.EQ. 0) WRI TE (6,41) DSCR

$H H=H+D S C R$

C

C IF MAX. CHANNEL DEPTH NOH EXCEEDS LOWER BOUNOARY OF SECTION - JDB ENDS

IF (HH.GT. (FLOAT (IHS) *YCEL) ) GO TO 980

C FOR EVERY COLUMN OF POINT BAR GRADUALLY FILL TO ORIGINAL DEPTH

$370 Z=Z C E L L$

DD $390 \mathrm{~J}=1, \mathrm{NZCEL1}$

$N C O L=J+I B A N K$

IFIIFCOD 7.EQ.01GO TO 372

CALL BARI $(0, \mathrm{HH})$

GD TO 373

372 CALL BAR $(0, \mathrm{HH})$

$373 Z=Z+Z C E L I$

390 CONTINUE

$H H=H H-Y C E L$

IF (HH.GE.H)GO TO 370

C

C FILL SCOURED TALHEG

IFINRMIG.LT.I)GO TO 400

DO $380 \mathrm{~J}=1$, NRMIG

$N C O L=I B A N K+N Z C E L I+J$

$Y=-D S C R / 2.0 *(C D S(3.14 *(F L O A T(N R M I G-J) / F N R M I G))-1.0)$

NYCEL $=(H+Y) / Y C E L$

INDEX $=$ I HS-NYCEL

381 SEDG S ( I NDEX,NCOL) =SEDG (I NDEX, I BANK +NZCEL I)

SEOSTR ( INDEX,NCOL) $=$ SEDSTR ( INDEX, I BANK+NZCEL I )

$Y=Y-Y C E L$.

INOE $X=I$ NDE $X+1$

IFIY.GE.0.0)GO TO 381

380 CONT INUE

C

C FOR EVERY COLUMN IN CHANNEL SECTION,FIND GRAINSIZE AND BEDFORM ACROSS

C INNER BANK AND ERODE OUTER BANK

C INITIALISE PARAMETERS AND WRITE HEADINGS

$400 Z=Z C E L I$

$O B G S=0.0$

$B G S=0.0$

GRAV $=0.0$

$N R M I G I=N R M I G+N Z D I F+1$

I NDE XK $=$ I WS

KOUNT $=0$

INDEXO=IHS $-\mathrm{NH}+1$

IF I IPR I NT, EQ. 0 ) HRITE $(6,42)$ T ITLE, NFLD

C

C BEGIN MAJOR LOOP ENTERED ONCE FOR EVERY COLUMN OF CHANNEL SECTION

DO $450 \mathrm{~J}=1$, NZCEL

$N C O L=I B A N K+J+N R M I G$

IF (J.LE.NZCELI)GO TO 410

C

ERODE DUTER BANK 
C.

C

410 IF(IFCOD7.EQ.0)GO TO 411 CALL BAR $1(1, H)$ GO TO 412

411 CALL BAR $(1, H)$

412 IF (D. LE. 0.00625) BGS $=8 G S+1.0$ IF (ITPLOT.EQ. 0. AND.J.NE.NZCELI)TLPLOT (INDEX,NCOL) =DOT

c IFINFLD.EQ.0) GO TO 440

C IFILL. POINT BAR

IF (NRMIGI.LT.1)GO TO 440

DO $415 J J J=1, N R M I G I$

$J J=N C D L-J J J$

IFIJJ.LT.1IGO TO 415

IF INZCELI. L.T. NZCLID. AND. I FCOD5 . EQ. 1 )GO TO 413

IF ( SEDSTR ( I NDEX, JJ). NE. WATER. AND. SEDSTR (INDEX, JJ) .NE .FLODD.AND. SED

ISTR (INDEX, JJ). NE. OLDSEDIGO TO 415

413 SEOGS(INOEX, $J J)=$ SEDGS (INOEX, JJ+1)

SEDSTR (INOEX,JJ)=SEDSTR (INDEX,JJ+I)

C

415 CONT INUE

C

420 IF ( (INDEXK-INDEX) . LT. 2$)$ GO TO 424

INDE XK =INDE XK-1

DO $422 \mathrm{JJ}=1, \mathrm{NRMIG1}$

$N C O L K=N C O L-J J$

IFINCOLK.LT.1)GO TO 422

IF (NZCELL. LT.NZCLID. AND. IFCOD5.EQ. 1 )GO TO 421

IF ( SEDSTR I I NDEXK, NCOLK). NE. WATER. AND.SEDSTR (INDEXK, NCDLK), NE . OLDSE 1D) GO TO 422

421 SEDGS (INDEXK, NCOLK) =SEDGS (INDEX, NCOLK)

422 CONTINUE

GO TO 420

$\mathrm{C}$

424 INDEXK $=$ INDEX

C FILL NEW CHANNEL WITH WATER

C

440 INDEX $=$ I WS-NYCEL+1

IF (NYCEL.EQ.0) GO TO 450

DO 445 II = I NOEX, I WS

SEDSTR III, NCULI =WATER

445 SEDGS(II, NC OL) = WATER

$C$

$450 \quad L=Z+Z C E L 1$

C CALCULATE PERCENT SILT-CLAY IN PERIMETER OF CHANNEL C

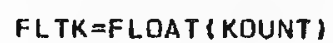

C FILL 2-D ARRAYS FOR THE SECOND CHANNEL IF A TWO CHANNEL DOWNVALLEY

C SECTION IS BEING USED 
C PRINT OUT CROSS SECTION SHOHING GRAIN SIZE DISTRIBUTION

IF(IFCO06. NE. 2$) 60$ TO 548

DO $545 \mathrm{~J}=1$. NZCELT

$J \mathrm{~J}=\mathrm{I} B \mathrm{BANK}+\mathrm{J}$

JJJ $J=J J+M D A V A$

DO $545 I=1$, I HS

TLPLOT $(I, J J J)=T L P L O T(I, J J)$

SEDGS $(I, J J J)=\operatorname{SEDGS}(I, J j)$

545 SEDSTR $(I, J J J)=\operatorname{SEOSTR}(I, J J)$

548 IF (IPRINT. NE. 0)GO TO 570

HR I TE $(6,35)$ TI TLE, NFLD

DO $550 \mathrm{~J}=1$, NCOLS

550 HRITE $(6, F M T 4)$ ( SEDGS I I J), I=1, NROWS), (TLPLOT $(I, J), I=1$, NROWS)

C PRINT OUT CROSS SECTION SHOWING SEDIMENTARY STRUCTURE DISTRIBUTION

WR I TE (6,36) TI TLE , NFLD

DO $560 \mathrm{~J}=1, N C O L S$

560 WRITE $(6, F M T 4)$ ( SEDSTR (I,J), I =1, NROWS ), (TLPLOT $I, J), I=1, N R O H S$ ) GO TO $(570,990,992)$, I CUT

$c$

570 IBANK $=I B A N K+N R M I G$

C FIND PLANIMETRIC FORH OF MEANDER AT END OF THIS YEAR

IF (NF LD.EQ.0) GO TO 575

IF ( (TDMIG+WVL).GT.XMAX)GO TO 986

C FIND Changes IN AMPLITUDE

IF ILIM.EQ.1/GO TO 572

$A M P=A M P+R L M I G=2,0$

$S S=($ AMPLI $[M-A M P) / 2.0$

IFISS.GT.0.0)GO TO 573

$L I M=1$

RLMIG $=0.0$

AMP $=$ A MP LI $M$

$S S=0.0$

CALL MEANDR

IFIIFCOD6.LE.0IGO TO 971

WR I TE $(6,49)$ NF LO

GO TO 575

572 IFIRDMIG.LE.0.0IGO TO 575

CALL MEAND 1

GO TO 575

573 CALL MEANDR

IF IGAP.LE. HWIGO TO 992

575 NF LD $=N F L D+1$

IF INFLD.LE.NTIMIGO TO 169

c

c$$
\text { GO TO } 999
$$

460 WRITE $(6,43)$ GRAVI GO TO 999

585 HRITE $(6,39)$ GO TO 999

971 HR I TE $(6,972)$ NF LD GO TO 999

976 WR I TE $(6,977)$ GO TO 1000

978 WR I TE $(6,979)$ GO TO 1000

980 WR I TE $(6.981)$ GD TO 1000

982 WRITE $(6.983)$ GO TO 1000

984 HRITE $(6,985)$ GO TO 999

986 HRITE $(6.987)$ GO TO 999

98 B WR ITE $(6,989)$ GD TO 1000

990 WR I TE $(6,991)$ NFLO GO TO 999

994 WRITE $(6,995)$ GD TO 1000

992 HRITE $(6,993)$ NFLD

999 CALL PLOT(7)

1000 STOP

END 
c

0001

SUBRDUTINE MEANDR

0002

0003

0004

0005

0006

C

C CONTROL STATEMENTS

EXTERNAL FUNC 2

DI MENSION COSPHI (50), SINPHI (50)

COMMON/COM1/I PRINT , RINB , FROUD1, FROUD2, VAR 2S, RC

COMMON/COM3 /NFLD, TI TLE (15), HVL, AMP, VS, GAP, NFPLOT, CHS, WH, R , RO, VAR 2 , 1F28,F18,SS, TDMIG, SN

C FORMAT STATEMENTS

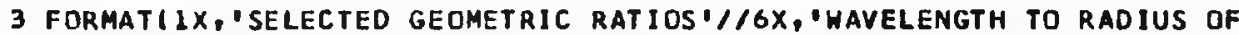
1 CUR VATURE ', $12 X, F 10.3 / 6 X$, HAVELENGTH TO CHANNEL WIDTH, $17 X, F 10,3 / 6$ $2 X$, 'RADIUS OF CURVATURE TO CHANNEL WIDTH, 8X,F10.3/6X, 'AMPLITUDE TO 3 CHANNEL WIDTH', $18 \times, F 10.3 / / / 1$

0007

4 FORMATIIHI, $1 X, 15 A 4, "$ TIME INCREMENT', $15 / 11 \mathrm{X}$, 'PLANIMETR IC FORM OF M

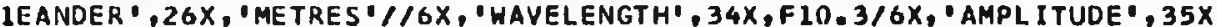
$2, F 10.3 / 6 X$, 'SINUDSITY",45X,F10.3/6X, "RADIUS OF CURVATURE AT BEND AX $315,12 x, F 10.3 / 6 x$, 'WIDTH DF MEANDER NECK', $23 x, F 10.3 / 6 x,{ }^{\circ}$ CHANNEL LEN 4GTH ALONG MEANDER , 16X,F10.3/6X, 'VALLEY SLOPE',42X,F10,8/6X, 'LONGI STUDINAL HATEK SURFACE SLOPE $, 22 X, F 10,8 / / /)$

0008

6 FORMAT $(14,100 X)$

C REGRESSION EQUATION RELATING SINUOSITY TO AMPLITUDE/WAVELENGTH

0009

0010

IFINF LD.EQ.OIGO TO 5

$A O H=A M P / W V L$

$S N=(1-0.4301562 * A 0 H+1.674662) * A O H+0.337086) * A O W+0.9634151$

C

C CALCULATE CHL, CHS,AND R

0012

0013

0014

0015

0026

0017

0018

0019

0020

0021

0022

0023

0024

0025

0026

0027

0028

0029

0030

0031

0032

0033

0034

0035

0036

$5 \mathrm{CHL}=W V L \cdot S N$

CHS $=V S / S N$

$R=W V L *(S N * * 1.5) /(13.0 * S Q R T(S N-1.0))$

c

C Calculate miscellane ous parameters

$R$ I NO $=R-H W / 2.0$

$\mathrm{RC}=\mathrm{RO}=\mathrm{CHS}$

VAR $25=$ VAR $2 *$ CHS

$c$

C Calculate froude numbers

FRDUD $1=S O R T(E H S * F 28)$

FROUD 2 = SOR $T(C H S * F 28)$

C

C Calculate selected ratios

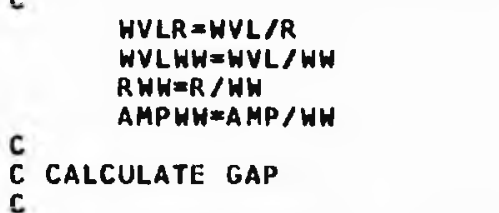

OMEGA $=2.2 * 5 O R T((S N-1.0) / S N I$

IF (OMEGA.LE. 1.57)GO TO 8

CALL SIMINT 0.0 , OMEGA,1.57,0.005, SIMP2, FUNC2)

$G A P=W V L *(1,0-S N / 3,14 * S I M P 2)$

GD TO 9

B GAP $=99999999.0$

ENTRY MEAND 1

C PRINT GEOMETRIC PARAMETERS AND RATIOS IF REQUIRED

C

9 IFIIPRINT. NE.0IGO TO 20

WR I TE $(6,4)$ TITLE, NF LD, WVL, AMP, SN, R, GAP, CHL, YS, CHS

WR I TE $(6,3)$ WVLR, WVLHW, RHW, AMPWH

c

C PARAMETER INITIALISATION FOR PLOTTING IF NFLD(MOD NFPLOT) EQUALS ZERO

20 IF (MOO (NFLD.NFPLOT).NE. 0160 TO 55

DSI $=$ CHL 1100.0

$S=0 S 1$ 


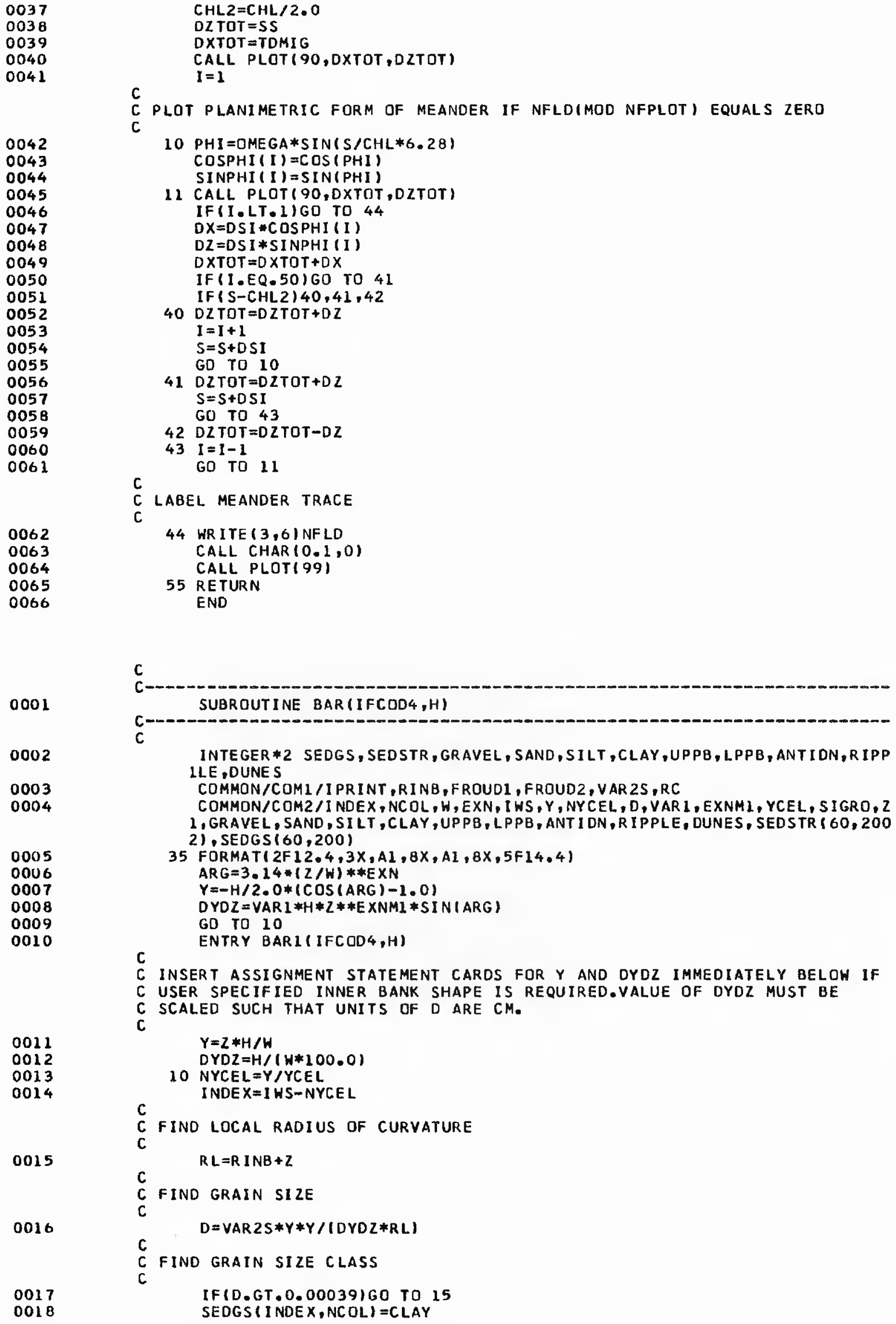

41. $D Z T O T=D Z T O T+D Z$ $S=S+D S I$

c

0001

0002

0003

0004

0005

0006

0007

0008

0009

0010

C

SUBROUT INE BAR (IFCOO4, H) 


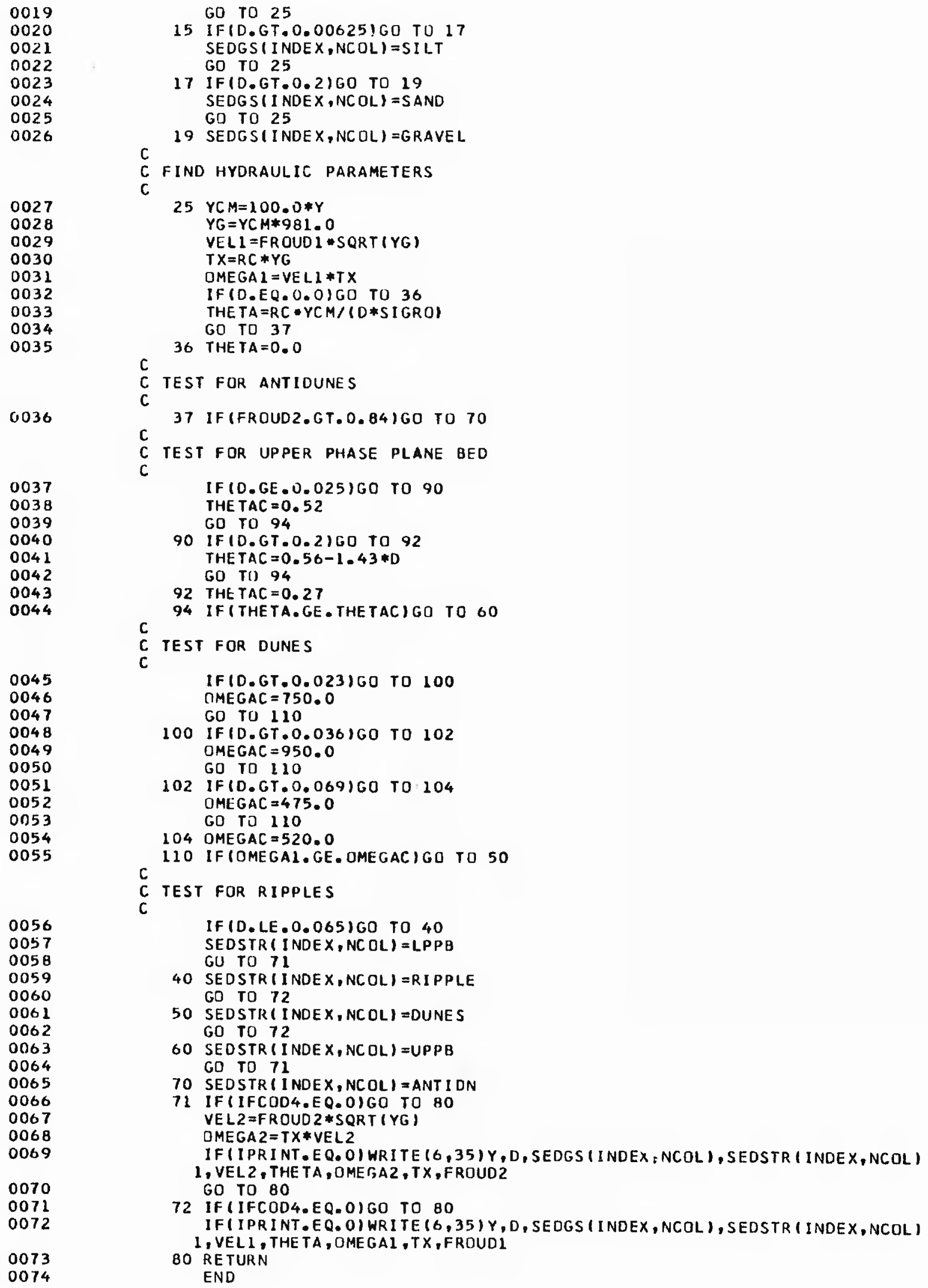
c

15 IFID.GT.0.00625!G0 TO 17 SEDGS (INDEX, NCOL) $=S I L T$ GO TO 25

17 IF(D.GT.0.2)GO TO 19 SEDGS (INDEX, NCOL $)=$ SAND GO TO 25

$19 \operatorname{SEDGS}($ INDEX, NCQL) = GRAVEL

FIND hydRaulic parameters

$25 Y C M=100.0 * Y$

$Y G=Y C M * 981.0$

VELL $=F R O U D I * S Q R T(Y G)$

$T X=R C$ * $Y G$

DMEGA $1=V E L I * T X$

IF (D.EQ.0.0) GO TO 36

THE TA $=R C * Y C M /(D * S I G R O)$

GO TO 37

36 THE TA $=0.0$

$\mathrm{c}$

C TEST FOR ANTIDUNES

$c$

37 IF(FROUD2.GT.0.84)GO TO 70

C TEST FOR UPPER PHASE PLANE bED

c

IF (D.GE.0.025)GO ro 90

THE TAC $=0.52$

GD TO 94

90 IFID.GT.0.2)GO TO 92

THE TAC $=0.56-1.43 * D$

GO TI) 94

92 THE TAC $=0.27$

C

94 IF (THETA.GE.THETAC)GO TO 60

C TEST FOR DUNES

IF(D.GT.0.023)GO TO 100

IMEGAC $=750.0$

GO TO 110

100 IF(D.GT.0.036)G0 TO 102

$O M E G A C=950.0$

GD TO 110

102 IF(D.GT.0.069)GO TO:104

OMEGAC $=475.0$

GD TO 110

104 OMEGAC $=520.0$

c

110 IF (OMEGAL.GE. OMEGACIGO TO 50

C TEST FOR RIPPLES

IF (D.LE.0.065)GO TO 40

SEDSTR ( INDEX, NCOL) $=$ LPPB GU TO 71

40 SEDSTR ( INDEX,NCOL) = RI PPLE GO TO 72

50 SEDSTR ( I NDEX, NC OL) =DUNES GO TO 72

60 SEDSTR ( INDEX, NC OL) $=U P P B$ GD TO 71

70 SEDSTR ( I NDEX, NCOL I = ANT I DN

71 IFIIFCOO4.EQ.0IGO TO 80 VEL2 $=F R O U D 2 * S Q R T$ (YG) OMEGA2 $=T X * V E L 2$

IFI I PRI NT . EQ. O) WRITE $(6,35)$ Y, D, SEDGS IINDEX , NCOL I, SEOSTR (INDEX, NCOL) 1, VEL2, THE TA, OME , A 2, TX, FROUD2 GO TO 80

72 IFIIFCOO4.EQ.0IGO TO 80 IF ( I PR I NT.EQ. O) WRI TE $(6,35) \mathrm{Y}, \mathrm{D}, \mathrm{SEDGS}$ ( I NDEX, NCOL), SEDSTR (INDEX, NCOL) 1 , VELI, THE TA, OMEGAI, TX, FROUDI $B O$ RETURN

END 
C

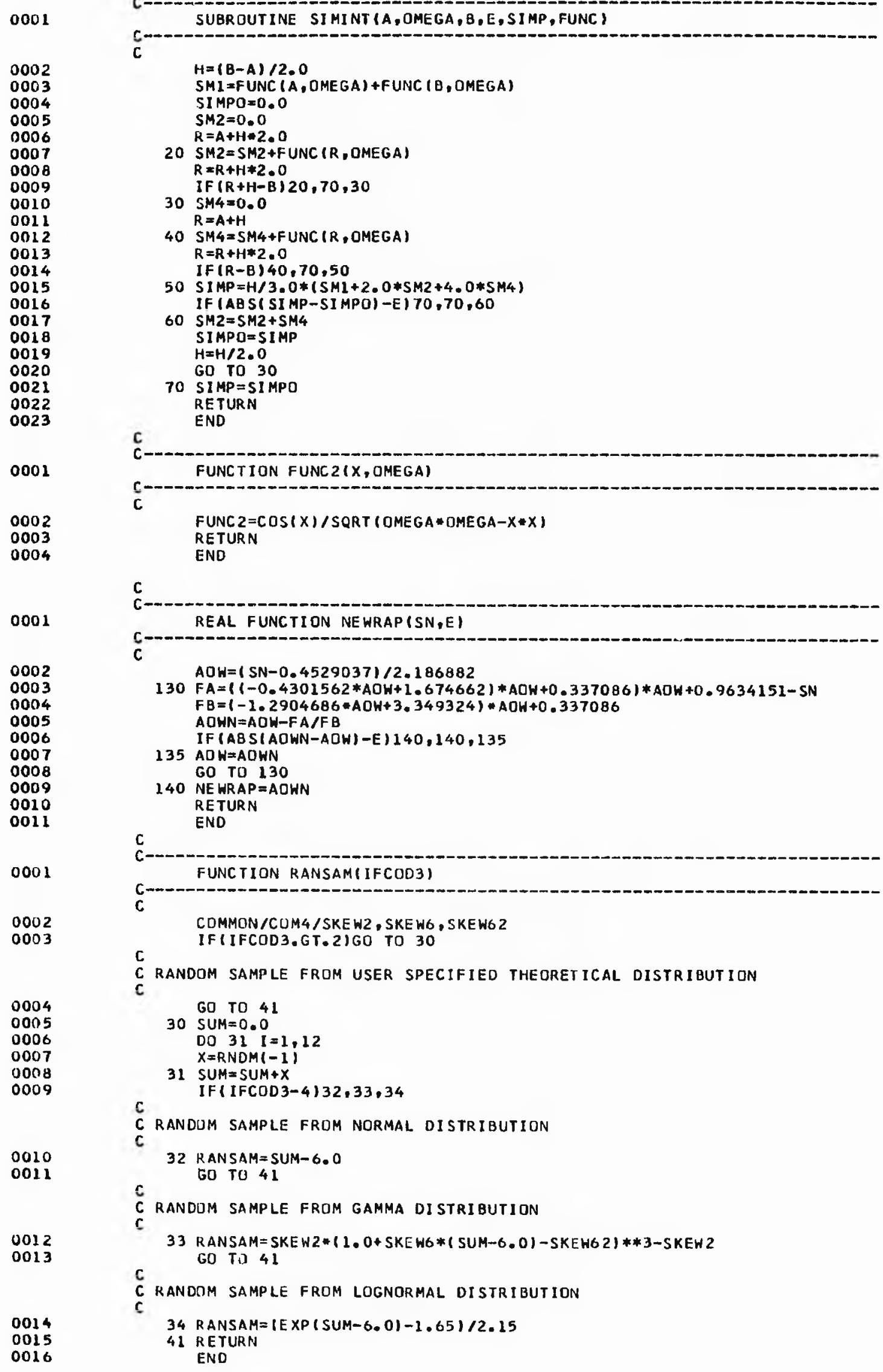




\subsection{Main Program (using disc storage)}

The structure of this program is essentially the same as the main program without a disc, therefore only those steps and comments which are not the same are listed below.

6. Alphameric characters are read into array SEDIN(I) and the grain size, sedimentary structure and time line cross sections are initiallsed and stored on disc. For every column of the cross sections the following operations are executed:Array TEMPGS(I) is filled from the bottom of the section up to the row IWS with the grain size characters read into SFDTN, one character of SLDIN specifying the character for the complete row, I, of the grain size cross section. TEMPST(I) is filled up to Irs with the OLDSED character and the remaining rows in TEMPST and TEMPGS are filled with blanks, Every element in TEMPTL(I) is filled with blanks. Each set of three columns of data, one from each cross section, is then stored in one record on disc. operations 19 to 32 are involved with recording on SEDGS (INDEX, NCOL) and SEDSTR (INDEX,NCOL) the resulting erosion and deposition after this years 'floods', and putting a time line on TLPLOT (INDEX, NCOL) if ITPLOT $=0$. These arrays are then stored on disc in the appropriate place.

20. Find total number of cell widths/colums, NZCELT, required for the channel section and corresponding array elements needed in the core store at once. If NZCEL' exceeds the specified maximum number, MNCOLs, the job is terminated, as is the case if the right hand edge of the cross sections is exceeded. The necessary columns of the cross sections are read off disc into SliDGS (INDEX, NCOL), SEDSTR (INDNX, NCOL) and TLPLOT (INDEX, NCOL).

23. Every column of the inside bank of the channel section (1 to 
NZCEL1) is now filled to the original depth by successive recalculation of the transverse profile, the depth at the old talweg being progressively decreased by one cell depth row until filling is complete. Subroutine BAR or BARl is called during these operations in order to fill the appropriate elements of SEDGS and SEDSTR with alphameric characters.

24. The area bounded by the position of the old talweg, the maximum scour depth below the old talweg, and the position of the new talweg is now filled by allocating for each row in this area the grain size and bed form symbols calculated for the row elements of the old talweg column, NZCEL1, in step 23.

Fig. 14.2 illustrates the sequence of events in the scouring and filling operation described above. Steps 25 to 32 constitute a major loop and are concerned with erosion of the outer bank and deposition on the point bax. As a result of the erosion of the outer bank and changes in the projected channel width in the cross sections, the whole transverse profile is shifted accordingly, and the left hand side of the new point bar profile is started at column NRMIG+1 of the cross section. For every column, NCOL, of the new point bar (up to NZCEL1+NRMIG) steps 26 to 31 are executed.

For every colum of the outer bank (NZCELI+NRMIG+1 up to NZCELT) the following step is executed. (This is step 32). 35. The arrays SEDGS, SEDSTR, and TLPLOT containing the channel section clata just computed, are written onto disc in the appropxiate place with respect to the whole cross section. If a two-channel downvalley section is being used, this same information is ajso written on the disc, NDAVA records further on. 
c

C CONTROL STATEMENTS

0001

0002

0003

0004

0005

0006

0007

0008

0009

0010

0011

0012

0013

0014

0015

0016

0017

0018

0019

0020

0021

0022

0023

REAL NEWRAP

DI MENSI ON FMT $4(5), A(6), B(6), S A(6)$, SB $(6)$, FMSUM $(365)$, FSSUM( 365$)$

INTEGER *2 SEDGS, SEDSTR, TLPLDT $(60,50)$, GRAVEL, SAND, S ILT, CLAY, UPPB, LP 1PB, ANTI DN, RI PPLE, DUNES, OLDSED, WATER, DOT, BLANK, SED IN (60), TEMPG S(60) 2, TEMPST 160$)$, TEMPTL $(60)$, FLOOD

COMMON/COM1 /I PRINT, RI NB, FROUD1, FROUD2, VAR2S, RC

COMMON/COMZ / I NOEX, NCOL, W, EXN, IWS, Y, NYCEL, D, VAR I, EXNM I, YCEL, S IGRO, 2 1, GRAVEL, SAND, SI LT, CLAY,UPPB, LPPB, ANT I DN, R IPPLE, DUNES, SEDSTR $(60,50)$ $2, \operatorname{SEDGS}(60,50)$

COMMON/COM3/NFLD,TITLE (15), WVL, AMP, VS, GAP, NFPLOT, CHS, WW, R, RO, VAR 2 , IF $2 B, F 18, S S, T D M I G, S N$

COMMON/COM4/SKEW2, SKEWG, SKEWG 2

DATA RMIG,TLMIG,AGG,DEV,NDAVA,LIM, MARK, ICUT / $4 * 0.0,2 * 0,2 * 1 /$

$C$

DEFINE FILE $4(200,360, L, I D I$

C FORMAT STATEMENTS

\footnotetext{
1 FORMAT(15A4,110,12)

2 FORMAT (11,3F12,0,11)

3 FORMAT (4I 4,5A4)

5 FORMAT (BOAl)

6 FORMAT (6F12.0)

7 FDRMAT ( $8 F 8.0, I 4)$
}

21 FORMATI $1 H 1,1 \times, 15 A 4 / / 1 X$, 'CROSS SECTION PARAMETERS',49X, 'METRES',5X, 1 'CELLS $\% / 6 X, \cdot H I D T H$ OF SECTION , $48 X, F 10,3,110 / 6 X$, 'THICKNESS OF SECT $2 I O N \cdot 44 X, F 10,3,110 / 6 X, \cdot$ INITIAL DISTANCE OF INNER CHANNEL BANK FROM 3 L.H.S. OF SECTION $, 3 X, F 10,3, I 10 / 6 X, \cdot$ INITIAL BANKFULL STAGE MEASUR 4ED FROM SECTION BASE , 15X,F10.3,110/6X, CELL SIZE IN VERTICAL(Y) D 5IRECTION', $30 X, F 10.3 / 6 X,{ }^{\circ}$ CELL SILE IN HORIZONTAL $(Z$ OR $X)$ DIRECTION' $6,23 \times, F 10.3 / 1 / 1$

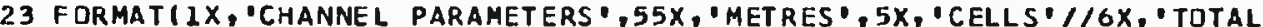
IWIDTH OF CHANNEL(H) $, 39 X, F 10,3,110 / 6 x, \cdot H I D T H$ OF FLOH BETWEEN INNER 2 BANK AND TALHEG (HI) $, 17 X, F 10,3,110 / 6 x$, 'RAT IO OF $W 1$ TO $W, 68 X, F 10$. $33 / 6 X$, MAXI MUM FLOH DEPTH MEASURED ABOVE TALWEG', $24 X, F 10,3 / 6 X$, 'DENS 41 TY DF SEDI MENTARY PARTICLES $, 52 X, F 10.3,{ }^{\circ}$ GM/CM3'/6X, "FLUID DENSIT $5 Y 1,71 X, F 10.3,0^{\circ}$ GM/CM3 $16 X, \circ$ DARCY -WEISBACH FRICT IDN COEFFIC IENT FOR 6 DUNES AND RI PPLES $, 27 X, F 10.3 / 6 X, \cdot D A R C Y-W E I S B A C H$ FRICTION COEFFICI TENT FOR PLANE BEDS AND ANTIDUNES, $20 X, F 10,3 / 6 X, '$ EXPONENT $N 1 \%, 73 X, F$ BI0.3///1

24 FORMAT(IX, 'SYNTHETIC HYDROLOGY PARAMETERS (UNITS NOT NECESSARY) $/ / 6$ $1 X$, MEAN OF ALL DAILY MEAN VALUES , $25 X, F 10.3 / 6 X$, "STANDARD DEVIATION 2 OF DAILY MEAN VALUES $, 15 X, F 10,3 / 6 X, " M E A N$ OF YT SER IES $, 37 X, F 10.3 /$ $36 X$. "STANDARD DEVIATION OF YT SERIES:, $23 x$, F10,3/6X, COEFFICIENTS IN 4 AUTOREGRESSI VE MODEL', 15X,'Al $=1, F 10,3,7 X, \cdot A 2=\circ, F 10,3 / 60 X$, 'HARMON I 5CS FROM 1 TO $6 \% / 6 X$, "FOURIER COEFFICIENTS FOR DAILY MEANS(A) $, 15 X, 6$ 6F $10.3 / 42 X$, , (B) $1.15 \times, 6 F 10.3 / 6 X$, , FOUR IER COEFFICIENTS FOR DAILY STD TOE VIATIONS (SA) " .5X, 6F10.3/51X," (SB)",5X,6F10.3/6X,"MAXIMUM VALUe 0 BF QVOL', 33X,F $10.3 / 1 / 1$

25 FJRMAT $11 X$, "BANK MIGRATION PARAMETERS $, 16 X, 0^{\circ}$ EXPONENT N2",53X,F10.3/ $16 x,{ }^{\circ}$ VALUE OF CONSTANT IN LATERAL MIGRAT ION RELATION', $14 \mathrm{X}^{\circ} \mathrm{\circ} \mathrm{K} 2=\circ, \mathrm{E} 10$ 2. 3/6X,"VALUE OF CONSTANT IN DOWNVALLEY MIGRATION RELAT ION', $11 \mathrm{X}, " \mathrm{~K} 3$ $3=\bullet$, E $10.3 / 6 x, \cdot$ LI MI IING PERCENTAGE OF GRAVEL ALLOWABLE IN OUTER BANK $4 \cdot, 11 X, F 10.3 / 1 / 1$

26 FORMAT $1 \mathrm{X}$, " SCOUR AND FILL PARAMETERS $/ 6 \mathrm{X}, \circ$ CONSTANT $K 4^{\circ}, 43 \mathrm{X}, \mathrm{E} 10,3 / 6$ $1 X$, 'EXPONENT $N 33^{\prime}, 39 \mathrm{X}, \mathrm{F} 10.3 / 6 \mathrm{X}$, 'STANDARD DEVIATION OF ERROR TERM', 18 $2 X, F 10.3 / 1 / 1$

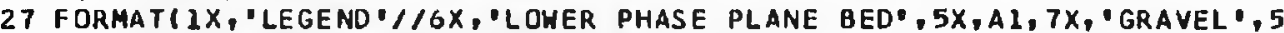

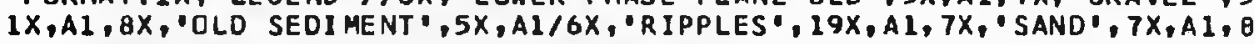
$2 X, ' H A T E R ', 12 X, A 1 / 6 X$, 'DUNES ', 21X,AL, 7X, 'SILT', 7X, A I, 8X, 'TIME LINE', $38 X, 5 A 1 / 6 X$, 'UPPER PHASE PLANE BED',5X,A1,7X, CLAY', 7X, A1, 8X, AIR', 1 $44 X$, 'BLANK' $/ 6 X$, 'ANT I DUNES' $17 X, A 1,7 X$, 'OVERBANK $^{\prime}, 3 X, A 1 / 40 X$, 'DEPOSITS $5, / / 1$

28 FDRMATI $1 X$, 'CUT-DFF CONTROL PARAMETERS $/ 6 x, ' L I M I T I N G$ WIDTH OF MEAND IER NECK',24X,F10.3," METRES $/ 6 X,{ }^{\circ}$ EXPQNENTS IN NECK CUT-OFF RELATIO $2 N^{\prime}, 16 X,{ }^{\circ} E N 1={ }^{\circ}, F 10,3,6 X,{ }^{\circ} E N 2=1, F 10,3 / 6 X,{ }^{\circ} L$ IMITING SINUDSITY', $36 X, F 1$

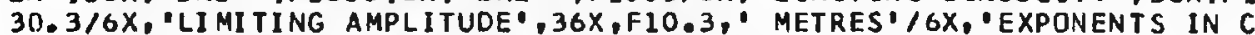
4HUTE CUT OFF RELATION', $15 X,{ }^{\prime} E C I=1, F 10.3,6 X_{1}{ }^{\circ} E C 2=1, F 10.3 / 1 / 1$

29 FORMATIIHI, $1 X, 25 A 4$, "TIME INCREMENT', I5//2X,"FLOOD PER IOD VOLUME FO IR THIS YEAR $, 26 X, F 10,3 / 11 X, 0^{\circ}$ OUTER BANK GRAINSIZE INDEX AT BEGINNIN $2 G$ OF YEAR $, 12 X, F 10,3 / 11 X$, ' INNER BANK GRAINSIZE INDEX AT BEGINNING $30 F$ YEAR $, 12 X, F 10.3 / 11 X, 0 \%$ SILT-CLAY IN CHANNEL PERIMETER AT BEGINN 4ING OF YEAR $, 6 X, F 10,3 / 11 X, 0^{\circ}$ ISTANCE FRDM LIMITING AMPL. ITUDE AT BEG $5 I$ NNING OF YEAR $, 6 X, F 10,3,{ }^{\circ}$ METRES $/ / 1 X, 1 L A T E R A L$ MIGRATION DUR ING $T$ GHIS YEAR', $25 X, F 10.3$, METRES $/ / 1 X, ' T O T A L$ LATERAL MIGRATION AT END TOF THIS YEAR $, 16 X, F 10,3, \cdot$ METRES $/ / 1 X, \cdot$ DOWNVALLEY MIGRATION DUR ING

Table 14.2. Listing of main program (using additional disc storage). 
8 THIS YEAR',22X,F10,3," METRES $/ / 1 \mathrm{X}$, 'TOTAL DOWNGLLEy MIGRATION AT 9 END OF THIS YEAR $, 13 \times, F 10.3,1$ METRES $/ / 1$

30 FORMATI $L X$, 'TOTAL AGGRADATION AT END UF THIS YEAR', 22X,F10.3,' METR IES $\% / 1$

0025

31 FORMAT////1X,'A DOWNVALLEY SECTION IS REPRESENTED IN THIS TEST'/IX 1, DISTANCE OF LINE OF SECTION FROM POINT OF INFLECTIDN OF LOOP IS* $2, F 10.3,1$ METRES $\because / 1$

0026

0027

0028

0029

0030

0031

0032

0033

0034

0035

0036

0037

0038

0039

0040

0041

0042

0043

0044

0045

0046

0047

0048

0049

0050

0051

0052

0053

0054

0055

0056

0057

0058

0059

0060

0061

0062

0063

33 FORMATI///IX, "A LATERAL SECTION IS REPRESENTED IN THIS TEST $/ / 1$

35 FORMATI $1 H I, 1 X, 15 A 4, '$ TIME INCREMENT', I5//1X, 'CROSS SECTION SHOWING 1 DISTRIBUTION OF GRAIN SIZE ACROSS MEANDERING RIVER FLOOD PLAIN // 2()

36 FDRMATIIHI, 1 X, $15 A 4, '$ TIME INCREMENT', I $/ / 1 \mathrm{X}$, 'CROSS SECTION SHOWING 1 DISTRIBUTION OF SEDIMENTARY STRUCTURE ACROSS MEANDER ING RIVER FLO 200 PLAIN $/ / / 1$

37 FORMATIIX, THE NUMBER DF COLUMNS REQUIRED FOR THE SPECIFIED CHANNE IL WIDTH IS GREATER THAN//IX,'THE CORE STORE WILL HOLD - RESCALING 2 IS REQUIRED 1

38 FORMATIIX, 'A DEVICE ERROR CONDITION WAS ENCOUNTERED DURING DATA TR IANSFER FROM DEVICE TO STORAGE'I

39 FORMATIIX, 'THE SPECIFIED SECTION HIDTH HAS BEEN EXCEEDED-THE WIDTH 1 MUST BE INCREASED IF MIGRAT IDN IS TO PROCEED')

41 FORMATI1X,'DEPTH OF SCOUR AT TALHEG FOR THIS YEAR', $21 X, F 10.3, \cdot$ MET IRE S $/ 11$

42 FORMAT(1HO, 1X,15A4, 'TIME INCREMENT $\bullet, 15 / / 1 X,{ }^{\circ}$ VAR IATION DF GRAINSI 2 IE AND BED FORM OVER CHANNEL CRDSS PROFILE'//7X, 'DEPTH', $3 X$, 'GRAINSI 2ZE', 8X, 'BED FORM',6X,'LOCAL MEAN', 4X, 'LOCAL', 10X, 'LOCAL STREAM', 2X 3.' LOCAL BED', 5X, 'LOCAL FROUDE'/7X, '(H)',5X,'(CM)', 27X, 'FLOW VELOC I 4 TY', $1 X$, 'OI MENSI ONLESS', $2 X$, 'POWER', $9 X$, 'SHEAR STRESS', $2 X$, 'NUMBER'/46

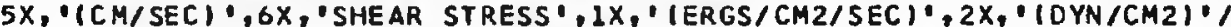
6)

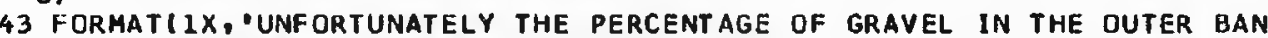
IK IS OF 10.31

45 FORMATI'SCALE-1 INCH TO',F 10.2," METRES', 100X)

47 FDRMAT("MEANDER GEOMETRY', 100X)

49 FORMAT////1X,"TIME INCREMENT $, 15, "$ LIMITING SINUOSITY/AMPLITUDE IHAS BEEN REACHED 1

972 FORMAT////IX,' TIME INCREMENT $1,15, / 1 X, ' L$ IMIT ING SINUDSITY/AMPL ITU IDE REACHED IN A LATERAL SECTION - TEST TERMINATED')

977 FORMATI///1, ,INITIAL BANKFULL STAGE MEASURED FRJM BASE OF SECTION 1 EXCEEDS SPECIFIED SECTION THICKNESS - TEST TERMINATED')

979 FORMAT/ $/ / 1 X$, 'ERROR IN SECOND DATA CARD - LAST THREE VARIABLES MUS $1 T$ BE NONZERO'

981 FORMATI $1 X$, THE LOHER BOUNDARY OF THE CROSS SECTION HAS BEEN EXCEED IED - $/ 1 X, " A D J U S T M E N T$ IS REQUIRED IN EITHER INITIAL BANKFULL STAGE, 2DEPTH AT TALWEG,OR SCOUR AND FILL PARAMETERS'?

983 FORMATIIX, 'INITIAL SINUOSITY IS OUTSIDE THE SPECIFIED LIMITS - TES $1 T$ TERMINATED')

985 FORMATIIX, 'THE SPECIFIED SECTION THICKNESS HAS BEEN EXCEEDED - RES ICALING IS REQUI RED IF AGGRADATION IS TO CONT INUE')

987 FORMATIIX. 'THE SPECIFIED LENGTH DF THE $X$-AXIS ON THE GRAPH PLOTTER 1 HAS BEEN EXCEEDED - RESCALING IS REQUIRED')

989 FORMAT////1X, 'RATE OF AGGRADATION PER FLODD INCREMENT IS GREATER T IHAN UNE VERTICAL CELL - RESCALING IS REQUIRED'

991 FORMATI///1X, TIME INCREMENT $\because 15, "$ - TEST TERMINATED DUE TO CHUT IE CUT OFF')

993 FORMATI///1X," TIME INCREMENT " I5," - TEST TERMINATED DUE TO NECK 1 CUT OFF 1

995 FORMATI///1X. DEFINITION OF LINE OF SECTION IS IN ERROR - TEST TER IMINATED $\%$

C

\section{READ INPUT PARAMETERS}

READ $(5,1)$ TITLE, IX, IDISK

READ ( 5,3$)$ NT I M, NPRI INT, NF PLOT, NT PLOT

IF (NPRINT.EQ. O.OR. NFPLOT.EQ.0.OR.NTPLOT . EQ.0)GO TO 978

KEAD $(5,3)$ NCDLS, NROHS, MNCOLS, IFCOD6, FMT 4

READ $(5,6) Z$ TOT, YTOT, BANK, WS , DWS, $25 E C T$

IFIWS.GT.YTOTIGO TO 976

READ $(5,6) S N, W V L, V S, X M A X$

READ $(5,6) C 1, C 2, E 1, G R A Y L M$

READ $(5,6)$ EC 1, EC 2, ENL ,EN2, GAPLIM, SNLIM

IF (SN.GT. SNLIMIGO TO 982

READ $(5,7) W, H, E X N, C 5, F 1, F 2, S I G M A, R D, I F C O D 7$

IF (H.GT.WS)GO TO 980

READ (5, 5$)$ GRAVEL, SAND, SI LT, CLAY, UPPB, LPPB, ANT I DN, R IPPLE , DUNES, OLD SE 1D. WATER, DOT, DLANK, FLOOD

READ $(5,2)$ IFCOD 3 , QVOLMX, SKEH

READ $(5,6) D M, D S, Y M, Y S, A 1, A 2, A, B, S A, S B$ 


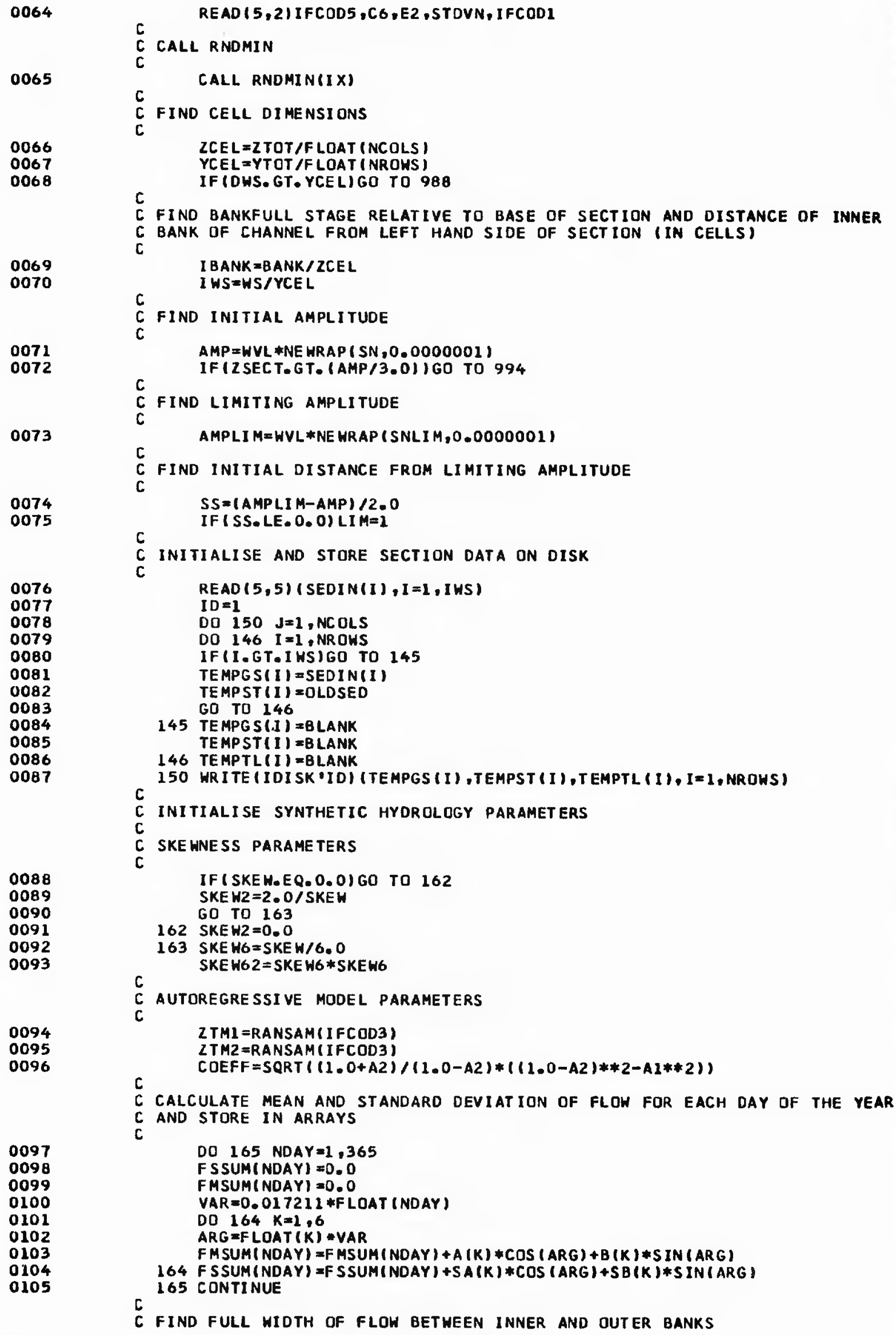

ZCEL =ZTOT/F LOAT (NCOLS) YCEL $=$ YTOT /F LOAT (NRONS)

$c$

C Find BanKFull STAGE RELATIVE TO BASE OF SECTION AND DISTANCE OF INMER C BANK DF CHANNEL FROM LEFT HAND SIDE OF SECTION (IN CELLS) 


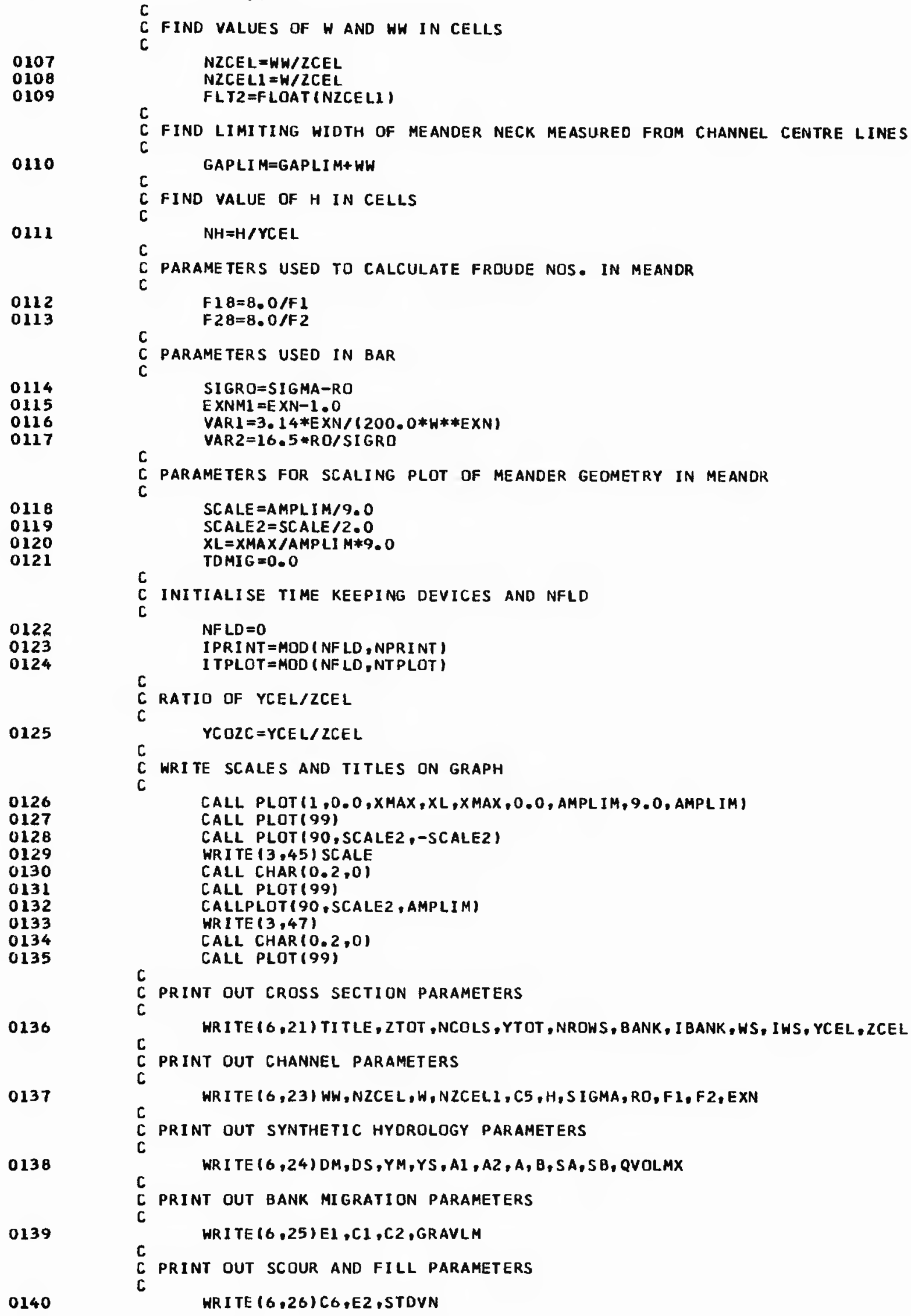


$\stackrel{C}{c}$ PRINT DUT CUT OFF CONTROL PARAMETERS

0141

HR I TE $(6,28)$ GAPLI M,EN1, EN2, SNL IM, AMPLIM, ECI, EC2 C PRINT OUT TYPE OF SECTION

0142

0143

0144

0145

0146

IFIIFC006.GT.01G0 TO 167

HR I TE $(6,33)$

GO TO 168

167 WR I TE $(6,31) 2$ SECT

C

PRINT LEGEND

168 WR I TE $(6,27)$ LPPB, GRAVEL, OLDSED, R IPPLE, SAND, HATER, DUNES, SILT, DOT,DOT $1, D O T$,DOT, DOT, UP PB, CLAY, ANT I DN, FLOOD

C FIND AND PLOT INITIAL PLANImEtRIC FDRM OF MEANDER

0147

0148

0149

0.150

0151

0152

0153

0154

0155

0156

0157

CALL, ME ANDR

C INITIAL OPERATIONS CONCERNING CROSS SECTION DEFINITION - THEN BRANCH

C TO INITIALISE AND PRINT CHANNEL SECTION

0158

0159

0160

0161

0162

0163

0164

0165

0166

0167

0168

0169

0170

0171

0172

0173

0174

0175

0176

0177

0178

0179

0180

0181

0182

0183

0184

0185

0186

\footnotetext{
IFIIFCOD6-1)170,172,174
}

170 NZCELO=NZCEL

ZCELI $=2 C E L$

IFILIM.EQ.2)GO TO 972

GO TO 218

172 IFILIM.NE. I) IGO TO 175

PAR $1=3.14159 *(A M P-2.0 * 2 S E C T) /(S N * W V L)$

PHI $=(0.0505 * S N+P A R I+0.0692) / 0.6371$

PAR2 $=\{(-0.0292 * S N+0.2132) * S N-0.4651\} * S N-P A R 1+0.2668$

$130 \mathrm{FA}=((\mathrm{PHI} * 0.2804+(0.2244-0.1713 * S N)) * P H I+((0.1139 * S N-0.552) * S N+0.88$ $1951) * P H I+P A R 2$

$F B=(P H I * 0.8412+(0.4488-0.3426 * S N)) * P H I+(0.1139 * S N-0.552) * S N+0.8695$ PHIN=PHI -FA/FB

IF (ABS (PHIN-PHI) -0.0001$) 140,140,135$

$135 \mathrm{PHI}=\mathrm{PHIN}$

GO TO 130

140 SINPHI $=$ SIN $(P H I N)$

GO TO 176

$c$

C STRAIGHT LINE DISTANCE BETHEEN POINTS OF INFLECTION OF LOOP

174 NDAVA $=$ WVL $/ 12.0 * Z C E L)$

175 SINPHI $=\operatorname{SIN}(2.2 * \operatorname{SQRT}((S N-1.0) / S N))$

176 ZCELI $=$ SINPHI $*$ ZCEL

NZCEL $=$ WH $/ Z C E L I$

NZCELI $=W / Z C E L 1$

GO TO 216

C

C BEGIN MAJOR LOOP, ONCE THROUGH EVERY YEAR

c

C INITIALISE TIME KEEPING DEVICES

169 IPRINT $=$ MOD (NF LD, NPR INT)

I TPLOT $=$ MOD ( NF LD,NTPLOT )

C FINN FLOOD PERIOD VOLUME

QVOL $=0.0$

DO 180 NDAY $=1,365$

$2 T=A 1 * 2 T M 2+A 2 * Z T M 2+C O E F F * R A N S A M(I F C O D 3)$

$X T=D M+F M S U M(N D A Y)+(D S+F S S U M(N D A Y)) *(Y M+Y S * Z T$

IF $(X T \cdot G T \cdot D M) Q V D L=Q V O L+X T$

ZTM2 $=2$ TM1

$180 \quad 2 T M I=2 T$

C

C TEST FOR CUT OFF

$P C=(Q V D L / Q V O L M X) * E C) *(S N / S N L I M) * E C 2$

$P N=(Q V O L / Q V O L M X) * * E N 1 *(G A P L I M / G A P) * * E N 2$

$X=R N D M(-1)$

IF $\left(X_{0}\right.$ LE $P$ PC $) \backslash C U T=2$

$X=R N D A(-1)$

IF ( X. LE.PN $)$ ICUT $=3$

IFIICUT.EQ.1)GO TO 182 
C

C CUT-OFF HAS OCCURRED - OUTPUT REQUIRED INFORMATION AND TERMINATE PROG.

HRI TE $(6,29)$ TI TLE, NF LD, QVOL, OBGS I , BGS I, SCHUMM, SS, RLM I G, TLMIG , RDMIG, 1 TOMIG

0188

0189

0190

0191

0192

0193

0194

0195

0196

0197

0198

WR I TE $(6,30)$ AGG

I PRINT $=0$

NFPLOT $=$ NF LO

CALL MEANDR

GO TO 548

$\mathrm{C}$

C FIND AMOUNT OF LATERAL AND DOHNSTREAM BANK MIGRATION

182 IF(LIM.EQ.1)GO TO 185

$R L M I G=S S * C 1 * 0$ VOL/OBGSI $* * E I$

$T L M I G=T L M I G+R L M I G$

185 RDMIG $=C 2 * 0$ VOL/OBGSI *EI

TDMI G $=$ TDMI G + RDMI G

C

C PRINT OUT REQUIRED DATA FOR THIS TIME INCREMENT

c c

0199

0200

0201

0202

0203

0204

0205

0206

0207

0208

0209

0210

0211

0212

0213

0214

0215

0216

0217

0218

0219

0220

0221

0222

0223

0224

0225

0226

0227

0228

0229

0230

0231

0232

IF (I PR I NT. EQ. O) WRI TE $(6,29)$ I IT LE, NFLD, QVOL, OBGS I, BGS I, SCHUMM, SS, RLM IIG,TLMIG,ROMIG,TDMIG

C AGgrade the FloodPlain if REQUiRED

$A G G=A G G+D H S$

C

WRITE TOTAL AMOUNT OF AGGRADATION SO FAR

IF ( I PR I NT. EQ. O) WR I TE $(6,30)$ AGG

NAGG $=A G G / Y C E L$

IF(NAGG. LT. MARK)GO TO 210

C

6 IF ROW OF CELLS IS FILLED, ADJUST BANKFULL STAGE AND FILL ROW WITH

C ALPHAMERIC CHARACTERS

C

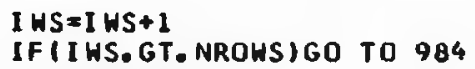

1 HS $\approx I H S+1$

IF I I HS. GT. NROHSIGO TO 984

MARK $=$ MARK+1

$I D=1$

DO $200 \mathrm{~J}=1, \mathrm{NCOLS}$

READ (IDISK'ID) (TEMPGS (I),TEMPST (I),TEMPTL (I),I=1,NROWS)

$I D=J$

TEMPGS (I HS) $=F$ LOOD

TEMPST (IWS) $=F$ LOOD

200 WRITE (IDISK IID) (TEMPGS (I), TEMPST I I),TEMPTL (I), I= I, NROWS )

C RECORD ON 2-D ARRAYS THE RESULTING EROSION AND DEPOSITION AFTER THIS

C YEAR - STORE ARRAYS ON OISK

C FIND AMOUNT OF BANK MIGRATION IN CROSS SECTION REPRESENTED.ADJUST

C CHANNEL WIDTH(IN CELLS) REPRESENTED IN CROSS SECTIONIAND RELATED

C PARAMETERSI, DEPENDING DN TYPE OF CROSS SECTION AND CHANGES IN SHAPE

C OF MEANDER

210 IF(IFCOD6.GT. 0)GO TO 215

RMIG $=S Q R T$ (RLMIG*RLMIG+RDMIG*RDMIG)

$A A=A T A N$ (RDMIG/RLMIG)

TANA =RDMI G/RLMIG

$P=A T A N(I R+W W / 2.0-S Q R T((R+W W / 2.0) * 2+2.0 * R * W W * T A N A=T A N A)) /(-2.0 * R * T$ LANAI)

$Z C E L I=Z C E L * C O S(A A-P) / \operatorname{COS}(P)$

60 TO 216

215 RMIG =RDMI G

SINPHI $=$ SIN $(2.2 * S Q R T((S N-1.0) / S N))$

ZCELI $=Z C E L * S I N P H I$

216 NZCELO $=$ NZCEL

NZCLIO $=$ NZCELI

NZCEL $=W H / Z C E L 1$

N2CELL $=W / 2 C E L 1$

FLT2 = FLOAT (NZCELI)

$Y Z O Z C=Y C E L / Z C E L I$

IF (NZCEL1-NZCLI O) 218,218,217

$217 N 2 D I F=N Z C E L 1-N Z C L I O$

GO TO 225

218 NZOIF $=0$

c 
C

C FIND BANK MIGRATION(IN CELLS) IN CROSS SECTION, AND CALCULATE ERROR

C DUE TO SMOOTHING, OEV 
C INITIALISE PARAMETERS AND WRITE HEADINGS

$400 Z=Z C E L I$

OBGS $=0.0$

$B G S=0.0$

GRAV $=0.0$

NRMI GI = NRMI G+NZDIF +1

I NDE XK $=$ I HS

KOUNT $=0$

I NDE $X O=$ I $W S-\mathrm{NH}+1$

0281

0283

0284

0285

0286

0288

0289

0290

0291

0292

IF I I PRI NT. EQ. 0$)$ HRI TE $(6,42)$ TITLE, NFLD

$c$

C BEGIN MAJOR LOOP ENTERED ONCE FOR EVERY COLUMN OF CHANNEL SECTION

DO $450 \quad J=1, N Z C E L$

$N C O L=J+N R M I G$

IF (J.LE. N2CEL1)GO TO 410

C

C ERODE OUTER BANK

0293

0294

0295

0296

0297

0298

0299

0300

0301

0302

0303

0304

0305

0306

0307

0308

0309

0310

0311

0312

0313

0314

0315

0316

0317

0318

0319

0320

0321

0322

0323

0324

0325

0326

$Y=-H / 2.0 *(C O S(3.24 *(H W-Z) /(H H-W))-1.0)$

NYCEL $=Y / Y C E L$

I NDE X $=I$ I S-NYCEL

IF (I TPLOT.EQ. O) TLPLOT I INDEX, NCOL -1$)=D O T$

I NDE XK = I NDEX

405 IF ( SEDG S I I NDEX, NCOL) .EQ.CLAY. OR.SEDGS (INDEX, NCOL ) .EQ.5ILT . OR . SEDGS

1 ( I NDEX, NC OL). EQ.FLOOD) OBGS =OBGS +1.0

IF ( SEDG S(I NDEX,NCOL).EQ.GRAVEL) GRAV = GRAV+1.0

KOUNT $=$ KOUNT +1

INDE $X=I$ NDE $X-1$

IF (INDEX.GT. I NDEXO)GO TO 405

INDEXD $=$ I NDEXK

GO TO 440

c

C DEPOSIT SEDIMENT ON INNER BANK

410 IF (IFCODT.EQ.0)GO TO 411

CALL BARI $(1, H)$

GO TO 412

411 CALL BAR (1, H)

412 IF (D. LE . 0.00625) BGS $=8 G 5+1.0$

IF I I TPLOT.EQ. O. AND. J.NE. NZCELI ITLPLOT (INDEX,NCOL) =DOT

IF (NFLD.EQ.0)GO TO 440

C 'FILL' POINT BAR

IF (NRMIGI.LT.1)GO TO 440

DO $415 J J J=1$, NRMIG

$J J=N C D L-J J J$

IFIJJ.LT.I)GO TO 415

IF INZCELI.LT. NZCLID. AND. IFCODS.EQ.1) GO TO 413

IF ( SEDSTR (I NDEX, JJ). NE. WATER. AND.SEDSTR (INDEX, JJ) . NE . FLODD, AND . SED ISTR (INDEX,JJ).NE. OLDSED)GO TO 415

413 SEDG S (INDEX,JJ) =SEDGS (INDEX, JJ+1)

SEDSTR $($ INDEX, $J J)=S E D S T R(I N D E X, j J+1)$

C

415 CDNTINUE

C FILL IN "EMPTY" ROWS

420 IF ( ( I NDEXK-1 NDEX). LT. 2 ) GO TO 424

I NDE XK $=1$ NDE XK-1

DO $422 \mathrm{~J} J=1, N R M I G 1$

$N C O L K=N C O L-J J$

IF (NC OLK. LT.1)GO TO 422

IFINZCELI. LT. NZCLIO. AND. IFCODS.EQ.1)GO TO 421

IF ( SED STR (I NDEXK, NCOLK) . NE. WATER.AND.SEDSTR (INDEXK, NCOLK) , NE .OLDSE 10) 60 TO 422

421 SEDGS ( I NDEXK, NC QLK) =SEDGS (INDEX, NCOLK)

SEDSTR (INDEXK, NCOLK) $=S E D S T R$ (INDEX, NCOLK)

422 CONTINUE

GD TO 420

424 I NDEXK = I NDEX

$\checkmark$ FILL NEW CHANNEL WITH WATER

440 I NDEX $=I$ HS-NYCEL+1

IF (NYCEL.EQ.0)GO TO 450 


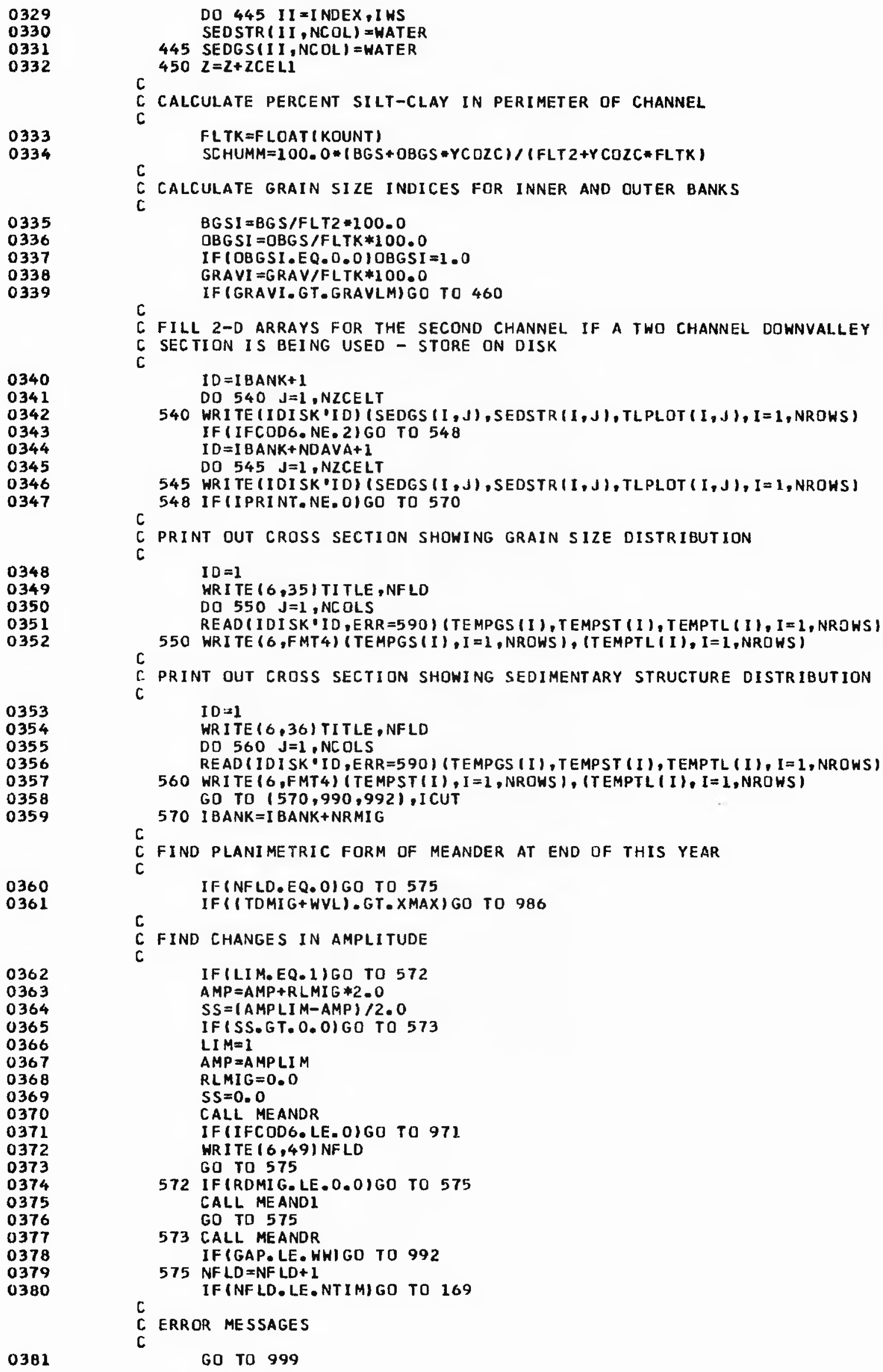



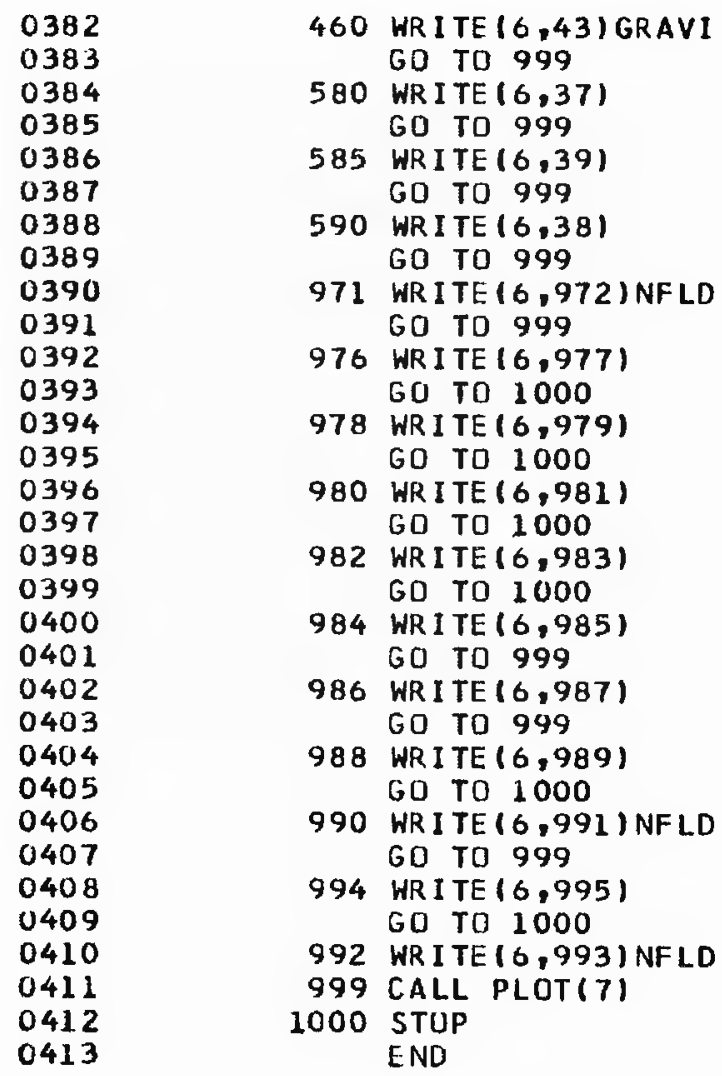
36. If IPRINT=O the contents of the disc data set corresponding to the cross sections are read and printed out as sections showing grain size and sedimentary structure distribution. 14.3 Subroutine BAR (with entry point BARl)

This subroutine calculates the grain size and bed form present at a particular station on the point bar using the somewhat modifled version of Allen's (1970a,b) model.

1. Calculates the argmment, ARG, required in the SIN and Cos functions of the next two operations.

2. Calculates depth of water, Y, at a station (column NCOL), using equation $(5 \cdot 1)$.

3. Calculates local transverse slope of point bar, DYDZ, using equation $(5.18)$.

If a user specified transverse profile is required, the above operations are skipped by using entry point BARI. Arithmetic assifnment FORTRAN statements defining $Y$ and DYDZ must be added immediately below the ENTRY BARI statement (see listing). The value of DYDZ must be scaled such that the units of $D$ are cm. In this respect it should bo noted that units of length are everywhere metres, except where otherwise specified, and VAR2S is dimensionless.

4. Finds row, INDEX, in the two-dimensional arrays corresponding to calculated depth.

5. Finds local radius of curvature, RI,.

6. Calculates grain size, D, at the station, using equation (5.20). Units are in $\mathrm{cm}$.

7. Allocate grain size to Wentworth scale division, clay, silt, sand or gravel, and fill appropriate element of SEDGS (INDEX, NCOL) with corresponding alphameric character, Changing the program to accommodate furthex subdivision of grain size classes would be a simple task if required. 


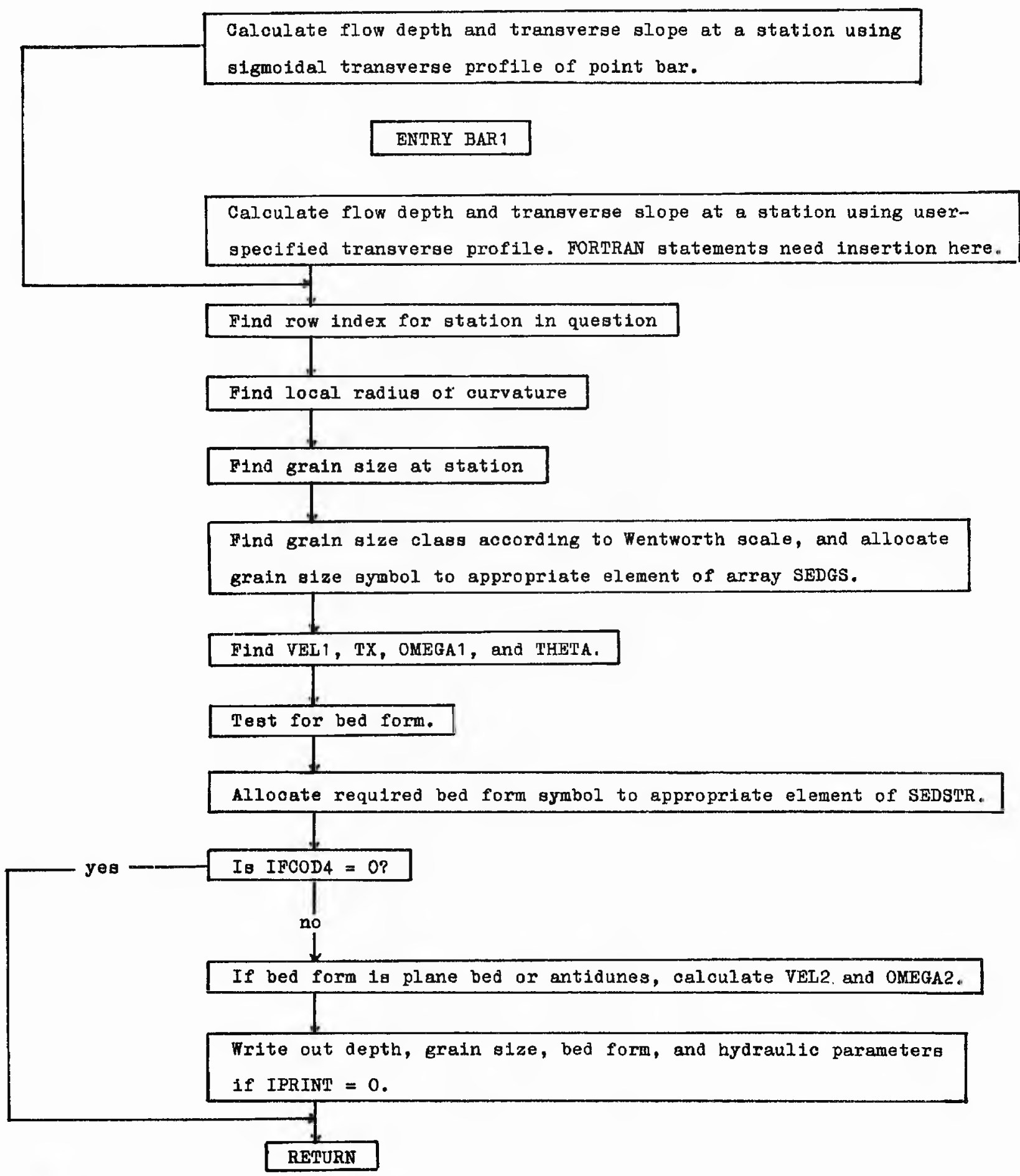

Fig. 14.4. Flow diagram for subroutine BAR. 
8. Calculates local. depth of water expressed in cm., XCM.

9. Calculates product of YCA and acceleration due to gravity, $G$, where the unjts of $G$ are $\mathrm{cm} . / \mathrm{sec} / \mathrm{sec}$.

10. Calculates local mean flow velocity, vell, using equation and using the rriction coefficient for dunes and ripples, fl Units are $\mathrm{cm} / \mathrm{sec}$.

11. Calculates local bed shear stress parallel to the channel centre line, TX. Units are dynes/sq.cm.

12. Calculates local stream power, OMEGA1, as the product of TX and VEIl. Units are ergs/sq.cm./sec.

13. Calculates local dimensionless shear stress, THETA, using equation $(5.23)$.

14. Tests to see if bed form is antidunes, upper phase plane bed, dunes, ripples, or lower phase plane beds by applying a series of inequalities, as in $\Lambda 11$ en's (1970a) model. The appropriate element of SEDSTR (INDEX, NCOL) is filled with an alphameric character corresponding to the bed form chosen .

15. If IPRINT=0 writes out grain size, bed form and other hydraulic variables, calculating VEL2 and OMEGA2 where necessary. VEL2 and ONEGA2 axe the local mean flow velocity and stream power respectively, calculated using the friction coefficient for plane beds and antidunes, $\mathrm{F} 2$. If IFCOD4=0 step 15 is skipped, as BAR is being called during a scour and flll operation.

A simplified flow diagram for subroutine BAR is given in fig. 14.4

1.4.4 Subroutine MEANDR (with entry point MEANDI)

This subroutine calculates the planimetric geometry of the meander for every time increment and plots a trace of the channel centre line whenever required. If entry point MEAND1 is used, the planimetric geometry is not calculated. 


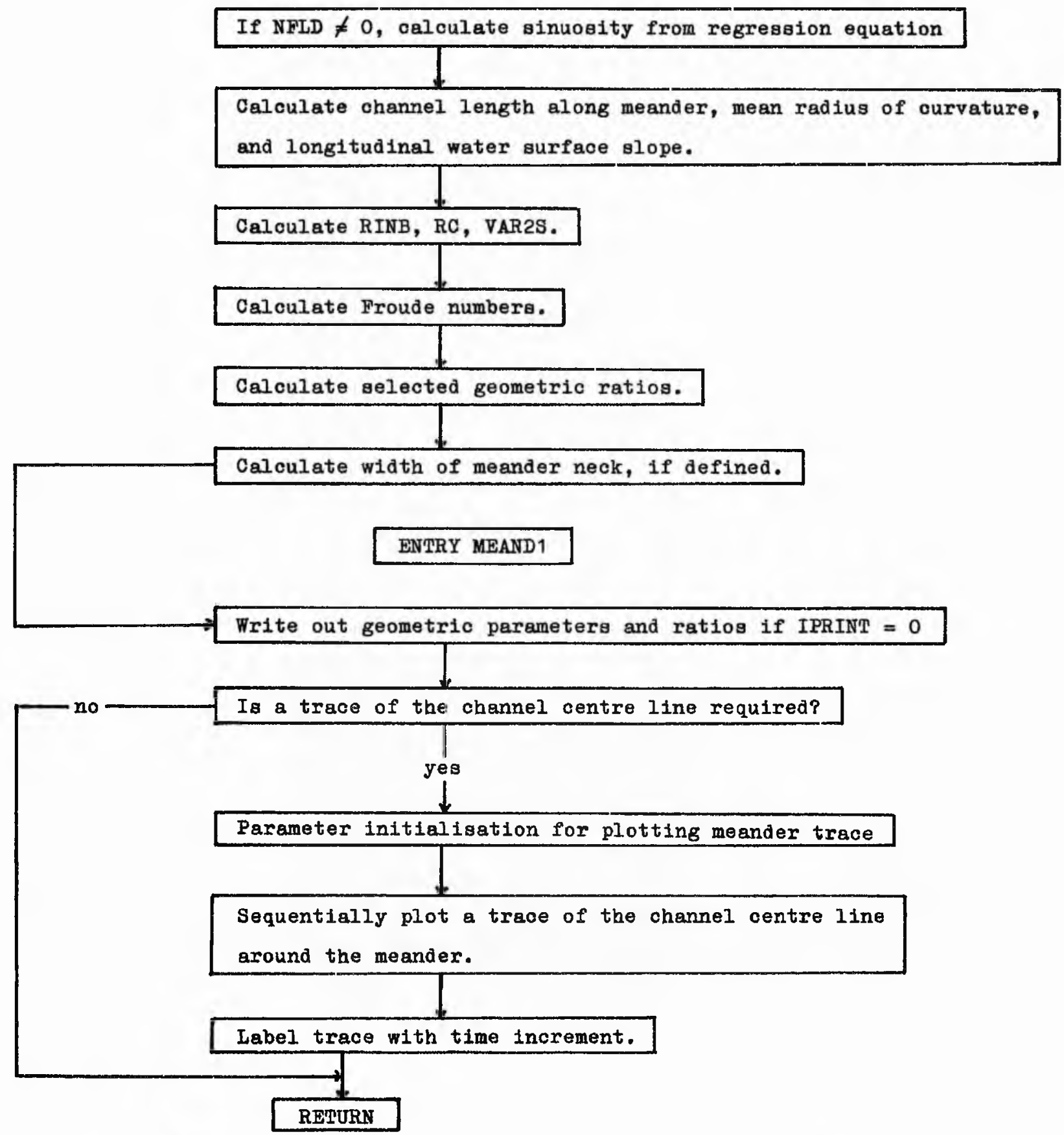

Figr. 14.5. Flow diagram for subroutine MEANDR. 
1. Struosity, SN, is calculated, given amplitude, AMP, and wavelength, IVL, using the regression equation (2.12). If $N F L D=0$ this step is omitted as $S N$ is road initially as input data.

2. Channel length along the meander centre line, CHL, is calculated as the product of WVL and SN.

3. Longitudinal water surface slope, CHS, is calculated as valley slope, VS, divided by SN.

4. Radius of curvature of the channel centre line at the bend axis, $R$, is calculated from equation (2.5).

5. Radius of curvature of the inner bank at the bend axis, RINB, is calculated for use in subroutine BAR.

6. Calculates product of fluid density, Ro, and cHs for use in subroutine BAR. Units in gm./cu.cm.

7. Calculates product, VAR2S, of VAR2 and CHS for use in BAR. Dimensionless variables.

8. Calculates Froude numbers, FROUDl and FROUD2 (calculated using friction coefficients Fl and F2, respectively), for use in BAR.

9. Cajculates selected geometric ratios.

10. Calculates the maximum angle (radians) that the path of the chamnel centre line makes with the mean downvalley direction, OMFGA, using equation $(2.4)$. If ONEGA is less than $\pi / 2$, the width of the meander neck, GAP, is set to a very large number as an indication of being underined, and the next step is omitter.

11. Calculaties width of meander neck measured to channel centro lines, CAP, aftex solving the necessary integral by calling subroutine SIMTNT (and FUNC2).

The preceding operations are skipped, by using entry point MEAND1, if amplitucle growth of the meander has ceased, the variables above xemaining unchanged. The following operation 
is skipped if no printed output is required.

12. If IPRINT=0 write out planimetric geometry parameters and selected geometric ratios.

The following; operations axe skipped if no trace of the channej centre line is to be plotted.

13. Divides CHL into 100 incremental lengths and, by calculating the deviation angle, PHI, from the downvalley direction (using equation (2.3)) for each incremental length, sequentially plots the meander trace. The sines and cosines of the deviation angles for the first half of the bend ( 50 of each) are stored in COsPHI (50) and SINPHI (50) and then used for plotting the second half. Subroutine plot is used during this step.

14. Labels the trace with the appropriate time increment. Subroutines PLOT and CHAR are called here.

All units of length are metres unless otherwise specified A flow diagram of subroutine MEANDR is shown in fig. 14.5 .

14.5 Subroutine SIMINT (and FUNC2)

This subroutine is called by MEANDR and evaluates integrals numericaliy using simpson's rule. The interval is successive 1y halved until estimates of the integral do not differ by more than the specified limit, E. This is set to 0.005 . The function to be integrated, given in equation (2.11) is specified in a FUNCTION subroutine FUNC2, which is called by SIMINT. See appendix 1 for description of simpson's rule.

\subsection{Subroutine RANSAM}

This FUNCTION subroutine returns random samples from specified frequency distributions. It is called by the main program, and is used when finding the flood period volume, also In the scour and fill relation, equation (9.4). A flow diagram tis shown in $f i g .14 .6$.

1. If IfCoI3 equals 3,4 or 5 , twelve pseudorandom numbers are 


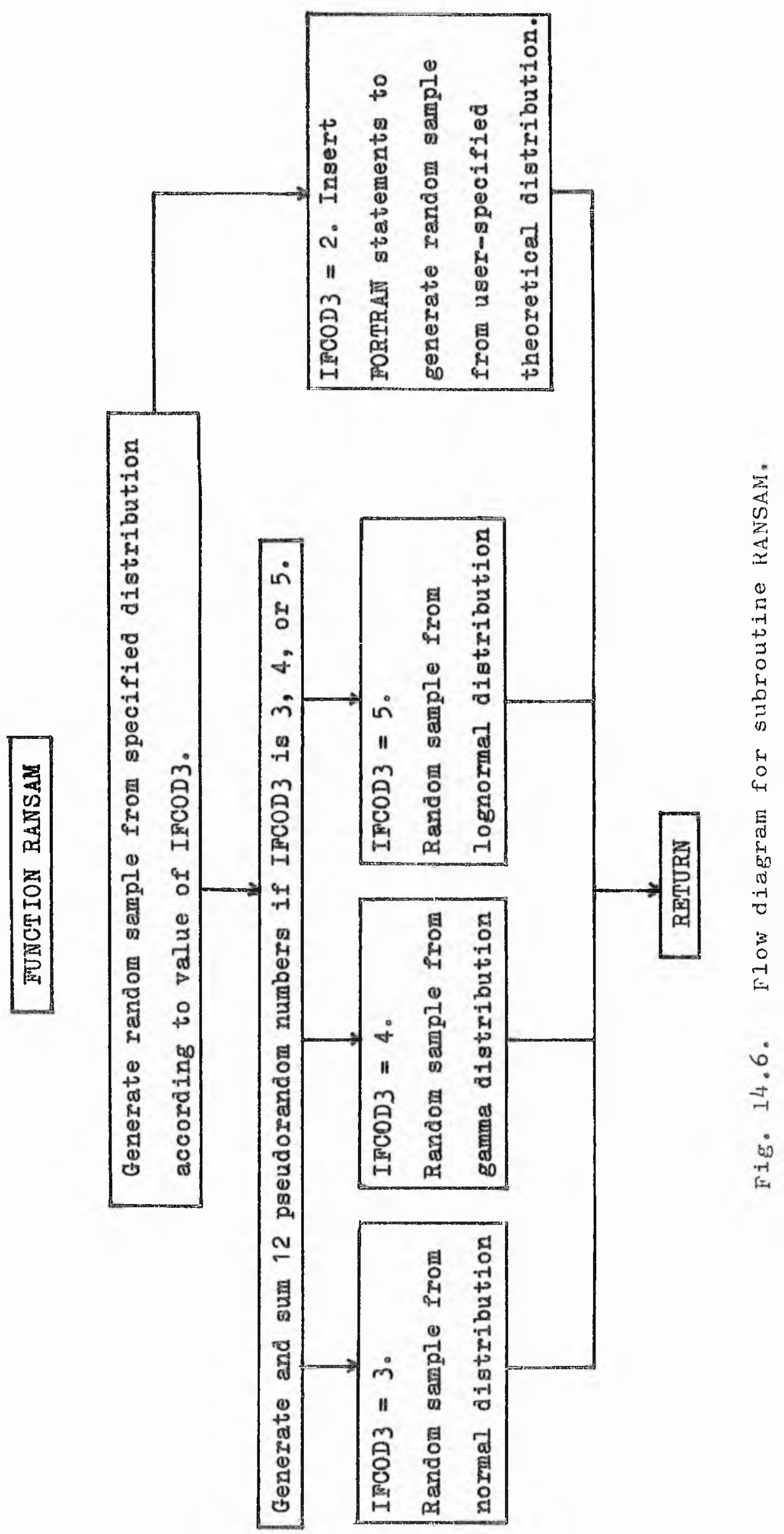


Generated, using subroutine RNDM, and sunmed.

2. This sum is then converted to a nomally distributed random variable, using equation (Al.2). If $\operatorname{IFCOD3=3}$ this variable is returned, but may be further converted to a gamma distributed (sec equation $(\Lambda 1.7)$ ) or a lognormally distributed (see equation ( 11.6$)$ ) random variable, if IFCOD3 equals 4 or 5 , respectively. These random samples are distributed with zero mean and standard deviation unity.

If a random sample from a user specified distribution is required, FORTRAN statements must be added imnediately arter the appropxiate comment card (see listing). This is before step 1. Additional data will be required if more than tho fixst three moments or any other parameters are required to describe the distribution.

\subsubsection{Subroutine NETRAP}

This REAL FUNCTION subroutine is called by the main program during initialisation, and evaluates a root of the polynomial, given in equation ( 2.12$)$, by the Newton-Raphson iteration procedure (see appendix 1). It is required to find a particular value of AMP/WVL, which satisfies the equation. Whence AMP is round given the initial value of SN and WVL as input.

The initial estimate of the root required is given by the Inear approximation of the third degree polynomial, given in appendix 2. The estimate is sufficiently close to the required root, compared"with other roots, to avoid any complications in the iteration procedure. The iteration continues until successive estimates differ by less than or equal to the specifjed amount, If. This is set to 0.0000001 . Only a few iterations are requixed as the convergence js fairly rapid. 


\subsection{Subroutines RNDMIN and RNDM}

These subroutines are involved in the generation of pseudorandom numbers (i.se. practically uniformly distributed random numbers). The puxpose of RNDMTN is to read a starting variable, IX, an integer preferably 9 or less digits, on which the random floating point number generation will be based. RNDM does the actual random floating point number generation using the number declaxed above. It uses the same generating sequence as RANDU, in the JBM scientific subroutine package (1971, p.77), to produce numbers between 0.0 and 1.0 . RNDMIN and RNDM are automatically available on the IBM $360 / 44$ system 1ibraries.

\subsection{Subroutines PLOT and CHAR}

These are subroutines used for controlling the CIL graph plotter. PJoT is used for drawing and scaling of graph axes, and the actual plotting of the graph. CHAR is used for annotat.ion. PLOT and CHAR are specific to the installation, and are automatically available in the system libraries of st. Andrews University Computing Laboratory. Different installations will be expected to use diffexent graph plottor routines. 
15 INPUT REQUIREMENTS AND PROGRAM MODIFICATION INSTRUCTIONS ForThan 'froknt codes are given for each variable to be read. With the I code all numbers must be right justified in the specified fields. With the $F$ code, numbers can be anywhere in tho specified field as long as a decimal point is present. In this program $F$ codes have been used such that a decimal point is always required unless no places of dectmals are needed, in which case the numbers must be right justified. Input requirements are given first for the program which uses no disc, and subsequent1y, those data cards which are different when using a disc are described. 1. Title Card

Column

1-60 TITLE Alphameric title (15A4). Can be placed anywhere in this 60 column field.

61. 70 IX Starting number for pseudorandom number generator, preferably large. (I10).

2. Time and output control card

Column

1.4 NTIM Number of time increments ( 14 ).

6-8 NPRINT Line printer output is printed every NPRINT-th time increment (It).

9-12 NFPLOT Graph plottex output is every NFPLOT-th time increment (I4).

13-16 N'TPLOT A time line is plotted on the cross sections every NTPLOT-th time increment (x4).

The last three variables must not be set to zero. 
3. Cross section control card (see fig. .14.1)

Column

1-4 NCOLS Number of columns in a cross section (I4). If this if greater than 200 the dinensional information Given in the INTEGER*2 and the labelled COMMON/COM2 statiements must be changed.

5-8 NROWS Number of rows (I4). If this is greater than 60 the dimensional information given in the INTEGER*2 the labelled COMNON/COM2 statements must be changed. The maximum possible sizes of NCOLs and NROWS will depend on the addressable storage (core store) available at a particular installation. If a reduction in the anount of core store used in the listed program is required, the same adjustments as outlined above apply. The physical size of the line printer will also limit NRoWs (i.e. 132 in IBM 1403).

9-12 Ircod6 condition code to determine type of cross section represented $\left(I^{4}\right)$. That is, o - Lateral section

1 - One-channel downvalley section 2 - Twomchannel downvalley section.

1.3-20 ZTOT Wiath of section, in metres (F8.0)

21-38 YTOT Thicliness of section, in metres $(F 8,0)$.

29-36 BANK Initial distance of inner channel bank rrom left hand side of section, in metres (F8.0).

37-44 WS Initial bankfult stage measured from section base, in metrés $(F 8.0)$. Must not exceed XTOT.

45-52 DWS Rate of aggradation, in metres per year $(F 8,0)$. Value must not be greatex than the cell depth, given by YTOT/NROWS.

53-60 ZSECT Normal distance of line of section from line joining points of inflection of loop (F8.0). Applies only to one-channel downvaley section, thererore if 
Ircov $6 \neq 1$, leave blank. Must not exceed $\Lambda \mathrm{MP} / 3.0$.

61-80 FMT't object time Format code used for printing out cross sections $(5 \mathrm{~A} 4)$. Must be of the rorm $(1 \mathrm{x}, \mathrm{nA} 1)$ where $n$ equals NROWS

4. Meander Geometry card

Column

1-12 SN Initial sinuosity (F12.0). Must not exceed Iimiting sinuosity, SNLIM, which is read from card 6 . 13-24 WVL Meander wavelength, in metres (F12.0). 25-36 VS Valley slope (F12.0) 37-48 XNAX Maximuin length in the $X$ (downalley) direction required for plotting the plan form of the meander as it migrates downvaliey, in metres (F12.0). XMAX must not be less than WVL+total amount of downvalley migration.

\section{Bank mifration card}

Column

1-12 C1 Constant $\mathrm{k}_{2}$ in bank misration relation, equation $(6.3) \cdot(F 12,0)$

13-24 C2 Constant $k_{3}$ in bank migration relation, equation $(6.4) \cdot(612.0)$

25-36 E1 Exponent $\mathrm{n}_{2}$ in bank inigration relations above. $(F 12.0)$

37-48 GRAVLM Limiting percentage of grave1 (grain diameter 2mm. + ) allowable in outer bank $(\mathrm{P} 12.0)$. Measured as amount of areal exposure.

6. Cutwore control caxd

Column

1-12 EC1 Exponent ec 1 in chute cutmoff relation, equation $(10.1)(\mathrm{F} 12.0)$

13-24 EC2 Exponent $e c_{2}$ in chute cut-off rejation (Fi2.0). 
25-36 ENl Exponert en ${ }_{1}$ in neck cut-off relation, equation $(10.2)(\mathrm{F} 12.0)$

37 ar 48 EN2 Exponent; en 2 in neck cut-off relation (F12.0).

49-60 GAPLIM Limiting width of meander neck, in metres, measured as the shortest distance between the adjacent banks of each meander $1 \mathrm{imb}($ F.2.0).

61-72 SNLIM Limiting sinuosity (F12.0).

\section{Channe1 parameter card}

Column

$1-8 \quad W$

$9-16 \quad H$

$17-24 \quad \mathrm{EXN}$

$25-32 \quad C 5$

$33-40 \quad F 1$

$41-48 \quad+2$

49-56 SIGMA (F8.0).

57.64 RO

Fluid density, in gm/cu.cm. (F8.0).

$65-70 \quad \operatorname{IFCOD} 7$ in metres $(F 8.0)$. $(\mathrm{F} 8.0)$ equation $(5.2))($ F8.0). ripples, $f_{1}(F 8.0)$. and antidunes, $f_{2}(\$ 8.0)$.
Width of flow between inner bank and talweg, $w_{1}$,

Maximum unscoured depth of flow measured above talweg, $h$, in metres (F8.0). Must be less than or equal to VS, as specified in card 3 . Exponent $\mathrm{n}_{1}$ in transverse profile equation (5.1)

Ratio of $w_{1}$ to full width, w (constant $k_{1}$ in

Darcy-Weisbach friction coefficient for dunes and

Darcy-Weisbach friction coefficient for plane beds

Density of sedimentary particles, in $\mathrm{gm} / \mathrm{cu} . \mathrm{cm}$.

Condition code to determine type of transverse profile of point bar (I4). That is, o - sigmoidal profile as given by equation (5.1). $l$ - user specified profile. Extra parametexs may be required to describe this. 
8. Symbols caxd

Colum

1 GRAVEL Alphaneric character used in line printer output to represent grave1 (A1).

2 SAND Character for sand (A1).

3 SILT Character for silt (A1).

4 Clay character for clay (AI).

5 UPPB Charactex for upper phase plane bed (Al).

6 LPPB Character for lower phase plane bed (A1).

7 ANTIDN Character for antidunes (A1).

8 RIPPLE Charactex for ripples (A1).

9 DUNES Character for dunes (Al).

10 OLDSED Character for old sediment (A1).

11 WATER Character for water (A1).

12 DOT Character used for plotting time lines (A1). Should ideally be a full stop.

13 BLANK Blank space.

14 FLOOD Charactex used for overbank sediments, produced with aggradation (A.1)

9. Synthetic liydrology cards - any units allowable as long as they are consistent

Card 9a

Column

1 Ircon3 Condition code used to determine the frequency distribution of the independent residual series, as given in equation $(11.7)$ (I1). That is, 2 - User specified theoretical

3 - Norma1

4 - Gamma

5 - Lognoxmal.

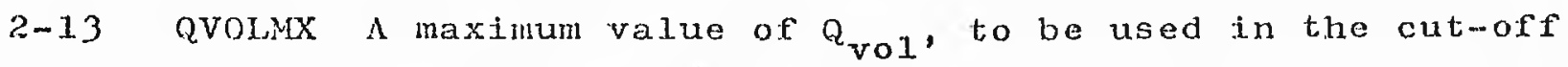
$\operatorname{tasts}(F .2,0)$ 
14-25 SKET

Skewress of eamma distribution (or user specified distribution) if condition code in column 1 is appropriate. Otherwise leave blank (F12.0).

Card $9 b$

Colunn

$1-12 \quad D M$

Mean of all daily flow values $(F .2 .0)$.

$13-2^{4}$ DS

$25-36 \quad \mathrm{YM}$

$37-48 \quad Y S$

$49-60 \quad$ A 1

$61-72 \quad$ A2

Standaxd deviation of all daily flow values (F12.0) Mean of $Y_{t} \operatorname{series}(F 12.0)$.

Standard doviation of $Y_{t}$ series (F12.0).

Coefficient $a_{1}$ in autoregressive model (FI2.0).

Coefficient $a_{2}$ in autoregressive model (Fl2.0).

Card 9c

Column

$1-72 \quad A(6)$

Array of Fourier coefficients, $A_{k}$, for the cosine texms of the hamonic representation of the daily means. Six coefficients corresponding to the first through to the sixth hamonic $(k=1,6)$, each having $(12.0)$

Card $9 \mathrm{~d}$

Column

$1-72 \quad B(6)$

Array of Fouriex coefficients, $B_{k}$, for the sine terms of the harmonic xepresentation of the daily means. Six coefficients corresponding to the first through to the sixth harmonic (6F12.0).

Card $9 e$

Co1umn

$1-72 \quad \mathrm{SA}(6)$

Array of Fourier coefficients ${ }_{s} \Lambda_{k}$ for the cosine terms of the liarmonic representation of the dajly standard deviations. Six coefficients corresponding to the fixst through to the sixth harmonic (6F12.0);

Card $9 f$

Column 
1-72 $\mathrm{SB}(6)$ Array of Fourier coefficients, $\mathrm{s}^{\mathrm{B}_{\mathrm{k}}}$, for the sine texms of the harmonic representation of the daily standard deviations. Six coefficients corresponding to the first through to the sixth harmonic (6F12.0).

\section{Scour and fill card}

Column

1

IFCOD5 Condition code to deternine whether scour and fill process is required. If 1 , the process is required, If 0 , the process is not required and the whole caxd may be left blank.

2-13 c6 Constant $k_{4}$ in scour and fill rolatión, equation $(9.4)(F 12.0)$.

14-25 E2 Exponent $n_{3}$ in scour sand fill relation (F12.0).

26-37 STDVN Standard deviation of error term in scour and fill relation $(\mathrm{F} 12.0)$.

38 IFCODl Condition code to determine rrequency distribution of error term in scour and fill relation (II). The codes available are as for TFCoD3 on card 9 .

\section{Flood plain sediments card(s)}

Column

1-IVS SEDIN(60) Array containing alphameric characters to specify the initial grain size distribution in the cross section. One symbol for each row acoording to the symbols read from card 8 , each in (A1) format There will be IWS symbols, where IWS is the integer part of WS $x$ NROrS/YTOF. The symbol in column 1 is allocated to the base of the section, and then successively up the section until the last character is read into row IWS. If IWS is greater than 60 the dimensional information given in the INPEGER*2 statement must be chaniped. If IWS is gxeater than So the data nust be continued onto anothex card using the same format. 
If a disc is being used a slightly different data input is required. The title card (1) and the cross section control card (3) must be replaced, the latter with two cards (3a and $3 b$ ). These replacement cards are as follows:

1. Title card

Column

1-60 TITLE Alphameric title (15A4). Can be placed anywhere in this 60 column field.

61-70 IX Starting number for pseudorandom number generator, preferably large (IIO).

71-72 IDISK Data set reference number for disc data set (I2). The number will depend on the programming system, and whether a system or private disc is being used. If not equal to 4 , the DEFINE FIIE statement in the main program must be changed.

3. Cross section control cards

Card 3a

Column

1-4 NCOLS Number of columns (I4). If this is greater than 200 the number of records in the DEFINE FILE statement in the main profram must be changed. NCOLS records must be stored on the disc data set.

5-8 NROWS Number of rows (I4). If this is greater than 60 the dimensional information given in the INTEGER*2 and the labelled COMMON/COM2 statements must be changed. This will also entail changing the record size in the DEFINE FILE statement. The size of one record is NROWS $x 6$ bytes.

9-12 MNCOLS Maximum number of columns that can be held in the core store at any one time (I4). If more than 50 dimensional information given in the INTEGER*2 and the labelled COMMON/COM2 statement must be changed. 
The actual maximum number of columns required in the program will depend on the cell width, given by ZTOT/NCOLS, the channel width and the maximum rate of bank migration in the cross section represented (see fig. 14.1).

The maximum possible sizes of NROWS and MNCOLS will depend on the addressable (core) storage at a particular installation.

a reduction in the anount of core store in the listed program is required, the same adjustments as outlined above apply. NROWS will also be limited by the physical sizo of the line printer. 13-15 IFCOD6 Condition code to determine type of cross section represented (I4). That $1 \mathrm{~s}$, o - Lateral section

1 - One channel downvalley section

2 - Two channel downvalley section.

17-37 FMT4 object time FORMAT code used for printing out cross sections $(5 \mathrm{~A} 4)$. Must be of the form ( $1 \mathrm{X}$, nAl) where $n$ equals NROwS.

\section{Card 3b}

Column

1-12 ZTOT Width of section, in metres (F12.0).

13-24 YTOT Thickness of section, in metres (F12.0).

25-36 BANK Initial distance of Inner channel bank from left hand side of section, in metres (F12.0).

$37-48$ WS Initial bankfull stage measured from section base, in metres, $(\mathrm{F} 12.0)$. Must not exceed YTOT. 49-60 DWS Rate of aggxadation, in metres per year (F12.0). Value must not be greater than the cell depth given by YTOT/NROWS.

61-72 zSECT Normal distance of line of section from line joining points of inflection of loop (F12.0). 
Applies only to one-channel downvalley section, therefore if IFCOD $6 \neq 1$, leave blank. Must not exceed $\operatorname{AMP} / 3.0$ 


\section{SAMPLE RUN}

Table 16.1 contains the data input for an illustrative sample run of the program. Output is the same with or without the use of a disc. Tables 16.2 to 16.5 and figs. 16.1 to 16.3 illustrate the output from this run as follows.

Table 16.2 shows data which is output once for each separate run of the program, and this precedes al1 subsequent output for the run. The information given is partly that supplied as input and part1y computed during initialisation. The notation used is as used in the development of the mathematical model, part 2. The cross section parameters listed are shown in fig. 14.1, and some of the channel parameters listed are shown in fig. 5.2. As already stated the synthetic hydrology parameters require no units to be specified. The data used in this case are taken from the data of Quimpo (1967) for the oconto River as listed in table 11.1, and the maximum value of $Q_{\text {vol }}$ was inferred from inspection of 500 years of simulated streamflows. With respect to the bank migration parameters, the word 'lateral' here refers to bank migration in the direction normal to the mean downvalley direction. This word is normally reserved for erosion or deposition 'lateral' to the local mean current direction. Limiting width of the meander neck specified here is the distance measured between channel centre lines, and therefore not as originally input. The legend refers to symbols used in the cross sections. It should be noted here that any number of symbols could be used (within the limitations of the line printer) and an increase in the number of the qualities (i.e. grain sizes, bed forms, etc.) used in the program would entail simple and straightforward modifications. The symbols used, however, are considered sufficient for present purposes. 


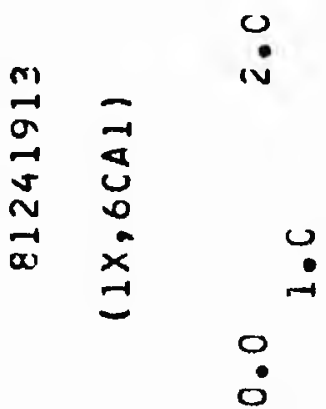

21
0
0

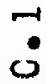

$000 \mathrm{un}$ $\rightarrow 0$

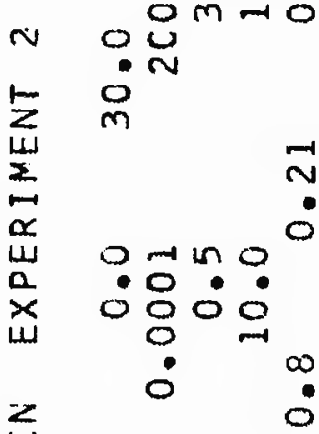

$\sum_{1}^{2}$

$\prod_{i} \quad 0 \quad 000$

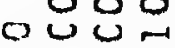

ज $5 \%$

س

$\omega N$

$\propto$

N

ONO

U

mo u

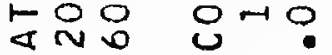

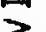

$\exists \omega$

$\rightarrow \circlearrowleft 4$

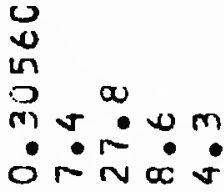

N

o $\infty$ แn $N$ in $\dot{a} \dot{n} \dot{m}$

- inara

0,1 i

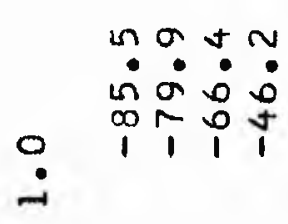

vi

$0 v \omega 0 N$

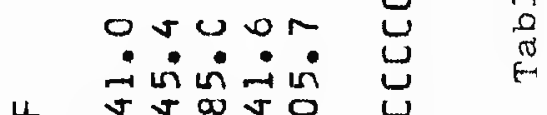
L $T$ T 2 T - 0 $\therefore 0$

更怘

$x 0$

$\pm \rightarrow m v m$

$?$ In $\dot{0} 0$

v) muiniviv

c $v \pi n \rightarrow \infty$ 
Fluviatile process Simulatiom experimeat 2

CRDSS SECTION PARAMETERS

\begin{tabular}{|c|c|c|}
\hline METRES & CELLS & \\
\hline $\begin{array}{r}1750.000 \\
60.000 \\
0.0 \\
30.000 \\
1.000 \\
5.000\end{array}$ & $\begin{array}{r}350 \\
60 \\
6 \\
30\end{array}$ & \\
\hline METRES & CELLS & \\
\hline $\begin{array}{l}125.000 \\
100.000\end{array}$ & $\begin{array}{l}25 \\
20\end{array}$ & $0.8 \mathrm{CO}$ \\
\hline 200000 & & $\begin{array}{l}2.650 \mathrm{GM} / \mathrm{CH}_{3} \\
1 . \mathrm{CCO} \mathrm{GM} / \mathrm{CN3} \\
0.21 \mathrm{C} \\
0.150 \\
1 . \mathrm{CCC}\end{array}$ \\
\hline
\end{tabular}

WIDTH OF SECTION

THICKNESS OF SECTION INNER CHANNEL GANK FRCM L.H.S. OF SECTION

IN ITAL OISTANCE UFTN

CELL SIZE IM VERTICALIY) OIRECTION

CELL SIZE IN VERTICALTYLIZ OR XI DIRECTICN

CHANMEL PARAMETERS

TOTAL HIOTH OF CHANNEL(h)

HIDTH OF FLOH BETWEEN INNER BANK AND TALHEGIWII

MAX IMUM FLOW DEPTH MEASLRED ABOVE TALWEG

DENSITY OF SEDIMENTARY PARTICLES

FLUID DENSITY

DARCY-WEISEACH FRICTION COEFFICIENT FOR PLANE BEOS AND ANTICUNES EXPONENT ML

0.306

STANDARD DE YIATION OF DAILY MEAN YALUES

MEAN OF YT SERIES

STANDARD DEVIATION OF YT SERIES

COEFFICIENTS IN AUTOREGRESSIVE MODEL

FDURIER COEFFICIENTS FOR DAJLY MEANSIA)

FOURJER COEFFICIENTS FOR DAILY STO DEVIATICNS ISA]

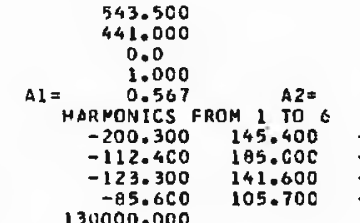

MAXIMUM VALLE OF QVOL

130000.000

$79.9 \mathrm{CC}$

$-66.4 \mathrm{CC}$

$58.0 \mathrm{CO}-39.800 \quad 7.400$

$65.600 \quad-72.500 \quad 27.800$

BANK NIGRATION PARAMETERS

EXPONENT N2

VALUE OF CONSTANT IN LATERAL MIGRATICA RELATICA

VALUE DF CONSTANT IN DOHIVALLEY MIGRATICA RELATICA

LIMITING PERCENTAGE OF GRAVEL ALLOHABLE IN GUTER BANK

0.500
$\times 2=0.100 E-05$

$K_{2}=0.100 E-05$

$100 E-03$
30.000

SCOUR AND FILL. PARAMETERS

CONSTANT KA

STANOARD OEVIATION OF ERROR TERH

0.0

0.0

CUT-DFF CONTROL PARAMETERS

LIMITING WICTH OF MEANOER NECX

EXPONENTS IN NECK CUT-OFF RELATICN

LIM IT ING SINUOSITY

LIMIT ING AMPLITUDE

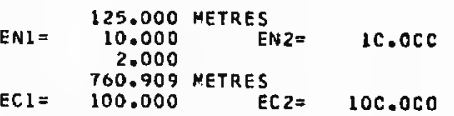

A DOHNVALLEY SEC TOM IS REPRESENTED IN THIS TEST

OISTANCE OF LINE OF SECTION FRON PCINT OF INFLECTICN CF LCOP IS 0.0 METRES

LEGEND

LOHER PHASE PLANE BED RIPPLES

UPPER PHASE PLANE BED

ANT IDUNES

$L$
$R$
$D$
$U$
$A$

$\begin{array}{llll}\text { GRAYEL } & G & \text { OLE SEOIMENT } & x \\ \text { SAND } & G & \text { HATER } & 1 \\ \text { SILT } & S & \text { TIME LINE } & \text { \#.... } \\ \text { CLAY } & - & \text { AIR } & \text { BLANK } \\ \text { OVERBANK } & F & & \end{array}$

Table 16.2 .

Sample output from main program during initialisation. 


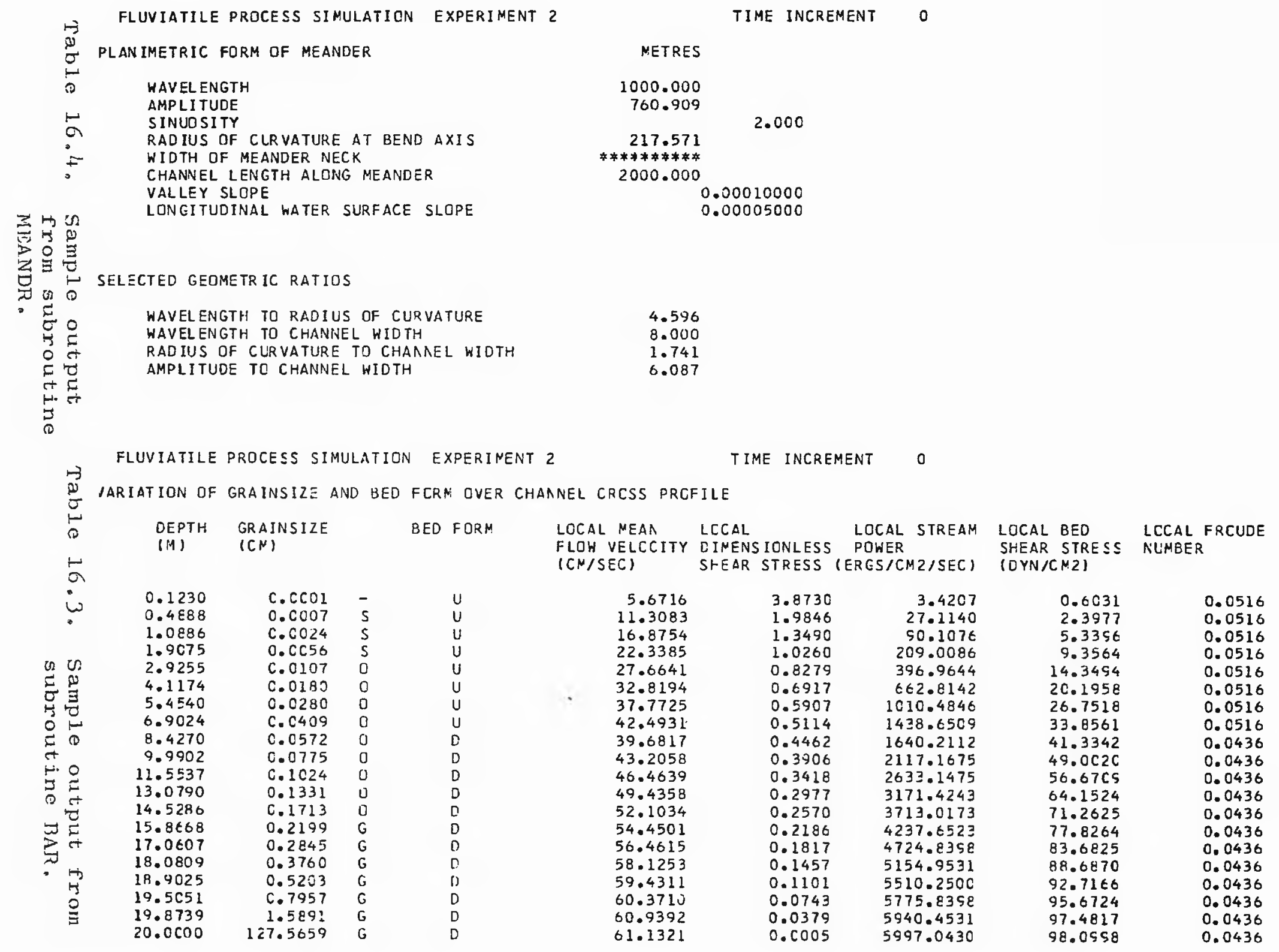




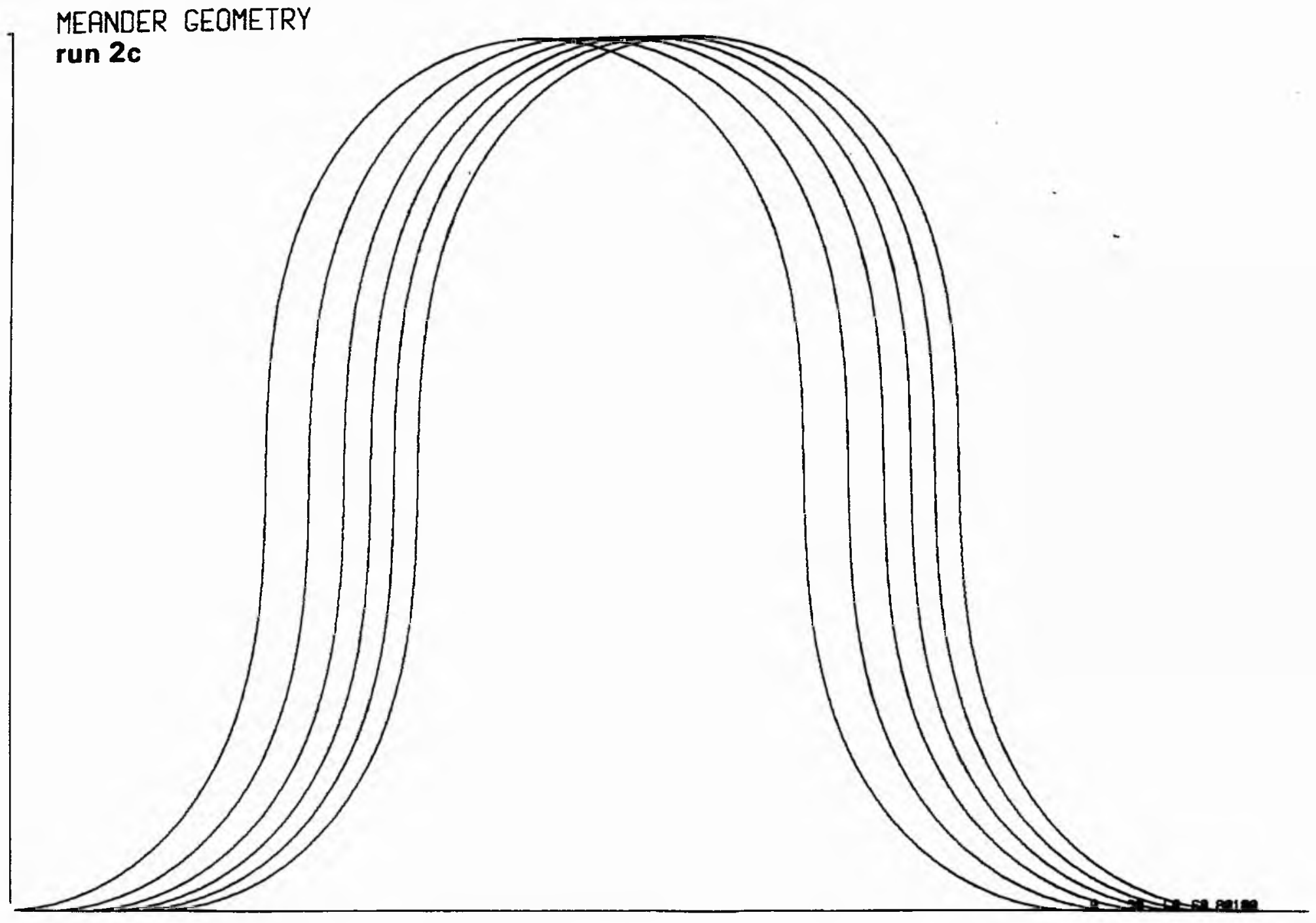

SCALE-1 INCH TO 84.55 METRES

Fig. 16.1. Example of graph plotter output from subroutine MiEANDR. 
Tables 16.3 and 16.4 are printed at the beginning of the run and every NPRINT-th time increment. Table 16.3 represents an example of output from subroutine BAR and shows the computed grain size, bed form and various hydraulic parameters (measured parallel to the $x$ direction) for selected depths over the point bar profile. These depths correspond to stations located one cell width apart over the profile, from the top of the profile (starting at a distance of one cell width from the inner bank) down to the talweg. The profiles pertain to the unscoured channel which exists before and after scouring and filling, and before slope adjustments are made ( 1 n the case of a developing meander). The depth at the talweg is therefore $h$. The symbols shown for grain size and bedform at a given depth are allocated to the cross sections in the appropriate place. The actual depth, grain size and bedform for a station, as seen approximately in the cross sections, correspond to the right hand edge of the appropriate cell. As previously mentioned, the very top parts of these profiles, where clay is predicted, may not be theoretically correct, but should be qualitatively acceptable.

Table 16.4 is an example of output from the subroutine MEANDR and refers to the planimetric form of the meander after a high water period, that is, at the end of the time increment in question. Similar information is also printed out at the beginning of the run during initialisation. If the width of the meander neck is not defined, asterisks are printed to indicate this. The longitudinal water surface slope is assumed constant all round the meander, as well as across the channel, during the water stages being considered. Fig. 16.1 shows an example of the graph plotter output from MEANDR. A trace of the channel centre line is produced during intialisation (and labelled with a zero), and subsequentiy every NFPLOT-th time increment. Each trace is 
요

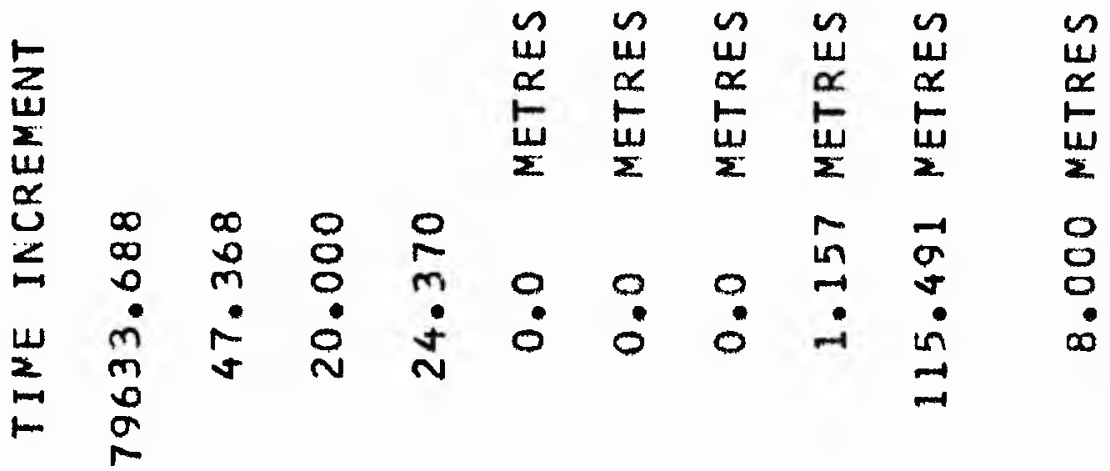

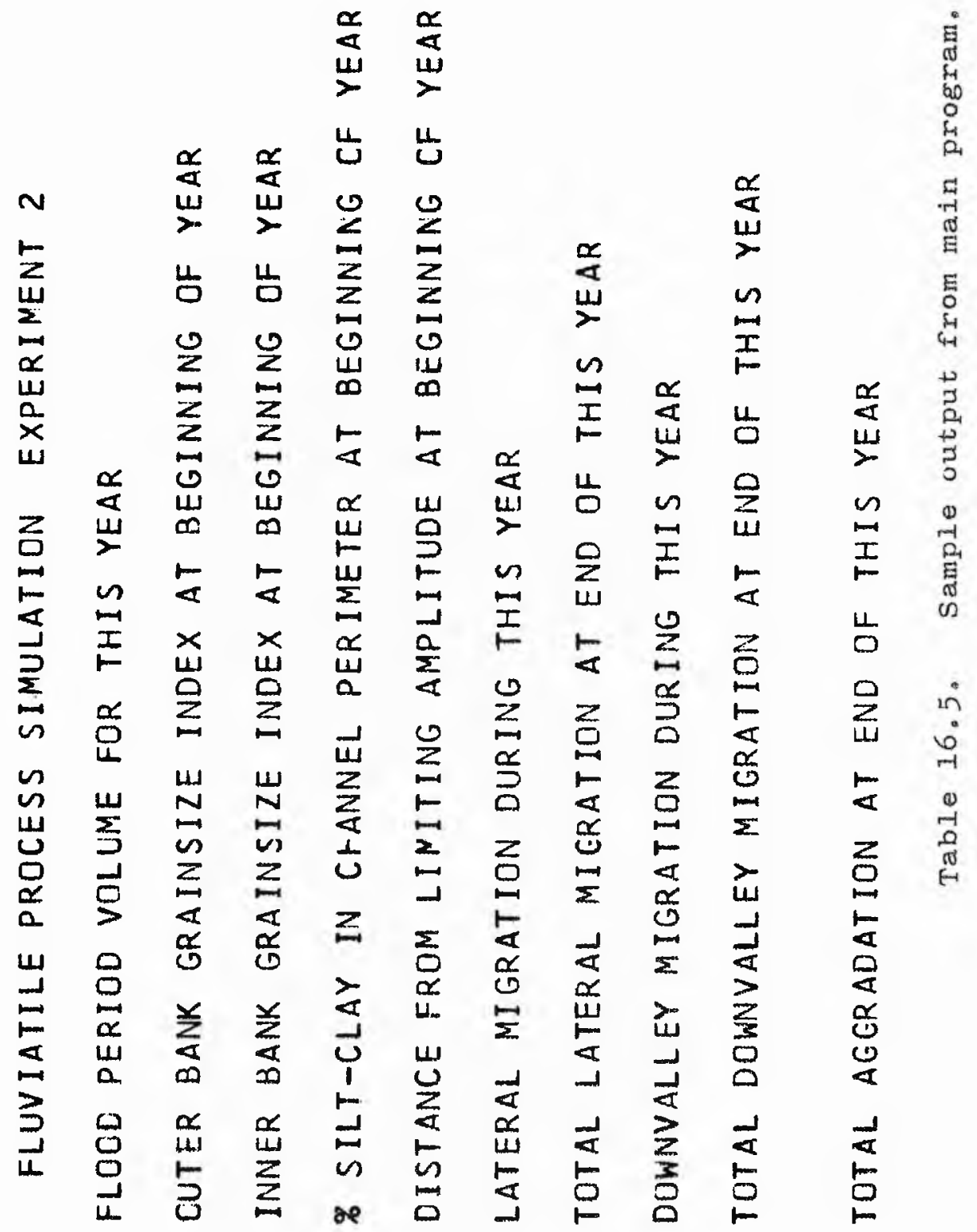


annotated with the appropriate time increment, and represents the position at the end of the time increment. When an experimental run was stopped due to cut-off, the appropriate cut-off infornation has been added to these planform figures. The run numbers are also added. The scales shown at the bottom of these figures are those of the orlginal graph plotter output and are not appropriate for the figures reproduced here. As the dimensions of the meander plans are specified in the text, the scales of the reproduced figures can be obtained.

Table 16.5 is printed every NPRINT-th time increment but not during initialisation. The grain size indices and the total percent of silt and clay (including overbank deposits) in the channel perimeter represent actual percent areal exposure in the cross section defined, not necessarily in the transverse profile defined at right angles to the local mean current direction. As already stated, the percent silt and clay in the channel perimeter is not the same as that used by Schumm (1960). The distance from the limiting amplitude at beginning of year refers to the distance normal to the mean downvalley direction measured from the channel centre line at the bend axis. Again, 'lateral' migration refers here to bank migration in the direction noxmal to the mean downvaliey direction.

Fig. 16.2 and fig. 16.3, respectively, show representative partis of the grain size and bed form distribution oross sections produced after a certain number of time increments of this sample run. Each symbol marked (except for the time line dots) occupies one ce11, the dimensions of which are given in table 16.2. The past positions of the unscoured channel bed are picked out with the dots of the time lines. These are plotted for the initial position, and subsequently every NTPLOT-th time increment, and they represent the situation at the end of the time increment. In the forthcoming experiments these time lines are annotated 


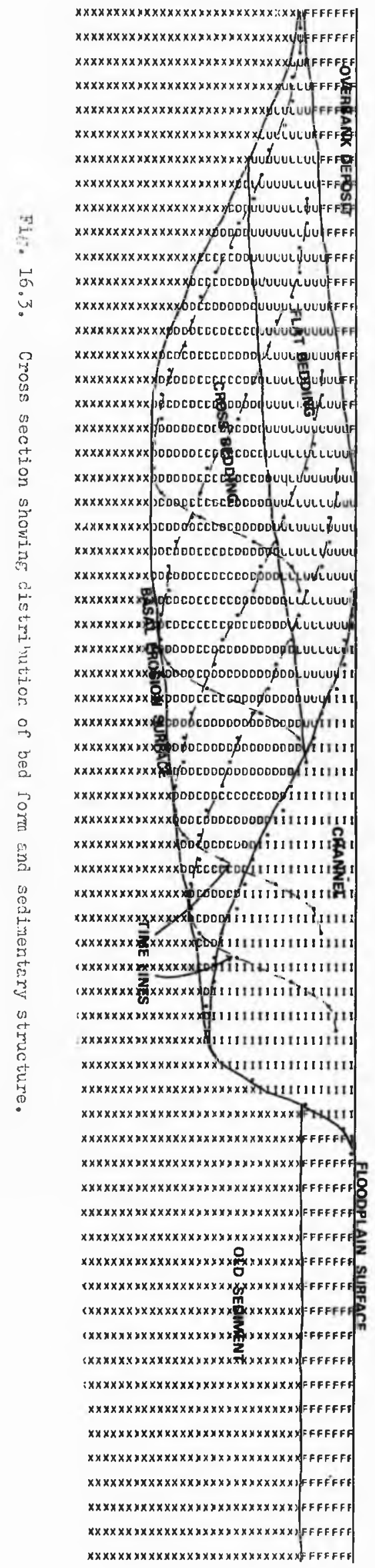

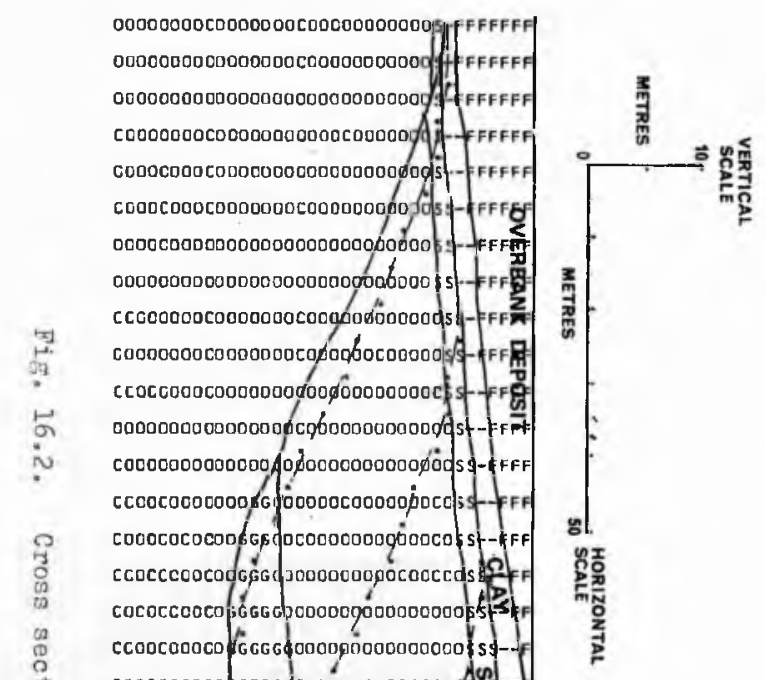

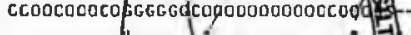

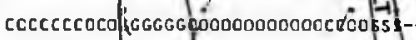

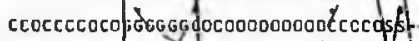

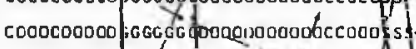

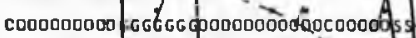

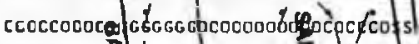

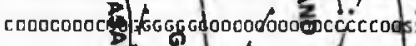

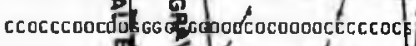

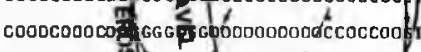

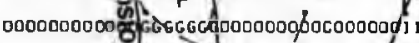

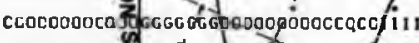

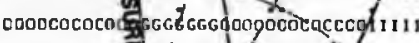

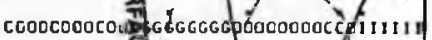

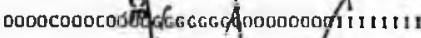

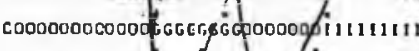

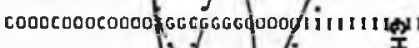

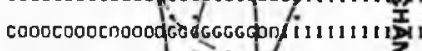

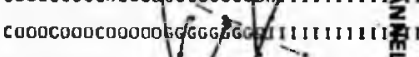

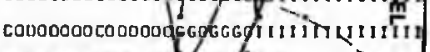

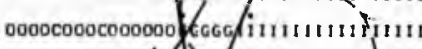
ccocoooncoocong

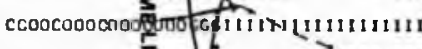

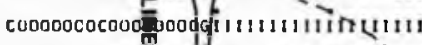

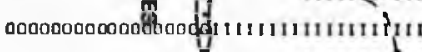
co000000co0000000 $11111111+11111$

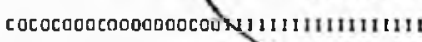

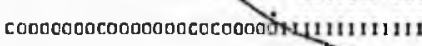

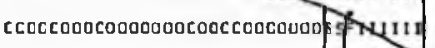
CO00000000000000000000000000

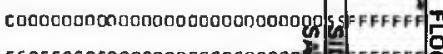

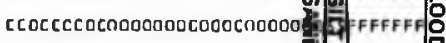

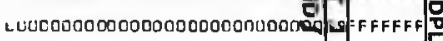
CCOC COLODO000000CDO00000000 d SFFFFF ECCCCOOOCOOUOOOOCOOOOOOOOOOQ SFFFFF US CCCCCCCOCD000000C0000000000 SfFFFF

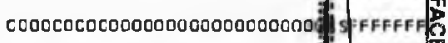

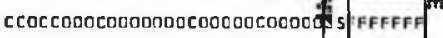
CUOC COUOCOOOQU0UCDO00000CODCSS:FFFFF CCCCCCCODOCODOOOCCOQDODOOOOC OS S FFFFFF DOD0000000000000000000000000 SS FFFFFF CLOLCLCCCOQUOOOOCOCOCOODOOOOSSSFFFFFF

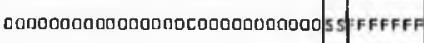
CCCCCCCCOO00000000000000000D SS FFFFFF

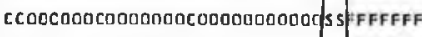
CCOC COCOOJ00000000000000000तSS SFFFFF C000000000000000 C0000000000dS SFFFFFF 
with the appropriate time.

clearly the cross sections only record the response of the model to the processes as simplified from the real world system. However, an increasing degree of accuracy in the cross sections, within the simplified model system as it stands, could be obtained, for instance, by increasing the number of grain size divisions or decreasing the cell size. The symbols for dunes and ripples should be assumed to represent cross bedding and cross lamination respectively, and those for upper and lower phase beds to represent flat bedding. The symbol for overbank deposits must be thought of as baing internally variable in both grain size and sedimentary structure, probably with some characteristics similar to the immediately underlying point bar sedinents (particularly the grain sizes).

In the originals of cross sections shown and in subsequent sections produced in the experiments (except fig. 25.1), the vertical and horizontal scales were $1 \mathrm{~cm}$. to YCEL/0.25 metres and ZCEL/0.85 metres, respective1y. YCEL and ZCEL are the depth (size in vertical dixection) and width (size in horizontal direction) of the cells, and in these cases their values are 1.0 and 5.0 metres respectively. The vertical exaggeration is therefore $(\mathrm{ZCEL} / 0.85) \times(0.25 / \mathrm{YCEL})$, which equals 1.47 . These scales only apply to the original line printer output (produced on IBM 1403). Other line printers may have different spacing and size of characters. During reproduction the cross sections were reduced: The horizontal and vertical scales are therofore calculated as, respectively, physical width of one cell (units) to ZCEL metres, and physical depth of one cell (units) to YCEL metres. The vertical exaggeration remains unchanged. This vertical exaggeration implies that the relief of any of the grain size, sedimentary structure, or sediment-water boundaries will be exaggerated. 
Associated with the use of discrete cells which are filled with a particular quality, is a smoothing error, as all lengths in the cross section are rounded down to the nearest number of cell units. As a result of this, the positioning of any quality is only within an accuracy of one cel1. Confidence should not be placed, therefore, in, say, irregularity in factes boundaries with only one cell relief.

It should be noticed that in neither lateral nor downvalley sections is the valley slope explicitly accounted for $1 \mathrm{n}$ the cross sections. This is largely due to the negligible slopes compared to the scales of the cross sections. It is a simple matter, however, in the case of the downvalley sections, to tilt the cross sections the required amount, as the bahkfull level of the cross sections corresponds to the land surfaco. Also, no control is made of the erosive effect of an upstream channe1, which will be present in all sections. It is therefore necessary to look at the plots of the channel centre lines to discover how much of the deposits to the left of the current channel in the cross sections can be sensibly assumed correct. All lines separating different symbols or joining time line dots are added by hand, thus subjectivity in the positioning of such lines are the responsibility of the individual. In future, lines separating different grain size classes, sedimentary structures, etc., w111 be termed 'facies boundaries'. 
PART FOUR

EXPERIMENTATION AND RESULTS 
The development of the overall mathematical model gives a more intimate insjght into the natural system to be simulated, and it is interesting to look at the modes of sedimentation expected under particular conditions on the basis of this insight. Such an insight has also been important in the designing of selected experiments with the computer program, without the necessity of experimenting with every possible combination of variables. Broadly the experiments fall into two categories; those where the meandering is developing to a stable form and those already in dynamic equilibrium. Data input is such that all dependent and independent variables are in conformity with those found in natural situations, as far as these are known. This aspect was discussed in part 2. 


\section{EXPERIMENT 1 - MEANDERS IN DYNAMIC EQUILIBRIUM}

It was noted that over a high-water period the bed in the pool of a meandex characteristically scours and subsequently fills to the same position as existed before the high stages. When such changes occur in the channel bed, together with lateral movement associated with bank recession, it is expected that some degree of relief will exist in the grain size and sedimentary structure boundaries and in the basal erosion surface. This experiment is therefore designed to examine the nature of sedimentation as the rates of bank migration and depth of scour vary.

The experinent consists of nine runs of the program corresponding to all possible combinations of three different average rates of downvalley bank migration and three different averago depths of scour at the talweg. The input data varied for each run are shown in table 17.1, and these correspond to average rates of bank migration of about 2.4, 12 and 48 metres per year (or, more precisely, per time increment), and average net depths of scour at the talweg of about $0.78,7.8$ and 15.6 metres. All other parameters, shown in table 17.2 and 17.1 , are constant for all runs. The meanders are assumed in dynamic equilibrium with a sinuosity of 2.0 , the meander and channel geometry being constant for all runs. Table 17.3 shows the variation of grain size, bed form and hydraulic parameters over the point bar profile (at bankfull stage) before and aftex the scouring and filling of the bed. This profile and the cross sections produced were defined in the mean downvalley direction. The input data deck set-up is listed in appendix 3 . In this experiment a disc was used, the cross sections were made up of 200 by 60 , cells, and $78 \mathrm{k}$ bytes of core store were required. Average running times (CPU times) were 10 seconds per time increment. 


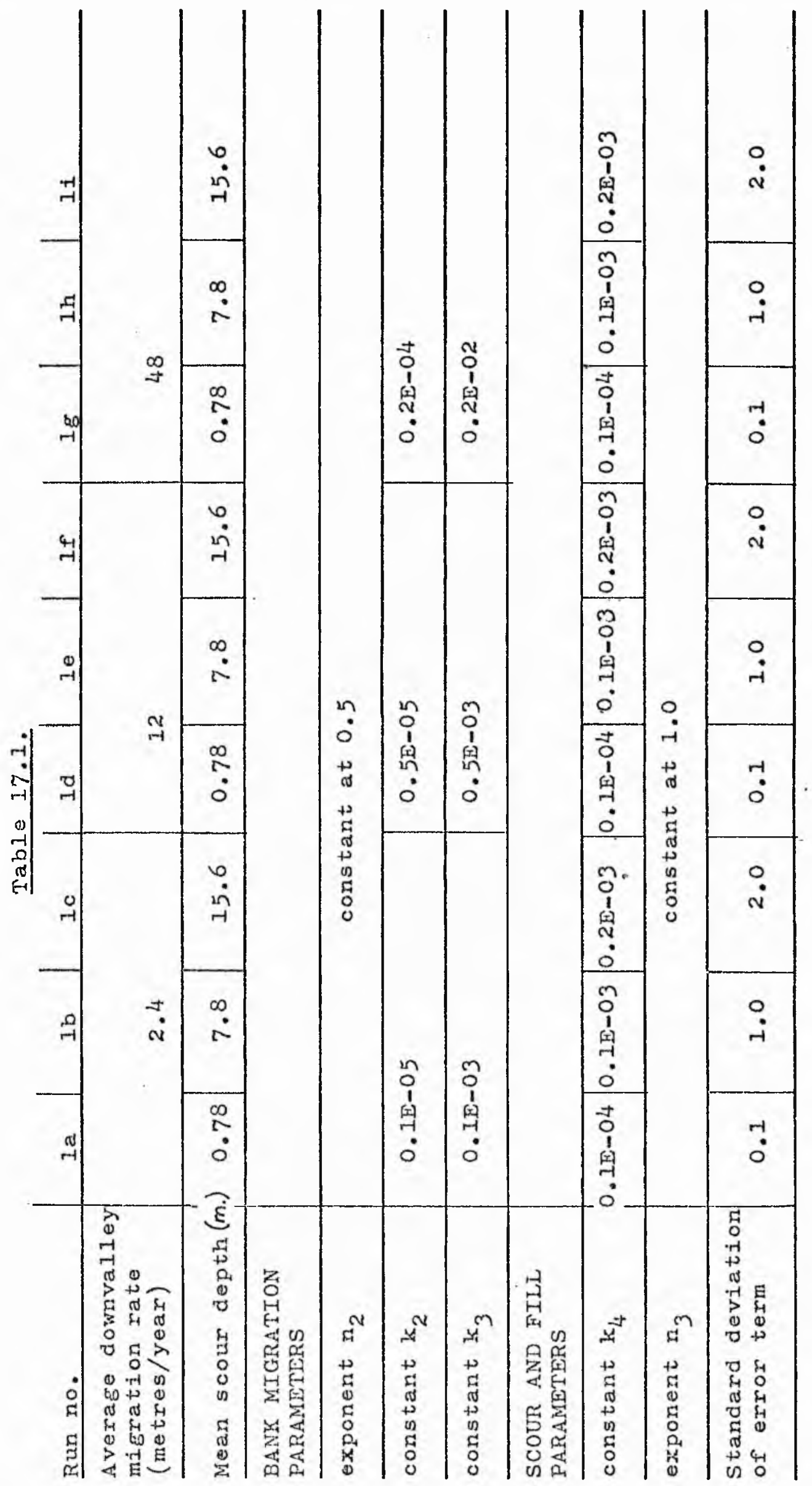


FLUVIATILE PROCESS SIMULATION EXPERIMENT I

CHOSS SECTION PARAHET:RS

1000.000

INITIAL BANKFULL STAGE MEASUREO FROM SECTION BASE

CELL SIZE IN VERTICALIYI DIRECTION

CELL SIZE IN HORIZONTALIZ OR XI DIRECTION

Channel, parametizs

TOTAL WIOTH CIF CHANNELIWN

WIDTH OF FLGW BETHEEN INNER BANK AND TALHEG (HI)

RAT IO UF HI TO H

MAX IMUM FLOh DEPTH MEASURED ABOVE TALHEG

DENSITY UF SEDIMENTAKY PARTICLES

FLUID DEISITY

DARCY-HEI SBACH FRICTION COEFFICI CNT FOR DUNES AND RIPPLES

DARCY-HEISBACH FKICTIUN COEFFICIENT FUR PLANE BEOS AND ANTICUNES EXP ONENT $\mathrm{NI}$
METRES 60.000

60.000
0.0

0.0
00.000

1.000

5.000

CELLS

200

60
0
60

METRES CELLS

$\begin{array}{ll}125.000 & 25 \\ 100.000 & 20\end{array}$

0.800

2.650 GH/CH3

0.210

0.150

$1.0 C C$

SYNTHETIC HYOROLCGY PARAMETERSIUNITS NCT NECESSARYI

MEAN OF ALL DAILY MEAN VALUES

STANDARD DEVIATIUN OF DAILY MEAN VALUES

MEAN OF YT SER IES

STANDARO DEYIATILN UF YT SERIES

COEFFICIENTS IN AUTOREGRESSIVE MUDEL

FOURIER COEFFICIENTS FOR DAILY MEANSIA)

FOURIER COEFFICIENTS FUR DAILY STD DEVIATIONSISA)

MAX IMUM VALUE OF QVOL

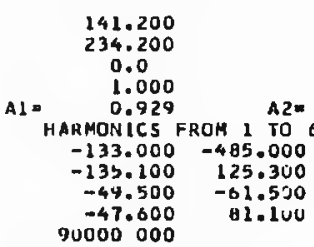

$-0.151$

$\begin{array}{rrr}62.460 & -14.36 c & -7.100 \\ -9.800 & -29.500 & 1.000\end{array}$

$\begin{array}{rrr}-9.600 & -29.500 & 1.000\end{array}$

$\begin{array}{rrr}9.960 & 2.800 & 2.900 \\ 4.960 & -31.400 & -10.200\end{array}$

CUT-OFF CONTROL PARAMETER'S

LIMITING WICTH OF HEANDER NECK

EXPONENTS IN NECK CUT-JFF RELATION

LIHITING SINUOSITY

LIMITING AMPLITUDE
EXPONENTS IN CHUTE CUT OFF RGLLATICN

$\begin{array}{cc}125.000 & \text { METRES } \\ \text { EN1 } & 10.000 \\ 2.000 & \text { EN2 } \\ 760.909 & \text { METRES } \\ 50.000 & \text { EC2= }\end{array}$

10.000

$E C l=$

90.909

EC 2a

50.000

a domnYalley SECTION 15 hepREsented IN THIS TEST

DISTANCE OF LINE UF SECTIUN FROM POINT OF INFLECTION CF LOOP IS 0.0 METRES

LEGEND

LOWER PHASE PLANE BeU RIPPLES

DUNES PPPER PHASE PLANE BED

ANT IOUNES

$$
\begin{array}{ll}
\text { L } & \text { GRAVEL } \\
R & \text { SAND } \\
D & \text { SILT } \\
U & \text { CLAY } \\
\Lambda & \text { OVERBANK } \\
\text { UEPQSITS }
\end{array}
$$

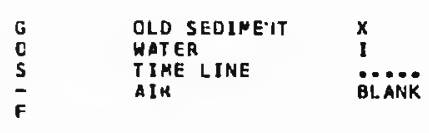

planimetric fomm of meander

METRES

MAVEL ENGTH

AMPLI TUDE

SINUOSITY

RADIUS OF CUBYATURE AT BEND AXIS

HIOTH OF MEAMDER HICK

CMANNEL LENGTH ALONG MEANOER

LONGITUDHNAL WATER SURFACE SLOPE

1000.000
760.909

2.000

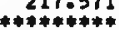

2000.000

0.00010000

0.00005000

SELECTED GEOHETR IC RATIIIS

WAVELENGTH IU RADJUS UF CURVATURE

MAVELEMGTH TO CHAINNEL MIOTII

WAVELENGTH IU RADIUS UF CURVATURE

AMPLITUDE TU CHANNEL WIDTH

4.596

8.000

1.741
6.087

Table 1.7.2.

Initial data for experiment 1 . 


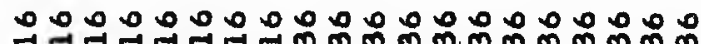

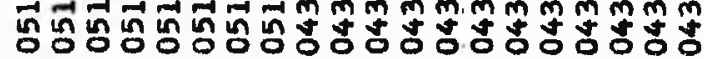

on-10NN-1

Nin

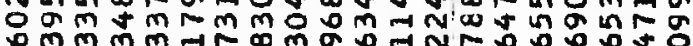
-

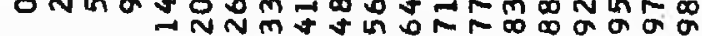

mm in

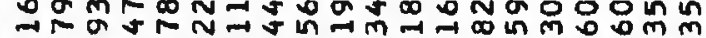

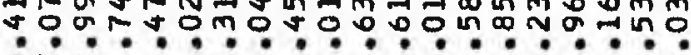
mRa No $\frac{\alpha}{4}$

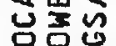

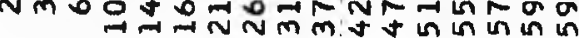

눈

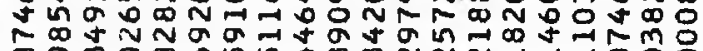
कom 0 o in in

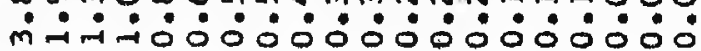

.

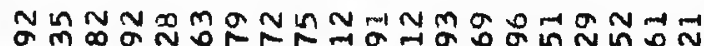

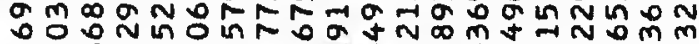

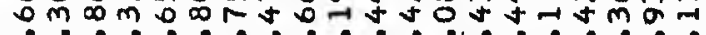

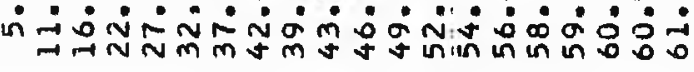

से $x \geq$

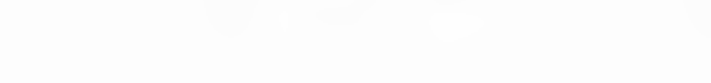

$$
\text { 咅 }
$$

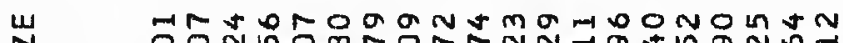

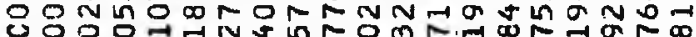

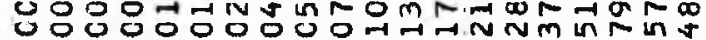

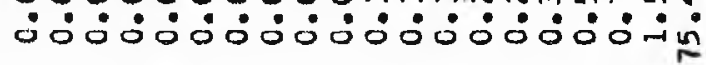

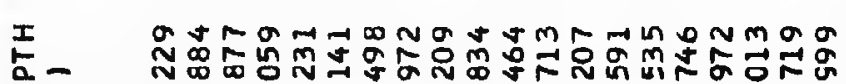

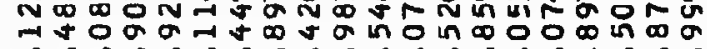

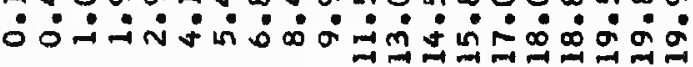


With regard to the simulated cross sections, the errors involved due to smoothing (section 16) and the vertical exaggerat.. ion should be borne in mind. Unfortunately the cross sections do not record the position of the scoured bar profile for each time increment. These profiles may be thought of as representing the boundaries of sigmoidal units which will subsequently be termed epsilon units. Each unit marks the deposition during one flood period. In the case of no scouring and filling the time lines givo the shape of these units. Where possible, however, profiles of tho scoured bar for a number of time increments, have been added to the cross sections in order to show the variable distribution of grain size and sedimentary structure within and between the units. The grain size distribution cross sections of runs 1 a,b and c, average downvalley bank migration about $2.4 \mathrm{~m} . /$ year, show very little relief in the facies boundaries separating clay, silt and sand sizes (fig. 17.1). The sand-gravel facies boundary and the scoured basal surface, however, show increasing degrees of relief as the scour depth increases. A relief corresponding to up to about $5 \%$ of the unscoured talweg depth of the channel does not seem unrealistic for those channels that scour to the degree suggested in the literature. The scoured bar profiles for the final time increment are shown in the sections, and an interesting feature is the sloping of all facies boundaries up to the contemporary point bar surface. The upward slope represents the effect of filling of the scoured bar for the final time-increment with only a small amount of lateral migration. This feature is, of courso, the basis for the relief of facies boundaries within the bar. If the channel is abandoned after cut-off or avulsion fairly rapidly such a feature may be preserved. It is interesting to note that the grain-size facies boundaries do not slope down into the talweg as filling and migration proceed. This is a function of the 


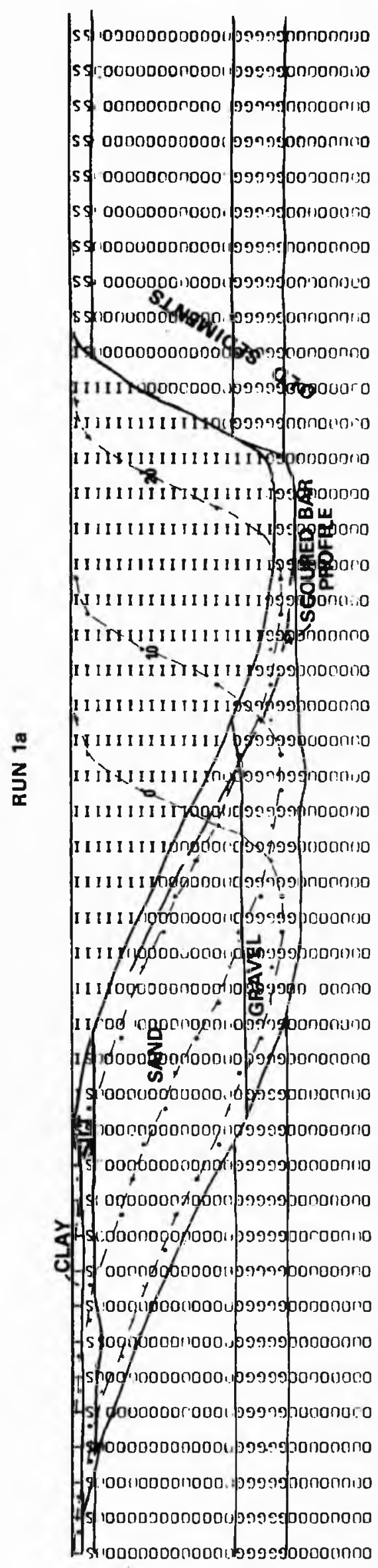

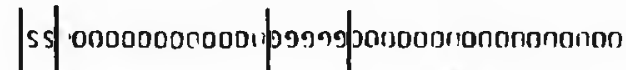

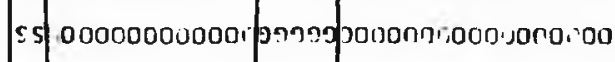

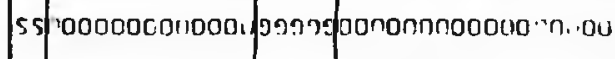

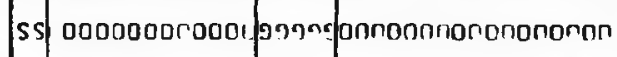

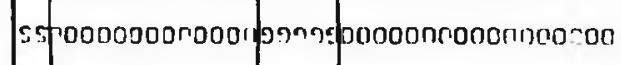

Ss :0000000no00י

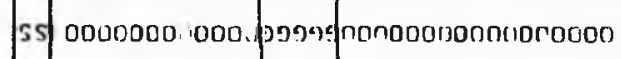

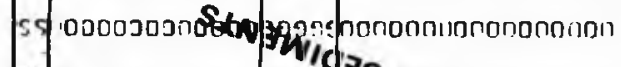

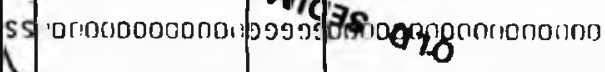

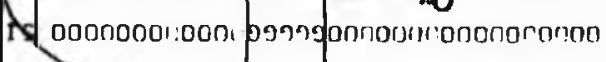

II III

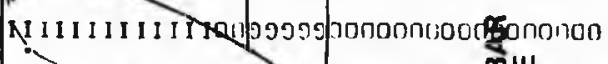

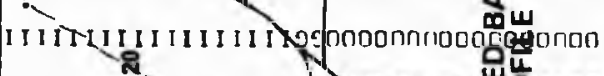

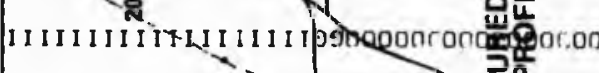

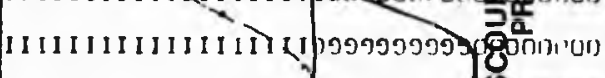

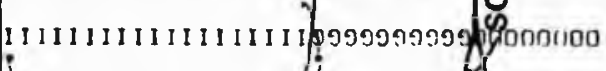

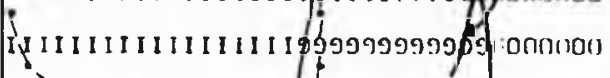

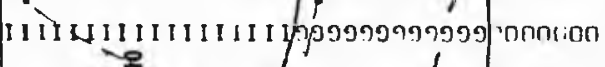

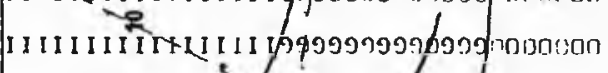

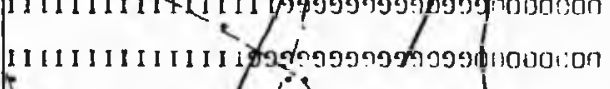

1.

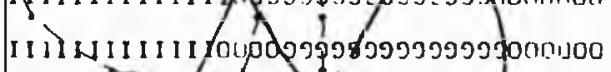

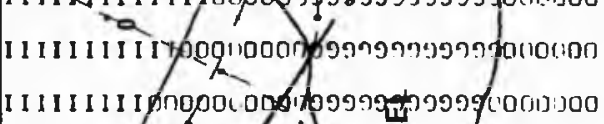

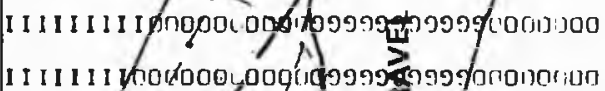

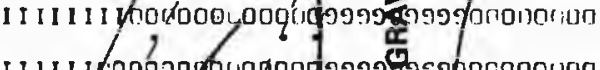

I 11 il 1 ir o.

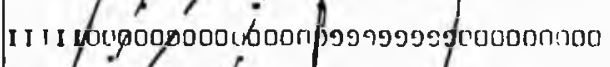

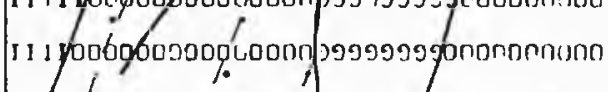

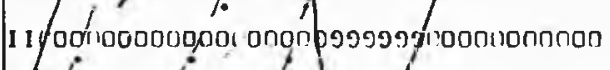

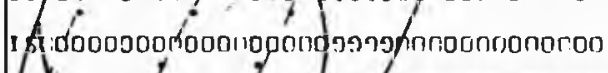

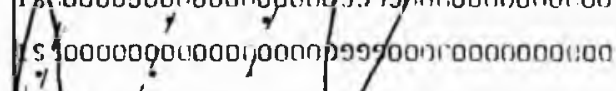

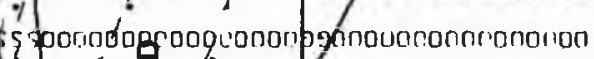

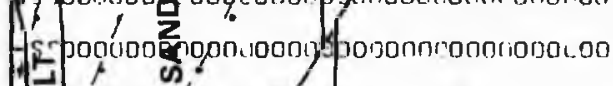

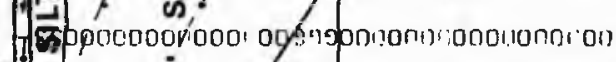

就

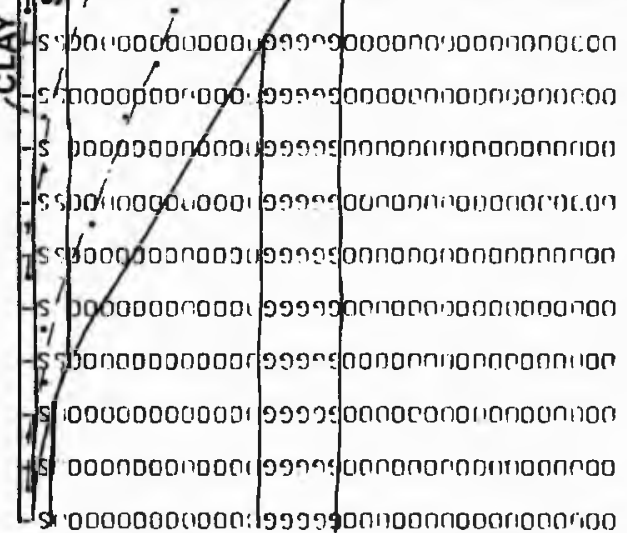

ss 10000000n00000porogponor

s. 90000000000040929030001

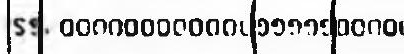

S. 000003070000 ग099s ponor

s. 00000000000 J99gono

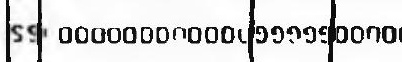

s. 000000000800 (209090000

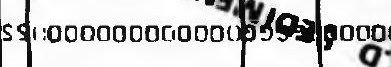

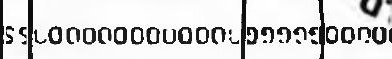

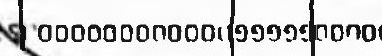

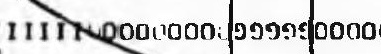

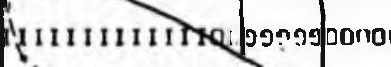

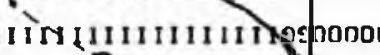

IIIIIIIITH

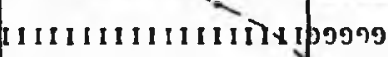

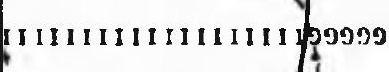

dy

IIILIIIIIIIII DOP09990

ㄴ.

11 g.

I IIIIIIIIIIIIIF⿻0

$\stackrel{0}{2}$

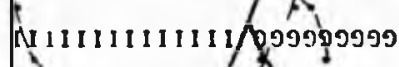

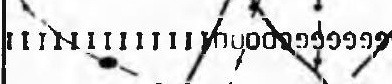

I I I I I I I I r rpootionoopbesos

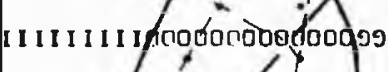

I I I I I I y teoponourog̣boop

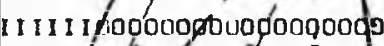

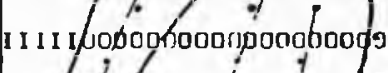

I I lyogogononootionopooobo

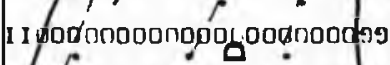

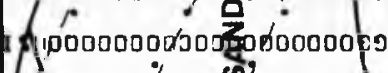

S) ponoogonoonyounononods

s. Junod

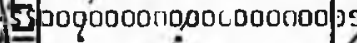

(4)

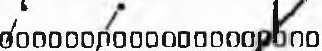

otonooduongsabono

sspono,

5 spodoooncamo

is sṕonogrono possospono

- s \$onoo6onono posos0000

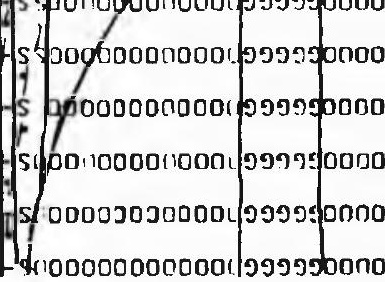




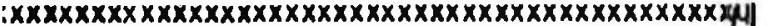

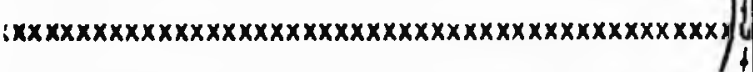

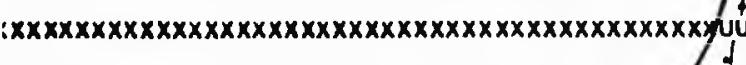

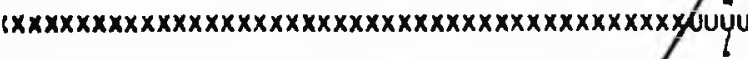

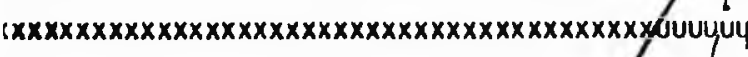

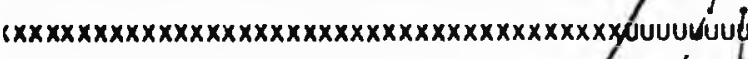

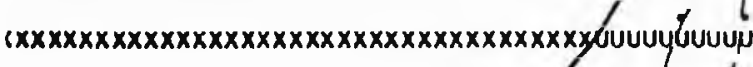

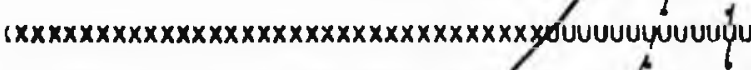

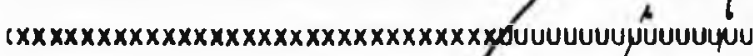

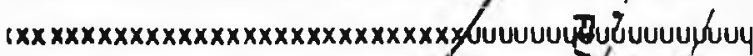

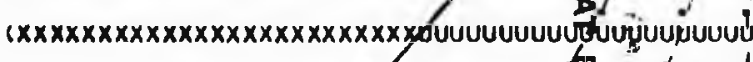

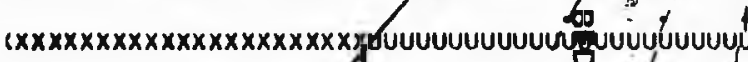

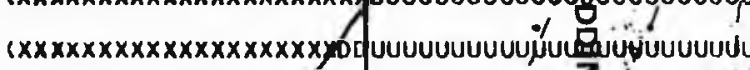

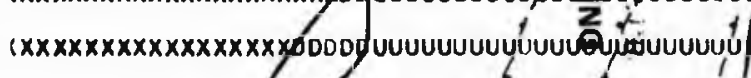

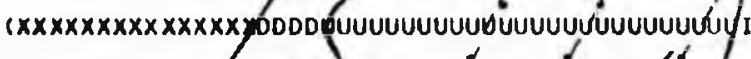

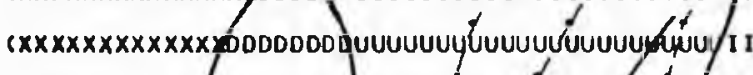

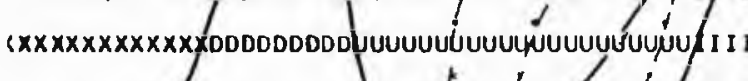

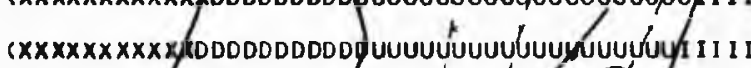

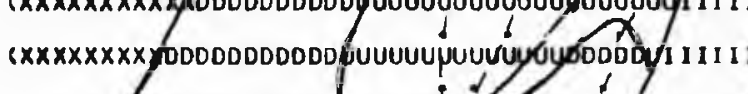

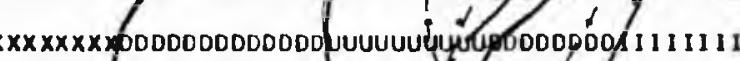
作

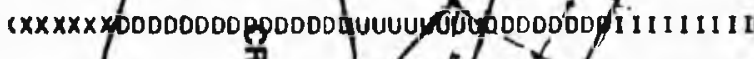

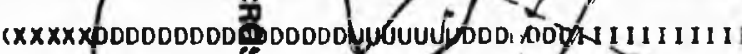
s $\times x \times x \times 100000000 \frac{6}{6}$.

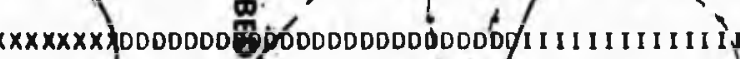

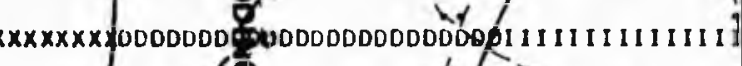

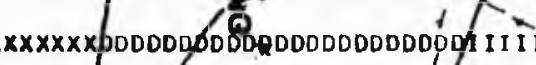
Xx.x

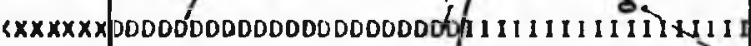

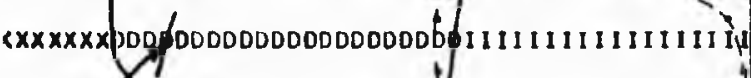

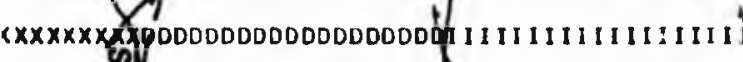
( xxxxxx:

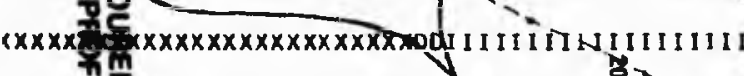

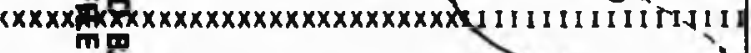

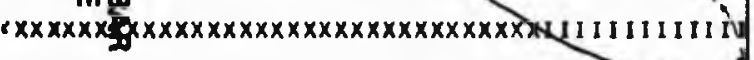

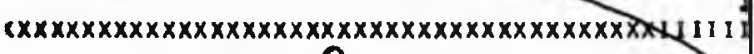

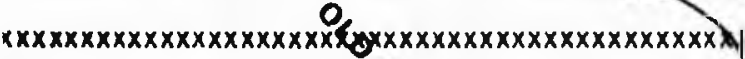

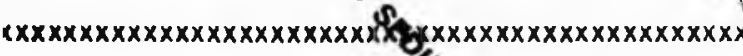

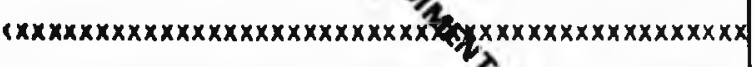

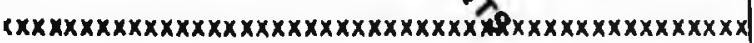

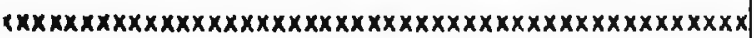

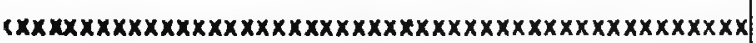

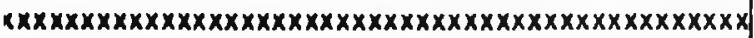

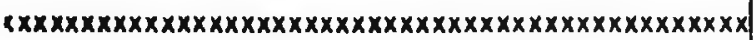

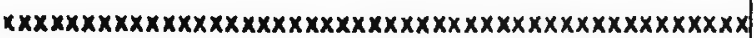
$x \times x \times x \times x \times x \times x \times x \times x \times x \times x \times x \times x \times x \times x \times x \times x \times x \times x \times x \times x \times x \times x \times x \times x$ $x \times x \times x \times x \times x \times x \times x \times x \times x \times x \times x \times x \times x \times x \times x \times x \times x \times x x \mid$

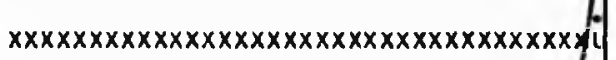
$x \times x \times x \times x \times x \times x \times x \times x \times x \times x \times x \times x \times x \times x \times x \times x \times x \times x$ di $x \times x \times x \times x \times x \times x \times x \times x \times x \times x \times x \times x \times x \times x \times x \times x \times x$ foll

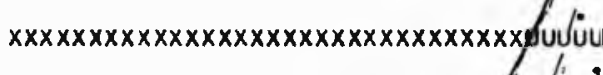

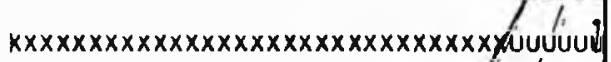

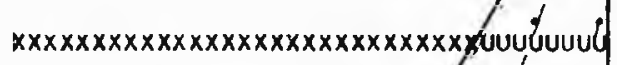

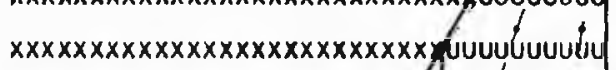

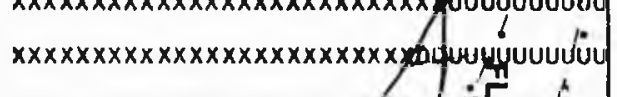
$x \times x \times x \times x \times x \times x \times x \times x \times x \times x \times x \times x$ boopuif $x \times x \times x \times x \times x \times x \times x \times x \times x \times x \times$ DODDo ruvinuUut $x \times x \times x \times x \times x \times x \times x \times x \times x y$ Q 0000 (b) $x \times x \times x \times x \times x \times x \times x \times x \times$ c popoqyoqus

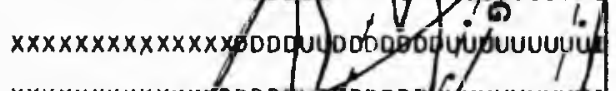

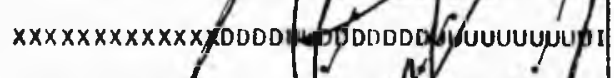

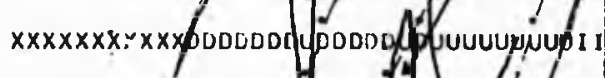

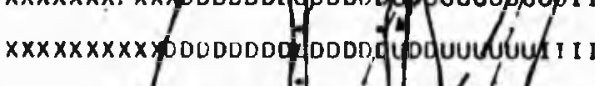

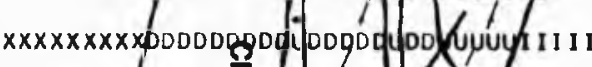

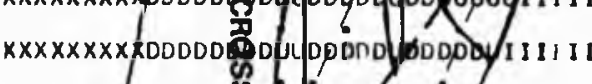
$\times x \times x \times x \times x 000000000$

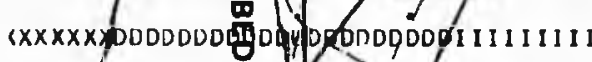

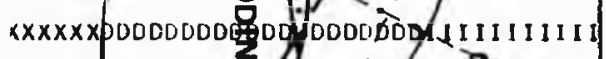

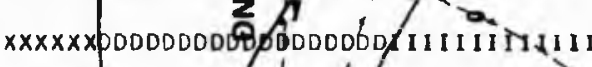

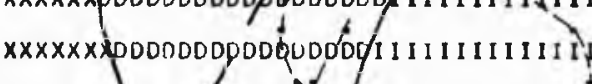
$\times x \times x \times x \times x=0000000000,000$ I I I I I I I I I I I I I I I

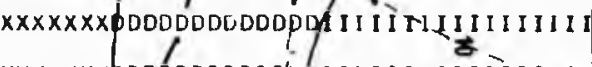

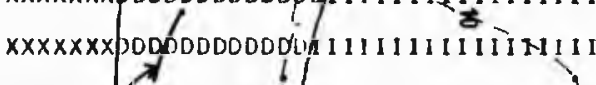
$x \times x \times x \times x \times 000000000 b 0$

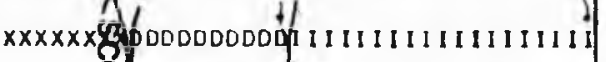
$x \times x \times$ 무을

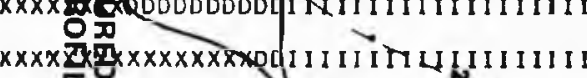

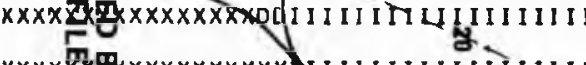

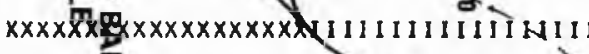

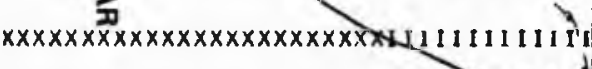

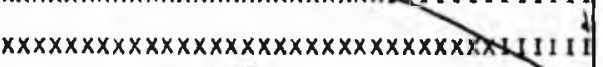

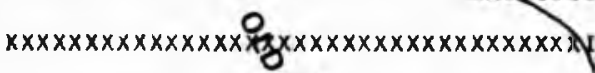

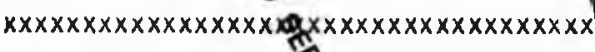

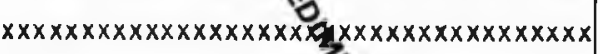

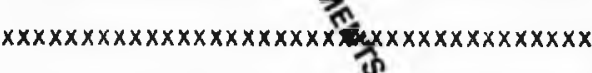
$x \times x \times x \times x \times x \times x \times x \times x \times x \times x \times x \times x \times x \times x \times x \times x \times x \times x x x$

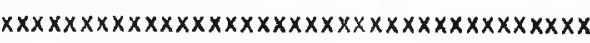
$x \times x \times x \times x \times x \times x \times x \times x \times x \times x \times x \times x \times x \times x \times x \times x \times x \times x \times x$

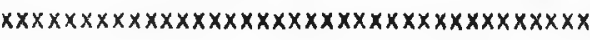
$x \times x \times x \times x \times x \times x \times x \times x \times x \times x \times x \times x \times x \times x \times x \times x \times x \times x \times x$ $x \times x \times x \times x \times x \times x \times x \times x \times x \times x \times x \times x \times x \times x \times x \times x \times x \times x \times x$ $x \times x$

$\mathbf{x x x}$

$\mathrm{x} \times \mathrm{x}$

$\mathrm{x} \times \mathrm{X}$

$x \times x$

$x \times x$

$x x x$

$x \times x$

$x x x$

$x \times x$

$x \times x$

$x x x$

$x x x$

$x x x$

$x x x$

$x x x$

$x x x$

$x \times x$

$x \times x:$

$x \times x$.

$\langle x \times$

$8 x x$

\&xx:

$x \times x:$

$x \times x$

$x \times x$;

$x \times x$

$x \times x$

$x \times x=$

$x \times x$

$x x x y$

$x x x y$

$x x x y$

$x x x$

$x x x x$

$x x x x$

$x \times x$

$x \times x x$

$x \times x x$

$x x x x$

$x x x x$

$x x x x$

$x x x x$ 


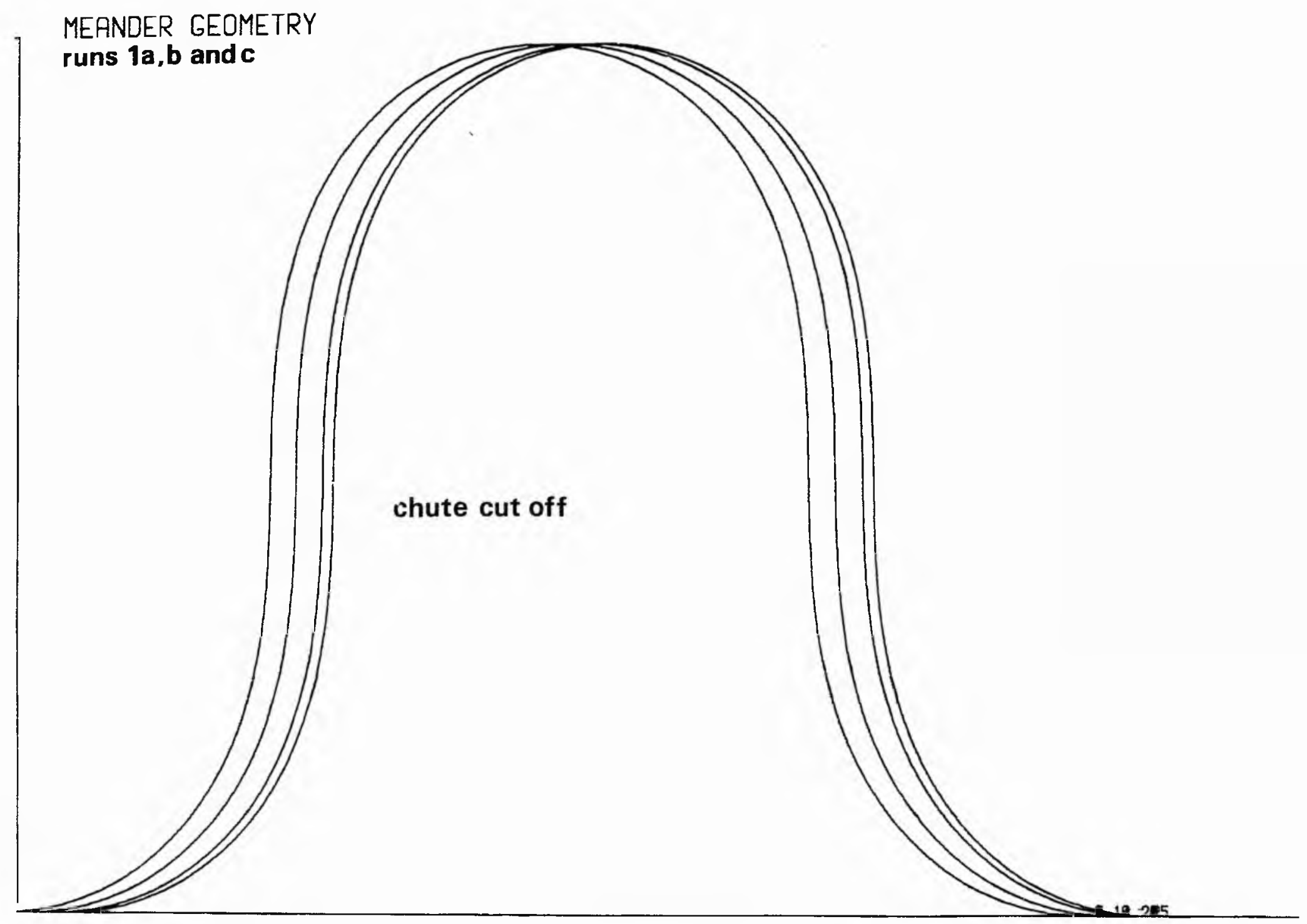

SCALE-1 INCH TO 84.55 METRES

Fis. 17.3. Neander plan forms for runs $1 a, b$ and $c$. 
construction of the model and must be viewed in the light of approximations made. This point 1 s returned to in part 5 . Increasing scour depth has'the effect of increasing the thicknesses of silt and sand, but increasing gravel thickness to a relatively greater extent.

The effect of increasing the depth of scour is more marked on the sedimentary structure sections for runs $1 a, b$ and $c$, fig. 17.2. The relief of the facies boundaries separating flat bedding and cross bedding is again very small with little scouring. As scour depth increases the relief of the boundary becomes as much as $10 \%$ of the channel talweg depth. The tendency for development of lenses of flat bedding within the cross bedding is apparent in fig. 17.2, run Ib. As well as thickening the deposit, increasing scour depth has the effect of increasing total thickness of flat beds at the expense of cross bedding. Hence the sloping of the facies boundary up to the latest point bar surface is present to such an extent in run lc that flat beds effectively interfinger with cross beds. Fig. 17.3 shows the meander movement in plan which produced the deposits in figs. 17.1 and 17.2 .

In the grain-size distribution cross-sections of $1 d$, e and f, (fig. 17.4; corresponding to an average downvalley bank migration rate of about $12 \mathrm{~m}$./year) the sand-gravel boundary and the basal scoured surface again show increasing relief as scour depth increases. With the basal scoured surface the relief ranges from virtually planar to about 20-30\% of the unscoured talweg depth as the scour depth increases, while the relief of the grave1sand facies boundary increases from a few to $20 \%$ of the unscoured talweg depth. Furthermore, in le and if the basal scoured surface is not regularly undulating, but has stretches several tens of metres in length where there is no appreciable change in relief. The relief of the clay-silt boundary does not vary and the regularly undulating relief is up to a few percent of the unscoured 


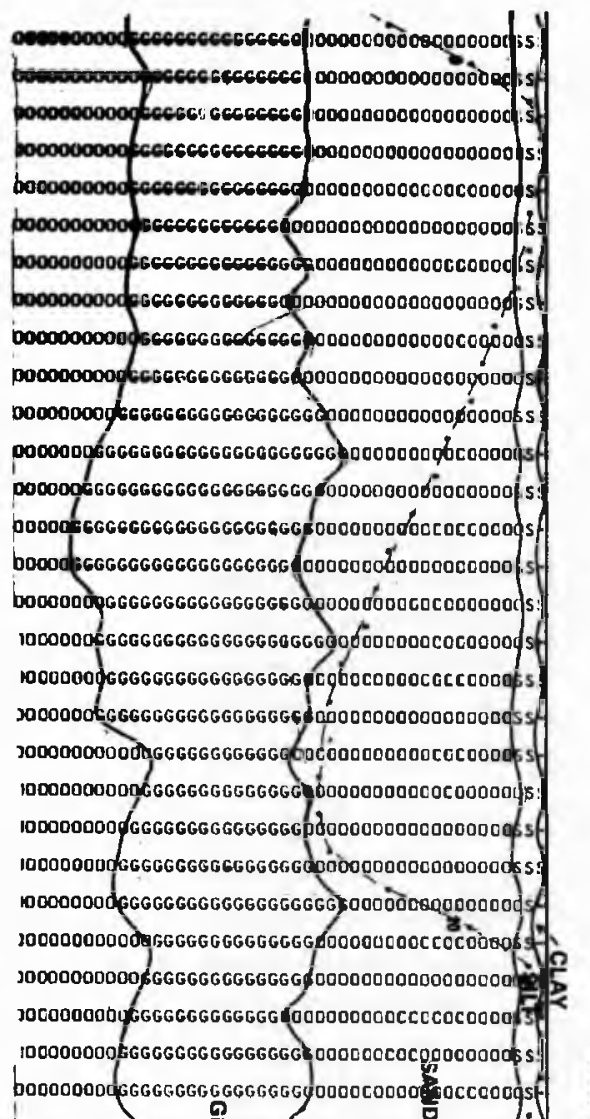

D000000000dGGGGG FGGG bono000000otgGGGG no0000000 ofGGGGGGGGGGGGGGG000000000 $0000 / 25$

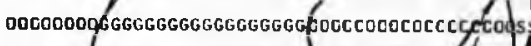

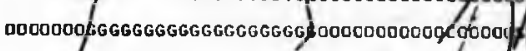

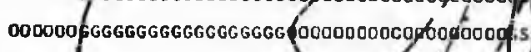

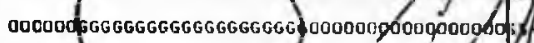
DOconodGG GGGGGGGGGGGGGG donooganooo proc ptogo

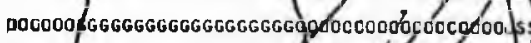

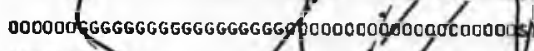
0000000000gGGGGGGGGGgGGGdoooognopótceccooo

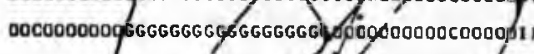

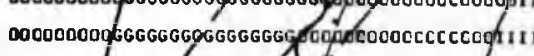

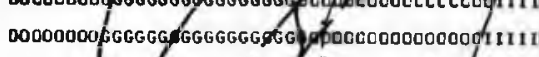

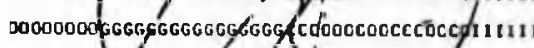

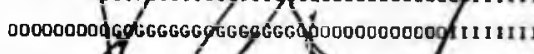

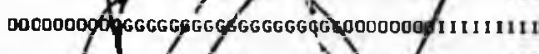

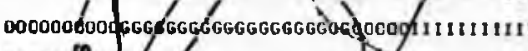

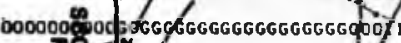

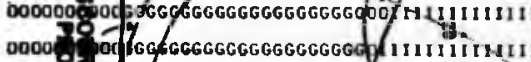

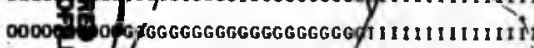
0000 a 00C00000 TGGGGGGGGGGGGGGGG OF 111111111111111 D0000001GGGGGGGGGGGGGGGG 111111111111111 00000000000d0GGGGGGGGGGGGG GIIIIIIIIIIIIIIII

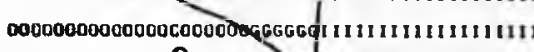

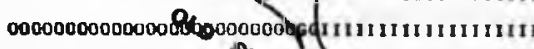
0000000000000000000000 . 0000000000000000000000000 (bestomerimititi

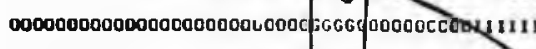
00000000000000000000000000 ;66 600000000000000 0000000000000000000000000 of:GGG 0000000C00000 is

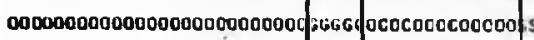
00000000000000000000000000366600000000000000 00000000000000000000000000 GG6G 0000000000000 , 00000000000000000c c000000d G6G C000000000000 कs 00000000000000000000000000 GGGG00000c00000000 5 s:

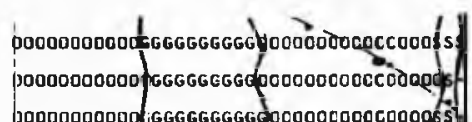
poo0000C000tGGGGGGGGG0000000000000000as,

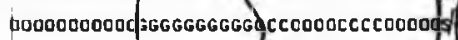
p0000000000):G6GGGGGGCOOOCC00000000000 है है:

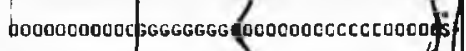
00000000000/GGGGGGGGG5,000000000000gós b0000000000 3GGGGGGGG0000000COOCOOgCods: 0000000000d GGGGGGGGG\%00000000000000gs noD00000000́GGGGGGGGG0000000000060000ns:

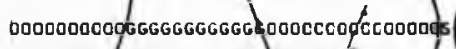
0000000006GGGGGGGGGGG\%00000,0000000o s

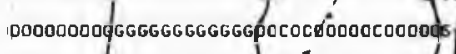
D00000000GGGGGGGGGGG ccobocooccoooofs

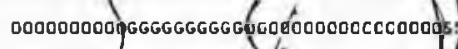
D0000000U GGGGGGGGGGGgCCO000006000000/sf

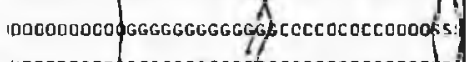
UD0000000 QGGGGGGGGGGFDO0000000000000ds

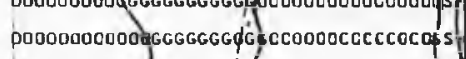
OUDOD000000 ofGGGGGG

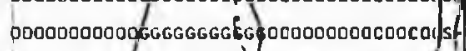
DOQ000000 OEGGGGGGGGGCOQ0000000000000|s:

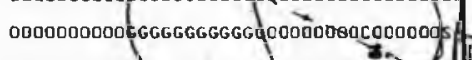

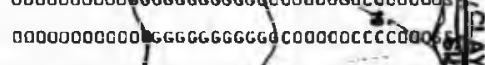
0000000000 ofGGGGGGGG000000 0000G0000d

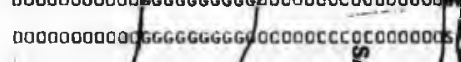

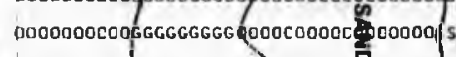

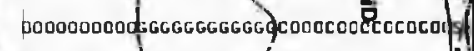

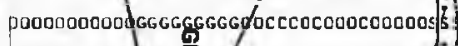
poodouacoootgGC

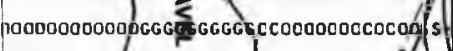

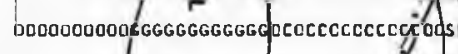
DO0000000U6GGGGGGGGGG O00000000/:GGGGGGGGGGG DOEDC00000000 O s:

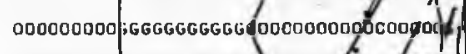

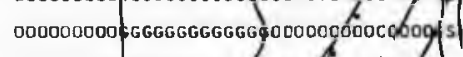

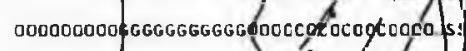
00c000000 GGGGGGGGGGapt ptcooboc proooc s

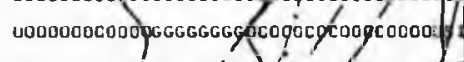

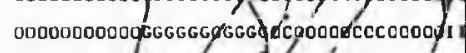
0000000000 GGGGógGGG

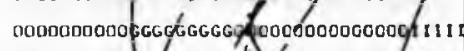

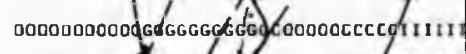
no000000006:GGGGGGG $0000000000 / 111111$

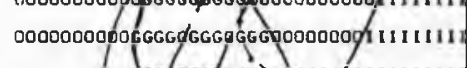

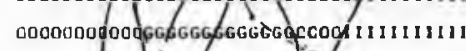

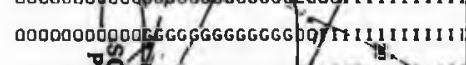

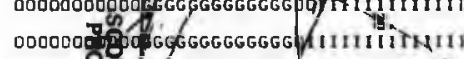
000000 Of 000000融,GGGGGGGG/111111131 0000000c 0000000000-GGGGGGGG IIIIIIIIIIIIIII 00000000000dgGGGGGo क111111111111111

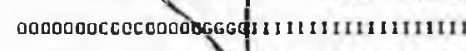

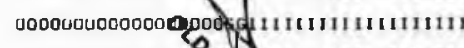
$00000000000000000051111+1111111$

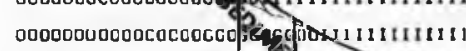

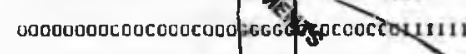

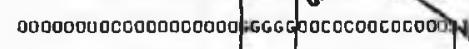

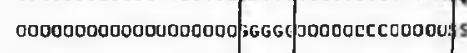

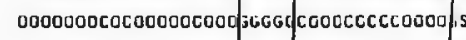

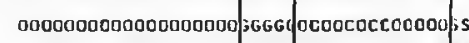
D000000000000000000/3GGG00000000000000)is

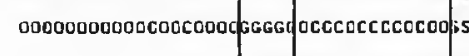

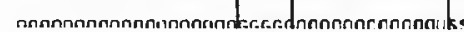

co00000d;GGGG/00000ancooood D0000000/36GGGro00000000000 $00000 \mathrm{CO}$ क;GG

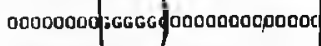
ODODO000);GGG :0000CECCOOCOC 00000000/:GGGG0000000000000 0000000 中GGGG ouco000000000d

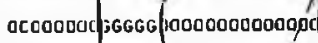
00000000/3GGGG Ja00000cooqbac 00000000;:GGGG:000ccocco600 00000000pGGGG \$0000000 ocoonouopsGGGhooocog000000r D0000000;GGGG LU0000ช́์0000000 acoonoougGGGGdooṕoccccooooc 00000006GGGGGodónooco00000 0000000 ofGGGggoo00coccoocos 0000000otGGGapopo0000000000

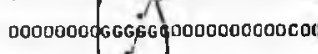
0000000 CGGFGG 0000000000000 0000000r GQGG 1000000000000

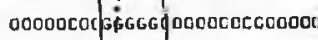
Do00000dedGg 00000000 a GGogd cococcocccoof

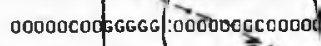

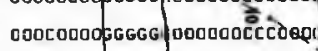
100000000tGGGG CoCODO0LCOOO ocooceodgagedecececececea 000000udGGGG \$000000000000

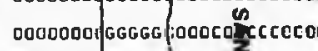

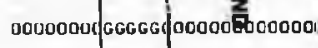
oovoonoc arogo oconooncooca

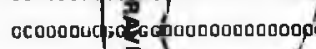
D0000000 iGGGGCODODOOCCOOp 00000000);GGGG(cosccecódo 00000000 ;GGGG doconoocéfoboa occooouc GGGGG o000 obfonod o000000r GG GG fococe d́focogáo ocodocodgGgGailocóccecclood ou000000 GGGG \$oćóoccocooto ono0000 oGGGG offocecolecoloco 0000000r GGGG pooogccotoooo onoonond sGg. acooocuc);ct:GG goodtoooogh 1

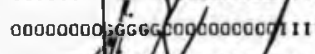

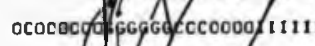

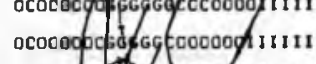

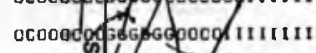
o000Q0

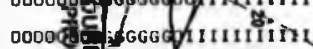
ccood. ocondog oGg 111111111111 ocooocacoghtint111111

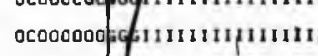

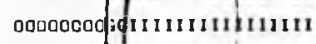
0000000 q $111111111+1111$

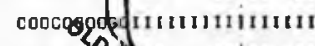

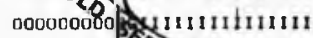

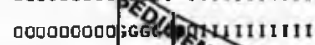

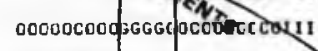
$00000000 \mathrm{c}$ GGGGCh00000000000 000000000/;GGGeco00000c0000

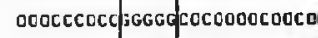
OB0000000/3GGG J00000000000 00000000 OFG GGG J000000CC000 coocococojgGG Jcooccoccoco nnemnroneutageghegececteono 


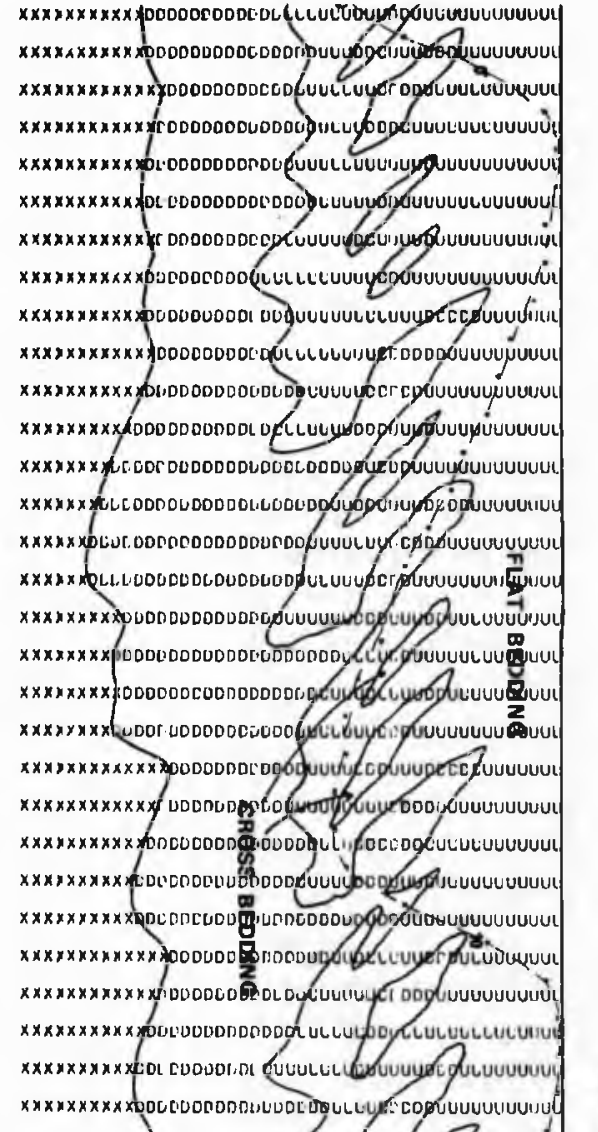

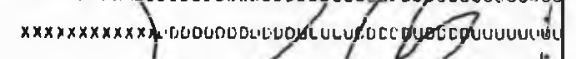

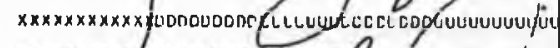

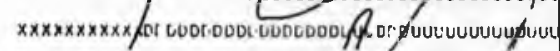

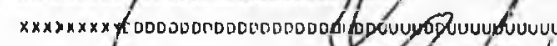

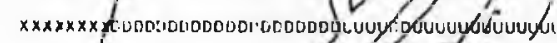

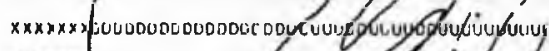

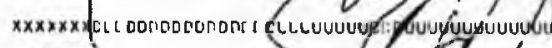

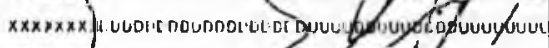

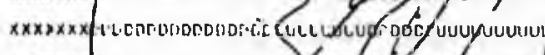

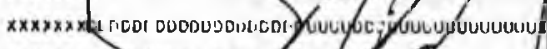

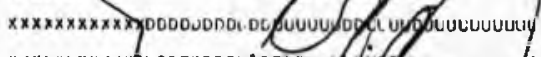
$x \times x \times x \times x \times x \times$ pl.

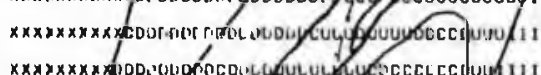

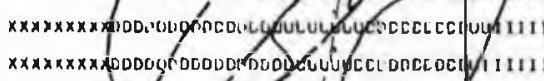
$\times x \times x \times x \times x \times$ fod

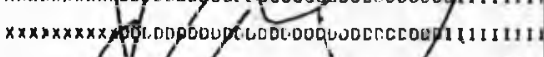
$x \times x \times x \times x \times x$ for for

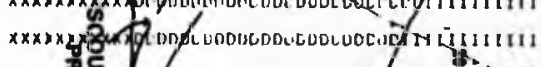

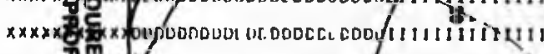

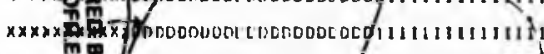

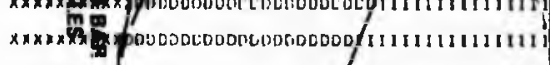

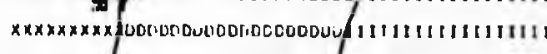

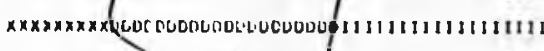

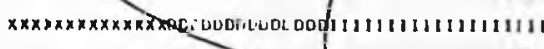

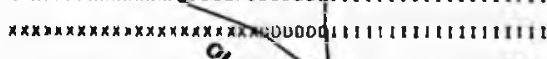

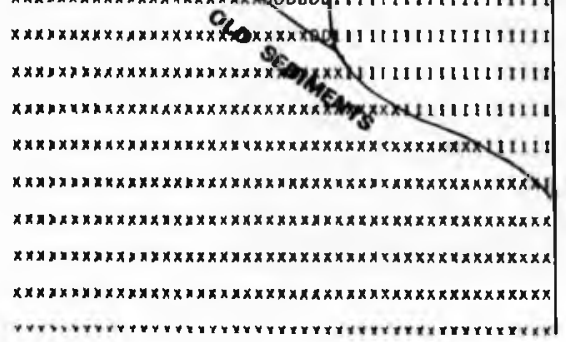

$x \times x \times x \times x \times x$ pDonèd

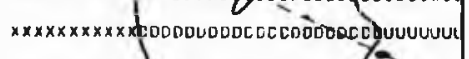

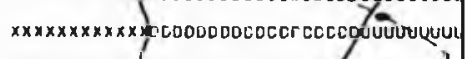

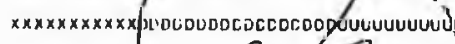

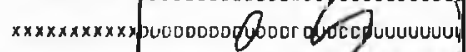

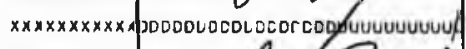

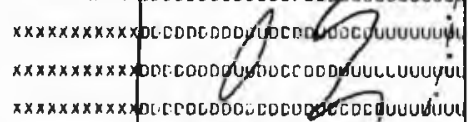

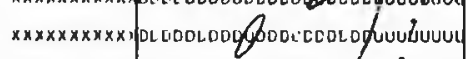

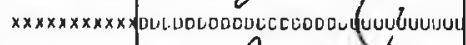

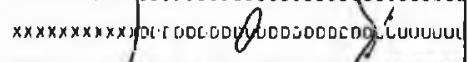

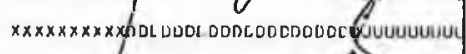

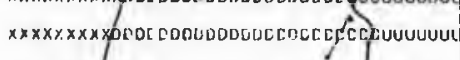

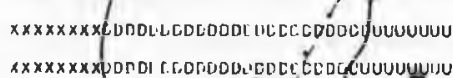

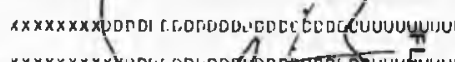

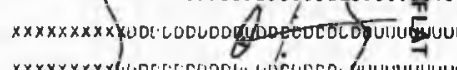

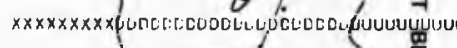

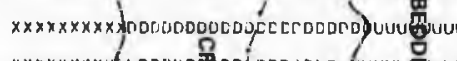

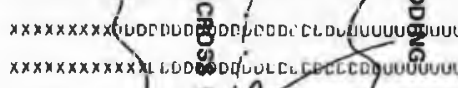

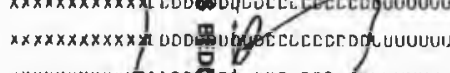
$x \times x x x \times x \times x x$ yor cro

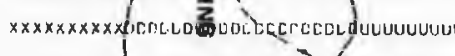

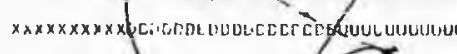

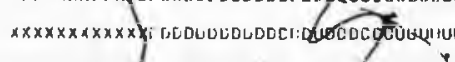
$x \times x \times x \times x \times x \times x \times$ Q

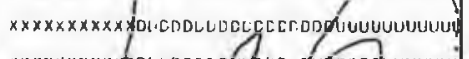$$
=x \times x x y
$$

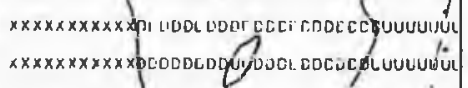

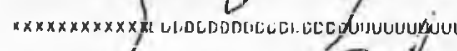

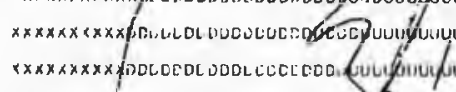

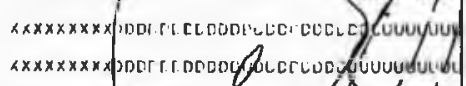

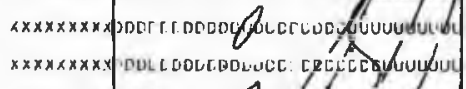

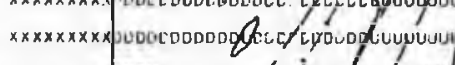

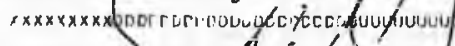

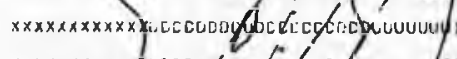

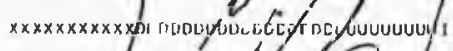

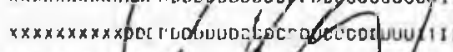

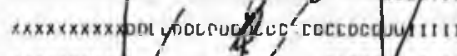

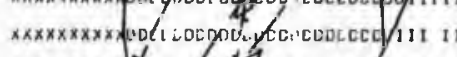
$x \times x \times x \times x \times y$ ooc 0 on

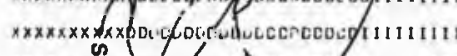

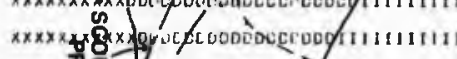

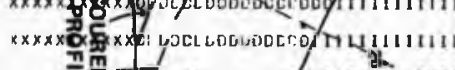

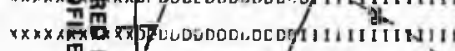

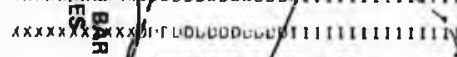

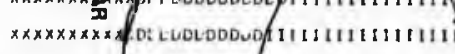

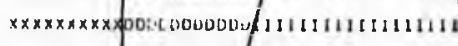

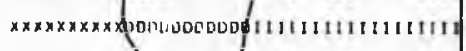

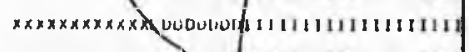

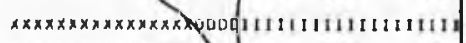
$x \times x \times x \times x \times x \times x \times x \times x \times x$ An⿴囗十)

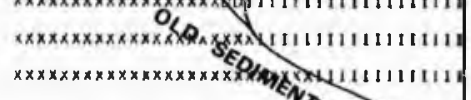

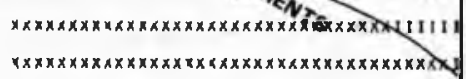

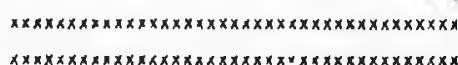

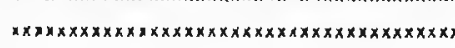

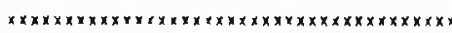

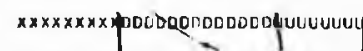
$x \times x \times x \times x \times$ podoacroonosecqueuueut $x x x x x x x x x p 000065000 c 00$ quevouvue

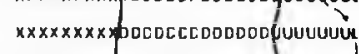

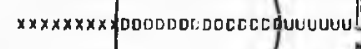

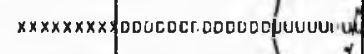
$x x x x x x x x$ y

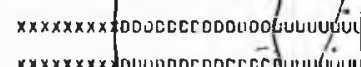

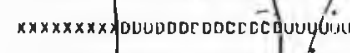

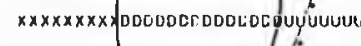

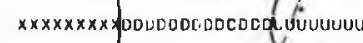

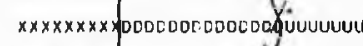

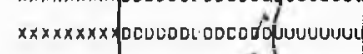

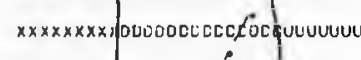

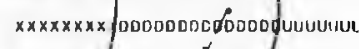

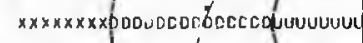

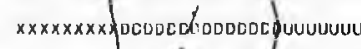

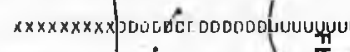

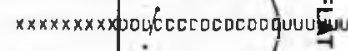

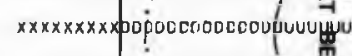

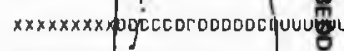

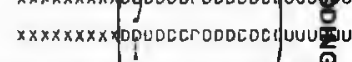

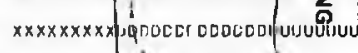

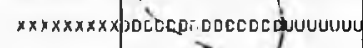

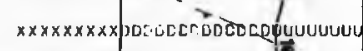

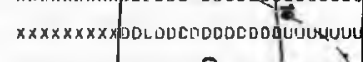

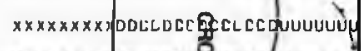

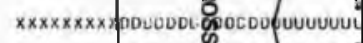

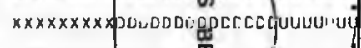

宏 $\vec{a}$

$x \times x \times$

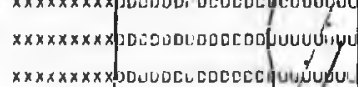

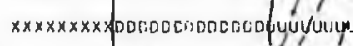

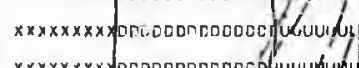

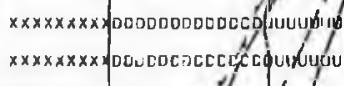

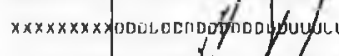

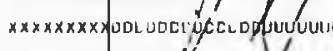

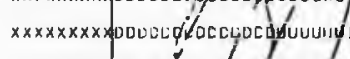

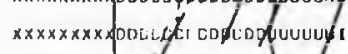

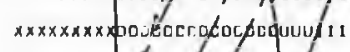

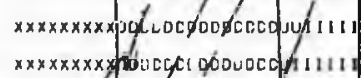

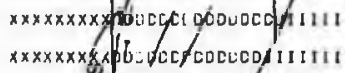

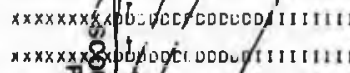

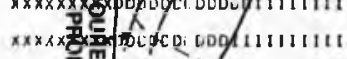
$x \times x \times$ 竞

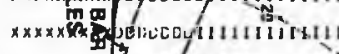

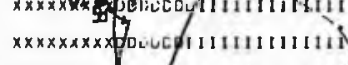

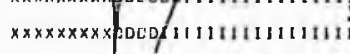
$x \times x \times x \times x \times x \times 001 / 1113+113111111$ $x \times x \times x \times x \times x$ ) $111+1111+1111$

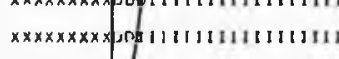

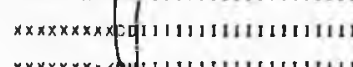

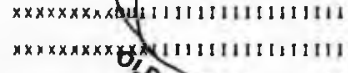

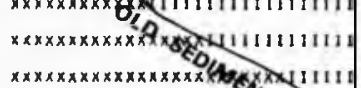

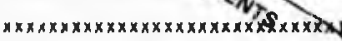

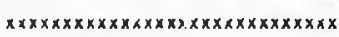

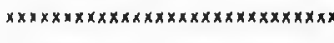

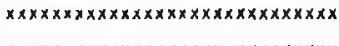




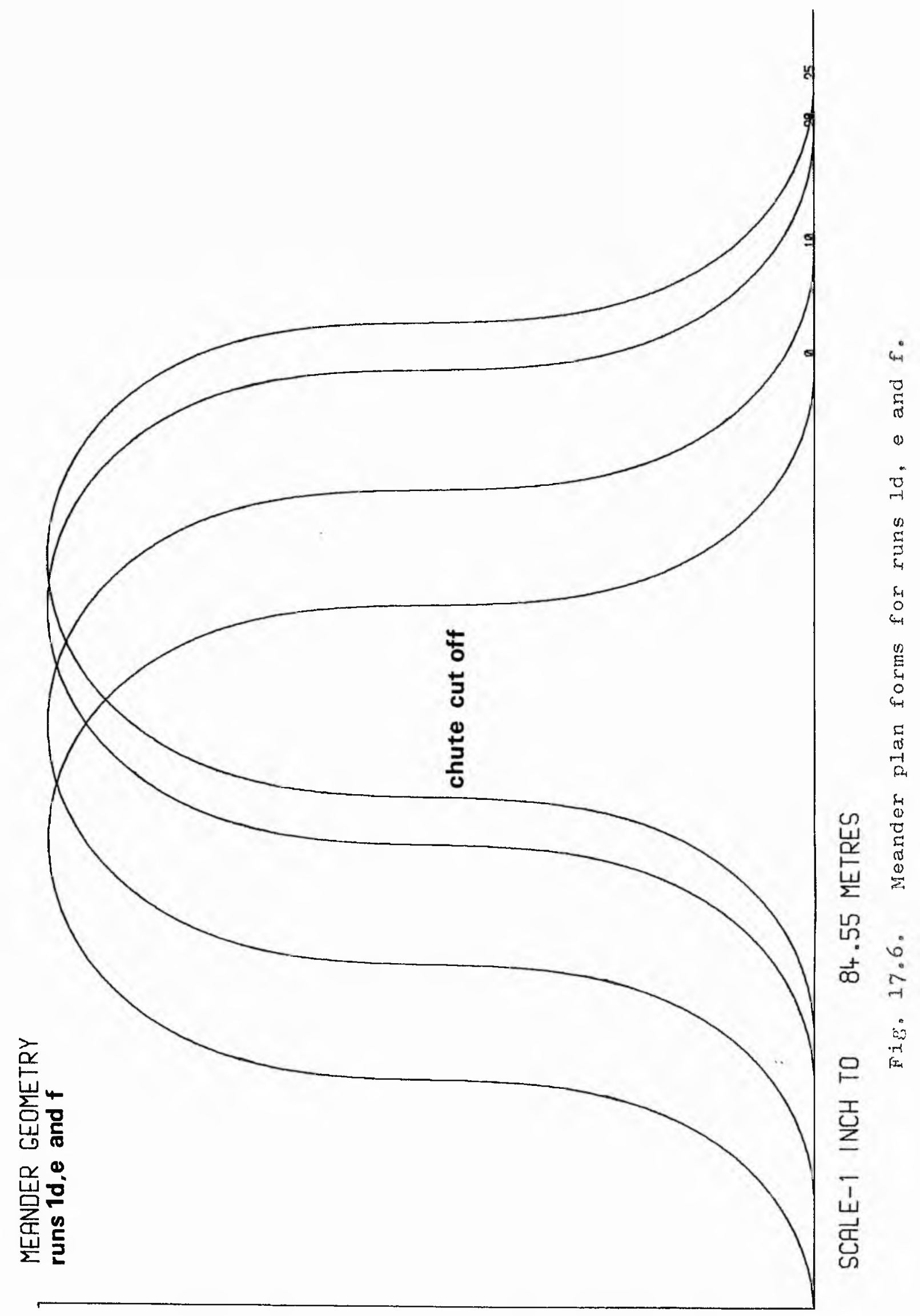


talweg depth. Similarly with the sand-silt boundary, except in case ld where there is very little relief at all. In some cases, each separate undulation may mark the separate successive flood periods, particularly in the case of silt-clay and sand-gravel boundaries. As before, increasing scour depth involves increasing the thickness of sand and silt, with gravel thickening a relatively greater amount than the sand and silt.

The sedimentary-structure sections for runs $1 d, e$ and $f$ show a very marked variation in the flat bedding-cross bedding facies boundary with scoux depth (fig. 17.5). In case 1d the relief is only a few percent of the unscoured talweg depth, but as scour depth increases, the relief increases until a complex system of interfingering and associated lensing becomes evident. The scale of the interfingering in case if is comparable with the maximum unscoured channel depth. The trend of the lensing and interfinfering is associated with the scoured bar profiles. The relief and degree of lensing and interfingering is probably exaggerated a certain amount due to approximations involved in the mathematical model and computer program. However it seems likely that under similar conditions in the natural situation a noteworthy degree of scoured basal surface and facies boundary relief would be present, with associated facies lensing and interfingering. Such features would be associated with discrete seasonal periods of erosional and depositional activity. The thickness of flat beding increases relative to cross bedding as scour depth increases. Fig. 17.6 shows the meander movement in plan relating to these sections.

The grain-size distribution cross-sections of $l_{g}, h$ and $i$, shown in $f i g .17 .7$, correspond to the cases with average downvalley bank migration of about $48 \mathrm{~m}$./year. The basal scoured surface and sand-gravel facies boundary increase in relief from virtually nil up to about $35 \%$ of the unscoured talweg depth, with increase 


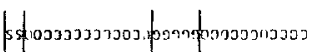
"\$

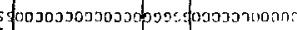

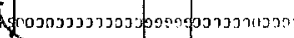
4 1 11

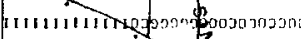
111,m!1 1 1

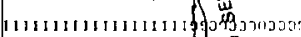

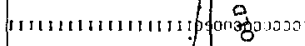

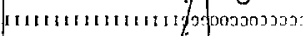

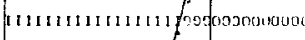

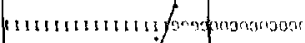
1111 แ11 แ แ 11111แ11 ห11 -11

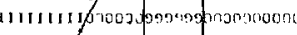
111114 II11

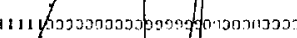
שו

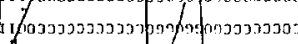

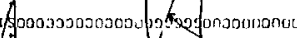
-

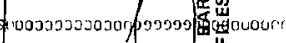

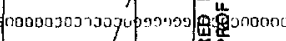
רכבכבר 0000ว כ3 600

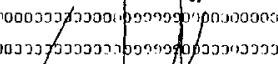
ס ס 5. ه

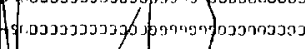

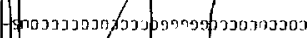
בכ חо

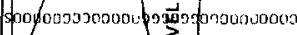

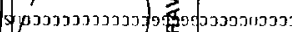

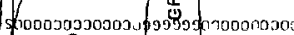
\&h000ว

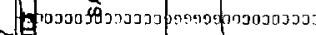
䊈 o. 月-

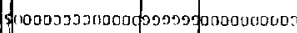
A)

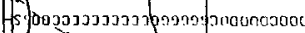
Sh \$ ด

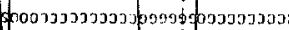
בכ" \$ \$ מכט |

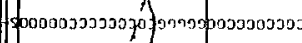

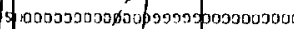

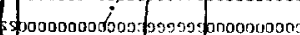

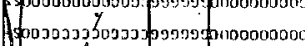

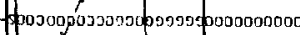

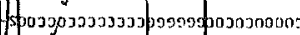

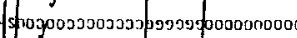

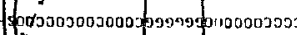

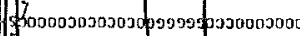

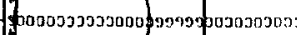
(9000000000000

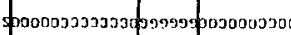
\$ Н

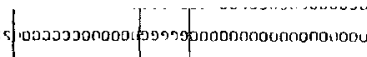
5) 0.3วכว

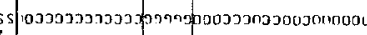

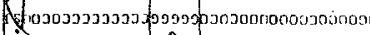
तो

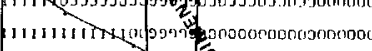

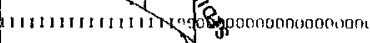

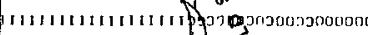

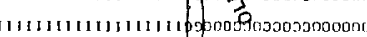

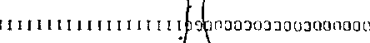

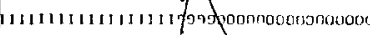

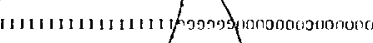

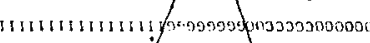

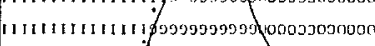

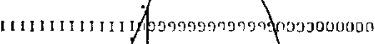
1 11111111111 1. 1111111111 \%00.

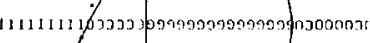

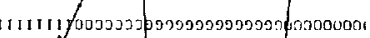

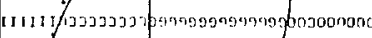
II 1 il 1 / 11 : 1 מ. 1

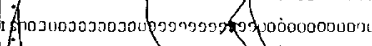
is s) 5. -

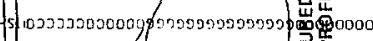

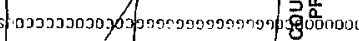

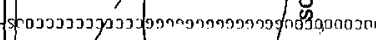
Hะว

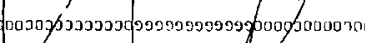

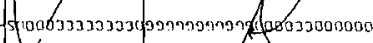
s do 2. A בטים \$1000303000000 s5

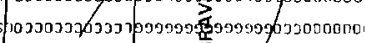
1100030000000

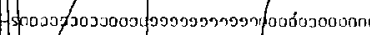

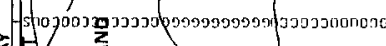
ล -

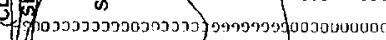

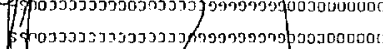
A

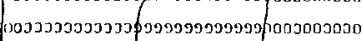
-

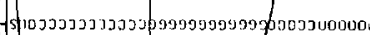

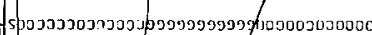
As

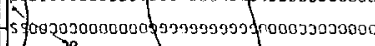
sh

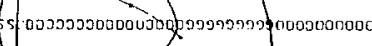
100005000000150 רon

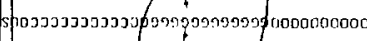

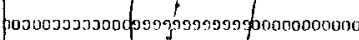

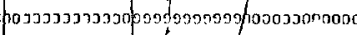

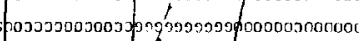
מั sh

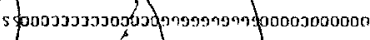
s

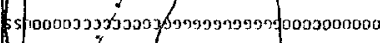
\$

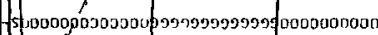
\$h

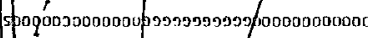

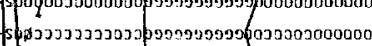

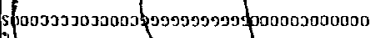
4. s.

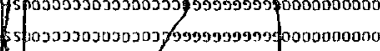
5 100003000ว303 99999990999900000000000 年

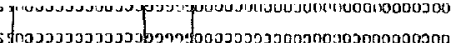
:

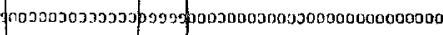
A 11 T

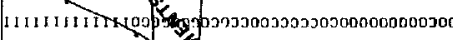

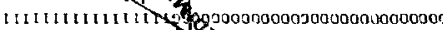

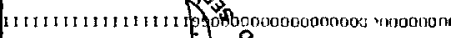

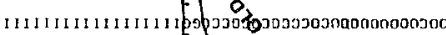

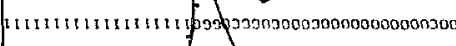

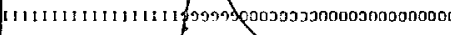

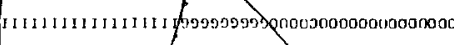

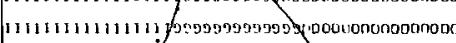

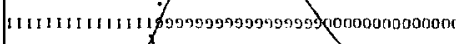

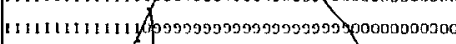

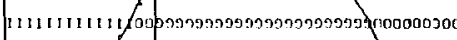

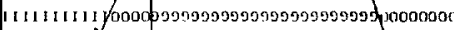
HIIIIII I ז111111303ว 11 11 1 211 11 11 11 (000500ก0030 (1)

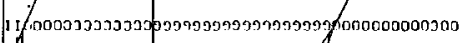

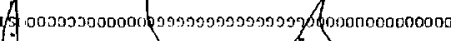

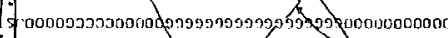

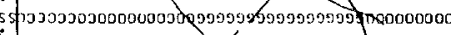
As (4000ว

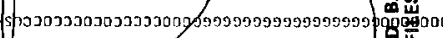
H. \& 10 ב0อวองอ3

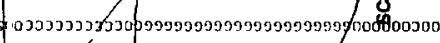
s

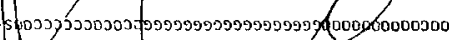

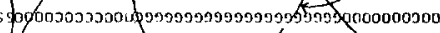
5 他 S

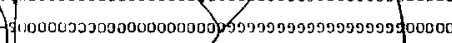
-

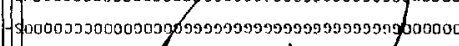
Hกอว H. \$ s)

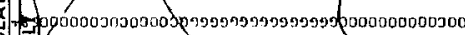
\%) St.

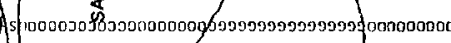
-spoכว - 5 p H -

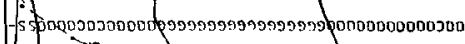

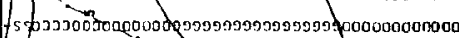

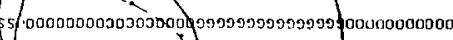
\$ \$ S\$1000วบ30530303693990909090000900600000000000

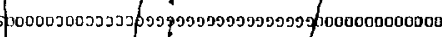
sด 100000000000 व s -5 po

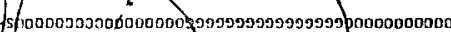
\$ \$0000วด90000อง $\$$ \$ \$ As shdo0000000000 f) - fono050000000Jd f (\$) 55000033ว03000000\%

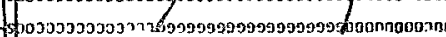




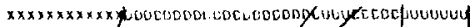
$x \times x \times x \times x \times x \times$ uet

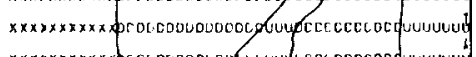

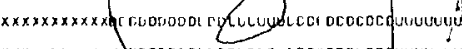

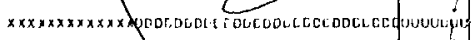

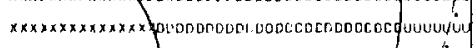

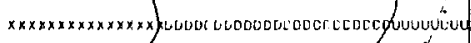

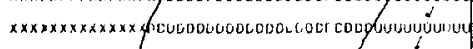

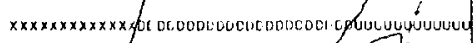

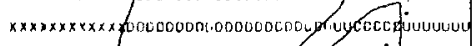

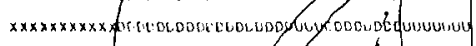

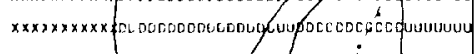

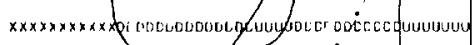
$x \times x \times x \times x \times x \times x \times x$ yocosoch LL. $x \times x \times x \times x \times x \times x \times x \times x$ (re floor nophood bor.

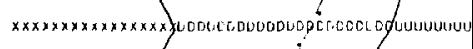

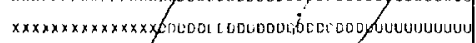

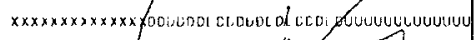

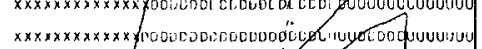

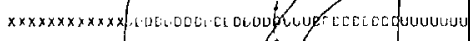

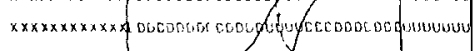

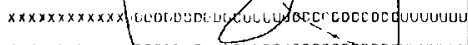

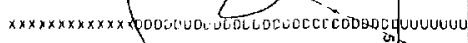

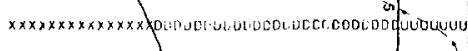

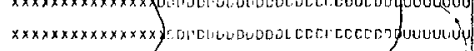

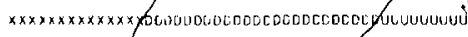

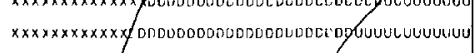

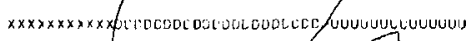

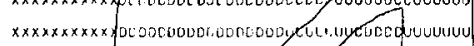

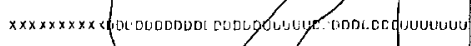

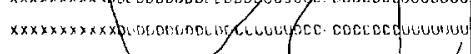

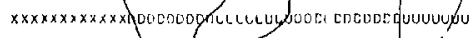

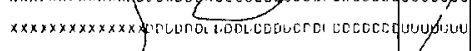

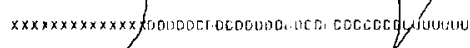

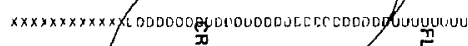
$x \times x \times x \times x \times x$ fordonouon

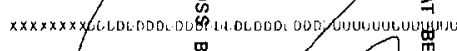
$x \times x \times x \times x$ Lut vocosonco

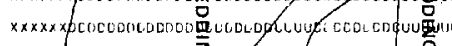

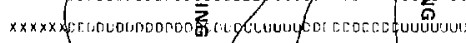

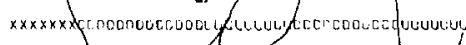

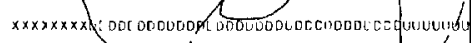

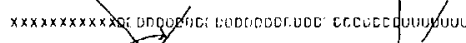

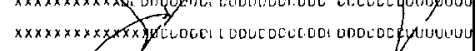

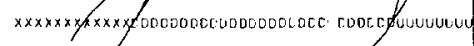

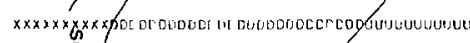

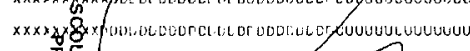
$x \times x \times$ 蛋 $x \times x \times m$ in

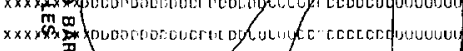

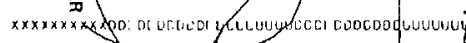

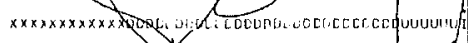

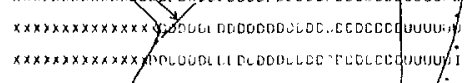

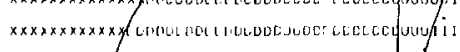

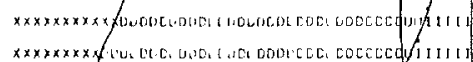

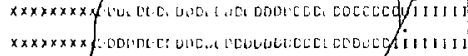

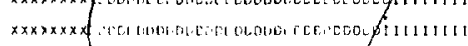

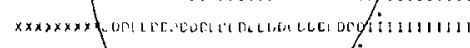

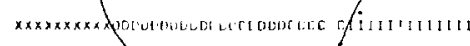

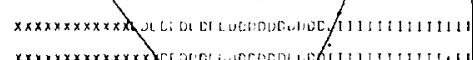

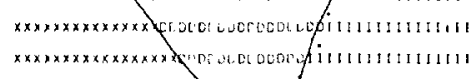

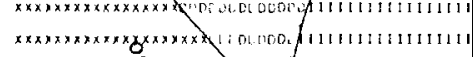

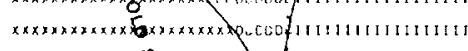

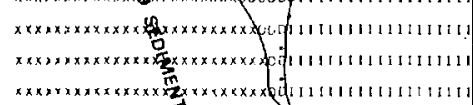

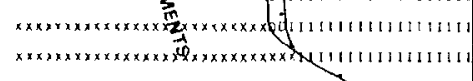

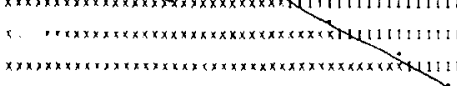

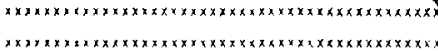

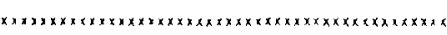

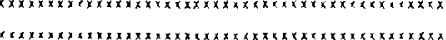

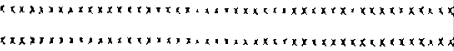

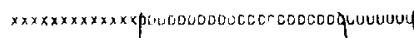

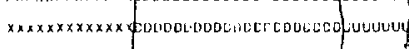

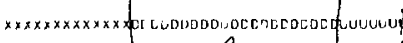
$x \times x \times x \times x \times x \times x \times x$ fovo00000 of foocr. 000000 quvuvue

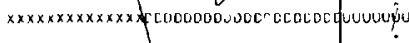

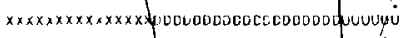

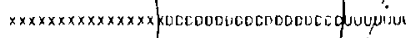

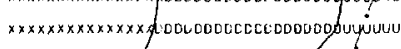

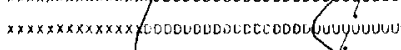

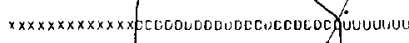

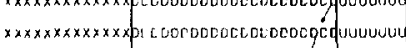

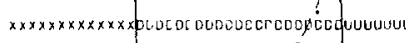

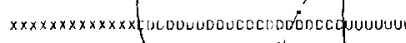

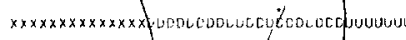

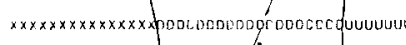

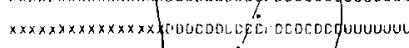

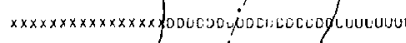

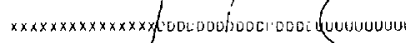

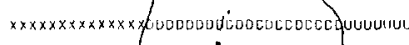

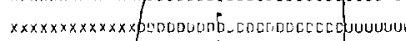
$x \times x x x \times x \times x \times x \times y$ ore

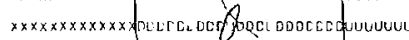

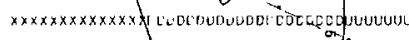

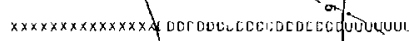

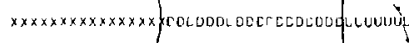

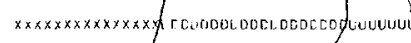

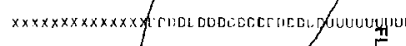

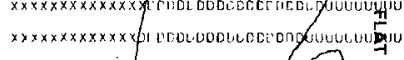

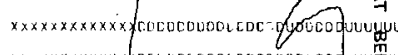

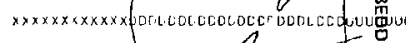

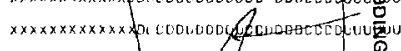

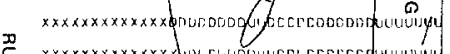
$\sum_{=}^{\pi}$

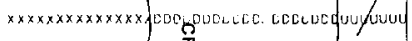
$x \times x \times x \times x \times x \times x \times x \times x$ o

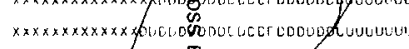
$x \times x \times x \times x x x x x$ for

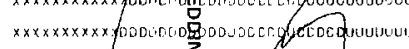

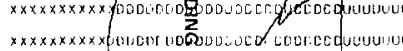

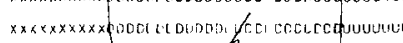

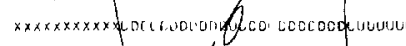

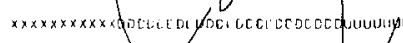

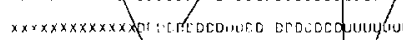

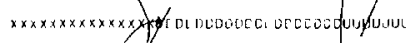

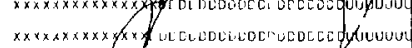

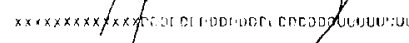

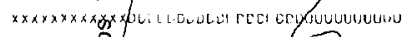

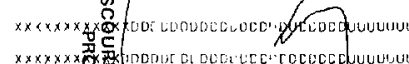
$x \times x \times x \times x$ 鱼 $x \times x \times x \times$ Fen
$x \times x \times x \times$

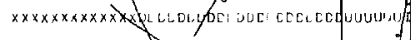

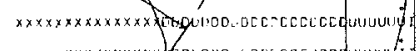

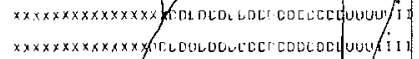

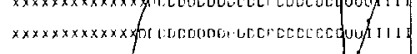

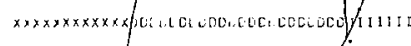

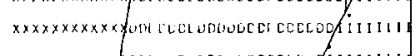

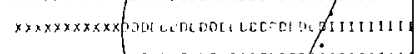

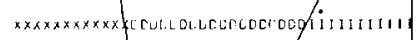

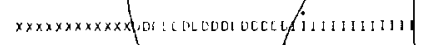

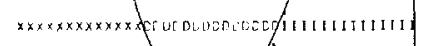

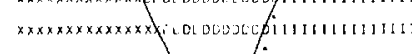

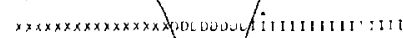
$x \times x \times x \times x \times x \times$ 量 $\times x \times x \times 40000111111111111111$

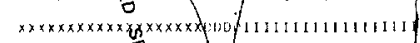

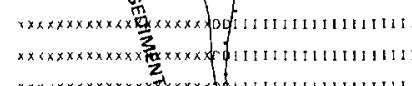

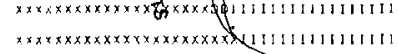

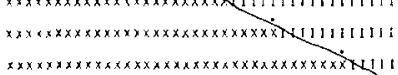
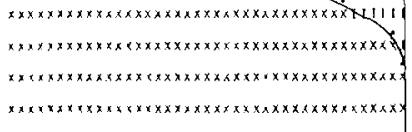

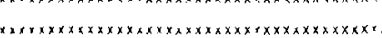

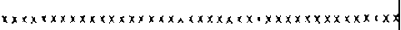

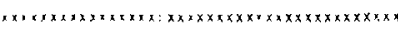

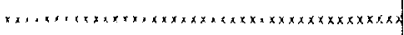

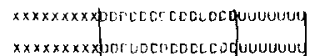
$x \times x \times x \times x \times x$ pojocer cocecc

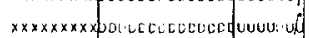

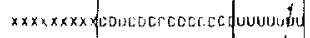

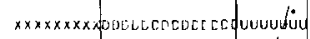

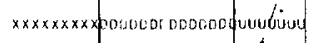

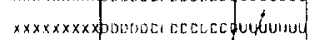

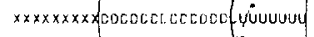

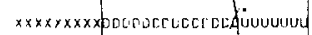
$x \times x \times x \times x \times x$ pouccoccecolón duvuueruy

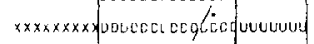

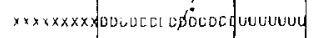

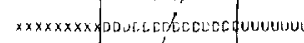
$x \times x \times x \times x x$ oristifí acoloo quevuuu

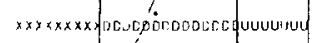

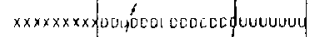

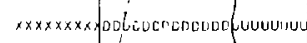

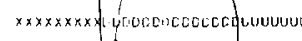

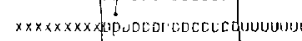

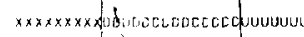

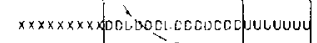

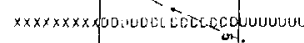

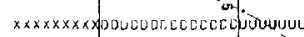

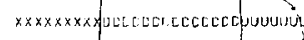

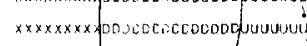

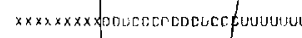
$x \times x \times x x x x \times$ poicecr oocr coluuvesures $x x x \times x x x x x$ bin tecteccoos huvuuter

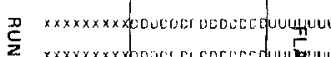

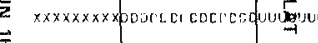

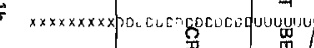

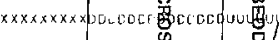

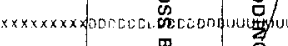
$x x x \times x x x \times x \times$ xisil.

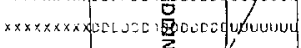

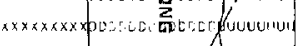

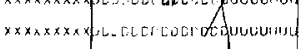

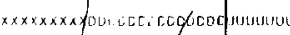

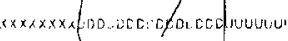

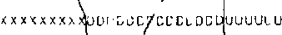

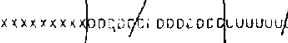
$x \times x \times x \times x x$ for poc cocudoon wous

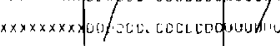

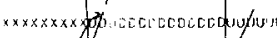
$x \times x \times x \times x \times x$, $x \times x \times x \times$

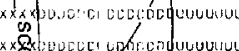

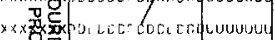

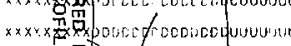
$x \times x \times x$ 然 $x \times x \times x \times x \times x$ cosfoc. sonucecustu if

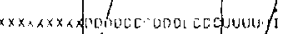

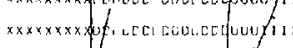

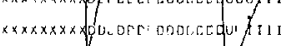

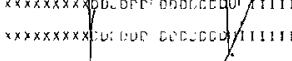

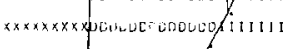

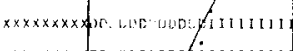

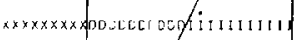
$x \times x \times x \times x \times x=0,100$.

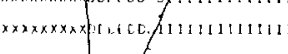

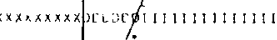

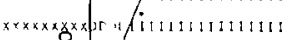

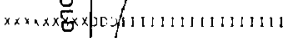

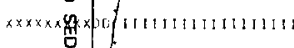
$x \times x \times x \times 2 \times 0$ of

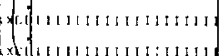
$x \times x \times x \times x \times x \times x+111111111: 11111$

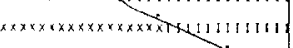

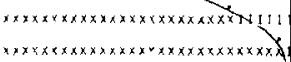

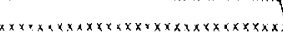

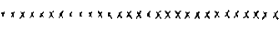

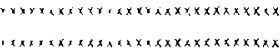

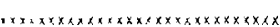
$\cos x \operatorname{sen} x \cos x \cos x \cos x \cos x \cos x \cos x$

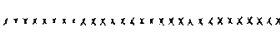




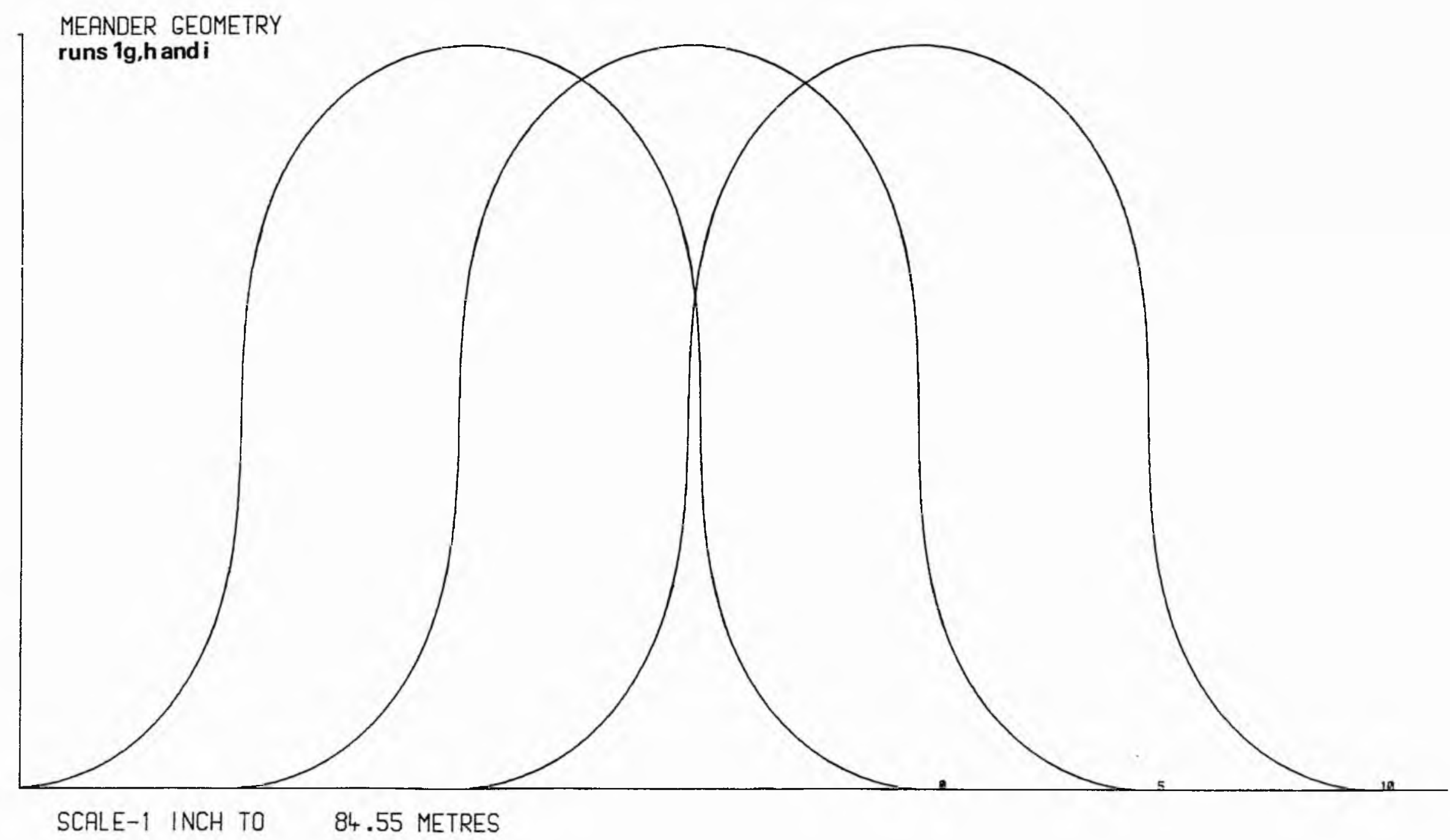

Fig. 17.9. Meander plan forms for runs $1 \mathrm{~g}, \mathrm{~h}$ and $i$. 
in scour depth. The relief of the clay-silt boundary does not vary and the regularly undulating relief is up to about 5\% of the unscoured talweg depth. Similarly, with the sand-silt boundary, except in the case $1 \mathrm{~g}$ where the boundary is essentially planar. Where any relief is developed it is of considerably greater wavelength than in the previous sections discussed, and each complete wave corresponds to a discrete flood period. It is noteworthy here that the peakedness of the depressions in facies boundaries is probably exaggerated due to the construction of the computer program, and becomes more so as the rate of migration increases. They should probably be nore asymmetrical with less steep riverward sides. Thus, in general, the degree of relief will not be quite as marked as shown. It is expected, nevertheless, that regular long wavelength undulations of grain-size boundaries and scoured basal surfaces will be present where bank migration is very rapid, each complete wavelength corresponding to a discrete period of erosional and depositional activity. By virtue of the scale of the undulations, these surfaces may appear broadly planar when seen only in limited lateral extent.

As scour depth increases in the sedimentary structure cross sections for runs $1 \mathrm{~g}, \mathrm{~h}$ and 1 , the flat bedding-cross bedding boundary develops from virtually planar to an undulating pattern with interfingering and lensing until, finally, large scale interfingering is present on a scale comparable with the maximum unscoured channel depth ( $1 \mathrm{ig} \cdot 17.8$ ). The disturbances in this boundary are again associated with seasonal floods, their trend corresponding to the scoured bar profile. The degrees of interfingering and undulation are again exaggerated, and in particular the upper, riverward boundaries of the interfingering flat-bedded areas are expected to expand riverward at the expense of the cross bedding. Nevertheless, a macked degree of undulation, interfingering and lensing is expected under similax conditions in 
the natural situation, despite the approximations nade. Silt, sand and gravel thicknesses increase as in the provious runs with increase in scour depth, and, in general, cross bedding thickens at the expense of the flat beds. Fig. 17.9 shows meander movement in plan associated with sections $1 \mathrm{~g}$, $h$ and $i$. Experiment 1 demonstrates that the relief and nature or the facies boundaries and exosion surfaces are dependent on the rate of bank migration relative to the depth of scour. 
As meanders migrate systematically downstream, point bax and overbank deposits are eroded by the channel of the upstream meander limb. If there is no net vertical deposition there will obviously be no preservation of sediment. If there is continuing net deposition (ageradation) the level of the upstream channel will be above the basal exosion surface of the bar sequence it is truncating. Whether the land surface is being raised during aggradation or whether net vertical deposition is being accommodated by subsidence with land level remaining constant, or a combination of both, is not relevant here. It is only necessary to know the relative levels of the basal erosion surfaces being considered. Bluck (1971) has indicated that an inordinate amount of aggradation would be required to preserve a complete point bar sequence between erosion surfaces, but that it is possible for a sma1l part of the sequence to be preserved. Furthermore, during aggradation, sediment deposited outside the channel is expected to be characteristically fine-grained silts, clays and some fine sands. As aggradation proceeds, therefore, the proportion of fine-grained alluvium exposed in the cut bank may increase, thus reducing the rate of channel migration calculated in the model. Experiment 2 is designed to examine the nature of the sedimentation in this 'moving phase' situation as the rates of downalley migration and aggradation vary.

The experiment consists of nine runs of the program corresponding to all possible combinations of three diffexent average rates of downvalley bank migxation and three different rates of aggradation. The input data that are different for each run are shown in table 18.1 , and correspond to average rates of bank migration of about 2,10 and $42 \mathrm{~m}$./year, and rates of agrradation of $0.001,0.01$ and $0.1 \mathrm{~m} . /$ year. A11 other parameters, shown in table 18.2 and 18.1 , are constant for all runs. The meanders are 


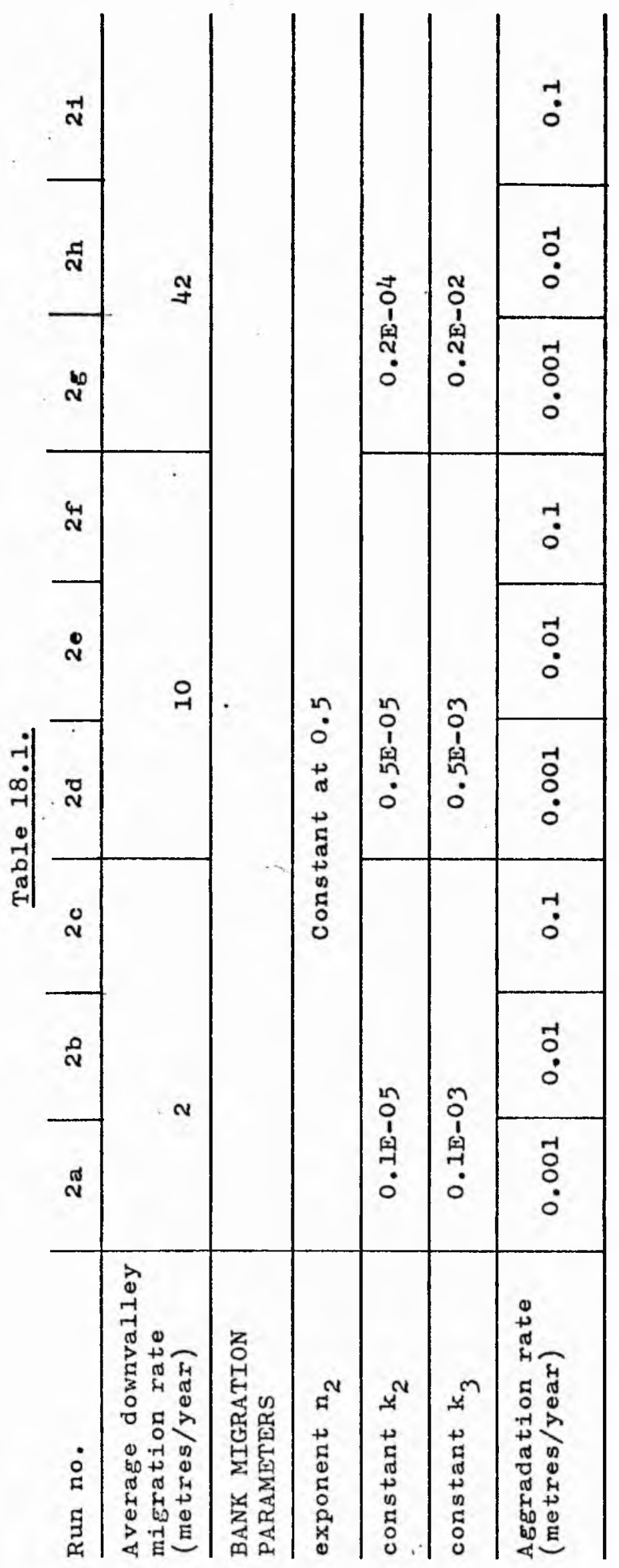


FLUUIATILE PROCESS SIMULATICN EXPERIMENT 2

CROSS SECTION PARAMETERS

METRES CELLS

1750.000

60.000

0.0

30.000

5.000

INITIAL OISTANCE OF INNER CHANNEL BANK FRON L.H.S
INITIAL BANKFULL STAGE MEASURED FROM SECTICN BASE

CELL SIZE IA VERTICAL $(Y)$ DIREC TION

CELL SIZE IN TORILONTALI $Z$ OR $X$ I DIRECTICN

METRES CELLS

$\begin{array}{ll}125.000 & 25 \\ 100.000 & 20\end{array}$

TOTAL HIDTH OF CHANNEL(h)

RIDTH OF FLOH BETHEEN IANER BANK ANO TALWEGIHI)

RATIO OF HI TO $h$

MAX IMUM FLOW DEPTH MCASURED ABOVE TALHEG

DENSITY OF SED IMENTARY PARTICLES

FLU $1 D$ DENSI TY

DARCY-WEISBACH FRICTION COEFFICIENT FCR DUNES ARD RIPPLES

DARCY-WEISBACH FRICTION COEFFICIENT FOR PLANE BEDS AND ONTIDUNES EXPONENT N1

$0.80 \mathrm{C}$

$2.65 \mathrm{C} \mathrm{GN/CN3}$

$1.0 C C G M / C N 3$

0.210

c. $15 \mathrm{C}$

1.000

SYNTFETIC HYDROLOGY PARAMETERSIUNITS NOT NECESSARYI

MEAN OF ALL CAILY MEAN VALUES

STANDARO DEVIATION OF OAILY MEAN VALUES

MEAN OF YT SERIES

DEYIATION OF YT SERIES

COEFFICIENTS IN AUTOREGRESSIVE MCOEL

FDURIER COEFFICIENTS FOR DAILY MEANSIAI

FOURIER CUEFFICIENTS FOR DAILY STD DEVIATICMSISAI

MAXIMUM VALLE OF QVOL

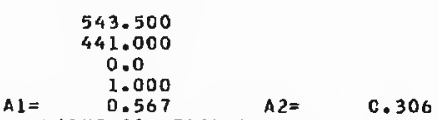

ARNONICS $A 2=\quad 0.30$

$-200.300 \quad 145.400 \quad-85.5 C C$

$-85.5 C \quad 58.0 C C \quad-39.800$

$\begin{array}{lll}-123.300 & 141.600 & -66.4 C C \\ & 105.700 & -48.2 C C\end{array}$

130000.000

SCOUR ANC FILL PARAMETERS

CONST ANT K4

STAMDARE CEVIATION OF ERROR TERM

0.0

0.0

CUT-CFF CONTROL PARAMETER

LIMITING WICTH OF MEANDER NECK

EXPONENTS IA NECK CUT-OFF RELATIGA

LIMITING SINUOSITY

LIMITING AMPLITLDE

EXPONENTS IN CHLTE CUT CFF RELATICN

125.000 METRES

EN $1=10.000 \quad$ EN2 $=10.000$

760.909 NETRES

00.000 ECTRES $=100.0 \mathrm{CO}$

A OCHNYALLEY SECTION 15 REPRESENTEO IN THIS TEST

DISTANCE OF LINE OF SECYION FRON PCIAT CF INFLECTICA CF LCCP IS 0.0 METRES

LEGEAD

Lower phase plane bed RIPPLES

DUNES PHASE PLANE DED

$\begin{array}{ll}\text { GRAVEL } & \text { G } \\ \text { SAND } & \text { C } \\ \text { SILT } & \text { S } \\ \text { CLAY } & - \\ \text { OVEREAAK } & \text { F } \\ \text { DEPCSITS } & \end{array}$

$\mathrm{X}$

TIRE LINE BOBNE

PLANIMETRTC FORM OF MEANDER

METRES

HAVEL ENGTH

AMPLITURE

SINUDSITY

RACIUS OF CLHVATURE AT BEND AXIS

HICTH IIF MEANCER NECK

CHAANEL LENGTH ALONG MEANOER

PALLEY SLDPE

LONGITUDINAL hATEK SLRFACE SLOPE

1000.000

$909-2.000$

217.571

2000.000

0.00010000

0.00005000

SELECTEO GEUHETRIC RATIUS

HAVELENGTH TO RADIUS OF CURVATURE

WAVELENGTH TO CHANNEL WIDTH

RADIUS OF CLRVATURE TO CHAMAEL hIDTH

AMPLITUCE TC CHANNEL HIOTH

4.596
8.000
1.741

1.741
6.087

Tab1e 18.2. Initial data for experiment 2. 
assumed in dynanic equilibrium with a sinuosity of 2.0 . No scouring and filling was assumed in order to simplify examination. of the cross sections. The cross sections were defined in the downvalley direction (TWO-CHANNEL DOWNVALLEY SECTIONS). In this experiment no disc was used, the cross sections were made up of 350 by 60 cells, and $182 \mathrm{k}$ bytes of core store were required. Average running times were $2-3$ seconds/time increment.

Unfortunately the model does not explicitly record basal erosion surfaces in the cross sections. These are normally inferred by cross cutting of previously specified floodplain deposits by the simulated point bar sediments. This may not be obvious when the channel is cutting across sediment of a similar type, especially in the case where the channel is truncating previously deposited point bar sediments. With the simplification of no scour and fill the basal erosion surfaces, if not already obvious after inspection of the sections, can be drawn as straight lines joining successive positions of the channel bed at the talweg, as picked out with time lines. Where scouring and filling is present the nature of the cross cutting would be more difficult to infer.

Runs 2a, b and c refer to average downvalley migration of $2 \mathrm{~m} . /$ year. After 100 years of simulation the channels have not moved through half a wavelength and therefore the upstream channel has not 'caught up' with the recent deposits of the channel immediately downstream (see fig. 18.1). Another 100 years or so would suffice. Vertical accretion over this period of time is $0.1,1.0$ and 10 metres respectively for runs $2 \mathrm{a}, \mathrm{b}$ and c. Projecting these rates of movement and aggradation in time, it is seen that about $0.2,2$ and 20 metres of sediment would be preserved between erosion surfaces. The two smaller rates of aggradation are not sufficiently great after 100 years to be recorded as vertical sedimentation on the simulated cross sections, figs. 18.2 


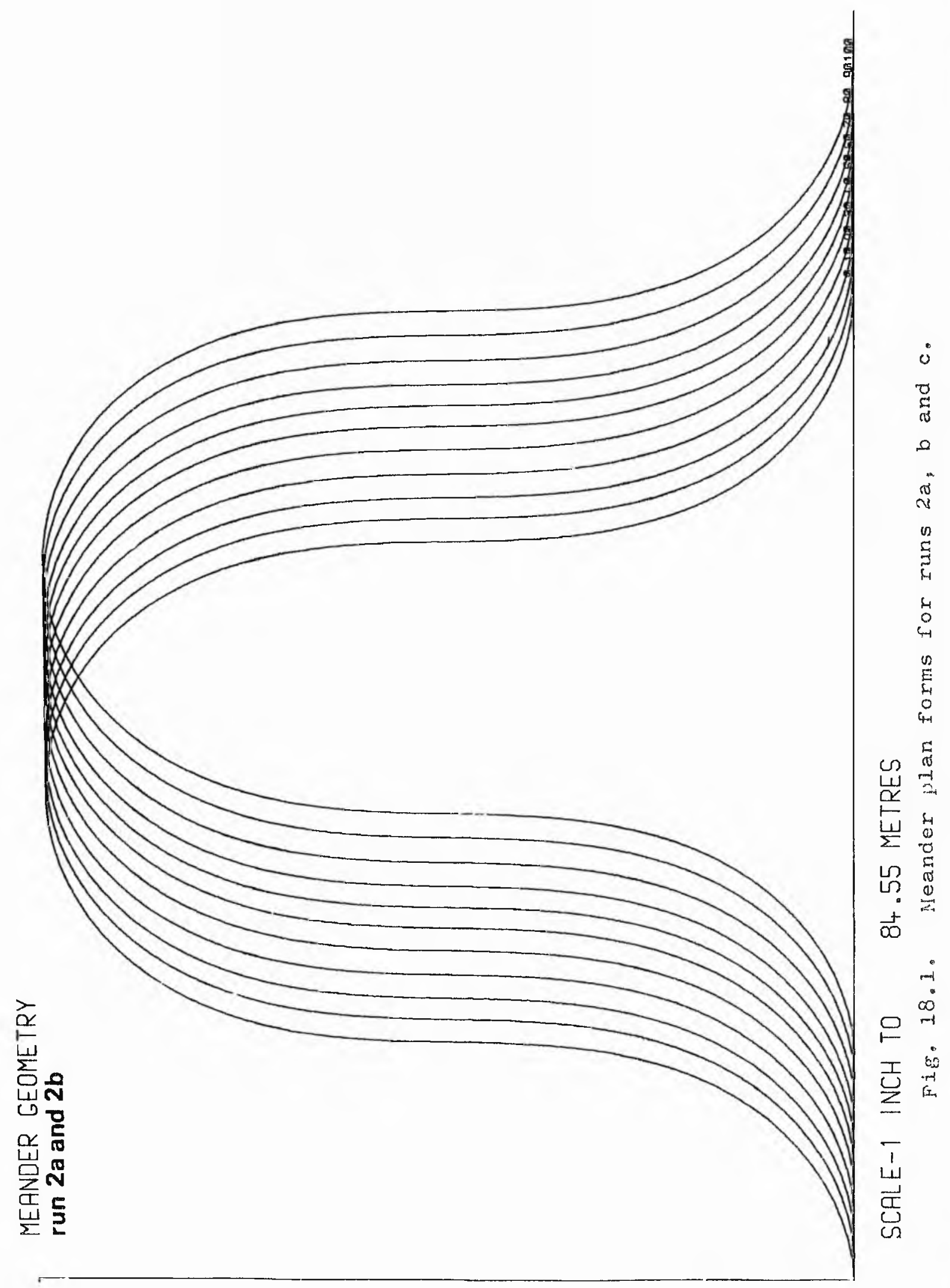




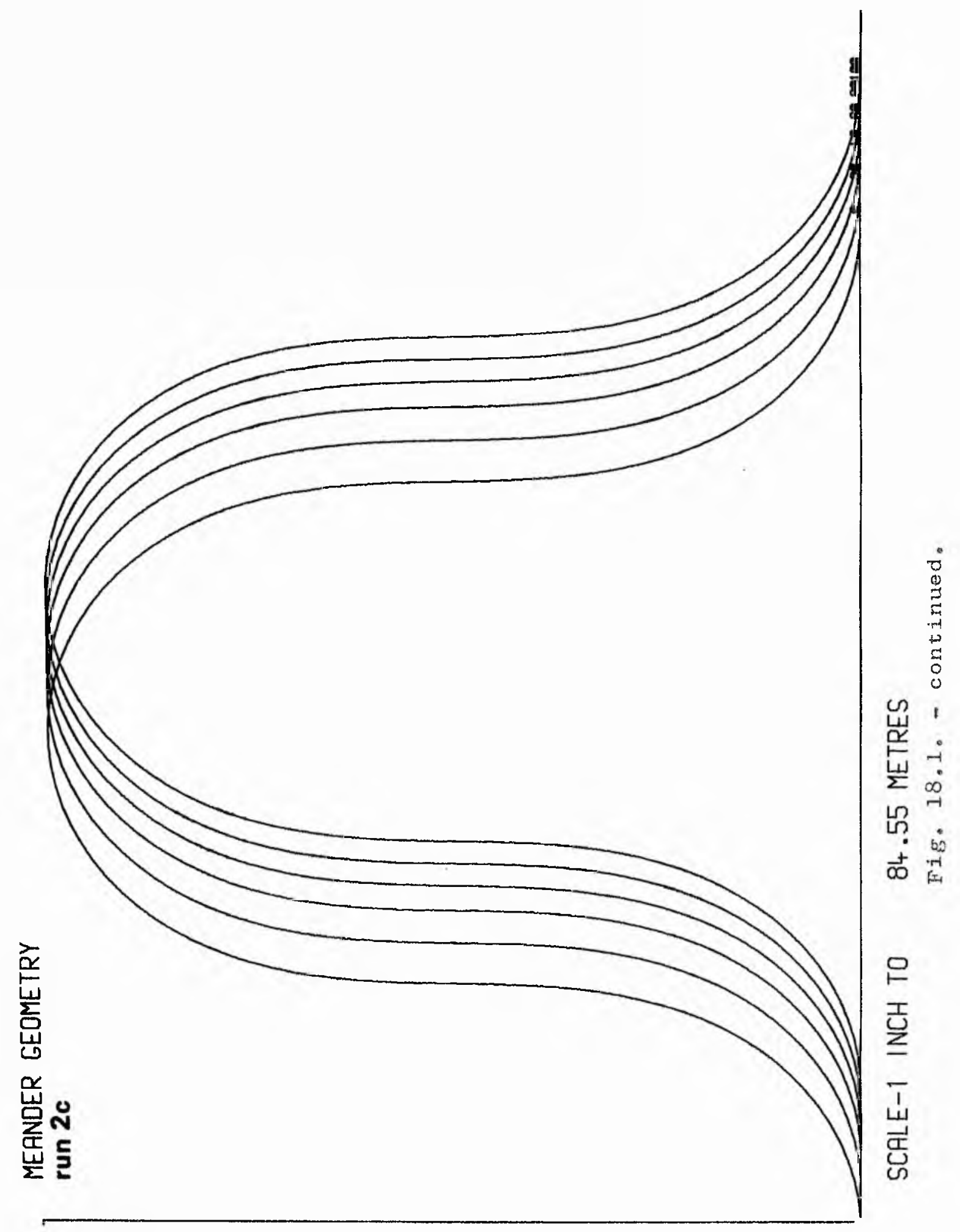


0000000000000000000000000000 -FFFFFFFF CCO00000C0000000C000C000000 C0000000C0000000C00000000000 : FFFFFFFF

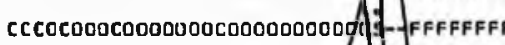
0000000000000000000000000000 SF FFFFFF 0000000000000000000000000000 S -FFFFFF

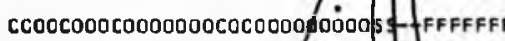
CC00000000000000C0000000DDOO S5-FFF EFF ccoccooacroononotococonocos 0000000000000000000000 000000000000000000000

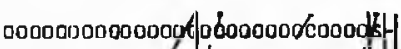

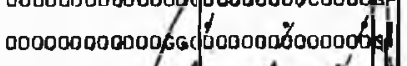
0000000000qtadecoodocacodods

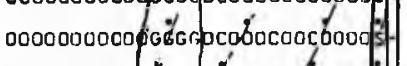

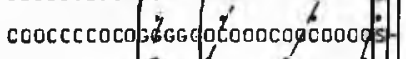

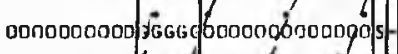

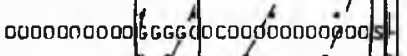

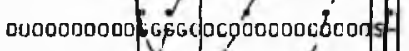

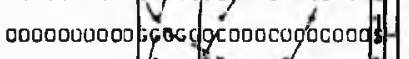

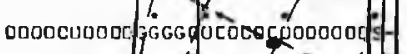

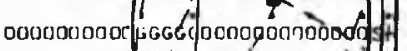

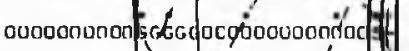

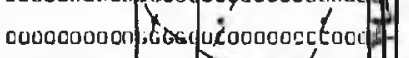
oovoununoo fGar focubecuóconods oouccuoocopgga ncooocuccesofo

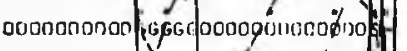

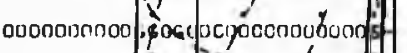

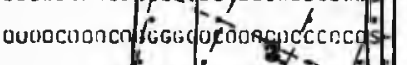

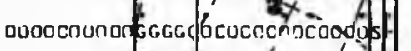

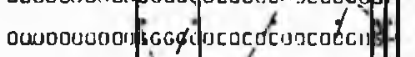
ounonenonos ;çacocónnonoybur

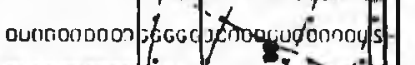

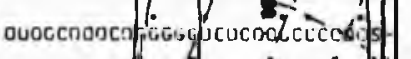

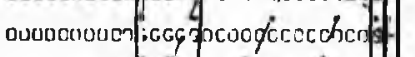

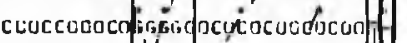

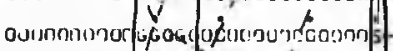

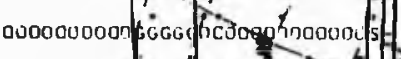

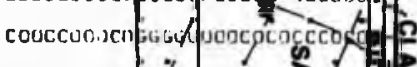

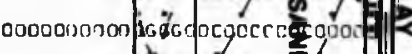

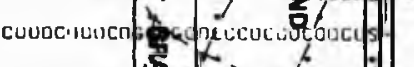

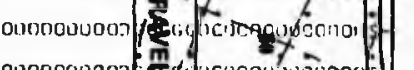
00000ก1า00ว

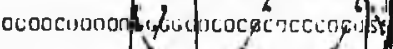

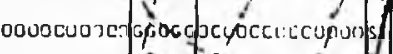

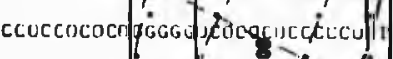

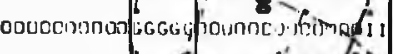

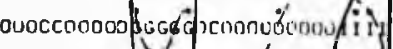

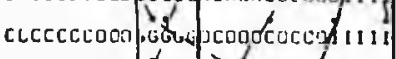

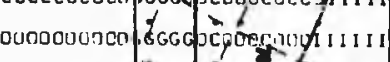

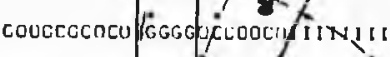

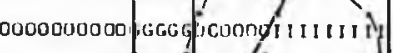

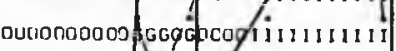

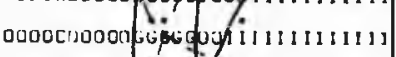

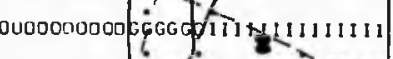

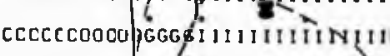

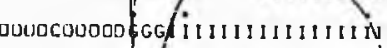

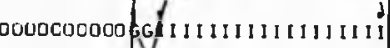
cuocccoocop Ni1111111111111

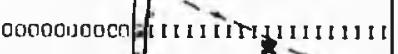

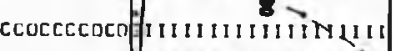
Qu00000000 111111111111111 tो 000000000000y+1t11111111111 00000000Cono000000 11111111111 auvo0o00co000000cooccodơ Duoocuonogodouodecuoccocucoun

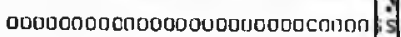
0000000000000000000000000u00/s oudooouncouvouducucodunudocus 
and 18.3 , because of the scale of an individual cell. All grain size and sedimentary structure facies boundaries and basal erosion surfaces on these particular sections appear as level, despite a very slight slope, and the effect of overbank deposits on rate of migration is negligible for this time span. With the largest aggradation rate (0.1 m./year) the overbank deposits can be seen as wedges of sediment, and all boundaries in the grain size and sedimentary structure sections are seen to slope up in the direction of migration. With this appreciable aggradation the rate of channel migration has slowed down (see fig. 18.1) and the slope of the facies boundaries and basal erosion surfaces increases with time. Thus in run $2 \mathrm{c}$ more than 20m. of sediment could be preserved, giving a complete point bar and some overbank deposits on top. Such rates of continuing aggradation are not common (see section 12.2 ) and the smallex rates are much more realistic. clearly with scouring and filling acting as well, the pattern of sedimentation would be very complex at the base of the bar sequences.

Runs $2 d, e$ and $f$ correspond to an average downvalley migration of about $10 \mathrm{~m}$./year. With rates of aggradation of 0.001 and $0.01 \mathrm{~m} . /$ year (runs $2 \mathrm{~d}$ and $2 \mathrm{e}$ ), about 0.04 and 0.4 metres of sediment, respectively, are preserved between the erosion surfaces as the time taken to move through half a wavelength is a little over 40 years (see fig. 18.4). The thicknesses deposited vertically were not great enough to appear in the cross sections for the time span considered, and there is no effect of these extensive thin sheets of overbank alluvium on bank migration rates. With an aggradation rate of $0.1 \mathrm{~m} . /$ year, run $2 f$ (figs. 18.5 and 18.6 ), the thickness preserved is between 6 and 7 metres, as the slowing down of bank migration, due to increasing thicknesses of overbank deposit, has increased the time span to over 60 years for movement of a half meander wavelength. The wedging of the overbank deposits 


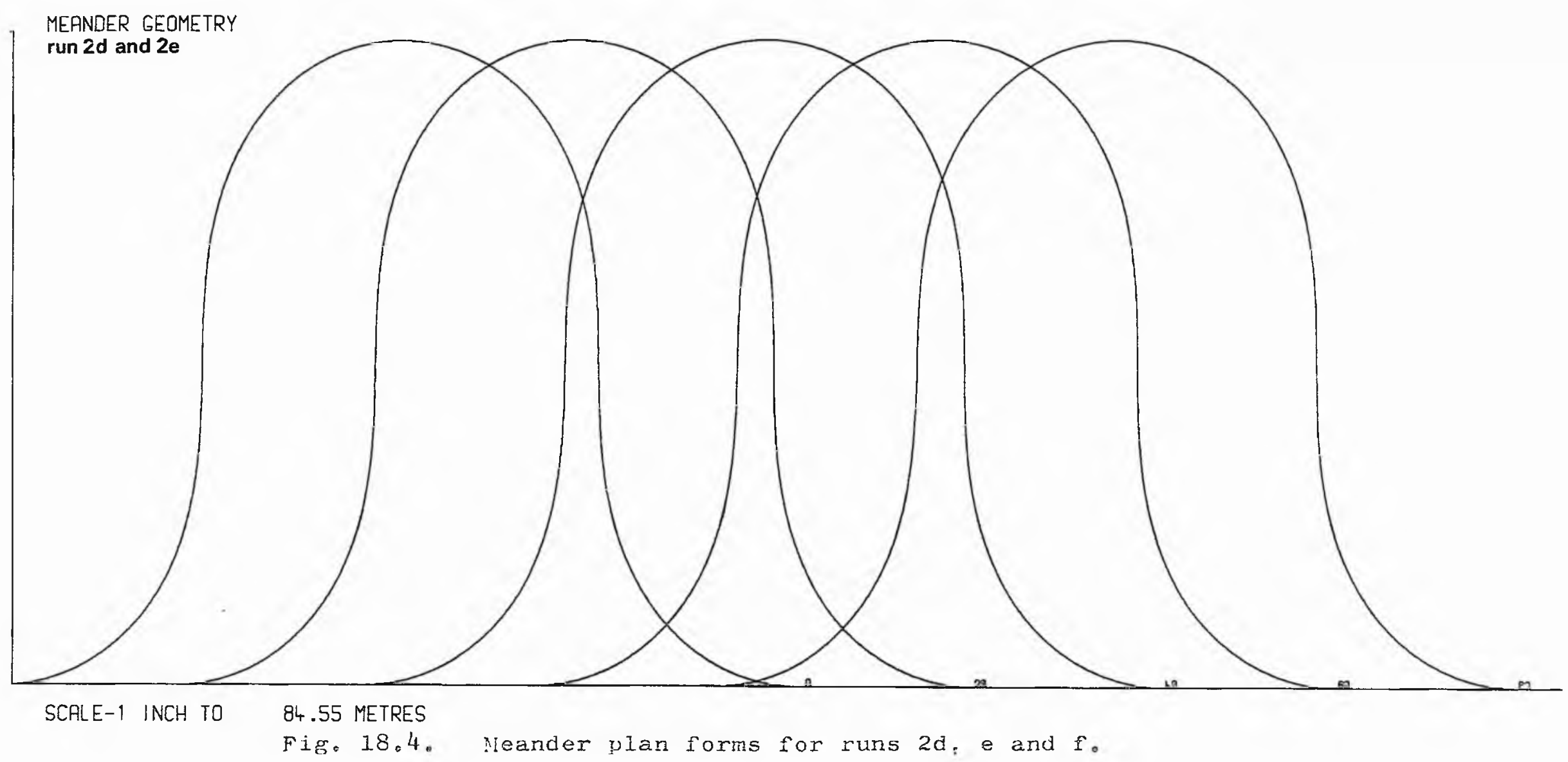




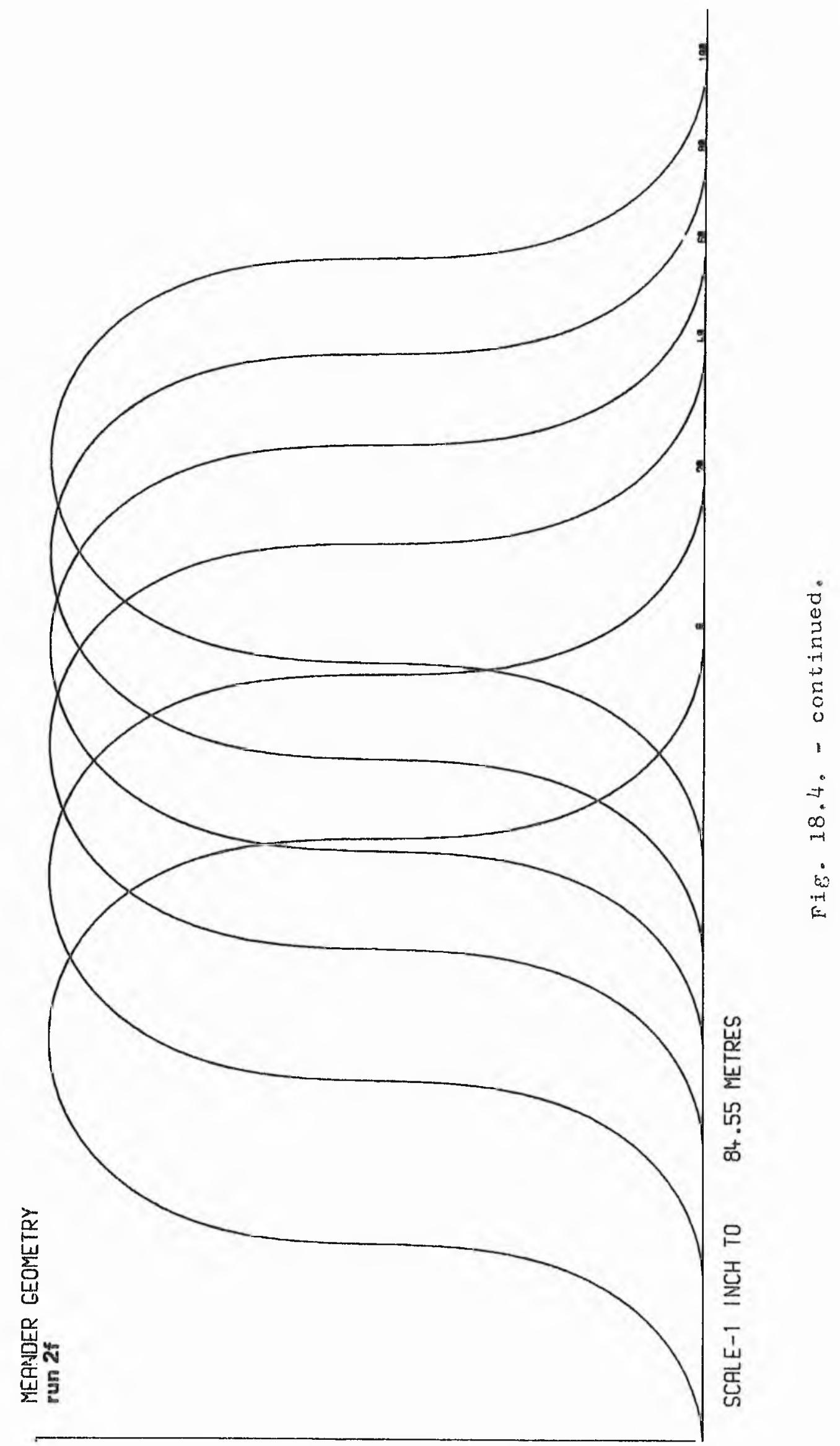



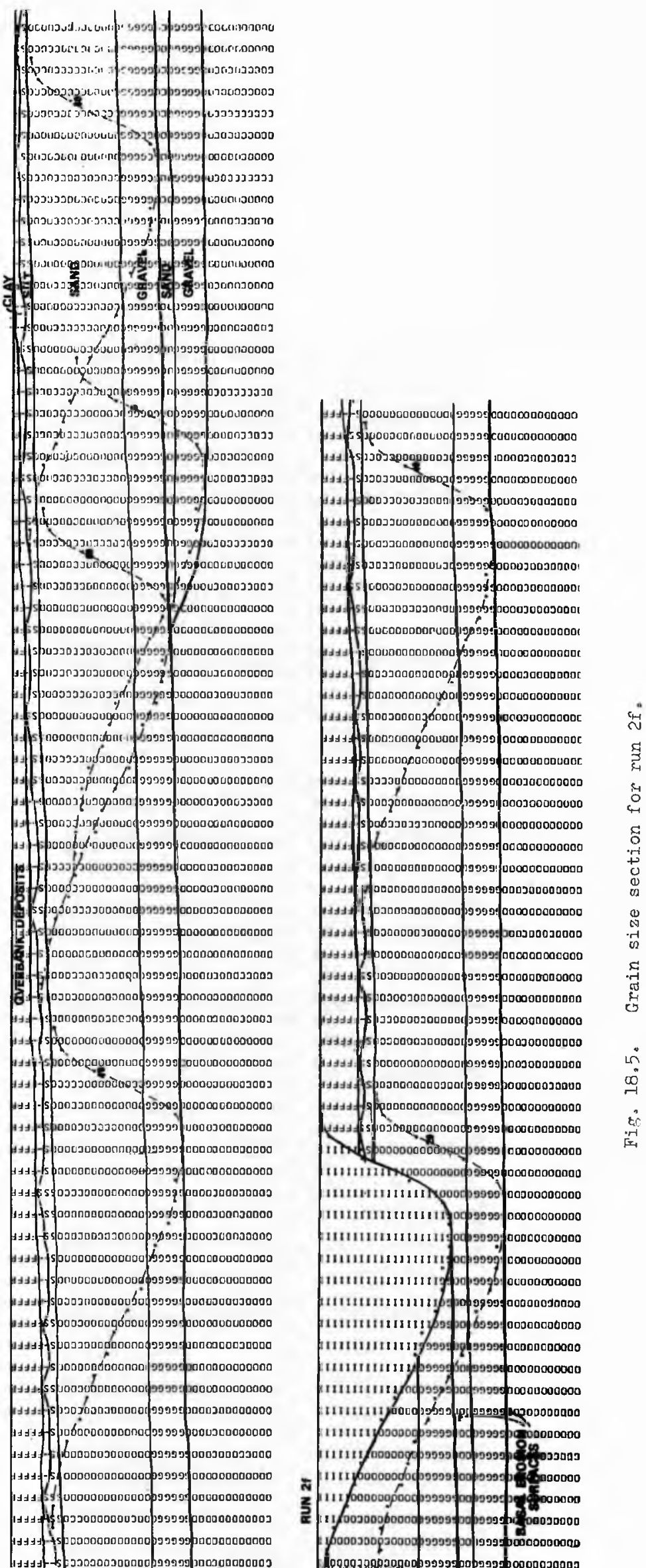


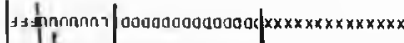
ม.ง

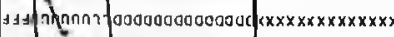
13. กnกำ 13: - in

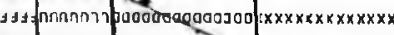

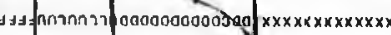

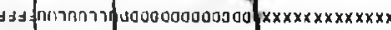

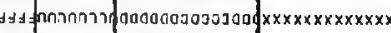

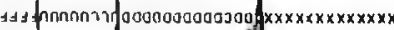

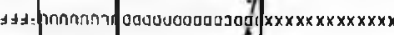

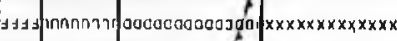

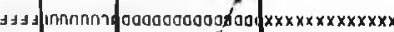
11.

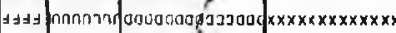

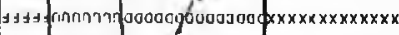
4⿻ง

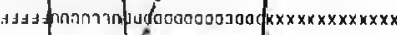

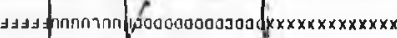

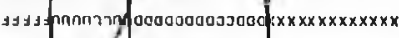
มy A3y

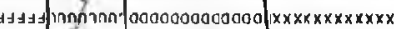

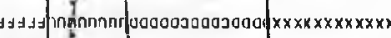

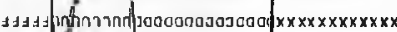

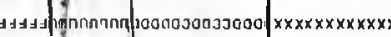

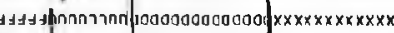
1.

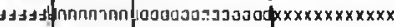

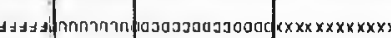

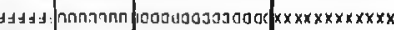

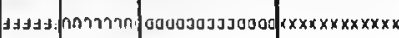

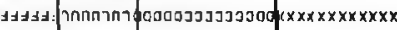
ม⿻上丨 HH.

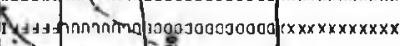

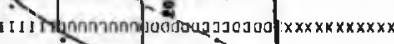
111111317n 1211111111111111) I111111111111111111100030000 $x \times x \times x \times x \times x \times x x$ :

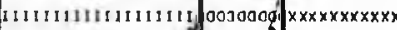

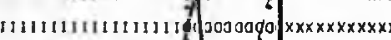
11111611111111 pod oosodoo $x x x x x x x x x x x$

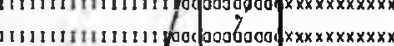

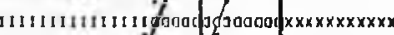

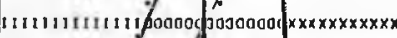

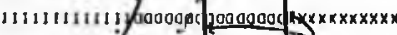

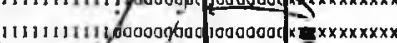

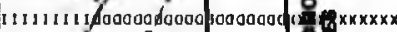

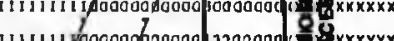

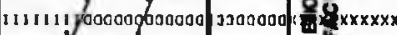

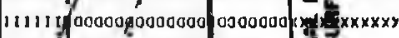

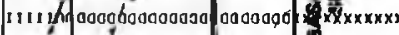

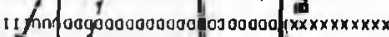

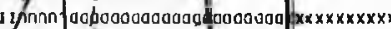

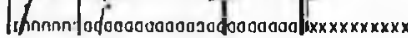


and the upward sloping of the facies boundartes and basal erosion surfaces in the downvalley direction can be seen as in run 20 but with a less steep slope. However the gradual increase in the slope of these surfaces with time as the bank migration rate is reduced is not easily seen. Noteworthy is the sequence produced in this moving phase situation, gravel-sand-gravel-sand-silt(clay)-overbank sediment. Again it should be noted that an aggradation rate of $0.1 \mathrm{~m}$./year is very rapid and rather improbable, also that scouring and filling would obscure the simple patterns shown in figs. 18.5 and 18.6 .

Runs $2 \mathrm{~g}, \mathrm{~h}$ and $i$ correspond to an average downvalley migration of about $42 \mathrm{~m}$ / /year. The time taken to move half a wavelength is a little over 10 years (see fig. 18.7), therefore there is about $0.01,0.1$ and 1 metres of sediment preserved between erosion surfaces, respectively with aggradation rates of 0.001 , 0.01 and 0.1 metres/year. The aggraded thicknesses are not great enough to appear in the cross sections for $2 \mathrm{~g}$ and $2 \mathrm{~h}$, and the effect of aggradation on bank migration rate is negligible. The grain size and sedimentary structure cross sections for the greatest rate of aggradation are shown in $f i g .18 .8$ and $f i g .18 .9$ respectively. The cenexal features are similar to runs 2 c and $2 f$, however, even in this extreme case, the slopes of the facies boundaries and basal erosion surfaces are not particularly marked. The dependence of the shape of the wedge of overbank deposits and the upward slopes in the downvalley direction of the facies boundaries and basal erosion surfaces on the relative rates of bank nigration and aggradation have been illustrated. The General upward slope in the downvalley direction of the facies boundaries and basal eroston surfaces (in the cross sections) is naturally equal to rate of agrradation divided by rate of downvalley migration; this slope inust be corrected for valley slope, if an absolute value is required. It is expected, given the 


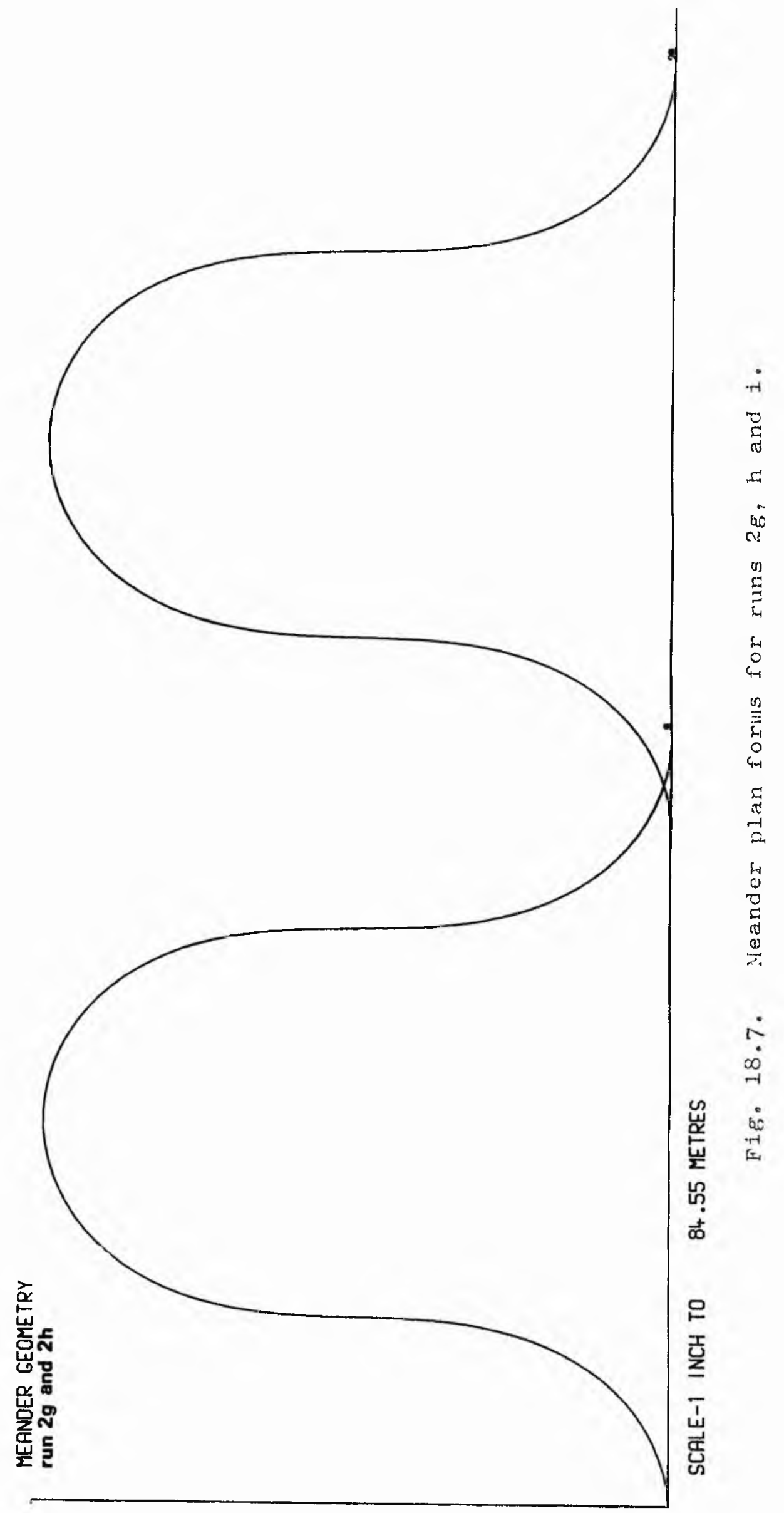




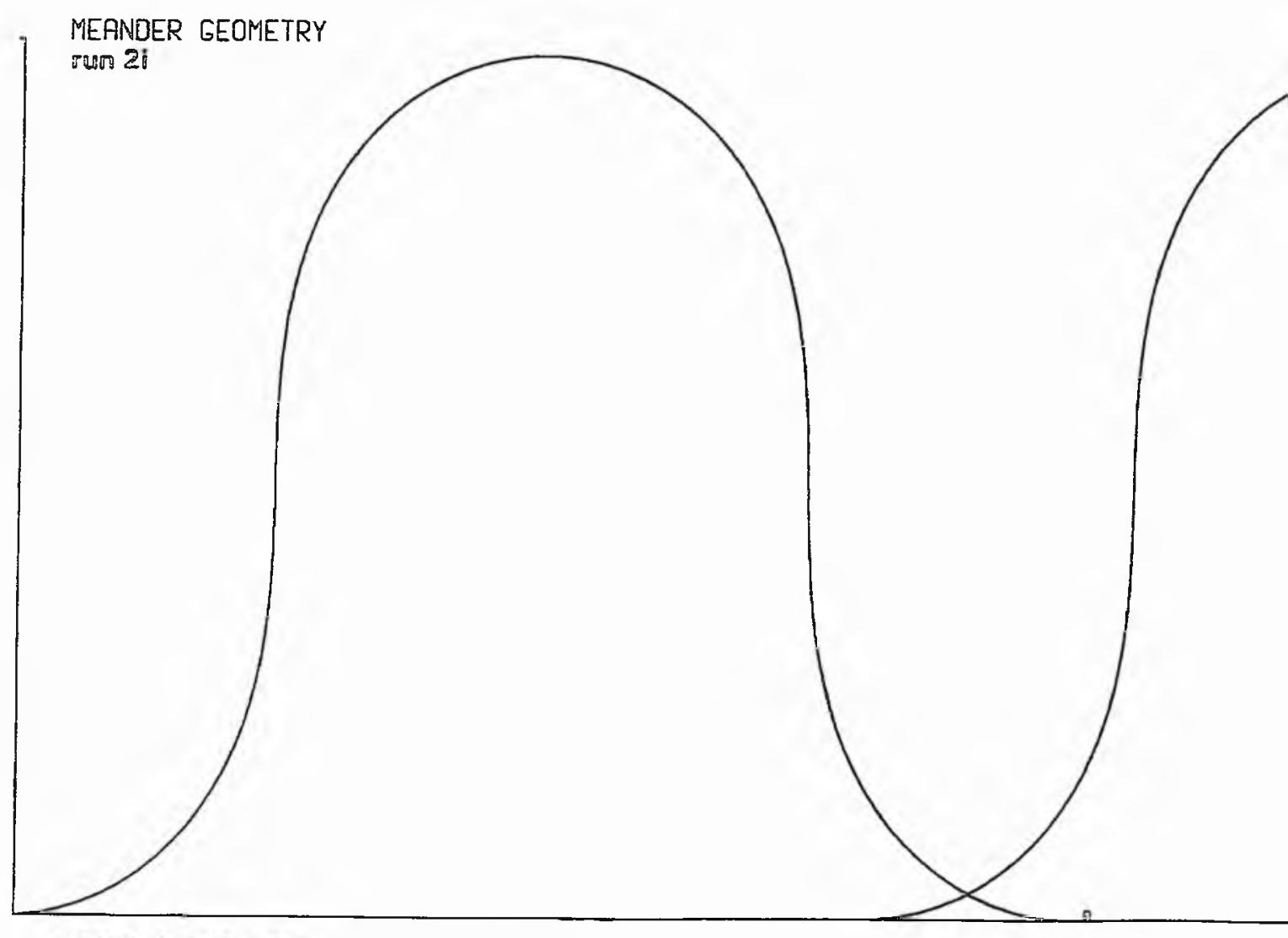

SCALE-1 INCH TO 84.55 METRES

Fig, 18.7. - continued. 

natural observed rates of aggradation, that in the moving phase situation the thin overbank deposits will probably appear virtually constant in thickness (on the larger scale) although basically wedge shaped. Facies boundary slopes on the large scale will also be negligible. Smaller scale complications are introduced when considering scouring and filling, or if the rellef of the floodplain and variability of overbank sedimentation and erosion were accounted for.

The thickness of the deposit preserved in a moving phase situation, in these cross sections, follows the simple algorithm,

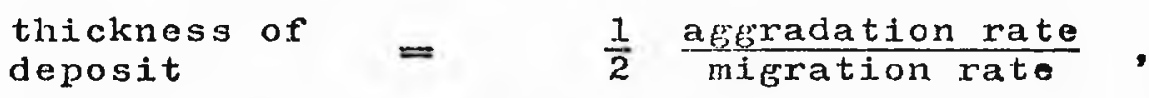
although this is only approximate as there may be scouring and filling to complicate matters. Preserved thicknesses of sediment are expected to be only fractions of the total point bar thickness in the moving phase situation, and Bluck (1971) notes that many sequences of believed fluvial origin have many erosion surfaces at their base. Clearly greater thicknesses or complete sequences may be built up if the eroding medium does not act on the sediments for a long interval of time with respect to the aggradation rate. This may apply in the case of the avulsion situation or in the case of a cut-off which lies out of range of the main channel for a sufficient length of time, as discussed by Bluck (1971). It would appear that if the general slope of facies boundaries or basal erosion surfaces relative to the land surface is not great and complete bar sequences are preserved, then a process other than purely moving phase must be responsible. 
According to the model, as meanders increase in amplitude and sinuosity, wavelength remaining constant, both longitudinal water surface slope and radius of curvature will change. In natural rivers with the Independent variables unchanging, width, depth at the talweg, the friction cocfficients, and the value of $n_{1}$ may be expected to vary to some extent, although such variation cannot be accounted for and is assumed absent for our present purposes. There may also be a systematic variation in the average scour depth as the meander develops, which is also not accounted for in the model. By inspection of equation 5.20, the model would predict a general decrease in the calibre of load as sinuosity exceeds 1.5 . Up to a sinuosity of 1.5 , depending on the relative changes in $S$ and $r_{1}$, the general calibre could increase, decrease or remain about constant. It is noteworthy that increasing depth or width would always tend to increase general calibre of load.

In general, stream power will decrease as slope decreases at constant discharge (Bagnold, 1966, p. 15) and the dimensionless shear stress will vary with the ratio $r_{\mathrm{m}} / \mathrm{w}$ (see equation 5.25 ). Such variation, combined with variation in $D$, will be expected to affect the distribution of bed form and sedimentary structures over the bar as the meander develops. In general, lower flow-regime forms are expected to increase at the expense of upper plane beds as sinuosity increases.

As the meander develops, the angle at which the mean channel direction cuts the line of section (lateral or downvalley) will cause the projected channel width to vary. This will be expected to affect the facies patterns within the bar in the cross sections represented in this experiment. An interesting point in this respect is that field sections of fluvial sedimentary rocks may suggest different channel widths due to varying channel direction 
Tab1e 19.1.

\begin{tabular}{l|c|c|c} 
Run number & $4 \mathrm{~A} / \mathrm{a}$ and $4 \mathrm{~B} / \mathrm{a}$ & $4 \mathrm{~A} / \mathrm{b}$ and $4 \mathrm{~B} / \mathrm{b}$ & $4 \mathrm{~A} / \mathrm{c}$ and $4 \mathrm{~B} / \mathrm{c}$ \\
\hline $\begin{array}{l}\text { Average initial rate of } \\
\text { downation normal to mean } \\
\text { (Metres/year) }\end{array}$ & 3 & 9 & 30 \\
\hline BANK MIGRATION PARAMETERS & & & 9.5 \\
\hline exponent $\mathrm{n}_{2}$ & & $0.9 \mathrm{E}-06$ & $0.3 \mathrm{E}-05$ \\
\hline constant $\mathrm{k}_{2}$ & $0.3 \mathrm{E}-06$ & $0.1 \mathrm{E}-03$ & \\
\hline constant $\mathrm{k}_{3}$ & & &
\end{tabular}

Same parameters for experiment 4A (downvalley section) and experiment $4 \mathrm{~B}$ (1ateral section). 
MLUIATILE PROCESS SIMULATION EXPERIMENT 4B

CROSS SECTION PARAMETERS

\begin{tabular}{|c|c|c|}
\hline METRES & CELLS & \\
\hline $\begin{array}{r}1000.000 \\
60.000 \\
0.0 \\
60.000 \\
1.000 \\
5.000\end{array}$ & $\begin{array}{r}200 \\
60 \\
0 \\
60\end{array}$ & \\
\hline METRES & CELLS & \\
\hline $\begin{array}{l}125.000 \\
100.000\end{array}$ & $\begin{array}{l}25 \\
20\end{array}$ & 0.800 \\
\hline & & $\begin{array}{l}2.050 \mathrm{GM} / \mathrm{CH} \\
1.000 \mathrm{GM} / \mathrm{CH} \\
0.210 \\
0.150 \\
1.000\end{array}$ \\
\hline
\end{tabular}

HIDTH OF SECTION

THI CKNESS DF SECTION

INITIAL DISTANCE OF INNER CHANNEL BANK FROM L.H.S. OF SECTION

INITIAL. BANKFULL STAGE MEA SURED FROM SECTION BASE

CELL SIZE IN HORIZONTALIZ OR XI DIRECTION

Channel parameters

TOTAL WIDTH OF CHANMELIM)

HIOTH OF FLOH BETHEEN INNER BANK AND TALHEG(HI)

RATIO OF WITO W

DENSITY OF SEDIMENTARY PARTICLES

FLUID DENSITY

DARCY-WEISBACH FRICTION CDEFFICIENT FOR DUNES AND RIPPLES
DARCY-HEI SBACH FRICTION COEFFICIIENT FOR PLANE GEDS AND ANTIDUNES

1.000

SYNTHETIC HYDROLOGY PARAMETERSIUNITS NOT NECESSARYI

MEAN DF ALL DAILY MEAN VALUES
STANDARD DEYIATION OF DAILY MEAN VALUES

MEAN OF YT SERIIES

STANDARD DEVIATION OF YT SERIES

COEFF ICIENTS IN AUTOREGRESSIVE MODEL

FOURIER COEFFICIENTS FOR DAILY MEANS(A)

FOURIER COEFFICIENTS FOR DAILY STO DEVIATIONS ISAI

MAXIMUM VALUE OF QVOL

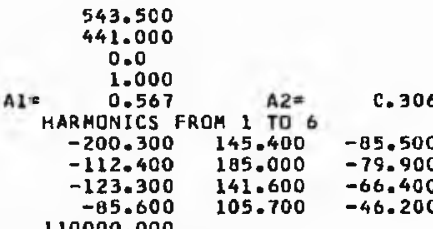

$58.000-39.800$

$65.600-72.500 \quad 7.400$

$75.700 \quad-47.200 \quad 27.800$

$\begin{array}{lll}75.700 & -47.200 & 8.600 \\ 31.700 & -43.200 & 4.300\end{array}$

110000,000

$41.600-66.400$

SCOUR AND FILL PARAMETERS

CON STANT K4

STANDARD DEVIATION OF ERROR TERM

$0.100 E-03$

1.000

CUT-OFF CONTROL PARAMETERS

LIMITING WIDTH OF MEANDER NECK

EXPONENTS IN NECK CUT-OFF RELATION

LIMITING SINUOSITY

LIMIT ING AMPLIIUDE

EXPONENTS IN CHUTE CUT OFF RELATION

$\begin{array}{lrll} & 225.000 & \text { METRES } & \\ 5.000 & \text { EN2 }= & 5.000 \\ 3.000 & & \\ 1185.529 & \text { METRES } & \\ 20.000 & \text { EC2 }= & 20.000\end{array}$

LEGEND

LOWER PHASE PLANE BED RIPPLES

DUNES

UPPER PHASE PLANE BED

ANT IDUNES

$\begin{array}{ll}\text { L } & \text { GRAVEL } \\ R & \text { SAND } \\ \text { D } & \text { SILT } \\ U & \text { CLAY } \\ \text { A } & \text { OVERBANK } \\ & \text { DEPOSITS }\end{array}$

\begin{tabular}{l} 
G \\
S \\
\hline \\
\hline F
\end{tabular}

WATER
TIME LINE

A IR

I

OVERBANK

A

planimetric forh of meanoer

METRES

HAVEL ENGTH

AMP LI TUDE

SINUSITY

RADIUS OF CURVATURE AT GEND AXIS

MIOTH OF MEANDER NEC

CHANNEL LENGTH ALONG MEANDER

VALLEY SLOPE

LONGI TUDINAL WATER SURFACE SLUPE

1000.000

205.834

280.638

1099.999

0.00010000
0.00009091

SELECTED GEDMETRIC RATIOS

HAVELENGTH TO RADIUS OF CURVATURE

NANEL MIOTH

RAD IUS OF CURVATURE TO CHANMEL HIOTH
AMPLITUDE TO CHANNEL HIDTH

3.563

8.000

2.245

1.64

Table 19.2. Initial data for experiment $4 \mathrm{~A}$ and $4 \mathrm{~B}$. 
relative to the plane of the section. Furthermore, in lateral sections only, the effect of slowing down of bank migration normal to the mean downvalley direction will be expected to influence the sedimentary facies patterns.

The experiment consists of three runs of the program using a lateral section and another three similar runs using a downvalley section. The three separate runs for each type of section correspond to three diffexent average initial rates of bank migration normal to the mean downvalley direction as the meander develops from a sinuosity of 1.1 to a limiting value of 3.0 . The average downvalley migration rate is constant for all runs (about $2.3 \mathrm{~m} \cdot /$ year), as is the selected average depth of scour. The input data that are different for each run are shown in table 19.1, and these correspond to average initial rates of about 3,9 and 30 metres per year. All other input parameters, shown in table 19.2 and 19.1, are the same for all runs. Fig. 19.1 shows the meander movement simulated which are responsible for the sedimentary deposits in the forthcoming cross sections. The data deck setup for all six runs is listed in appendix 3.

In runs $4 \mathrm{~A}$ and $4 \mathrm{~B}$ the cross sections were comprised of 200 by 60 cel1s. In $4 \mathrm{~A}$ a disc was used, $78 \mathrm{k}$ bytes of core store were required, and approximate running times were $7 \frac{1}{2}$ seconds per time increment. In $4 \mathrm{~B}$ no disc was used, $129 \mathrm{k}$ bytes of core store were required, and approximate running times were 3 seconds per time increment.

Fig. 19.2 shows the vaxiation of hydraulic parameters, at a specific station, as the meander develops, for the three separate input conditions. The station corresponds to a depth of 10 metres, or half the maximum unscoured talweg depth. The curves for $D$ indicate a general increasing calibre of load followed by a more substantial decrease in calibre. The turning point here 


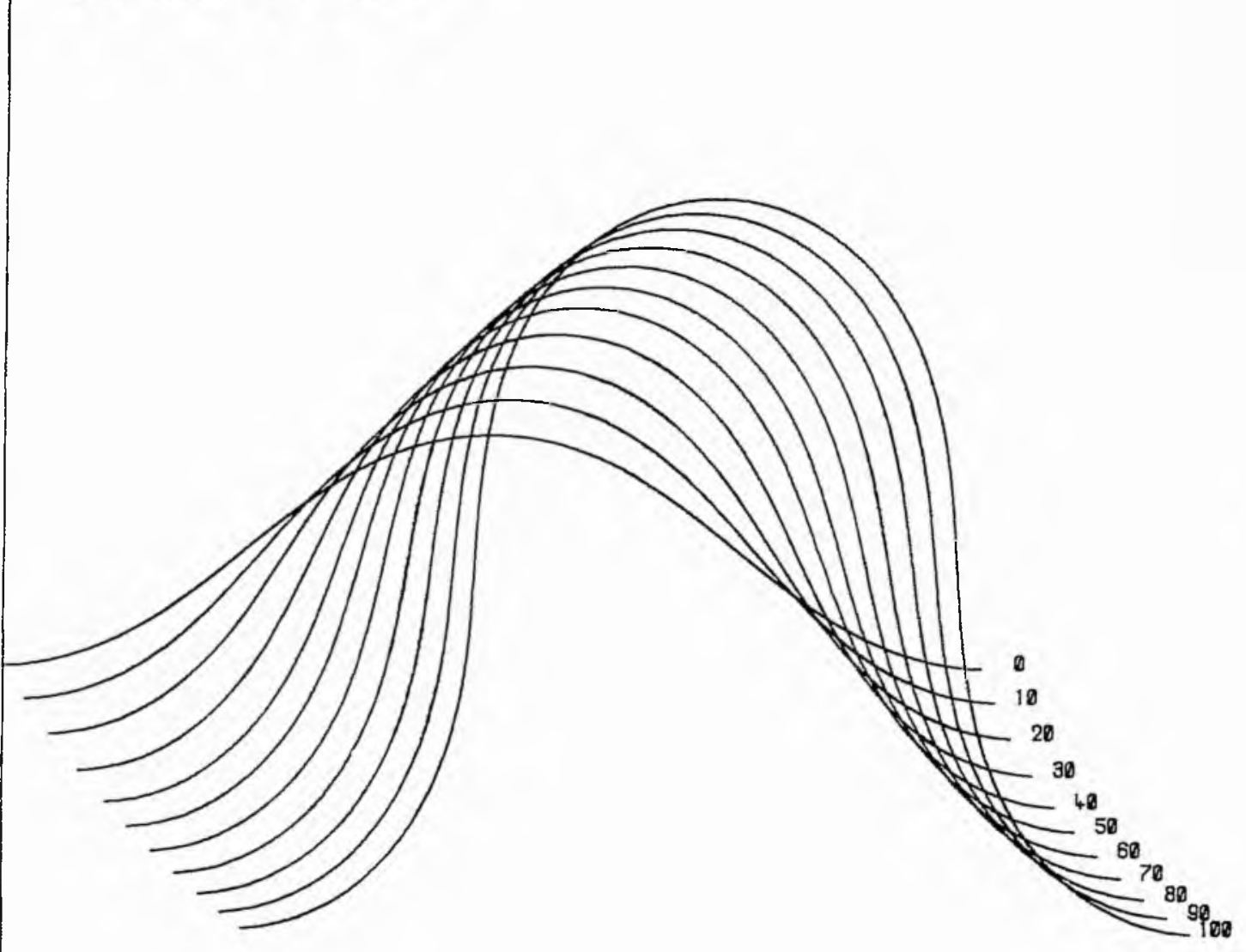

\section{SCALE-1 INCH TO 131.73 METRES}

Fig. 19.1. Meander plan forms for experiments $4 \mathrm{~A}$ and $4 \mathrm{~B}$. 


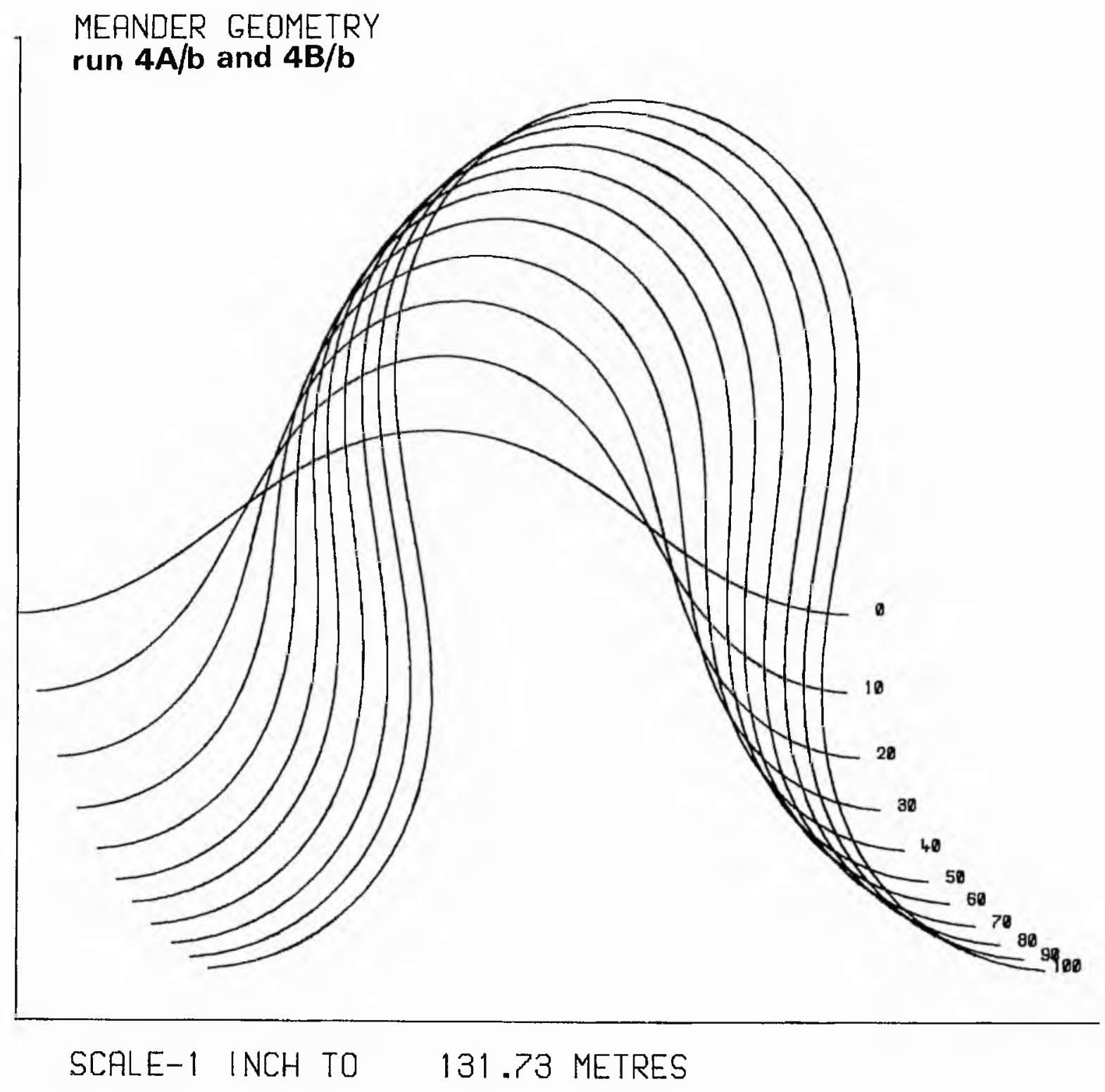

Fig. 19.1. - continued. 


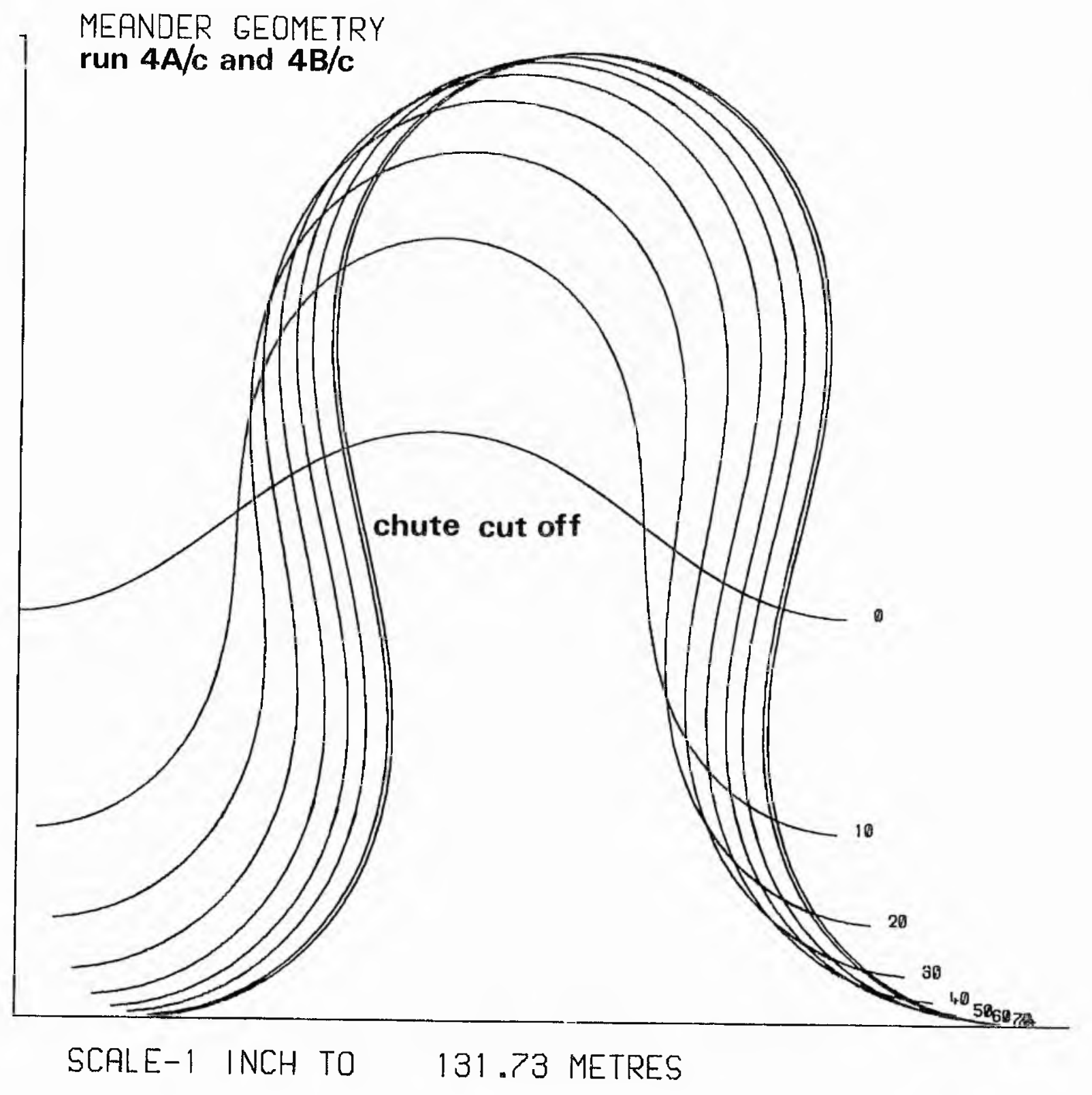

Fig. 19.1. - continued. 

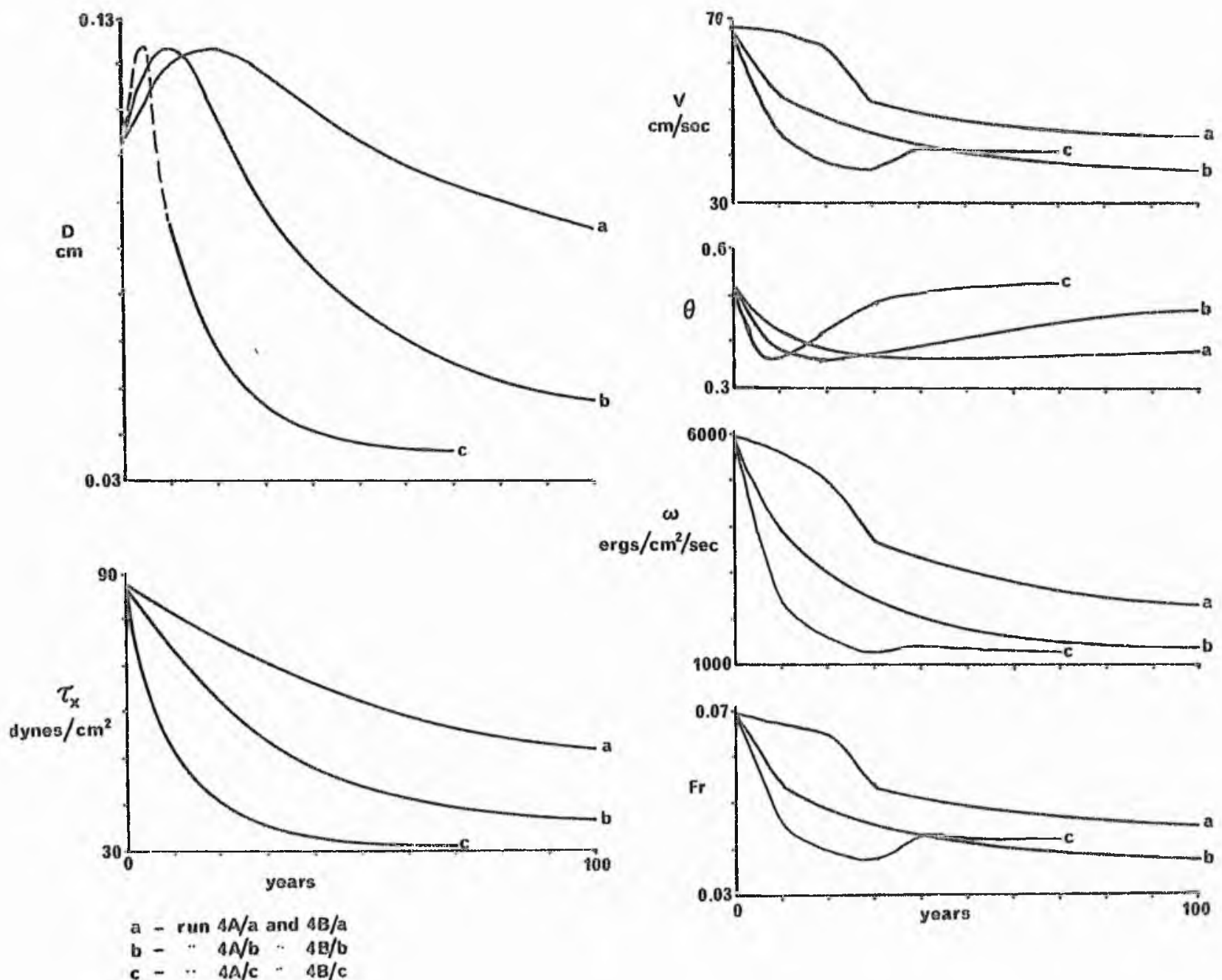

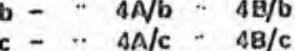

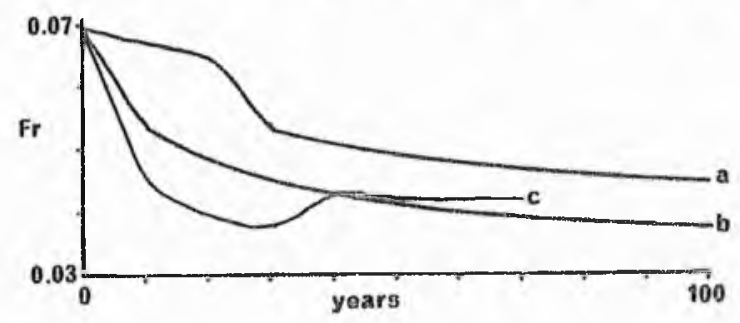

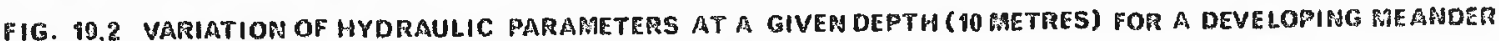
WITH TMREE DIFFERENT RATES OF AFAPLITUDE GROWTH. 

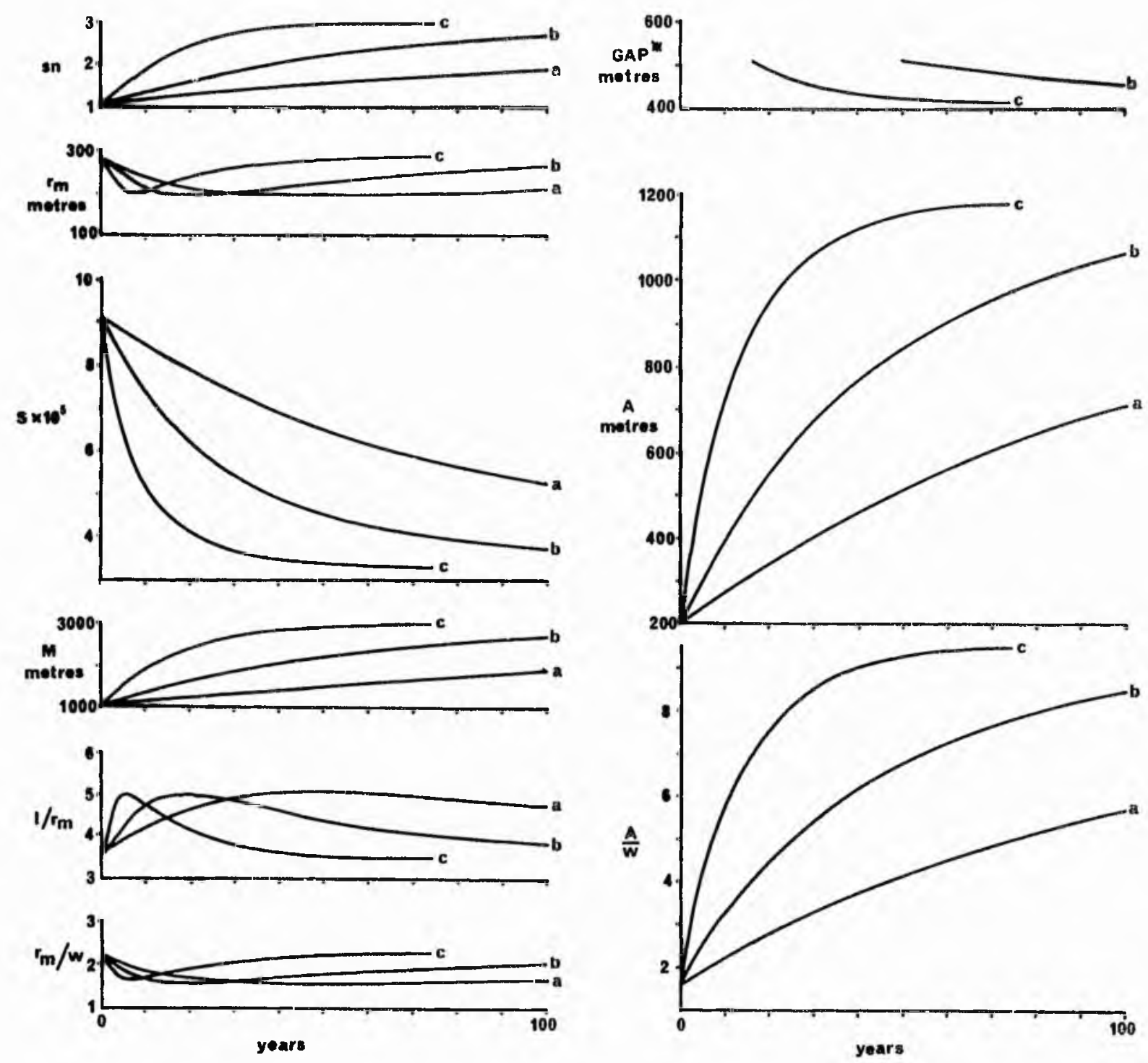

- run MA/a and AB/a

b - $44 / b \cdots 48 / b$

c $-\cdots 4 \mathrm{~A} / \mathrm{AB} / \mathrm{c}$

- GAP Is measured bstween channel centre lines here

FIG. 19.3 VARIATION OF GEOMETRIC PARAMETERS FOR DEVELOPING HEANDER WITH THREE DIFFERENT RATES OF AMPLITUDE GROWTH. 
corresponds to a sinuosity of about 1.3 . The curves for $D$ would be expected to be influenced slightly by changes in width, depth at talweg and $\mathrm{n}_{1}$. The curves of $\tau_{x}^{\prime}$ show a systematic decreasing trend, and these, being defined for a given depth of 10m., will remain independent of variation in width, depth at talweg, friction factors and $n_{1}$. Curves for $\theta$ show the expected trend as they depend on the ratio $r_{m} / w$ (see fig. 19.3). Changes in width or $n_{l}$ will influence these curves. The curves of $\omega, v$ and $\mathrm{Fr}$ are all generally decreasing, the 'kinks' being the result of change in bed form from upper phase plane beds to dunes. These curves will only be influenced by changes in $f$. Fig. 19.3 shows the variation of certain geometric parameters as the meander develops for the three separate input conditions. Parameters involving $x_{m}$ show the characteristic turning point at sinuosity of 1.5 . Those involving amplitude show the effect of the gradually decreasing rate of amplitude growth. The width of the meander neck here refers to the distance between channel centre lines, not adjacent banks.

Runs $4 \mathrm{~A} / \mathrm{a}, \mathrm{b}$ and $\mathrm{c}$, figs. 19.4 and 19.5, are downvalley sections, with the average rate of downvalley migration being constant at about $2.3 \mathrm{~m}$./year. The main feature of the grain size sections is the gradual lateral decrease in thickness of gravel and increase in sand, silt and clay thicknesses, after a small initial increase in general calibre (as indicated in fig. 19.2). The degree of lateral change increases from section $4 \mathrm{~A} / \mathrm{a}$ to $4 A / c$ as the initial rates of amplitude growth increase. Section $4 \Lambda / c$ shows a tendency for interfingering to develop. The previous channel positions in all sections show the changes in the projected channel width in the cross sections as the meander develops. The relief of the basal scoured surfaces and facies boundaries were discussed earlier.

The main feature of the sedimentary structure cross sections 


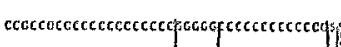

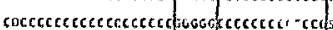

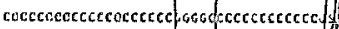

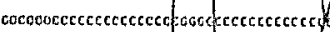

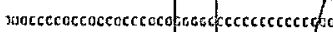

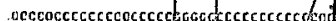

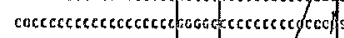

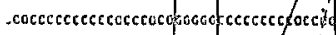

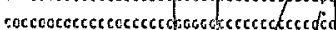

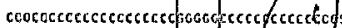

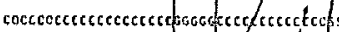

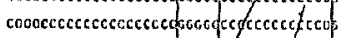

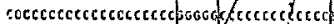

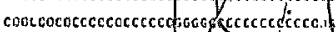

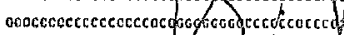

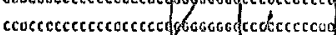

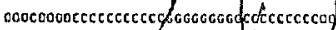

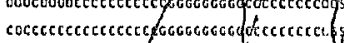

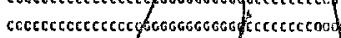

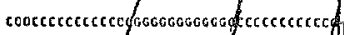

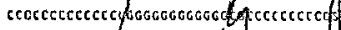

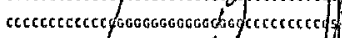

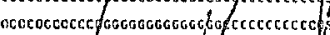

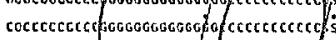

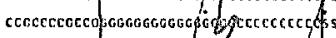

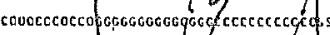

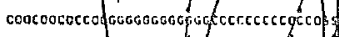

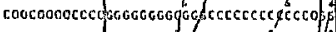

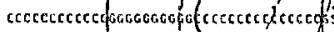

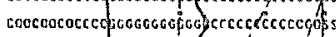

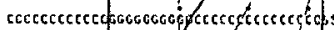

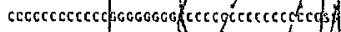

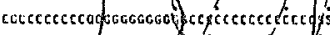

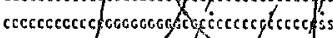

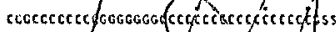

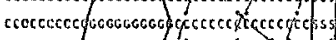

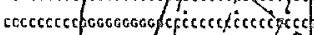

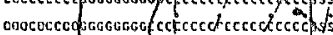

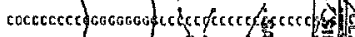

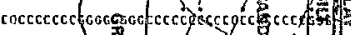

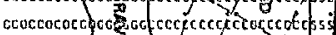

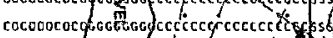

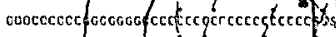

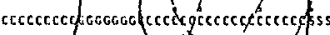

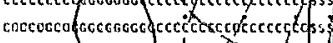

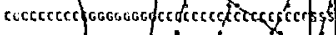

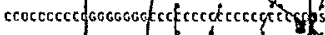

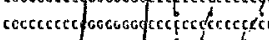

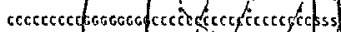

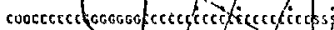

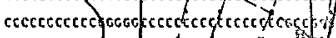

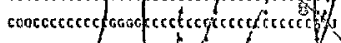

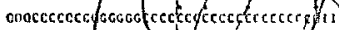

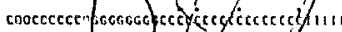

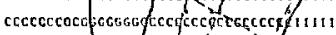

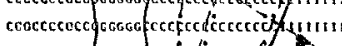

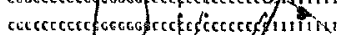

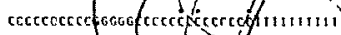

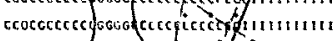

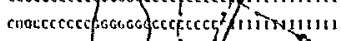

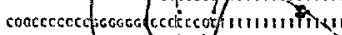

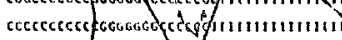

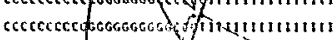

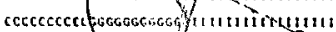

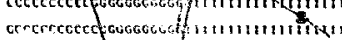

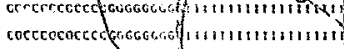

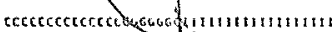

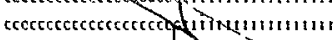

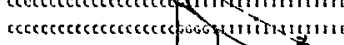

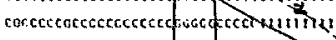

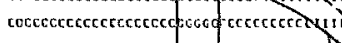

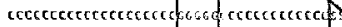

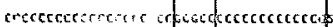

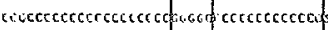

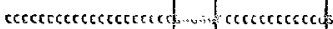

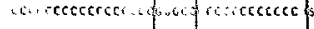

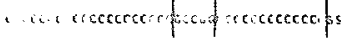

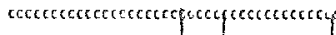

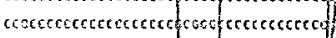

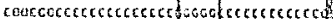

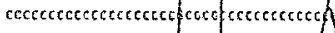

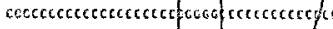

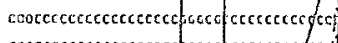

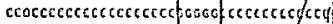

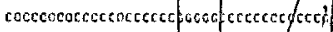

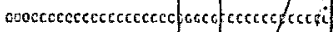

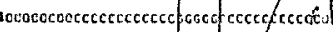

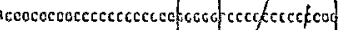

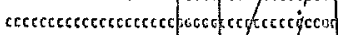

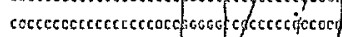

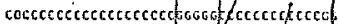

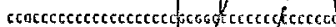

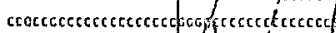

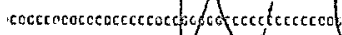

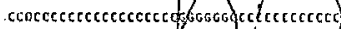

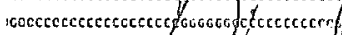

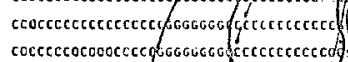

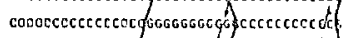

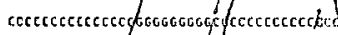

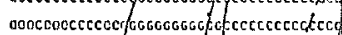

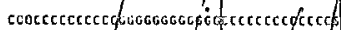

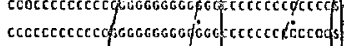

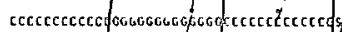

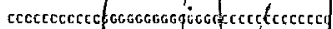

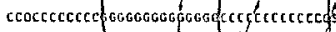

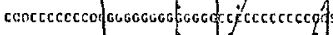

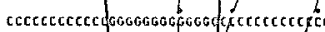

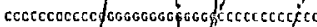

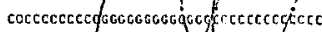

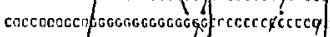

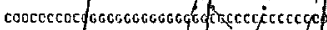

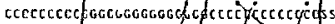

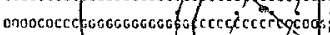

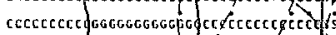

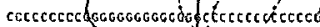

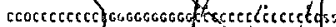

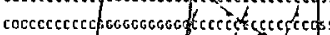

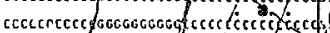

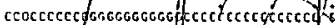

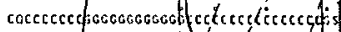

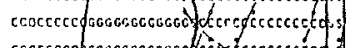

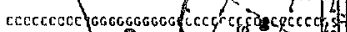

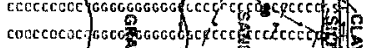

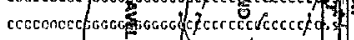

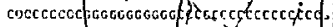

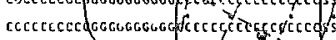

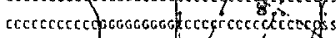

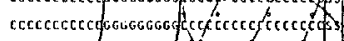

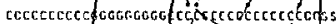

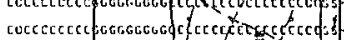

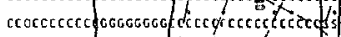

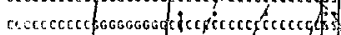

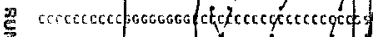

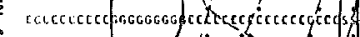

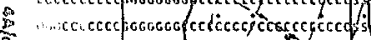

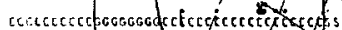

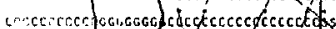

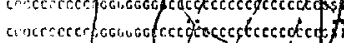

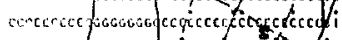

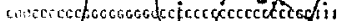

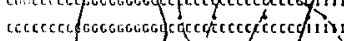

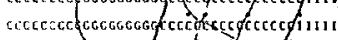

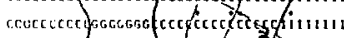

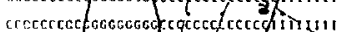

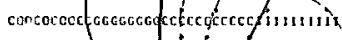

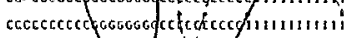

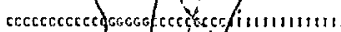

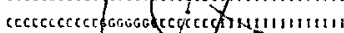

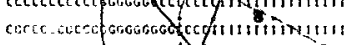

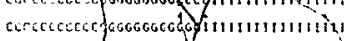

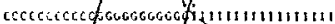

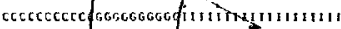

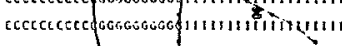

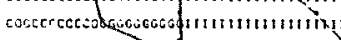
corccocoscececcosces

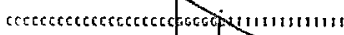

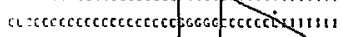

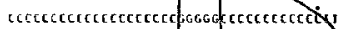

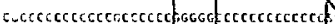

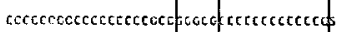

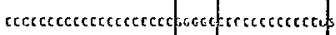

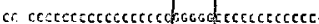

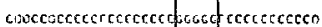

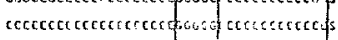

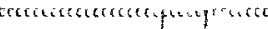

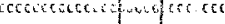

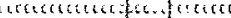

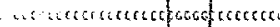

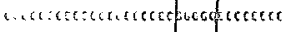

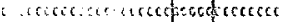

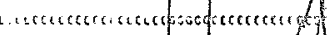

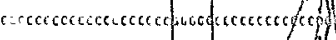

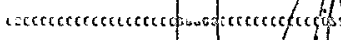

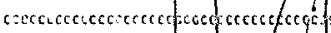

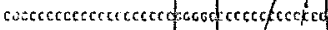

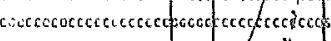

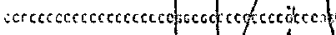

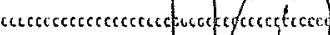

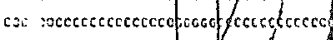

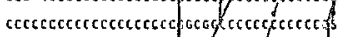

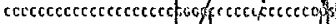

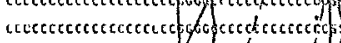

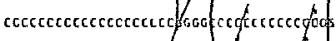

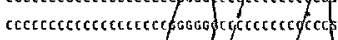

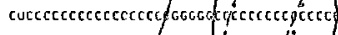

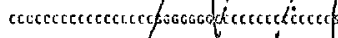

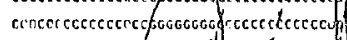

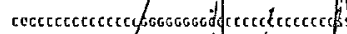

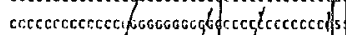

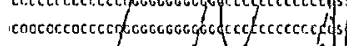

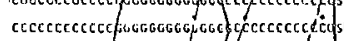

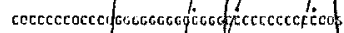

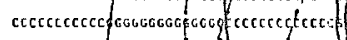

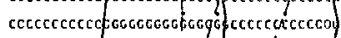

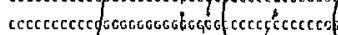

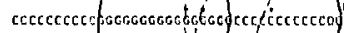

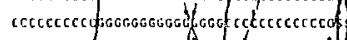

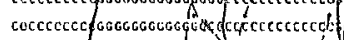

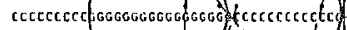

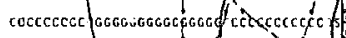

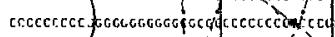

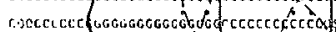

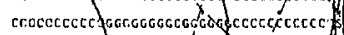

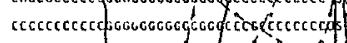

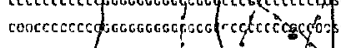

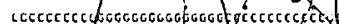

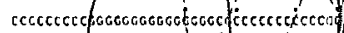

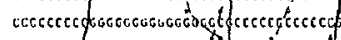

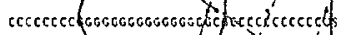

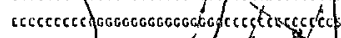

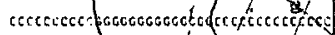

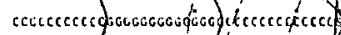

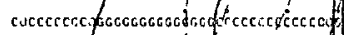

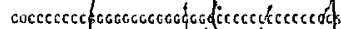

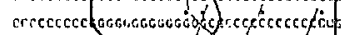

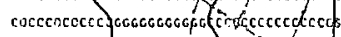

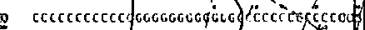

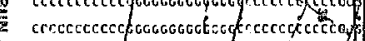

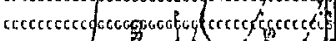
cercececes ace o

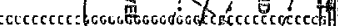

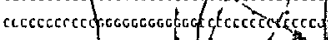

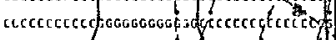

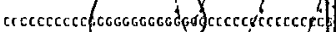

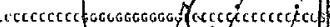

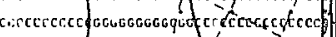

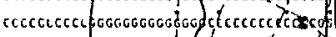

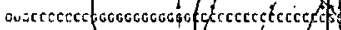

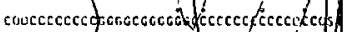

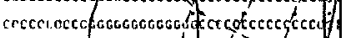

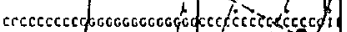

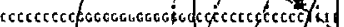

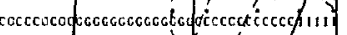

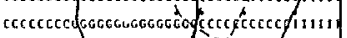

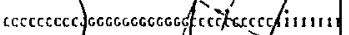

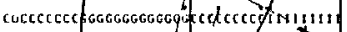

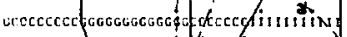

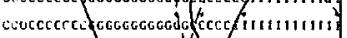

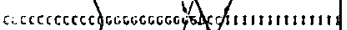

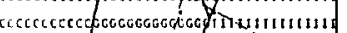

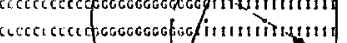

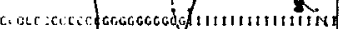
rececerace fogagegeosh

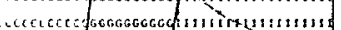

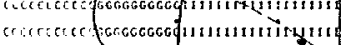

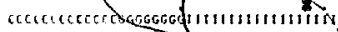

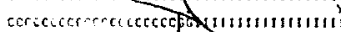

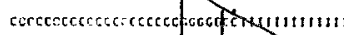

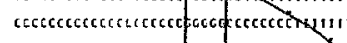

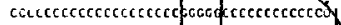

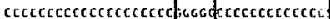

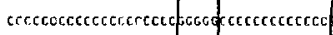

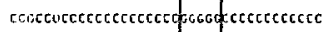

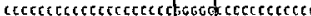

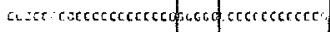

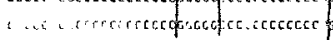



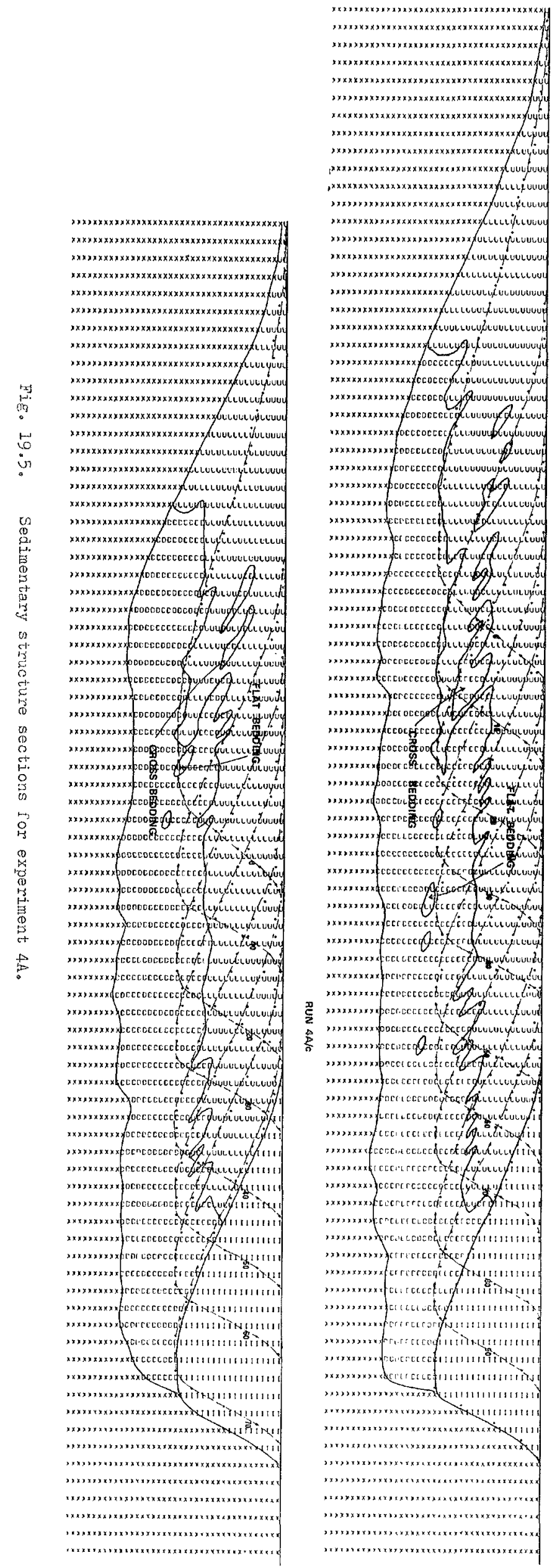

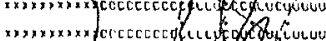

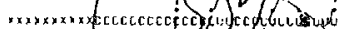

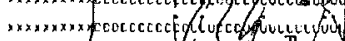

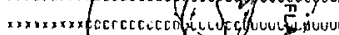

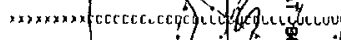

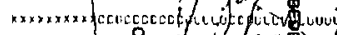

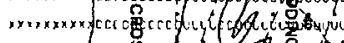

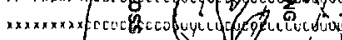

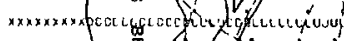

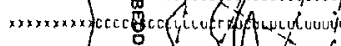
$x \times \times \times \times x \times x$ ect

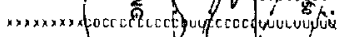
$\times \times \times \times \times \times \times x \times$ receces LO

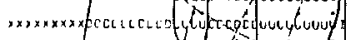

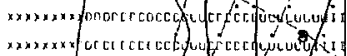

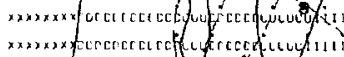

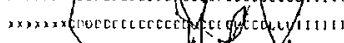

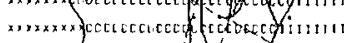

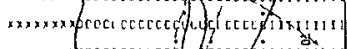

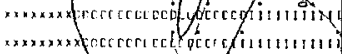

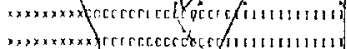

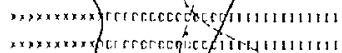

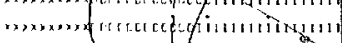

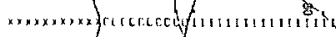

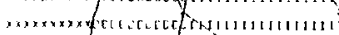

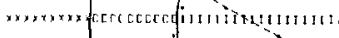

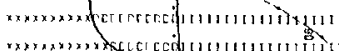

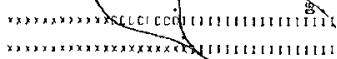

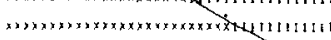

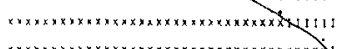
제 ‥

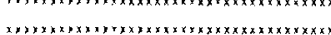
$x \times x \times x \times x \times x \times x \times x \times x \times x \times x \times x \times x \times x \times x \times x \times x \times x \times x \times x x$

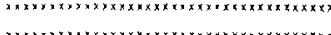


of $4 \mathrm{~A} / \mathrm{a}, \mathrm{b}$ and $\mathrm{c}$ is the lateral increase of cross beding at the expense of flat bedring. With gradual increase in the amount of cross bedding there is a transition area of considerable lateral extent which is a large-scale interfingering of flat beds and cross beds. The interfingering wedges of flat bedding or cross bedding break down laterally into lenses. The lateral extent of the transitional area clecreases as the rate of amplitude growth increases. The smaller scale relief of the flat-bedding-crossbedding boundary has been discussed elsewhere. It is noteworthy that such large scale interfingering produces a cyclical vertical sequence of cross-flat-cross-flat bedding continuously for a considerable lateral extent.

Runs $4 \mathrm{~B} / \mathrm{a}, \mathrm{b}$ and $\mathrm{c}$, figs. 19.6 and 19.7 , are lateral sections, and thexefore, by virtue of their definition, the projected channel widths are gradually increasing as the meander develops (as opposed to runs 4A). The grain size distribution cross sections are broadly.similar to runs $4 \mathrm{~A}$ except the same changes in grain size as occurred in $4 \mathrm{~A}$ have in general occupied a greater lateral extent. The different rates of channel migration in these sections have also affected the smaller scale relief of the grain size facies boundaries, as opposed to $4 A$ where channel migration is identical for each section. In these cases, therefore, the overall thinning or thickening of the various grain-size units may be overshadowed in field sections by local variation in thickness, the general. trend only appearing over sections with large lateral extent. Similar comments can be made with regard to the comparison of the sedimentary structure cross sections of $4 \mathrm{~B}$ with $4 \mathrm{~A}$. In particular, the transition involved with the increase in cross bedding extends laterally for a greater extent, and the different migration rates have affected the smaller scale relief of the flatcross bedding boundary. 


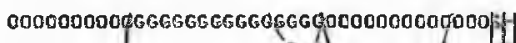

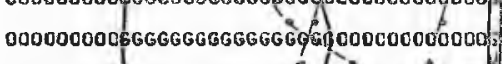

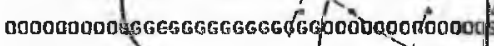

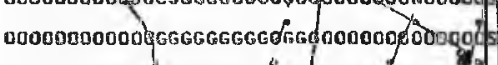

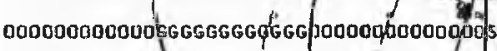

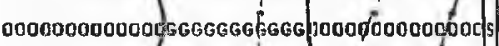

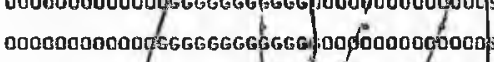

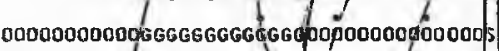

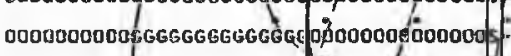
0000000000:3GGGGGGEGGGGG of 6000Q000000000:

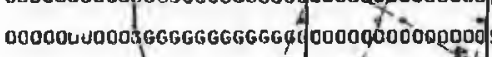

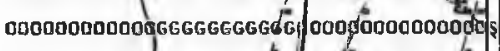

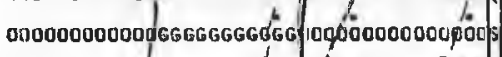

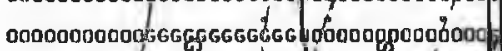

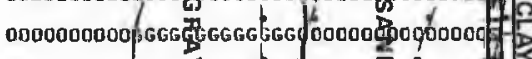

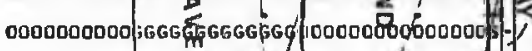

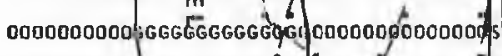

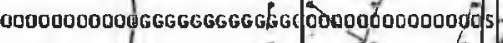

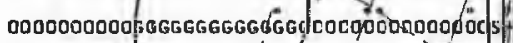

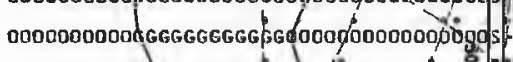

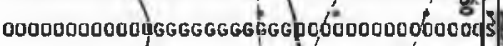

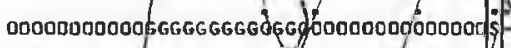

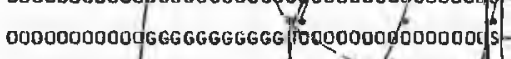

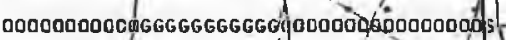

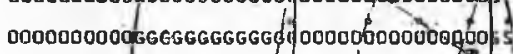

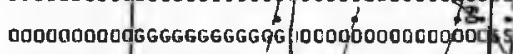

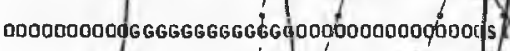

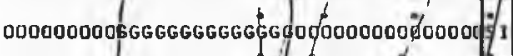
00000000qGG GGGGGGGGG\$GQ60000000000000 11 000000006GGGGGGGGGGGGG

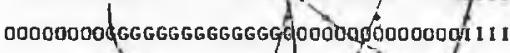

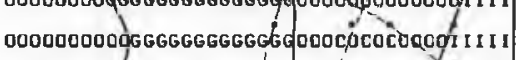
000000000GGGGGGGGGGGGGo006000000d I H I I I 00000000 opGGGGGGGGGGGdo ofo00000 i I I I I I 0000000000GGGGGGGGGGG foo00000 / IIIIIIIII 0000000000010GGGGGGGogdo000000 111111111 000000000000 00000000000dGGGGGGGGGGd000 OTNIIIIIIIII 000000000006GGGGGGGGGGGלم/IIIIIIINIIII 00000000000 GGGGGGGGGGGGG IIIIIIIIIII 00000000000006 GGGGGGEGGG111111111111111 0000000000006GGGGGG GefG I I I I I I I I I I I I I I I I I 00000000000dGGGGGGGGGkJ I I I I I I I I I I I I I I 000000000006GGGGGGGGIIITHIIIIIIIII 00000000000GGGGGGGGGi I IIIIIII I th I IIIII 0000000000 dGGGGGGGGG IIIIIIIIIIIIIIIt 000000000000GGGGGGGGit1111111111111112 000000000000000000Q6 1 I IIIIIIIIIII11111 00000000000000000000 1 I I I I I I I I I I I I I I I I I I

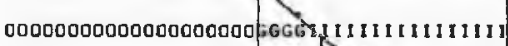

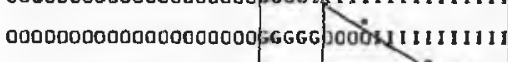

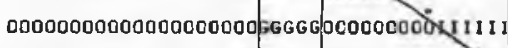
00000000000000000000 36 GG GJ00000000000 00000000000000000000/GGGG0000000000005S 00000000000000000000/3GGGGJ000000000000;s $00000000000000000000566 G$ G000000000000/s 00000000000000000000 360600000000000000/ss 00000000000000000000 )3GGG(0000000600000) is

00000000000000000000EGGGGP000000000000500000000000000000000366600000000000000 $\begin{array}{lll}00000000000000000000 & =6 G G G & 300000000000 / 5\end{array}$

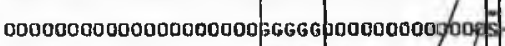
c00000000000000000006GGG

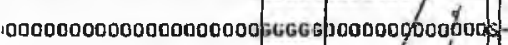

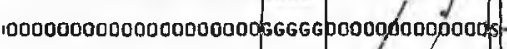

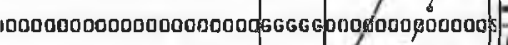
100000000000000000000 GG GG EDóo0000000000 s 1000000000uJ00000000036G6C6000ð60000000

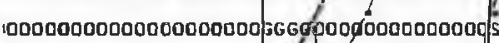
00000000000000000000 3GGG Q gl000000000ots 00000000000000000000GG Ge \$000000000006

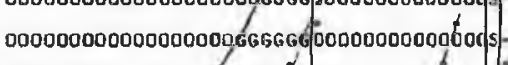
$0000000000000000 \mathrm{GGGGG} 6 \mathrm{~g} 00000000$ gonoch 00000000000000\%́rGGGGGGG

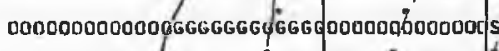
00000000000qGGGGGGGG FGGGGDODOd00000000 5.

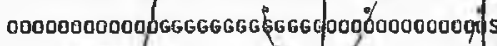

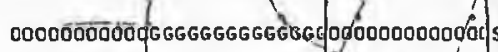

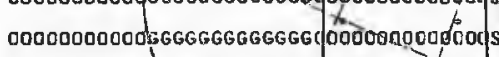

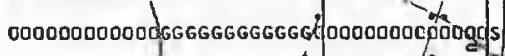
00000000000dGGGGGGGGGGQGG(0000000,00000r \$

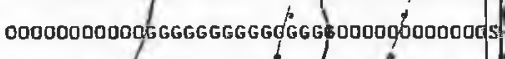

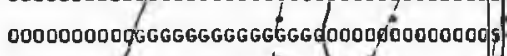
Do000000oGGGGGGGGGG ó GGGG\$ogononoononds

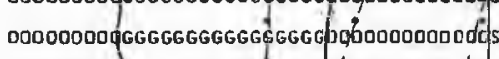

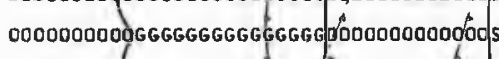

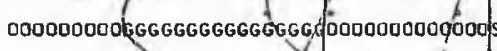

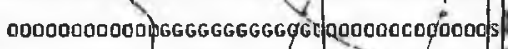
1000000000006GGGGGGGGGGGGGODODODOQQ0́000C/s 1000000000006 GGGGGGGG 10000000000G GGGGGGGGGG'GGG D000000000000

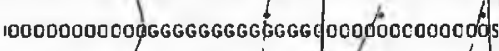

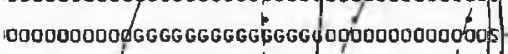
00000000 dGGGGGGGGGGGGGG 6do00000000600 st

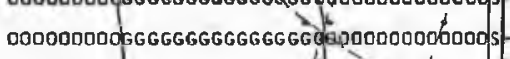

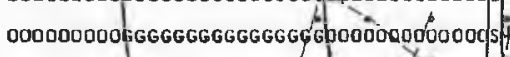

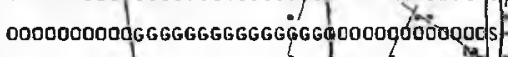

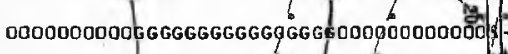
CODD0000006GGGGGGGGGGGGCOCOPDC0000000 00000000000 G GGGGGGGGGGG 00,000000000 of

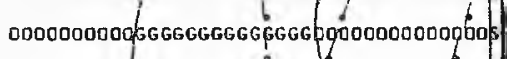




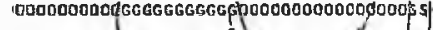

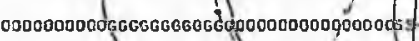

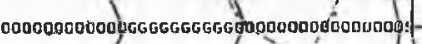

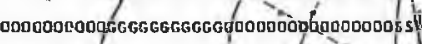

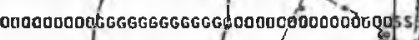
00000000OGGGGGGGGGGGG00000000000000OS:

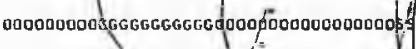

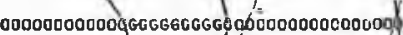

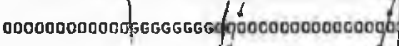

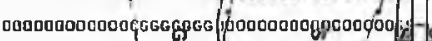
000000000000 GGG

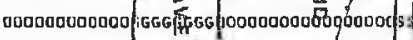
000000000000 GGGEGGG

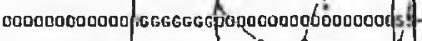

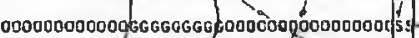

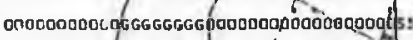
0000000000 GGGGGGGGG GO000 g6000000000g00

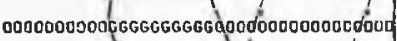

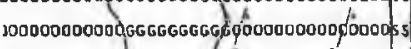

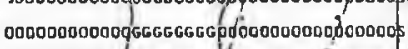

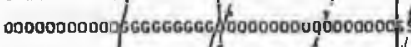

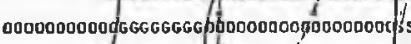
0000000000\$GGGGGGGGPDo000000600000000d ss

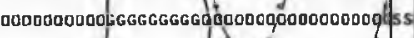

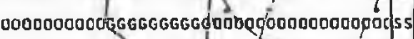

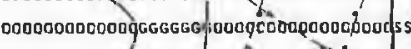

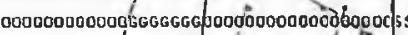

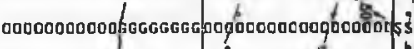

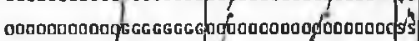

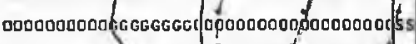

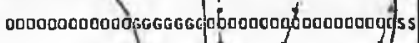

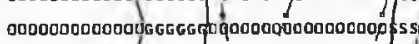
000000000000 G6GGGGocod QQ

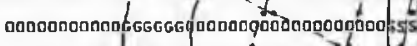

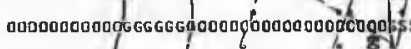
an00000000

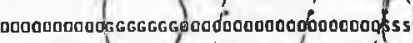

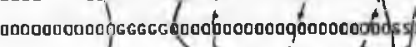

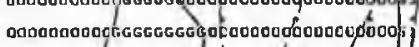

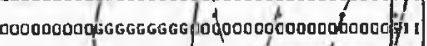

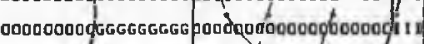

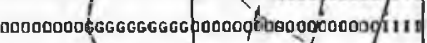

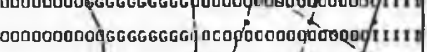

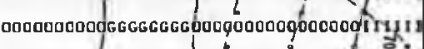

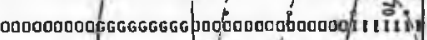

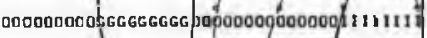

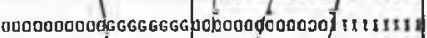

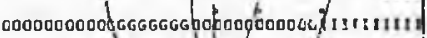

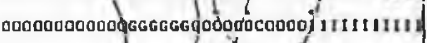

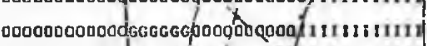

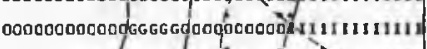

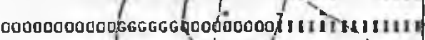

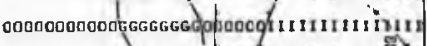

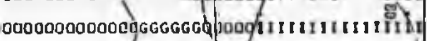

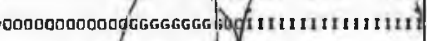

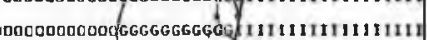

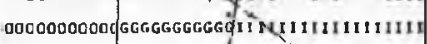

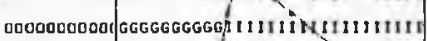
0000000000dGGGGGGGGGIIIIIIIII) H11111 00000000000GGGGGGG)1111111111 ig:

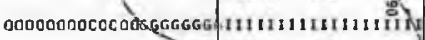
00000000000000000000 111111111111111

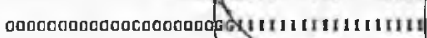

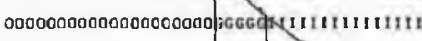

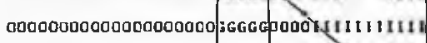

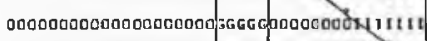

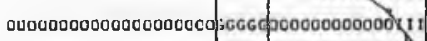

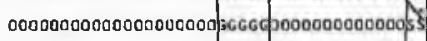

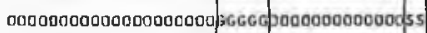

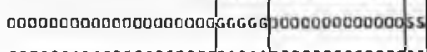

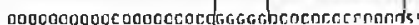

00000000000000000000FGGG600000000000005 Q00000000000000000000:G6GG I000000000000r) 000000000000000000000 OGGG Gh000000000000 IS DO00000000000000000000 GGG G0000000000\$0d 00000000000000000000 GGGGGD00000000000of

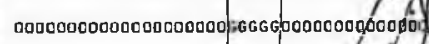

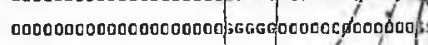

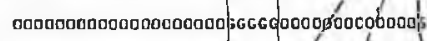

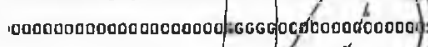
10000000000000000000006060 dro000006000000 $\$$

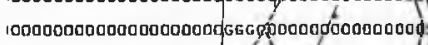
100000000000000000000 (696 G o) 090000000000 100000000000000000000 GGG G0000000000000000

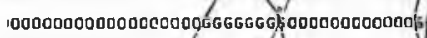

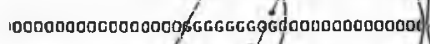

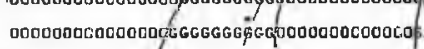
0000000000000 G GGGGGGG

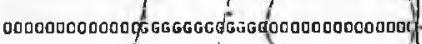

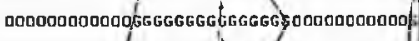

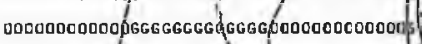

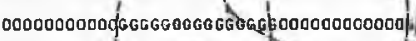

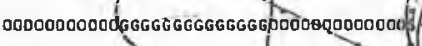

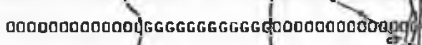

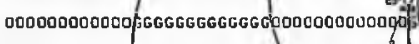

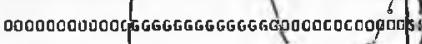

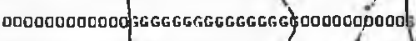
000000000000 |

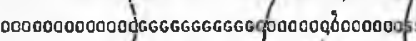

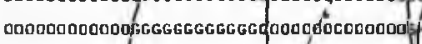
DO0000000000 GGGGGGGGGGGGG do000000000000 O 0000000000006GGGGGGGGGGGGG 50 50000000000 DOODOODOOdGGGGGGGGGGGGGGĞ

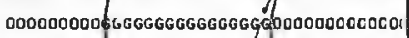

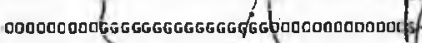
000nOCO00LobersGGGGGG oGGGG\$00000000000 o

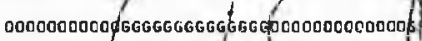

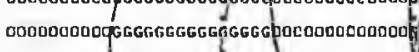
D000000000GGGGGGGGGGOGGGGDO0000CCCOCOD

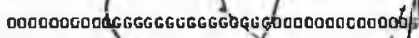

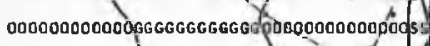

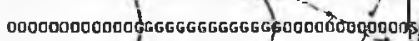

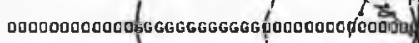

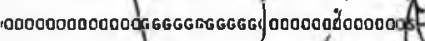
0000000000000 UGGGGGGGGGG h0000 ó 00000000

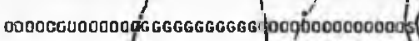
00000000000qG6GGGGGGGGGG DO QDOCDODOCOOdGGGGGGGGGG co0000 DonondGGGGGGGGGGGG 0000000000 \$GGGGGGGGGGG 角00000000000000 की 00000000000GGGGGGGGGGGG G0000000000000045 00000000000 dGG GGGGGGGGGGGG500000000000 os DOO00000000:FGGGGGGGFGGG\$0000000000000

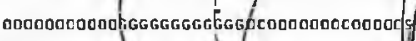
000000000000GGGGGGGGGC do0000000000000

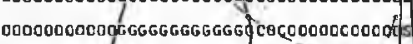

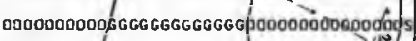
aU0000000 000000000\$GGGGGGGGGGGG 0000000000 pDo $\$$ ss 00000000000 Qे GG GGGGRGGUGO000000006000005s

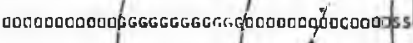

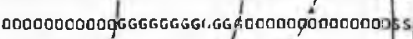

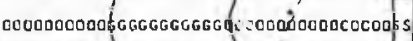

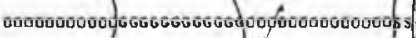

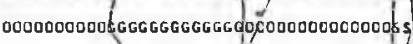

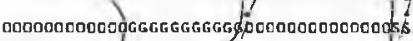

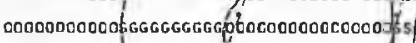

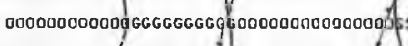
onounooocodGGGGGGGGG \& cocoocococcogands- 
0000000000 of $6666066660602000000000 c$ coc of st

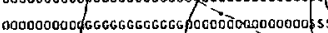

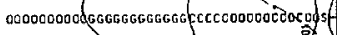
$00000000000006,6 G 66666000000060000000$ o⿰ 3 s 000000000000 dGGGGGGGG000000000 0000000 os Q0000000000006GGGGGGGG d000000000000000 of S? 000000000000 BG6GG606 0000000000000000 o s 000000000000 \$6GGGGGGG05000000000C 0000 a sf

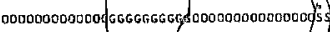

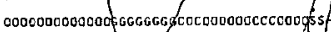

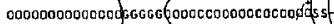

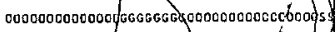
0000000000000 OGGGGGGGO $00000000 \mathrm{Cr}$

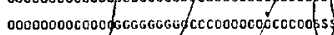

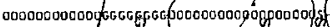

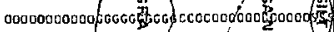

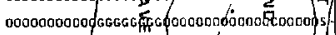

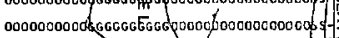

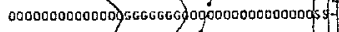

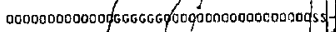

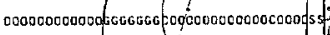

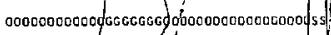
$00000000000606660 \mathrm{G} 606000000000000000055$

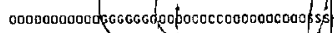

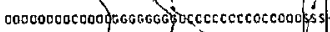

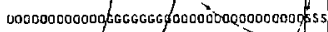

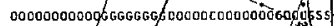

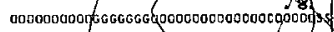
000000000 (60060 6000$) 000000000009000$ os s s

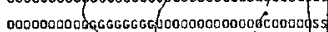
00000000 00000000000 OGGGGGG 500000000000000000055

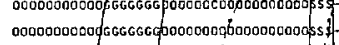

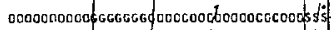
0000000000 0666006 000000060000000000065 s

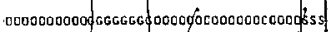

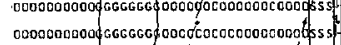

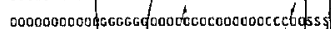

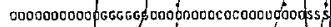

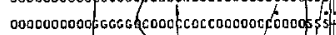

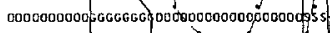
0000000000

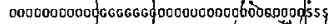

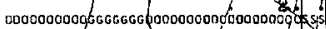

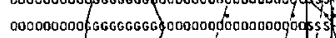

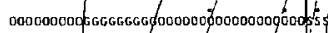

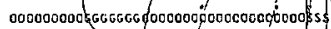

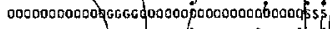

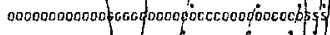

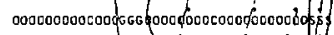

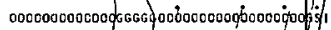

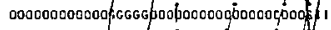

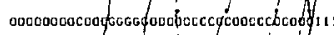

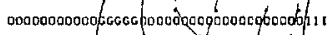

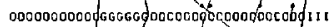

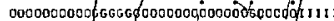

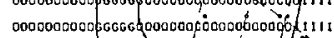

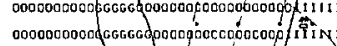
00000000000 do

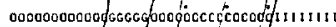

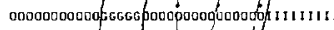

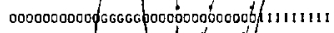

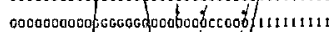

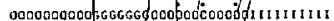

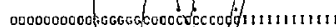

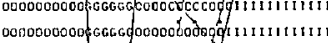
ou 000000000 oG G

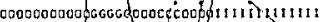

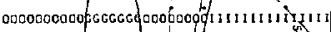

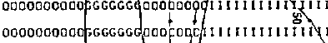

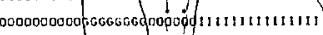

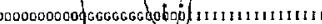

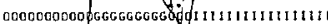

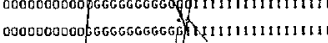

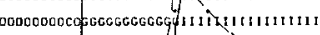

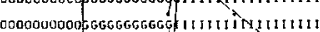

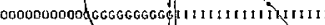

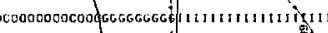

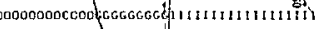

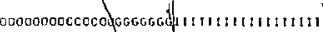

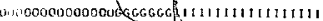

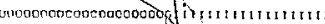

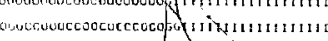

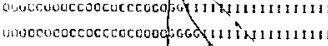

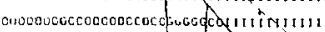

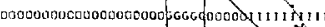

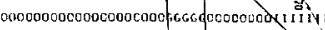

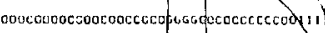

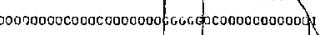

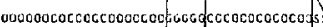

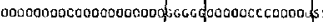

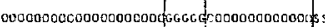

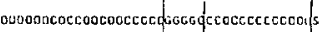

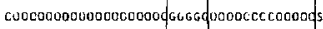
concounocsocroccco

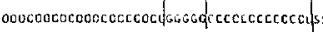

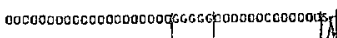

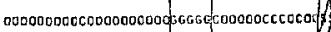
0000000000000000000050660 p00000000000 f

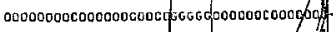
0000000000000000000006060000000000000 is

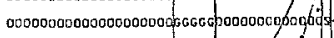

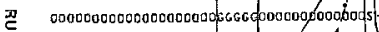

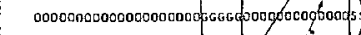

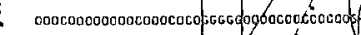

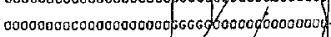
00000000000000000000600000000000000000 क

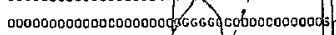

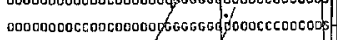

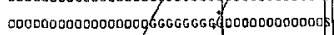

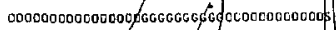

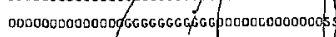

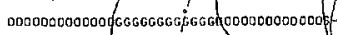
$000000000000606666604656606 G 600000000000$

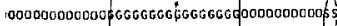

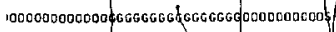

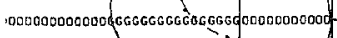

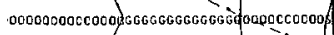

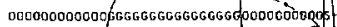

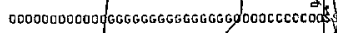

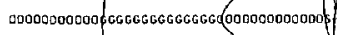
00000000000 \&GGGGGGGGGGGGGGG\$00000000000

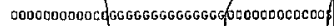
1000000000000656666666G6G600000000000000 100000000000000 GGGGGOGGGOGGOG0000000.000

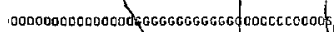
$0000000000000000066 G G 6 G G G 60600000000000$

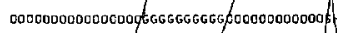

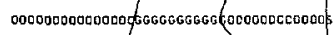
00000000000000 SECGGGGGGGGG G 0000000000 .

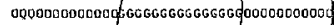

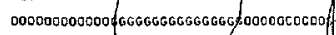

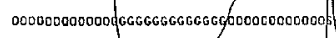

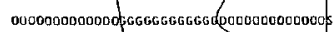
DODD0000000000 GGGGGGGGGGGGGy0000000000

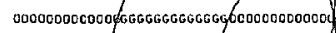

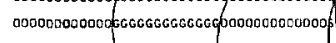
000000000000 GGGGG6G6GG6e.00000000000000

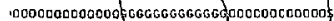

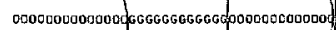
$0000000000000006 G 60 G 6 G 66000000000000000$

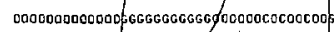
000000000000 OGGGGGG GGG G60000000 C000000)

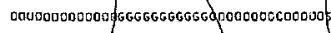

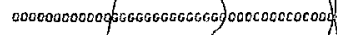

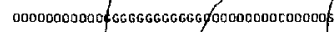

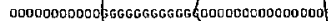

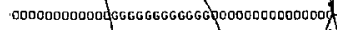

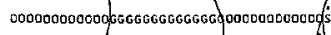

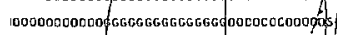

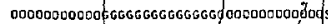

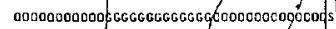

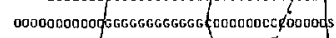

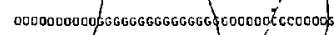

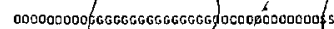

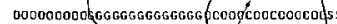

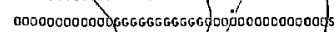

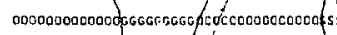

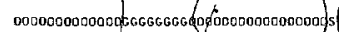

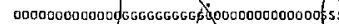

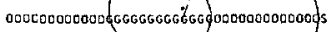

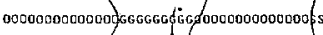

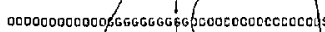

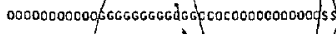




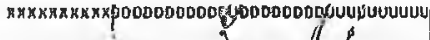

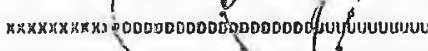

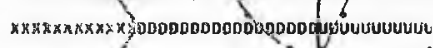

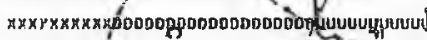

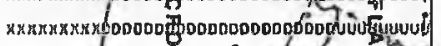

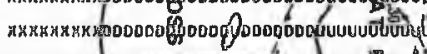

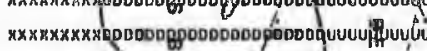

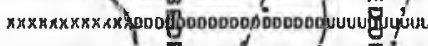

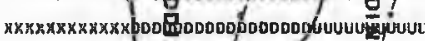

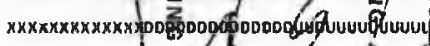

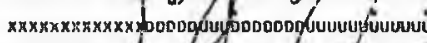

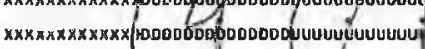

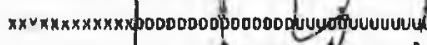

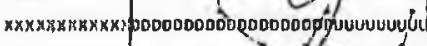

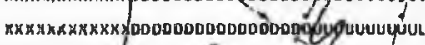

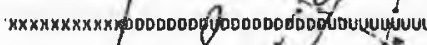
$x \times x \times x \times x \times 3 \times x 60000000000000$ g

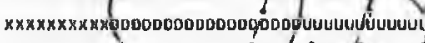

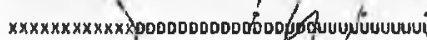
$x \times x \times x k x \times x \times x \times x>0000000000$ fod

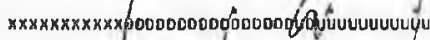

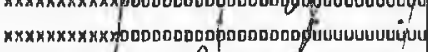

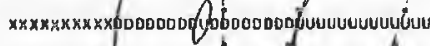

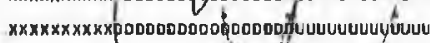
$x \times x \times x \times x \times x \times 4$ Q

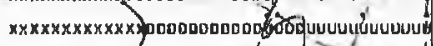

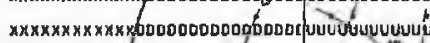

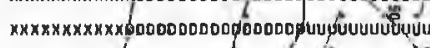

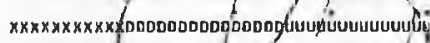

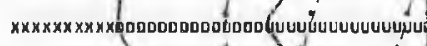

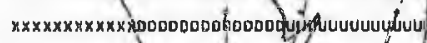

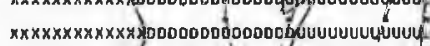

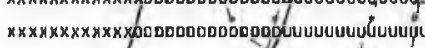
$x \times x \times x \times x \times x \times x \times p 0 a 000000000$ of

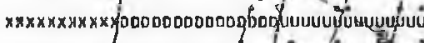

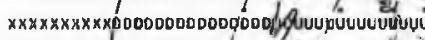
$x \times x \times x \times x \times x \times 000000000000000$ jón $x \times x \times x \times x \times x \times x=00000000+00000 a g$ a

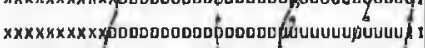

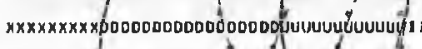

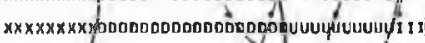

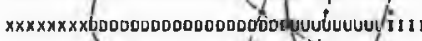

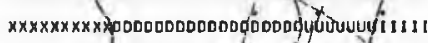
$x \times x \times x \times x \times x \times 0000000000090000$ étutuUuบ I $N$ II

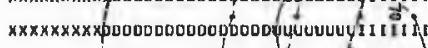

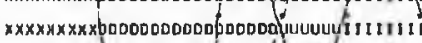

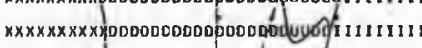

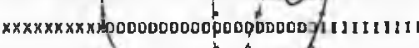

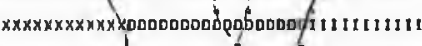
x $x \times x \times x \times x \times x \times$ p

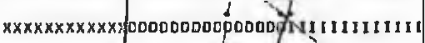

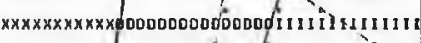

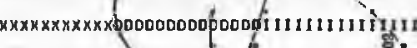

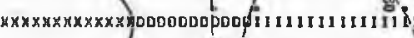
$\times x \times \times \times x \times x \times x \times x=0000000 p 0 \%$ $x \times x \times x \times x \times x \times x p 000000000011111111+111$

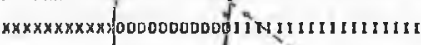

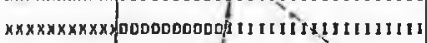

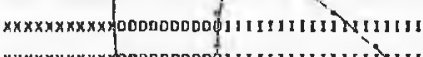

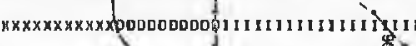

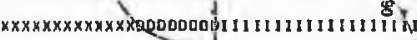

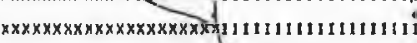

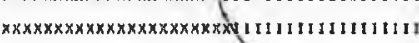

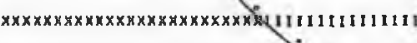

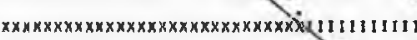

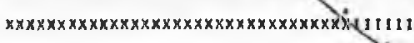

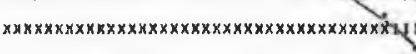

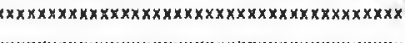


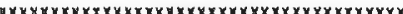

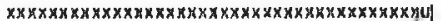

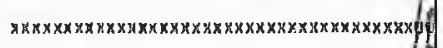

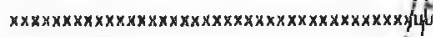

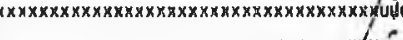

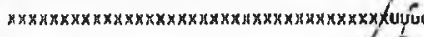

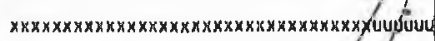

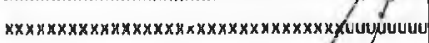

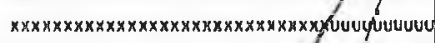

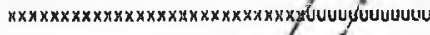

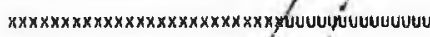

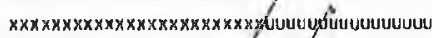

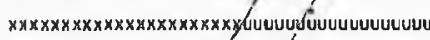

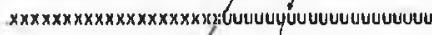

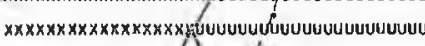
$x \times x \times x x x x \times x \times x \times x \times x$ x

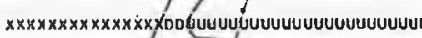
$x \times x \times x x x x \times x \times x \times \phi 000000$ of

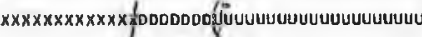

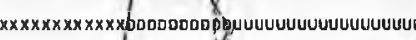

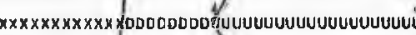

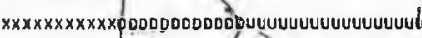

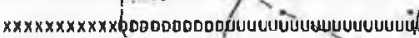
$x x x x x x x x x x x x \neq 000000$ od

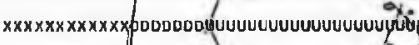

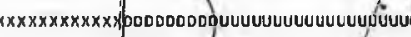

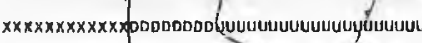

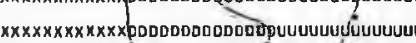

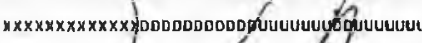

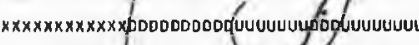

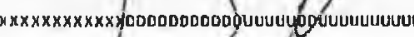

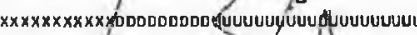
$x \times x \times x \times x x \times x \times 0000000000000$ a $x^{2}$ UuU

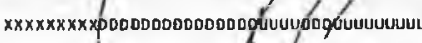

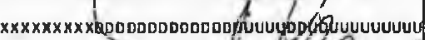

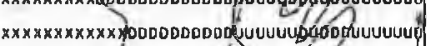

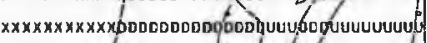
$x \times x \times x \times x \times x \times$ x $x \times x \times x \times x \times x \times y 0000000000$ pop suvuuu

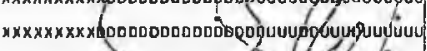

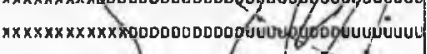

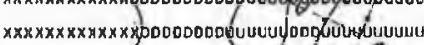
$x x x x x x x x x x x x 60000000000$ yu

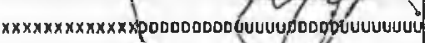

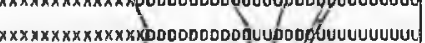

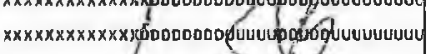

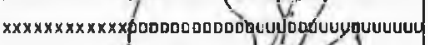

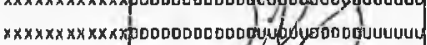

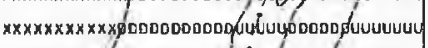

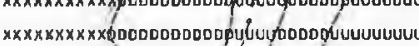

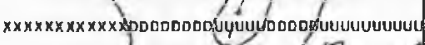

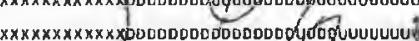

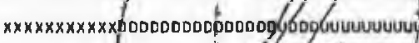

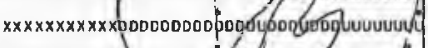
$x x x x x x x x x x x x=0000000 d$ Jun $x x x \times x \times x \times x \times x$ x

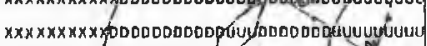
$x \times x \times x \times x \times x=000000000000, y y 00000$ a

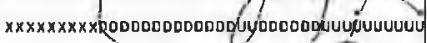

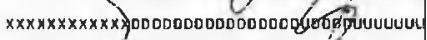
$x x x x x x x x x x x p 000000000000000000,0$ (U)บบบUUUน

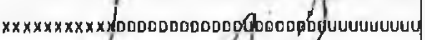
$x \times x \times x \times x \times x \times 0000000000$ gúp $x \times x \times x \times x \times x \times$ x $x \times x \times x \times x \times x \times$ apoodo00000000

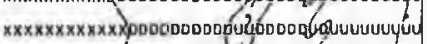

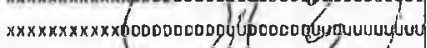
$x \times x \times x \times x \times x \times x=00000$ quy $x \times x \times x \times x \times x \times y 00000000000000000069$ yuyuuuue 


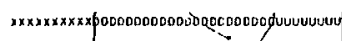

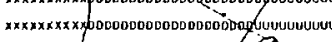

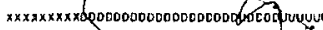

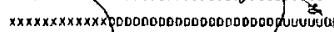

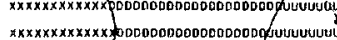
$x \times x \times x \times x \times x \times x \times x=000000000000000009$ Jusueuve

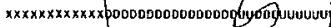
$x \times x \times x \times x \times x \times x \times x>000000000000000000$ (uvuUuvu

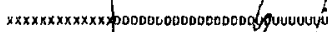
$x \times x \times x \times x \times x \times x \times x \times 10000000000000000$ Juevueuyvu

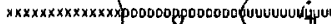

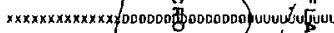

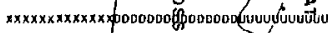

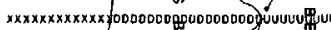

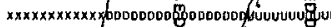

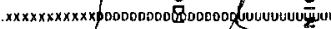

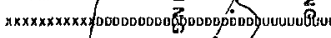
$x \times x \times x \times x \times x \times a p 000000000000 p 6000$ ovuvuvouvu $x \times x \times x \times x x \times x \times x \times x>0000000000$ ớ

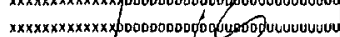

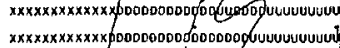

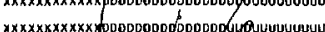

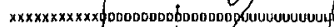

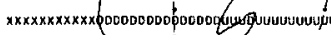

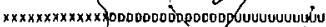
$x \times x \times x \times x \times x \times x \times 600000000000$ o

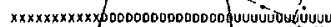

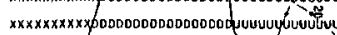
$x \times x \times x \times x \times x \times 0000000000000000$ fuv

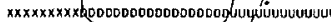

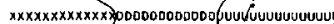

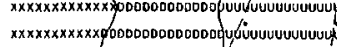

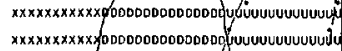
$x \times x \times x \times x \times x \times 000000000000000$ xx $x \times x \times x \times x \times x y 00000000000006$ fun

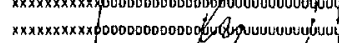

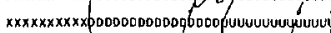

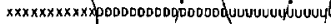

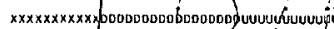

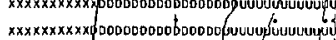
$x \times x \times x \times x \times x \times p 00000000000000000$ $x \times x \times x \times x \times x \times 6000000000000000$ $x \times x \times x \times x \times x \times x$ food

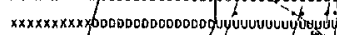
$x \times x \times x \times x \times x$ x $x \times \times \times \times x \times x \times 000000000000000$ of $x \times x \times x \times x \times \times x=0000000000000$ o' $x \times x \times x \times x \times x \times x \times 1000000000$ po

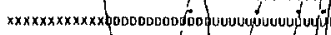

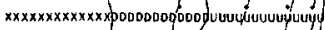

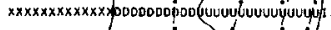

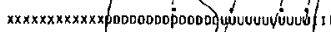
$x \times x \times x \times x \times x \times x \times 00000000$ ponod

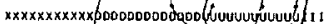

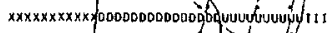

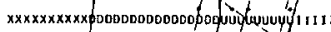
$x \times x \times x \times x \times x \times p 00000000000$ do

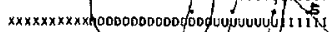

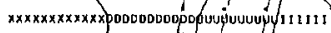
$x \times x \times x \times x \times x \times x \times x=0000000 p$ p

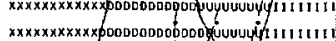
$x \times x \times x \times x \times x \times$ Yo00000000 $x \times x \times x \times x \times x \times p 000000000 b 00600000111111111$

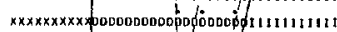

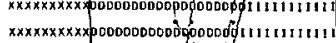
$x \times x \times x \times x \times x \times 60000000000 p 0000$.

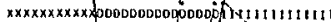
$\times x \times x \times x \times x \times x \times 0000000000000$ B

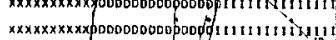

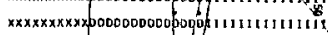
$\times \times \times \times \times \times \times \times \times \times$ ond

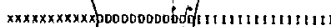

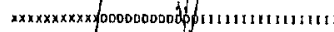

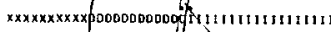
$x \times x \times x \times x \times x \times 30000000000$.

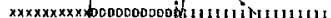

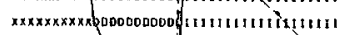

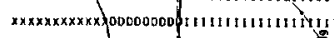

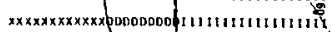

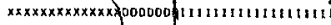

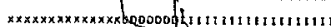

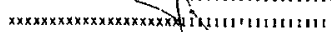

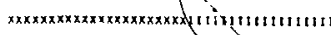

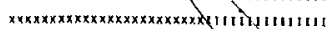

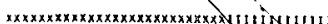

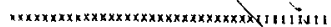

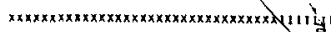

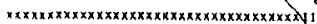

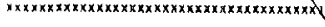

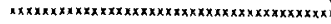

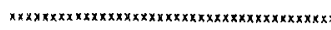

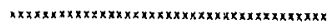

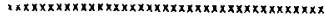

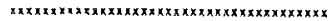

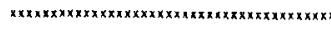

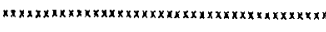

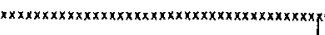

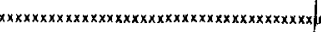

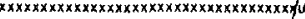

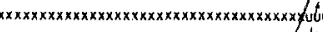
$x \times x \times x \times x \times x \times x \times x \times x \times x \times x \times x \times x \times x \times x \times x \times x \times x \times x \times x \times d u$

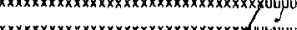

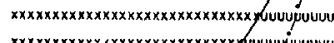

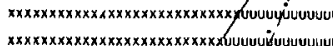
xxx

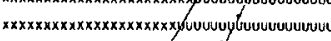
$x \times x \times x \times x \times x \times x \times x \times x \times x \times x x$ fou $x \times x \times x \times x \times x \times x \times x \times x \times x x$ 近

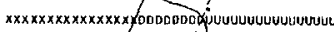
$x \times x \times x \times x \times x \times x \times x \times$ ond

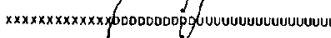
$x \times x \times x \times x \times x \times x \times$ popodono

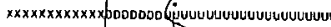

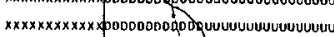

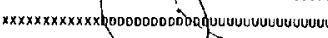

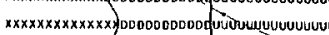

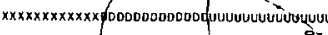

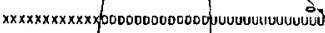

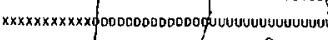

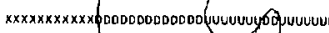

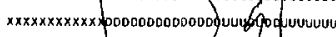

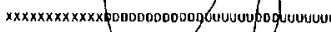

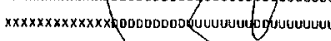

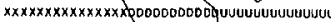

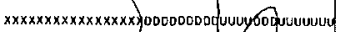
$x \times x \times x x \times x x x x x x x x x$ fod

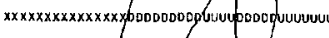

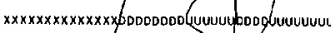

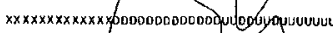

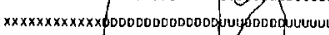

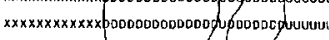

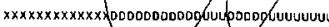

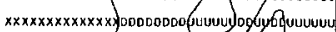

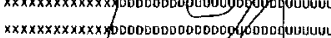
$x \times x \times x \times x \times x \times x \times x=0000000000000 y=000$.

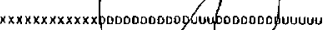

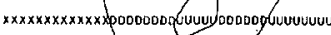

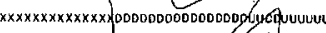

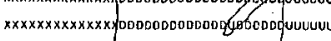

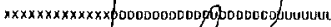
$x \times x \times x \times x \times x \times x \times x$ b000000000

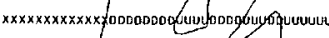
$x \times x \times x \times x \times x \times x y 00000000000000$ for

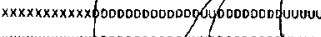

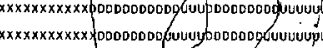

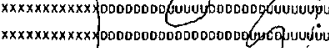
$x \times x \times x \times x \times x \times x \times 0000000000000000000000$

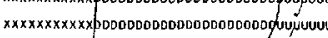

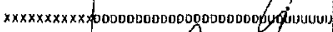
$x \times x \times x \times x \times x \times$ yoco00000000 fuy $x \times x \times x \times x \times x \times 000000000000000000000$ uvvuvuvuv

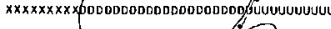

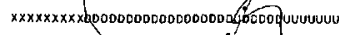

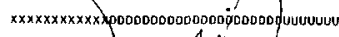

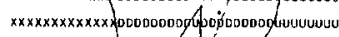

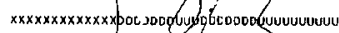

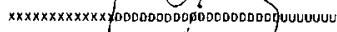
$x \times x \times x \times x \times x \times x \times 600000000$

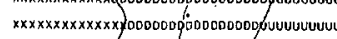
$x \times x \times x \times x \times x \times x \times x=00000000000000000000$

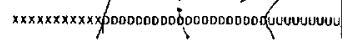


Experiment 5 is designed to illustrate the sedimentation patterns associated with aggradation as meanders are developing: from a sinuosity of 1.1 to a limiting sinuosity. A lateral section is used for each of nine runs of the program, which correspond to al1 combinations of three different rates of aggradation $(0.001,0.01$, and 0.1 metres/year) and three different modes of meander migration. In this experiment the downalley migration rates are not constant for all runs. The data which are different for each run are shown in table 20.1, and the bank migration parameters correspond to average initial rates of movement normal to the mean downvalley direction, and average downvalley migration, of about 3 and $2.2 \mathrm{~m} . /$ year, 9 and $2.2 \mathrm{~m} \cdot /$ year, and 30 and $7 \mathrm{~m} . /$ year, respectively, for the three different modes of meander movement. All other input parameters, shown in table 20.2, and 20.1 , are the same for all nine runs. No scouring and filling is assumed, as in experiment 2 , in order to simplify examination of the cross sections. Again, therefore, the sedimentary structure and grain size cross sections should be viewed bearing in mind all the simplifications and approximations involved. The data deck set-up for all nine runs is listed in the appendix 3. This experiment used no disc, the cross sections comprised 200 by 60 cells, and 129k of core store were required. The appxoximate running times were 2 seconds per time increment.

The simulated point-bar deposits, as shown in figs. 20.1 and 20.2, show essentially the same features as in experiment 4. The main points are the gradual lateral decrease in the general calibre of sediment, after an initial increase, and the lateral increase in cross bedding at the expense of flat bedding, with a large scale interfingering within a transition zone. The grain size and sedimentary-structure facies boundaries and basal erosion surfaces are more simplified, however, because of the lack of 


\begin{tabular}{|c|c|c|}
\hline Run number & $5 a$ & $5 b$ \\
\hline $\begin{array}{l}\text { Average initial } \\
\text { migration rate } \\
\text { normal to mean } \\
\text { downvalley } \\
\text { direction } \\
\text { (metres/year) }\end{array}$ & \multicolumn{2}{|c|}{3} \\
\hline $\begin{array}{l}\text { Average downvalley } \\
\text { migration rate } \\
\text { (metres/year) }\end{array}$ & \multicolumn{2}{|c|}{2.2} \\
\hline \multicolumn{3}{|l|}{$\begin{array}{l}\text { BANK MIGRATION } \\
\text { PARAMETERS }\end{array}$} \\
\hline exponent $\mathrm{n}_{2}$ & & \\
\hline Constant $\mathrm{k}_{2}$ & \multicolumn{2}{|c|}{$0.3 \mathrm{E}-06$} \\
\hline \multicolumn{3}{|l|}{ Constant $\mathrm{k}_{3}$} \\
\hline $\begin{array}{l}\text { Aggradation rate } \\
\text { (metres/year) }\end{array}$ & 0.001 & 0.01 \\
\hline
\end{tabular}


Table 20.1

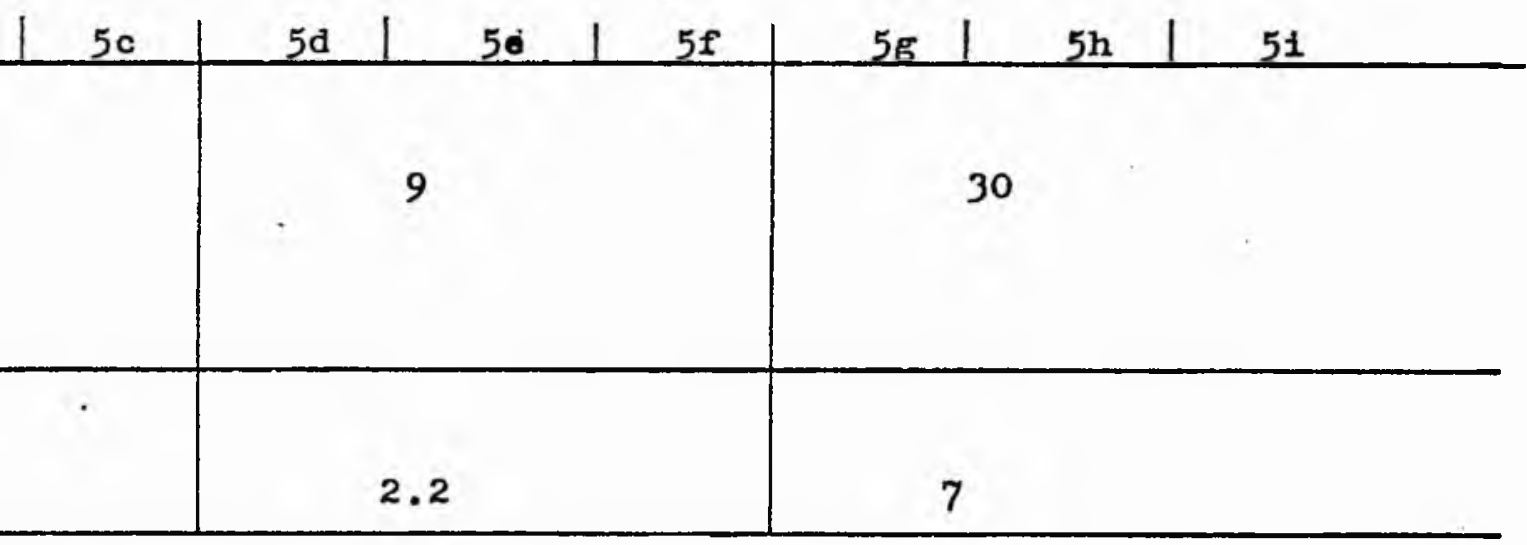

0.5

$0.9 \mathrm{E}-06$

$$
0.3 E-05
$$

$0.3 \mathrm{E}-03$

$0.1 E-03$

\begin{tabular}{l|l|l|l|l|l|l|}
0.1 & 0.001 & 0.01 & 0.1 & 0.001 & 0.01 & 0.1 \\
\hline
\end{tabular} 
rLUYIATILE PROCESS SIMULATICN EXPERIMENT 5

CROSS SECTION PARAMETERS

HIOTH OF SECIION

THICKNESS UF SECTIDN

IN INIIAL BANKFULI. STAGE MEASURED FRON SECTION BASE

CELL SIZE IN VERTICAL(Y) DIRECTION

CELL SIZE IN HORIZONTAL ( $Z$ OR $X)$ DIRECTICN

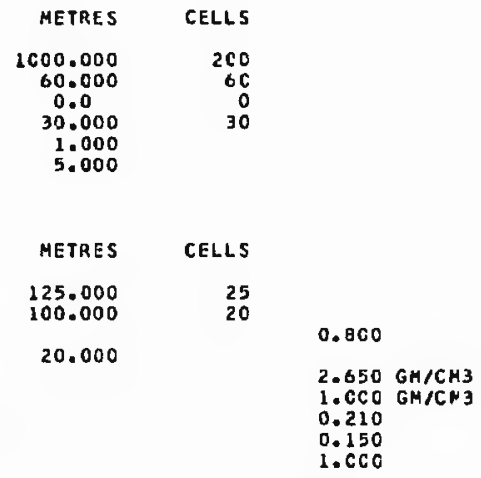

SYNTHET IC HYDROLOGY PARAME TERSIUNITS NCT NECESSARYI

MEAN OF ALL DAILY MEAN YaLUeS

STANOARD DEVIATIDN OF DAILY MEAN YALUES

MEAN OF YT SERIES

STANDARD DEVIATION OF YT SERIES

COEFF ICIENTS IN AUTOHEGRESSIVE MODEL

FOURIER CUEFFICIENTS FOR DALLY MEANS(A)

FQURIEH COEFFICIENTS FOR DAILY STD DEVIATIONSISAI

HAX IMUM VALUE OF OVOL

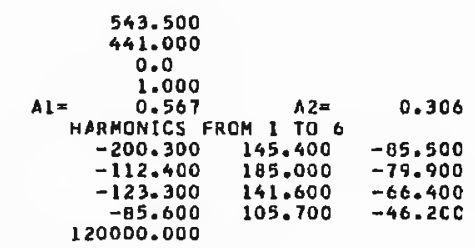

$\begin{array}{ll}58.600 & -39.800 \\ 65.600 & -72.500 \\ 75.700 & -47.200 \\ 31.700 & -43.200\end{array}$

7.400

0.0

0.0

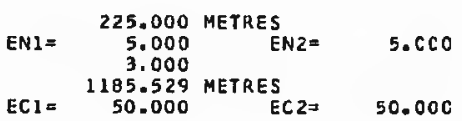

A LATERAL SECTION IS REPRESENTED IN THIS TEST

LEGEND

LDYER PHASE PLANE BED RIPPLES

DUNES PHASE PLANE DED

UPPER PHASE

\section{L
$R$
$D$
$U$
$A$}

PLANIMETRIC FORM OF hEANDER

MAVELENGTH

AMPLITUDE

RADIUS OF CURVATURE AT BEND AXIS

HIOTH OF MEANDER NECK

CHANYEL LENGTH ALONG MEANDER

YALLEY SLOPE

LONGITUDINAL HATER SURFACE SLOPE

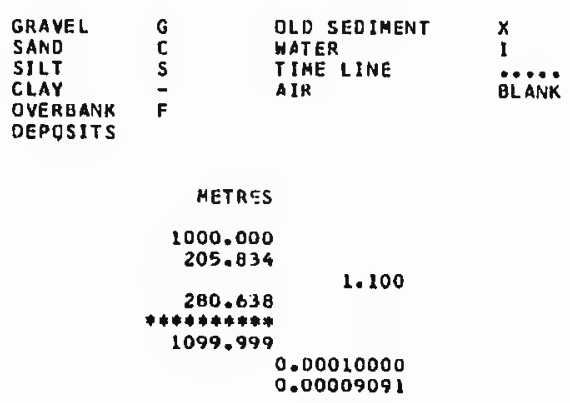

SELECTED GEOHETRIC RATJOS

WAVELENGTH TO RADIUS OF CURVATURE

WAYEL ENGTH TU CAANNEL HIDTH

AMPLUS OF CLRVATURE TO CHANNEL HIOTH

AMPLI TUDE TO CHANNEL WIOTH

3.563

2.245
1.647

Table 20.2. Initial data for experiment 5. 


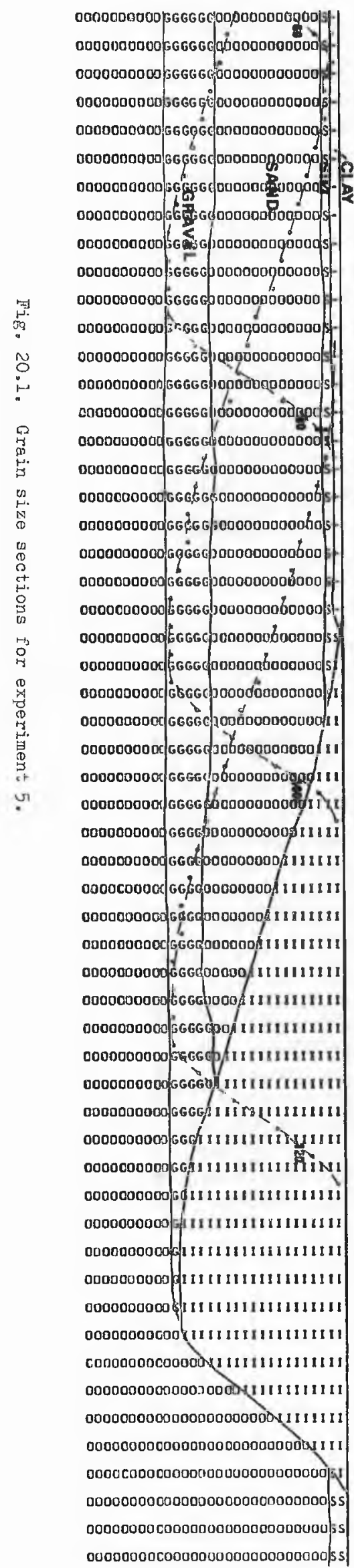

D000000000 GGGGGG G00000000000O-

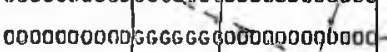

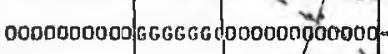
0000000000 GGGGGG CO0000p00000 COD0000000/56GGGG G0000D00000000000000000 /FGGGG CODODODOOODDI-

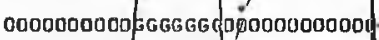
0000000000 GGGEGG DODODODO0000 00000000006G6GG od topo00000000 0000000000 GGGG\&G (J00000000000:

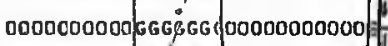
00Q0000000; GFGGG G00000000000 

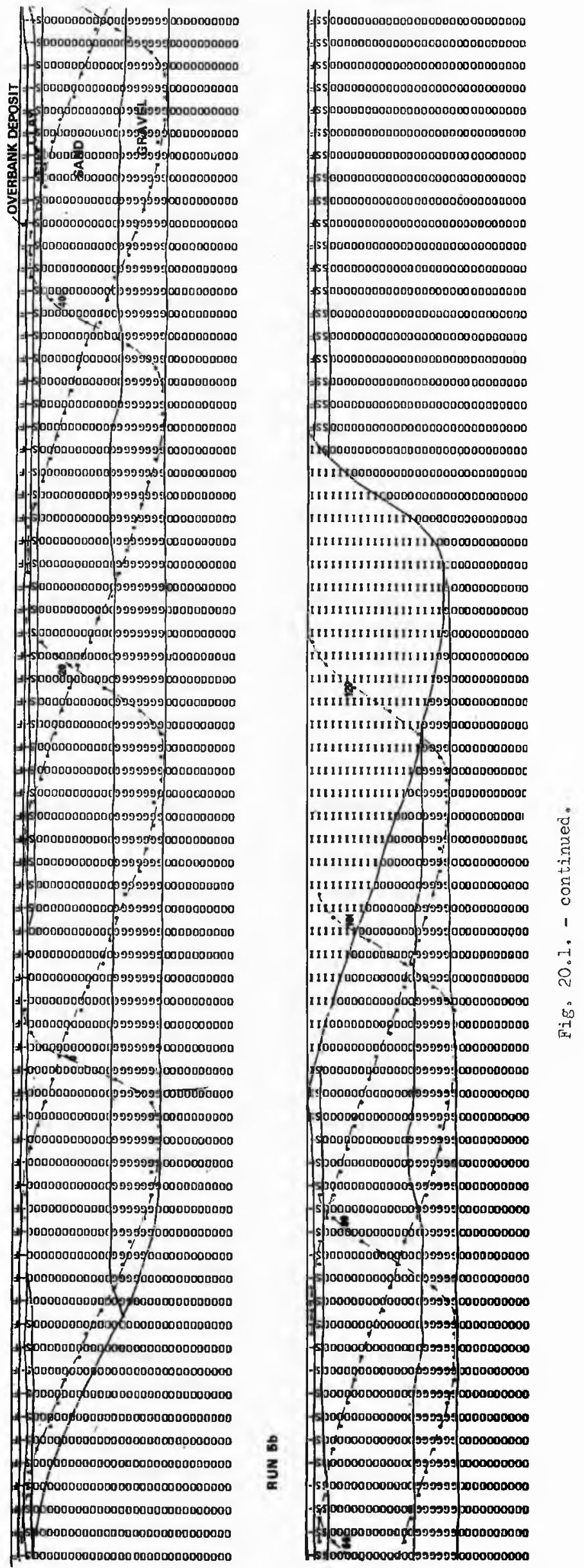


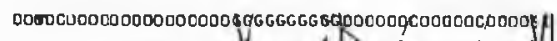

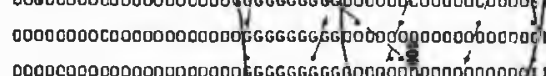

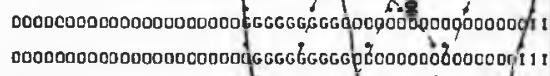

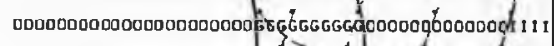

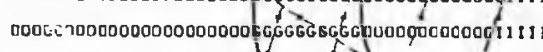

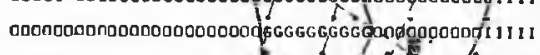

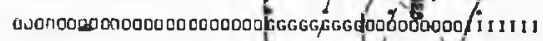
00000000000000000000000 GGGGGGGGU0000000/ 1111111 00000000000000000000000d GGGGGGGG000000 11111 I I I I I

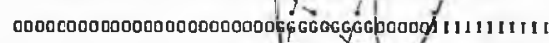

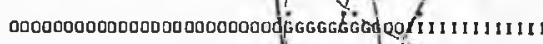

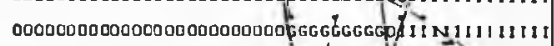

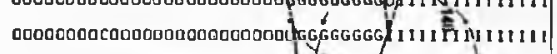

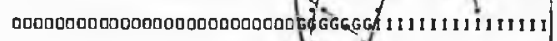
00000000000000000000000000 oGGG of

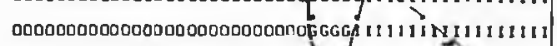
000000000000000000000000000 व6 0000000000000000000000000000 of 1111311111111111

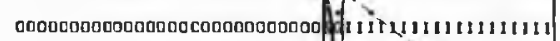

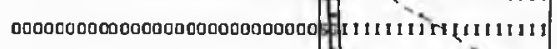
0000000000000000000000000000 | 1111111111 ì 1111

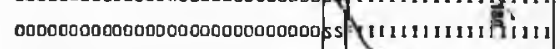

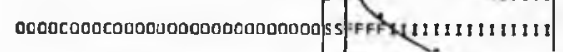
DOQ0000000000000000000000000 S\$FFFFFFT'W 11111111 O000000000000000000000000000 SSFFFFFFFFFFY I I II

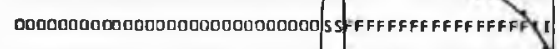
DODO000000000000000000000000 SSEFFFFFFFFFFFFFFF 0000000000000000000000000000 SS FFFFFFFFFFFFFFFF 0000000000000000000000000000 SS FFFFFFFFFFFFFFF C0000000000000000000000000000 SS: FFFFFFFFFFFFFFF

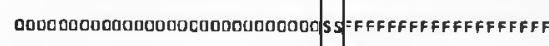
DONCCOOODO00000000000000000D5s -FFFFFFFFFFFFFFF 0000000000000000000000000000 S $\$$ FFFFFFFFFFFFFFFF OC0000000 D0000000000000000000 5s SFFFFFFFFFFFFFFF QC00000000000000000000000000 STFFFFFFFFFFFFFFF
000000000000000000000000000QS FFFFFFFFFFFFFTFFF D000000000000000000000000000 SI FFFFFFFFFFFFFFF

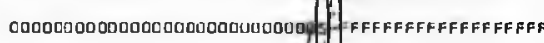

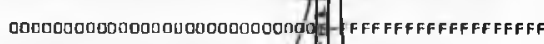

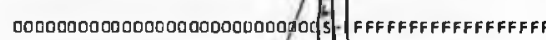
00000000000000000000000000 OS I I FFFFFFFFFFFFFFFF 0000000000000000000000060 ODOD STEFFFFFFFFFFFFF Q0000000000000000000000008 00 SS-FFFFFFFFFFFFFF 000000000000000000000000́0000/SS S- FFFFFFFFFFFFFFF

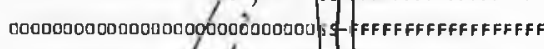
OD0000000000000Q 00000000000 SFFFFFFFFFFFFF ano00000000000 6 G

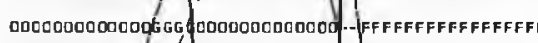

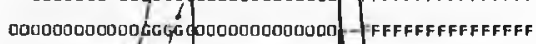

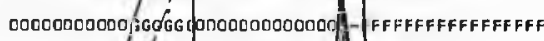

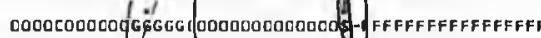

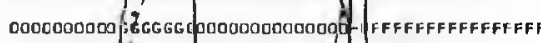
D000000000 OOOCO0O0OD GGGGG Q 0000000000; GGGGG G CO00CU0000 $606 G G G 0000000$ g 0000 - FFFFFFFFFFFFF

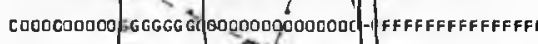

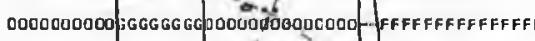

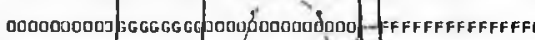

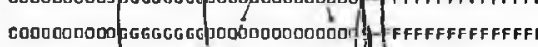
00000000000GGGGGGGhp'000000000000 मf FFFFFFFFFFFF 0000000000 $0 G G G G G E G\} 0000000000000$. FFFFFFFFFFFF 0000000000 GGGGGG

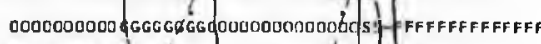

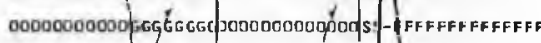

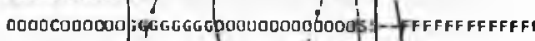

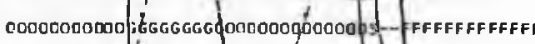

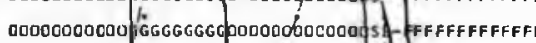

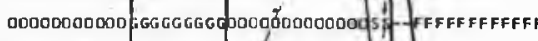
00000000000 GGGGGGGG0090000000000 JST-FFFFFFFFFF

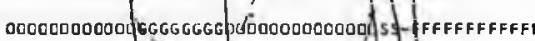

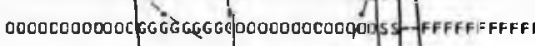

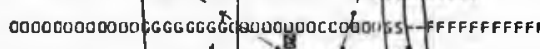

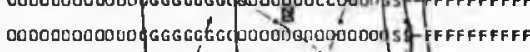
000000000000 oGGGGGGG

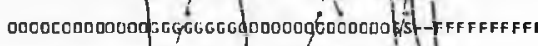

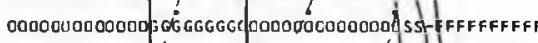

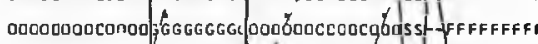

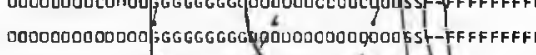

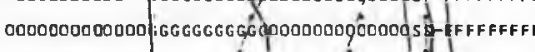

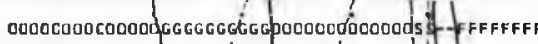

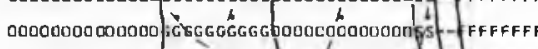

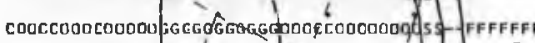

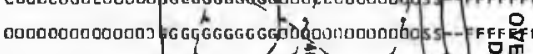

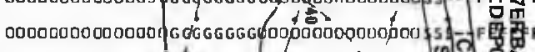

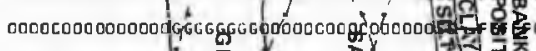
000000000000000 GGG co000000000000

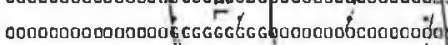
0000000000000000 Gे

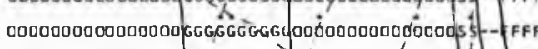

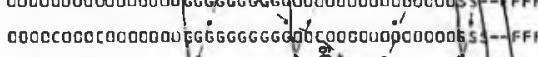

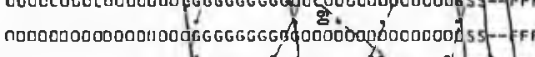

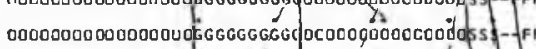

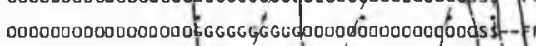

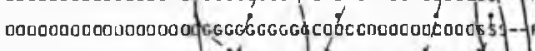

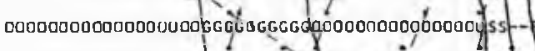

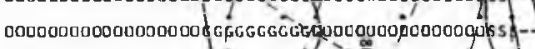

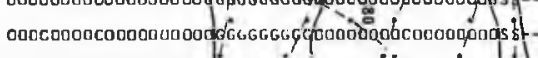

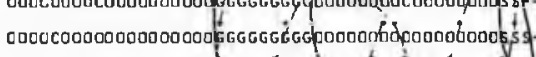

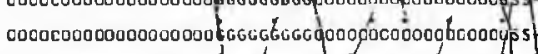

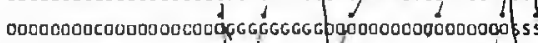

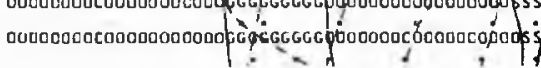




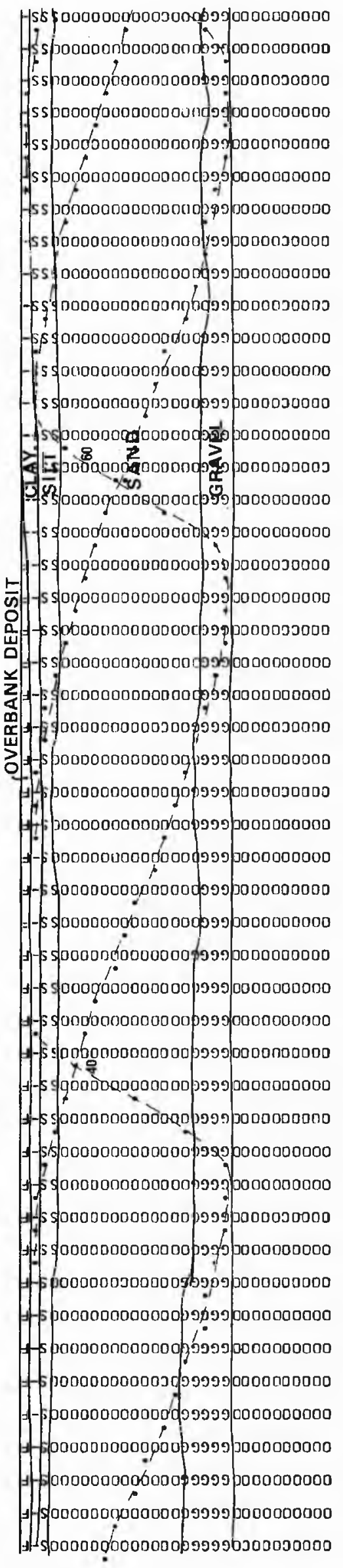

非5/000000000000000000000003003 非s 0000000000000000000000000000 Is $\$ 0000000000000000000000000000$ Is s 0000000000000000000000000000 S5 0000000000000000000000000000 \$) 550000000000000000000000000000 "\$ \$ 50000000000000000000000000000 550000000000000000000000000000 I I k000000000000000000000000000

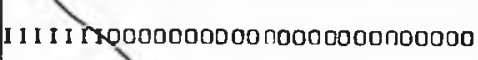
I 1 I I I I I I I kpoopono0000000000000 1111IIIIIIIX00000000000000000 I1111IIIIIIIII 00000000000000 I I I I I IIIIIIIIIIII I I I P00000000000 I I I I I I IIIIIIIIIIIII I I I I P00000000000 I I I I IIIIIIIIIIIIIIIIII I P00000000000 I I IIIIIIIIIIIIIII I I DD0000000000 FIIIIIIIIIIIIIIIIII I I IY I I I I I I I I I I I I I I I IIIIIN IIIIIIIIIIIPS00000000000 IIIIIIIIN IIIII IO900300030000 I I I I I I I IIIIIII N I I pod00000000000 I I I I I I I I I I I I I I II I Y Y popo0000030000 I II IIIIIIIIIIIII J00 \$00000000000 I IIIIIIIIIIIIIII 00p900000000000 I I I I I I I I I I I I I I I/O000pg00000000000 I I I I I I I I I I I I I I y00000popo000000000 I I I I I I I I I I I I I/b000 opopono00000000

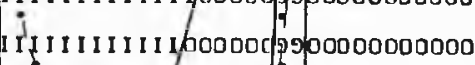

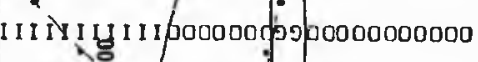
IIIIIII I I I I I I I I I I I fodono0000 900000000000 I I I I I I I I yoononoighopospo000000000

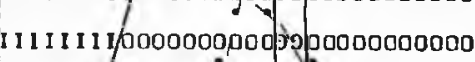

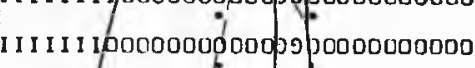
I 1 I I I Ippo0000p300 posp0000000000 I I I I I $p 000000$ d00000 900000000000 11 1p000000diono000p9/ 0000000000

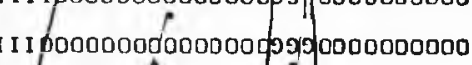
I younoonónoonoonópís 0000000000

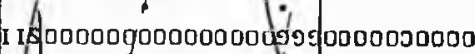

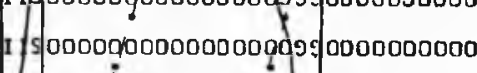

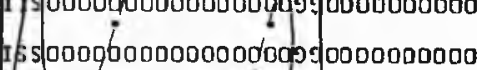

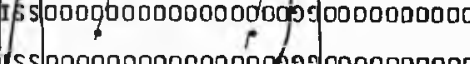

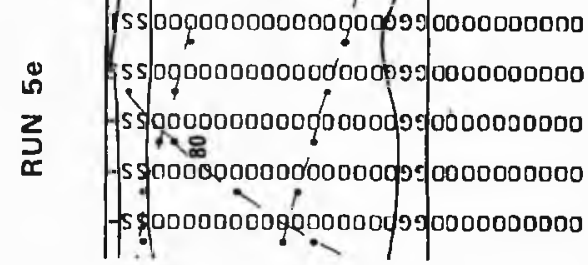




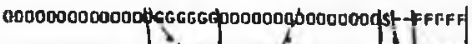

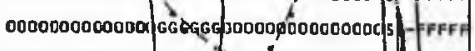
00000000000000 GGGGGifo00g6́0000000000 Bi FFFF 00000000000000 ; 6 GGG J0000000000000000 5ी FFFF 00000000000000 GGGG

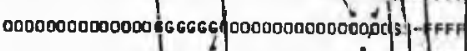
0000000000000006G6gGr 000000000000600

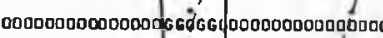

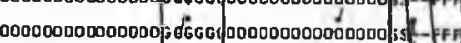

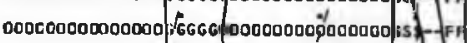
000000000000000 ;66GGE000000020000000/55 C00000000000000 GGGGC000000000000000 tsst-

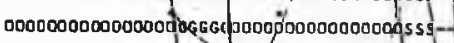

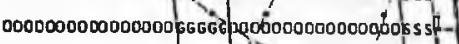

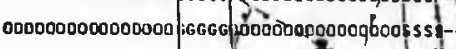
0000000000000000 isGe कq

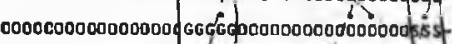

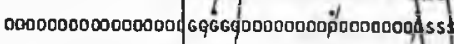

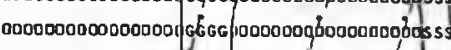
0000000000000000 66G6 10000000000000 aboos 53

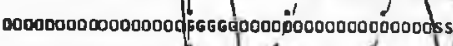

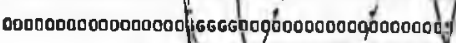

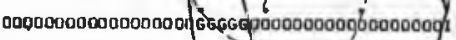

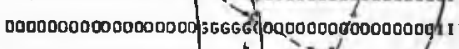

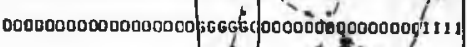

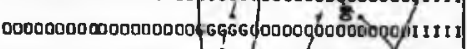
0000000000000000000 of 6 G 00000,0000000 of 111111

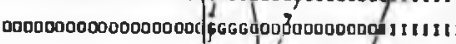

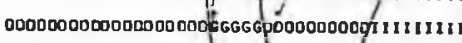

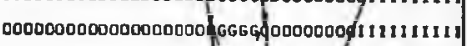

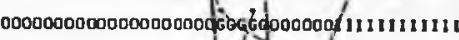

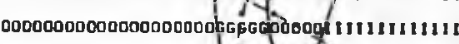

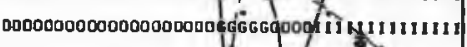

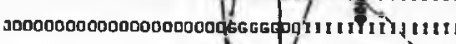
J00000000000000000000 06600 fil111111111111 300000000000000000000dpGG 111111111111111 s000000060008000000000 G

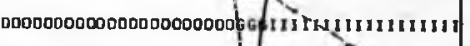

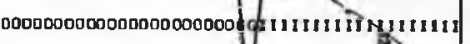

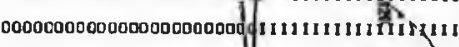
00000000600000000000000 h11111111113111 000000000000000000000000 1 I IIIIIIIIIIIIt 000000000000000000000000000111111111111] 0000000000000000000000000000 35 ㄴ11111111111 OD00000000000000000000000000/5SFFFFARIIII! 0000100000000000000000000000 /SS FFFFFFFPY OCO0C0000000000000000000000D SS FFFFFFFFFFF 0000000000000000000000000000) ISSFFFFFFFFFF D000000000000000000000000000 55 /FFFFFFFFFFF 0000000000000000000000000000 | SS FFFFFFFFFFF Q0000000000000000000000000000/ SSFFFFFFFFFFF" D000000000000000000000000000/SSTFFFFFFFFFF DO0000000000000000D00000000D SS:FFFFFFFFFFF 00000DOODODO0000000000000000 STFFFFFFFFFFF O000000000000000000000000000 SS: TFFFFFFFFFF 00000000000000000000000000000 SS SFFFFFFFFFFF O000000000000000000000000000 SOFFFFFFFFFFF

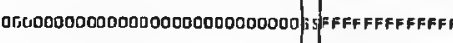
O000000000000000000000000000 \$FFFFFFFFFFF
Q000000000000000000000000000dSTFFFFFFFFFFF DO000000000000000000000000 If EFFFFFFFF 000000000000000000000000000 OFFFFFFFFF DFFFFFFFF C000000000000000000000000000 0 ( FFFFFFFFFFI O00000000000000000000000 00 OOO OO FFFFFFFFFF

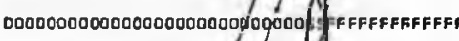
0000000000000000000000 gonono st FFFFFFFFFA

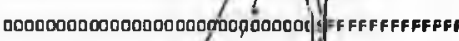
000000000000000000000000000 OfFFFFFFFFFA

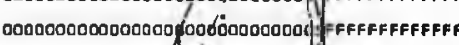
c00000000000000 6 óno00000000 FFFFFFFFFF 0000000000000960000000000000 o $)$ FFFFFFFFFFI $000000000000 \mathrm{~g} 6060$ QDO000000000 $\$$ FFFFFFFFFF O00000000000 OGGGGG Q00000000000D EFFFFFFFFFF

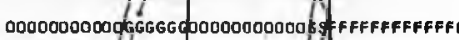

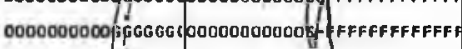
C000000000 SGGGGECDOOOOODODOO OFFFFFFFFFA

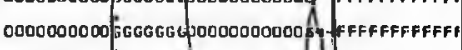
D000000000 0 GGGGGGI0000000000S $\$$ FFFFFFFFFI

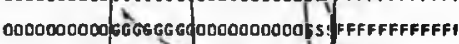
0000000000 3GGGGG

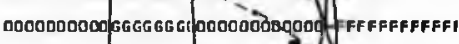

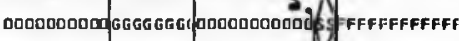
0000000000 GGGGGGG(D000000000 of FFFFFFFFFFI

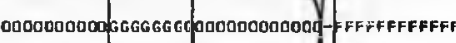
000000000 ;GGGGGG 000000000 FFFFFFFFFI

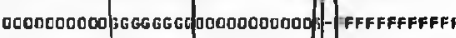
DODO000000 :GGGGGGCDOOODOODOOO OA FFFFFFFFFI

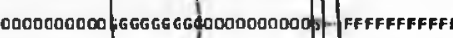
COODOOOODO GGGGGGGPO00000000 O FFFFFFFFF

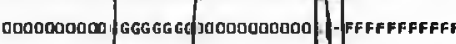
0000000000 GGGGGCOODO0000000 IAFFFFFFFFI

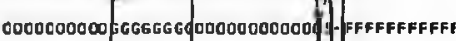

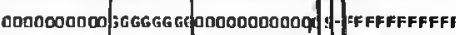

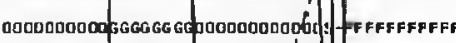
0000000000 EGGGGGGCDO000000DOOOO OHFFFFFFFFI

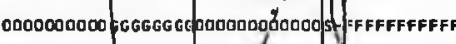

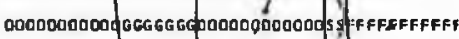

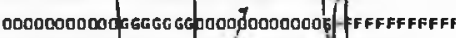

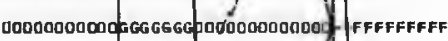
OOOD000000DGGGGGGG

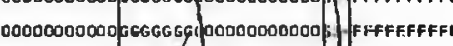
Q000000000QGGG GG GG G0000000000U HFFFFFFFI

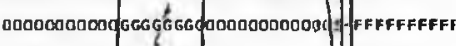
00000000000 GGGGGGG00000000000 OD FFFFFFFP

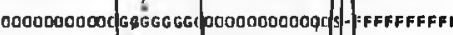

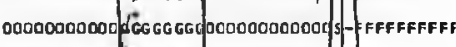

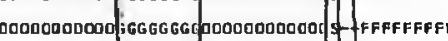

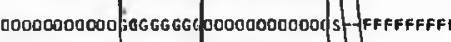
D000000000O GGGGGG(00000000000dS -FFFFFFFFF

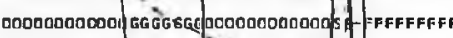

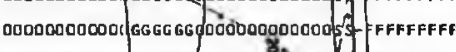
000000000002 GGGGGG0000000000000 S. FFFFFFF

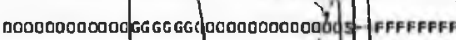
Q0n00000000Q GGGGGG

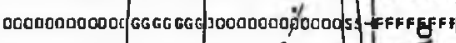
000000000000 GGGG GGIOD00000600000 S I FFEF FF

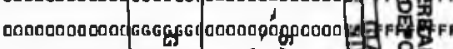
000000000000 GGG

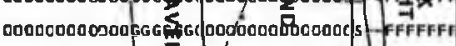

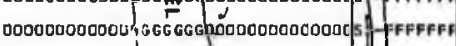

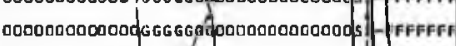
0000000000000 GGGGG00000000000000 of FFFFFF

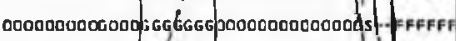

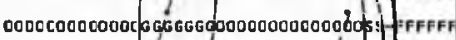

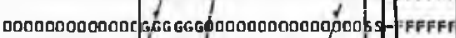
0000000000000 GGGGCD00000000006000 इS FFFFI

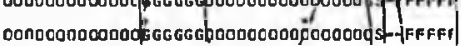


0000040000600000 ofsGq0000000/11111111!1

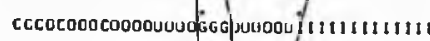

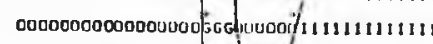

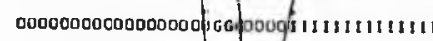

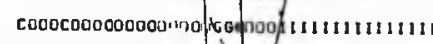

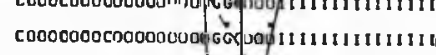

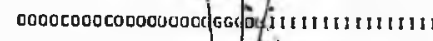
000000000000000000/600

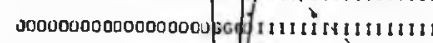
$00000000000000000 / 66$ (1111411tivilin 00000000000000 0000 5011111111111!

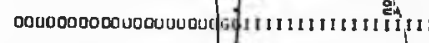
0000000000000000000 1111111111111111 0000000000000000004 651111111111111111 0000000000000000004 F11111111111111111 000000006000000000 月111111111111111111 c000000000000000000 di1111111111111111 000000000000000000/111111411111111

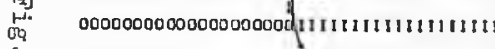
Q9 000000000000000000000 11111111111:11 O $00000000000000000000000 \times 111+11111111$ i. 0000000000000000000000000 रा 14111111 - 0000000000000000000000000000 हैंस1111111
$00000000030 \% 666$ / 0000000000000 /S: H FFFF

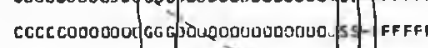

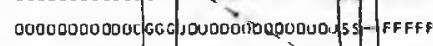

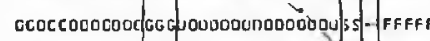

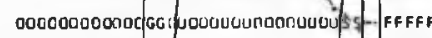

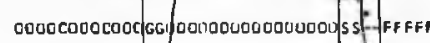

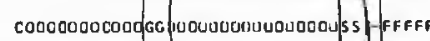

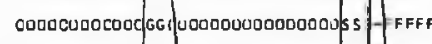

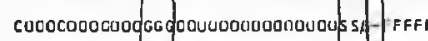

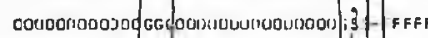
000000000000 GGdOCUUDONDU000000 is

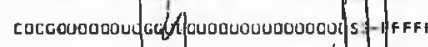

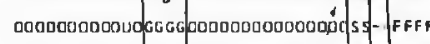

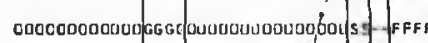

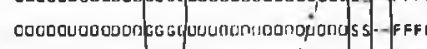

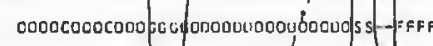

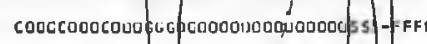

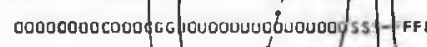

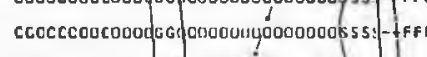

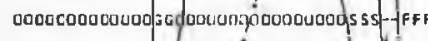
0000000003000 GG G000000

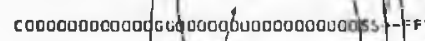
0000C0000000x G Ge0ucó000000000000 ss:- -

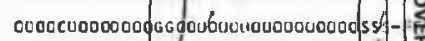

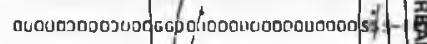

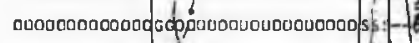
0000000000000 G Ganano000000000u0000 lss:

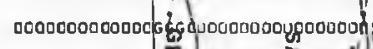

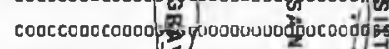

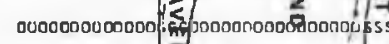
0000000000000); Gr Dono00no0000000phosss

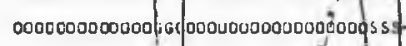

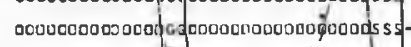

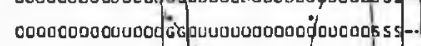

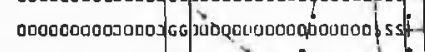

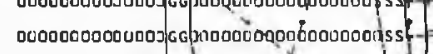
0000000000000u|cogovo0uv0g000000000ulss i-

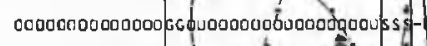

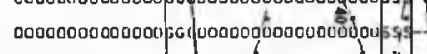

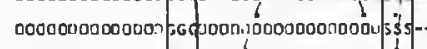
$0000000000000=6(j 00 y 000000000000$ J

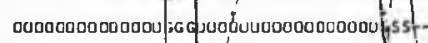

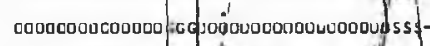

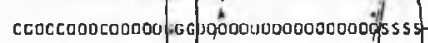

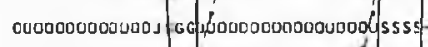

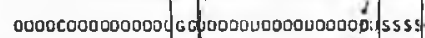

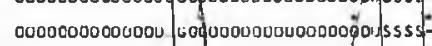

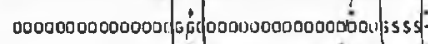
ocoocoooconuoor fiduouuvoouvuocpoousss

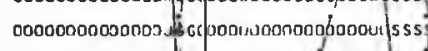

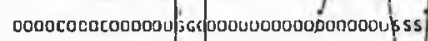

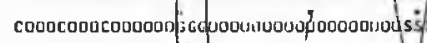

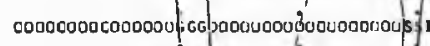

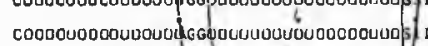

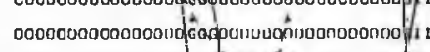

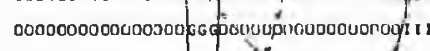

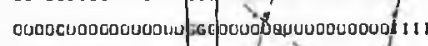

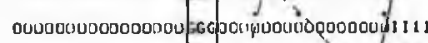

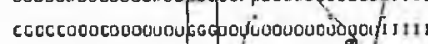

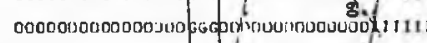

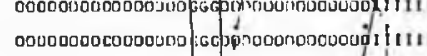

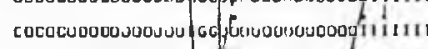

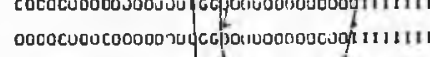

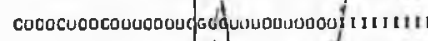

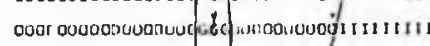

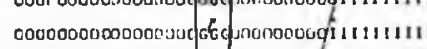

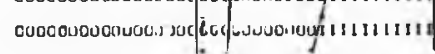




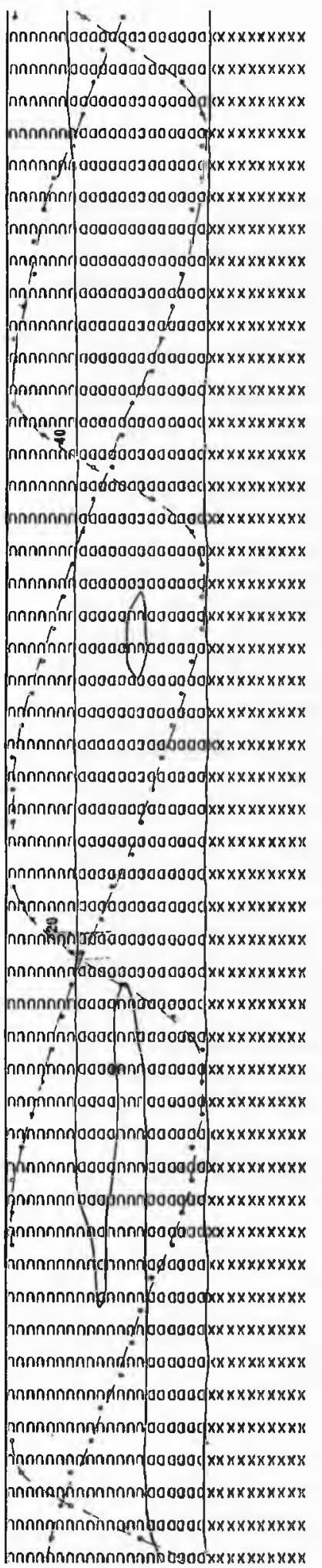

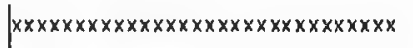

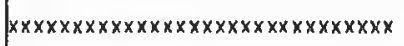

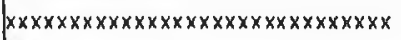

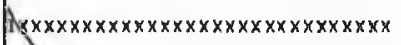

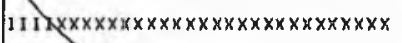

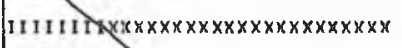

IIIIIIIIII $x x \times x \times x \times x \times x \times x \times x \times x \times x \times x$

I111111111111 $y \times x \times x \times x \times x \times x \times x \times x x$

IIIII111111]1111 $x \times x \times x \times x \times x \times x \times$

IIIIIIIIIIIIIIII If $x \times x \times x \times x \times x \times$

1111111111111111 $x \times x \times \times 4 \times x \times x$

11111111111111111 $x \times x \times x \times x \times x \times x$

I11111111111]111) $x \times x \times x \times x \times x \times$

III11111111111111pc) $\times x \times x \times x \times x \times x$

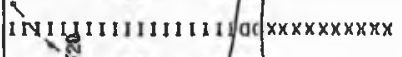

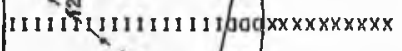

IIIIIItigII

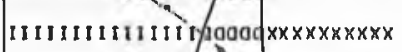

II IIIIIIIIII

I I I I I I I I I I I I I

II IIIIIIIII

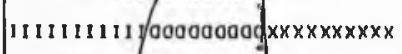

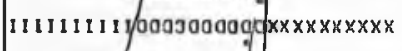

II IIIIII I Y Y000000000 $x \times x \times x \times x \times x \times$

I11111 $1000000000000 \times x \times x \times x \times x \times x$

IIIIIIfoooonoogoboc $x x \times x \times x \times x \times x$

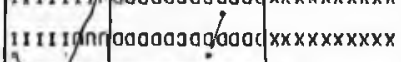

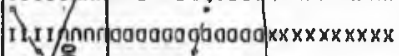

11 1 ifing $000007000000 \times x \times x \times x \times x \times x x$

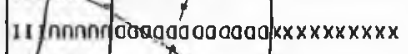

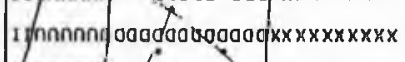

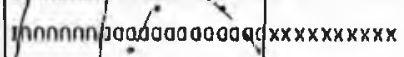

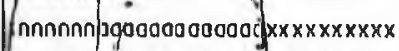

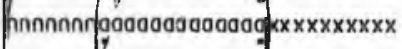
nกnกnกn boo000000000g $x \times x \times x \times x \times x \times$

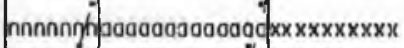
กกกากรกก| $0000003000000 \times x \times x \times x \times x \times x$ nnnกinn ooooadoooogoop $x \times x \times x \times x \times x x$ nกกกnก $0000000000000 \times x \times x \times x \times x \times x$

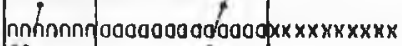

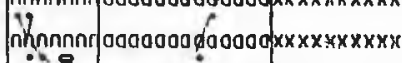
dnnning aoooogd aocood $x \times x \times x \times x \times x \times$

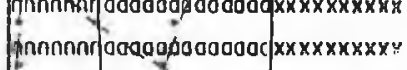
it

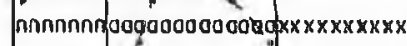
2 nnnกnกn $0000000000000 \times x \times x \times x \times x \times x$ nnnnnnn boo000000000 $x \times x \times x \times x \times x x$ nnnnnnh Jo0e0000000 $\times x \times x \times x \times x \times x$

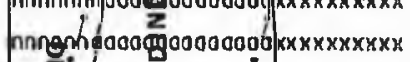
nnn nnr Annoooad nnf finn

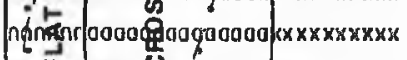

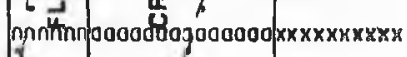

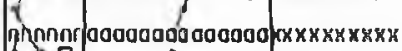

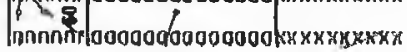




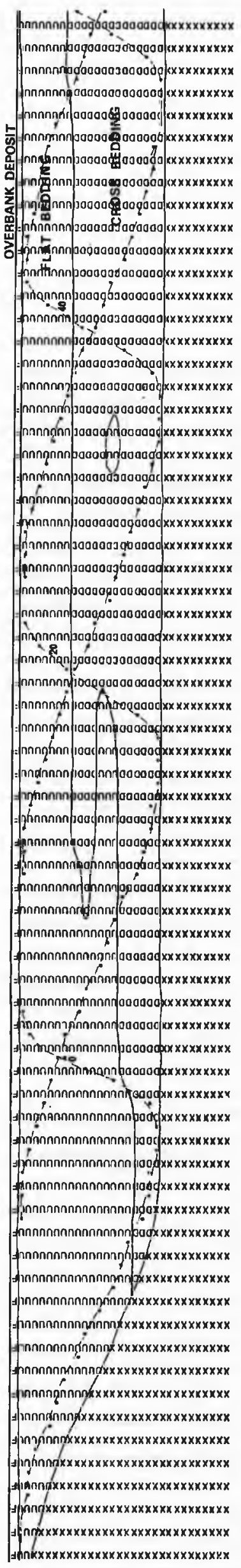




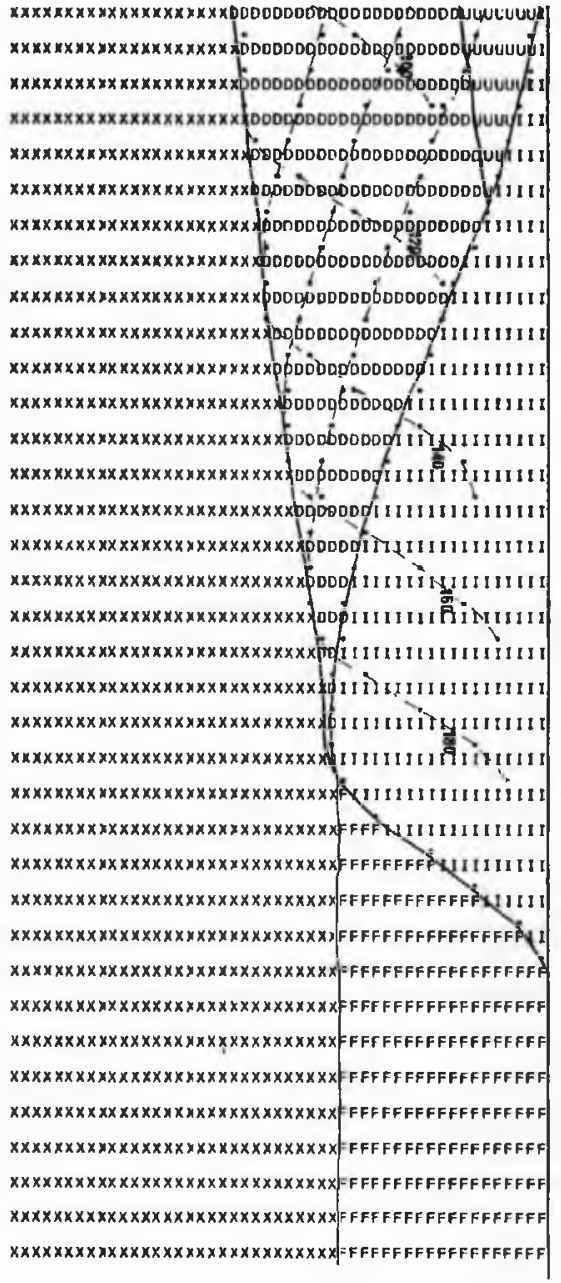

$x x x x x x x x x x x x x x x x x x x x x x x x x x x x x x x$ UUY FFFFFFFFFFFFFFF $x x x x x x x x x x x x x x x x x x x x x x x x x x x x x x x d$ UL $f$ FFFFFFFFFFFFFFFFF $x x x x x x x x x x x x x x x x x x x x x x x x x x x x x x$ uUư $x x x x x x x x x x x x x x x x x x x x x x x x x x x$ uUU: fFFFFFFFFFFFFFFFFF

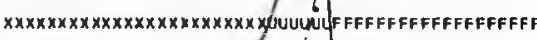
$x x x x x x x x x x x x x x x x x x x x x x x x x x$ UUUUyUUU

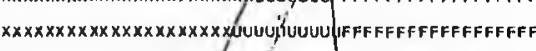
$x x x x x x x x x x x x x x x x x x x x$ wUUU

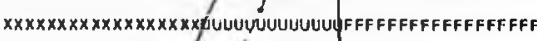

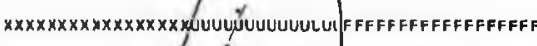
$x x x x x x x x x x x x x x x x x x$ LuUviluUUUUUUUU FFFFFFFFFFFFFFFF $x x x x x x x x x x x x x x x x$ buL vUUUUUUUUUU

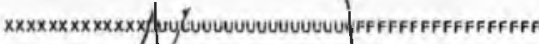

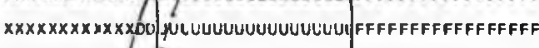

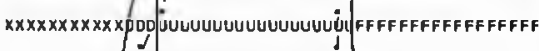

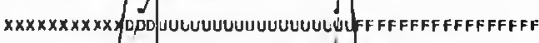
$x x x x x x x x x x$, boc

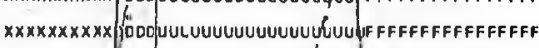

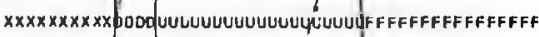
$x x x x x x x x x x x$ ADDD HUL UUUUUUUUUU $x x x x x x x x x x$ DDDO

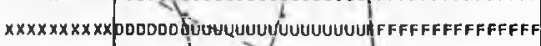

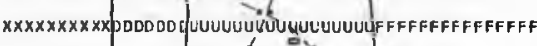

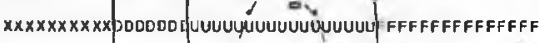

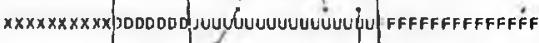
$x x x x x x x x x x x$ DODODD op UULUUUUUUULUUUUUU FFFFFFFFFFFFF $x x x x x x x x x x=00000$ c L (UUUUUUUUUUUUUUUUU $x x x x x x x x x x x$ ODDDDC DqUUUUUUUUUUUUUNUUCFFFFFFFFFFFF

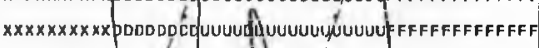
$x x x x x x x x x x x \times D D D$ PDCr UUUUUL

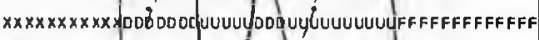

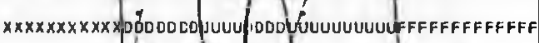

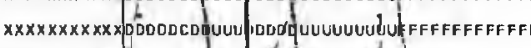
$x x x x x x x x x x \times b 0000$ CoqUU $x x x x x x x x x x x x$ PDDCOCCD

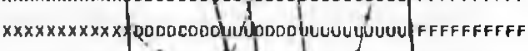

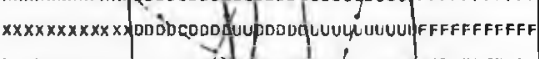

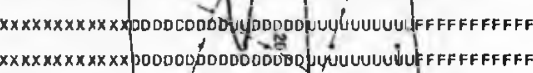

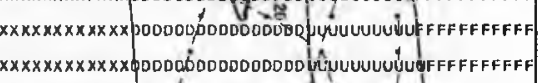

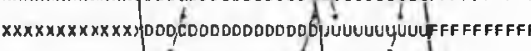

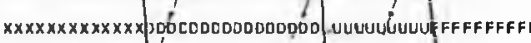
$x \times x \times x \times x \times x \times x \times x$,

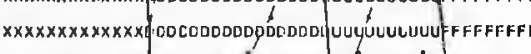

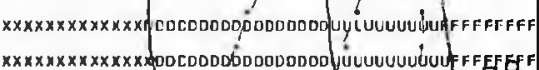

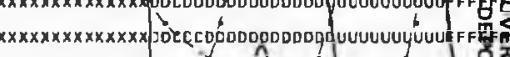

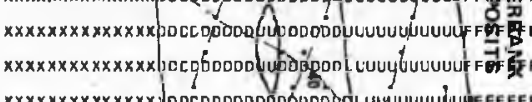

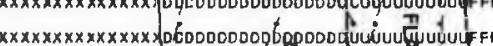

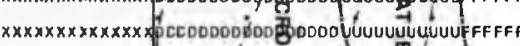

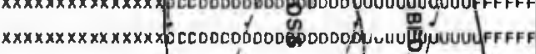

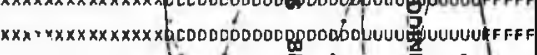

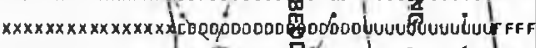

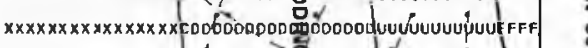

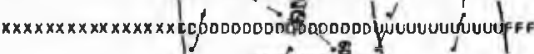
$x x x x x x x x x x x x x x x x x \times 100000000000000$. $x \times x \times x \times x \times x \times x \times x x x \times x \times 0000000000000000$ D

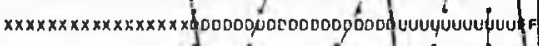

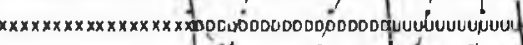

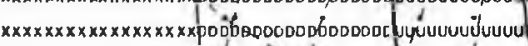

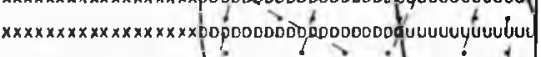

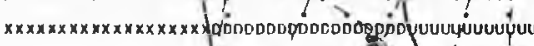
$x \times x \times x \times x \times x \times x \times x \times x \times x \times x$,

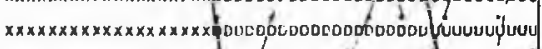

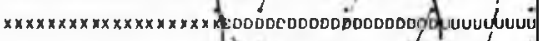

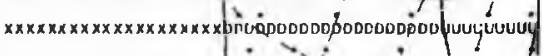




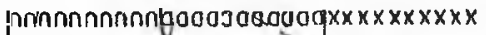

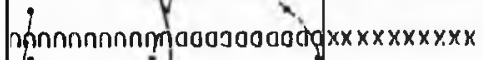

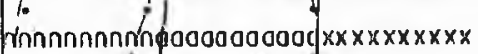

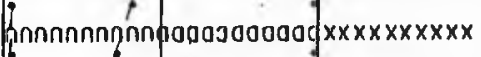

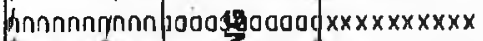

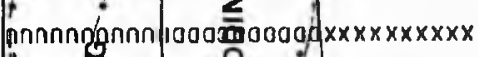

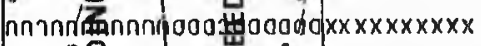
年 is is nnmynกำ

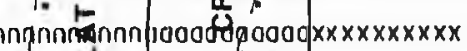

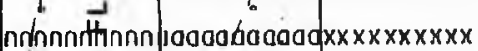
nnnnnnnnnnd loogboogoool $x \times x \times x \times x \times x x$

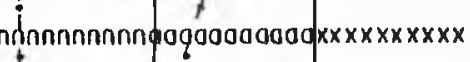

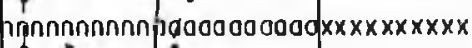

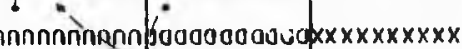

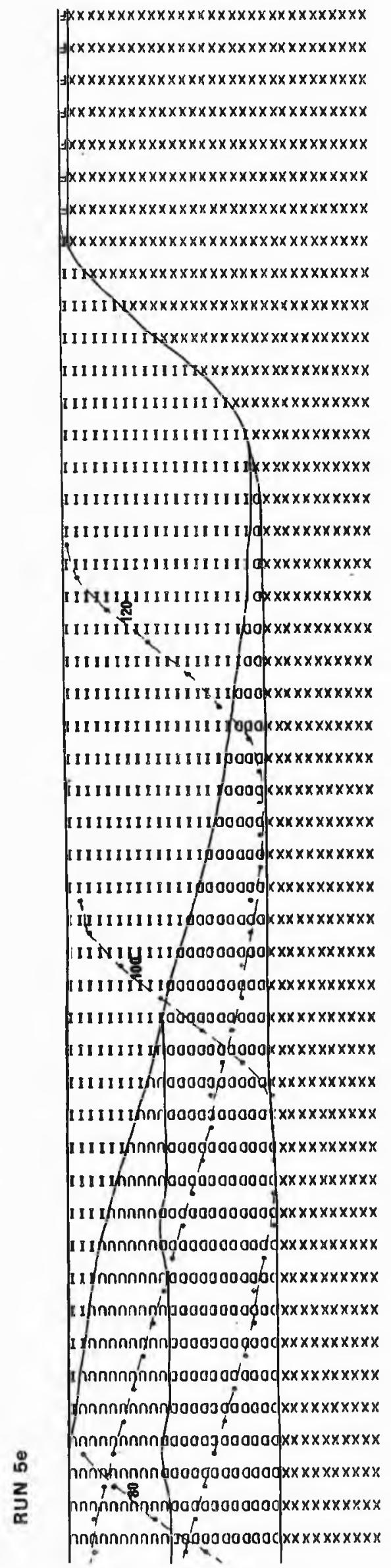




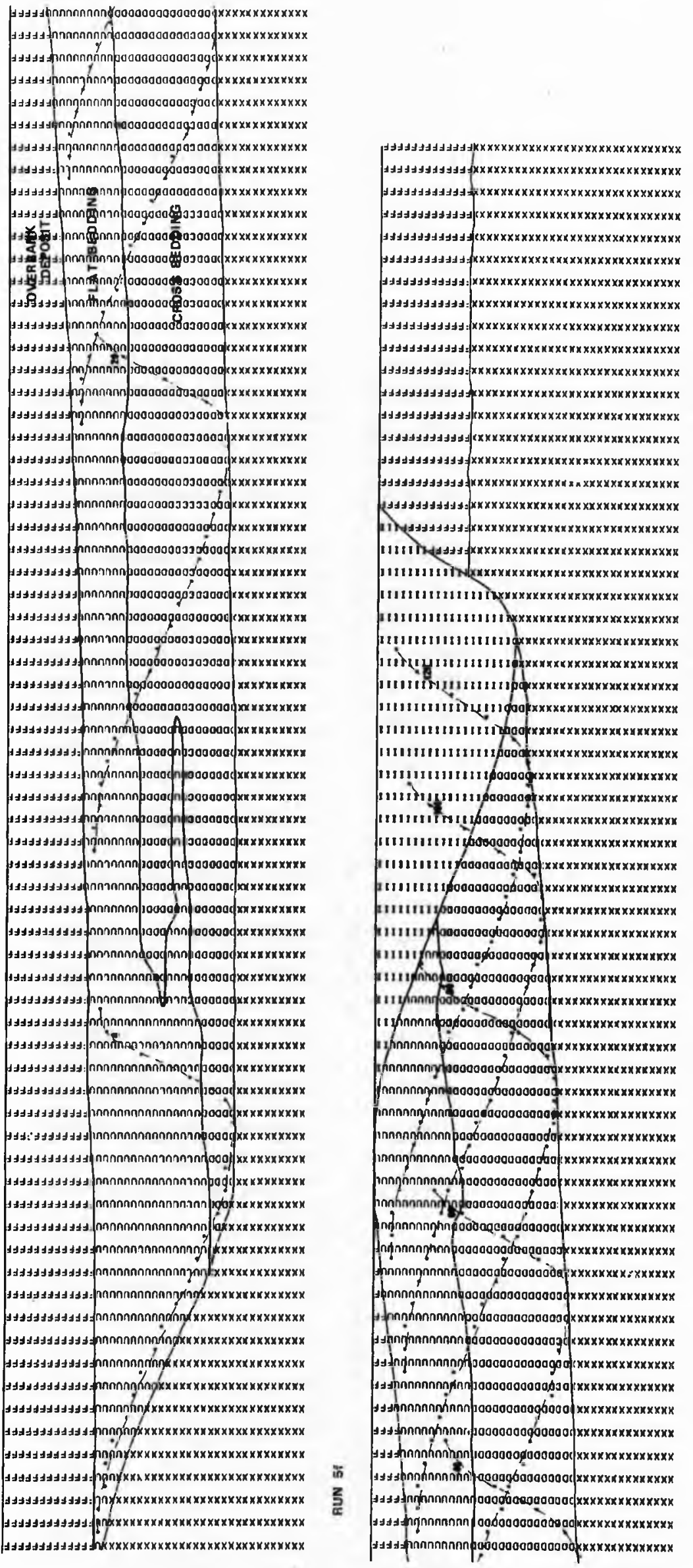




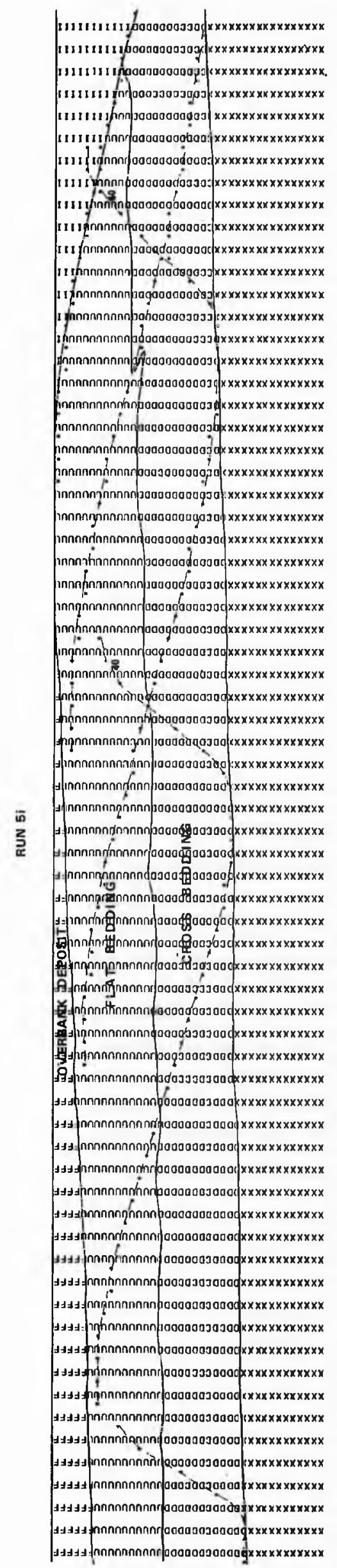

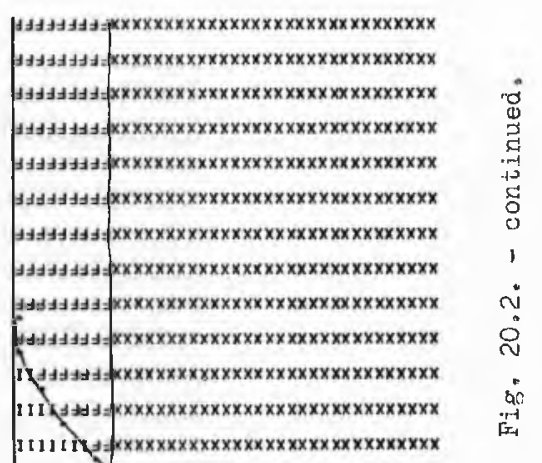

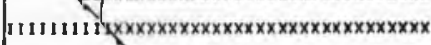

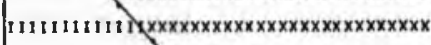

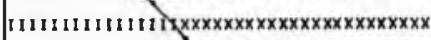

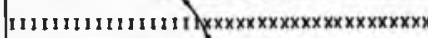

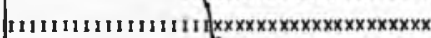

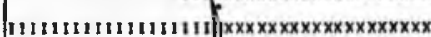

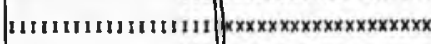

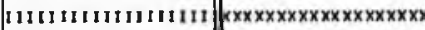

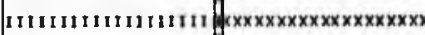

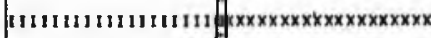

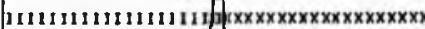

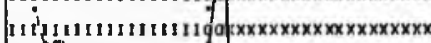

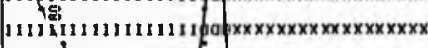
111 13:11 in1 1111 in

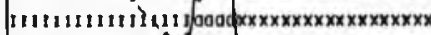

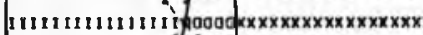

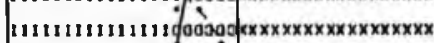

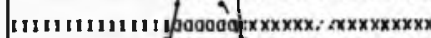
เim

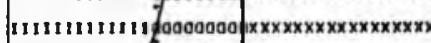

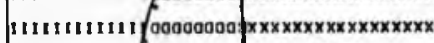

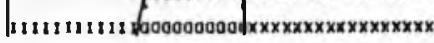




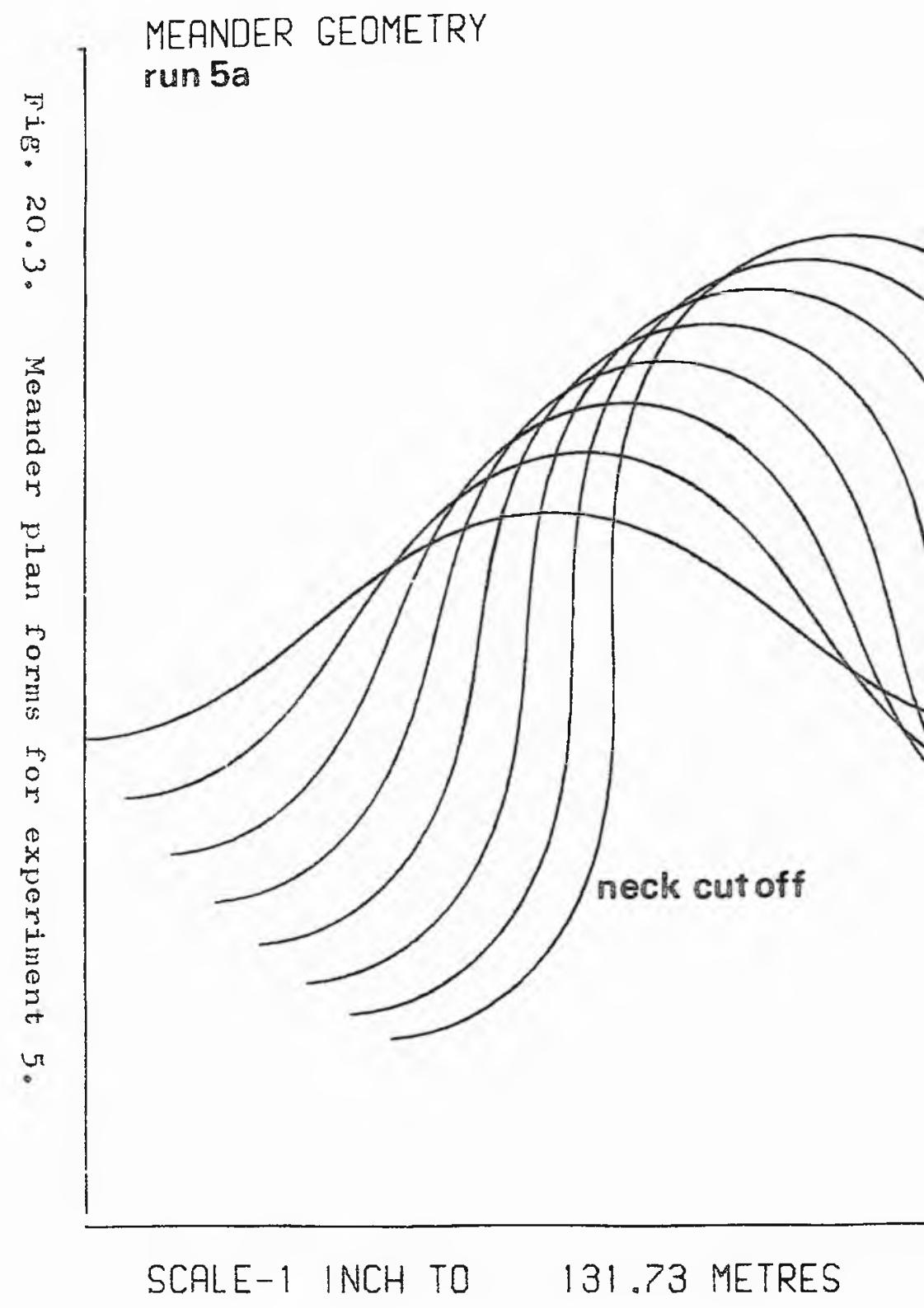




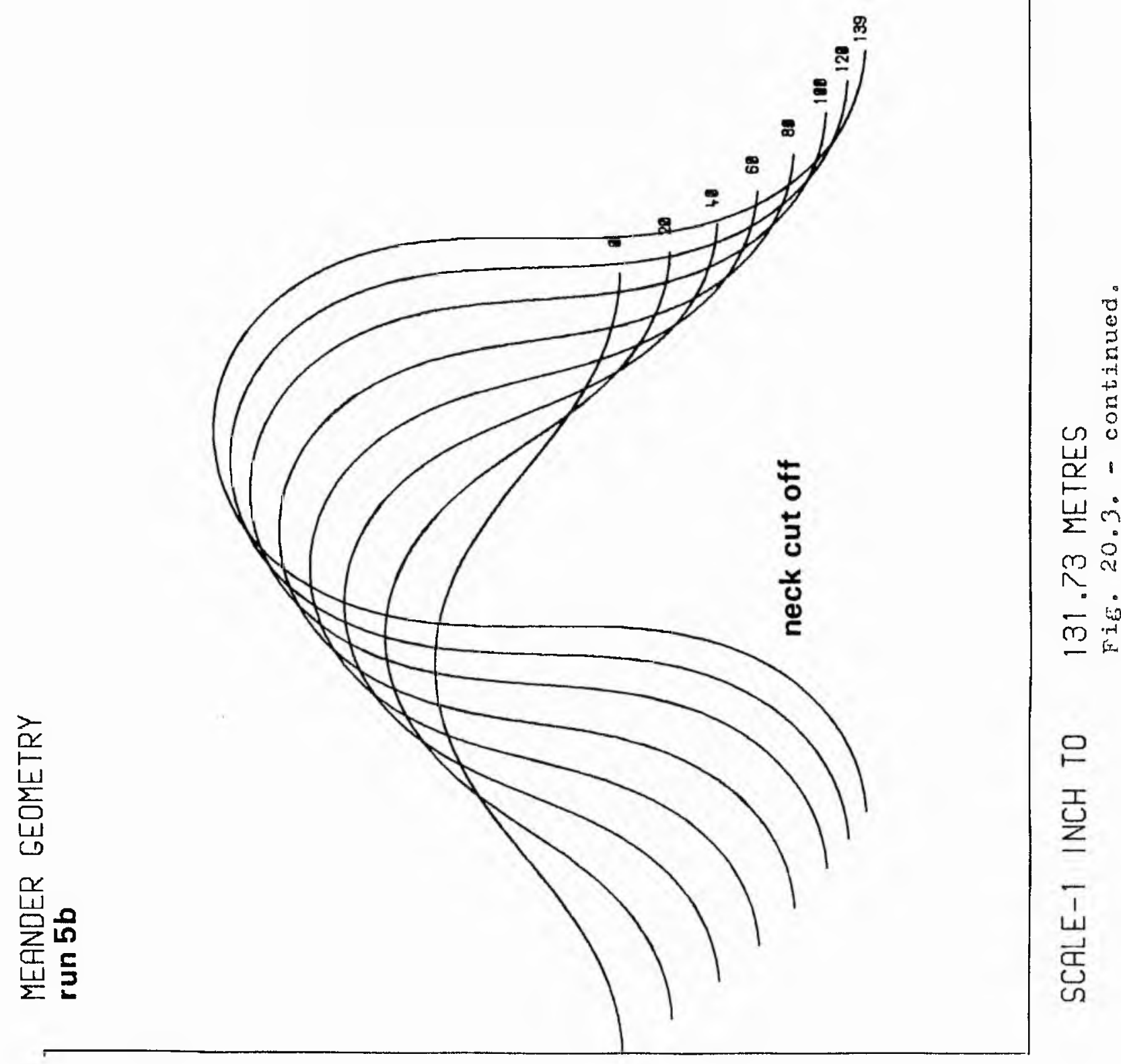




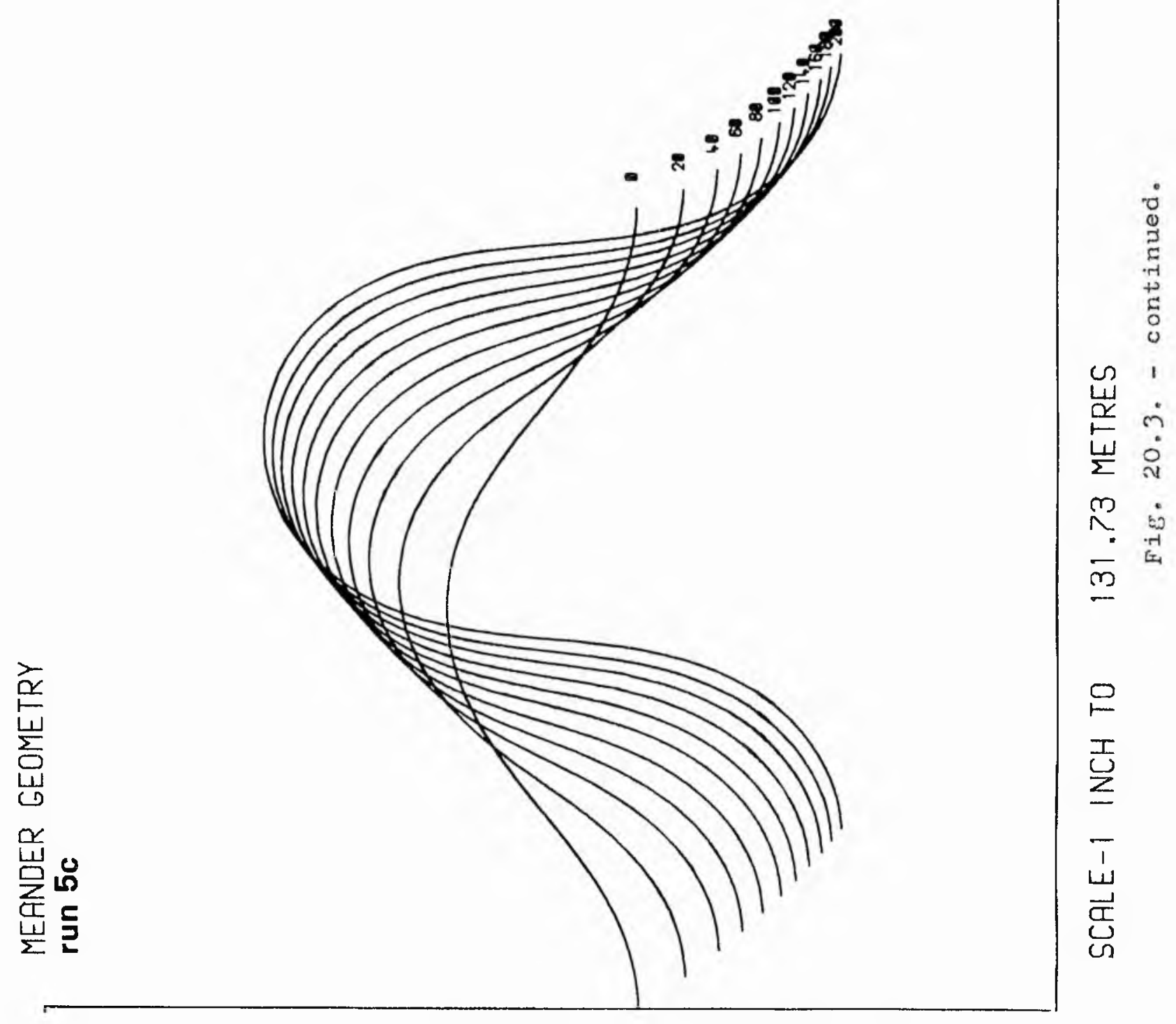




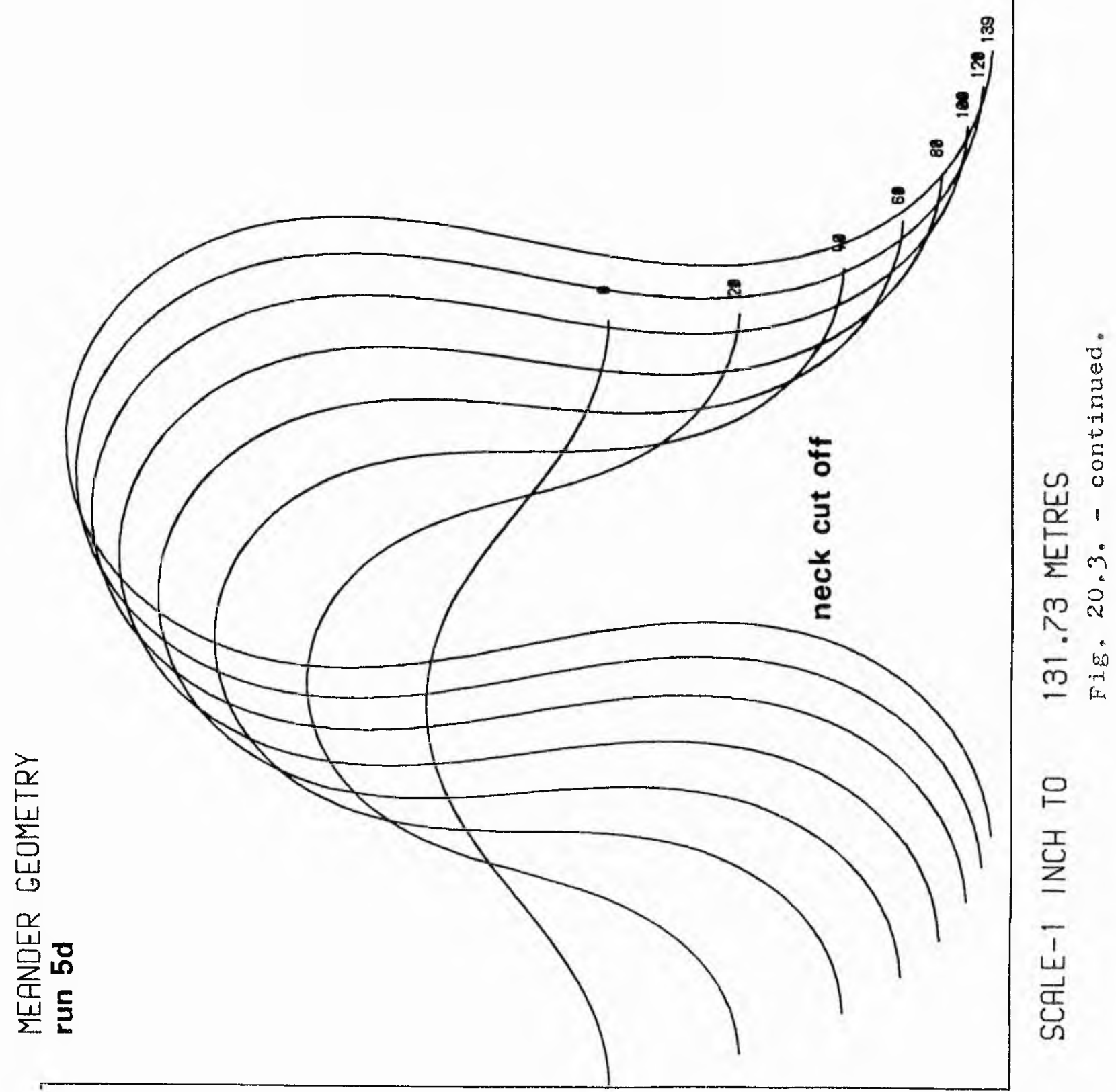




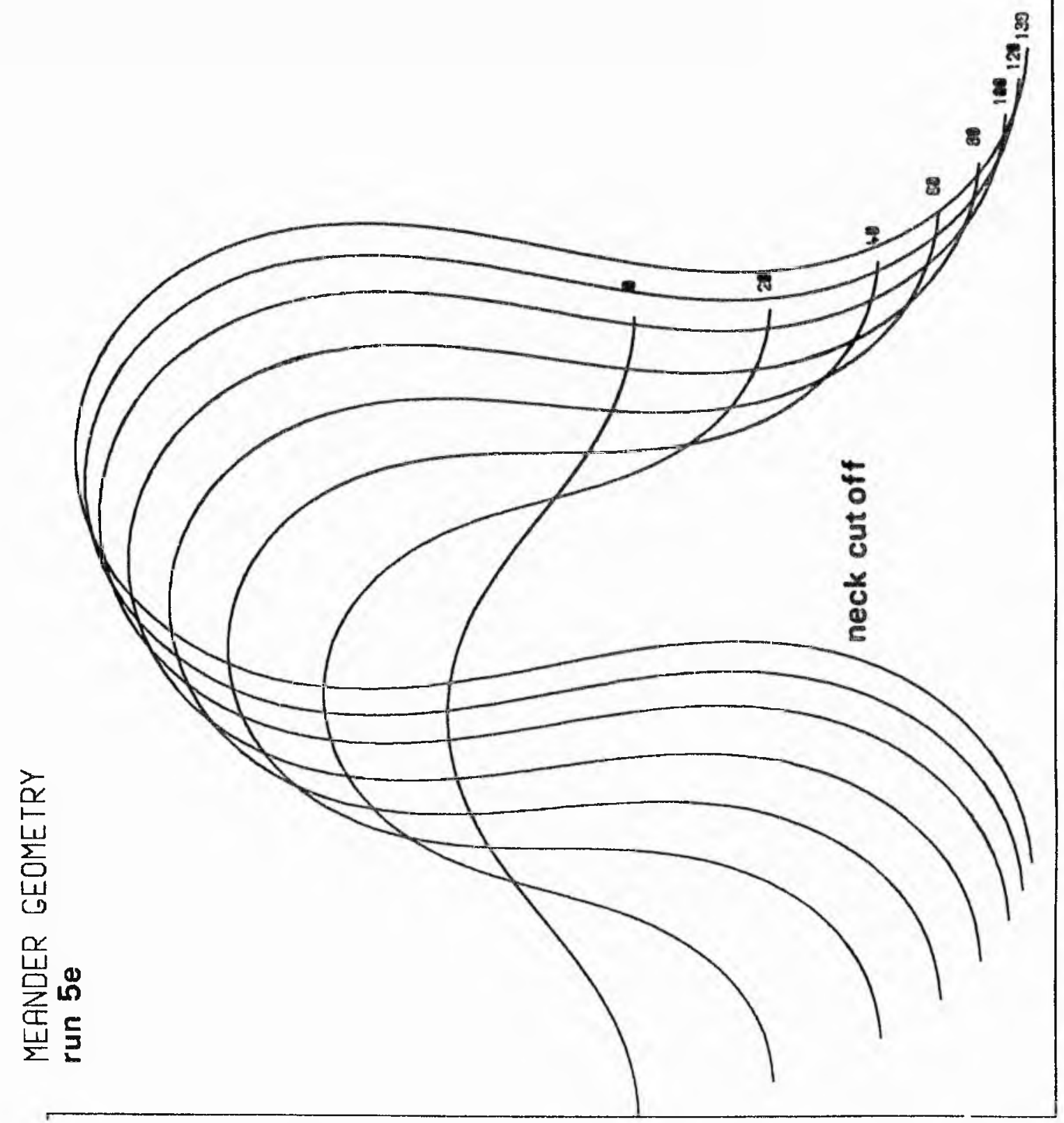

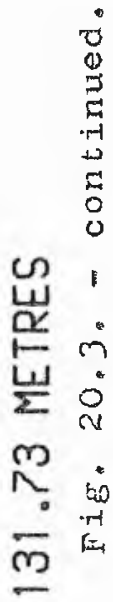

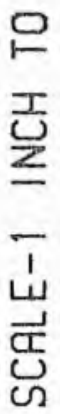




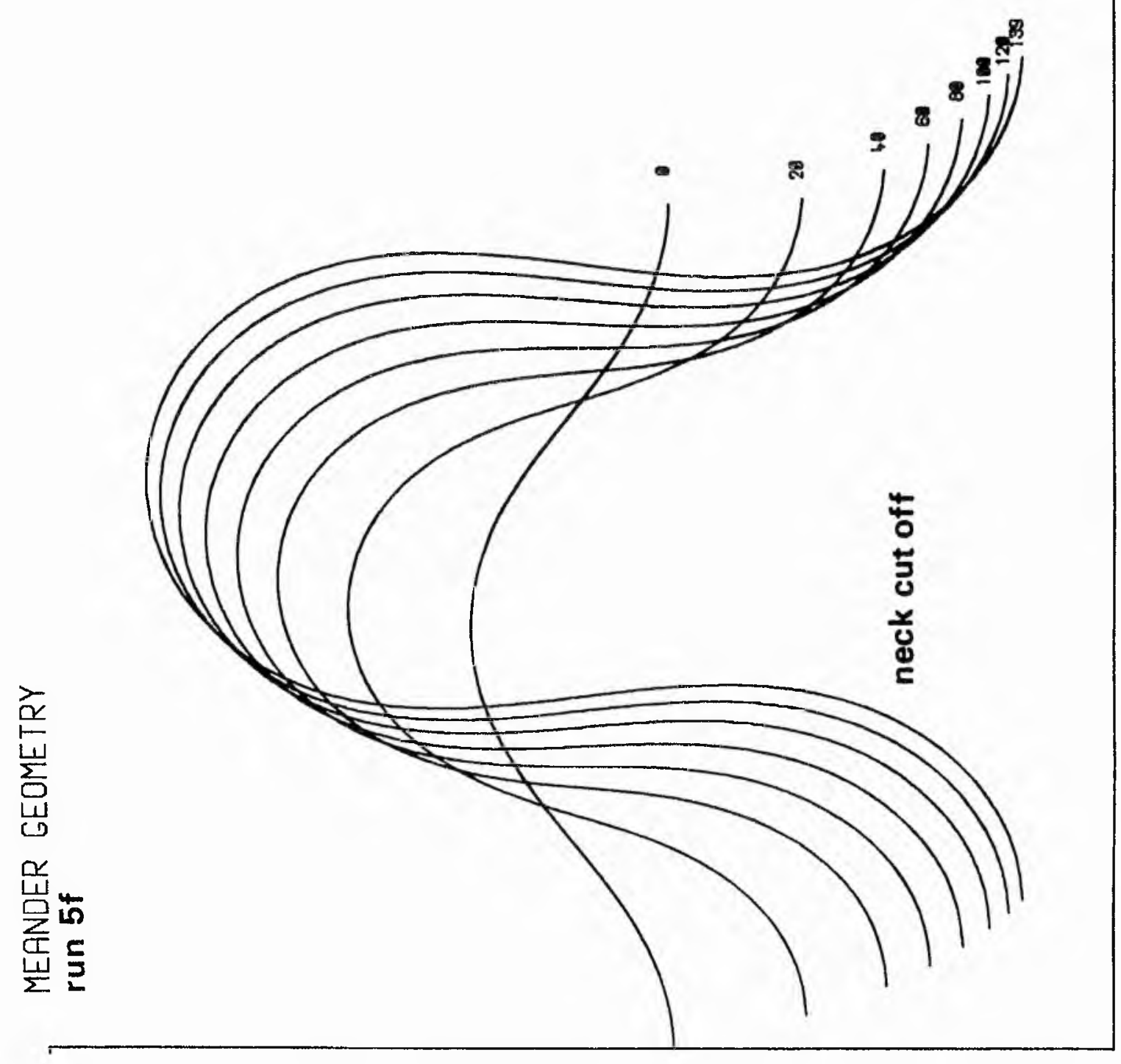

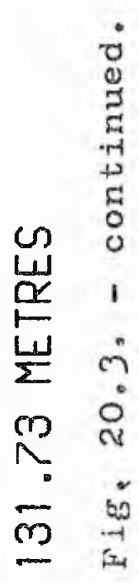

잉

$\stackrel{5}{Z}$

J 


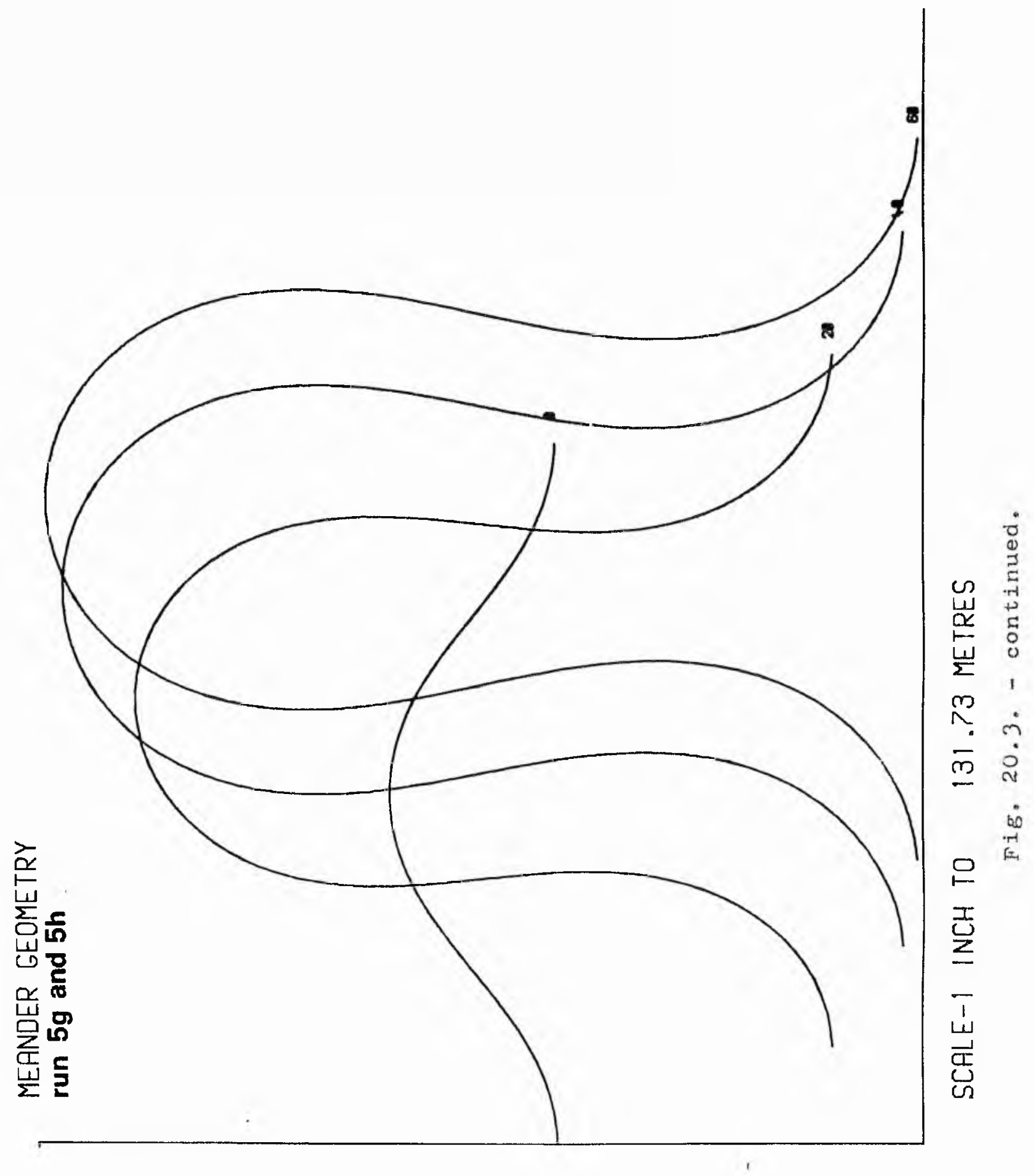




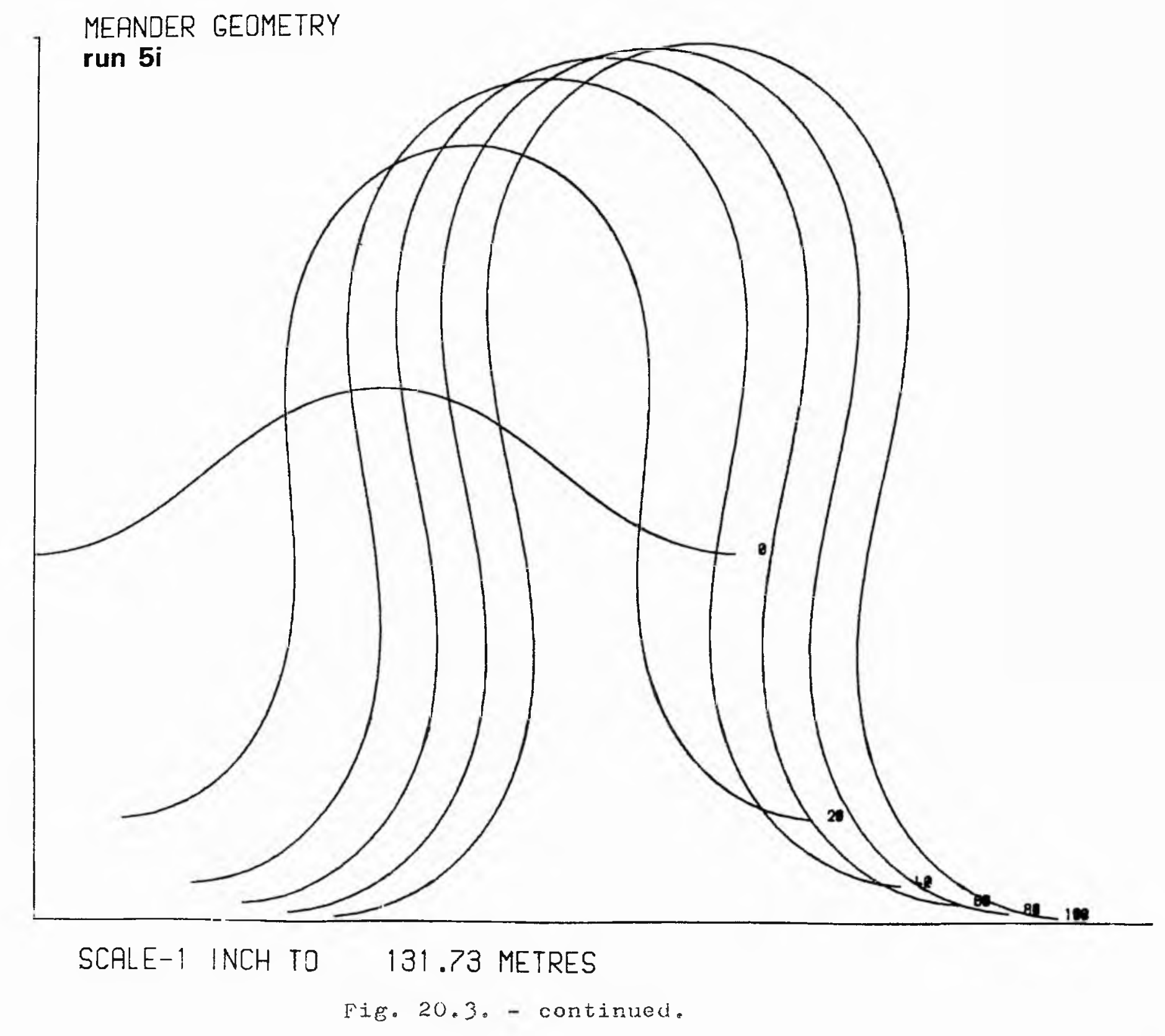


scouring and filling.

The overbank deposit wedges and the upward sloping of facies boundaries and basal erosion surfaces in the direction of migration are similax to those in experiment 2. The slowing down of bank migration rates due to the present overbank fines, with increasing aggradation rate, is enhanced by the slowing down of the amplitude growth anyway (see figs. 20.3). Thus the increase in slope of the facies boundaries and basal erosion surfaces is nore marked than in experiment 2 , as demonstrated particularly in the sections with an aggradation rate of 0.1 metres per year. In this simple moving phase situation the greatest thicknesses of overbank deposits, if preserved, will occur as aggradation rate increases relative to channel migration, as in experiment 2. Some of the sections with the more realistic slower aggradation rates are not shown because there is not enough vertical deposition to be visually recorded on the sections. The maximum accumulated thicknesses of overbank deposit can easily be calculated for the different aggradation rates. Figs. 20.3 show that, by virtue of the direction of definition of the lateral sections, the deposits are soon obliterated by the meander limb immediately upstream from the bend axis. This is particularly marked where the rate of amplitude growth is small, or becomes smalli relative to the downvalley migration rate. As a result, there is, in general, less chance of preservation of deposits in this part of the bend than other parts, perhaps leading to a bias in current directions represented in preserved deposits, notably lacking in directions around the mean downvalley direction. The occurrence of cut-off will probably increase the preservation of point bar and overbank deposits produced in these sections. Some of the cross sections in figs. 20.1 and 20.2 are roughly ledited to account for truncation by the upstream limb. Unfortunately, the model cannot do this automatically. 


\section{PART FIVE}

DISCUSSION AND CONCLUSION 


\section{GENERAL REMARKS}

The computer simulation model yields an abunclance of information. By matching this output with field observations the overall model can be evaluated, and its ability to provide a useful analogue to the real system can be judged. The closeness of the model to the real system will inevitably depend on whether important processes observed in nature have been accounted for and the adequacy of their treatment in mathematical and logical terms. The validity of the component mathematical models, and the approximations made, have been discussed in part 2. Approximations involving the representation of space in discrete form have been discussed in part 3. It is noteworthy that the development of the component mathematical models was based on observation, and subsequent theory, on natural meandering streams and scale model experiments. purely theoretical models are therefore considerably subordinate to empirical relationships. Data input is supplied bearing in mind the restrictions and mutual compatibilities of the system variables as imposed by the overall natural system. The necessary first step is to compare the output with information from modern free meandering stream systems, and subsequently apply the model to the understanding and prediction of modern erosion and deposition, and the interpretation of ancient fluvial systems. There are, however, inlerent difficulties in obtaining data from many geological dynamic systems and, even if available, data may be sparse or unsuitable in forn. The output from the model is conveniently in the form of laterally continuous cross sections showing simulated distribution of grain size and sedimentary structures. Invariably field data, when available, are also in the form of sections in a 1imited number of directions and of limited horizontal extent, or alternatively as a series of discrete sections ox boreholes from which a continuous 
section may be built up. It will be seen in the following discussion that although a reasonable qualitative comparison of the simulated sediments with real world abservations can be made, there are many instances where a substantial amount of data 1 s not available or is of unsuitable form. 


\subsection{Shape of point bar deposits}

The experiments indicate that when bank migration is fairly rapid, point bar deposits extend along the length of the valley, but their extent across the width of the valley is limited to the width of the meanders. If there were slow bank migration the deposits would be beaded along the length of the valley. The edges of the deposits have the shape of a channel bank. Their thickness remains approximately constant and corresponds to the depth of the stream measured from bankfull stage to the scoured base of the channel, as is the case in real streams (e.g. Bernard and Major, 1963).

Alien (1965a) reports that the shape of a point bar deposit complex depends on the extent of channel wandering as controlled by channel sinuosity. The point bar deposits are broadly shoet like with rapid migration and low sinuosity and are long, narrow beaded belts which are narrow compared to the floodplain when sinuosity is higher. Presumably the ability to construct a sheet of point bar material depends on the movement of the meander belt continuously or discontinuously (avulsion etc.). Movement of the meander belt cannot be simulated in the model, therefore sheet like deposits cannot be produced at this stage.

In the model the extent of channel wandering, for a given size of stream, is controlled to the extent that the floodplain sediment calibre is supplied as input. Thus if the meanders have low limiting sinuosity the floodplain sediment may be assumed to be sandy in nature. If the meanders have high limiting sinuosity the floodplain sediments may be designated as silt and clay, perhaps corresponding to fine grained channel fills produced with the more frequent cut-off (e.g. Allen, 1965a). The model will respond to these variations in floodplain sediments in a 
realistic manner.

The geometric nature of point bar and overbank deposits within a thick ageraded sequence of alluvium is only approximately known, and Allen (1965a) has presented some hypothetical alluvial facies models describing this. Detailed quantitative study of the shape of point bar and overbank deposits in thick sequences with relation to rates of aggradation and lateral channel movements is not at hand, and the processes involved with net vertical deposition and such large scale channel movements as avulsion are not fully understood. It has not been possible therefore, to generate thick sequences of alluvial sediment at this stage. Although not strictly comparable with any recent examples, the model has been able to simulate sections through the meander belt in the mean downvalley direction, and normal to this direction, which show the effects of net aggradation combined with channel migration. The results show a general slope of facies boundaries and scoured basal surfaces upwards in the direction of channel movement, depending on the relative rates of aggradation and migration, and record the effects of increasing amounts of overbank deposits. In general, the slopes involvod may be so small that they would not readily be recognised in an alluvial complex. There are some interesting features of the sections in the mean downvalley direction. With no aggradation or degradation the basal scoured surfaces would represent the slope of the valley, assuming negligible downstream changes in channel depth over the section represented. If there were slow and continuous degradation, the slope of the basal surfaces would be greater than the valley slope, and with aggradation the slopes would be less than the valley slope and may even dip in the opposite direction. clearly many factors would complicate this nalve situation. The experiments show a 'stabilisation' effect as thicknesses of fine sediment are deposited on the floodplajn with agradation. 
Stabilisation of meander belts is also effected by cut off and subsequent filling of abandoned channels with fine sediment (e.g. Allen, 1965a); such processes cannot be simulated at present, although, as previously indicated, abandoned channelfills can be defined implicitly at the outset. It is noteworthy that the stabilisation with aggradation would not be simulated if it was assumed that the overbank deposits were sand or gravel grade material.

\subsection{Epsilon cross stratification}

It is implicit in the model that successive deposits for a given flood event are bounded by sigmoidal boundaries which mark the position of the bar before being filled on falling stages. These surfaces must delineate the epsilon-cross-stratification of Allen (1963a). An important point here is that the maximum angle of the transverse slope of the epsilon-cross stratification must represent the scoured shape of the point bar, when scouring and filling is present. This may go some way to explaining the relatively steep angles of the few examples of epsilon-crossstratification found in ancient sediments when compared with recent bar surfaces (Al1en, 1970b). Recent and ancient examples may therefore be directly comparable. Allen (1970b) also notes that epsilon-cross-stratification may be very difficult to see in rocks unless the transverse slope is reasonably large.

Epsilon-cross-stratification is implied in recent point bar sediments by virtue of their mode of lateral growth, with the units being deposited at discrete periods of time and concomitant with concave bank recession. It would thus appear that the thickness and regularity of development of the units will depend on rate of bank inigration, variation in degree of scouring, and variation of the shape of the surface on which sediment is being deposited. In the model the shape of the bar profile is constant 
and only its maximum slope varies with degree of scouring, therefore the sigmoidal boundaries are very regular, although they may not be exactly parallel at all levels in the bar. In real streams, given sufficient bank migration to develop successive units, a greater degree of irregularity may be expected due to variation in the shape and slope of the point bar profile on which sediment is being deposited. This may or may not be associated with scour and fill. Purthermore, distinct epsilon unit boundaries may be obscured by small scale scouring over the profile, perhaps in the lee of dunes; this feature is not represented in the model sections.

Sundborg (1956) records very regular epsilon-cross-stratification in the point bar deposits of the klaralven, southern. Sweden, at least in the lower parts of the bar. Leopold and Wolman (1960) also note that '...approximate contact surfaces between materials of different textures are more or less parallel to past surface profiles'. Other examples in tidal meandering streams include Van Straaten (1954), Reineck (1958) and Klein (1963).

A final point is that the epsilon-cross-stratification may be visible over the total vertical thickness of the point bar. If this is the case it will represent a vertical thickness measured from about bankfull stage down to the base of the scoured channe1.

\subsection{Distribution of grain size and sedimentary structure}

Attention may be directed to that part of the model which predicts the grain size and sedimentary structure over the pointbar profile, using the conventional hydraulic equations. There are, unfortunately, inadequate experimental observations or data from present day river or tidal meandering channels by which to test this mode1. The qualitative features of the deposits produced in the model with lateral bar growth (with and without scour and fi1j) are however consistent with the general characters of lateral 
deposits formed in comparable tidal systems and streams. In these deposits grain size generally decreases vertically upwards and bed forms change from types indicative of large stream power upwards to forms denoting a small power (e.g. Allen, 1965a;

Bernard and Major, 1963; Bernard and LeBlanc, 1965; Evans, 1965; Fisk, 1944, 1947; K1ein, 1963; Oomkens and Terwindt, 1960; Reineck, 1958; Sundborg, 1956; Van straaten, 1954). It was pointed out earlier that the prediction of silt and clay at the tops of the bars was not strictly correct. Qualitative justification is afforded by observations of fine sediment on top of bars to such an extent that they often cannot be distinguished from the overlying levee deposit (e.g. Bernard and Major, 1963; Visher, 1965a; Wolman and Leopold, 1957).

The model does not record the expected variation in size of dunes with flow characteristics, or the scoured bases to the individual cross bedded units. McDowell (1960) describes crossbedded units in recent point-bar deposits which become on average thinner upwards in the bar. other structures not simulated include convulute lamination and various types of small scale scours.

Sundborg (1956) and Leopold and Wolman (1960) note that with falling discharge after some flood events, suspended sediment is deposited on bars over the coarser bed load material, thus leading to alternate coarse and fine sediment as individual layers are traced laterally and upward. This variation would be in addition to the general upwards fining in the point bar. Such small scale variation cannot be described within the model (1.) by virtue of the scale of variation involved and, probably more significant, (2) because only events at bankfull stage are considered and (3) no explicit account is taken of fine suspended sediment.

Thus when there is little or no scouring and filling, no small scale variation can be simulated. Some vindication lies 
in the fact that as most sediment is deposited on bars from bed load at high stage, such fine sediment may be insignificant and may be scoured during rising stages of the next flood anyway. Indeed, sometimes the fine sediment is just in the form of mud drapes (e.g. Bluck, 1971: McGowen and Garner, 1970). Fig. 22.1 ijlustrates the expected variation in grain size in a single natural depositional unit compared with the simulated variation, for the case where no scouring and filling occurs.

When there is no scouring and filling, lateral bar growth in the model produces no relief in the grain size and sedimentary structure facies boundaries or the basal scoured surface. When scouring and filling occurs in conjunction with lateral deposition, small scale variation in grain size and sedimentary structure both within and between individual epsilon units can be simulated, in addition to the general fining upwards of grain sizes and systematic distribution of sedimentary structure. This feature of the model gives rise to a relief, over the whole deposit, in the boundaries separating different grain size classes or sedimentary structure, and may take the form of lensing and interfingering. Obviously scouring and filling is also associated with a degree of relief in the basal erosion surface. The wavelength, amplitude and shape of such relief, and the nature of lensing and interfingering, are important features indicating the amount of scouring and filling relative to the amount of bank migration. In this respect it seems necessary to distingulsh the large scale relief in the basal erosion surfaces, as mentioned above, from smaller scale 'within channel' scours. The former are genetically related to processes operating only in meandering streams, whereas the latter may also form, for instance, at the base of levee or crevasse deposits (A11en, 1970c).

In general, there is not enough quantitative information available to test these aspects of the model, but there are many 


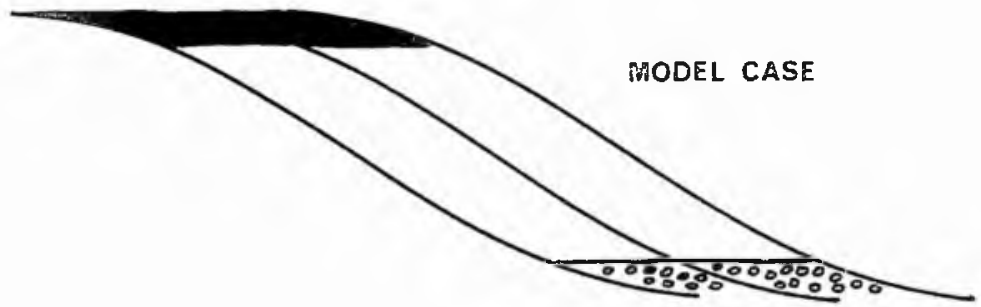

siltt and clay

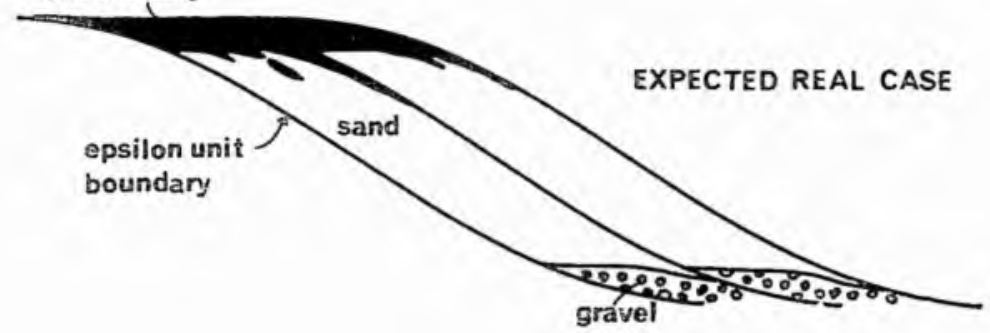

Fig. 22. 1 Schematic variation of grain size within single depositional units when no Scour-and-Fill. Expected rea! world and model cases. 


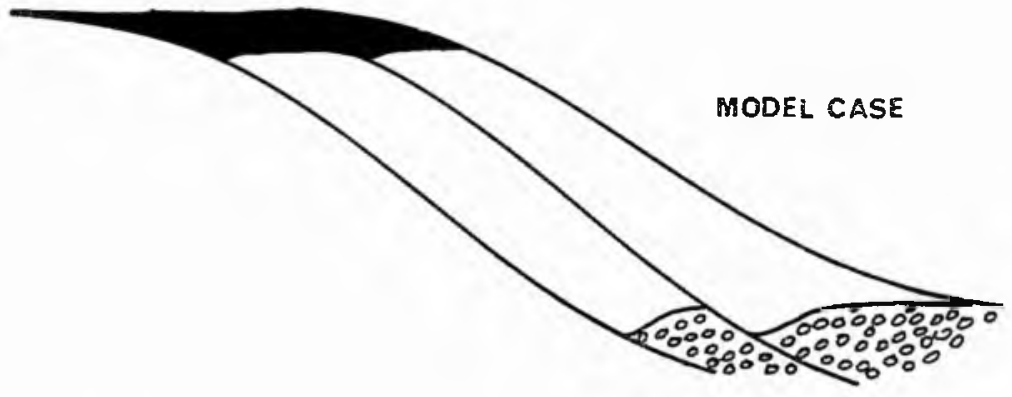

silt and clay,

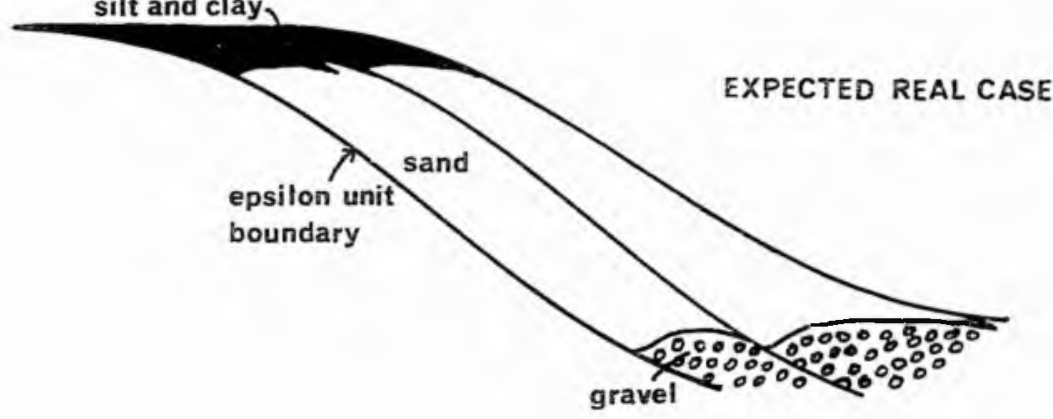

Fig. 22.2 Schematic variation of grain size within single depositional units, with Scour-and-Fill. Expected real world and mode! cases 
examples in recent deposits of large scale relief in basal scoured surfaces and facies boundaries (e.g. Bernard and LeB1anc, 1965; Fisk, 1947; Leopold and Wolman, 1960). Invariably 1ensing and interfingering of particular types of sedimentary structure and sediment size classes occur (e.g. Allen, 1965a; Frazier and Osanik, 1961; Leopold and Wolman, 1960; Sundborg, 1956).

Fig. 22.2 represents a comparison of the nature of variation in grain size within an individual epsilon unit between the model and that expected in the real situation. It is easy to see from the figure how, for instance, lenses of gravel occur. If there is not significant deposition of fines with falling stages in the real situation or if the fines are subsequently scoured the 'real' example may tend to the simulated situation.

With changes in the slope and radius of curvature of a developing meander in the model there were additional very largescale variations in grain size and sedimentary structure. In genera1, grain size decreased and the amount of lower regime forms increased at the expense of upper plane beds as sinuosity increased. Such changes were not simple and an intertonging transition zone was involved. To test this aspect of the model in the light of the assumptions made would require a considerable amount of information over an extensive continuous section. Such information is not forthcoming at present. Furthermore, the variation in all the dependent hydraulic variables in developing meanders needs to be examined in considerably more detail in the field before this aspect of the mathematical model could be used with confidence. Recent studies have indicated that different processes are acting to give dissimilar sediment deposits in fine and coarse grain point bars (B.uck, 1971; McGowen and Garner, 1970). In coarse grain point bars strong currents develop over the top of the bar during high flood stages in conjunction with those acting in the pool. These currents over the top of the point bar are 
responsible for localised scouring and deposition of coarse material as bars in this area. Transverse profiles across the point bar become complex and variable along the length of the bar. Sequences produced by bar migration do not always exhibit the general fining upwards as in fine grained point bars. Sequences of sedimentary structures characteristic of particular subenvironments within the bar can be recognised, but may differ from fine grained point bar sequences. Differences will also be expected to exist in the facies geometry. The present study deals essentially with processes operative in fine grained point bars, although there are obvious common features. In general, however, the present model cannot be thought of as truly representing the coarse grained point bar deposition as described by Bluck (1971) and McGowen and Garner (1970).

\subsection{Times taken to cut off}

No specific experiments were run to test this aspect of the model, although cut-off information is entered in the meandex plan figures where relevant. Observations on some of the experiments have shown that the times taken from inception of a meander to cut off is of the order of hundreds of years, which is supported by observational data (see section 10.3). To produce this situation the exponents in the cut off relations must be fairly large, and a realistic value of $Q_{\text {volmax }}$ must be specified. 
Various aspects of the model can also be compared with the coarse members of the fluviatile 'fining upwards' cycles, known abundantly from ancient sediments, and which are known or strongly believed to have been accumulated through processes of lateral deposition (e.g. Allen, 1963b, 1964, 1965b, c; Allen and Friend, 1968; Beutner et al., 1967; Moody-Stuart, 1966; McGowen and Garner, 1970; Potter, 1967; Visher, 1965a,b). Such interpretation is based on comparison with the textures, sedimentary structures, detailed stratigraphic succession and organic content of recent channel and overbank deposits.

The coarse units are commonly tabular in shape or broadly lens shaped. Epsilon-cross-stratification is only rarely observed, there being only three published occurrences to date (i.e. Allen, 1965b, Beutner et 리., 1967; and Moody-stuart, 1966). This may either be due to lack of preservation, or, as previously mentioned, due to difficulties in recognition. The coarse members exhibit the characteristic vertical patterning of grain size and sedimentary structure, and such vertical patterning has also been recorded from tidal upward fining sequences (e.g. Allen and Friend, 1968; Klein, 1965). Various other obvious recorded and characteristic features which have not been simulated have been the decrease in thickness of cross bedded units upwards, detailed grain fabric and texture, convolute lamination, and the presence of oriented and nonoriented sole structures (i.e. grooves, flutes, pot holes, Load structures, etc.).

Relief in grain size class and sedimentary structure boundaries (facies boundaries) is common, although not always present. Lensing of particular sedimentary structures or lithologies is also recorded frequently. Relief of the basal scoured surface is common, although it is not always evident in the literature, whether the relief is due to local small scale 
scouring, or is genetically related to the processes of scour and fill combined with bar migration. In general, only the amplitude of this relief is recorded but the shape and wavelength is often omitted. The reason for this may be due to lack of exposure, especially when the relief has very long wavelength.

A Downtonian coarse member at Ludlow, Shropshire (Allen, , 1964, p. 170) is of uniform thickness, 3.6-3.7 m., and has an essentially flat scoured base, except for scoured hollows with a maximum relief of $15 \mathrm{~cm}$. It would thus appear that scour-and-fill was not prominent in this case. This strongly contrasts with, for example, the high amplitude/wavelength basal scoured surface described by Heutner et al. (1967) in a Pennsylvanian channel sand stone.

The detailed organisation of the grain sizes and structures in the published examples of coarse members is not generally traced laterally in continuous sections. Thus even reasonable qualitative comparison with the simulation model deposits becomes difficult. A rare opportunity to compare the model in detail with ancient sediments occurs when epsilon-cross-stratified units are preserved in extensive outcrops cut perpendicular to the current direction. These structures are the most obvious indicator of the presence of lateral deposition. Allen (1965b) described examples from the Porth-y-Mor beds on the northeast coast of Anglesey. The units average 6' $3^{\prime \prime}$ in thickness and were traced for $30 \mathrm{~m}$. or more along the shore. Fig. 23.1 shows Allen's schematic representation of the chief features of lithology and sedimentary structure in the epsilon cross-stratified units. Many of the sigmoidal bedding surfaces are recorded as erosional contacts, indicating scour of the previous bar profile. The cross-stratified units are hetero1ithic. Although statistically there is an upward decrease of grain sizes with each unit, as well as an upward and lateral decrease in coarseness of beds between major bedding surfaces, the 

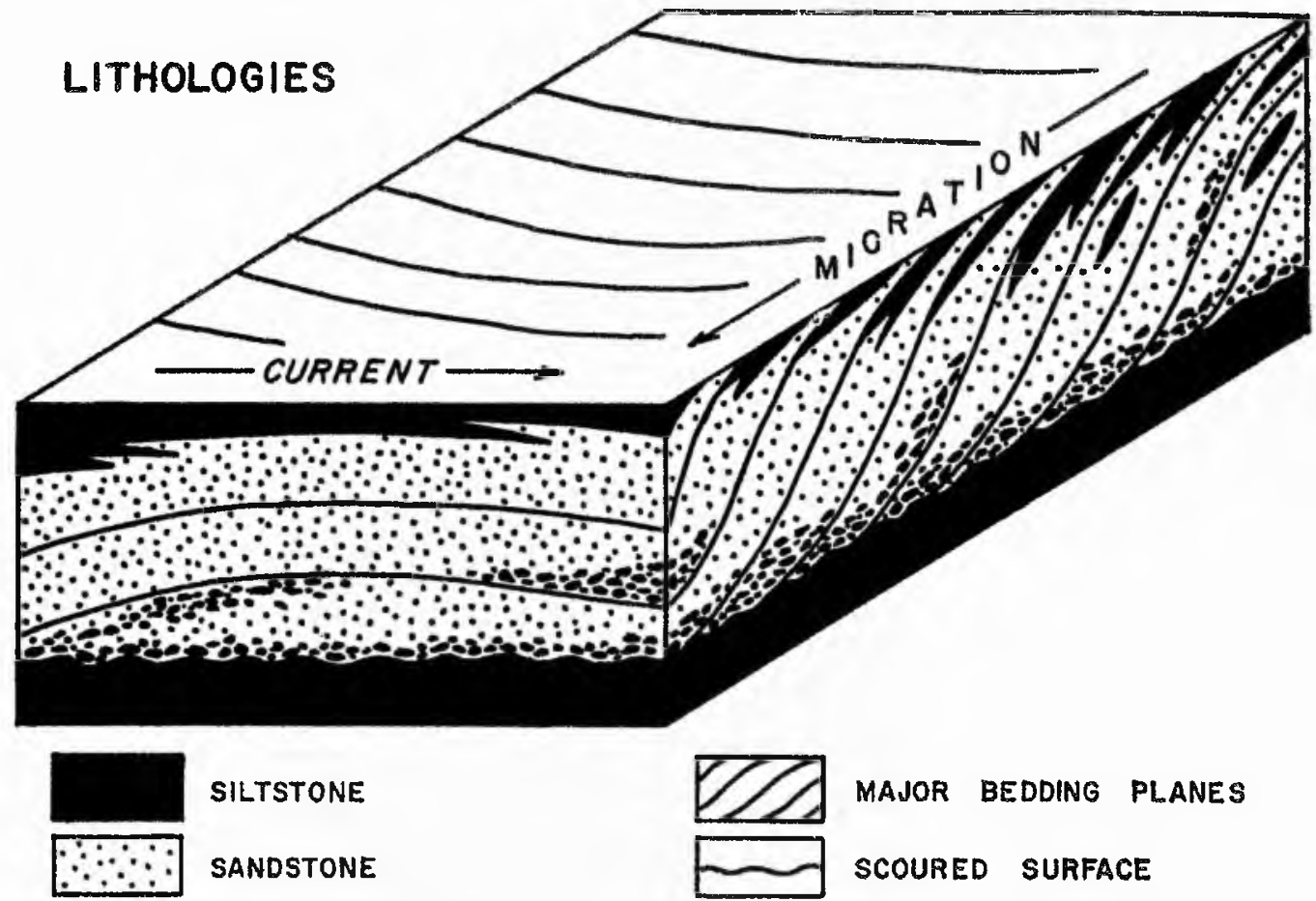

\section{INTRAFORMATIONAL \\ E-T. CONGLOMERATE}

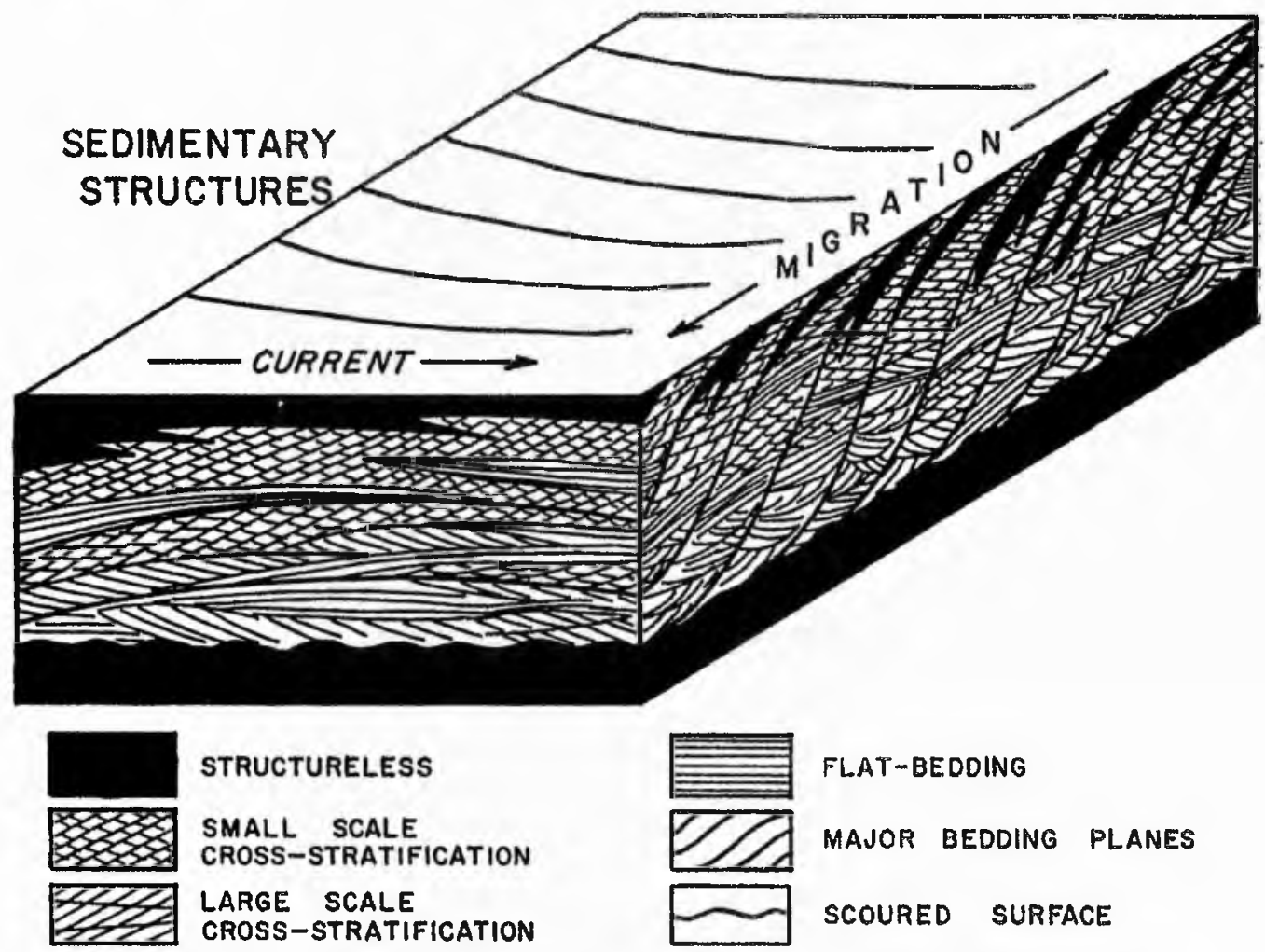

Fig. 23.1. Schematic block diagrams showing the

characteristics of epsilonmcrossostratified units in the old Red Sandstone of Anglesey, North Wales. Unit thickness is from three feet six inches to nine feet. Major bedding surfaces have maximum slopes from four to fourteen degreos. Vertical scale much exaggerated. (from Allen, 1965b). 
relationship between lithologies is one of complex intertonging. Sedimentary structures also show complicated spatial relationships with the development of lensing. The nature of these deposits is broady comparabie with simulated deposits, but fig. 23.1 shows up the inability of the model to treat deposition over a range of stages and points to the approximations both in the mathematical model and the computer program. Probably the example of epsilon-cross-stratification that best lends itself to comparison with the model is cited by Beutner et al. (1967). This is discussed more fully in the section dealing with the application of the model to interpretation of ancient fluvial sediments.

Allen (1963b, 1964) described two cases where conglomerate passes laterally gradually into gravel-free rock, despite the underlying basal scoured surfaces persisting laterally for considerable distances. Detailed data unfortunately are not available to determine whether these occurrences represent broad flat lenses of gravel associated with scour and fill of whether they are genetically related to a gradual decrease in load carrying ability of the channel as it moves laterally.

Ancient fluviatile sequences are normally made up of many repeated cyclothens, from which it must be concluded that a channel occupied a given site at successively higher levels relative to an original datum. Such an occurrence can only be attributed to channel migration of continuous or discontinuous nature in conjunction with net vertical deposition, which may also be continuous or discontinuous in nature. The mechanisms responsible for net vertical deposition (aggradation) cannot be simulated at present, however, irrespective of the causative processes involved, a constant rate of aggradation can be simulated. It is only possible to simulate continuous movoment of a channel. within a meander belt, and not channel abandonment with cut off or 
meander belt movement in a continuous way or by avulsion. At present therefore it is not possible to simulate repeated channel and overbank sequences. A complex distribution of channel. sediments would be expected in a thick alluvial succession (e.g. Allen, 1965a; Potter, 1967), and details of the three dimensional form of the cyclothens would be required before either the controls underlying the cyclicity could be solved or comparison with simulation models is made possible. 
The experiments show, despite their limitations, that a complete sequence of cliannel. sediments capped by overbank sediments would rarely be preserved in the moving-phase situation, rather that only basal fractions of the total point bar thickness would be preserved (c.f. Bluck, 1971). Clearly the preservation of a 'complete cyclothem' becomes more likely as the section lies out of range of any eroding channel for the sufficient time span. The channel movement may be continuous or discontinuous, and the occurrence of avulsion in particular appears to favour preservation of thick and complete sequences. The experiments suggest that if the general slope of facies boundaries or basal erosion surfaces relative to the land surface is not great and complete bar sequences are preserved, then a process other than purely movingplase must be responsible.

Complete vertical sequences are rarely preserved in modern and ancient coarse-grained point-bar deposits produced in streams subject to flash floods, because of the rapid channel migration (McGowen and Garner, 1970). Complete vertical sequences are more common in ancient fine-grained point-bar successions of the types described above. Because of the time scales involved in channel movement associated with discontinuous avulsion and cut-off and continuous moving phase, many erosion surfaces would be expected to exist in the lower parts of coarse members; Bluck (1971 states '...many sequences of believed fluvial origin have many erosion surfaces at their base'. In this respect also, the fills of the deeper scoured channels have a greatex preservation potential than contenporary shallower ones. 


\section{ANCIENT FLUVIATILE COARSE MEMBERS}

Allen (1970a) applies his original 'static' grain size and sedimentary structure model to various Devonian coarse members from Britain and North America, which are strongly believed to have accumulated through processes of lateral deposition. It appears in many cases that the only absolute control on data input used is via the density of the sedimentary particles and the maximum flow depth, which is taken as the thickness of the coarse member. This maximum flow depth must in reality be the maximum scoured flow depth. If scouring and filling has been an important process in the formation of such coarse members the application of this static model invites an additional caution because of the expected lateral variation in member thickness, grain size and bedding geometry. Allen has overcome complications due to major erosion surfaces at the bases of these members by choosing simple coarse members free of evidence of multi-storey character. other parameters defining channel geometry were chosen, being consistent with experience of sand-bed rivers, in order to give the closest fit with the observed coarse members. Additional control may have been forthcoming if epsilon cross-stratified units could be traced laterally to the extent that the width of the bar could be discerned (e.g. Moody-stuart, 1966). Caution is invited here with regard to the definition of the true width when looking at such units as projected in one cross section.

Where exposure limits examination of sections to any great lateral extent the application of the present model will necessarily follow the same general lines as Allen (1970a), thus restricting the use of all aspects of the model. An opportunity to apply substantially more interpretive aspects of the model lies in the pennsylvanian channel sandstone described by Beutner et al. $(1967)$, which represents some ryoo feet in lateral extent of sandy 
point-bar deposits exposed in a section cut approximately nornal to the mean downvalley direction. Directives for use of the model in this application, and a comparison of the simulated and actual section, will follow.

By inspection of the basal scoured surface it seems obvious that channel scouring and filling was continuing with bar migration (see Beutner et a1. 1967, p. 913)., The unscoured channel depth can be inferred by inspection of the relief of the scoured surface and the shape of the epsilon units. A value of 12 metres was chosen, and thus an idea of the amount of scouring below the talweg could be assessed; average about 4 netres. The width of flow between the inner bank and talweg was approximately 80 metres, by inspection of the width of epsilon units from the top to the bottom of the bar. The individual. units actually vary in length and show varying degrees of development, recording variation in the channel direction cutting the section combined with variable scouring. The ratio of partial width to full flow width (at bankfull stage) was arbitrarily taken as 0.8 thus giving a full width of 100 metres. The rate of bar migration can be inferred by measuring the horizontal thickness of certain well developed epsilon units. Average rates of migration are about 10-12 metres per time increment. A value of the exponent $\mathrm{n}_{1}$ was taken as 1.5 and this provides good agreement with the shape and maximum slope of the epsilon units $\left(10-20^{\circ}\right)$. In some runs a straight inner bank profile was used. Parameters for use in the scour and fill and bank migration relations were defined, bearing in mind the required average vảlues of scour and migration required, using an arbitrary flow pattern.

The remaining parameters required for input were defined, being consistent with observations from modern streams, such that the model could best simulate the main features of the section. The sands are medium grained on average but fine upwards. In the 
basal parts coarse sand and gravel size fragments are found. Most of the deposit is tabular cross bedded and is attributed to deposition by transverse bars migrating over the bar surface. There were a few examples of trough cross-bedding. Occurrences of cross lamination may also be seen towards the top of the section, and lenses of flat bedding sometimes occur in the deepest scours.

Figure 25.1 shows the simulated cross sections which are probably the closest obtainable at present. The grain size distribution section is made up predominantly of sand. Inspection of table 25.1, which shows the variation of various hydraulic parameters over the unscoured bar cross profile for the initial sediment deposition, indicates that the mean grain size is medium sand. The section fines upwards from fine gravel and coarse sand at the base to a sinall thickness of silt and clay at the top. The correspondence in grain size between the simulated and actual sections appear to be very good.

The correspondence between the simulated sedimentary structure section and the actual section is not as satisfactory. A thick set of cross bedding (due to dunes) is predicted, however, in general, the thicknesses of cross lamination and flat beding are overemphasised. Furthermore, no flat bedding is predicted in deeper scour hollows. The reason for the discrepancy probably lies in the fact that prediction of bed form was not as readily based on general principles as grain size prediction, and relied heavily on empirical flume data. Not until moro ricrorous and generalised methods of prediction are developed, which are applicable to natural streams, will this situation be remedied. It has already been pointed out that values of orit used are probably too low. The shape of the basal scoured surfaces agree well 
morizontes and

vortical acalo
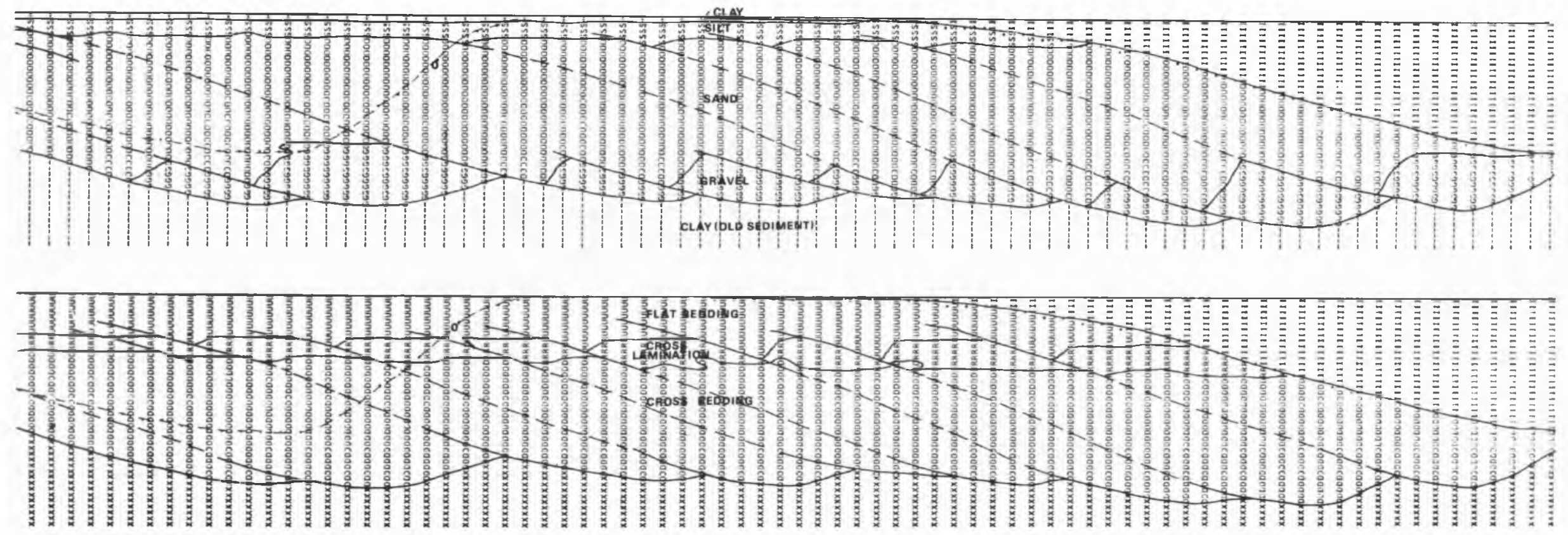

Fig. 25.1. Simulated cross sections comparable with ancient fluviatile coarse member shown in Fir. 25.2. 
Scale in Feel

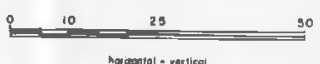

Aaciental - vartical

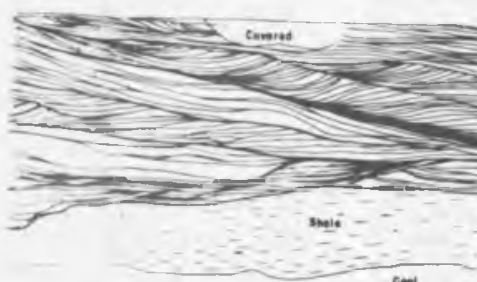

śó sóe

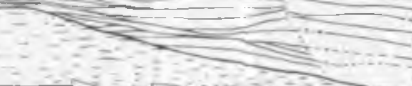

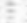

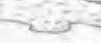

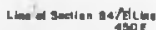

Sives

400

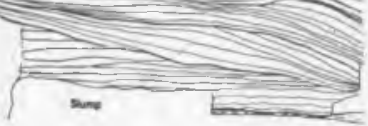

xśe

$F i_{i g} \cdot 25.2$.

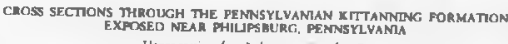

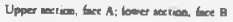

(after Beutner et el., 1967). 


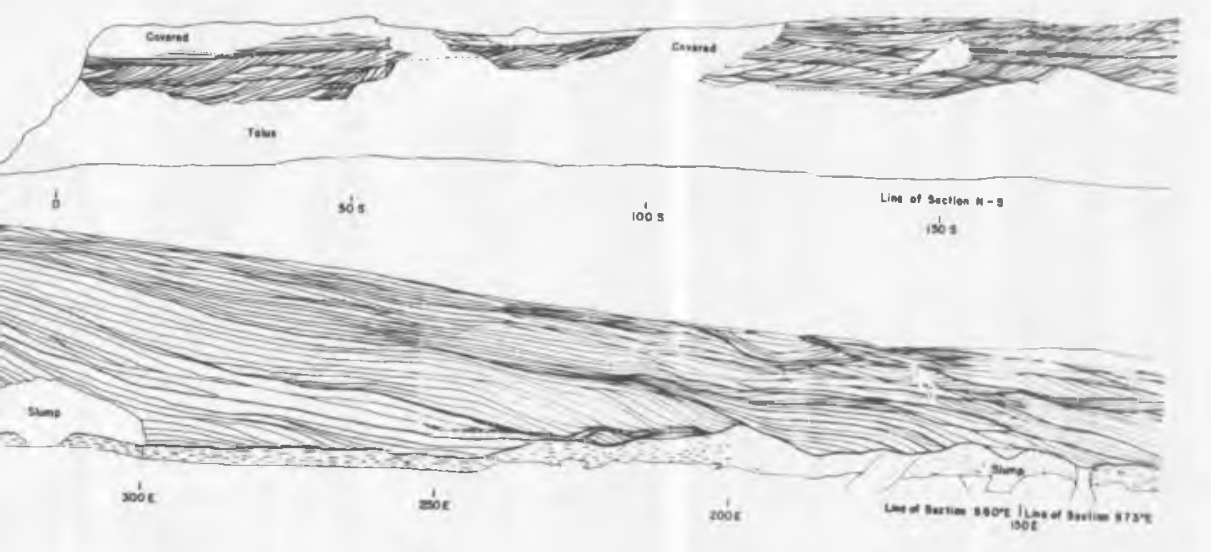


DVARIATION OF GRAINSIZE AND BED FORM OVER CHANNEL CROSS PROFILE

DEPTH GRAINSIIE BED FORM LOCAL MEAN LOCAL

TIME INCREMENT

0

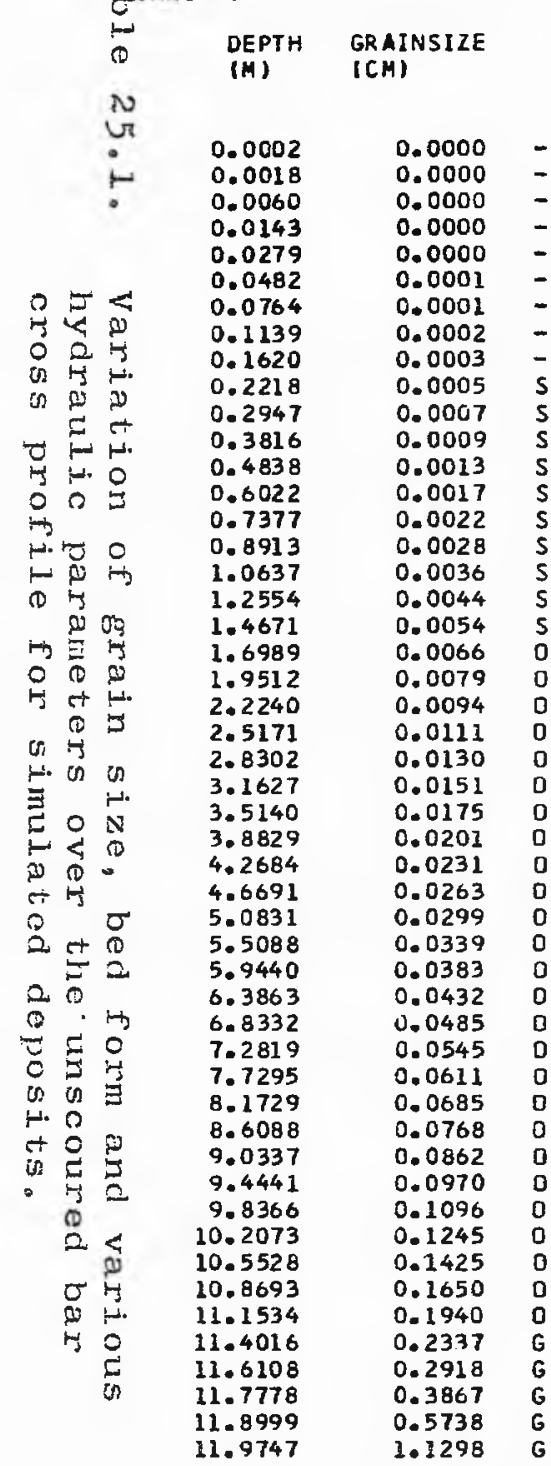

LOCAL.

LOCAL STREAM LOCAL BED LOCAL FROUDE (CM/SEC)

DIMENSIONLESS POWER
SHEAR STRESS (ERGSICHII

SHEAR STRESS NUMBER

\begin{tabular}{l}
0.6126 \\
1.7329 \\
3.1833 \\
4.9006 \\
6.8475 \\
8.9987 \\
11.3352 \\
13.8417 \\
16.5054 \\
19.3152 \\
22.2608 \\
25.3330 \\
28.5232 \\
31.8226 \\
35.2230 \\
38.7164 \\
42.2943 \\
45.9485 \\
49.6705 \\
53.4519 \\
57.2836 \\
61.1568 \\
65.0620 \\
68.9898 \\
72.9302 \\
76.8732 \\
40.4041 \\
42.3623 \\
44.3057 \\
46.2287 \\
48.1255 \\
49.9902 \\
51.8167 \\
53.5991 \\
55.3310 \\
57.0062 \\
58.6184 \\
60.1611 \\
61.6280 \\
63.0125 \\
64.3083 \\
65.5090 \\
66.6084 \\
67.5999 \\
68.4776 \\
69.2354 \\
69.8676 \\
70.3684 \\
70.7333 \\
70.9542 \\
\\
\hline
\end{tabular}

10.7323
5.4555
3.6973
2.8176
2.2896
1.9372
1.6850
1.4955
1.3477
1.2289
1.1313
1.0494
0.9796
0.9193
0.8664
0.8196
0.7777
0.7398
0.7053
0.6737
0.6443
0.6170
0.5914
0.5671
0.5441
0.5220
0.5008
0.4803
0.4604
0.4410
0.4219
0.4030
0.3844
0.3658
0.3473
0.3288
0.3101
0.2913
0.2722
0.2529
0.2332
0.2130
0.1924
0.17111
0.1493
0.1267
0.1034
0.0791
0.0539
0.0275

0.0006

0.1309

1.7329

0.0130

0.0009

0.1309

$0.0806 \quad 0.0253 \quad 0.1309$

$0.2942 \quad 0.0600 \quad 0.1309$

$\begin{array}{lllll}4.9006 & 2.8176 & 0.2942 & 0.0600 & 0.1309 \\ 6.8475 & 2.2896 & 0.8027 & 0.1172 & 0.1309 \\ 8.9987 & 1.9372 & 1.8217 & 0.2024 & 0.1309\end{array}$

3.6411

6.6300

11.2414
18.0150

27.5779

40.6444

58.0140

109.2499

145.0859

189.1414

189.1414
242.5242

306.3630

381.7937

469.9277

571.8396

68.05310

869.7542

969.7542

659.5916

760.2202

869.7217
987.9524

1114.6179

1249.2634

1391.2673

1539.8291

1693.9739

1852.5410

$0.3212 \quad 0.1309$

$0.4790 \quad 0.1309$

$0.6811 \quad 0.1309$

$0.9327 \quad 0.1309$

$1.2389 \quad 0.1309$

$1.6044 \quad 0.1309$

2.03390 .1309

$3.1017 \quad 0.1309$

$3.7474 \quad 0.1309$

$4.4720 \quad 0.1309$

$5.2782 \quad 0.1309$

$6.1679 \quad 0.1309$

$7.1428 \quad 0.1309$

$8.2035 \quad 0.1309$

$9.3504 \quad 0.1309$

$10.5827 \quad 0.1309$

$11.8990 \quad 0.1309$

$13.2970 \quad 0.1309$

$14.7737 \quad 0.1309$

17.32490 .0655

$19.6300 \quad 0.0655$

0.0655

$24.9902 \quad 0.0655$

$26.8498 \quad 0.0655$

$28.7287 \quad 0.0655$

$30.6153 \quad 0.0655$

$32.4972 \quad 0.0655$

$\begin{array}{lll} & 34.3612 & 0.0655 \\ 2014.1970 & 3.1936 & 0.0655\end{array}$

$2340.6377 \quad 37.9801 \quad 0.0655$

$\begin{array}{lll}2501.9636 & 39.7058 & 0.0655\end{array}$

$2659.5139 \quad 41.3557 \quad 0.0655$

$2811.2822 \quad 42.9144 \quad 0.0655$

$\begin{array}{lll}2955.1968 & 44.3668 & 0.0655\end{array}$

$\begin{array}{lll}3089.1436 & 45.6975 & 0.0655\end{array}$

3211.0422
37.9355

3910.8774 48.8149

$3484.4385 \quad 49.517100 .0655$

$3538.7820 \quad 59.517106 \quad 0.0655$

70.7323
70.9542

3572.1873

50.3450

0.0655 
FLUViatile PROCESS SIMULATION EXPERIMENT $X$

CRDSS SECTION PARAMETERS

METRES CELLS

HIOTH OF SECTION

THI CKNESS DF SECTION

INITIAL DISTANCE OF INNER CHANNEL BANK FROM L.H.S. OF SECTION

INITIAL BANKFULL STAGE MEASURED FRON SECTION BASE

CELL SILE IN VERTICAL(Y) DIRECTION

CELL SILE IN HORIZONTALIL OR X) DIRECTION

$340.000 \quad 200$

$30.000 \quad 60$

$\begin{array}{cc}0.0 & 0 \\ 20.000 & 40\end{array}$

0.500

1.700

CHANNEL PARAMETERS

METRES CELLS

TOTAL. WIDTH DF CHANNELI $W$

HIDTH OF FLOW BETWEEN INNER BANK AND TALHEG(WI)

RAT ID OF HI TO

$\begin{array}{ll}00.000 & 58 \\ 80.000 & 47\end{array}$

MAXIMUM FLOW DEPTH MEASUREO ABOVE TALHEG

12.000

0.800

SEDIMENTARY PARTICLES

DARCY-HEISBACH FRICTION COEFFICIENT FOR DUMES AND RIPPLES

DARCY-WEISBACH FRICTION COEFFICIENT FOR PLANE BEDS AND ANTIDUNES EXPONENT N2

$2.650 \mathrm{GH} / \mathrm{CH3}$

$1.000 \mathrm{GH} / \mathrm{CH}$

0.080

0.020

1.500

SYNTHETIC HYDROLOGY PARAMETERSIUNITS NOT NECESSARY I

MEAN OF ALL DAILY MEAN VALUES

STANOARD DEVIATION OF DAILY MEAN VALUES

MEAN DF YT SERIES

STANDARD DEVIATION DF YT SERIES

COEFFICIENTS IN AUTOREGRESSIVE MODEL

FOURIER COEFFICIENTS FOR DAILY MEANSIA

FOURIER COEFFICIENTS FOR DAILY STD DEVIATIONS (SA)

MAXIMUA VALUE OF QVOL

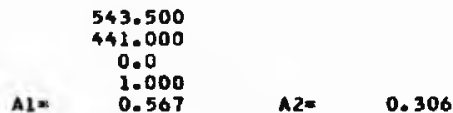

HARMONICS FROM 1 TO

$-200.300^{145.400}-85.500$

$\begin{array}{lll}-112.400 & 185.000 & -79.900 \\ -123.300 & 141.600 & -66.400\end{array}$

$\begin{array}{ll}142.600 & -66.400 \\ 105.700 & -46.200\end{array}$

$\begin{array}{rrr}58.000 & -39.600 & 7.400 \\ 65.600 & -72.500 & 27.800 \\ 75.700 & -47.200 & 0.600 \\ 31.700 & -43.200 & 4.300\end{array}$

EXPONENT N2

VALUE OF CONSTANT IN LATERAL MIGRATION RELATION

VALUE OF CONSTANT IN DOWNYALLEY MIGRATI ON RELATION

LIHITING PERCENTAGE OF GRAVEL ALLOHABLE IN OUTER BANK

110000.000

SCOUR AND FILL PARAMETEHS

CONSTANT $K 4$

EXPONENT N3

STANDARD DEVIATION OF ERROR TERM

$0.500 E-04$

1.000

CUT-OFF CONTROL PARAMETERS

LINITING MIDTH OF MEANDER NECK

EXPONENTS IN NECK CUT-OFF RELATION

LIMITING SINUDSITY

EXPONENTS IN CHUTE CUT OFF RELATION

EN I

200.000 METRES

$5.000 \quad$ EN 2 $=\quad 5.000$

29.870 METRES
20.000 EC 2

20.000

A DOMNYALLEY SECTION IS REPRESENTED IN THIS TEST
DISTANCE JF LINE OF SECTION FROM POINT OF INFLECTION OF LOOP IS 0.0 METRES

Table 25.2. Initial data used for simulation. 
FLUVIATILE PROCESS SIMULATION

EXPERI MENT $X$

PLANIMETRIC FORH OF MEANDER

WAVEL ENGTH

AMPLITUDE

SINUDSITY

RADIUS OF CURVATURE AT BEND AXIS

WIDTH OF MEANDER NECK

CHANNEL LENGTH ALONG MEANDER

VALLEY SLOPE

LONGI TUDINAL WATER SURFACE SLOPE

SELECTED GEOMETRIC RATIOS

WAVELENGTH TO RADIUS OF CURVATURE WAVELENGTH TO CHANNEL WIOTH

RADIUS OF CURVATURE TO CHANNEL WIDTH

AMPLITUDE TO CHANNEL WIDTH

FLUVIATILE PRDCESS SIMULATION EXPERIMENT $X$

PLANIMETRIC FDRM OF MEANDER

HAVELENGTH

AMPLITUDE

SINUOSITY

RADIUS OF CURVATURE AT BEND AXIS

WIDTH OF MEANDER NECK

CHANVEL LENGTH ALONG MEANDER

VALLEY SLOPE

LONGITUDINAL WATER SURFACE SLOPE

SELECTED GEOMETRIC RATIOS

HAYELENGTH TO RADIUS OF CURVATURE

WAYELENGTH TO CHANNEL WIDTH

RADIUS OF CURVATURE TO CHANNEL WIDTH

AMPLI TUDE TO CHANNEL WIDTH

Table 25.3. Various geometric variab. simulation. 
METRES

700.000

308.311

141.032

1.400

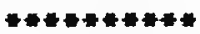

980.000

$$
\begin{aligned}
& 0.00006000 \\
& 0.00004286
\end{aligned}
$$

4.963

7.000

1.410

3.083

\section{TIME INCREMENT 10}

METRES

700.000

$501 \cdot 700$

148.890

$$
1.907
$$

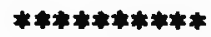

1334.817

$$
\begin{aligned}
& 0.00006000 \\
& 0.00003146
\end{aligned}
$$

$$
\begin{aligned}
& 4.701 \\
& 7.000 \\
& 1.489 \\
& 5.017
\end{aligned}
$$

les at the beginning and end of the 


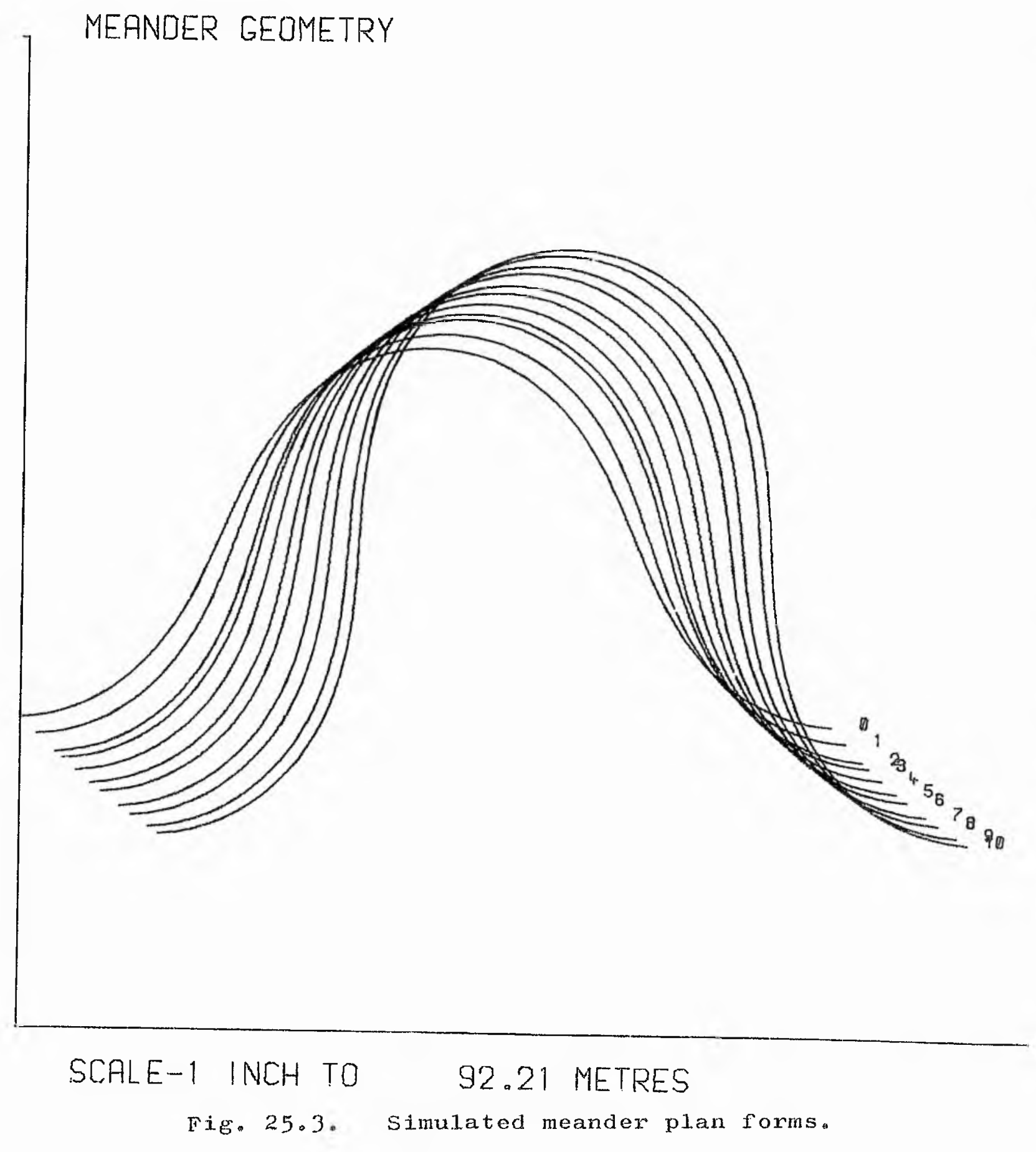


qualitatively with the observed sequence (c.f. fig. 25.2), which indicates that the amounts of bank migration and scour and fill used in the model are appropriate. Where possible approximate outlines of epsilon unit boundaries have been added.

Table 25.2 shows the input data used and various other initial data. Table 25.3 shows various geometric variables at the beginning and end of the simulation, and fig. 25.3 shows the meander movement in plan which produced the deposits. The low valley slope and high sinuosity indicate that the stream was very close to its base level. An estimate of the bankfull discharge can be obtained fairly easily by assuning a realistic value of the friction coefficient for the outer bank; computed values of velocity and the cross sectional area of the channel will then give the discharge. Various other hydrological parameters may be estimated using, for instance, the regression equations developed by Schumm (1972). 
As far as the model can be tested against nature it appears to operate realistically. In view of the many simplifications made in its development, the results and implications are encouraging, although no pretence is made to quantitative exactness. Unfortunately the model has not been able to be tested rigorously, and comparison of real examples with solutions produced with the simulation model do not involve any form of optimisation or statistical measure of closeness.

The development of the mathematical model and subsequent experimentation with the computerised version has led to some significant conclusions helpful in the understanding and interpretation of the lateral deposits attributable to meandering streams. The computer simulation model permits, in a matter of minutes computing time, the exploration of the behaviour of the system under a wide variety of physical conditions and over long periods of time. Such data are not easily obtainable from the natural situation by virtue of the time scale involved or the prohibitive costs of field surveys. Scale physical model expeximents also have severe limitations on time and often have stringent scaling qualifications.

The model may be used qualitatively as a guide to the recognition of ancient fluviatile sediments which were deposited under conditions of lateral sedimentation on the inside of meander bends. A quantitative picture of certain aspects of the physical. environment respsonsible for such deposits may then be built up by comparison with simulated deposits. However, until the model has been tested more rigorously and further developed, any quantitative interpretation must be treated with caution. There are a sufficient number of input variables to provide several solutions in any specific example, and thus any one overall answer 
cannot always be assuned to be correct in its entirety. Furthermore, at the level of the present study, any 'quantitative' results must be thought of as broad guidelines demarcating a 1ikely physical situation.

Both the matlematical model and computer program provide a framework for the construction of further simulation mode1s of more quantitative validity and embodying more complex and generalised fluvial systems. Obvious improvements would be the development of adequate mathematical models for erosion and deposition over a range of river stages within the channel and over the rloodplain. The reasonable treatment of the sequence of events involved with channel abandonment and relocation (cut-off and avulsion) would clearly be valuable. The model is very restrictive in its range of application; riffle deposits, overbank deposits, etc. require attention, and the deposits of coarsegrained point bars and braided rivers must surely merit consideration. Many of the mathematical relationships used in the model are empirical in nature; theoretical relationships will be more desirable in future because of their greater versatility.

As computer simulation models are developed and become more complex, core store requirements may be expected to rise. Fortunately there does not appear to be a problem here in view of the recent advances in computer technology. Associated with further development of such mathematical models will also come a deeper understanding of the processes involved in the natural system. As well as being parasitic on the vast amount of field, laboratory and theoretical information that exists, the models also direct research to areas that are inadequately explored. It should be realised that to test a model adequately, a large amount of clata of the appropriate form must be available. The interpretive and predictive potential of computer simulation warrants such further work. 


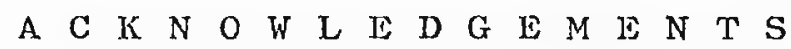

This research was carried out whilst under the receipt of a N.E.R.C. research studentship, for which I an grateful.

I would like to express my sincere gratitude to Professor E.K. Walton for his friendly encouragement and guidance during his supervision of this research. Thanks are also due to Dr. B.J. Bluck, Dr. D.G. Farmer and Professor J.R.L. Allen, for their continuing interest and stimulating discussion. My colleagues in the Geology Department, st. Andrews have continued to show a friendly interest and $I$ am indebted in particular to Dr. W. Edryd stephens for his help in various statistical problems.

Mr. R.W. Benson of the Hydraulics Research station, Wallingford, kindly allowed me to examine the results of laboratory experiments conducted at wallingford.

I would like to express my gratitude to Professor A.J. Cole of the st. Andrews University Computing Laboratory for his patient and friendly co-supervision during the early periods of the research. The fine computing facilities at st. Andrews and the invaluable help given by various members of the academic and technical staff of the computing laboratory is gratefully acknow 1 edged.

Thanks are due to Mrs. J. Galloway for palnstakingly typing the script and to Mr. J. Allan for producing excellent photographic results.

It has not been possible to give full credit to all the people who have advised, encouraged, criticised, hindered or otherwise shown interest in the research. I would like to extend my gratitude to all those not mentioned specifically above. 
LIST OF SYMBOLS USED IN MATIEMATICAL MODEL.

Symbol

a

$a_{1}, a_{2}$

$a(t)$

A

$A(t)$

$\mathbf{A}_{\mathbf{k}}$

$\mathbf{s}^{A_{k}}$

$\mathbf{B}_{\mathbf{k}}$

$\mathrm{s}^{\mathrm{B}} \mathrm{k}$

$c_{1}, c_{2}, \ldots$

C

C

$\mathrm{C}_{\mathrm{s}}$

d

D

DSCR

e

er

$e c_{1}, e c_{2}$

${ }^{e n_{1}}, e n_{2}$
Description

Cross sectional area of stream.

Coefficients in autoregressive synthetic

hydrology model.

Amplitude of bed wave.

Meander amplitude.

Amplitude of surface wave.

Fourier coefficients for cosine terms in

harmonic representation of daily mean flows.

Fourier coefficients for cosine terms in

harmonic representation of daily standard

deviations about daily means.

Fourier coefficients for sine terms in

harmonic representation of daily mean flows.

Fourier coefficients for sine terms in

harmonic representation of daily standard

deviations about means.

Any constants.

Chezy C.

Dimensionless parameter from Hayashi (1970).

Welght concentration of sediment.

Mean depth of flow.

L

Diameter of sedimentary particle.

L

Depth of scour at the talweg.

L

Base of naperian logarithms.

Error term in scour and fill relation.

$\mathbf{L}$

Exponents in chute cut-off relation.

Exponents in neck cut-off relation.

$-$
L

L

L

$L^{\frac{1}{2}} T^{-1}$

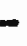

Dimensiona 1 formula

2

$* M=$ Mass, L=Length, $\mathrm{T}=\mathrm{T} 1 \mathrm{me}$. 
Darcy-Weisbach friction coefficient.

$f_{1} \quad$ Darcy-Wetsbach friction coefficient for ripples and dunes in a straight channel.

$\mathrm{f}_{2}$ Darcy-Weisbach friction coefficient for

plane beds and antidunes in a straight

channe1.

$f_{b}$ Part of Darcy-Weisbach friction coefficient representing form losses due to addition

of a bend.

$\mathbf{f}_{\mathbf{s}}$

Darcy-Weisbach friction coefficient in the straight channel that is comparable with a given bend.

Upsiope component of fluid force on a point $\mathrm{MLT}^{-2}$ bar.

$F$
$F r$
$F r_{1}$
$F r_{2}$
$F r_{a}$
$F_{m}$
$F_{m}$

Width-depth ratio.

Froude number.

Maximum Fr for formation of dunes.

Maximum Fr for formation of antidunes.

Minimum Fr for formation of antidunes.

Upper stability limit for 2-D bed waves.

Fr at change from transition to upper flow

regine.

$\mathrm{Fr}$

g

Fr at change from lower flow regime to transition.

Gravitational acceleration.

$L T^{-2}$

G

Body force component acting on a particle.

$M L T^{-2}$

GAP

Width of meander neck.

L

GSI

Grain size index.

h

Maximum unscoured flow depth measured above talweg.

L

1

Sediment transport rate (immersed weight

per unit width).

$\mathrm{ML}^{-1} \mathrm{~T}^{-1}$ 
j Kennedy j factor.

k Wave number $(=2 \pi / L)$

$k_{1} \quad$ Ratio of full width to partial width of channe1.

$k_{2}, k_{3}$ Dimensional constants in bank migration relations.

$\mathbf{k}_{4} \quad$ Dimensional constant in scour and fill relation.

1 Neander wavelength.

$\mathbf{L}$

L Wavelength of ginusoidal bed waves.

L

m

Dimensional coefficient from Hayashi's

(1970) sediment transport relation.

Mean daily flow for day $\tau, \tau=1,365$.

Nean of all the $\mathrm{m}_{\tau}$.

Continuous representation of $m_{\tau}$ using Fourier analysis.

M

Weighted mean percentage of silt and clay

in channe1 perimeter.

M

Total path length in a meander wavelength

$\mathbf{L}$

n Exponent in Kennedy's (1963, 1969) transport

rate equation.

$n_{1} \quad$ Exponent in transverse profile shape equation. -

$\mathrm{n}_{2} \quad$ Exponent in bank migration relations.

$\mathrm{n}_{3}$ Exponent in scour and fill relation.

N

An exponent.

NS

Average net scour at a channel cross section. $\mathrm{ML}^{-2}$

P A probability.

p(c) Probability of chute cut-off.

$p(n) \quad$ Probability of neck cut-off.

Q

Discharge.

$\mathrm{L}^{3} \mathrm{~T}^{-1}$

Qvol Flood period rolume. 
$Q_{t}$

$\mathbf{r}$

$\mathbf{r}_{\mathbf{m}}$

$r_{1}$

$r_{L}$

R

RMIG

RIMIG

3

${ }^{3} \tau$

$\bar{s}_{\tau}$

s $\mathbf{y}$

$\mathbf{s}$

sn

stdvn

$S$

5

Total sediment load that is sand or bed

load at mean annual discharge.

Factor by which $f_{s}$ may have to be multiplied

to account for change in relative roughness

(arising from bed features) due to change

in hydraulic radius with meandering.

Radius of curvature measured to channel

centre lines.

$\mathbf{L}$

Local radius of curvature.

$\mathbf{L}$

Lth order serial correlation coefficient

of sample $\mathrm{z}_{t}$

Hydraulic radius (= hydraulic mean depth).

L

Bank migration rate in specific cross

section.

$\mathrm{LT}^{-1}$

Bank migration rate normal to mean downvalley

direction.

$\operatorname{LT}^{-1}$

Bank migration rate in mean downvalley

direction.

Distance along meander path.

L

Standard deviation of daily flow for day. $\tau$.

Mean of all ${ }^{3} \tau$.

Standard deviation of $Y_{t}$ series.

Continuous representation of $s_{\tau}$ using

Fourier analysis.

Sinuosity.

Standard deviation in error term.

Longitudinal slope of water surface.

Distance of channel at bend axis from an

assumed equilibrium position (i.e. position

of limiting amplitude) - measured normal to

mean downvalley direction. 
So

\begin{tabular}{|c|c|c|}
\hline & above. & L \\
\hline $\mathrm{s}_{\mathbf{b}}$ & Longitudinal bed slope. & - \\
\hline t & Time. & $\mathbf{T}$ \\
\hline $\tan$ & Dynamic solid friction coefficient. & - \\
\hline $\mathrm{T}$ & $\begin{array}{l}\text { Constant value of } t \text { in scour and fill } \\
\text { analysis. }\end{array}$ & $\mathbf{T}$ \\
\hline$\overline{\mathrm{T}}$ & $\begin{array}{l}\text { Net forward sediment transport rate for } \\
\text { whole stream. }\end{array}$ & \\
\hline $\mathrm{V}$ & Mean fluid flow velocity for whole stream & $L \mathrm{~S}^{-1}$ \\
\hline$v_{c}$ & $\begin{array}{l}\text { Critical velocity for initiation of } \\
\text { sediment motion. }\end{array}$ & $L T^{-1}$ \\
\hline $\mathrm{V}_{\mathrm{b}}$ & Bed form velocity. & $\mathrm{LT}^{-1}$ \\
\hline $\mathrm{V}_{*}$ & Shear velocity. & $L T^{-1}$ \\
\hline$v_{*} \operatorname{crit}$ & Critical shear velocity. & $L T^{-1}$ \\
\hline
\end{tabular}

w Full width of flow between inner and outer banks.

$\mathbf{L}$

$w_{1}$ Width of flow between inner bank and talweg. I $w_{s}$ Channel width projected in a specific cross section.

Meander width.

L

Downchannel distance measured parallel to channel centre line.

$x$ Coordinate of reference axis in downvalley direction.

$x_{t}$ rime series.

y Flow depth measured positively downward from water surface at any transverse distance $z$ across channe1.

$Y_{t} \quad$ Standardised fitted series or Fitted series. 
Mean of $Y_{t}$.

Perpendicular transverse distance across

the water surface measured from edge of

water at inner bank.

Coordinate for reference axis normal to

$X$ axis in horizontal plane.

$\mathbf{z}$

$Z_{\text {sect }}$

$\propto$

$\alpha$

$\beta$

$\Gamma$

$\delta$

$\Delta_{\mathrm{s}}$

$\Delta \phi$

$\epsilon_{t}$

$\eta_{t}$

$\theta$

$\theta_{\text {crit }}$

$\nu$

$\pi$

$\rho$

$\rho_{\mathrm{w}}$

$P_{\text {L }}$ standardised series.

Normal distance of line of section from

line joining points of inflection of 100 .

$\mathbf{L}$

Angle on bed between channel centre line

and tangent to a skin-friction line.

Angle that line of section makes with normal

to mean downvalley direction.

Slope in degrees of channel cross profile.

Expression in Hayash's (1970) analysis.

Lag distance.

$\mathbf{L}$

Elemental distance alang path of a meander

i.e. small change in s.

$\mathbf{L}$

Sma11 change in $\phi$.

Independent residual series.

standardised independent stochastic variable

(primary variable)

Dimensionless shear stress.

Critical dimensionless shear stress.

Kinematic viscosity.

$\mathrm{L}^{2} \mathrm{~T}^{-1}$

Pi, radians.

Fluid density (including suspended sediment). $\mathrm{ML}^{-3}$

Fluid density of water.

$\mathrm{ML}^{-3}$

Lth order serial correlation coefficient

of the population from which $z_{t}$ is dxawn. 
$\sigma$

$\sigma_{s}^{\prime}$

$\tau_{\mathbf{x}}$

$\tau_{s}$

$\varnothing$

$\omega$

$\omega$

$\omega_{\text {crit }}$
Density of sediment particle in bed load.

205.

Density of suspended sediment.

Component of bed shear stress parallel to channel centre line.

$\mathrm{ML}^{-1} \mathrm{~T}^{-2}$

Bed shear stress parallel to skin friction

line.

$\mathrm{ML}^{-1} \mathrm{~T}^{-2}$

Deviation angle of meander path from mean downvalley direction.

Maximum value of $\varnothing$.

Stream powor.

Critical stream power.
$\mathrm{MT}^{-3}$

$\mathrm{ML}^{-3}$

$\mathrm{ML}^{-3}$

$\mathrm{MT}^{-3}$ 
ACKRRS, P. and CIARLTON, F.G. 1970a. The geometry of smal1 meandexing streams. Proc. Instn. Civ. Engrs. supplenent (xii). paper 7328s, 289-317.

1970b. Dimensional analysis of aliuvial channels with special reference to meander length. J.Hydraul. Res., 8, 287-314.

19700. Meander geometry arising from varying flows. J. Itydrol., 11, 230-252.

1970d. The slope and resistance of smali meandering channels, proc. Instn. Civ. Engrs. Supplement (xv). paper $7362 \mathrm{~S}, 349-370$.

ADANOTSKI, K. 1971 .

Spectral density of a river flow time series. I. IIydrol., 14, 43-52.

ALBERTSON, M.L. and SIMONS, D.B. 1964. Fluid mechanics. In V.T. Chow (Ed), Handbook of Applied llydrology, sect. 7. BcGraw Hi.1.

ALLEN, J . 1939.

The resistance to flow of water along a tortuous stretch of river and in a scale model of the same. J. Instn. Civ. Engra., 11, 115 .

ALIEN, J. and SIAHWAN, A. 1954. The resistance to flow of water along a tortuous stretch of the river Irwell (Lancashire) - an investigation with the aid of scale-model experiments. Proc. Instn. Civ. Engrs., 25, $244 \ldots 165$.

ALLEN, J.R.L. $1963 a$.

The classification of cross-stratified units with notes on their origin.

Sedimentolory, 2, 93-1.24. $1963 \mathrm{~b}$.

Depositional features of Dittonian rocks: Pembrokeshire compared with the Velsh Borderland. Geo1. Mag., 100, 385-400.

$1963 \mathrm{c}$.

Henry clifton Sorby and the sedimentary structures of sands and sandstones in relation to flow conditions. Geol. Mijnbouw, 42, 223-238.

1964.

studies in fluviatile sedimentation: six cyclothcus from/the Lower old Red Sandstone, Anglo-Welsh Basin. Sedimentology, 3, 163-198. 19652.

A review of the origin and characteristics of recent alluvial sediments. Sedimentology, 5, 89-191.

$1965 \mathrm{~b}$

Sedimentation and palaeogeography of the old Red Sandstone of Anglesey, North Wales. Proc. Yorks. Geol. Soc., 35, 139-185. 19650 . Fining upwards cycles in alluvial successions. Liverpool Manchester Geol. J., 4 , $229-246$. 
ALLEN, J.R.L. 1968.

Current Ripples. North-Holland

publishing Co., Ansterdam. 433 p.

$1970 \mathrm{a}$.

A quantitative model of grain size and sedimentary structures in lateral deposits. Geol. J., I, 129-146.

$1970 \mathrm{~b}$.

Studies in fluviatile sedimentation:

A comparison of fining upwards cyclothems, with special reference to coarsemember composition and interpretation. J. Sedim. Petrol., 40, 298323.

$1.970 \mathrm{c}$. $\therefore$ George Allen and Unwin, London. $248 \mathrm{pp}$.

1971.

Rivers and their deposits.

Oxf., 59, 109-122.

ALLEN, J.R.L. and FRIEND, P.F. 1968. Deposition of the CatskiII facies, Appalachian region: with notes on some other old Red Sandstone Basins. Spec. Pap. Geol. Soc. Am., 106, $21 .-74$.

ANDERSON, A.G. 1953. The characteristics of sedinent waves formed by flow in open channels. Proc. 3rd Midwest. Conf. Fluid Mech., Minneapolis, 379--395. 1967. On the development of stream meanders. Proc. Int. Ass. Hydraul. Res., 1, 370.-378.

ATHAULlAH, M. and SIMONS, D.B. 1970. Prediction of bed forms in alluvial channels. In press.

BAGNOLD, R.A. 1954.

Experiments on a gravity free dispersion of large solid spheres in a Newtonian fluid under shear. Proc. R. Soc., 225A, $49-63$.

1956.

The flow of cohesionless grains in fluids, Phil. Trans. Roy. Soc. London, Ser. A, 249, 235-297.

1960.

Some aspects of the shape of river meanders. Prof. Pap.U.S. Gool. Surv. No. 282E, 135-144.

1966.

An approach to the sediment transport problem from general physics. Prof. Pap.U.S. Geo1. Surv., No. 422-I, 1-37.

BECKINSALE, R.P. 1969. River regimes. In R.J. Chorley (Ed), Water, Earth and Man, 455-471. Methuen.

BEERBOWER, J.R. 1964 . Cyclothems and cyclic depositional mechantsms in alluvial plain sedimentation. Bul1. Kansas Geol. Surv., No. 169, 31-42.

BERNARD, H.A. and LeBLANC, R.J. 1965. Resume of the Quaternary geology of the northwestorn Gulf of Mexico Province. In The Quaternary of the United States (Ed. H.I. Wright and D.G. Frey). Princeton University press, Princeton, N.J., 137. 
BERNARD, H.A. and MAJOR, C.F. 1963. Recent meander belt deposits of the Brazos River: an alluvial 'sand' model. Bu11. Am. Ass. Petrol. Geol., 47, 350 .

BEUTNER, E.C., FLUECKINGISR, L.A. and GARD, T.M. 1967. Bedding geometry in a Pennsylvanian channel sandstone. Bull. Geol. Soc. Am., 78, 911-916.

BLUCK, B.J. 1971.

Sedimentation in the meandering River Endrick. Scott. J. Geol., 7, 93-138.

CALLANDER, R.A. 1969. Instability and river channels.

J. Fluid. Mech., 36, $465-480$.

CARLSTON, C.W. 1965. The relation of free meandex geometry to stream discharge and its geomorphic

implications. Am. J. Sci., 263, 864-885.

CHANG, H-Y., STMONS, D.B., and WOOLHISER, D.A. 1971. Flune experiments on alternate bar formation. J. Waterways, Harbours and Coastal Engrg. Div., Am. Soc. Civ. Engrs., $27,155-165$.

CHANG, T.P. and TOEBES, G.H. 1970. A statistical comparison of meander plan forms in the Vabash Basin. Water. Resources Res., 6, 557-578.

CHARLTON, F.G. and BENSON, R.T. 1966. Effect of discharge and sediment charge on meandering of small streams in alluvium. Hydraulics Research Sta., Wallingford, England. CHITALE, S.V. 1970. River channel patterns, J. Hydraul.
Div.,An. Soc.Civ.Engrs., 26, 201-221.

CHORLEY, R.J. and KENNEDY, B.A. 1971. Physical Geography: a systems approach. Prentice Hall, london. 370pp. CHOW, V.T. 1959. $680 \mathrm{pp}$.

Open Chamel Hydraulics. McGraw Hill. 1964. Statistical and probability analysis of hydrologic data. pt. 1. Frequency analysis. In Chow, V.T. (Ed), llandbook of Applied Hydrology, sect. 8,1. McGraw Hill.

1967. Simulation of the hydrologic behaviour of watersheds, a general report on new ideas and scientisic methods in hydrology. Proc. Int. Hydrol. Symp. 50-65. Colorado State University, Fort Collins, Colorado.

and KARRLIOTIS, S.J. 1970. Analysis of stochastic hydrologic systems. Water Resources. Res., 6, 1.569-1582.

COLBY, B.R. 1964. Scour and Fill in sand bed streams. Prof. Pap. U.S. Geol. Surv., No. 462-D. 32pp.

COLAMAN, J.M. 1969. Brahnaputra River: channel processes and sedimentation. Sediment. Geol., 3, 129-239. 
ClRAWFORD, N.H. and LINSLEY, R.K. 1966. Nigital simulation in hydrology: Stanford Watershed model IV. Stanford Univ. Dept. Civ. Engrg. Tech. Rept. 39.

CRESS, P., DIRKSIN, P. and GRAHAM, J.W. 1970. FORTRAN IV with WATFOR and WATFIV. Prentice Hall.

CULBERTSON, J.K. and DAKDY, D.R. 1964. A study of fluvial characteristics and hydraulic variables, Middle Rio Grande, New Mexico. U.S. Geol. Surv. Water Supply Pap., No. 1498-F, 74pp.

SCOTT, C.H. and BENNETT, J.P. 1972.' Summary of alluvial..channel data from Rio Grande Conveyance Channel, New Mexico, 1965-69. Pror. Pap. U.S. Geol. Surv., No. 562-J. $49 \mathrm{pp}$.

DANTEL, J.F. 1971. Channel movement of meandering Indiana streams. Prof. Pap. U.S. Geol. Surv., No. 732-A. 18pp.

DAWDY, D.R. and MATALAS, N.C. 1964. Statistical and probability analysis of hydrologic data. pt. III. Analysis of variance, covariance and time series. In Chow, V.T. (Ed), Handbook of Applied Hydrology, sect. 8-III. McGraw Hili.

ENGELUND, F. 1970.

Instability of erodible beds. J. Fluid. Mech. 4t2, 225-244.

and FREDSOE, J. 1971. Three dimensional stability analysis of open channel flow over an erodible bed. Nordic Ifydrology, 2, 93-108.

and HANSEN, E. 1966. Investigations of flow in alluvial streams. Acta Polytech. Scand. Civil Engrg. B1d. Construct. Ser., No. 35, 1-100.

1967. Comparison between similarity theory and regime formulae. Tech. Univ. Denmark, Copenhagen, Coastal Engrg. Lab. (Hydraulic Lab.), Basic Research Progr. Rep. 13, $14 \ldots 16$.

EVANS, G.

1965.

Intertidal flat sediments and their enviromments of deposition in the Wash. Q.J. Geo1. Soc. Lond., 121, 209-245.

FISK, H.N. 1944 .

Geological investigation of the alluvial valley of the lower Misstssippi River. Mississippi River Commission, Vicksburg, Miss. $78 \mathrm{pp}$.

1947.

Fine grrained alluvial deposits and their erfects on Mississippi rivex activity. U.S. Waterways Expt, Sta., Vicksburg, Miss. 2 vols.

FRAZIER, D.F. and OSANIK, A. 1961. Point bar deposits, old River Locksite, Louisiana. Trans. Gulf Coast Assoc. Geol. Soc., 11, 121-1.37. 
FRTEDKIN, J.F. 1945.

A laboratory study of the meandering of alluvial rivers. U.S. Waterways Expt. Sta., Vicksburg, Miss.

FUJIYOSIII, Y。 1950 .

Theoretical treatise on the meandering of river. Japan Sci. Rev., 1, 29-34.

GHOSI, A.K. and SCHITDEGGER, A.E. 1971. A study of natural wiggly lines in hydrology. J.Hydro1. 13, 101-126.

GRADOWCZYK, M.H. 1968. Wave propagation and boundary instability in erodible-med channels. J. Fluid Moch., 33, 93-112.

GRAF, W.H. 1971. Hydraulics of sediment transport. McGraw Hi11. 513pp.

GUY, H.P, SIMONS, D.B., and RICILARDSON, E.V. 1966. Summary of alluvial channel data from flume experiments, 1956-61. Prof. Pap.U.S.Geo1. Surv., No. 462-I. $96 \mathrm{pp}$.

ILAMISTN, M.J. 1971.

$\Lambda$ study of synthetic flow generation techniques using Elan valley data. J. Instn. Water Engrs., 25, 355-370.

HANDY, R.L. 1972 .

Alluvial cutoff dating from subsequent growth of a meander. Bull. Geol. Soc. Am. 33, $475-480$.

HANSEN, E. 1967. On the formation of meanders as a stability problem. Tech. Univ. Denmark, Copenhagen, Coastal Engrg. Lab. (IIydrauljc Lab.), Basic Research Progr. Rep. 13, 9-13.

HARBAUGH, J.W. and BONIAM-CARTER, G. 1970. Conputer simulation in goology. Wiley.

HARMS, J.C. and FAHNESTOCK, R.K. 1965. Stratification, bed forms and flow phenomena (with an example from the Rio Grande). In Primary sedimentary structures and their hydrodynamic interpxetation (Ed. G.V. Middieton). S.E.P.M. Spec. Pub1n. 12, 84-115.

HAYASIII, T. 3970. Formation of dunes and antidunes in open channels. J. Ilydraul. Div. Ail. Soc. Civ. Engrs., 26, 357-366.

HILL, II.M. 1966. Bed forms due to a fluid stream. $\frac{\mathrm{J} \cdot \mathrm{Hydraul} \text {. Div. Am. Soc. Civ. Engrs. }}{1 \mathrm{i} 3 \text {. }}$, $127-$ , SRINIVASAN, V.S. and UNNY, T.E. 1969. Instability of a flat bed in alluvial channels. J. Ilydraul. Div. Am. Soc. Civ. ingrs., 25, $1545-1558$.

IBADE-ZADE, YU.A. and KIYASBEILJ, T.N. 1967. The bed form on rectilinear and curvilinear river and big channel sections. proc. XII Congress, Int. Ass. Hydxau1. Res., $1,3 \overline{45-353 .}$

IBM 1968.

System/360 FORTRAN IV 1anguage. 
INGLIS, C.C. 1947. Meanders and their bearing on river training. Inst. Civil Engrs. (Lonclon). Maritime and Vaterways Engrg. Div., Session $1946-47$

1949.

The behaviour and control of rivers and canals. Res. Publ. Central liaterpower and Irrigation Navigation Res. Sta., Poona (India), 13, pt. I, 143-157; pt. II, 459-467.

IPPEN, A.T. and DRTNKER, P.A. 1962. Boundary shear.stresses in curved trapezoidal channels. J. Hydraul. Div. Am. Soc. Civ. Engrs., 88, 143-180.
JAINS, R.H. 1947.
Geologic features of the connecticut Valley, Massachusetts, as related to recent floods. U.S. Geol. Water Supply Papers, 296 , $158 \mathrm{pp}$.

KENNEDY, J.F . 1963. The mechanics of dunes and antidunes on erodible-bed channels. J. Fluid Mech., 26, $521-544$.

1969.

The formation of sediment ripples, dunes and antidunes. Annual Review of Fluid Mech., $1,147-168$.

KINOSITA, R. 1961 . Study of the channel evolution of the Isikari River. Bureau of Resources, Department of Science and Technology, Japan (In Japariese).

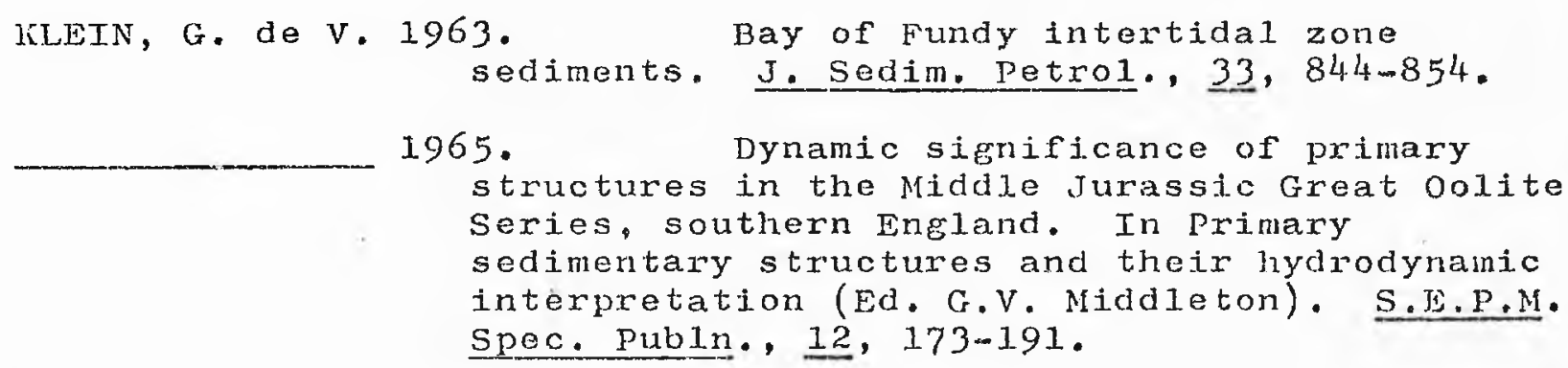

KOLB, C.R. $\quad 1963$.

Sediments forming the bed and banks of the Lower Mississippi River and their effect on river migration. Sedimentology, 2, 227-235.

KONDRATEV, N.G. 1962.

River flow and river channel format. ion. Israel prograin for scientific translations, Jerusalem.

KREITZBERG, C.B. and SHNEIDLRMAN, B. 1972, The elements of FORTRAN style: techniques for elficient programing. Ifarcourt Brace Jovanovich, Inc. 
LANE, I.W. and BORLAND, W.M. 1954. River bed scour during floods. Trans. Am. Soc. Civ. Engrs. 119. 1069-1080.

LANGBEIN, W.B. 1964. Geometry of river channels. J. Hydraul. Div. Am. Soc. Civ. Engrs., 20, 301-312.

LANGBEIN, W.B. and LEOPOLD, L.B. 1964. Quasi-equilibrium states in channel morphology. Am. J. Sci., 262, 782794.

1966. River meanders-theory of minimum variance. Prof. Pap. U.S. Geol. Surv., No. 422-H. 15pp.

LATHRAP, D.W. 1968. Aboriginal occupation and changes in river channel on the Central Ucayali, Peru. American Antiquity, 33, 62-79.

LEOPOLD, L.B., BAGNOLD, R.A., WOLMAN, M.G. and BRUSH, L.M. 1960. Flow resistance in sinuous or irregular channels. Prof. Pap.U.S. Geol. Surv., No. 282D, $111-134$.

LEOPOLD, L.B. and LANGBEIN, W.B. 1962. The concept of entropy in landscape evolution. Prof. Pap. U.S. Geol. Surv., No. 500-A, 20pp. American, $214,60-70$.

1966. River meanders. Scientific

LEOPOLD, L.B. and MADDOCK, T. 1953. The hydraulic geometry of stream channels and some physiographic implications. prof. Pap. U.S. Geol. Surv., No. $252,1-57$.

LEOPOLD, L.B. and MILLER, J.P. 1956. Tphemeral streams-hydraulic factors and their relationship to the drainage net. Prof. Pap. U.S. Geo1. Surv., No. 282-A, $1-36$.

I.EOPOLD, I.B. and WOLMAN, M.G. 1960. River meanders. Bul1. Geol. Soc. Am., 71, 769-794.

LEOPOLD, L.B., WOLMAN, M.G. and MILIER, J.P. 1964. Fluvial processes in geomorphology. W.H. Freeman and Co., San Francisco. 522p.

MTALAS, N.C. 1967. Mathematical assessment of synthetic hydrology. Water Resources Res., 3, 937-946.

MATTHES, G.H. 1941. Basic aspects of stream meanders. Trans. Am. Geophys. Union $(1941), 632-636$.

MERGER, A.G. 1971. Analysis of alluvial bed forms. In Shen, H.W. (1id), River Mechanics, 1., ch. 10, 126. Fort Collins, Colorado, water Resources Pubs. 
MOODY-S'TUART, M. 1966. High and Jow sinuosity stream deposits, with examples from the Devonian of Spitsbergen. J. Sedill. Petro1., 36, 1102-1117.

McDOTELL, J.P. 1960. Cross beddinf formed by sand waves in Mississippi River point bar deposits. Bu11. Geol. Soo. Am., 71, 1925.

MCGOWEN, J.H. and GARNER, L.T. 1970. physiographic features and stratification types of coarse grained point bars: moderr and ancient examples. Sedinentology, $14,77-111$.

McKEE, E.D., CROSBY, E.J. and BERRYHILL, H.L. 1967. Flood deposits, Bijou Creek, Colorado, June 1965. J. Sedim. Petro1., 37, 829-851.

NAGABHUSHANAIAH, H.S. 1967. Meandering of rivers. Bu11. Tnt. Ass. Scient. Ilydrol., 12, $28-43$.

NEDEC 0 .

1959.

River studies and recommendations on improvement of $\mathrm{Niger}$ and Benue. North-Holland, Amsterdam, $1000 \mathrm{pp}$.

NORDIN, C.F. 1964. Aspects of flow resistance and sediment transport Rio Grande near Bernalilio, New Mexico. U.S. Geol. Surv, Water Supply Pap., $1498-\mathrm{H} \cdot 41 \mathrm{pp}$.

OOMKENS, E. and TERWINDT, J.H.J. 1960. Inshore estuarine sediments in the Haringvleit (The Netherlands). Geol. Mijnbouw, 39, 701-710.

PARTHENIADES, E. 1971. Erosion and deposition of cohesive materials. In Shen, Jf.W. (Ed), River Mechanics, 2 , ch. 25, 1-91. Fort Collins, Colorado, water Resources Pubs.

PARTHENIADES, E. and PAASWELI, R.E. 1970. Erodibility of channels with cohesive boundary. J. Hydraul. Div. Am. Soc. Civ. Fners., 26, 755-771.

POKISRARYAN, M.S. 1957. Non eroding current velocities. IzV. Akad. Nauk. Armyan S.S.R., Ser. Tekh. Nauk., 10,

1958. Transverse profiles of natural river channels. Izv. Akad, Nauk. Armyan. S.S.K., Sex. Tekh. Nauk., 11, 31-38.

POTTER, P.E. 1967. Sand bodies and sedimentary environm ments: a review. Bull. Am. Ass. petrol. Geol. $51,337-365$.

POTTER, P.E. and BLAKELY, R.F. 1967. Generation of a synthetic vertical profile of a fluvial sand body. J. Petroleum Technology, 1, 2/3-251.

QUIMPO, R.G. 1967. Stochastic model of daily river flow sequences. Colorado state University, Hydrology Paper No. 18, Fort Collins, Colorado. 
QUIMPO, R.G. 1968 a.

Stochastic analysis of daily river

flows. J. Hydraul. Div. Am. Soc. Civ. Engrs. $24,43-5 \overline{7}$.

$1968 \mathrm{~b}$.

Autocorrelation and spectral analysis in hydrology. J. Hydraul. Div. Am. Soc. Civ. Engrs. 94, 363-373.

RAUDKIVI, A.J. 1967 .

Loose boundary hydraulics. Pergamon press. 331pp.

REINECK, H.E. 1958. Longitudinale Schragschicht im watt. Geol. Rdsch.. 47. 73-82.

REYNOLDS, A.J. 1965. Waves on the erodible bed of an open channel. J. Fluid Mech., 22, 113-133.

RIPLEY, H.C. 1927. Relation of depth to curvature in channe1s. Trans. Am. Soc. Civ. Fingrs., 20 , $207-238$.

RODRIGUEZ-ITURBE, I. 1968. A modern statistical study of monthly levels of the Orinoco River. Bull. Int. Ass. Scient. Ilydro1.. 13, 25-41.

ROESNER, L.A. and YEVDJEVICH, V. 1966. Mathematical models for time series of monthly precipltation and monthly runoff. Colorado State University, Hydrology Paper No. 15. Fort Collins, Colorado.

ROZOVSKII, I.L. 1961. Flow of water in bends of open channels. Israel program for scientific translations, Jerusalem.

RUSSELL, R.J. 1954. Alluvial morphology of Anatolian rivers. Ann. Ass. Am. Geographers, 44, 363391.

1.967.

River and delta morphology.

Louisiana State Univ. Press, Coastal studies Series, No. 20 .

SCHIIDEGGER, A.E. 1967. A thermodynamic analogy for meander systems. Water Resources Res., 3, 1041-1046.

1970. Theoretical geomorphology, 2nd edition. George Allen \& Unwin Ltd., London. SpringerVerlag, Berlin, 435pp.

SCHUMM, S.A. 1960. The shape of alluvial channels in relation to sediment type. Prof. Pap.U.S. Geo1. Surv., No. 352-B, 17-30.

1963. Sinuosity of alluvial rivers on the Great Plains. Bull. Geol. Soc.Am., 74, 1089-1100.

1967. Meander wavelength of alluvial rivers. Science, 157, 1549-1550. 
SCHUMM, S.A. I968.

River adjustment to altered hydrologic regimen - Murrumbidgee River and palaeochannels, Australia. prof. Pap. U.S. Geol. Surv., No.598. $65 \mathrm{pp}$.

1969.

River metamorphosis. J. Hydraul. Div. Am.-Soc. Civ. Engrs., 25, 255-273.

1971.

Fluvial geomorphology. In Shen, If.W. (Ed), River Mechanics, 1, ch. 4, 1-30. Fort Collins, Colorado, Watex Resources Pubs.

1972.

Fluvial palaeochannels. In Recognition of ancient sedimentary environments, S.E.P.M. Spec. Pub1. No. 16, 98-107.

SCHUMM, S.A. and KHAN, H.R. 1972. Experimental study of channel patterns. Bu11. Geo1. Soc. An., 83, 1755-1770.

SCHUMM, S.A., KHAN, H.R., WINKLEY, B.R. and ROBBINS, L.G. 1972. Variability of river patterns. Nature, 237, $75-76$.

SCHUMM, S.A. and LICHTY, R.W. 1963. Channel widening and floodplain construction along Cimarron River in southwestern Kansas. Prof. Pap. U.S. Geol. Surv. No. 352-D, 71-88.

SELLIN, R.H.J. 1964 .

A laboratory investigation into the interaction between the flow in the channel of a river and that over the floodplain. La Houille Blanche, No. 7, 793-801.

SHAHJAHAN, M. 1970.

Factors controlling the geometry of fluvial meanders. Bull. Int. Ass. Scient. Hydro1. , 15, 13-24.

SHUKRY, A. 1950.

Flow around bends in an open channel flume. Trans. Am. Soc. Civ. Engrs., 115. $751-779$.

SIMONS, D.B. 1971.

River and canal morphology. In Shen, H.W. (Ed), River Mechanics, 2 , ch. 20 , 1-60. Fort collins, Colorado, water Resources pubs.

SIMONS, D.B. and RICHURDSON, E.V. 1966. Resistance to flow in alluvial channels, Prof. Pap. U.S. Geol. Surv., No. 422-J, 1-61.

1971. Flow in alluvial sand channels. In Shen, H.W. (Ed), River Mechanics, 1, ch. 9, 1-89. Fort Collins, Colorado, Water Resources pubs.

SIMONS, D.B., RICIIARIISON, E.V. and ALBERTSON, M.L. 1961. Flume studies using medium sand $(0.45 \mathrm{~mm})$. U.S. Geo1. Surv. Water Supply Papers, 1498-A. 76pp. 
SIMONS, D.B., RICIIARDSON, E.V. and NORDIN, C.F. 1965. Sedimentary structures generated by flow in alluvial channe1s. Spec. Publs. Soc. Econ. Palaeont. Miner. Tulsa, 12, 34-52.

SOUTIARD, J.B. 1971. Representation of bed configurations in depth-velocity-size diagrams. J. Sedim. Petrol., 41 , 903-915.

SPEIGIYT, J.G. $1965 \mathrm{a}$. Meander spectra of the Angabunga River. J. Ilydrol., 3, 1-15.

1965b. Flow and channel characteristics of the Angabunga River, Papua. J.Hydrol., 3, 16-36.

1967. Spectral analysis of meanders of some Australasian rivers. In J.N. Jenniness and J.A. Mabbutt (Eds), Landform studies from Australia and New Guinea. Cambridge University Press, 48.63.

STALL, J.B. and FOK, YU-SI. 1968. Hydraulic geometry of Illinois Streams. Illinois Univ. Water Resources Centre Res. Rept. $15,47 \mathrm{pp}$.

SUNDBORG, A. 1956. The River Klaralven: a study of fluvial processes. Geogr. Annlx., 38, 127-316.

SURKAN, A.J. and VAN KAN, J. 1969. Constrained random walk meander generation. Water Resources Res., 5 , $1343-1352$.

TASK COMMITTEE ON EROSION OF COHESIVE MATERIALS. 1968. Erosion of cohesive sediments. J. Jydraul. Div. Am. Soc. Civ. Engrs., 24, $1017-1049$.

THAKUR, T.R. and SCHETDEGGER, A.E. 1968. A test of statistical theory of meander formation. Water Resources Res., 4, 317-329.

1970. Chain model of river meanders. J. Ilydrol., 12, $25-47$.

TOEBES, G.H, and CHANG, T.P. 1967. P1anform analysis of meandexing river. proc. 12th Congr. Int. Ass. Hydraul. Res., Fort Collins, Colorado.

TOEBES, G.H. and SOOKY, A.A. 1967. Hydraulics of meandering streams with flood plains. J. Waterways Harbours Div. Am. Soo. Civ. EMgrs. (1967), WW2, $213-236$.

TURNBULL, W.J., KRINITZSKY, E.L., and WIAVER, F.J. 1966. Bank erosion in soils of the Lower Mississippi Va11ey. J. Soil Mech. Found. Div. Am. Soc. Civ. Engrs: $22,121-136$.

VAN STRAATEN, L.M.J.U. 1.954. Composition and structure of recent marine sediments in the Netherlands. Leld. Geol. Meded., 19, $1-110$. 
VISHER, G.S. $1965 \mathrm{a}$.

Use of vertical profile in environmental reconstruction. Bull. Am. Ass. petrol. Geo1., 49, 41-61.

$1965 \mathrm{~b}$.

Fluvial processes as interpreted

from ancient and recent fluvial deposits. In primary sedimentary structures and their hydrodynamic interpretation (Ed. G.V. Middleton) S.E.P.M. Spec. Publn. 12, 116-132.

VON SCHELLING, H. 1951.

Most frequent particle paths in a plane. Trans. Am. Geophys. Union, 32, $222-226$. 1964 .

Most frequent random walks. Gen. Elec Co. Rept. 64GL92, Schenectady, N.Y.

WILLIAMS, G.P. 1967 .

Flume experiments on the transport of a coarse sand. Prof. Pap. U.S. Geol. Surv., No. 562-B. 31pp.

1970. Flume width and water depth effects in sediment transport experiments. Prof. Pap. U.S. Geo1. Surv., No. 562-H. 37pp.

WOLMAN, M.G. 1959.

Factors influencing erosion of a cohesive riverbank. Am.J.Sci., 257, 204-216.

WOLMAN, M.G. and EILER, J.P. 1958. Reconnaissance study of erosion and deposition produced by the flood of August 1955 in Connecticut. Trans. Am. Geophys. Union, 39, 1-14.

WOLMAN, M.G. and LEOPOLD, L.B. 1957. River flood plains: some observations on their formation. Prof. Pap. U. S. Geol. Surv., No. 282-C, 87-107.

WOODYER, K.D. 1968.

Bankful1 frequency in rivers. . Hydro. , $6,114-142$.

YANG, C.T. $1971 \mathrm{a}$.

Potential energy and stream morphology Water Resources Res., I, 311-322.

$1971 \mathrm{~b}$. $231-253$

on river meanders. J. Ilydrol., 13,

19710

Formation of riffles and pools. Water Resources Res., 7, 1567-1574.

YEN, B.C.

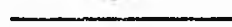
1971 . 1965.

Characteristics of subcritical flow in a meandering channel. Institute of rydraulic Research, University of Iowa, Iowa city, Iowa.

Spiral motion of developed flow in wide curved open channels. In Shen, H.W. (Ed), Sedimentation (Einstien), ch. 22, 1-33. Fort Collins, Colorado, Water Resources pubs.

YEN, C-L. 1970 meander. J. Irydraul. Div. Am. Soc. Civ. Engrs., 96, $57-73$. 


\section{A1.1 Newton-Raphson iterative formula}

This method is used to find approximate values of the real roots of equations. It can be applied to polynomials of any degree and also to nonpolynomial equations. The iterative fornula is as follows

$$
x_{r+1}=x_{r}-\frac{f\left(x_{r}\right)}{f^{\prime}\left(x_{r}\right)}
$$

Here $x_{r}$ is the approximate root of function $f(x)=0 . \quad f\left(x_{r}\right)$ is the value of the function $f(x)$ for $x=x_{r}$ and $f^{\prime}\left(x_{r}\right)$ is the first derivative of $f(x)$ for $x=x_{r}$. Then $x_{r+1}$ is a closer approximation to the real root. The formula is the basis for an iterative process that lends itself to use on a computer. The iterative process is continued until the difference between successive estimates is less than a specified amount. Although the process has the advantage of converging rapidy, an initial estimate is required and sometimes, under exceptional circumstances, convergence may not occur. Difficulties also occur if the equation has two or more nearly equal roots.

The development, geometrical Interpretation and reasons for failure of the method can be obtained from any standard text on numerical analysis.

\section{A1.2 Simpson's rule}

This is a numerical method for evaluating definite integrals when they cannot be evaluated exactly. The formula is as follows

$\int_{a}^{b} f(x) d x \approx \frac{h}{3}\left(y_{0}+4 y_{1}+2 y_{2}+4 y_{3}+--+2 y_{n-2}+4 y_{n-1}+y_{n}\right)$ 
The formula is obtained by dividing the curve $y=f(x)$ into $n$ equal parts between $x=a$ and $x=b$, of length $h=(b-a) / n$, where $n$ is always an even integer. Each separate piece of curve, covering an $x-$ subinterval of width $2 \mathrm{~h}$ is then approximated by an arc of a parabola through its ends and its mid point. These points correspond to values of $y=f(x)$ of $y_{0}, y_{1}, y_{2}$, then $y_{1}, y_{2}, y_{3}$, and so on up to $y_{n-2}, y_{n-1}, y_{n}$. The areas under each parabolic arc are then added to give the expression above.

on geometrical grounds the smaller the value of $h$ taken, the greater will be the accuracy of the approximation. Thus Simpson's rule may be applied successively, halving the interval on each application, until the difference between successive estimates is less than an arbitrary specified amount.

\section{A1.3 Generation of random samples from specified theoretical} distributions

This is usually done by generating uniformly distributed pseudorandom numbers and using these to draw random samples from the specified frequency distribution. This is known as Monte Carlo simulation (Harbaugh and Bonham-Carter, 1970).

The inverse transformation method can sometimes be used to transform the uniform distribution into a specified non-uniform distribution. A random number is simply equated with the cumulative frequency distribution, expressed either discretely or continuously, and a corresponding value from the specified distribution is obtained (see Harbaugh and Bonham-Carter, 1970). The initial step is to define the cumulative frequency distribution, obtained from the specified distribution either by summing over each discrete class (for empirical distributions), or by integration of a continuous distribution, if necessary dividing by the total to scale the range from 0.0 to 1.0 .

The normal probability density function cannot be directly 
integrated to give the cumulative distribution, unless by numerical methods, and so the inverse transformation method cannot be easily used. A much easier way of generating normally distributed random variables is to use the formula derivable from the contral limit theorem,

$$
y=\frac{\sum_{i=1}^{k} r_{i}-(k / 2)}{\sqrt{k / 12}}
$$

where $y$ is a random variable with standard normal distribution with mean=0, standard deviation $=1 ; r_{i}$ is the $i$ th element of a sequence of random numbers from a uniform distribution in the range 0.0 to $1.0 ; k$ is the number of values of $r_{i}$ to be used. As $k$ tends to $\infty$, y approaches a true normal distribution, but for most applications $k=12$ is adequate. Thus to generate a normally distributed random variable, $x$, with mean $\mu$ and standard deviation $\sigma$, sum 12 random numbers in the range 0.0 to 1.0 , subtract 6 , and apply the following formula

$$
\mathbf{x}=\mathbf{y} \sigma+\mu
$$

In the mathematical model samples are required to be generated in standardised form, as in equation (A1.3).

For a lognormally distributed random variable we perform the same process but replace the last equation by

$$
x=\exp (y \sigma+\mu)
$$

where $\mu$ and $\sigma$ are the mean and standard deviation of logx. Lognormally distributed random variables generated directly from standardised normal distributions, with mean of logarithms of $x$ zero and standard deviation of logarithms of $x$ equal to unity, have an actual mean of 1.65 and standard deviation of 2.15 . In order to transform such a variable $x$ into a standardised form $x_{s}$ the following transformation is necessary 


$$
x_{s}=\frac{x-1.65}{2.15}
$$

A normal standardised deviate, $y$, can be transformed to be distributed approximately as gamma using the following equation

$$
x=\frac{2}{\gamma}\left\{1+\frac{y \gamma}{6}-\frac{\gamma^{2}}{36}\right\}^{3}-\frac{2}{\gamma} \quad \text { (AI.7) }
$$

where $x$ is approximately gamma distributed with zero mean, standard deviation unity and skewness equal to $\gamma$ (Matalas, 1967). 


\section{A2.1 Polynomial regression}

The following tables show the relevant results from

polynomial regression analyses. Table A2.1 is for the regression of the ratio $\mathrm{A} / 1$ on $\mathrm{sn}$, as discussed in section 2.2 . Table. A2.2 refers to the regression of the parameter $\operatorname{gD}^{3} / \nu^{2}$ on $\mathrm{v}_{*} \mathrm{~J} / \nu$ as discussed in section 5.5.4. Tables A2.3 and A2.4 are for the regression of Kennedy's $j$ factor on Fr discussed in section 5.5.5. A2.2 polynomial surface fitting

The accompanying diagram, fig. A2.1, and table A2.5 show the results of fitting polynomial surfaces of degree 1,2 and 3 , by least squares, to the solution to the integral given in equation (4.2). The independent variables were sn and $\varnothing$, and 594 points were used. 
POLYMOHIAL REGRESSION......S 005

MUMBER OF DBSERVATIONS 36

POLYNOMIAL REgReSSION OF DEgREe

INTERCEPT $\quad 0.4529037 E 00$

REGRESSION COEFFICIENTS

O. $2186882 E$ OL.

ANALYSIS OF VARIANCE FOR $L$ DEGREE POLYNOMIAL

SOURCE DF VARIATION

DUE TO REGRESS ION

DEVIATION ABDUT REGRESSION TOTAL
SUM OF
SOUARES

38.33064

0.51912
36.84976

MEAN
SQUARE

36.33064 0.01527

$$
\text { VALUE }
$$

2510.48602
IMPROVEMENT IN TERHS

DF SUH OF SQUARES

38.33064

POLYNOMJAL REGRESSION OF DEGREE 2

INTERCEPT $0.8033228 E$ OO

REGRESSION COEFFICIENTS

0.1260494 E OL

$0.4695846 E$ DO

ANALYSIS OF VAHIANCE FOR 2 DEgREE POLYNOMIAL

SOURCE OF VALIATION

DUE TO REGRESSION

DEVIAT ION ABUUT REGHESSION TOTAL

DECREE UF
FREEDUM

SUM DF SOUARES

38.14538

0.10434 38.84976

$\begin{array}{cc}\text { MEAN } & \text { F } \\ \text { SQUARE } & \text { YALUE } \\ 19.37268 & 0124.40625 \\ 0.00316 & \end{array}$

IMPROVEHENT IN TERHS OF SUM OF SQUARES

0.41473

POLYNOHIAL REgReSSION TF LIEGRE 3

INTERCEPT $0.9034151 E 00$

REGRES SION COEFF IC IENTS
$0.3370860 E^{00}$
0.1674062502
$-0.6301562 E 00$

ANALYSIS OF VARIANCE FOR 3 DEGREE PULYNUMIAL

\begin{tabular}{|c|c|c|c|c|}
\hline SOURCE DF VARIATIUN & $\begin{array}{l}\text { DEGREF OF } \\
\text { FREEDUM }\end{array}$ & $\begin{array}{l}\text { SUM OF } \\
\text { SOUARES }\end{array}$ & $\begin{array}{l}\text { MEAN } \\
\text { SOUARE }\end{array}$ & Value \\
\hline $\begin{array}{l}\text { OUE TO REGAESSION } \\
\text { DEVI AT ION ABOUT REGRESSION } \\
\text { TOTAL }\end{array}$ & $\begin{array}{r}3 \\
32 \\
35\end{array}$ & $\begin{array}{r}38 . A 2158 \\
0.02210 \\
38.04976\end{array}$ & $\begin{array}{r}12.94252 \\
0.00069\end{array}$ & 18667.42576 \\
\hline
\end{tabular}

IMPRDYEMENT IN TERMS OF SUM DF SQUARES

0.08220

POLYNontal REgResSION UF DEGBEE 4

HB IMPROVERENT

Tab1e A2.1.

Results of polynomial regression. 


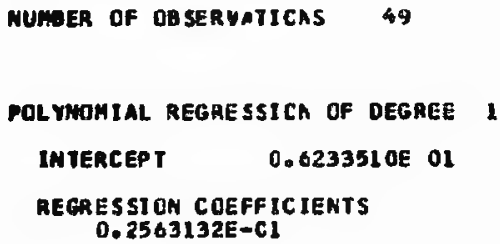

$2 \quad 5379.03906 \quad 2689.51953 \quad 580.94165$

309.64844 TOTAL 5592.00600
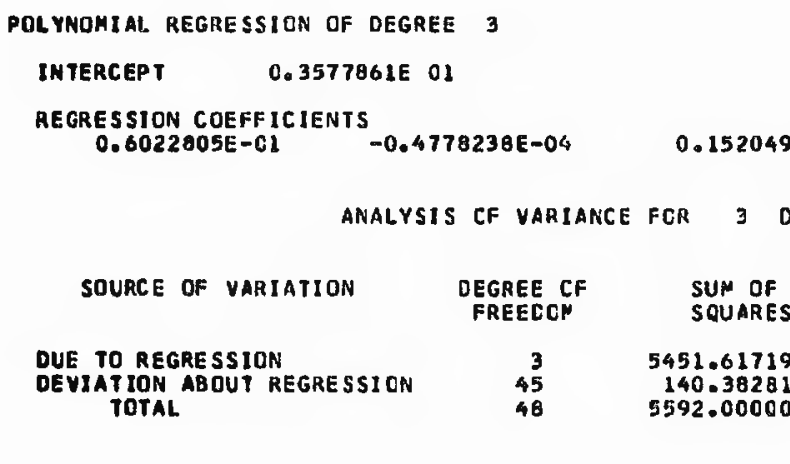

POLYNOHIAL REGRESSICA CF DEGREE 4

INTERCEPT O.3129055E 01

REGRESSION COEFFICIENTS
0. $7277495 E-02$
$-0.9190920 E-04$
$0.6242391 E-07$
$-0.1501976 E-16$

ANALYSIS OF VARIANCE FOR \& CEgREE POLYMOMIAL

\begin{abstract}
SOURCE OF VARIATIOA
OUE TO REGRESSICR
DEVIATION ABOUT REGRESSION TOTAL.
\end{abstract} DEGREE OF
FREEDCY REDCN

\section{SUM OF} SQUARES MEAN SQUARE

1365.51660 2.95304

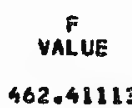

462.41113
IMPROVENENT IH TERPS OF SUP GF SQUARES

10.44922

\subsection{2}

polymomial Regressica cf degree 5

HO IMPROYEMENT 


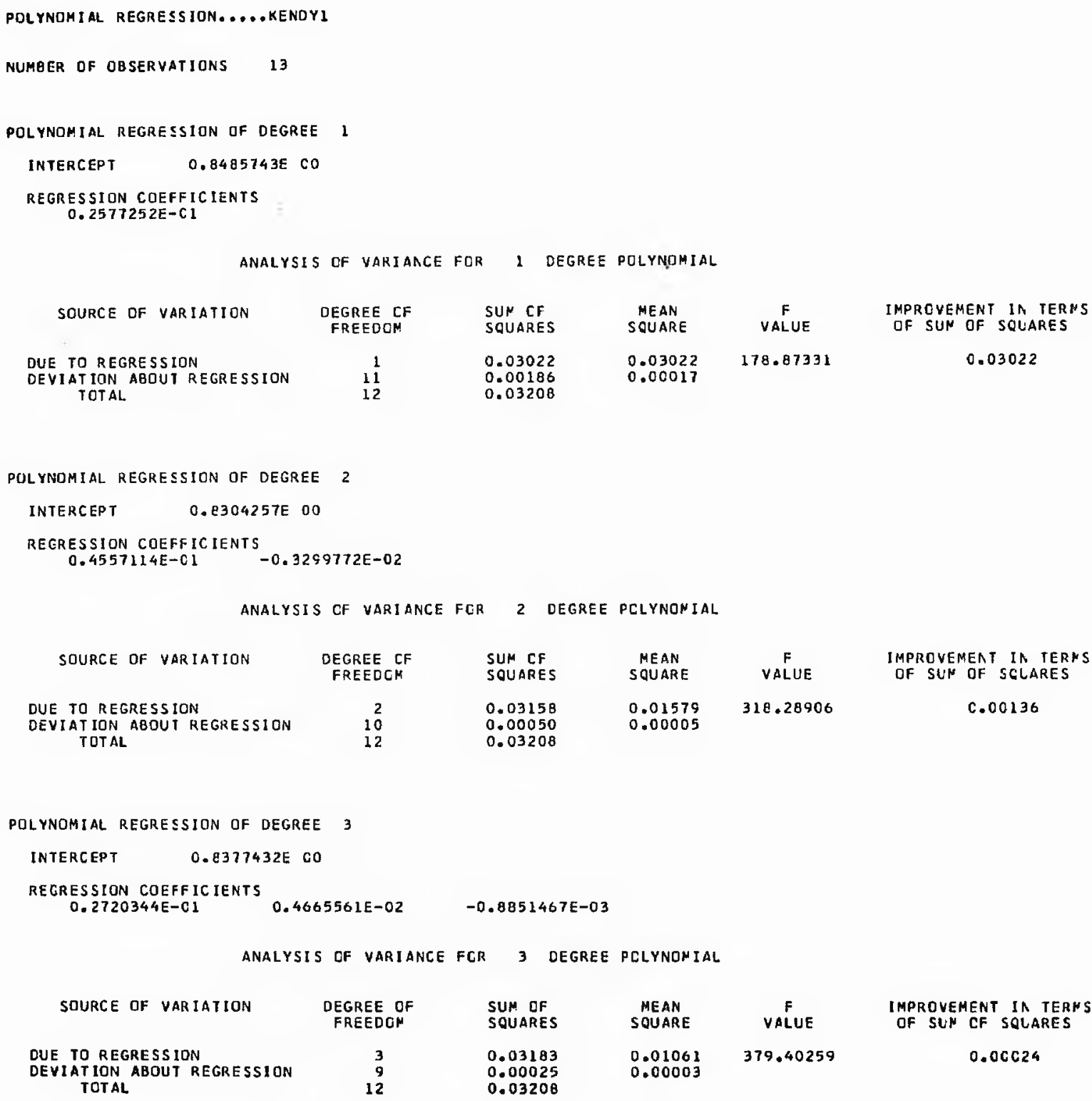

POLYNOMIAL REgRESSION OF DEGREE 4

NO IMPROVEMENT

Table A2.3. Results of polynomial regression. 
POLYNOMIAL REGRESSION......KERDY 2

NUMBER OF UBSERVATIONS

POLYNOMI AL REGRESSION OF DEGREE I

INTERCEPT C.332577OE CO

REGRESSION COEFFICIENTS

0.1197 CTSE OO

ANALYSIS CF VARIANCE FIR I CEgREE PCLYNDMIAL

SOURCE OF VARIATION

DLE TO REGRESSION

DEVIAT'ION AUOUT REGRESSION TOTAL
SUM of

SQUARES

0.65201

0.22743

0.87944
MEAN
SQUARE

0.65201 0.02068
VALUE

31.53506
IMPRQVEMENT IN TERNS OF SUM DF SQUARES

$0.652 C 1$

POLYNOMIAL REGRESSION OF DEGREE 2

INTERCEPT $\quad 0.1245201 E$ CO

REGRESSION COEFFICIENTS

$0.3466788 E$ CO $-C .3782854 E-01$

ANALYSIS CF VARIANCE FCR 2 CEGREE PCLYNCMIAL

SOURCE OF VARIATION

DUE TO REGRESSION

DEVIATION ABOUT REGRESSICN TCTAL

DEGREE OF
FREEDON
2
10
12

SUN OF

SCUARES

0.83106

0.04838 0.87944

\section{MEAN
SQUARE}

0.41553

0.00484

FALUE

VALUE

$B E . \varepsilon 8647$

IMPROVEMENT IN TERNS

OF SUN OF SOUARES

c. 17905

pulynomial REgression of degree
INTERCEPT
C. 31443 COE -O1

REGRESSION COEFFICIENTS
$0.5809163 \mathrm{E} \mathrm{CC}$
$-0.1394290 \mathrm{E} 00$
$0.1128839 E-01$

ANALYSIS OF VARIAACE FCR

3 cegree pclynomial

$\begin{array}{ccccc}\text { SOURCE OF VARIATION } & \begin{array}{c}\text { OEGREE CF } \\ \text { FREEDON }\end{array} & \begin{array}{l}\text { SUN CF } \\ \text { SCUARES }\end{array} & \begin{array}{c}\text { MEAN } \\ \text { SOUARE }\end{array} & \begin{array}{c}\text { F } \\ \text { VALUE }\end{array} \\ \text { DUE TO REGRESSION } & 3 & 0.87193 & 0.29064 & 347.96606 \\ \text { DEVIATION ABOUT REGRESSICN } & 9 & 0.00752 & 0.00084 & \\ \text { TOTAL } & 12 & 0.87944 & \end{array}$

IMPROVEMENT IN TERNS OF SUN OF SQLARES

c. 04086

POLYNOMIAL REGRESSION OF OEGREE

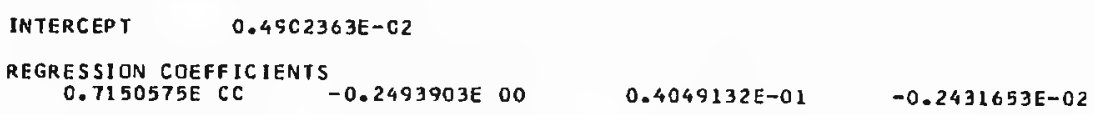

\begin{tabular}{|c|c|c|c|c|c|}
\hline SOURCE OF VARIATION & $\begin{array}{l}\text { DEGREE CF } \\
\text { FREEDON }\end{array}$ & $\begin{array}{l}\text { SUM CF } \\
\text { SQUARES }\end{array}$ & $\begin{array}{l}\text { MEAN } \\
\text { SOUARE }\end{array}$ & $\underset{\text { VALUE }}{\text { F }}$ & $\begin{array}{l}\text { IMPRCVEMENT IN TERHS } \\
\text { OF SUH OF SQUARES }\end{array}$ \\
\hline $\begin{array}{l}\text { DUE TO REGRESSION } \\
\text { CEVIAT ION ABOUT REGRESSION } \\
\text { TOTAL }\end{array}$ & $\begin{array}{r}4 \\
8 \\
12\end{array}$ & $\begin{array}{l}0.87817 \\
0.00127 \\
0.87944\end{array}$ & $\begin{array}{l}0.21954 \\
0.00016\end{array}$ & 1384.06030 & 0.06625 \\
\hline
\end{tabular}

POLYNOMIAL REGRESSION OF DEGREE 5

NO IMPROVEMENT

Table A2.4. Results of polynomial regression. 
TABLE A2.5 G1obal fit data.

\begin{tabular}{|c|c|c|c|}
\hline & plane & quadratic & cubic \\
\hline \multicolumn{4}{|c|}{ COEFFICIENTS } \\
\hline$\phi^{3}$ & - & - & 0.2804 \\
\hline$\phi^{2} \mathrm{sn}$ & - & - & -0.1713 \\
\hline$\phi \mathrm{sn}^{2}$ & - & - & 0.1139 \\
\hline $\sin ^{3}$ & - & - & -0.0292 \\
\hline$\phi^{2}$ & - & 0.4433 & 0.2244 \\
\hline$\not \phi_{\text {sn }}$ & - & -0.1348 & -0.5520 \\
\hline$s n^{2}$ & - & 0.0419 & 0.2123 \\
\hline$\varnothing$ & 0.6371 & 0.2269 & 0.8895 \\
\hline $\operatorname{sn}$ & -0.0505 & -0.1993 & -0.4651 \\
\hline Intercept & -0.0692 & 0.2281 & 0.2668 \\
\hline & $\begin{array}{l}\text { IT } \\
87.114\end{array}$ & 96.782 & 98.568 \\
\hline
\end{tabular}



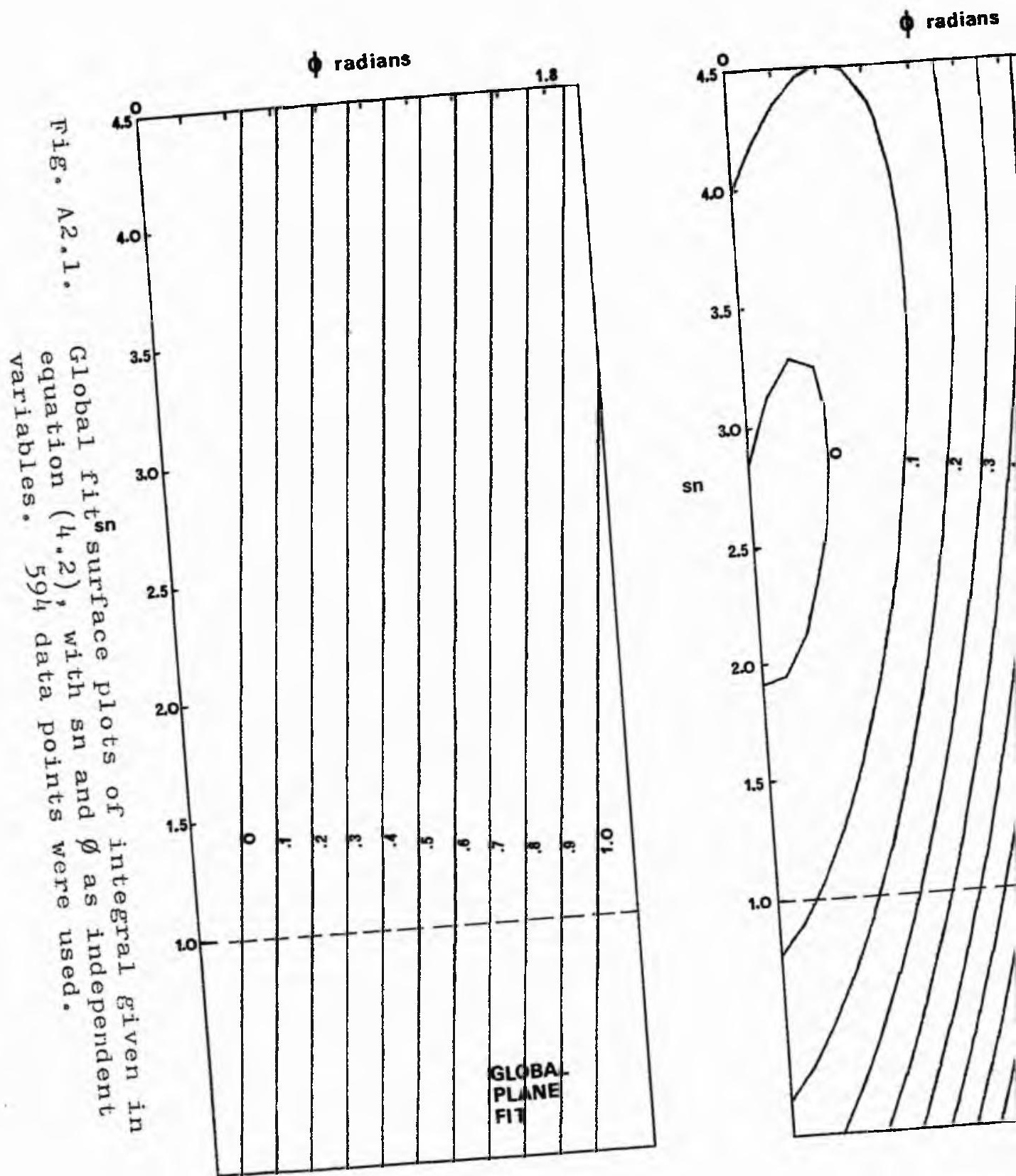


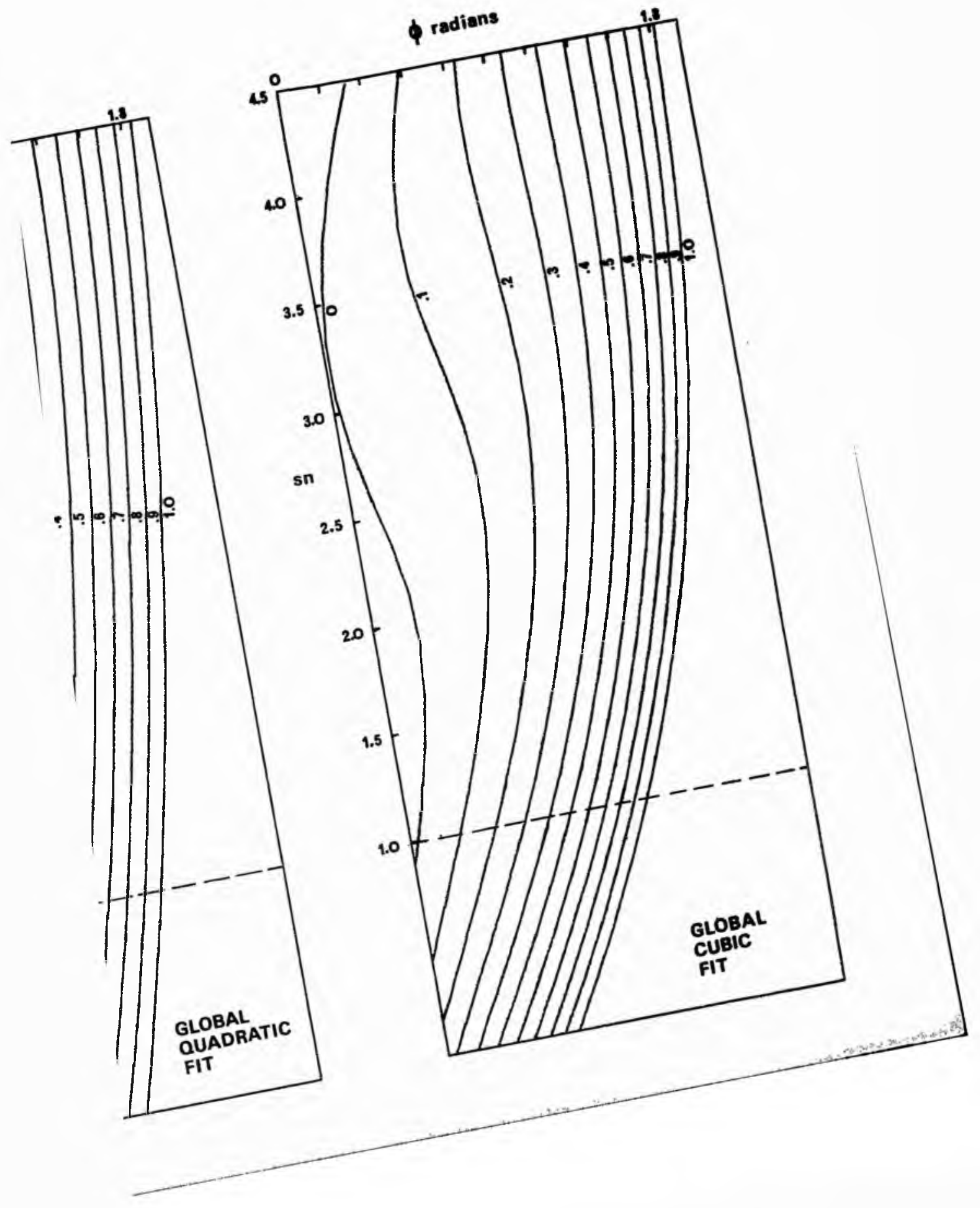


A6.

APPENDIX 3. DATA DECK SET UP FOR EXPERIMENTS. 


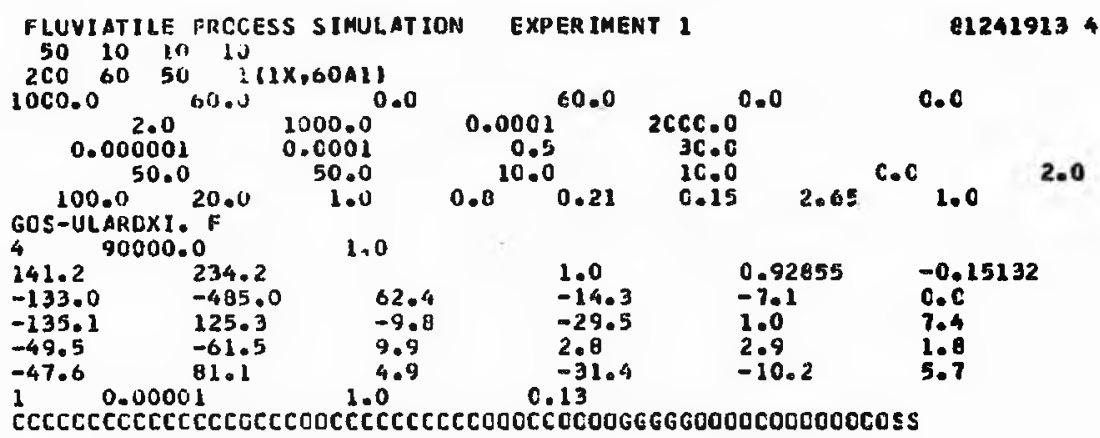

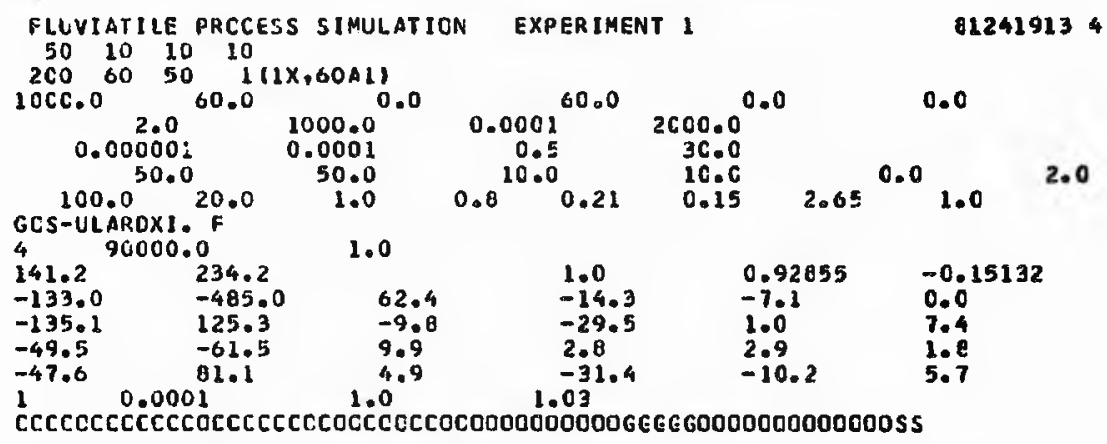

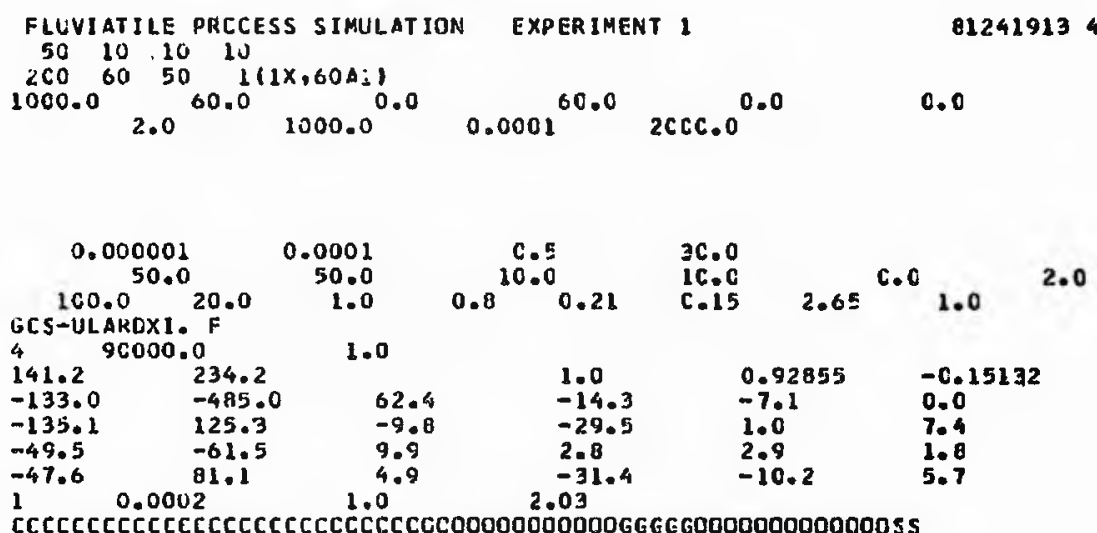
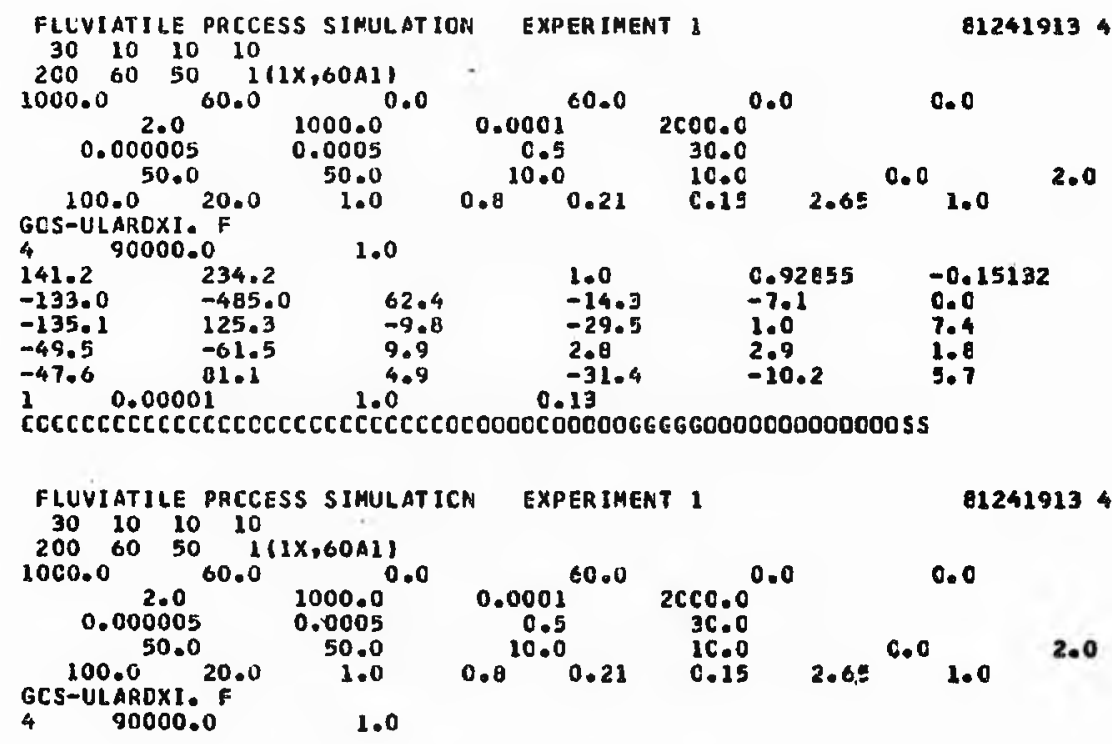


\begin{tabular}{|c|c|c|c|c|c|}
\hline $\begin{array}{l}141.2 \\
-133.0 \\
-135.1 \\
-49.5 \\
-47.6 \\
1\end{array}$ & $\begin{array}{l}214.0 \\
-40 \div .0 \\
1 .-3.3 \\
-61.2 \\
81.1\end{array}$ & $\begin{array}{l}62.4 \\
-9.8 \\
9.8 \\
4.9 \\
1.0\end{array}$ & $\begin{array}{c}1.0 \\
-14.3 \\
-29.5 \\
2.8 \\
-31.4 \\
1.03\end{array}$ & $\begin{array}{l}\text { C.92es5 } \\
-7.1 \\
1.0 \\
2.9 \\
-10.2\end{array}$ & $\begin{array}{l}-C .15132 \\
C .0 \\
7.4 \\
1.8 \\
5.7\end{array}$ \\
\hline
\end{tabular}

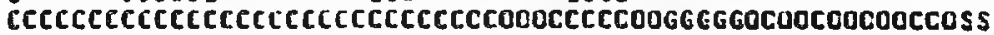

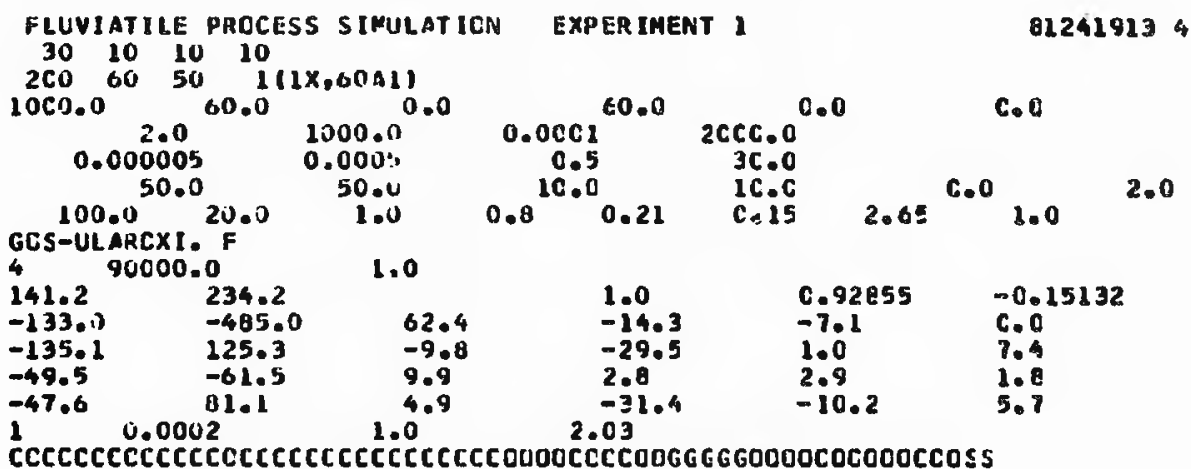

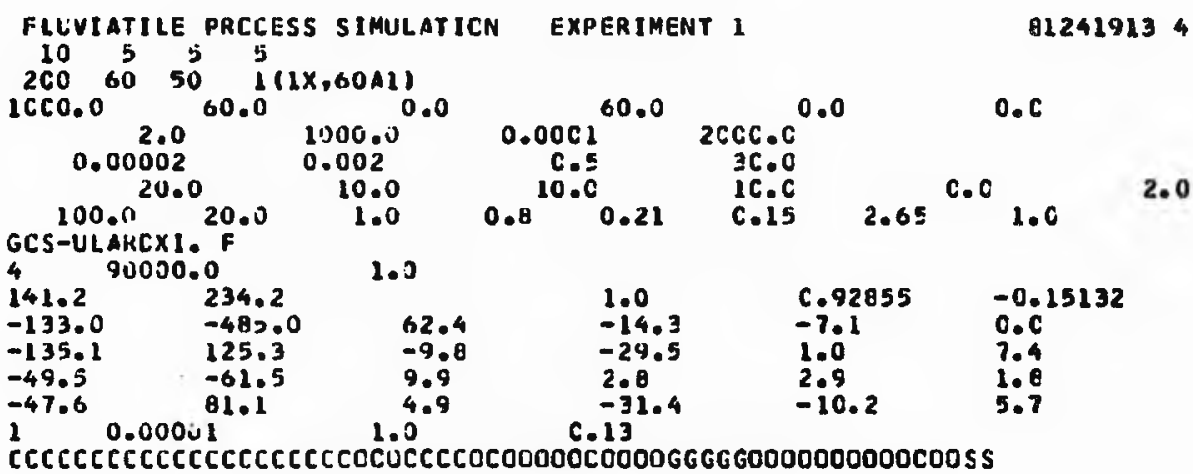
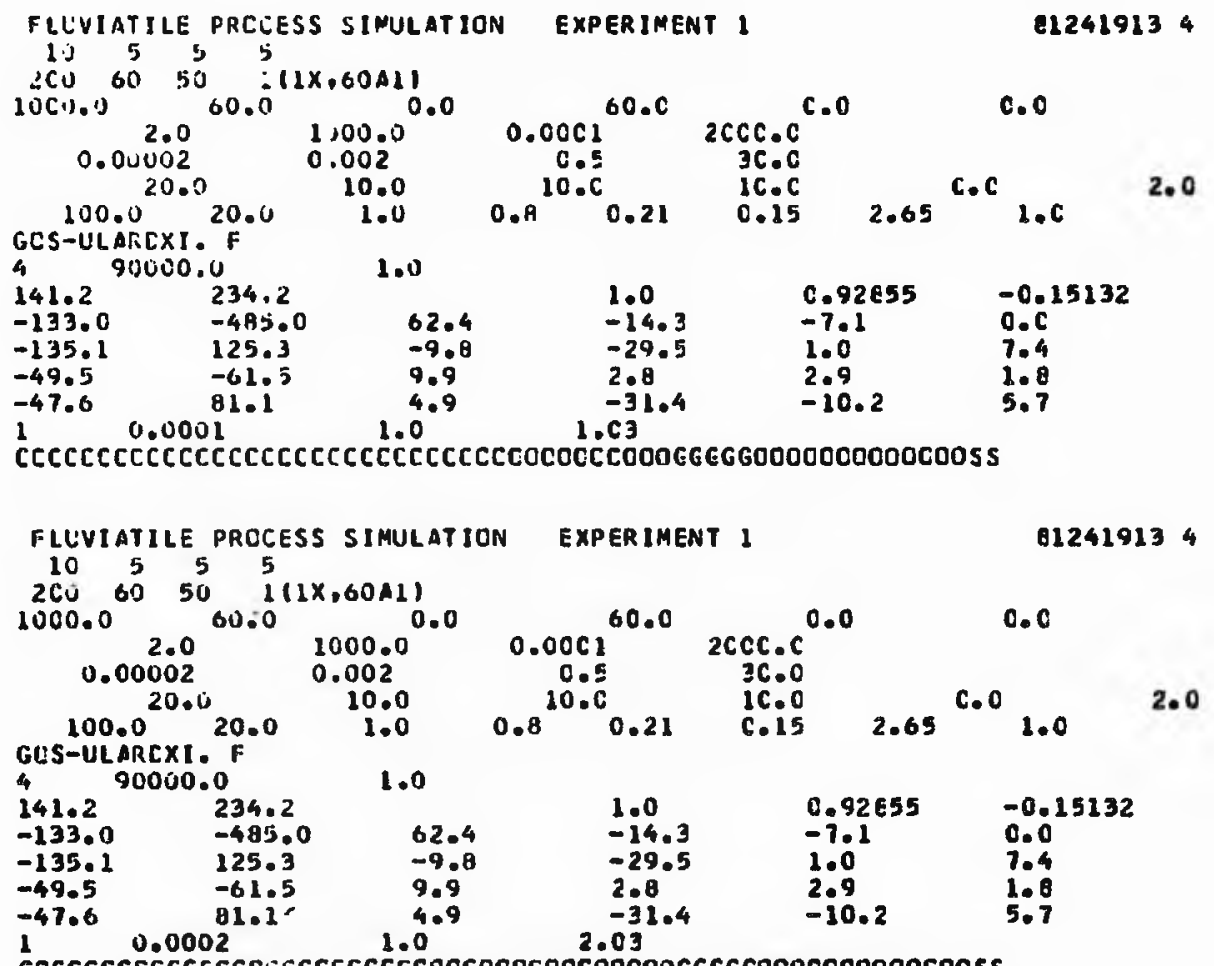
FLUVIATILE PRGCESS SIMULATICN EXPERIMENT 2

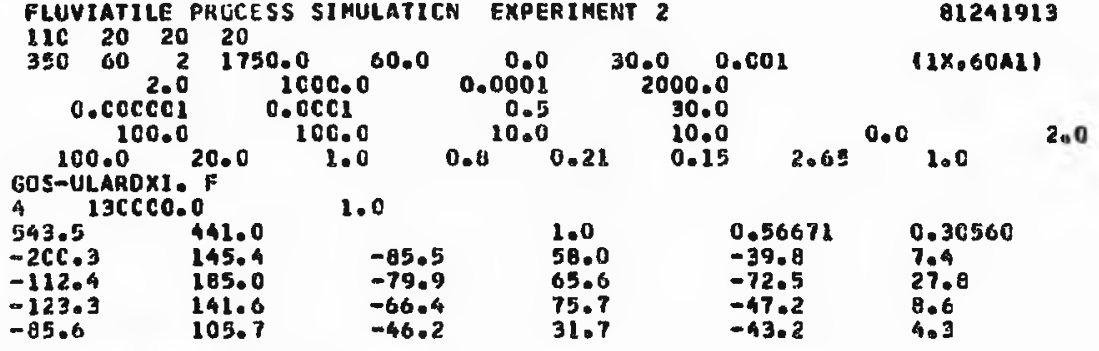

cor.coceooçecrocececcecceccess

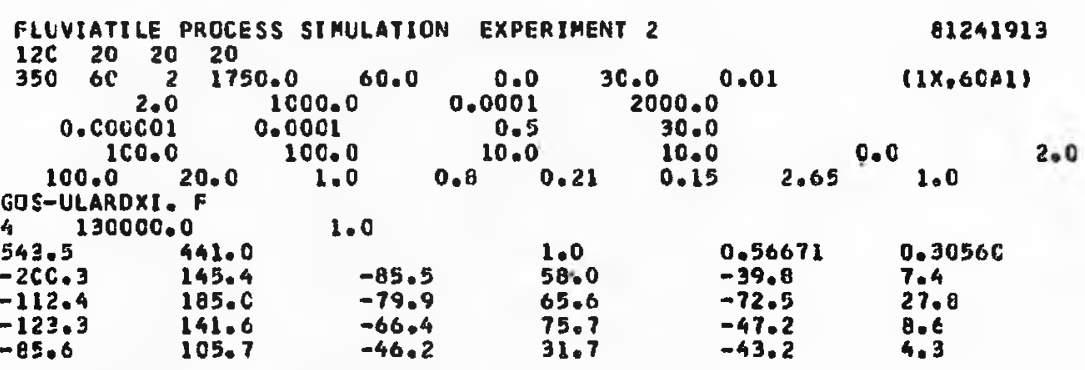

000600000600000000 cooceccecoss

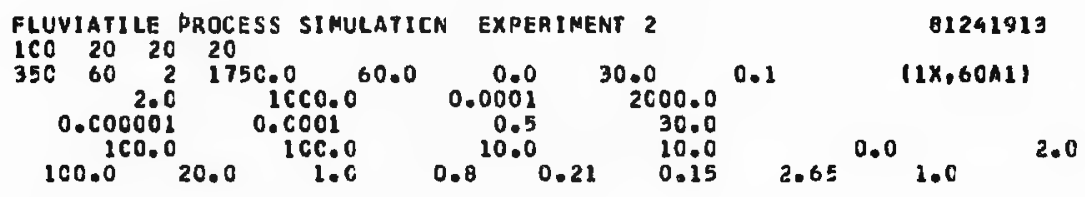

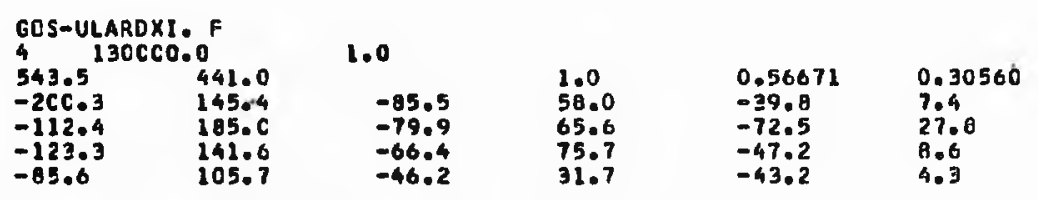

odecocecoococecececceccececess

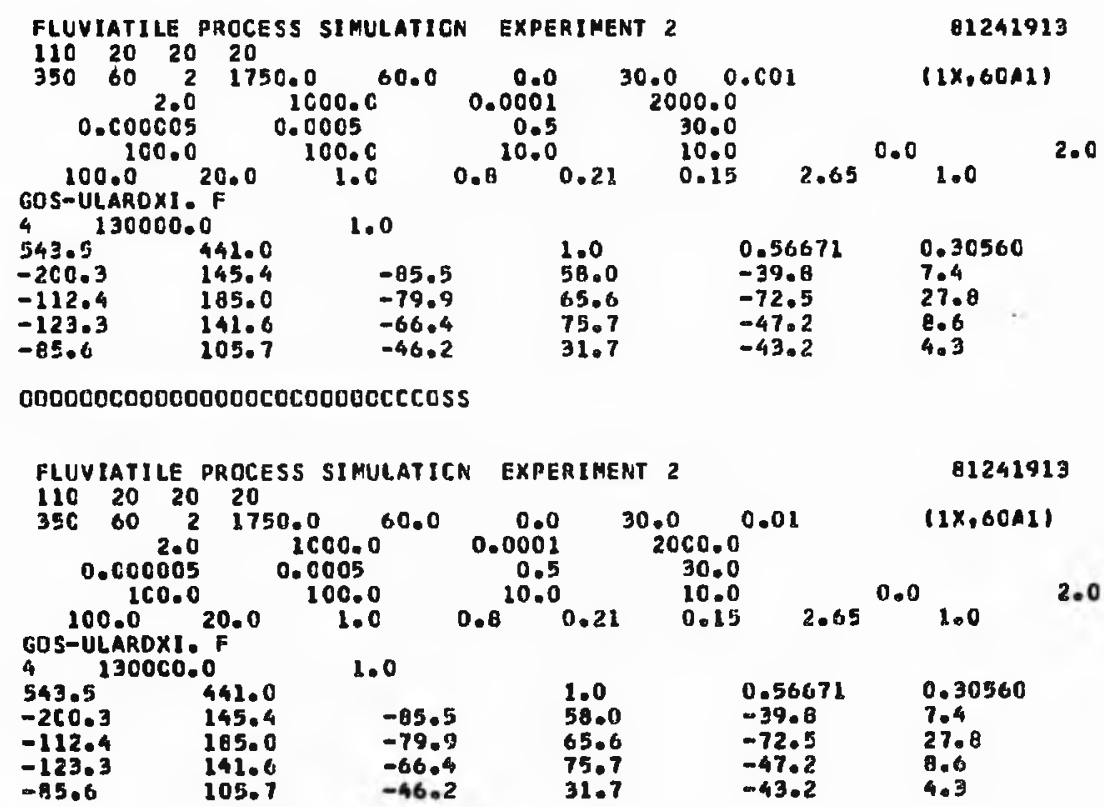


oocccocoonocecceceocreccecoss

Fluviatile process simulation experiment 2

01241923

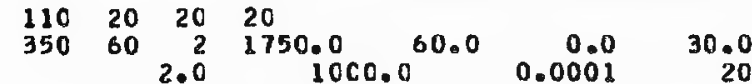

$\begin{array}{rr}0.000005 & 0.0005 \\ 100.0 & 100 .\end{array}$

100.0 $20.0 \quad 10 C . C$

$0.8^{0.00} 0.21$

2000.0
30.0
10.0
0.15

0.1

$(1 \times, 60 A 1)$

GOS-ULARDXI. F

a 130000.0

543.5 441.0

$-260.3 \quad 145.4$

1. 0

$-112.4 \quad 285.0$

185.0

105.7

$-85.5$

$-79.9$

$-66.4$

$-46.2$

1.0
58.0
65.6
75.7
31.7

0.56671
-39.8
-72.5
-47.2
-43.2

0.0

2.0

ooco0o0000ccocococococcceccoss

FLUVIATILE PROCESS SIMULATION EXPERIMENT 2

1) $20 \quad 20 \quad 20$

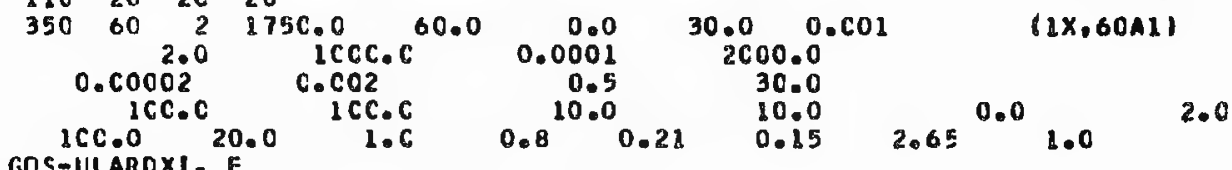

$13 \mathrm{CCCO} .0$

$543.5 \quad 441 . \mathrm{C}$

$-200.3 \quad 145.4$

$-112.4 \quad 185 . \mathrm{C}$

$-123.3 \quad 141.0$

$-85.6 \quad$ iC5.?

1.C

occececooocoeccecceccecccoss

FLLVIATILE PRCCESS SIAULATICA EXPERIMERT 2

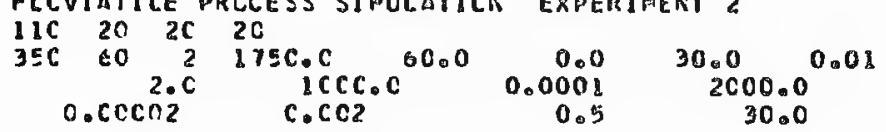

81241913

$(2 x, 6012)$

0.30560

7.4

27.0

4.3

61241913

$0.3056 \mathrm{C}$

27.8

4.3 

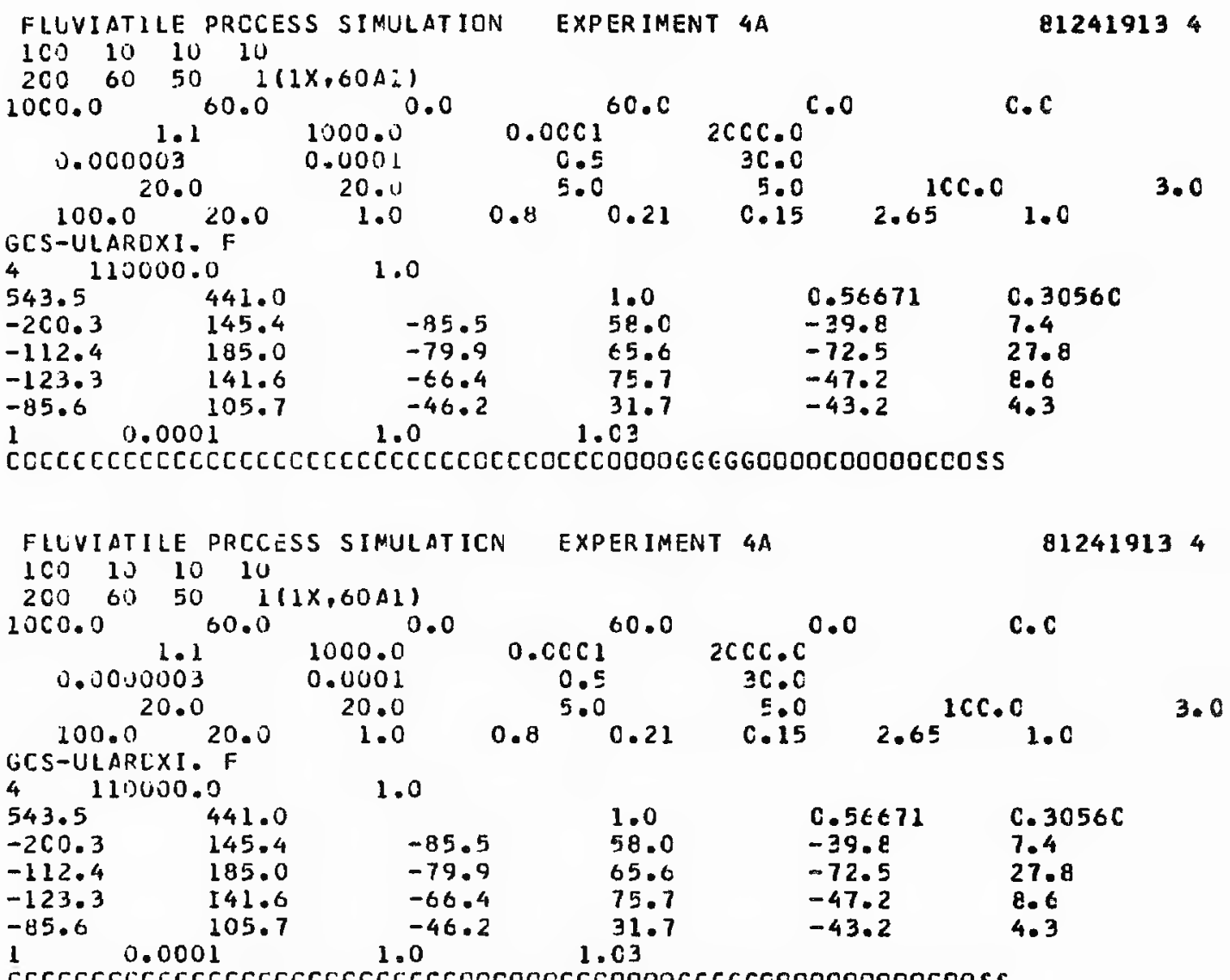

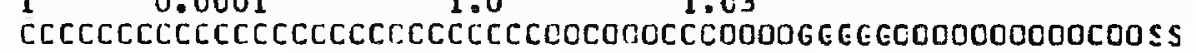

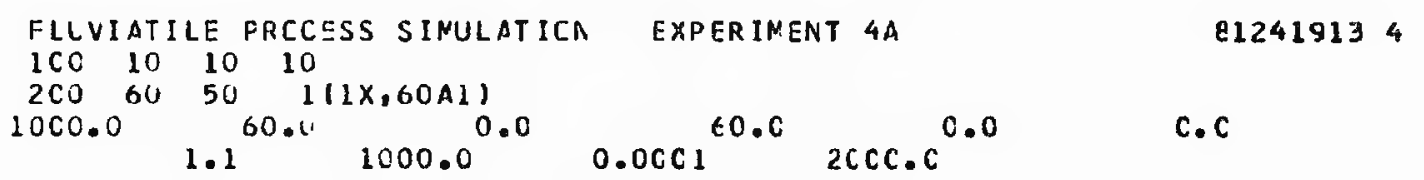

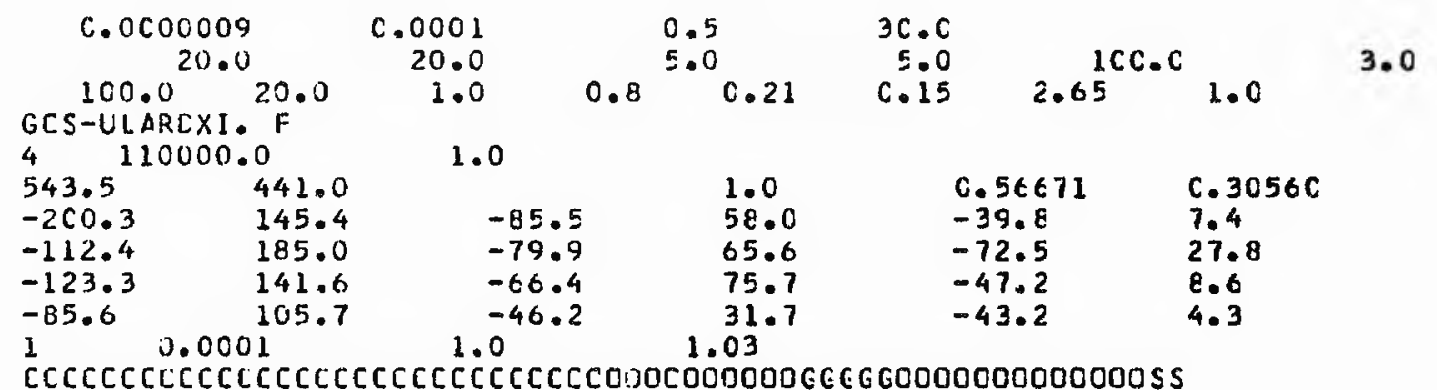



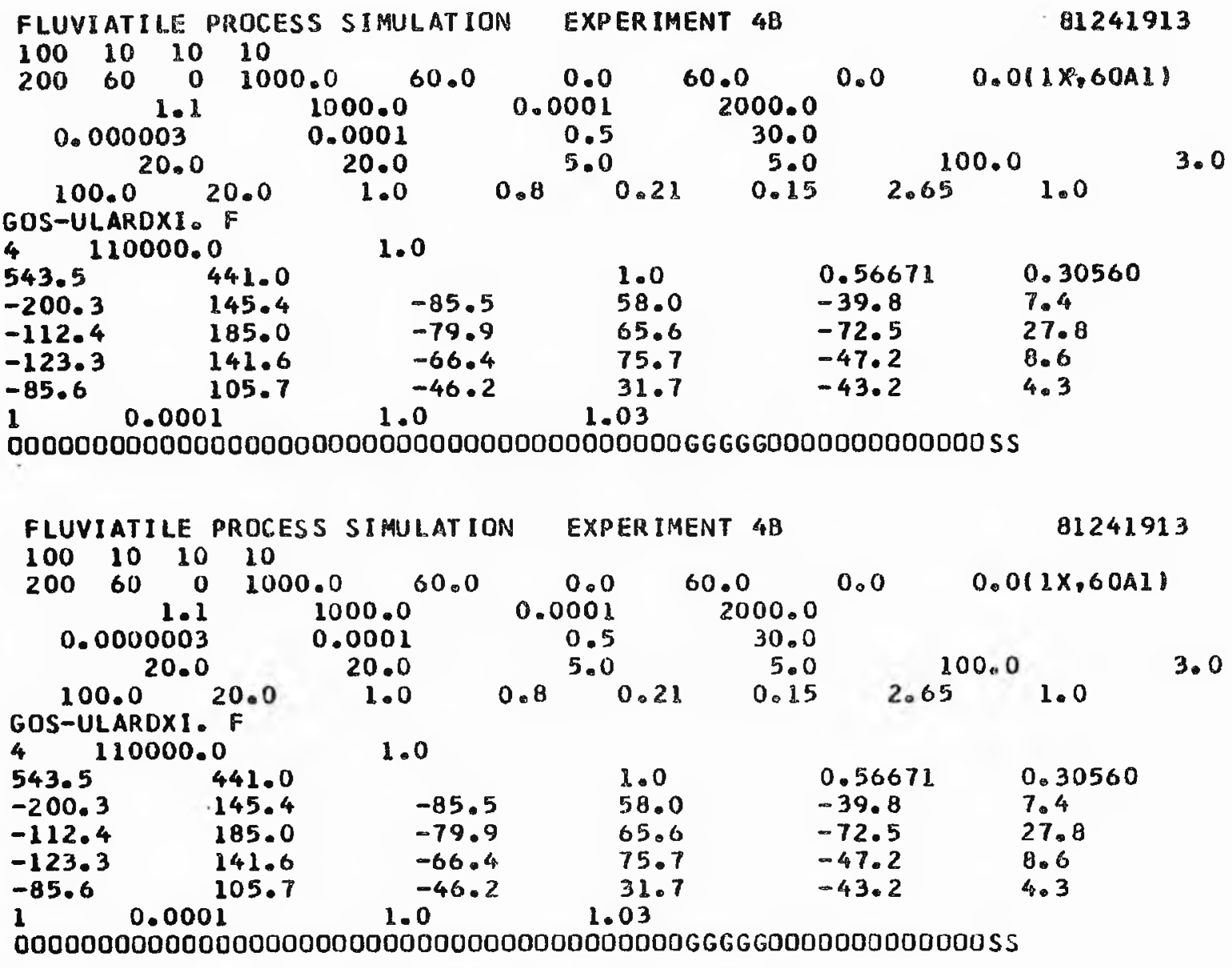

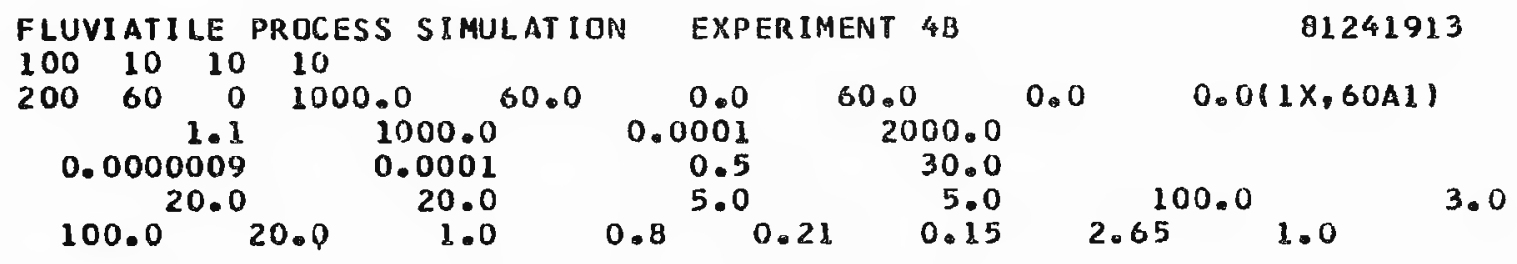

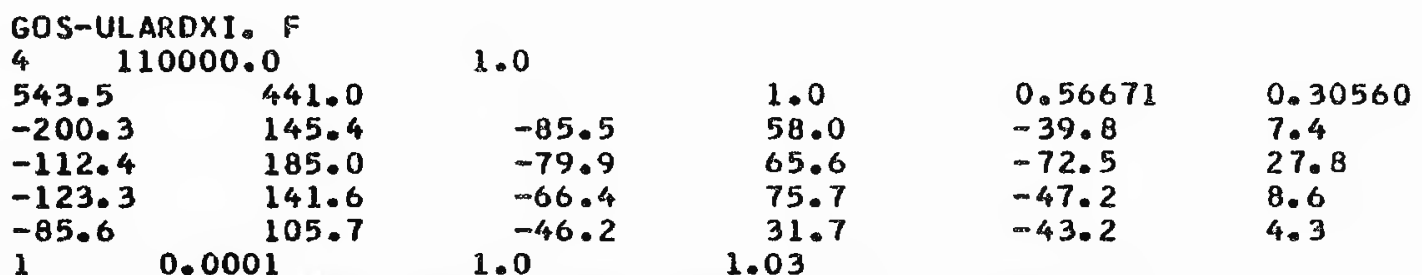

0000000000000000000000000000000000000000GGGGG0000000000000SS 


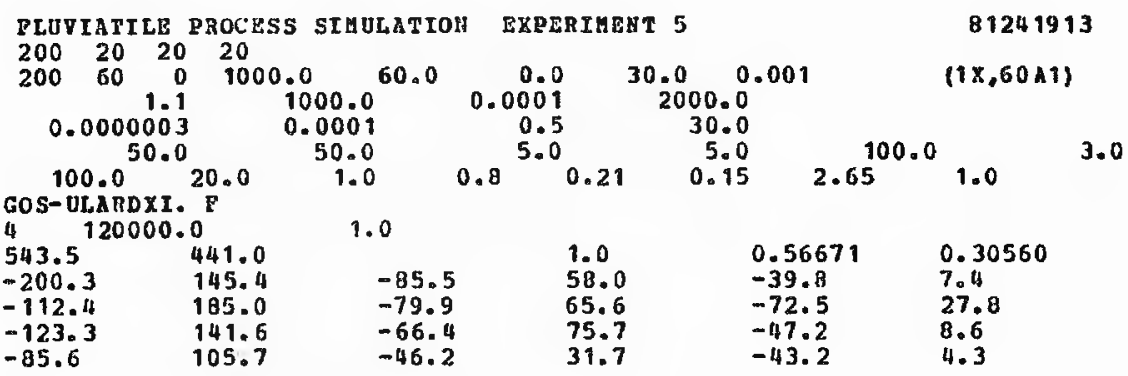

000000000000000000000000000055

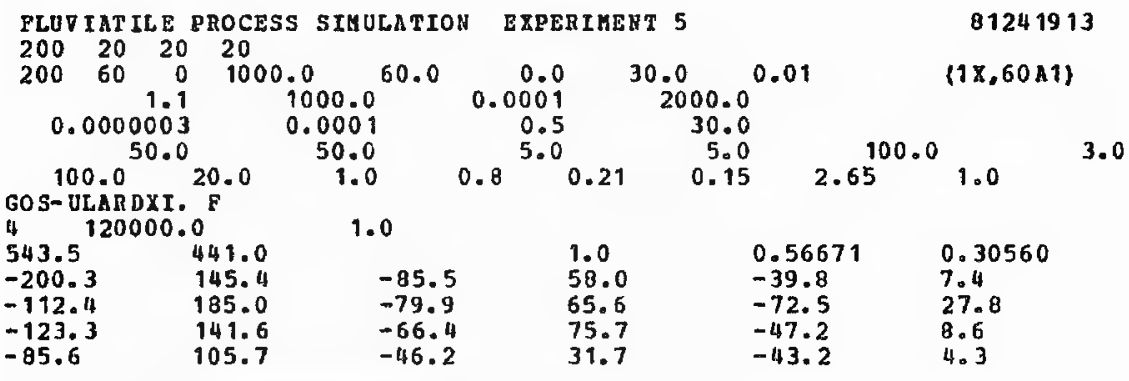

000000000000000000000000000055

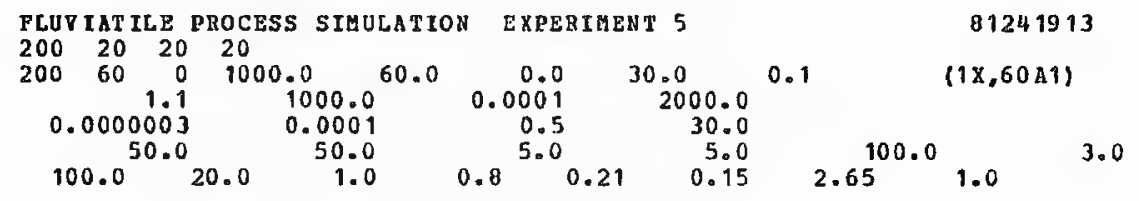

\begin{tabular}{|c|c|c|c|c|c|}
\hline $\begin{array}{c}60 S-0 L A \\
4\end{array}$ & $0^{F}$ & 1.0 & & & \\
\hline $\begin{array}{l}543.5 \\
-200.3 \\
-912.4 \\
-123.3 \\
-85.6\end{array}$ & $\begin{array}{l}141.0 \\
145.4 \\
185.0 \\
141.6 \\
105.7\end{array}$ & $\begin{array}{l}-85.5 \\
-79.9 \\
-66.11 \\
-46.2\end{array}$ & $\begin{array}{l}1.0 \\
58.0 \\
65.6 \\
75.7 \\
39.7\end{array}$ & $\begin{array}{l}0.56671 \\
-39.8 \\
-72.5 \\
-47.2 \\
-43.2\end{array}$ & $\begin{array}{l}0.30560 \\
7.4 \\
27.8 \\
8.6 \\
4.3\end{array}$ \\
\hline
\end{tabular}

0000000000000000000000000000 ss

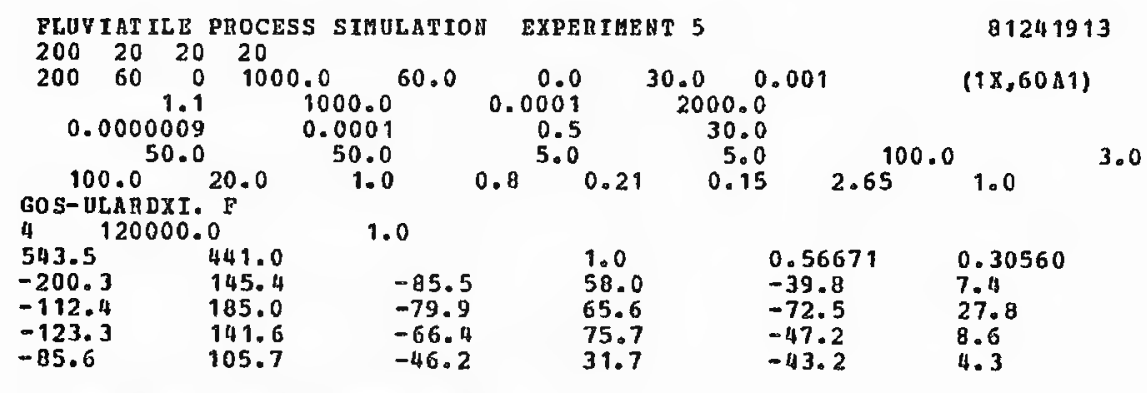

0000000000000000000000000000ss

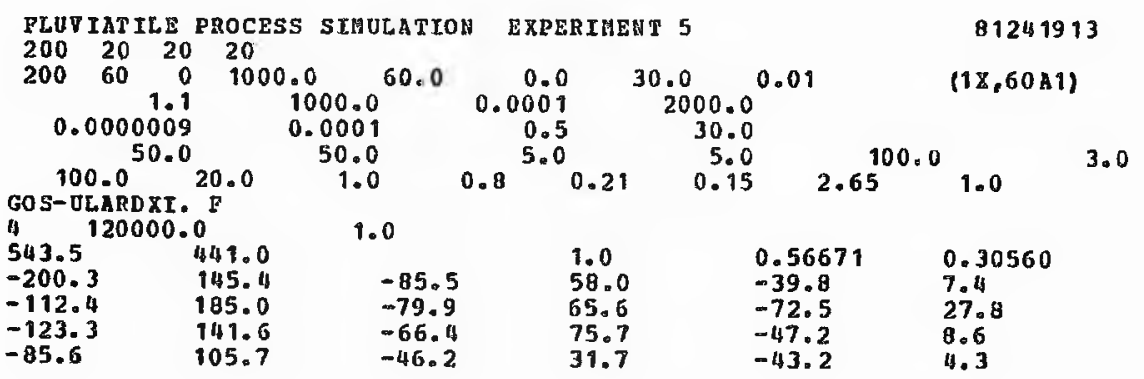




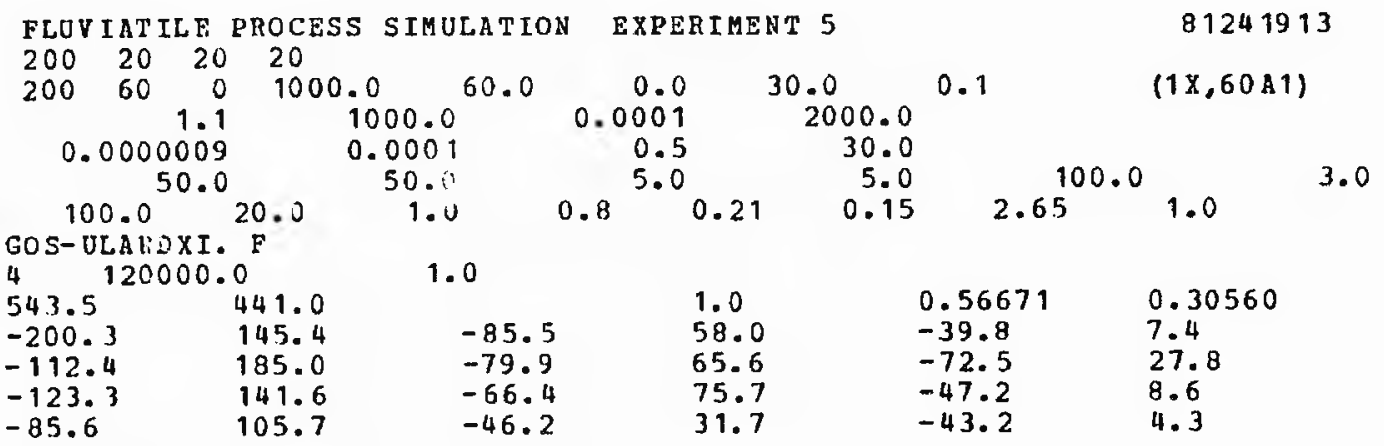

000000000000000000000000 ccooss

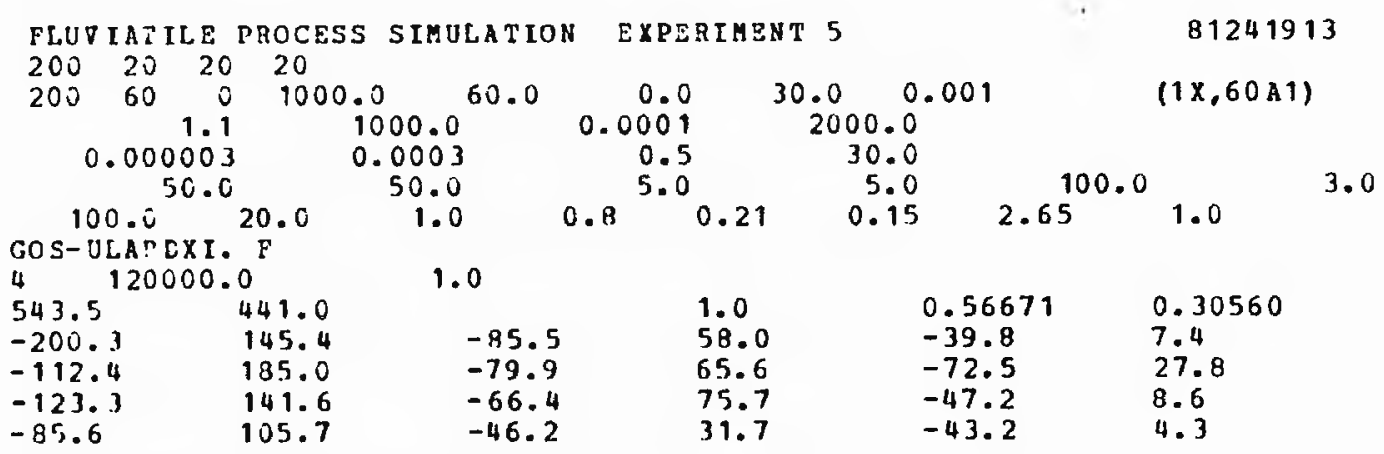

00000000000000000000 c000c00ss

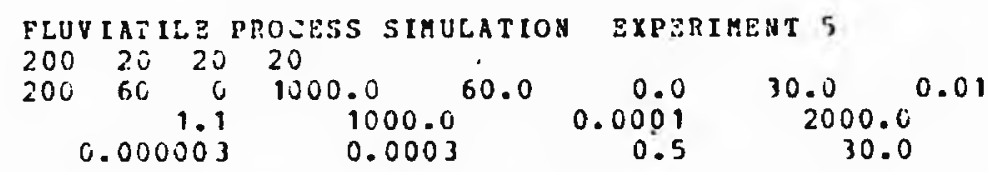

(18.60A1)

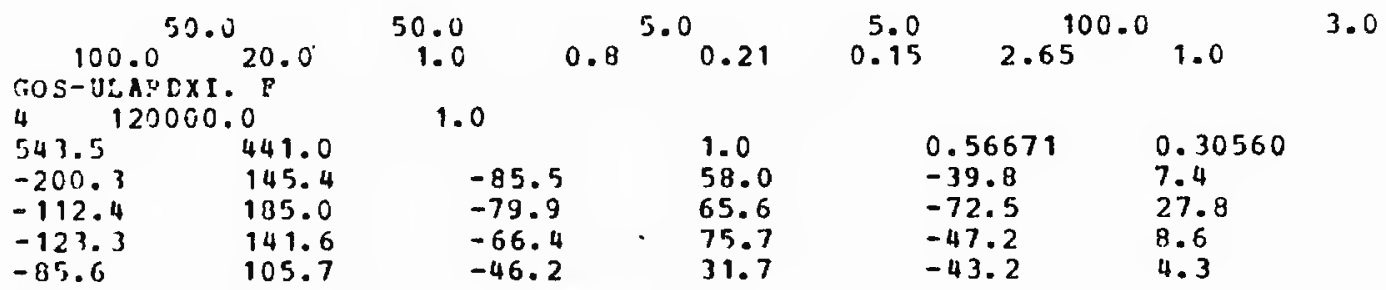

$000000000000000000000000 \mathrm{cccoss}$

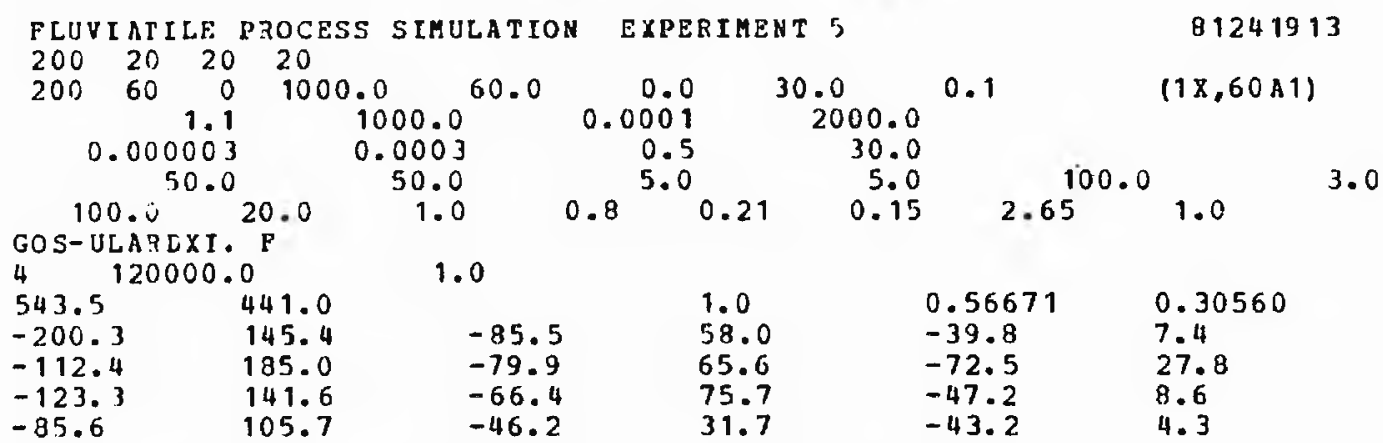

0000000000000000000000000000 SS 